\title{
HYDROLOGIC AND SEDIMENTOLOGIC DATA COLLECTED \\ DURING FOUR CRUISES AT HIGH WATER ON THE \\ MISSISSIPPI RIVER AND SOME OF ITS TRIBUTARIES, \\ MARCH 1989-JUNE 1990 \\ by John A. Moody and Robert H. Meade
}

U.S. GEOLOGICAL SURVEY

Open-File Report $92-651$

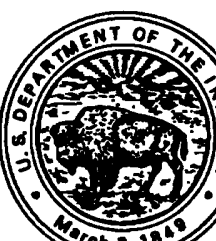




\section{U.S. DEPARTMENT OF THE INTERIOR}

BRUCE BABBITT, Secretary

U.S. Geological Survey

Dallas L. Peck, Director

The use of brand, trade, or firm names in this report is for identification purposes only and does not constitute endorsement by the U.S. Geological Survey.

For additional information write to:

Chief, Branch of Regional Research U.S. Geological Survey Box 25046, Mail Stop 418 Federal Center Denver, CO 80225-0046
Copies of this report can be purchased from:

U.S. Geological Survey Books and Open-File Reports Section Box 25425, Mail Stop 517

Federal Center

Denver, CO 80225-0425 
Page

Abstract-1

Introduction-1

Objectives of the study- 3

Purpose and scope-1 4

Acknowledgments-- 8

Sampling procedures and results- 12

Bed sediments-- 13

Water discharge-- 20

Water and suspended sediment-1

Pumped composite sample-15

Depth-integrated composite sample-- 26

Suspended-sediment analysis-_- 38

Concentration- 38

Particle size-1.

Surface-water properties- 52

References cited-1

Tabulated cruise data-1

Data listings for:

March-April 1989 cruise-- 61

June 1989 cruise-_. 105

February-March 1990 cruise-2

May-June 1990 cruise- 179

\section{FIGURES}

Figure 1. Map showing Mississippi River drainage basin and eight subbasins--.-.....-. 2

2. Map showing location of sampling sites on the Mississippi River and some of its tributaries-a 5

3. Map showing location of sampling sites upstream and downstream from the confluence of the Mississippi and Ohio. Rivers, May-June 1990 cruise-4-6. Graphs showing water discharge:

4. At two locations on the Upper Mississippi River and for three tributaries from February 1989 through July 1990-

5. At three locations on the Lower Mississippi River from February 1989 through July 1990-_... 10

6. At locations on three secondary tributaries of the Mississippi River (Wabash, Cumberland, and Tennessee Rivers) and two tributaries of the Lower Mississippi River from February 1989 through July 1990

7. Drawing showing suspended-sediment sampler with current meter

8. Flow diagram for processing the suspended-sediment sample collected by depth-integration method-_e 28

9. Graph showing the specific conductance of surface and depth-integrated water samples-_- 
Figures 10-26. Graphs showing depth, mean velocity, and specific

conductance of surface water versus the distance from the left edge of water or left edge of flow for the March-April 1989 cruise:

10. Mississippi River near Winfield, Mo._... 63

11. Illinois River at Hardin, Ill. 65

12. Missouri River at Hermann, Mo... 67

13. Mississippi River at St. Louis, Mo._. 69

14. Mississippi River at Thebes, I11._. 71

15. Ohio River at Olmsted, Ill. 73

16. Mississippi River below Hickman, Ky...... 76

17. Mississippi River below Fulton, Tenn. 79

18. Mississippi River at Helena, Ark._. 81

19. White River at Mile 11.5, Ark._8 83

20. Arkansas River at Pendleton, Ark._. 85

21. Mississippi River above Arkansas City, Ark.---.-- 88

22. Yazoo River below Steele Bayou, Miss._....... 91

23. Mississippi River below Vicksburg, Miss.-....... 94

24. Old River Outflow Channel near Knox Landing, La.--- 97

25. Mississippi River near St. Francisville, La.-...-- 100

26. Mississippi River below Belle Chasse, La....... 103

27-44. Graphs showing depth, mean velocity, and specific

conductance of surface water or depth and

depth-averaged suspended-sediment concentration

versus the distance from the left edge of water

for the June 1989 cruise:

27. Mississippi River near Winfield, Mo.-.... 107

28. Missouri River at Hermann, Mo._- 111

29. Mississippi River at St. Louis, Mo., June 8-...- 113

30. Mississippi River at St. Louis, Mo., June 9-....- 115

31. Mississippi River at St. Louis, Mo., June 9,

suspended-sediment concentration-_._. 116

32. Mississippi River at Thebes, I11._. 119

33. Ohio River at 0lmsted, I11. 121

34. Mississippi River below Hickman, Ky., June 12-...- 123

35. Mississippi River below Hickman, Ky., June 13-...- 125

36. Mississippi River below Hickman, Ky., June, 13, suspended-sediment concentration-_. 126

37. Mississippi River at Fulton, Tenn.- 129

38. Mississippi River at Helena, Ark. 131

39. Arkansas River at Pendleton, Ark._- 135

40. Mississippi River above Arkansas City, Ark.--..-- 137

41. Mississippi River below Vicksburg, Miss.-...... 141

42. Old River Outflow Channel near Knox Landing, La.-- 143

43. Mississippi River near St. Francisville, La.-..-- 145

44. Mississippi River below Belle Chasse, La...... 147 


\section{FIGURES (Cont.)}

Figures 45-57. Graphs showing depth, mean velocity, and specific con-

Page ductance of surface water versus the distance from the left edge of water or left edge of flow for the

February-March 1990 cruise:

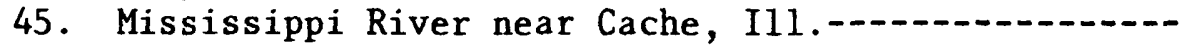

46. Ohio River at Uniontown, Ky.-...

47. Wabash River near New Haven, Ill.

48. Cumberland River near Smithland, Ky.

49. Tennessee River near Calvert City, Ky.---.-

50. Ohio River at Olmsted, I11.

51. Mississippi River below Hickman, Ky.......... 163

52. Mississippi River below Fulton, Tenn.-........ 165

53. Mississippi River at Helena, Ark._..... 168

54. Mississippi River above Arkansas City, Ark.-.-.-- 171

55. Mississippi River below Vicksburg, Miss........ 173

56. Mississippi River near St. Francisville, La...... 175

57. Mississippi River below Belle Chasse, La.--...-- 177

58-75. Graphs showing depth, mean velocity, and specific conductance of surface water or depth and depthaveraged suspended-sediment concentration versus the distance from the left edge of water for the first (mixing) leg of the May-June 1990 cruise:

58. Mississippi River near Cairo, I11._..... 181

59. Mississippi River near Cairo, Ill., suspendedsediment concentration-_.-. 182

60. Ohio River at Olmsted, I11... 184

61. Ohio River at Olmsted, Ill., suspended-sediment concentration-185

62. Mississippi River at Wickliffe, Ky., May 30-_-_.-. 187

63. Mississippi River at Wickliffe, Ky., May 30 , suspended-sediment concentration------------ 188

64. Mississippi River at Wickliffe, Ky., June 1-.-.-.- 190

65. Mississippi River at Wickliffe, Ky., June 1, suspended-sediment concentration--.-.-.- 191

66. Mississippi River at Wickliffe, Ky., June 4-_-_-- 193

67. Mississippi River at Wickliffe, Ky., June 4, suspended-sediment concentration-_-n 194

68. Mississippi River near Columbus, Ky.-- 196

69. Mississippi River near Columbus, Ky., suspendedsediment concentration--.-- 197

70. Mississippi River above New Madrid, Mo._-_-_-_-- 199

71. Mississippi River above New Madrid, Mo., suspendedsediment concentration-- 200

72. Mississippi River near Point Pleasant, Mo.-.-.--- 202

73. Mississippi River near Point Pleasant, Mo., suspended-sediment concentration-_-_.-.- 203

74. Mississippi River at Caruthersville, Mo.-....- 205

75. Mississippi River at Caruthersville, Mo., suspended-sediment concentration---.-- 
Figures 76-85. Graphs showing depth, mean velocity, and specific conductance of surface water versus the distance from the left edge of water or left edge of flow for the second (NASQAN) leg of the May-June 1990 cruise:

76. Illinois River at Valley City, Ill._. 209

77. Mississippi River below Grafton, Ill......... 211

78. Mississippi River at Thebes, Ill._.-...- 213

79. Ohio River at Olmsted, Ill. 215

80. Mississippi River below Memphis, Tenn.--_.-. 217

81. Mississippi River below Arkansas City, Ark.---.-- 219

82. Yazoo River below Steele Bayou, Miss.-... 221

83. Mississippi River below Vicksburg, Miss.-.... 223

84. Mississippi River near St. Francisville, La.-.-.- 225

85. Mississippi River below Belle Chasse, La.-...-.-- 227

\section{TABLES}

Table 1. Drainage area and mean annual discharges of water and sediment for eight subbasins in the Mississippi River basin-...-

2. Sampling sites for March-April 1989 cruise, June 1989
cruise, February-March 1990 cruise, and May-June 1990 cruise--

3-6. Particle size, determined by sieving, of bed material collected from the Mississippi River and some of its tributaries for:

3. March-April 1989 cruise-a 14

4. June 1989 cruise- 16

5. February-March 1990 cruise-- 18

6. Second leg of the May-June 1990 cruise-_- 19

7-10. Summary of discharge measurements made of the Mississippi River and some of its tributaries using the depth-integration method for:

7. March-April 1989 cruise- 22

8. June 1989 cruise-- 23

9. February-March 1990 cruise-_. 24

10. May-June 1990 cruise-14

11. Volumes of river water collected and processed and associated sampling parameters for March-April 1989 cruise--.- 30

12. Water volumes processed and masses of silt/clay and colloid fractions recovered from samples of the Mississippi River and some of its tributaries for March-April 1989 cruise-n

13. Volumes of river water collected and processed and associated sampling parameters for June 1989 cruise--.-- 32

14. Water volumes processed and masses of silt/clay and colloid fractions recovered from samples of the Mississippi River and some of its tributaries for June 1989 cruise- 
15. Volumes of river water collected and processed and associated sampling parameters for February-March 1990 cruise--- 34

16. Water volumes processed and masses of silt/clay and colloid fractions recovered from samples of the Mississippi River and some of its tributaries for February-March 1990 cruise-n

17. Volumes of river water collected and processed and associated sampling parameters for second leg of the May-June 1990 cruise-1 36

18. Water volumes processed and masses of silt/clay and colloid fractions recovered from samples of the Mississippi River and some of its tributaries for second leg of the May-June 1990 cruise-

19-22. Suspended-sediment concentrations in depth-integrated,
composite samples collected from the Mississippi River

19-22. Suspended-sediment concentrations in depth-integrated,
composite samples collected from the Mississippi River and some of its tributaries and the corresponding sediment discharges for:

19. March-April 1989 cruise- 39

20. June 1989 cruise- 40

21. February-March 1990 cruise-_. 41

22. Second leg of the May-June 1990 cruise-_ 42

23. Average difference in suspended-sediment concentration in duplicate depth-integrated, composite samples--.-.article-size distribution of suspended-sediment samples collected from the Mississippi River and some of its tributaries for:

24. March-April 1989 cruise- 46

25. June 1989 cruise-_. 47

26. February-March 1990 cruise-_-_ 48

27. Second leg of the May-June 1990 cruise-- 49

28. Suspended-sediment concentration, by particle-size class, obtained by different collecting methods and concentrated by different processing procedures-a 50

29-31. Specific conductance of surface water at locations across the:

29. Lower Mississippi River for first leg of the May-June 1990 cruise- 54

30. Lower Mississippi River for second leg of the May-June 1990 cruise- 55

31. Upper Mississippi River for second leg of the May-June 1990 cruise- 56 
CONVERSION FACTORS

Multiply

micrometer $(\mu \mathrm{m})$

millimeter $(\mathrm{mm})$

centimeter $(\mathrm{cm})$

meter $(\mathrm{m})$

kilometer $(\mathrm{km})$

square meter $\left(\mathrm{m}^{2}\right)$

square kilometer $\left(\mathrm{km}^{2}\right)$

milliliter (mL)

liter (L)

cubic meter $\left(\mathrm{m}^{3}\right)$

centimeter per second $(\mathrm{cm} / \mathrm{s})$

meter per second $(\mathrm{m} / \mathrm{s})$

kilometer per hour $(\mathrm{km} / \mathrm{h})$

cubic meter per second $\left(\mathrm{m}^{3} / \mathrm{s}\right)$

cubic meter per year $\left(\mathrm{m}^{3} / \mathrm{yr}\right)$

liter per minute (L/min)

metric ton per year

(metric ton/yr)

milligram (mg)

gram (g)

metric ton

degree Celsius $\left({ }^{\circ} \mathrm{C}\right)$
$B y$

To obtain

\section{Length}

0.00003937

0.03937

0.3937

3.281

0.6214

\section{Area}

10.76

0.3861

\section{Volume}

0.03382

0.2642

35.31

Velocity

0.03281

3.281

0.6214

Discharge

\subsection{1}

35.31

0.2642

1.102

\section{Mass}

0.00003527

0.002205

2,205

Temperature

${ }^{\circ} \mathrm{F}=1.8 \times{ }^{\circ} \mathrm{C}+32$ inch

inch

inch

foot

mile

square foot

square mile

ounces, fluid

gallon

cubic foot

foot per second

foot per second

mile per hour

cubic foot per second cubic foot per year gallon per minute ton per year

ounce, avoirdupois pound, avoirdupois pound, avoirdupois

degree Fahrenheit 


\section{HYDROLOGIC AND SEDIMENTOLOGIC DATA COLLECTED DURING FOUR CRUISES AT HIGH WATER ON THE MISSISSIPPI RIVER AND SOME OF ITS TRIBUTARIES, MARCH 1989-JUNE 1990}

By John A. Moody and Robert H. Meade

\section{ABSTRACT}

Bed-sediment, water, and suspended-sediment samples were collected from 33 sites on the Mississippi River and some of its tributaries. Four cruises were made at high water during a 16-month period from March 9, 1989, to June 27, 1990. The maximum measured water discharge was about 34,100 cubic meters per second on March 10, 1990, in the Mississippi River at Vicksburg, Mississippi, and the maximum measured suspended-sediment discharge was 1,330,000 metric tons per day in the Mississippi River at Thebes, Illinois, on June 13, 1990. Depth-integration and pumping methods were used at equally spaced locations across the river to collect two composite samples of river water containing suspended sediment. The depth-integrated composite samples were approximately 100 liters and the pumped composite samples were approximately 500 liters. Both sampling methods used Teflon or Teflon-coated parts to prevent chemical contamination.

This report contains the following data associated with the samples: cross-sectional area of the river, water depths, depth-averaged velocities, water discharge, surface temperature and specific conductance, concentrations of the suspended sand and silt/clay and colloid fractions, and particle sizes of bed material and suspended sediment. These data provide the framework for interpreting subsequent chemical analyses of the water and suspended-sediment samples collected during the four cruises and for calculating bed-load transport in the Mississippi River and some of its tributaries.

\section{INTRODUCTION}

The Mississippi River drains about 40 percent of the conterminous United States and commonly is divided into two parts--the Lower Mississippi River and the Upper Mississippi River. Distances on the Lower Mississippi River begin at zero where the mouth of the river divides into three separate channels at Head of Passes in Louisiana and increase upstream to the mouth of the Ohio River at Cairo, Ill. Distances on the Upper Mississippi River begin at zero at the mouth of the Ohio River (Lower Mississippi River mile 953.8) and increase upstream to the source of the Mississippi River in Minnesota.

At Vicksburg, Miss., the river has a mean annual water discharge of about $500 \times 10^{9} \mathrm{~m}^{3} / \mathrm{yr}$ and a mean annual sediment discharge of about $200 \times 10^{6}$ metric tons/yr. At 191 kilometers downstream from Vicksburg, approximately 
25 percent of the water discharge and sediment discharge is diverted from the Mississippi River by the Old River Control Structures into the Atchafalaya River and then into the Gulf of Mexico. The remaining water $\left(375 \times 10^{9} \mathrm{~m}^{3} / \mathrm{yr}\right)$ and sediment $\left(150 \times 10^{6}\right.$ metric tons/yr) are discharged by the Mississippi River directly into the Gulf of Mexico (Moody and Meade, 1992).

The U.S. Geological Survey (USGS) began a study of the sedimenttransported pollutants in the Mississippi River and some of its tributaries in 1987 and made three research cruises at low water in July-August 1987, in November-December 1987, and during the record low water in May-June 1988 (Moody and Meade, 1992). These cruises started about $50 \mathrm{~km}$ upstream from the confluences of the Upper Mississippi-Missouri-Illinois Rivers near St. Louis, Mo., and ended about $40 \mathrm{~km}$ downstream from New Orleans, La. On the basis of distinctive differences in water chemistries, the Mississippi River basin was divided into eight subbasins (fig. 1). The corresponding drainage areas and the mean annual discharges of water and sediment for the eight subbasins are listed in table 1.

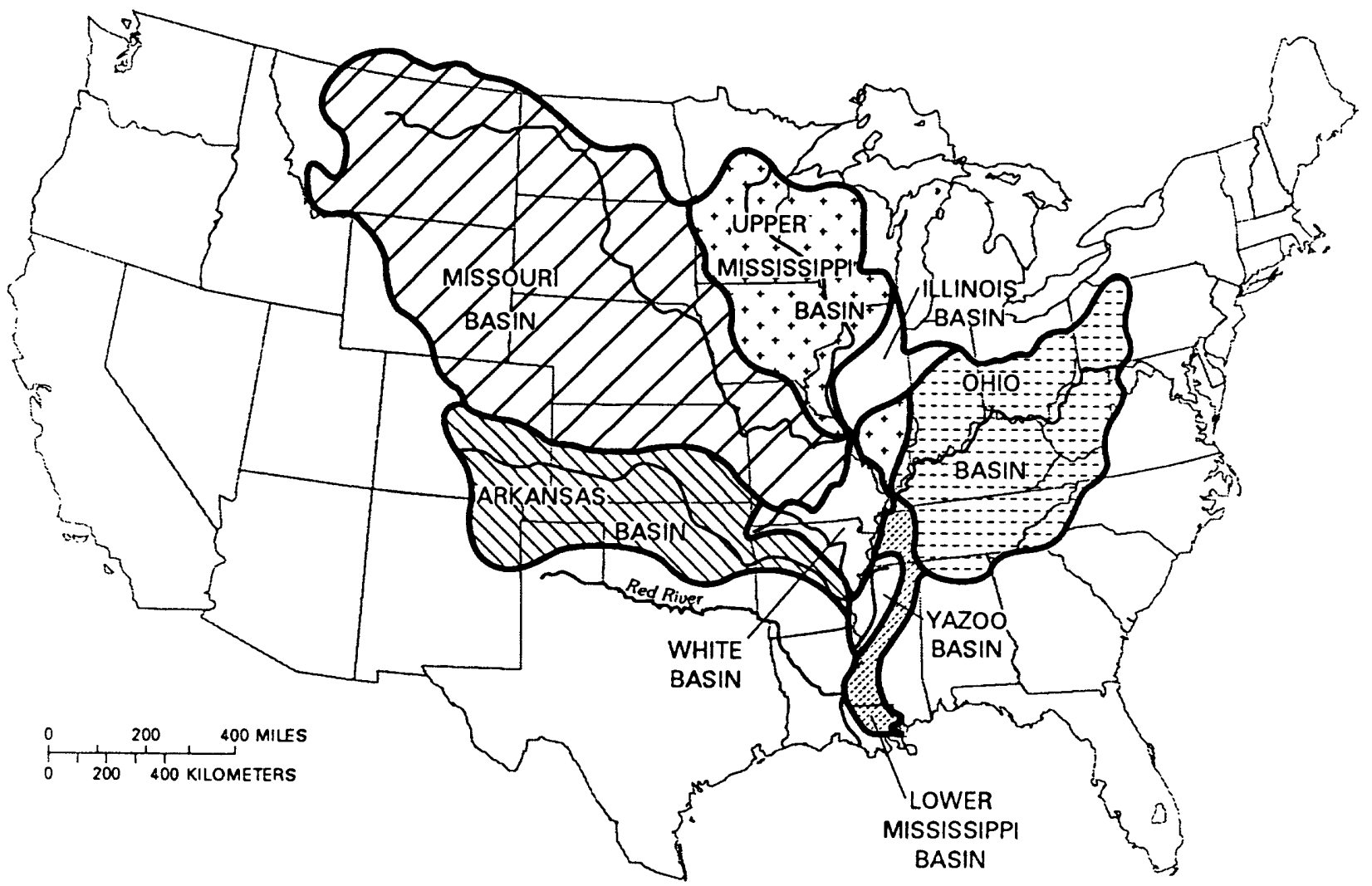

Figure 1.--Mississippi River drainage basin and eight subbasins. The Red River basin was not included because it is no longer a tributary to the Mississippi River. (Modified from Bragg, 1977.) 
Table 1.--Drainage areas and mean annual discharges of water and sediment for eight subbasins in the Mississippi River basin

[The basin areas and sediment discharges are taken from Keown and others, 1981, except where noted. Values of the water discharges were taken from the U.S. Geological Survey water-resources data reports for the appropriate States, except where noted; $\mathrm{km}^{2}$, square $\mathrm{kilometer;} \mathrm{m}^{3} / \mathrm{yr}, \mathrm{cubic}$ meter per year; /yr, per year]

\begin{tabular}{|c|c|c|c|c|c|c|c|}
\hline \multirow{3}{*}{ Basin } & \multirow{3}{*}{$\begin{array}{l}\text { Length } \\
\text { of record } \\
\text { (years) }\end{array}$} & \multirow{2}{*}{\multicolumn{2}{|c|}{$\begin{array}{c}\text { Drainage } \\
\text { area }\end{array}$}} & \multicolumn{4}{|c|}{ Mean annual discharge } \\
\hline & & & & \multicolumn{2}{|c|}{ Water } & \multicolumn{2}{|c|}{ Sediment } \\
\hline & & $\left(\mathrm{km}^{2}\right)$ & (percent) & $\left(10^{9} \mathrm{~m}^{3} / \mathrm{yr}\right)$ & (percent) & $\begin{array}{c}\left(10^{6} \text { metric }\right. \\
\text { tons } / y r)\end{array}$ & (percent) \\
\hline $\begin{array}{l}\text { Upper Mississippi River } \\
\text { less Illinois River and } \\
\text { Missouri River }\end{array}$ & 58 & 407,660 & 14 & 173 & 15 & 16.0 & 8 \\
\hline Illinois River & 48 & 80,810 & 3 & $2_{20}$ & 4 & 6.7 & 3 \\
\hline Missouri River & 88 & ${ }^{3} 1,357,670$ & 45 & 72 & 15 & 78.5 & 40 \\
\hline Ohio River & 58 & 528,310 & 18 & 243 & 50 & 472.6 & 37 \\
\hline White River & 2 & 566,190 & 2 & 19 & 4 & 3.4 & 2 \\
\hline Arkansas River & 60 & 416,600 & 14 & 37 & 8 & 10.3 & 5 \\
\hline $\begin{array}{l}\text { Lower Mississippi River } \\
\text { less the Yazoo River } 6\end{array}$ & $17-50$ & 80,530 & 3 & 11 & 2 & 6.7 & 3 \\
\hline Yazoo River & 20 & 35,840 & 1 & ${ }^{7} 12$ & 2 & 3.8 & 2 \\
\hline TOTAL & & $2,973,610$ & & 487 & & 198.0 & \\
\hline
\end{tabular}

${ }^{1}$ Discharge for Mississippi River at Alton, Illinois, minus discharge for the Illinois River.

${ }^{2}$ Discharge for the Illinois River at Meredosia, Illinois.

${ }^{3}$ Drainage area taken from the Missouri Water-Resources Data for water year 1986.

Sediment discharge was estimated by Keown and others (1981) by differences.

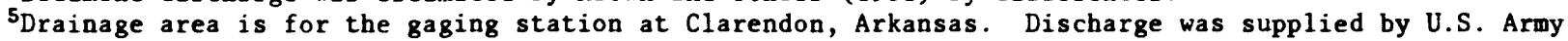
Corps of Engineers, Memphis, Tennessee (oral commun., 1988).

${ }^{6}$ Seven tributaries: St. Francis, Obion, Hatchie, Wolf, Big Black, Homochitto, and Buffalo Rivers.

${ }^{7}$ Combined discharges (1967-87) for the Yazoo River at Greenwood, Mississippi, and the Big Sunflower River at Sunflower, Mississippi (Henry Noble, U.S. Army Corps of Engineers, Vicksburg, Mississippi, oral commun., 1988).

\section{Objectives of the Study}

The broad objectives of this multidisciplinary research study are to investigate the movement, mixing, and storage processes of sediment-associated and dissolved pollutants in the Mississippi River system. Some specific objectives are to:

1. Understand the compartmentalization of organic and inorganic agrochemicals among the water, sediment, and biotic phases;

2. Investigate the mixing, partitioning, and redistribution processes of the various pollutants downstream from major river confluences;

3. Understand the movement, storage, and remobilization of suspended sediment and associated pollutants at seasonal or longer time periods;

4. Predict the location and travel time of water masses and the associated sediment and pollutants; and

5. Examine the geochemistry of the suspended silt, clay, colloidal material, and dissolved phases of river water. 


\section{Purpose and Scope}

The purposes of this report are (1) to provide a description of the sampling sites on the Mississippi River and some of its tributaries, (2) to describe the sampling procedures used to collect and process bed-sediment samples and a representative water and suspended-sediment sample for physical and chemical analysis, and (3) to publish the hydrologic and sedimentologic data collected during four cruises. The hydrologic data a re cross-sectional area of the river, water depths, depth-averaged velocities, and water discharge. The sedimentologic data are concentrations of suspended sand (greater than $63 \mu \mathrm{m}$ ), concentrations of silt/clay and colloidal material (finer than $63 \mu \mathrm{m}$ ), and particle-size distributions of the bed material and suspended sediment. Surface temperature and specific conductance are listed in this report, but inorganic and organic chemical data are published elsewhere. The hydrologic data that are published in this report provide some of the necessary framework for interpreting the chemical data.

The 17-m research vessel ACADIANA, owned and operated by the Louisiana Universities Marine Consortium, was used for collecting the samples because it has a shallow draft (about $1.2 \mathrm{~m}$ ), which permitted samples to be collected close to the river banks. The researchers, during each cruise, used a Lagrangian scheme that collected an initial sample from the Mississippi River near St. Louis, Mo., followed the water, and collected samples at additional sampling sites downriver. A 2-dimensional sampling scheme (different locations across the river) was used at each sampling site to collect individual water samples and to measure water surface properties. The individual water samples were combined to make a single composite sample for each sampling site so that the data consists of a primary data set that is 1-dimensional and a secondary data set that is 2-dimensional. This Lagrangian sampling scheme was limited by the logistical constraints of using a single vessel to sample the tributaries and the Mississippi River and by the time required to process samples (Moody, 1993). This report includes data from four cruises that collected samples from sites on the Mississippi River starting about $100 \mathrm{~km}$ upstream from St. Louis, Mo., and ending about $40 \mathrm{~km}$ downstream from New Orleans, La. (fig. 2). Samples also were collected from sites within $230 \mathrm{~km}$ of the mouths of the following tributaries: Illinois, Missouri, Ohio, Wabash, Cumberland, Tennessee, White, Arkansas, and Yazoo Rivers. The sampling-site locations for each cruise are listed in table 2 and shown in figures 2 and 3 .

The March-April 1989 cruise (March 9 through April 1, 1989) and the June 1989 cruise (June 4 through June 28, 1989) were planned to sample the Mississippi River when the water level and corresponding water discharge were high. The February-March 1990 cruise (February 23 through March 14, 1990) was designed to follow the high water created by the spring runoff of the Ohio River (starting at the confluence of the Wabash and Ohio Rivers) down the Ohio and Lower Mississippi Rivers. The May-June 1990 cruise had two purposes. The purpose of the first leg (May 30 through June 6, 1990) was to conduct a 2-dimensional study of the mixing processes downstream from the confluence of the Mississippi and Ohio Rivers during a period of high water (see fig. 3), and the purpose of the second leg (June 7 through June 27, 1990) was a joint project with NASQAN (National Stream Quality Accounting Network) to compare collection, processing, and analytical techniques for trace-element analysis of water collected from the Mississippi River and some of its tributaries. 


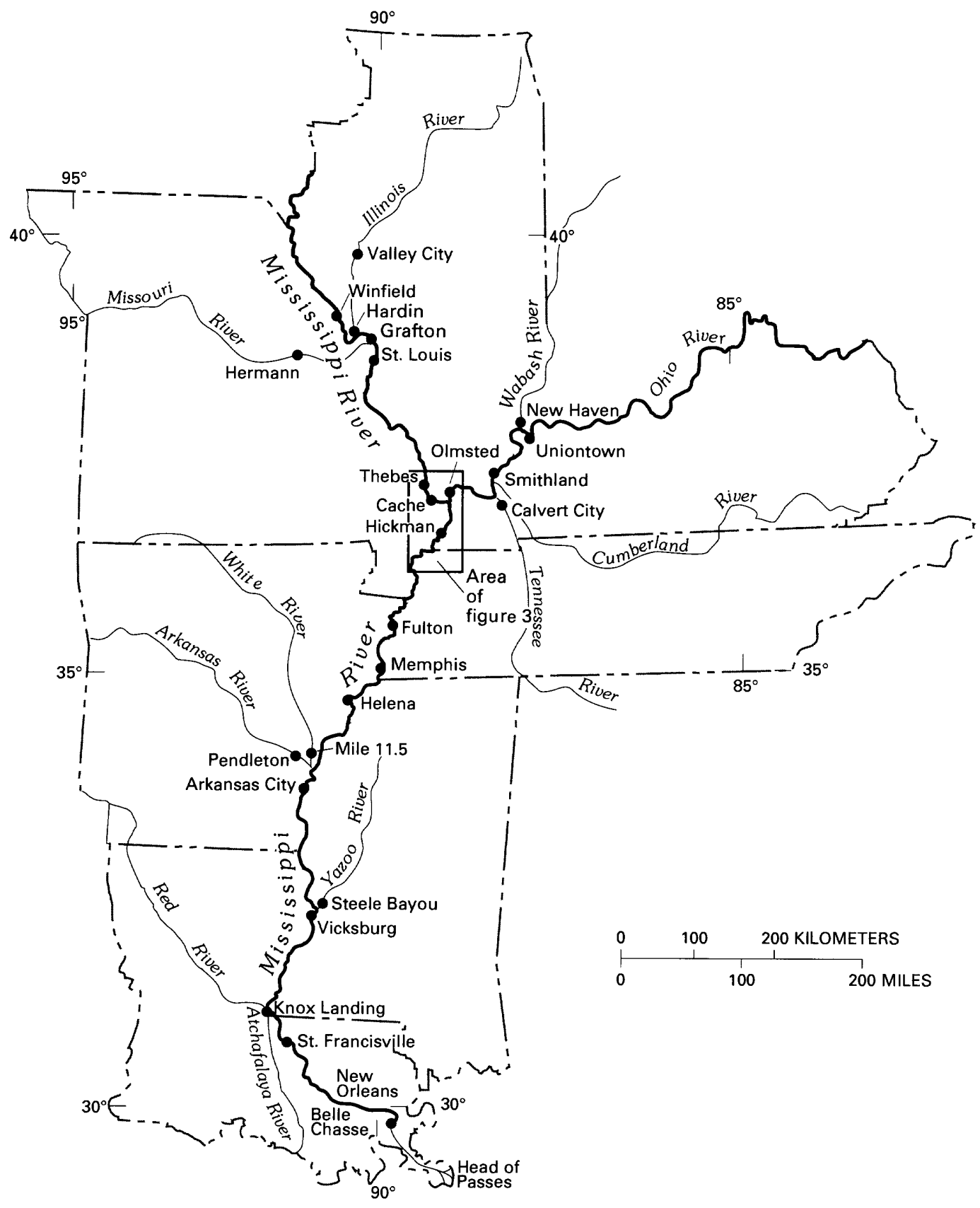

GULF OF MEXICO

Figure 2.--Location of sampling sites on the Mississippi River and some of its tributaries. Sampling sites are shown as solid circles. Sampling sites within the rectangular area that includes the Mississippi-Ohio confluence are shown in figure 3. 


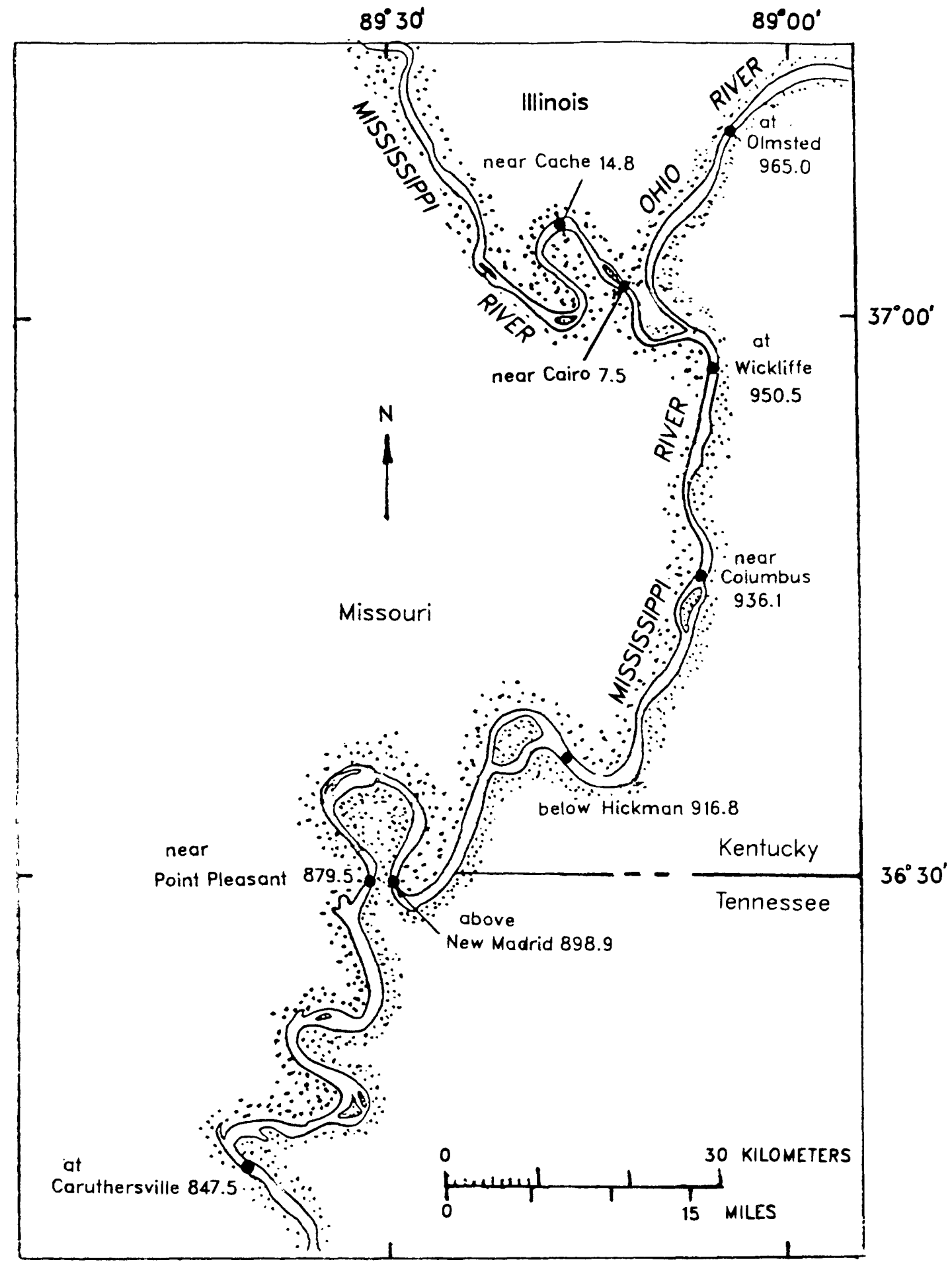

Figure 3.--Location of sampling sites near the confluence of the Mississippi and Ohio Rivers, May-June 1990 cruise. The number after the site name is the river mile [modified from 1989 Flood Control and Navigation Maps of the Mississippi River, U.S. Army Corps of Engineers River Commission (1989a?)]. 
Table 2.--Sampling sites for March-April 1989 cruise, June 1989 cruise, February-March 1990 cruise, and May-June 1990 cruise

[River miles taken from navigation charts, U.S. Army Corps of Engineers, 1983?, 1986?, $1987 \mathrm{a}, 1987 \mathrm{~b}, 1989 \mathrm{a}$ ?, 1989b?; the X designates that the site was sampled]

\begin{tabular}{|c|c|c|c|c|c|}
\hline \multirow[b]{2}{*}{ Site name } & \multirow[b]{2}{*}{ River mile ${ }^{1}$} & \multicolumn{4}{|c|}{ Cruise } \\
\hline & & $\begin{array}{l}\text { March- } \\
\text { April } \\
1989\end{array}$ & $\begin{array}{l}\text { June } \\
1989\end{array}$ & $\begin{array}{l}\text { February- } \\
\text { March } \\
1990\end{array}$ & $\begin{array}{l}\text { May- } \\
\text { June } \\
1990\end{array}$ \\
\hline
\end{tabular}

\section{Upper Mississippi River basin}

Mississippi River near Winfield, Mo.

Illinois River at Valley City, I11.

Illinois River at Hardin, Ill.

Mississippi River below Grafton, I11.

Missouri River at Hermann, Mo.

Mississippi River at St. Louis, Mo.

Mississippi River at Thebes, III.

Mississippi River near Cache, IIl.

Mississippi River near Cairo, I11.

$\begin{array}{lrll}\text { UM } & 239.2 & \mathrm{X} & \mathrm{X} \\ \text { IL } & 61.0 & & \\ \text { IL } & 21.8 & \mathrm{X} & \mathrm{X} \\ \text { UM } & 214.6 & & \\ \text { MO } & 97.9 & \mathrm{X} & \mathrm{X} \\ \text { UM } & 179.3 & \mathrm{X} & \mathrm{X} \\ \text { UM } & 43.9 & \mathrm{X} & \mathrm{X} \\ \text { UM } & 14.8 & & \end{array}$

$\mathbf{x}$

(1)

$x$

$x$

UM $\quad 7.5$

$\mathbf{X}$

$\mathbf{X}$

$\mathbf{x}$

$\mathrm{X}$

Ohio River basin

Ohio River at Uniontown, Ky.

Wabash River near New Haven, I11.

Cumberland River near Smithland, Ky.

Tennessee River near Calvert City, Ky.

Ohio River at 0lmsted, I11.

$\begin{array}{lrll}\text { OH } & 842.4 & & \\ \text { WA } & 13.8 & & \\ \text { CU } & 6.8 & & \\ \text { TE } & 11.1 & & \\ \text { OH } & 965.0 & X & X\end{array}$

$\mathbf{x}$

$\mathbf{x}$

$\mathrm{X}$

OH $965.0 \quad X \quad X \quad X \quad X$

Near Mississippi-Ohio River confluence

Mississippi River at Wickliffe, Ky.

Mississippi River near Columbus, Ky.

Mississippi River below Hickman, Ky .

Mississippi River above New Madrid, Mo.

Mississippi River near Point Pleasant, Mo.

IM 950.5

IM 936.1

LM 916.8

LM 898.9

LM 879.5

LM 847.5

$\begin{array}{ccc} & \mathbf{X} \\ \mathbf{X} & \mathbf{X} \\ & \mathbf{X} & \mathbf{X} \\ \mathbf{X} \\ \end{array}$

\section{Lower Mississippi River basin}

Mississippi River at Fulton, Tenn.

Mississippi River below Fulton, Tenn.

Mississippi River below Memphis, Tenn.

Mississippi River at Helena, Ark.

White River at Mile 11.5, Ark.

Arkansas River at Pendleton, Ark.

Mississippi River above Arkansas City, Ark.

Mississippi River below Arkansas City, Ark.

Yazoo River below Steele Bayou, Miss.

Mississippi River below Vicksburg, Miss.

old River Outflow Channel near Knox Landing, La.

Mississippi River near St. Francisville, La.

Mississippi River below Belle Chasse, La.

$\begin{array}{lr}\text { LM } & 777.3 \\ \text { LM } & 773.5 \\ \text { LM } & 731.2 \\ \text { LM } & 663.9 \\ \text { WH } & 11.5 \\ \text { AR } & 22.4 \\ \text { LM } & 566.0 \\ \text { LM } & 551.7 \\ \text { YZ } & 9.0 \\ \text { LM } & 433.4 \\ \text { OR } & 5.5 \\ \text { LM } & 266.4 \\ \text { LM } & 73.1\end{array}$

$\mathbf{x}$
$\mathbf{x}$
$\mathbf{X}$
$\mathbf{X}$
$\mathbf{X}$
$\mathbf{x}$
$\mathbf{X}$
$\mathbf{X}$
$\mathbf{X}$
$\mathbf{X}$

$\mathbf{x}$

$\mathrm{x}$

$\mathrm{x}$

$\mathbf{X}$

$x$

$\begin{array}{ll}x & x \\ x & x \\ x & x\end{array}$

$\mathrm{x} \quad \mathrm{x}$

$\mathrm{x} \quad \mathrm{x}$

${ }^{1}$ UM, Upper Mississippi River miles measured upriver from confluence with Ohio River.

IL, Illinois River miles measured upriver from confluence with Mississippi River (UM mile 218.0).

MO, Missouri River miles measured upriver from confluence with Mississippi River (UM mile 195.3).

OH, Ohio River miles measured downriver from Pittsburgh, Pa. Ohio-Mississippi confluence is at Ohio River mile 981.5 and Lower Mississippi River mile 953.8.

LM, Lower Mississippi River miles measured upriver from Head of Passes, La.

Wh, White River miles measured upriver from confluence with Mississippi River (LM mile 598.8).

AR, Arkansas River miles measured upriver from confluence with Mississippi River (LM mile 581.5).

YZ, Yazoo River miles measured upriver from confluence with Mississippi River (LM mile 437.2).

WA, Wabash River miles measured upriver from confluence with Ohio River (OH mile 848.0).

$\mathrm{CU}$, Cumberland River miles measured upriver from confluence with Ohio River (OH mile 923.2).

TE, Tennessee River miles measured upriver from confluence with Ohio River (OH mile 935.5).

OR, Old River Outflow Channel miles measured downriver from the 01d River Control Structure (LM mile 314.5) 
While all the cruises sampled high-water conditions, the proportions of water contributed by the Mississippi River and its tributaries were different for each cruise (figs. 4, 5, and 6). The first two cruises predominantly sampled Ohio River water, much of which was contributed by the Tennessee River (figs. 4 and 6). The third and fourth cruises sampled water with increasing proportions of Upper Mississippi and Missouri River water, and in samples collected downstream from the Arkansas River (fig. 5b and 5c), during the fourth cruise, there was a major contribution of Arkansas River water.

\section{Acknowledgments}

The U.S. Army Corps of Engineers representatives from the following districts and divisions provided extremely helpful and friendly assistance during and after the cruises: David Legg, Cincinnati District; Samuel Lehr, Whit Barton, and Janet Garner, Memphis District; Billy Garrett, John Miller, and Cecil Soileau, New Orleans District; Ray Kopsky, St. Louis District; Henry Noble, Vicksburg District; and James Farrell of the Lower Mississippi Valley Division.

Many people along the river provided facilities for docking, loading, and repairing the ship and equipment, and they often went out of their way to be helpful: Renee Ory and the people at Ory Brothers Marine Service of America in Hartford, Ill.; Rusty Sutton in Grafton, Ill.; Daniel Gipe and the people at Hermann Sand and Gravel Company in Hermann, Mo.; Captain William Carroll and the people at Gateway River Cruises in St. Louis, Mo.; Kent Hoffmeister and the workers at Missouri Dry Dock and Repair Company in Cape Girardeau, Mo.; the crew at Lock and Dam 53 near Olmsted, IIl.; the Coast Guard Station in Hickman, Ky.; John Janoush and the people at Janoush Marine, Inc., in Rosedale, Miss.; the crew at Norrell Lock 非 near Arkansas Post, Ark,; Mary and John Hoseman and the people working on the SPIRIT OF VICKSBURG in Vicksburg, Miss.; and the people running the New Roads Ferry at

St. Francisville, La., and the Belle Chasse Ferry at Belle Chasse, La.

The crew of the research vessel ACADIANA, Lee Black, Steve Rabalais, Wayne Simoneaux, Craig LeBoeuf, Chuck Guidry, and Wilton Delaune, were especially accommodating and showed an unusual interest and concern to maintain proper scientific operations.

Measuring river discharge or collecting suspended-sediment samples from large rivers is not a two-man operation, and the following individuals' collaboration was necessary to get the job done: Ellen Axtmann, Terry Brinton, Pat Brown, Daphne Frilot, John Garbarino, Lois Koehnken, Tom Kraemer, Jerry Leenheer, Tom Leiker, Debby Martin, Dick Martin, Ted Noyes, Dale Peart, Willie Pereira, Jim Ranville, Terry Rees, Colleen Rostad, Jim Seeley, Alan Shiller, Herb Stevens, Bob Stallard, Dick Tagg, and Howard Taylor. Particlesize analyses of suspended and bed sediments were made by Mike Werito, Carol Anderson, and her colleagues.

Jerry Leenheer, Colleen Rostad, Bill Emmett, Marjorie Pokladnik, Mary Kidd, and John Flager made so many helpful changes to the text, that whose words these are we do not know. 

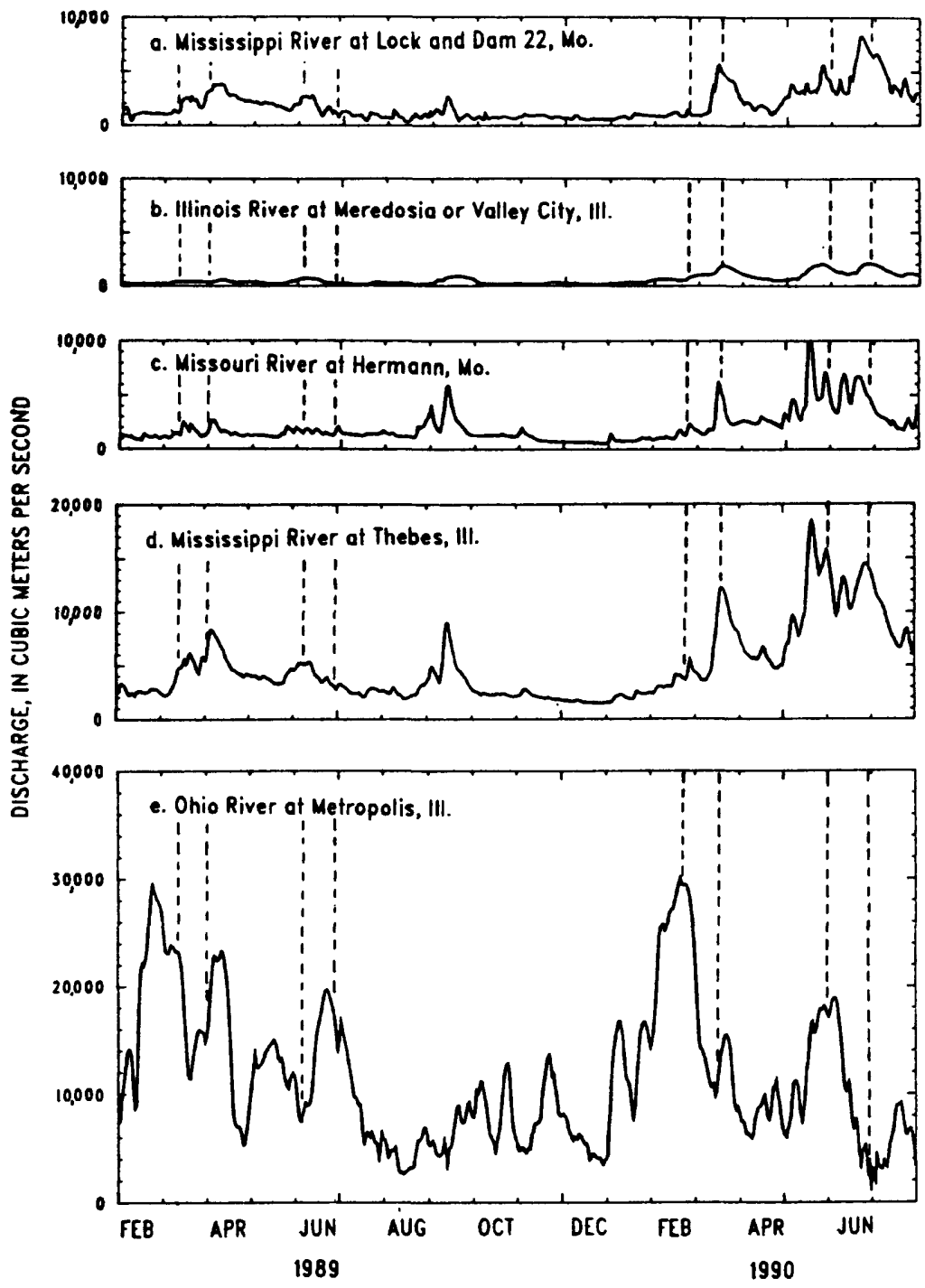

Figure 4.--Water discharge at two locations on the Upper Mississippi River and for three tributaries from February 1989 through July 1990. The period of time for each cruise is shown by two vertical dashed lines.

a. Lock and Dam 22 is on the Upper Mississippi River about 98 kilometers upstream from the sampling site near Winfield, Mo.

b. The discharge gaging station on the Illinois River was at Meredosia, Ill., from February 1989 through September 1989 and at Valley City, Ill., from October 1989 through July 1990. Meredosia is about 72 kilometers and Valley City is 63 kilometers upstream from the sampling site at Hardin, Ill.

c. Sampling site on the Missouri River.

d. Sampling site is on the Upper Mississippi River about 71 kilometers upstream from the confluence of the Upper Mississippi and Ohio Rivers.

e. The Ohio River at Metropolis, I11., is about 29 kilometers upstream from the sampling site at olmsted, Ill.

Discharge data are from the U.S. Geological Survey, except for the Mississippi River at Lock and Dam 22 (U.S. Army Corps of Engineers, Rock Island District, written commun., 1990). 


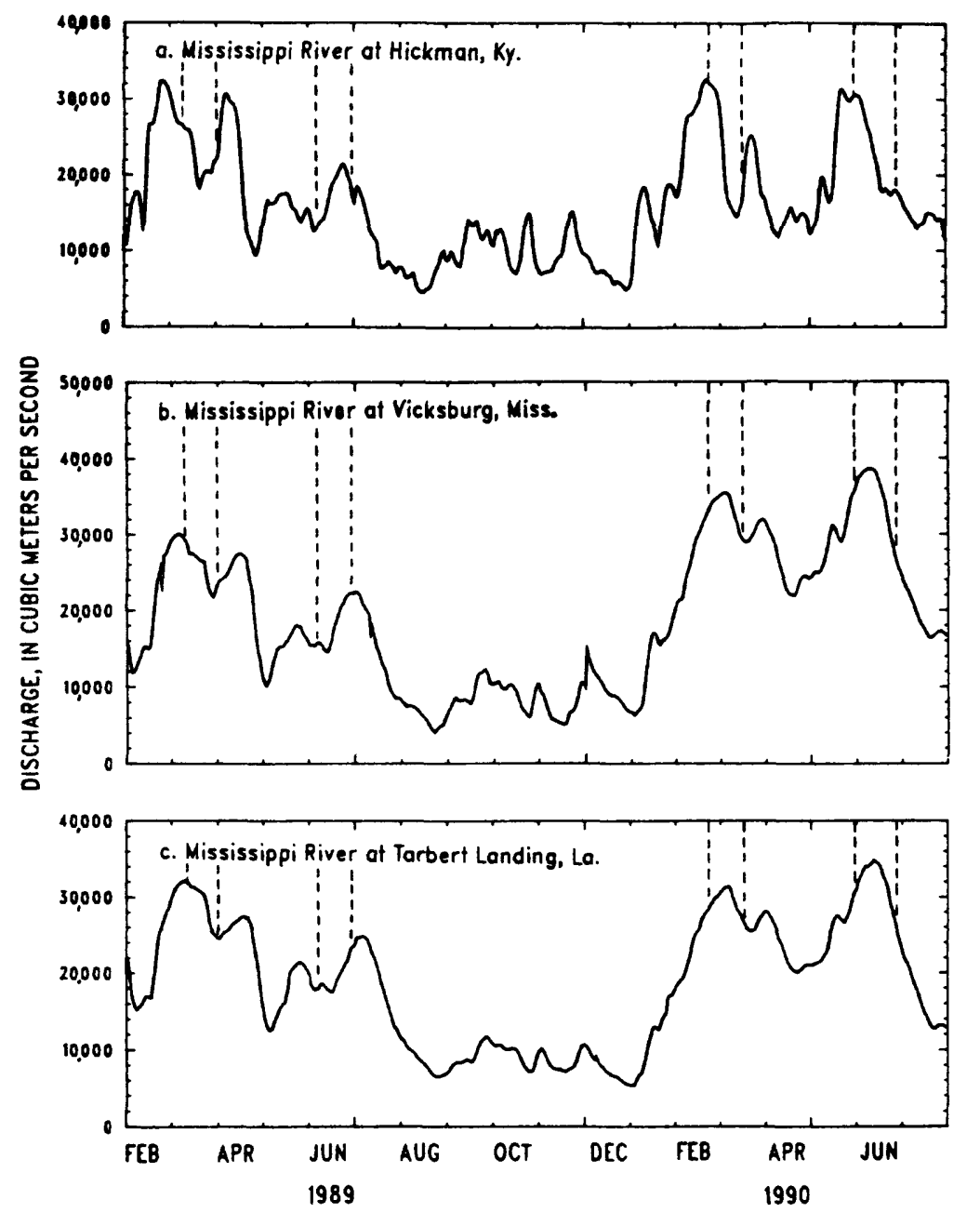

Figure 5.--Water discharge at three locations on the Lower Mississippi River from February 1989 through July 1990. The period of time for each cruise is shown by two vertical dashed lines.

a. Hickman, $\mathrm{Ky}$, is about $58 \mathrm{kilometers} \mathrm{downstream} \mathrm{from} \mathrm{the} \mathrm{confluence}$ of the Upper Mississippi and Ohio Rivers.

b. The discharge at Vicksburg, Miss., is near the maximum for the entire Mississippi River because, at 191 kilometers downstream from Vicksburg, approximately 25 percent of the water in the river is diverted through the 0ld River Outflow Channel into the Atchafalaya River.

c. Tarbert Landing, La., is about 13 kilometers downstream from the 0ld River Outflow Channel near Knox Landing, La., and 63 kilometers upstream from the sampling section near St. Francisville, La.

Discharge data are from the U.S. Army Corps of Engineers, Memphis District (Hickman) (1990?, 1991?); Vicksburg District (Vicksburg) (1990?, 1991?); and New Orleans District (Tarbert Landing) (1990?, 1991?). 

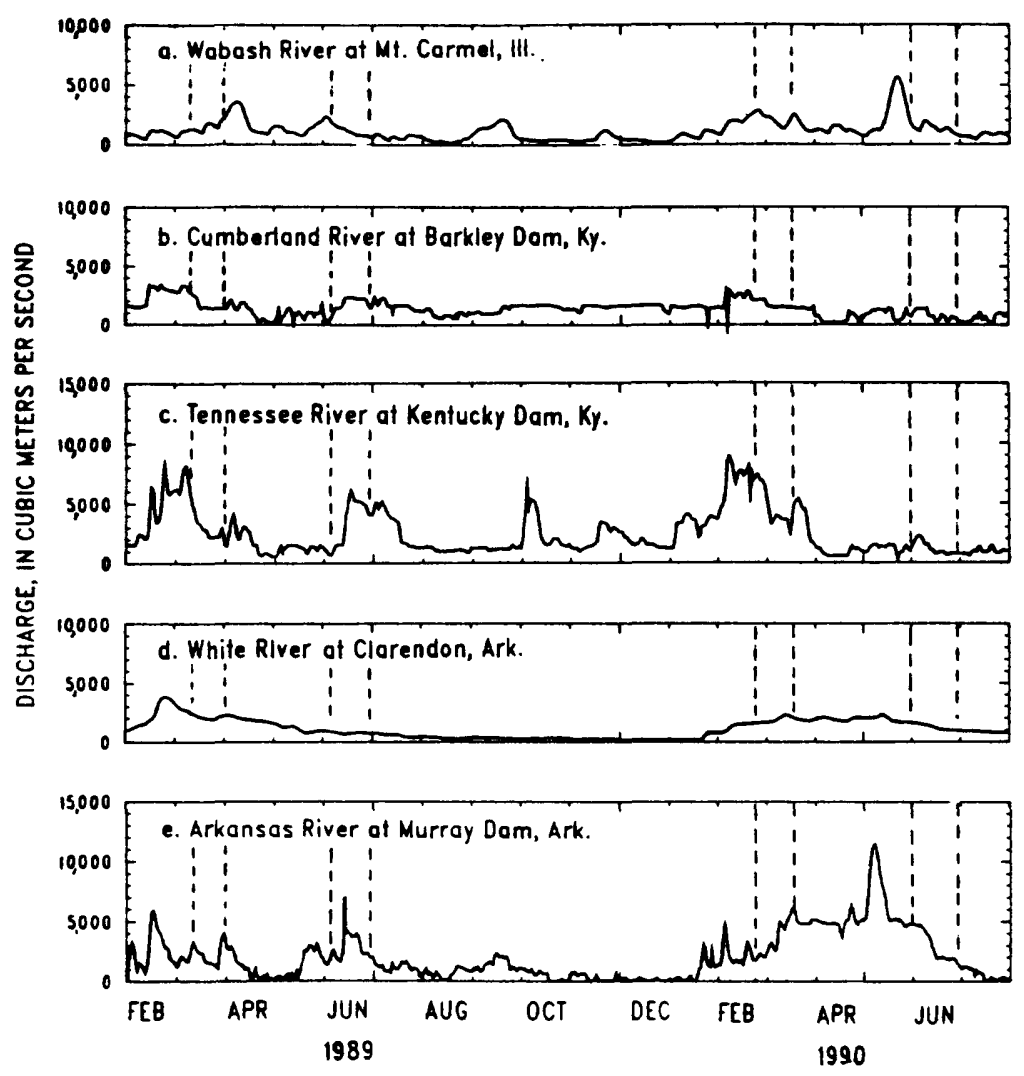

Figure 6.--Water discharge at locations on three secondary tributaries of the Mississippi River (Wabash, Cumberland, and Tennessee Rivers) and two tributaries of the Lower Mississippi River from February 1989 through July 1990. The period of time for each cruise is shown by two vertical dashed lines.

a. Mt. Carmel is about 130 kilometers upstream from the sampling site on the Wabash River.

b. Barkley Dam is about 39 kilometers upstream from the sampling site on the Cumberland River.

c. Kentucky Dam is about 18 kilometers upstream from the sampling site on the Tennessee River.

d. Clarendon is about 142 kilometers upstream from the sampling site on the White River at Mile 11.5, Ark.

e. Murray Dam is about 191 kilometers upstream from the sampling site on the Arkansas River at Pendleton, Ark. Bayou Meto is the primary tributary (mean discharge is less than 1 percent of Arkansas River) between Murray Dam and the sampling site on the Arkansas River.

Discharge data are from U.S. Geological Survey (Wabash, Cumberland, and Arkansas Rivers) and the U.S. Army Corps of Engineers, Memphis District (White River) (1990?, 1991?), and Cincinnati District (Tennessee River). 


\section{SAMPLING PROCEDURES AND RESULTS}

The onsite sampling procedures were carried out from the ACADIANA and consisted of collecting representative samples of bed sediment, water, and suspended sediment, and of measuring physical and simple chemical properties of the water. Bed sediments were sampled at three to five locations across the channel to provide information for velocity, suspended-sediment, and bed-load calculations. Suspended sediments were collected at a number of locations or verticals spaced across the river channel by two different methods: (1) pumping from fixed depths, and (2) depth integration. For each of the two suspended-sediment sampling methods, the individual samples from each vertical across the river were combined to form two representative samples, which were processed differently--a pumped composite sample and a depth-integrated composite sample. While the depth-integrated sample was being collected, the physical (the water depth, the depth-integrated or depth-averaged velocity, and the surface temperature) and simple chemical (surface $\mathrm{pH}$ and surface specific conductance) properties of the water were measured at each location across the river.

This report is organized in the order in which the data are needed to investigate the movement, mixing, and storage processes of sediment-associated and dissolved pollutants. The detailed bed-sediment procedures and results. are presented first because bed sediments affect velocities, suspendedsediment transport and bed-load transport. The water-discharge procedures and results are presented second because this information is needed to compute the flux of suspended sediment and dissolved constituents.

Suspended-sediment collecting methods, processing procedures, and results follow the "Water-Discharge" section. This section also briefly describes the procedures used for concentrating the suspended sediment finer than $63 \mu \mathrm{m}$. These procedures were carried out aboard the ACADIANA to reduce the sample volumes to a manageable size that could be shipped to the laboratory for chemical analyses. This report does not include a description of how each of the numerous chemical subsamples from the pumped and depth-integrated composites were prepared, treated, and processed in the laboratory.

The fourth section describes the procedures for determining the suspended-sediment concentration and particle sizes of both the suspended sand and the suspended silt and clay fractions. There also is a discussion of the reproducibility or precision and the accuracy of the depth-integration method as a function of the number of sampled verticals.

The last section discusses some surface-water properties measured at each sampling site and presents some additional data collected at sites where bed sediments and representative water and suspended-sediment samples were not collected. 


\section{$\underline{\text { Bed Sediments }}$}

A BM-54 sampler (Guy and Norman, 1970, p. 15) was used to collect bedsediment samples for particle-size analyses. At the beginning of the measurements at each sampling site, while equipment was being set up for the water-discharge measurement and suspended-sediment sampling, the ship drifted downstream, and as it crossed the line of section a bed-sediment sample was collected. A fathometer (Lowrance, Model X16) was used to obtain a continuous record of depth; at the time each bed sample was taken a mark was made on this continuous trace of the river bottom. Samples generally were collected at $0.1,0.3,0.5,0.7$, and 0.9 of the distance between the left and right banks. Particle-size analyses were done at the U.S. Geological Survey Sediment Laboratory in Lakewood, Colorado, for the March-April 1989 cruise and at the USGS Sediment Laboratory in Iowa City, Iowa, for the June 1989, February-March 1990, and May-June 1990 cruises. The particle sizes (expressed in millimeters) are listed in tables 3-6 along with the median diameter determined by straight-line interpolation between appropriate particle sizes. 
Table 3.--Particle size, determined by sieving, of bed material collected from the Mississippi

River and some of its tributaries for March-April 1989 cruise

[Analyses by M.J. Werito, U.S. Geological Survey, Colorado Sediment Laboratory; m, meter; mm, millimeter]

\begin{tabular}{|c|c|c|c|c|c|c|c|c|c|c|c|c|c|c|c|c|c|}
\hline $\begin{array}{l}\text { Date } \\
1989\end{array}$ & $\begin{array}{l}\text { Location } \\
\text { in cross } \\
\text { section } \\
\text { (fraction of } \\
\text { distance } \\
\text { between } \\
\text { left and } \\
\text { right banks) }\end{array}$ & $\begin{array}{l}\text { Depth } \\
\text { of } \\
\text { water } \\
\text { (a) }\end{array}$ & 0.063 & 0.090 & 0.125 & $\frac{P}{0.180}$ & $\frac{\text { ercent }}{0.250}$ & $\frac{\text { iner th }}{0.355}$ & $\frac{\operatorname{an} \text { ind }}{0.50}$ & $\frac{\text { licated }}{0.71}$ & $\frac{\text { size }}{1.00}$ & $\frac{(\mathrm{m})}{1.41}$ & 2.00 & 4.00 & 8.00 & 16.00 & $\begin{array}{l}\text { Median } \\
\text { diameter } \\
\text { (mm) }\end{array}$ \\
\hline $3-10$ & $\begin{array}{l}0.1 \\
0.3 \\
0.5 \\
0.7 \\
0.9\end{array}$ & $\begin{array}{l}7.5 \\
7.4 \\
5.6 \\
4.3 \\
3.8\end{array}$ & $\begin{array}{l}0.5 \\
0.2 \\
0.7 \\
1.0 \\
0.8\end{array}$ & $\begin{array}{l}0.8 \\
0.3 \\
0.9 \\
1.1 \\
1.1\end{array}$ & $\begin{array}{l}1.5^{M} \\
0.4 \\
1.0 \\
1.2 \\
1.3\end{array}$ & $\begin{array}{c}\operatorname{sgigsi} \\
2.6 \\
1.0 \\
2.0 \\
2.0 \\
1.9\end{array}$ & $\begin{array}{l}\text { i Rive } \\
4.6 \\
4.7 \\
6.0 \\
5.5 \\
5.4\end{array}$ & $\begin{array}{l}\text { near } \\
20.0 \\
26.3 \\
22.1 \\
23.5 \\
30.1\end{array}$ & $\begin{array}{c}\text { Winfie } \\
52.6 \\
55.0 \\
47.6 \\
51.7 \\
68.5\end{array}$ & $\begin{array}{l}1 \mathrm{~d}, \mathrm{Mi} \\
82.1 \\
75.2 \\
70.3 \\
79.2 \\
88.7\end{array}$ & $\begin{array}{l}\text { Egouri } \\
95.3 \\
82.9 \\
83.9 \\
93.0 \\
96.0\end{array}$ & $\begin{array}{l}{ }^{2} \\
98.7 \\
86.0 \\
90.9 \\
98.2 \\
98.0\end{array}$ & $\begin{array}{l}99.7 \\
87.5 \\
95.7 \\
99.6 \\
98.9\end{array}$ & $\begin{array}{r}100.0 \\
90.8 \\
99.5 \\
100.0 \\
99.5\end{array}$ & $\begin{array}{r}96.0 \\
100.0 \\
100.0\end{array}$ & 96.7 & $\begin{array}{l}0.48 \\
0.47 \\
0.52 \\
0.49 \\
0.43\end{array}$ \\
\hline $3-12$ & $\begin{array}{l}0.2 \\
0.3 \\
0.5 \\
0.7 \\
0.9\end{array}$ & $\begin{array}{l}5.9 \\
4.6 \\
4.2 \\
3.5 \\
4.2\end{array}$ & $\begin{array}{l}0.1 \\
0.0 \\
0.0 \\
0.0 \\
0.0\end{array}$ & $\begin{array}{l}0.1 \\
0.1 \\
0.0 \\
0.0 \\
0.0\end{array}$ & $\begin{array}{l}0.3 \\
0.5 \\
0.2 \\
0.0 \\
0.0\end{array}$ & $\begin{array}{l}\mathrm{Migsol} \\
3.6 \\
6.7 \\
2.8 \\
1.7 \\
0.8\end{array}$ & $\begin{array}{r}\text { i Rive } \\
43.2 \\
43.8 \\
17.6 \\
12.9 \\
7.2\end{array}$ & $\begin{array}{c}\text { at } \mathrm{He} \\
95.7 \\
75.9 \\
38.7 \\
27.9 \\
16.2\end{array}$ & $\begin{array}{c}\text { rmann, } \\
99.4 \\
93.5 \\
60.7 \\
49.1 \\
31.9\end{array}$ & $\begin{array}{l}\text { Missol } \\
99.7 \\
98.5 \\
74.4 \\
71.9 \\
53.2\end{array}$ & $\begin{array}{l}\frac{u r i}{99} .8 \\
99.6 \\
82.4 \\
87.7 \\
72.9\end{array}$ & $\begin{array}{l}99.9 \\
99.8 \\
87.3 \\
93.4 \\
85.0\end{array}$ & $\begin{array}{r}100.0 \\
99.9 \\
90.9 \\
95.7 \\
91.8\end{array}$ & $\begin{array}{l}99.9 \\
96.3 \\
96.9 \\
99.0\end{array}$ & $\begin{array}{r}100.0 \\
98.6 \\
98.2 \\
100.0\end{array}$ & $\begin{array}{l}100.0 \\
100.0\end{array}$ & $\begin{array}{l}0.26 \\
0.27 \\
0.43 \\
0.51 \\
0.68\end{array}$ \\
\hline $3-13$ & $\begin{array}{l}0.1 \\
0.3 \\
0.5 \\
0.7 \\
0.9\end{array}$ & $\begin{array}{r}10.1 \\
8.6 \\
6.5 \\
4.9 \\
4.9\end{array}$ & $\begin{array}{l}0.0 \\
0.0 \\
0.0 \\
0.0 \\
0.0\end{array}$ & $\begin{array}{l}0.0 \\
0.0 \\
0.1 \\
0.0 \\
0.0\end{array}$ & $\begin{array}{l}0.1 \\
0.2 \\
0.4 \\
0.2 \\
0.1\end{array}$ & $\begin{array}{r}\text { lississ } \\
1.2 \\
2.5 \\
3.4 \\
1.8 \\
1.8\end{array}$ & $\begin{array}{r}\text { ippi Ri } \\
10.6 \\
42.2 \\
27.1 \\
19.1 \\
5.6\end{array}$ & $\begin{array}{c}\text { rer at S } \\
39.5 \\
82.0 \\
40.6 \\
47.7 \\
15.2\end{array}$ & $\begin{array}{c}\text { t. Lou } \\
93.8 \\
95.3 \\
51.5 \\
63.1 \\
35.2\end{array}$ & $\begin{array}{c}\text { Is, Mi } \\
98.0 \\
97.1 \\
63.1 \\
78.3 \\
68.2\end{array}$ & $\begin{array}{l}\text { ssouri } \\
99.8 \\
97.5 \\
71.2 \\
86.5 \\
86.9\end{array}$ & $\begin{array}{l}{ }^{2} \\
99.2 \\
97.7 \\
75.3 \\
89.9 \\
93.1\end{array}$ & $\begin{array}{l}99.4 \\
97.9 \\
78.9 \\
91.5 \\
96.6\end{array}$ & $\begin{array}{l}99.8 \\
98.7 \\
84.1 \\
94.1 \\
99.1\end{array}$ & $\begin{array}{r}100.0 \\
99.6 \\
87.9 \\
97.6 \\
100.0\end{array}$ & $\begin{array}{r}100.0 \\
93.8 \\
100.0\end{array}$ & $\begin{array}{l}0.39 \\
0.27 \\
0.48 \\
0.38 \\
0.59\end{array}$ \\
\hline $3-15$ & $\begin{array}{l}0.3 \\
0.5 \\
0.7 \\
0.9\end{array}$ & $\begin{array}{l}9.4 \\
9.2 \\
7.5 \\
5.5\end{array}$ & $\begin{array}{l}0.0 \\
0.0 \\
0.1 \\
0.0\end{array}$ & $\begin{array}{l}0.0 \\
0.1 \\
0.1 \\
0.1\end{array}$ & $\begin{array}{l}0.1 \\
0.7 \\
0.4 \\
0.5\end{array}$ & $\begin{array}{c}\text { Missis } \\
0.6 \\
6.6 \\
3.3 \\
3.6\end{array}$ & $\begin{array}{r}\text { ippi } R \\
1.7 \\
35.3 \\
12.8 \\
25.7\end{array}$ & $\begin{array}{r}\text { ver at } \\
5.6 \\
79.5 \\
40.5 \\
79.8\end{array}$ & $\begin{array}{c}\text { Thebes } \\
22.9 \\
95.6 \\
74.5 \\
97.9\end{array}$ & $\begin{array}{l}\text { I11i } \\
42.7 \\
99.0 \\
93.3 \\
99.9\end{array}$ & $\begin{array}{l}\text { nois } \\
58.5 \\
99.6 \\
98.5 \\
99.9\end{array}$ & $\begin{array}{l}71.6 \\
99.7 \\
99.5 \\
99.9\end{array}$ & $\begin{array}{l}81.7 \\
99.8 \\
99.8 \\
99.9\end{array}$ & $\begin{array}{r}95.4 \\
100.0 \\
99.9 \\
100.0\end{array}$ & $\begin{array}{r}99.3 \\
100.0\end{array}$ & 100.0 & $\begin{array}{l}0.84 \\
0.28 \\
0.40 \\
0.30\end{array}$ \\
\hline $3-16$ & $\begin{array}{l}0.1 \\
0.3 \\
0.5 \\
0.7 \\
0.9\end{array}$ & $\begin{array}{l}11.8 \\
13.9 \\
15.6 \\
18.5 \\
18.1\end{array}$ & $\begin{array}{l}0.0 \\
0.0 \\
0.0 \\
0.0 \\
0.0\end{array}$ & $\begin{array}{l}0.0 \\
0.0 \\
0.0 \\
0.0 \\
0.0\end{array}$ & $\begin{array}{l}0.1 \\
0.0 \\
0.0 \\
0.0 \\
0.0\end{array}$ & $\begin{array}{l}\text { Ohi } \\
0.8 \\
0.3 \\
0.0 \\
0.1 \\
0.1\end{array}$ & $\begin{array}{c}\text { River } \\
7.9 \\
2.9 \\
0.7 \\
0.7 \\
0.5\end{array}$ & $\begin{array}{r}\text { te } 01 \mathrm{mg} \\
49.2 \\
16.9 \\
4.2 \\
5.8 \\
3.0\end{array}$ & $\begin{array}{c}\text { ted, I } \\
92.8 \\
65.2 \\
18.1 \\
31.4 \\
17.4\end{array}$ & $\begin{array}{l}11 \text { inoi } \\
95.5 \\
87.0 \\
42.7 \\
60.6 \\
42.3\end{array}$ & $\begin{array}{l}\frac{g^{2}}{95.7} \\
91.6 \\
60.6 \\
73.7 \\
58.3\end{array}$ & $\begin{array}{l}95.7 \\
93.2 \\
71.4 \\
81.1 \\
69.2\end{array}$ & $\begin{array}{l}95.8 \\
94.3 \\
78.0 \\
87.1 \\
77.1\end{array}$ & $\begin{array}{l}96.0 \\
95.8 \\
88.8 \\
94.8 \\
89.7\end{array}$ & $\begin{array}{l}97.9 \\
98.9 \\
96.8 \\
99.4 \\
97.7\end{array}$ & $\begin{array}{r}100.0 \\
100.0 \\
98.0 \\
100.0 \\
100.0\end{array}$ & $\begin{array}{l}0.36 \\
0.45 \\
0.83 \\
0.63 \\
0.85\end{array}$ \\
\hline $3-17$ & $\begin{array}{l}0.3 \\
0.5 \\
0.7 \\
0.9\end{array}$ & $\begin{array}{l}13.0 \\
11.0 \\
16.2 \\
16.2\end{array}$ & $\begin{array}{l}0.0 \\
0.0 \\
0.0 \\
0.0\end{array}$ & $\begin{array}{l}0.0 \\
0.0 \\
0.0 \\
0.0\end{array}$ & $\begin{array}{l}0.0^{M} \\
0.0 \\
0.0 \\
0.0\end{array}$ & $\begin{array}{l}\text { Bissi } \\
0.2 \\
0.4 \\
1.1 \\
1.2\end{array}$ & $\begin{array}{l}i \mathrm{Riv} \\
1.0 \\
2.7 \\
6.4 \\
8.4\end{array}$ & $\begin{array}{c}\text { r belor } \\
2.2 \\
10.5 \\
10.8 \\
26.2\end{array}$ & $\begin{array}{r}\text { Hickm } \\
6.0 \\
41.3 \\
17.4 \\
68.5\end{array}$ & $\begin{array}{c}\tan , \text { Ke } \\
20.0 \\
77.4 \\
31.3 \\
95.1\end{array}$ & $\begin{array}{c}\text { ntucky } \\
37.4 \\
93.3 \\
48.2 \\
99.4\end{array}$ & $\begin{array}{l}L^{2} \\
49.4 \\
98.3 \\
61.2 \\
99.8\end{array}$ & $\begin{array}{l}60.3 \\
99.5 \\
72.3 \\
99.9\end{array}$ & $\begin{array}{r}78.6 \\
99.9 \\
89.0 \\
100.0\end{array}$ & $\begin{array}{r}90.7 \\
100.0 \\
98.1\end{array}$ & $\begin{array}{r}96.9 \\
100.0\end{array}$ & $\begin{array}{l}1.42 \\
0.55 \\
1.06 \\
0.44\end{array}$ \\
\hline $3-19$ & $\begin{array}{l}0.1 \\
0.3 \\
0.5 \\
0.7 \\
0.9\end{array}$ & $\begin{array}{l}11.1 \\
11.7 \\
12.0 \\
12.5 \\
16.7\end{array}$ & $\begin{array}{l}0.0 \\
0.0 \\
0.0 \\
0.0 \\
0.0\end{array}$ & $\begin{array}{l}0.1 \\
0.0 \\
0.0 \\
0.0 \\
0.0\end{array}$ & $\begin{array}{l}0.7^{M i} \\
0.2 \\
0.8 \\
0.0 \\
0.0\end{array}$ & $\begin{array}{c}\text { gsisgi } \\
10.9 \\
1.8 \\
1.3 \\
0.4 \\
0.4\end{array}$ & $\begin{array}{c}\text { Pi Riv } \\
72.1 \\
9.4 \\
5.7 \\
3.4 \\
2.2\end{array}$ & $\begin{array}{l}\text { r belor } \\
89.4 \\
44.7 \\
23.6 \\
22.9 \\
11.9\end{array}$ & $\begin{array}{l}\text { Fulto } \\
92.7 \\
87.4 \\
59.7 \\
50.8 \\
27.8\end{array}$ & $\begin{array}{l}n_{2} \text { Ten } \\
95.7 \\
97.5 \\
83.0 \\
82.7 \\
48.6\end{array}$ & $\begin{array}{c}\text { nessee } \\
97.8 \\
99.4 \\
92.0 \\
96.2 \\
69.0\end{array}$ & $\begin{array}{l}98.9 \\
99.8 \\
95.9 \\
99.1 \\
78.9\end{array}$ & $\begin{array}{l}99.5 \\
99.9 \\
97.9 \\
99.6 \\
84.9\end{array}$ & $\begin{array}{r}99.9 \\
100.0 \\
99.1 \\
99.9 \\
91.0\end{array}$ & $\begin{array}{r}99.7 \\
100.0 \\
95.4\end{array}$ & $\begin{array}{l}100.0 \\
100.0\end{array}$ & $\begin{array}{l}0.22 \\
0.37 \\
0.46 \\
0.50 \\
0.73\end{array}$ \\
\hline $3-21$ & 0.3 & 17.8 & 0.0 & 0.0 & 0.0 & $\frac{M i s \mathrm{~s}}{0.1}$ & $\frac{p P i g}{0.4}$ & $\frac{\text { ver at }}{2.0}$ & $\frac{\text { Helena }}{13.2}$ & $\frac{1, \text { Arka }}{59.9}$ & $\frac{\text { ngas }}{91.8}$ & 97.0 & 98.0 & 98.5 & 99.0 & 100.0 & 0.67 \\
\hline $3-2$ & $\begin{array}{l}0.2 \\
0.5 \\
0.9\end{array}$ & $\begin{array}{l}13.8 \\
12.6 \\
10.5\end{array}$ & $\begin{array}{l}0.1 \\
0.2 \\
0.2\end{array}$ & $\begin{array}{l}0.2 \\
0.2 \\
0.3\end{array}$ & $\begin{array}{l}0.4 \\
0.3 \\
0.6\end{array}$ & $\begin{array}{l}\text { White } \\
2.2 \\
1.7 \\
1.4\end{array}$ & $\begin{array}{r}\text { River } \\
12.9 \\
11.1 \\
2.9\end{array}$ & $\begin{array}{c}\text { te Mile } \\
71.2 \\
33.8 \\
18.6\end{array}$ & $\begin{array}{l}11.5, \\
94.2 \\
72.4 \\
62.7\end{array}$ & $\begin{array}{c}\text { Arkans } \\
98.3 \\
92.1 \\
93.7\end{array}$ & $\begin{array}{l}\text { as } \\
98.9 \\
96.1 \\
98.3\end{array}$ & $\begin{array}{l}99.0 \\
97.5 \\
99.1\end{array}$ & $\begin{array}{l}99.1 \\
98.4 \\
99.4\end{array}$ & $\begin{array}{l}99.3 \\
99.5 \\
99.8\end{array}$ & $\begin{array}{l}100.0 \\
100.0 \\
100.0\end{array}$ & & $\begin{array}{l}0.32 \\
0.42 \\
0.46\end{array}$ \\
\hline $3-23$ & $\begin{array}{l}0.3 \\
0.5 \\
0.7\end{array}$ & $\begin{array}{l}5.1 \\
6.7 \\
8.8\end{array}$ & $\begin{array}{l}0.0 \\
0.0 \\
0.0\end{array}$ & $\begin{array}{l}0.1 \\
0.1 \\
0.0\end{array}$ & $\begin{array}{l}0.5 \\
0.8 \\
0.0\end{array}$ & $\begin{array}{c}\text { Arkans } \\
3.6 \\
5.6 \\
1.3\end{array}$ & $\begin{array}{c}18 \text { River } \\
23.7 \\
45.9 \\
19.1\end{array}$ & $\begin{array}{l}\text { at Pen } \\
60.7 \\
95.2 \\
90.1\end{array}$ & $\begin{array}{c}\text { dleton } \\
84.3 \\
99.1 \\
97.9\end{array}$ & $\begin{array}{c}\text { Arka } \\
96.3 \\
99.6 \\
99.6\end{array}$ & $\begin{array}{l}\text { nsas } \\
99.1 \\
99.6 \\
99.9\end{array}$ & $\begin{array}{r}99.7 \\
99.7 \\
100.0\end{array}$ & $\begin{array}{l}99.8 \\
99.7\end{array}$ & $\begin{array}{l}99.9 \\
99.7\end{array}$ & $\begin{array}{r}100.0 \\
99.9\end{array}$ & 100.0 & $\begin{array}{l}0.32 \\
0.26 \\
0.30\end{array}$ \\
\hline ? & $\begin{array}{l}0.1 \\
0.3 \\
0.5 \\
0.8 \\
0.9\end{array}$ & $\begin{array}{l}10.4 \\
16.2 \\
20.8 \\
18.3 \\
18.0\end{array}$ & $\begin{array}{l}0.0 \\
0.0 \\
0.0 \\
0.0\end{array}$ & $\begin{array}{l}\text { Mud } \\
0.5 \\
0.0 \\
0.0 \\
0.1\end{array}$ & $\begin{array}{l}\frac{\text { Missi }}{\text { wit }} \\
4.4 \\
0.0 \\
0.0 \\
0.3\end{array}$ & $\begin{array}{l}\text { ssippi } \\
23.9 \\
1.2 \\
0.5 \\
2.0\end{array}$ & $\begin{array}{r}\text { River } \\
\text { 30e } \\
94.6 \\
20.9 \\
2.3 \\
8.4\end{array}$ & $\begin{array}{c}\text { bove A } \\
\text { sand } \\
99.9 \\
62.5 \\
22.5 \\
21.8\end{array}$ & $\begin{array}{l}\text { kansas } \\
99.9 \\
87.1 \\
80.3 \\
53.0\end{array}$ & $\begin{array}{r}100.0 \\
91.4 \\
98.9 \\
79.9\end{array}$ & $\begin{array}{l}91.8 \\
99.6 \\
87.1\end{array}$ & $\begin{array}{l}92.1 \\
99.7 \\
91.2\end{array}$ & $\begin{array}{l}92.3 \\
99.7 \\
93.7\end{array}$ & $\begin{array}{l}93.0 \\
99.7 \\
97.1\end{array}$ & $\begin{array}{l}94.2 \\
99.7 \\
98.5\end{array}$ & $\begin{array}{l}100.0 \\
100.0 \\
100.0\end{array}$ & $\begin{array}{l}0.21 \\
0.32 \\
0.42 \\
0.49\end{array}$ \\
\hline
\end{tabular}


Table 3.--Particle size, determined by sieving, of bed naterial collected from the Mississippi River and some of its tributaries for March-April 1989 cruise--Continued

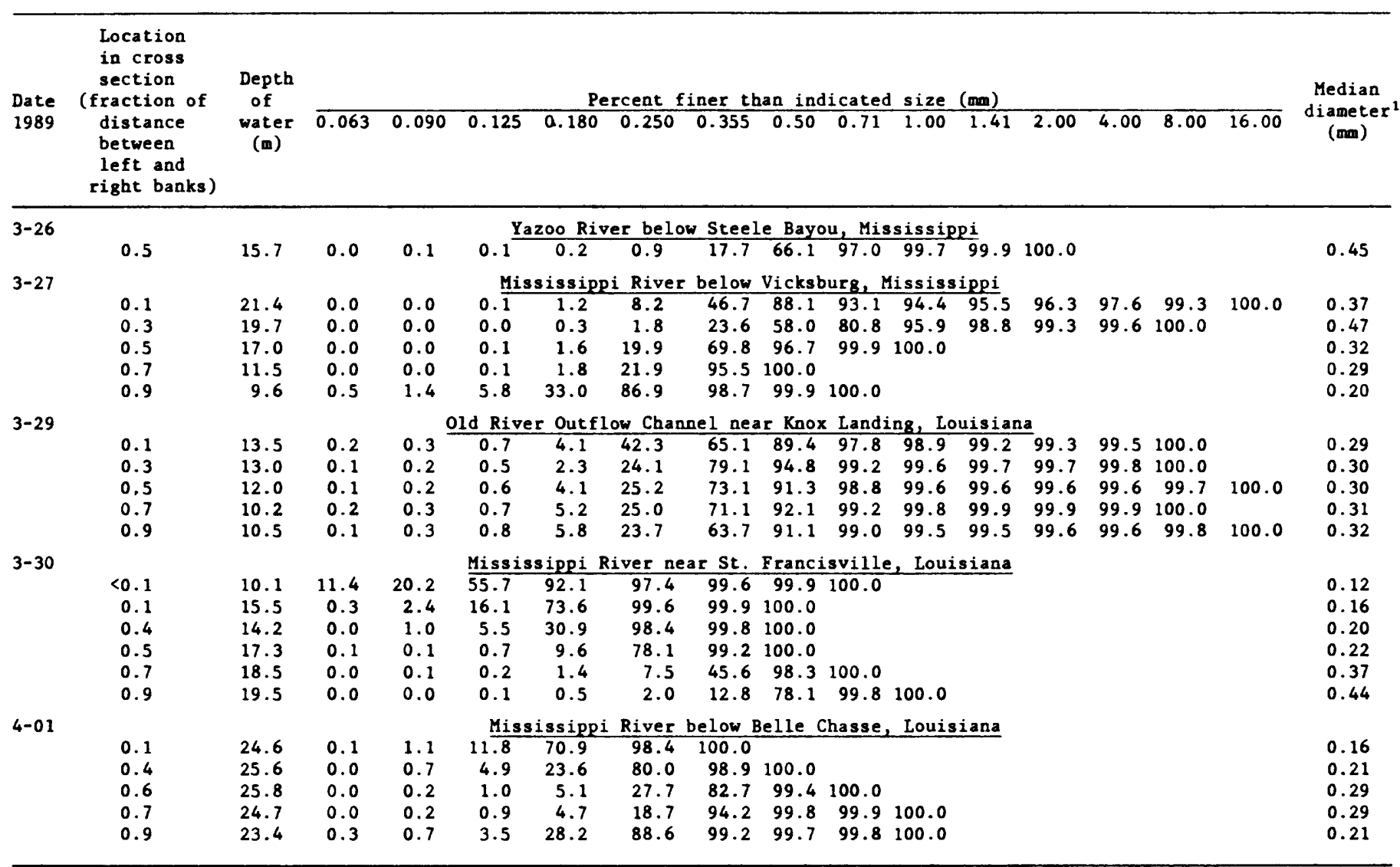

Median diameter determined by straight-1ine interpolation.

${ }^{2}$ Percent finer than $32.0 \mathrm{~mm}$ is 100.0 . 
Table 4.--Particle size, deterwined by sieving, of bed material collected from the Mississippi River and some of its tributaries for June 1989 cruise

[Analyses by U.S. Geological Survey Sediment Laboratory in Iowa City, Iowa; m, meter; ma, millimeter; --, no measurement]

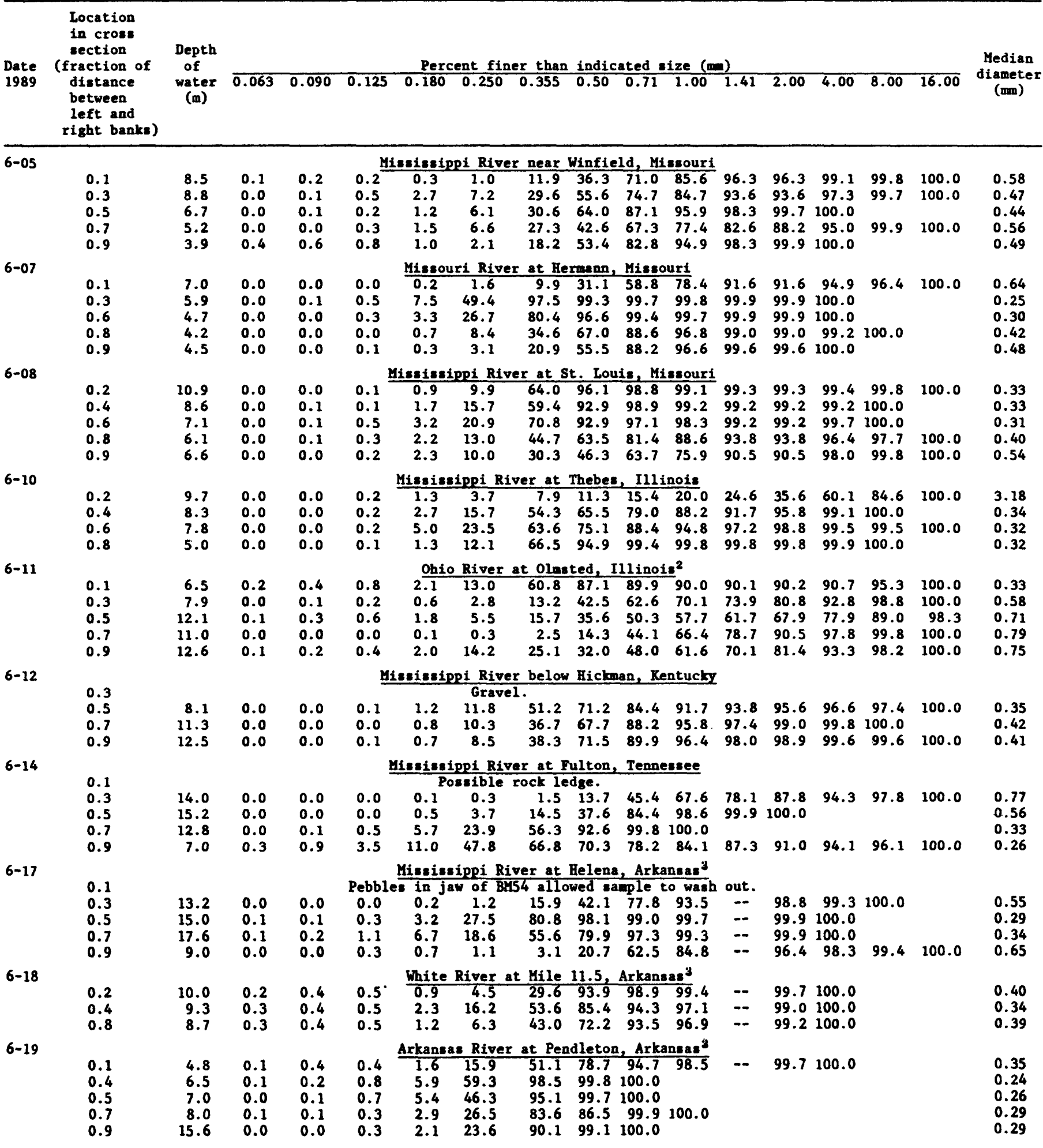


Table 4.--Particle size, determined by sieving, of bed material collected from the Mississippi River and some of its tributaries for June 1989 cruise--Continued

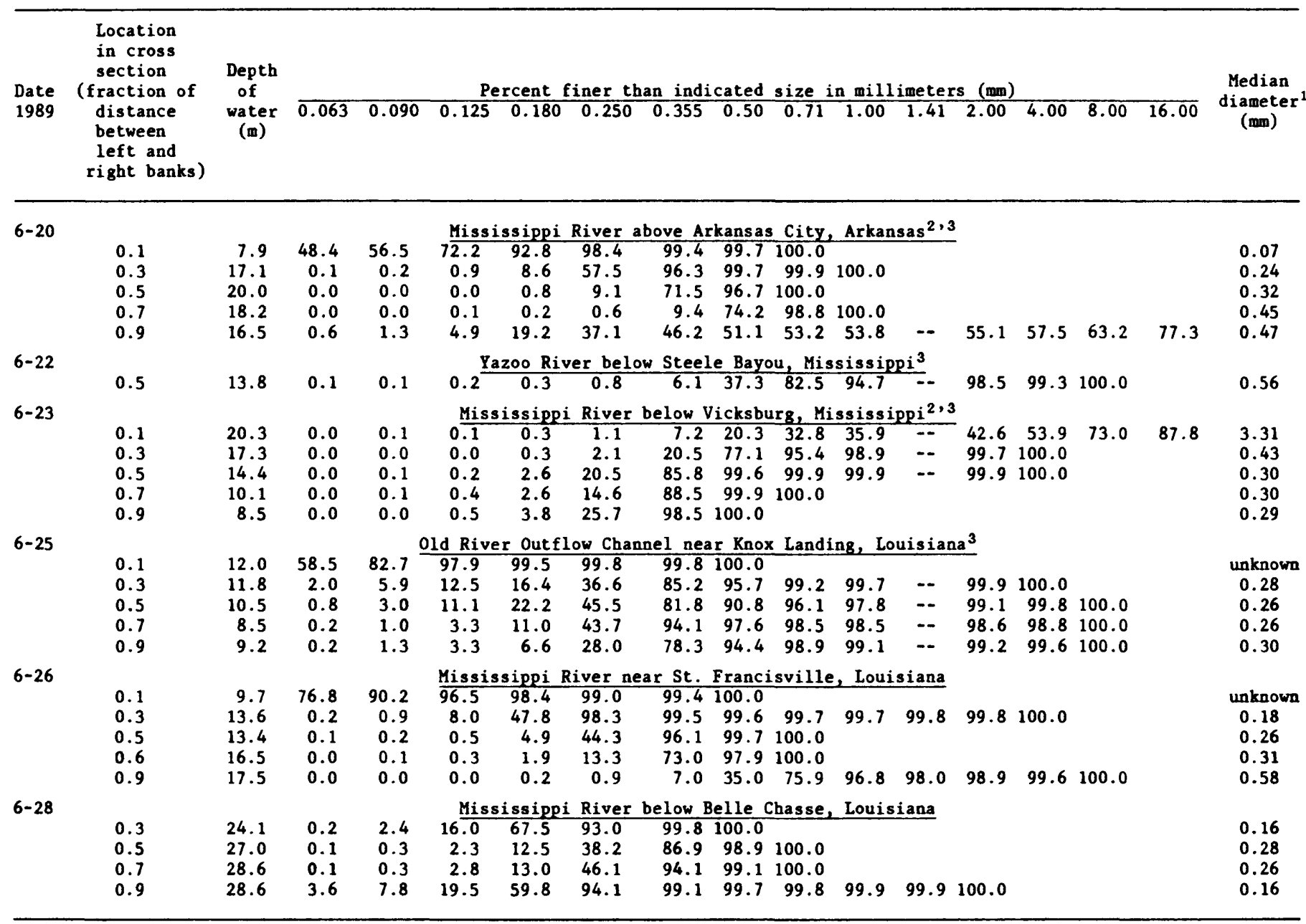

Median diameter determined by straight-line interpolation.

${ }^{2}$ Percent finer than $32 \mathrm{~mm}$ is 100.0 .

${ }^{3}$ The $1.41-\mathrm{mm}$ sieve was not used. 
Table 5.--Particle size, determined by sieving, of bed material collected from the Mississippi River and some of its tributaries for February-March 1990 cruise

[Analyses by U.S. Geological Survey Sediment Laboratory in Iowa City, Iowa; m, meter; m, millimeter]

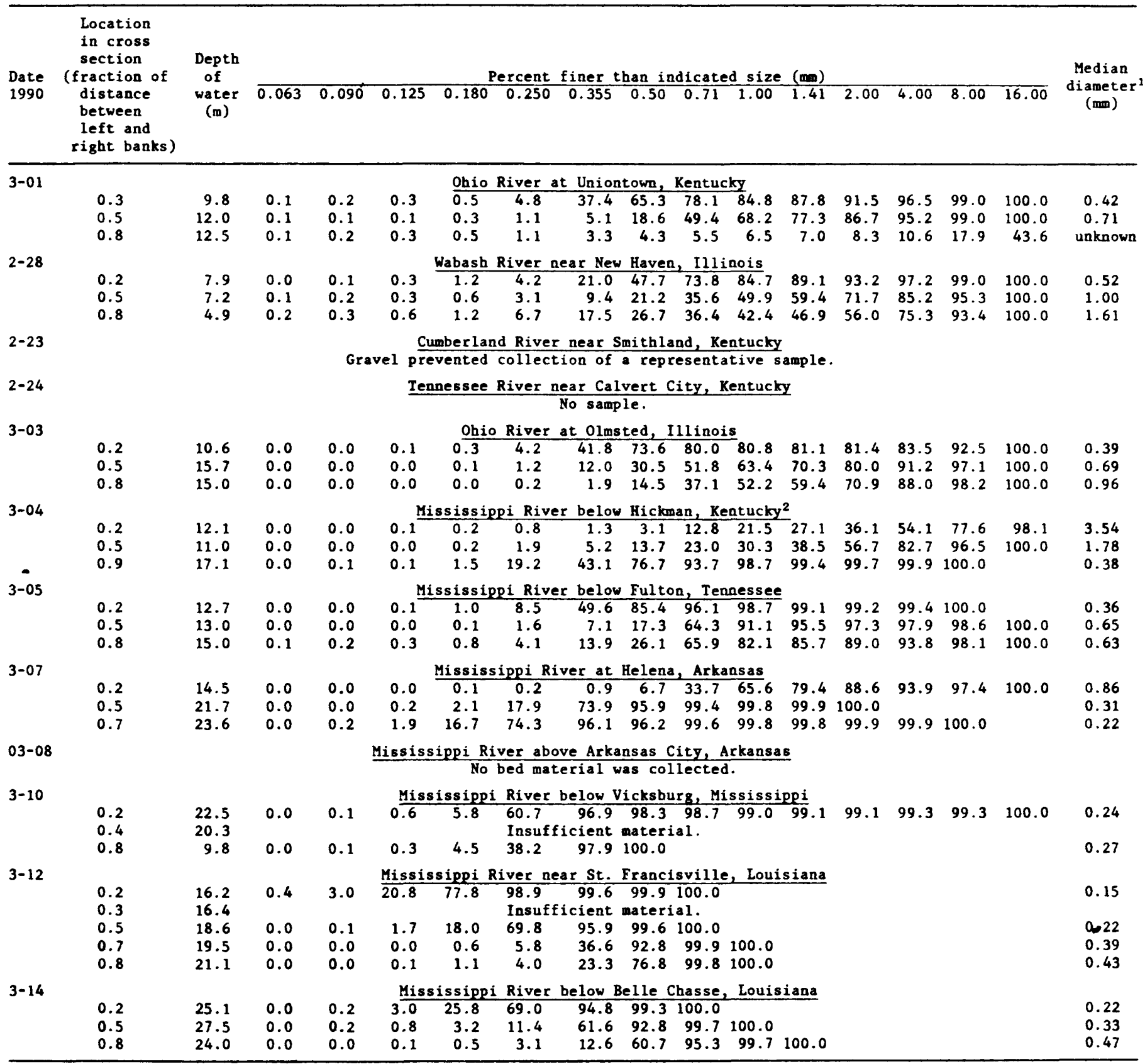

${ }^{1}$ Median diameter determined by straight-line interpolation.

${ }^{2}$ Percent finer than $32 \mathrm{~mm}$ is 100.0 . 
Table 6.--Particle size, determined by sieving, of bed material collected from the Mississippi

River and some if its tributaries for the second leg of the May-June 1990 cruise

[Analyses by U.S. Geological Survey Sediment Laboratory in Iowa City, Iowa; meter, mm, millimeter]

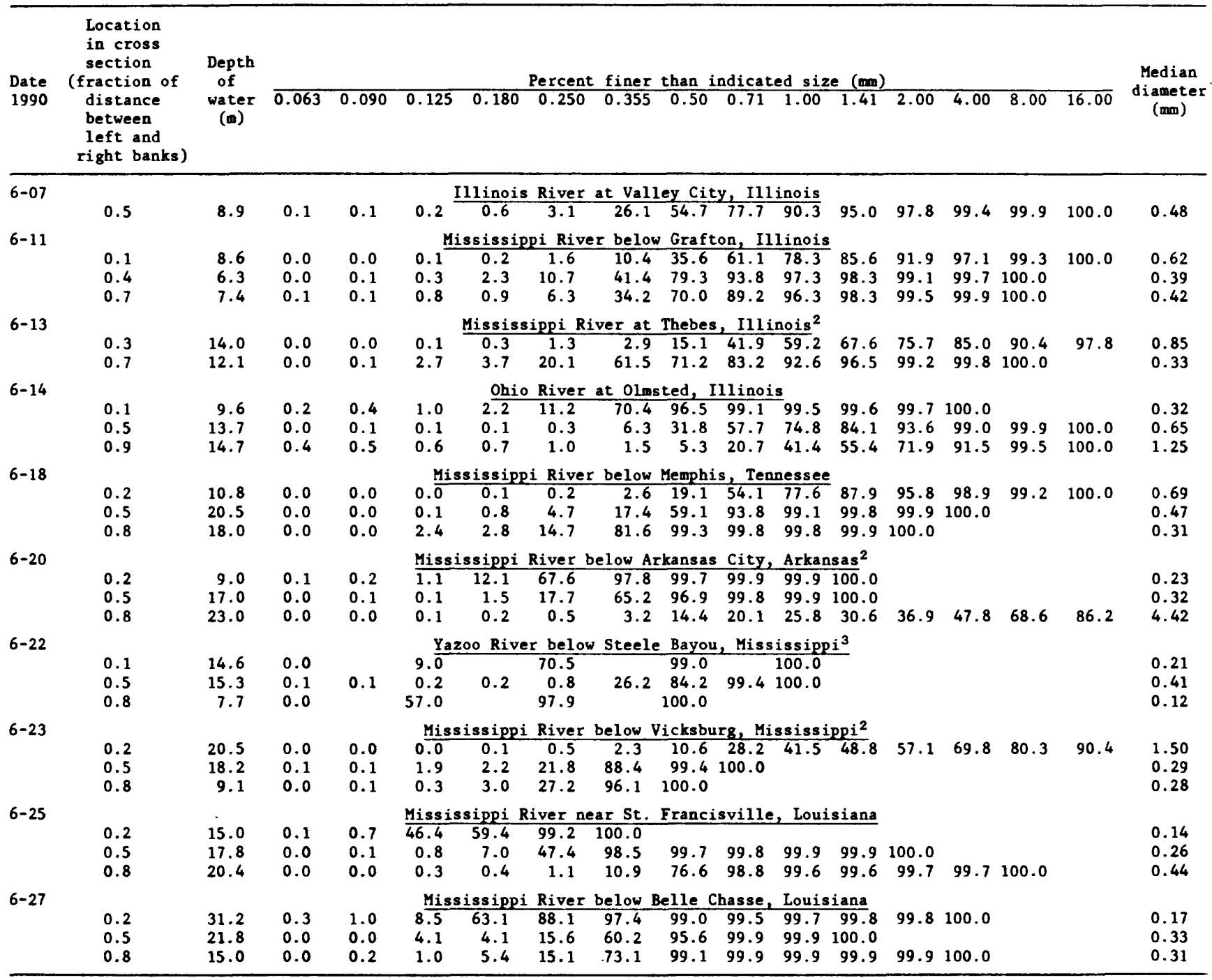

${ }^{1}$ Median diameter determined by straight-line interpolation.

${ }^{2}$ Percent finer than $32 \mathrm{~mm}$ is 100.0 .

Samples at depths of 14.6 and 7.7 were too small for sieving. Particle size was measured with the visual-accumulation tube and only whole phi sizes were determined. 


\section{Water Discharge}

The depth-averaged velocity was measured by depth integration from the river bottom to the river surface at several locations (referred to as verticals) across the river. The current meter (a Price AA with a solid, polymer bucket wheel; fig. 7) and suspended-sediment sampler were first lowered to the bottom at a constant transit rate. The depth-averaged velocity was determined only during the upcast because the current meter was not in the turbulent wake of the suspended-sediment sampler bottle. Ideally, for discharge measurements, the transit rate should be as slow as possible so that the integration time is as long as possible. However, at very slow transit rates the suspended-sediment sampler would overfill. The transit rate was therefore determined by the requirements for collecting a total composite sample of 100 to $130 \mathrm{~L}$ of water and suspended sediment (see Nordin and others, 1983) and keeping the transit rate less than 20 percent of the mean velocity (based on unpublished data for the Amazon River) in order to obtain a reliable measurement of velocity and hence discharge.

Because the ship usually was not anchored during the vertical sampling procedure, the measured velocities were corrected for ship drift according to procedures described by Moody and Troutman (1992). Two microwave transmitter/receiver stations on shore and a master station (Del Norte Technology Trisponder system) aboard the ship were used to measure the change in upriver-downriver and cross-river position. The mean depth was obtained from the fathometer, which produced a continuous strip-chart record of depth during the upcast. In the Mississippi River, the depth-averaged velocity was measured at 13 to 36 equally spaced vertical locations across the river. In most tributaries, which were narrower than the Mississippi River, the depth-averaged velocity was measured at 5 to 14 vertical locations across the river. At some sampling sites, especially those where cross sections were asymmetrical and the discharge per unit width was large, additional verticals were occupied in order to measure additional velocities. At these additional verticals, even though no suspended-sediment sample was collected, a sample bottle was put in the holder so that the current-meter calibration was not altered. The calibration equation had a standard error less than $1 \mathrm{~cm} / \mathrm{s}$.

This method of measuring discharge has associated bias and standard errors, as discussed by Moody and Troutman (1992). Most of the error in the discharge measurement results from errors in measuring the depth-averaged velocity. The major bias is the incomplete depth-integration error (2-5 percent) incurred because the measurement of velocity is started slightly above the bottom (see $h$ in fig. 7). This error can be estimated by assuming a theoretical velocity profile dependent upon the roughness length scale, $z_{0}$, the water depth, D, and the height, $h$, of the unmeasured zone. The major source of standard error comes from the natural variability (which is assumed to be random) of river velocities. The standard error of the natural variability is about 5-12 percent of the mean velocity. Typical standard errors in discharge are between 1 and 2 percent. Because the total discharge is essentially a statistically weighted mean of the random velocities, the total discharge error is reduced by the averaging process. The bias and standard errors have been estimated for each discharge measurement according to the methods discussed by Moody and Troutman (1992) (see tables 7-10). The estimated bias error generally is positive (overestimated) because the 
velocities in the unmeasured zone near the bottom (where velocities are lowest) have not been included in determining the mean velocity. However, at some sections where only a few verticals were measured, this positive bias error may be smaller than the negative bias error that results from neglecting the flow between the banks and halfway out to the first or last vertical; thus the total bias error is negative (for example see White River, tables 7 and 8 , and Mississippi River near Cache, I11., in table 9). The standard errors in discharge were 5 percent or less and generally were 1 or 2 percent.

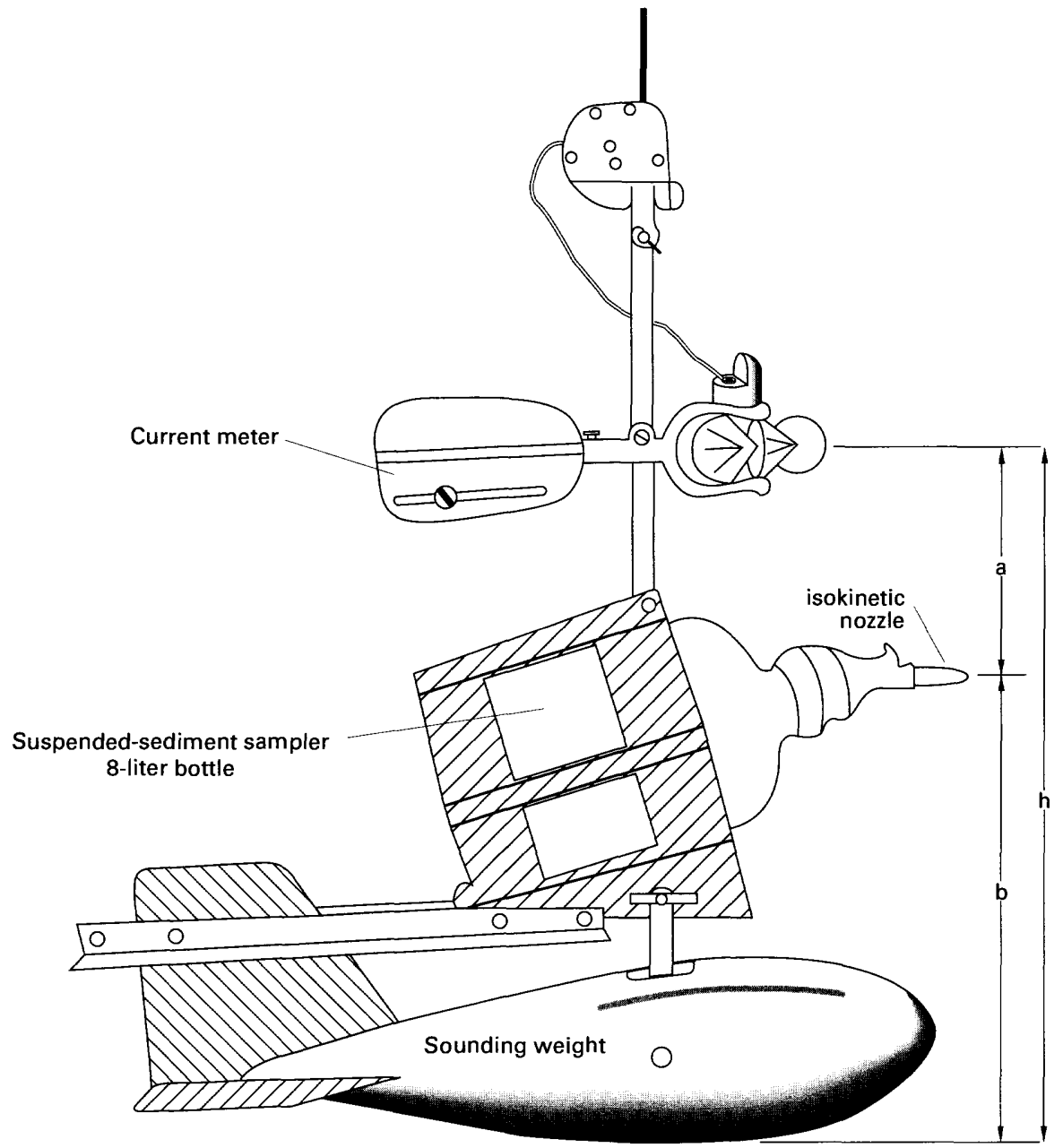

\begin{tabular}{|c|c|c|c|}
\hline WEIGHT, IN POUNDS & a & $\mathrm{b}$ & $\mathrm{h}$ \\
\hline \multirow{2}{*}{150} & 22 & 42 & 64 \\
200 & 22 & 45 & 67 \\
300 & 22 & 48 & 70 \\
\hline
\end{tabular}

Figure 7.--Suspended-sediment sampler with current meter. No water velocity is measured in a zone of height, $h$, above the bottom. 
Table 7.--Summary of discharge measurements made of the Mississippi River and some of its tributaries using the depth-integration method for March-April 1989 cruise

[All velocity measurements were made with a Price AA solid, polymer bucket-wheel current meter with calibration equation: velocity $(\mathrm{m} / \mathrm{s})=0.765 \times$ revolutions per second +0.006 ; NC indicates that discharge was measured by a different method and errors were not calculated; $m$, meter; $m^{2}$, square meter; $\mathrm{m} / \mathrm{s}$, meter per second; $\mathrm{m}^{3} / \mathrm{s}$, cubic meter per second; $\%$, percent]

\begin{tabular}{|c|c|c|c|c|c|c|c|c|}
\hline \multirow{3}{*}{$\begin{array}{l}\text { Date } \\
1989\end{array}$} & \multirow{3}{*}{ Site name } & \multirow{3}{*}{$\begin{array}{l}\text { Width } \\
\text { (m) }\end{array}$} & \multirow{3}{*}{$\begin{array}{l}\text { Area } \\
\left(\mathrm{m}^{2}\right)\end{array}$} & \multirow{3}{*}{$\begin{array}{l}\text { Mean } \\
\text { depth } \\
(m)\end{array}$} & \multirow{3}{*}{$\begin{array}{l}\text { Mean } \\
\text { velocity } \\
(\mathbf{m} / \mathbf{s})\end{array}$} & \multicolumn{3}{|c|}{ Discharge } \\
\hline & & & & & & \multirow{2}{*}{$\begin{array}{c}\text { Magnitude } \\
\left(\mathrm{m}^{3} / \mathrm{s}\right)\end{array}$} & \multicolumn{2}{|c|}{ Errors } \\
\hline & & & & & & & $\begin{array}{l}\overline{\text { Bias }} \\
(\%)\end{array}$ & $\begin{array}{c}\text { Standard } \\
(\%)\end{array}$ \\
\hline $3-10$ & Mississippi $R$, near Winfield, Mo. & 552 & 2,930 & 5.3 & 0.29 & 850 & 9 & 2 \\
\hline 3-09 & Illinois R. at Hardin, Ill. ${ }^{1}$ & 269 & 1,090 & 4.1 & 0.38 & 410 & NC & NC \\
\hline $3-12$ & Missouri R. at Hermann, Mo. & 270 & 1,220 & 4.5 & 1.21 & 1,480 & 7 & 2 \\
\hline 3-13 & Mississippi R. at St. Louis, Mo. & 490 & 3,380 & 6.9 & 1.16 & 3,940 & 6 & 1 \\
\hline $3-15$ & Mississippi R. at Thebes, Ill. & 602 & 4,390 & 7.3 & 1.11 & 4,890 & 6 & 1 \\
\hline $3-16$ & Ohio R. at Olmsted, III. & 1,070 & 14,690 & 13.7 & 1.39 & 20,400 & 4 & 1 \\
\hline $3-17$ & Mississippi R. below Hickman, Ky. & 1,165 & 14,960 & 12.8 & 1.65 & 24,700 & 4 & 1 \\
\hline $3-19$ & Mississippi R. below Fulton, Tenn. & 1,337 & 16,210 & 12.1 & 1.53 & 24,800 & 4 & 1 \\
\hline 3-21 & Mississippi $R$. at Helena, Ark. & 913 & 15,090 & 16.5 & 1.72 & 25,900 & 4 & 1 \\
\hline $3-22$ & $\begin{array}{l}\text { White } R \text { at Mile } 11.5 \text {, Ark. } \\
\text { estimate of in-channel flow, } \\
\text { no estimate of overbank flow }\end{array}$ & 200 & 2,060 & 10.3 & 0.73 & 1,500 & -3 & 4 \\
\hline $3-23$ & Arkansas R. at Pendleton, Ark. & 380 & 2,690 & 7.1 & 0.71 & 1,900 & 6 & 2 \\
\hline $3-24$ & Mississippi R. above Arkansas City, Ark. & 1,035 & 16,420 & 15.9 & 1.63 & 26,800 & 3 & 1 \\
\hline $3-26$ & Yazoo R. below Steele Bayou, Miss. & 182 & 1,890 & 10.4 & 0.79 & 1,500 & 4 & 2 \\
\hline $3-27$ & Mississippi R. below Vicksburg, Miss. & 1,210 & 17,510 & 14.5 & 1.52 & 26,600 & 3 & 1 \\
\hline 3-29 & $\begin{array}{l}\text { Old River Outflow Channel near } \\
\text { Knox Landing, La. }\end{array}$ & 556 & 6,180 & 11.1 & 1.00 & 6,160 & 5 & 1 \\
\hline $3-30$ & Mississippi R. near St. Francisville, La. & 1,011 & 15,710 & 15.5 & 1.47 & 23,100 & 3 & 1 \\
\hline 4-01 & Mississippi R. below Belle Chasse, La. & 800 & 17,190 & 21.5 & 1.31 & 22,500 & 2 & 1 \\
\hline
\end{tabular}

${ }^{1}$ Water and suspended sediment were collected from 1 vertical in midriver while the water discharge was measured at 27 verticals. 
Table 8.--Sumary of discharge measurements made of the Mississippi River and some of its tributaries using the depth-integration method for June 1989 cruise

[All velocity measurements were made with a Price AA solid, polymer bucket-wheel current meter with calibration equation: velocity $(\mathrm{m} / \mathrm{s})=0.765 \times$ revolutions per second $+0.006 ; \mathrm{m}$, meter; $\mathrm{m}^{2}$, square meter; $\mathrm{m} / \mathrm{s}$, meter per second; $\mathrm{m}^{3} / \mathrm{s}$, cubic meter per second; $\%$, percent]

\begin{tabular}{|c|c|c|c|c|c|c|c|c|}
\hline \multirow{3}{*}{$\begin{array}{l}\text { Date } \\
1989\end{array}$} & \multirow{3}{*}{ Site name } & \multirow{3}{*}{$\begin{array}{l}\text { Width } \\
\text { (m) }\end{array}$} & \multirow{3}{*}{$\begin{array}{l}\text { Area } \\
\left(m^{2}\right)\end{array}$} & \multirow{3}{*}{$\begin{array}{l}\text { Mean } \\
\text { depth } \\
(\mathbf{m})\end{array}$} & \multirow{3}{*}{$\begin{array}{l}\text { Mean } \\
\text { velocity } \\
(\mathrm{m} / \mathrm{s})\end{array}$} & \multicolumn{3}{|c|}{ Discharge } \\
\hline & & & & & & \multirow{2}{*}{$\begin{array}{l}\text { Magnitude } \\
\left(\mathrm{m}^{3} / \mathrm{s}\right)\end{array}$} & \multicolumn{2}{|c|}{ Errors } \\
\hline & & & & & & & $\begin{array}{l}\text { Bias } \\
(\%)\end{array}$ & $\begin{array}{l}\text { Standard } \\
\text { (\%) }\end{array}$ \\
\hline $\begin{array}{l}6-05 \\
6-04 \\
6-07 \\
6-08 \\
6-09\end{array}$ & $\begin{array}{l}\text { Mississippi R. near Winfield, Mo. } \\
\text { Illinois } R \text {. at Hardin, Ill. } \\
\text { Missouri R. at Hermann, Mo. } \\
\text { Mississippi R. at St. Louis, Mo. } \\
\text { Mississippi R. at St. Louis, Mo. } \\
\text { based on } 13 \text { verticals }\end{array}$ & $\begin{array}{l}562 \\
273 \\
270 \\
508 \\
508\end{array}$ & $\begin{array}{l}3,460 \\
1,110 \\
1,400 \\
3,890 \\
3,920\end{array}$ & $\begin{array}{l}6.2 \\
4.1 \\
5.2 \\
7.7 \\
7.7\end{array}$ & $\begin{array}{l}0.67 \\
0.70 \\
1.26 \\
1.22 \\
1.31\end{array}$ & $\begin{array}{r}2,320 \\
780 \\
1,760 \\
4,760 \\
5,120\end{array}$ & $\begin{array}{l}6 \\
2 \\
7 \\
5 \\
4\end{array}$ & $\begin{array}{l}1 \\
4 \\
2 \\
1 \\
2\end{array}$ \\
\hline $\begin{array}{l}6-10 \\
6-11 \\
6-12 \\
6-13\end{array}$ & $\begin{array}{l}\text { Mississippi R. at Thebes, Ill. } \\
\text { Ohio R. at Olmsted, Ill. } \\
\text { Mississippi R. below Hickman, Ky. } \\
\text { Mississippi R. below Hickman, Ky. } \\
\text { based on } 17 \text { verticals }\end{array}$ & $\begin{array}{r}609 \\
1,008 \\
1,135 \\
1,135\end{array}$ & $\begin{array}{r}4,020 \\
9,390 \\
10,640 \\
10,540\end{array}$ & $\begin{array}{l}6.6 \\
9.3 \\
9.4 \\
9.3\end{array}$ & $\begin{array}{l}1.30 \\
0.94 \\
1.33 \\
1.32\end{array}$ & $\begin{array}{r}5,230 \\
8,760 \\
14,100 \\
13,900\end{array}$ & $\begin{array}{l}5 \\
4 \\
4 \\
4\end{array}$ & $\begin{array}{l}1 \\
1 \\
1 \\
2\end{array}$ \\
\hline $6-14$ & Mississippi R. at Fulton, Tenn. 1 & 1,102 & 10,900 & 9.9 & 1.40 & 15,300 & 4 & 1 \\
\hline $\begin{array}{l}6-17 \\
6-18 \\
6-19 \\
6-20 \\
6-22\end{array}$ & $\begin{array}{l}\text { Mississippi R. at Helena, Ark. } \\
\text { White R. at Mile } 11.5 \text {, Ark. }{ }^{2} \\
\text { Arkansas R. at Pendleton, Ark. } \\
\text { Mississippi R. above Arkansas City, Ark. } \\
\text { Yazoo R. below Steele Bayou, Miss. }\end{array}$ & $\begin{array}{l}897 \\
195 \\
391 \\
990 \\
165\end{array}$ & $\begin{array}{r}11,750 \\
1,460 \\
2,810 \\
14,960 \\
1,520\end{array}$ & $\begin{array}{r}13.1 \\
7.5 \\
7.2 \\
15.1 \\
9.2\end{array}$ & $\begin{array}{l}1.44 \\
0.53 \\
1.28 \\
1.56 \\
0.70\end{array}$ & $\begin{array}{r}16,900 \\
770 \\
3,600 \\
23,300 \\
1,070\end{array}$ & $\begin{array}{r}4 \\
-3 \\
5 \\
3 \\
3\end{array}$ & $\begin{array}{l}1 \\
4 \\
1 \\
1 \\
3\end{array}$ \\
\hline $\begin{array}{l}6-23 \\
6-25\end{array}$ & $\begin{array}{l}\text { Mississippi R. below Vicksburg, Miss. } \\
\text { Old River Outflow Channel near } \\
\text { Knox Landing, La. }\end{array}$ & $\begin{array}{r}1,222 \\
562\end{array}$ & $\begin{array}{r}16,180 \\
5,410\end{array}$ & $\begin{array}{r}13.2 \\
9.6\end{array}$ & $\begin{array}{l}1.53 \\
0.90\end{array}$ & $\begin{array}{r}24,800 \\
4,890\end{array}$ & $\begin{array}{l}3 \\
5\end{array}$ & $\begin{array}{l}1 \\
1\end{array}$ \\
\hline $\begin{array}{l}6-26 \\
6-28\end{array}$ & $\begin{array}{l}\text { Mississippi R. near St. Francisville, La. } \\
\text { Mississippi R. below Belle Chasse, La. }\end{array}$ & $\begin{array}{r}1,020 \\
813\end{array}$ & $\begin{array}{l}14,540 \\
17,750\end{array}$ & $\begin{array}{l}14.3 \\
21.8\end{array}$ & $\begin{array}{l}1.31 \\
1.13\end{array}$ & $\begin{array}{l}19,000 \\
20,100\end{array}$ & $\begin{array}{l}3 \\
3\end{array}$ & $\begin{array}{l}1 \\
1\end{array}$ \\
\hline
\end{tabular}

${ }^{1}$ Estimates of width, area, and discharge in an overflow channel are included in the width, area, and magnitude of discharge listed here.

${ }^{2}$ Based on 7 verticals plus 23 depths from depth profile and assuming unit discharge is proportional to depth to $5 / 3$ power. 
Table 9.--Summary of discharge measurements made of the Mississippi River and some of its tributaries using the depth-integration method for February-March 1990 cruise

[All velocity measurements were made with. a Price AA solid, polymer bucket-wheel current meter with calibration equation: velocity $(\mathrm{m} / \mathrm{s})=0.744 \times$ revolutions per second $+0.011 ; \mathrm{m}$, meter; $\mathrm{m}^{2}$, square meter; $\mathrm{m} / \mathrm{s}$, meter per second; $\mathrm{m}^{3} / \mathrm{s}$, cubic meter per second; $\%$, percent]

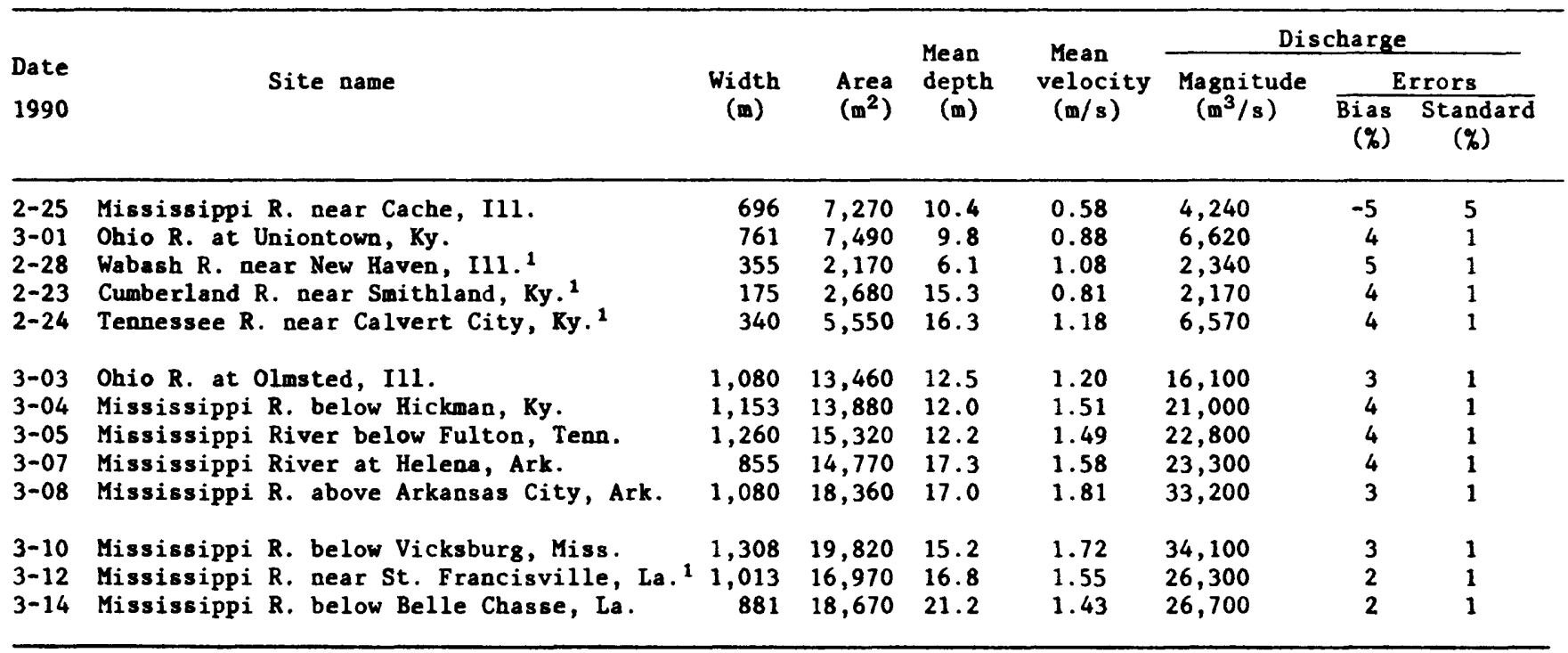
made.

${ }^{1}$ Discharge was measured from left edge of flow to right edge of flow. No overbank measurement was

Table 10.--Summary of discharge measurements made of the Mississippi River and some of its tributaries using the depth-integration method for May-June 1990 cruise

[All velocity measurements were made with a Price AA solid, polymer bucket-wheel current meter with calibration equations: velocity $(\mathrm{m} / \mathrm{s})=0.744 \times$ revolutions per second +0.011 from $6-07$ through $6-18$, and velocity $(\mathrm{m} / \mathrm{s})=0.755 \times$ revolutions per second +0.011 from $6-20$ through $6-27$; m, meter; $\mathrm{m}^{2}$, square meter; $\mathrm{m} / \mathrm{s}$, meter per second; $\mathrm{m}^{3} / \mathrm{s}$, cubic meter per second; $\%$, percent]

\begin{tabular}{|c|c|c|c|c|c|c|c|c|}
\hline \multirow{3}{*}{$\begin{array}{l}\text { Date } \\
1990\end{array}$} & \multirow{3}{*}{ Site name } & \multirow{3}{*}{$\begin{array}{l}\text { Width } \\
\text { (m) }\end{array}$} & \multirow{3}{*}{$\begin{array}{c}\text { Area } \\
\left(m^{2}\right)\end{array}$} & \multirow{3}{*}{$\begin{array}{l}\text { Mean } \\
\text { depth } \\
(\mathrm{m})\end{array}$} & \multirow{3}{*}{$\begin{array}{l}\text { Mean } \\
\text { velocity } \\
(\mathrm{m} / \mathrm{s})\end{array}$} & \multicolumn{3}{|c|}{ Discharge } \\
\hline & & & & & & \multirow{2}{*}{$\begin{array}{c}\text { Magnitude } \\
\left(\mathrm{m}^{3} / \mathrm{s}\right)\end{array}$} & \multicolumn{2}{|c|}{ Errors } \\
\hline & & & & & & & $\begin{array}{r}\text { Bias } \\
(\%)\end{array}$ & $\begin{array}{l}\text { Standard } \\
(\%)\end{array}$ \\
\hline $\begin{array}{l}6-07 \\
6-11 \\
6-13 \\
5-31 \\
5-31\end{array}$ & $\begin{array}{l}\text { Illinois R. at Valley City, Ill. } \\
\text { Mississippi R. below Grafton, III. } \\
\text { Mississippi R. at Thebes, III. } \\
\text { Mississippi R. near Cairo, Ill. } \\
\text { Ohio R. at Olmsted, Ill. }\end{array}$ & $\begin{array}{r}221 \\
941 \\
623 \\
696 \\
1,098\end{array}$ & $\begin{array}{r}1,370 \\
6,060 \\
7,120 \\
8,490 \\
15,410\end{array}$ & $\begin{array}{r}6.2 \\
6.4 \\
11.4 \\
12.2 \\
14.0\end{array}$ & $\begin{array}{l}0.90 \\
0.83 \\
1.77 \\
1.60 \\
1.01\end{array}$ & $\begin{array}{r}1,230 \\
5,040 \\
12,600 \\
13,600 \\
15,600\end{array}$ & $\begin{array}{r}5 \\
5 \\
4 \\
-2 \\
5\end{array}$ & $\begin{array}{l}2 \\
1 \\
1 \\
4 \\
2\end{array}$ \\
\hline $\begin{array}{l}6-14 \\
5-30 \\
6-01 \\
6-04 \\
6-01\end{array}$ & $\begin{array}{l}\text { Ohio R. at Olmsted, Ill. } \\
\text { Mississippi R. at Wickliffe, Ky. } \\
\text { Mississippi R. at Wickliffe, Ky. } \\
\text { Mississippi R. at Wickliffe, Ky. } \\
\text { Mississippi R. near Columbus, Ky. }\end{array}$ & $\begin{array}{r}1,046 \\
827 \\
827 \\
827 \\
912\end{array}$ & $\begin{array}{l}12,310 \\
13,940 \\
13,870 \\
14,120 \\
14,240\end{array}$ & $\begin{array}{l}11.8 \\
16.9 \\
16.8 \\
17.1 \\
15.6\end{array}$ & $\begin{array}{l}0.78 \\
2.17 \\
2.14 \\
2.04 \\
2.06\end{array}$ & $\begin{array}{r}9,550 \\
30,300 \\
29,700 \\
28,800 \\
29,400\end{array}$ & $\begin{array}{l}6 \\
2 \\
2 \\
2 \\
2\end{array}$ & $\begin{array}{l}1 \\
2 \\
2 \\
2 \\
2\end{array}$ \\
\hline $\begin{array}{l}6-02 \\
6-02 \\
6-03 \\
6-18 \\
6-20\end{array}$ & $\begin{array}{l}\text { Mississippi } R \text {. above New Madrid, Mo. } \\
\text { Mississippi R. near Pt. Pleasant, Mo. } \\
\text { Mississippi R. at Caruthersville, Mo. } \\
\text { Mississippi R. below Memphis, Tenn. } \\
\text { Mississippi R. below Arkansas City, Ark. }\end{array}$ & $\begin{array}{r}1,060 \\
1,350 \\
849 \\
1,002 \\
1,055\end{array}$ & $\begin{array}{l}17,690 \\
17,910 \\
13,010 \\
14,130 \\
15,840\end{array}$ & $\begin{array}{l}16.7 \\
13.3 \\
15.3 \\
14.1 \\
15.0\end{array}$ & $\begin{array}{l}1.69 \\
1.62 \\
2.23 \\
1.47 \\
1.61\end{array}$ & $\begin{array}{l}29,900 \\
29,000 \\
29,000 \\
20,800 \\
25,500\end{array}$ & $\begin{array}{l}2 \\
2 \\
1 \\
4 \\
3\end{array}$ & $\begin{array}{l}2 \\
2 \\
3 \\
1 \\
1\end{array}$ \\
\hline $\begin{array}{l}6-22 \\
6-23 \\
6-25 \\
6-27\end{array}$ & $\begin{array}{l}\text { Yazoo R. below Steele Bayou, Miss. } \\
\text { Mississippi R. below Vicksburg, Miss. } \\
\text { Mississippi R. near St. Francisville, La. } \\
\text { Mississippi R. below Belle Chasse, La. }\end{array}$ & $\begin{array}{r}200 \\
1,255 \\
1,025 \\
845\end{array}$ & $\begin{array}{r}1,950 \\
18,750 \\
16,340 \\
16,900\end{array}$ & $\begin{array}{r}9.8 \\
14.9 \\
15.9 \\
20.0\end{array}$ & $\begin{array}{l}0.64 \\
1.46 \\
1.42 \\
1.38\end{array}$ & $\begin{array}{r}1,250 \\
27,300 \\
23,200 \\
23,300\end{array}$ & $\begin{array}{l}4 \\
3 \\
3 \\
3\end{array}$ & $\begin{array}{l}2 \\
1 \\
1 \\
1\end{array}$ \\
\hline
\end{tabular}




\section{Water and Suspended Sediment}

Two methods were used for collecting composite water and suspendedsediment samples. One method was the pumping method from fixed depths, which yielded large volumes $(300-700 \mathrm{~L})$ of water and a representative sample of particles finer than $63 \mu \mathrm{m}$. The other method was the depth-integration method, which yielded about 100-130 L of water and representative samples of both sand (greater than $63 \mu \mathrm{m}$ ) and silt/clay fractions (finer than $63 \mu \mathrm{m}$ ) of suspended sediment. Different procedures were used to dewater the pumped and depth-integrated composite samples after collection.

\section{Pumped Composite Sample}

The pumped composite sample was collected by using a 12-mm-diameter, perfluorinated-alkoxy (PFA) Teflon tube inside a 12.7-mm-diameter, double-braided stainless-steel hose, which was attached to a 300-pound sounding weight to keep the nozzle directed into the current. A hydraulic winch was used to lower the nozzle and sounding weight at each vertical to a depth of $5 \mathrm{~m}$ below the surface or one-half the water depth (whichever was less). Water was pumped from the fixed depth by a double-diaphragm pump (Wilden MI/UP/TF/TF/TF), driven by compressed air and constructed entirely of PFA Teflon, through a $63-\mu \mathrm{m}$ sieve and into a calibrated funnel (45-L upside-down carboy with no bottom).

The sand that collected in the $63-\mu \mathrm{m}$ sieve was called the pumped, composite sand sample, and the water and remaining suspended sediment was called the pumped, composite silt/clay and colloid sample. The volume of river water that was pumped at each vertical was proportional to the estimated fractional water discharge at each vertical. The following steps were used to determine the volume of water to be pumped at each vertical:

1. Determine the depth of each vertical (where water will be pumped) from the continuous strip-chart record of the bottom provided by the fathometer.

2. Measure a few depth-averaged velocities at three to five locations with different depths.

3. Calculate the proportionality constant relating depth-averaged velocity to the depth to the $2 / 3$ power (Manning's formula, Chow, 1959; Sium, 1975; and Sayre and Caro-Cordero, 1977).

4. Interpolate the value of the proportionality constant at verticals (where water will be pumped) between the three to five locations where depth-averaged velocities were measured.

5. Compute the discharge, centered about each vertical, and its fraction of the total discharge.

6. Calculate the volume to be pumped at each vertical as the fraction of the total discharge at each vertical times the total volume to be pumped.

The total volume of river water collected by the pumping method is listed in tables 11 and 12 for the March-April 1989 cruise, in tables 13 and 14 for the June 1989 cruise, in tables 15 and 16 for the February-March 1990 cruise, and in tables 17 and 18 for the second leg of the May-June 1990 cruise. 
The water and suspended-sediment mixture in the funnel was processed by allowing it to drain at $2 \mathrm{~L} / \mathrm{min}$ into a continuous-flow centrifuge (Sharples, model AS-12; see Leenheer and others, 1989, and Rees and others, 1991, for more details) spinning at about $16,000 \mathrm{r} \cdot \mathrm{p} . \mathrm{m}$. The sediment that was retained on the centrifuge liner was called the pumped, composite silt/clay sample. The water and remaining sediment was processed further by passing it through an ultrafiltration unit with pore sizes of $0.005 \mu \mathrm{m}$. The sediment retained on the membranes of the ultrafilter was called the pumped, composite colloid sample. The mass of silt/clay and colloid material that was recovered is listed in table 12 for the March-April 1989 cruise, in table 14 for the June 1989 cruise, in table 16 for the February-March 1990 cruise, and in table 18 for the second leg of the May-June 1990 cruise.

\section{Depth-Integrated Composite Sample}

The equal-width-increment (equal-transit-rate), depth-integration method (Nordin and others, 1983; Richey and others, 1986; Meade and Stevens, 1990) was used to collect a depth-integrated composite sample of water and suspended sediment from 1 to 36 vertical locations across the river. A collapsible-bag sampler (Stevens and others, 1980) fitted with an isokinetic nozzle (Guy and Norman, 1970, p. 8-10) collected suspended sediment in sand, silt, and clay sizes (see tables $11,13,15$, and 17 for nozzle sizes). The sampler was held in a frame so that the nozzle was horizontal, faced into the current, and was about $45 \mathrm{~cm}$ above the bottom of a sounding weight (fig. 7). A Price AA current meter with a solid, polymer bucket wheel was mounted $22 \mathrm{~cm}$ above the nozzle of the sampler. The current meter was used to measure the depth-averaged velocity and to determine the nozzle efficiency (volume of water collected divided by the mean velocity, the cross-sectional area of the nozzle, and the integration time). The vertical transit rate was controlled by a hydraulic winch, which produced a constant rate for the downcast and for the upcast. The multiple gears on the hydraulic system allowed transit rates as slow as $2 \mathrm{~cm} / \mathrm{s}$ and as fast as $22 \mathrm{~cm} / \mathrm{s}$. The same transit rate was reproduced within $1 \mathrm{~cm} / \mathrm{s}$ for each vertical across the river and was verified with a hand-held tachometer at the beginning of each downcast and at the beginning of each upcast.

Two 8-L plastic bottles, identified as $A$ and $B$, with collapsible, perfluorinated-alkoxy (PFA) Teflon bags and fluorinated-ethylene-propylene (FEP) Teflon nozzles were used in the isokinetic sampler to collect individual samples at alternate verticals, which were combined into two separate, depth-integrated, composite samples. Generally, bottle A was used to collect samples at the odd-numbered verticals and bottle $B$ at the even-numbered verticals. The $A$ and $B$ samples were poured into separate glass, graduated cylinders through separate, nickel-mesh, $63-\mu \mathrm{m}$ sieves to remove the sand. This sand was saved and called the depth-integrated, composite sand sample. The water volume (see section "Tabulated Cruise Data") collected at each individual vertical was measured and then composited in two separate Tefloncoated, stainless-steel, churn splitters labeled A and B (see tables 11, 13, 15, and 17 for total volume of water collected). The only significant

difference between these procedures and those described by Leenheer and others (1989) and Moody and Meade (1992) for the low-water cruises is the substitution of the stronger, but less flexible, PFA Teflon bags for the 
previously used FEP Teflon bags. Leenheer and others (1989) described the Teflon bags, nickel sieves, and churn splitters in more detail and discussed the evaluation of materials considered for the fabrication of each item so they would meet the diverse requirements for suspended-sediment, trace-organic, and trace-element analysis.

The remaining water and suspended sediment was processed further by keeping the suspended sediment in suspension by churning, and different-sized subsamples were taken from the $A$ and $B$ churn splitters for determining suspended-sediment concentration (150-250 $\mathrm{mL}$ ) and particle-size distribution (8-22 L) and for analysis of major ions, trace elements, organic compounds, and nutrients. The remaining water and suspended sediment in the $A$ and $B$ churn splitters were combined and processed through the continuous-flow centrifuge and ultrafilter (discussed above) to obtain a depth-integrated composite silt/clay sample and a depth-integrated composite colloid sample. The mass of silt/clay and colloid material recovered is listed in tables 12 , 14,16 , and 18, and a generalized flow diagram for processing the depthintegrated composite sample is in figure 8 .

This procedure was modified for the mixing study near the confluence of the Upper Mississippi and Ohio Rivers during the first leg of the May-June 1990 cruise. Instead of compositing the samples at each vertical, the individual sample (approximately $5 \mathrm{~L}$ ) from each vertical was put into a small churn splitter and subsamples were taken for determining suspended-sediment concentration and particle sizes and for analysis of major ions, trace elements, and selected organic compounds. No samples were processed through the centrifuge or ultrafilter. 


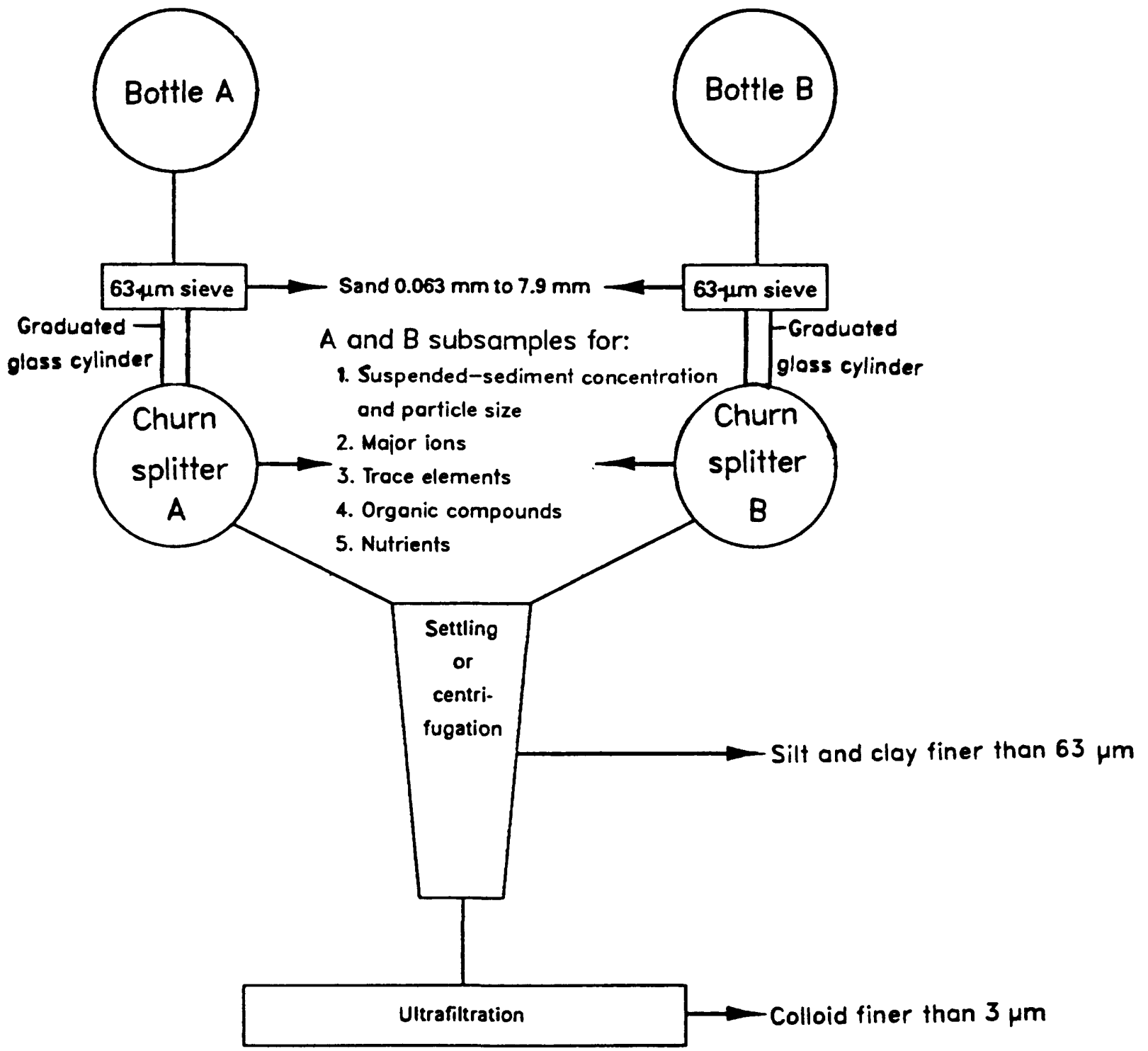

Figure 8.--Flow diagram for processing the suspended-sediment sample collected by depth-integration method. Abbreviations are $\mu \mathrm{m}$, micrometer and $\mathrm{mm}$, millimeters. 
Table 11.--Volumes of river water collected and processed and associated sampling parameters for March-April 1989 cruise

[ $V_{p}$ is the total volume collected by pumping; $V_{c}$ is the total volume collected by depth integration; $V_{d}$ is the total volume of depth-integrated sample that was dewatered by processing through the centrifuge and ultrafilter; L, liter; $\mathrm{cm} / \mathrm{s}$, centimeter per second]

\begin{tabular}{|c|c|c|c|c|c|c|c|}
\hline \multirow[b]{2}{*}{$\begin{array}{l}\text { Date } \\
1989\end{array}$} & \multirow[b]{2}{*}{ Site name } & \multicolumn{3}{|c|}{ Sample volumes } & \multicolumn{3}{|c|}{ Sampling parameters } \\
\hline & & $\begin{array}{l}V_{p} \\
(L)\end{array}$ & $\begin{array}{l}\mathrm{V}_{\mathrm{c}} \\
(\mathrm{L})\end{array}$ & $\begin{array}{l}\mathrm{v}_{\mathrm{d}} \\
(\mathrm{L})\end{array}$ & $\begin{array}{l}\text { Number } \\
\text { of } \\
\text { verticals }\end{array}$ & $\begin{array}{c}\text { Nozzle } \\
\text { size } \\
\text { (inches) }\end{array}$ & $\begin{array}{l}\text { Transit } \\
\text { rate } \\
(\mathrm{cm} / \mathrm{s})\end{array}$ \\
\hline $\begin{array}{l}3-10 \\
3-09 \\
3-12 \\
3-13 \\
3-15\end{array}$ & $\begin{array}{l}\text { Mississippi R. near Winfield, Mo. } \\
\text { Illinois R. at Hardin, Ill. } \\
\text { Missouri R. at Hermann, Mo. } \\
\text { Mississippi R. at St. Louis, Mo. } \\
\text { Mississippi R. at Thebes, Ill. }\end{array}$ & $\begin{array}{l}590 \\
597 \\
507 \\
500 \\
490\end{array}$ & $\begin{array}{l}119.4 \\
117.9 \\
127.1 \\
114.5 \\
133.0\end{array}$ & $\begin{array}{r}113.7 \\
93.7 \\
95.4 \\
95.7 \\
101.2\end{array}$ & $\begin{array}{r}120 \\
1 \\
30 \\
30 \\
27\end{array}$ & $\begin{array}{l}\text { none } \\
\text { none } \\
5 / 16 \\
1 / 4 \\
1 / 4\end{array}$ & $\begin{array}{r}6 \\
3-6 \\
11 \\
10 \\
8\end{array}$ \\
\hline $\begin{array}{l}3-16 \\
3-17 \\
3-19 \\
3-21 \\
3-22\end{array}$ & $\begin{array}{l}\text { Ohio R. at Olmsted, Ill. } \\
\text { Mississippi R. below Hickman, Ky. } \\
\text { Mississippi R. below Fulton, Tenn. } \\
\text { Mississippi R. at Helena, Ark. } \\
\text { White R. at Mile 11.5, Ark. }\end{array}$ & $\begin{array}{l}720 \\
505 \\
606 \\
499 \\
500\end{array}$ & $\begin{array}{l}112.9 \\
117.0 \\
101.3 \\
126.1 \\
108.6\end{array}$ & $\begin{array}{r}91.2 \\
98.3 \\
80.6 \\
107.3 \\
89.6\end{array}$ & $\begin{array}{r}30 \\
30 \\
30 \\
30 \\
5\end{array}$ & $\begin{array}{l}3 / 16 \\
3 / 16 \\
3 / 16 \\
3 / 16 \\
1 / 4\end{array}$ & $\begin{array}{r}16 \\
17 \\
17 \\
22 \\
6\end{array}$ \\
\hline $\begin{array}{l}3-23 \\
3-24 \\
3-26 \\
3-27 \\
3-29\end{array}$ & $\begin{array}{l}\text { Arkansas R. at Pendleton, Ark. } \\
\text { Mississippi R. above Arkansas City, Ark. } \\
\text { Yazoo R. below Steele Bayou, Miss. } \\
\text { Mississippi R. below Vicksburg, Miss. } \\
\text { Old River Outflow Channel near } \\
\text { Knox Landing, La. }\end{array}$ & $\begin{array}{l}600 \\
500 \\
500 \\
641 \\
503\end{array}$ & $\begin{array}{l}110.7 \\
116.7 \\
116.1 \\
113.9 \\
116.0\end{array}$ & $\begin{array}{r}98.8 \\
98.1 \\
109.4 \\
97.2 \\
97.3\end{array}$ & $\begin{array}{r}{ }^{3} 10 \\
30 \\
15 \\
36 \\
30\end{array}$ & $\begin{array}{c}1 / 4,5 / 16 \\
3 / 16 \\
1 / 4,3 / 16,5 / 16 \\
1 / 8 \\
1 / 4\end{array}$ & $\begin{array}{c}4-12 \\
22 \\
7-16 \\
11 \\
15\end{array}$ \\
\hline $\begin{array}{l}3-30 \\
4-01\end{array}$ & $\begin{array}{l}\text { Mississippi R. near St. Francisville, La. } \\
\text { Mississippi R. below Belle Chasse, La. }\end{array}$ & $\begin{array}{l}505 \\
499\end{array}$ & $\begin{array}{r}120.5 \\
98.3\end{array}$ & $\begin{array}{r}101.9 \\
84.7\end{array}$ & $\begin{array}{l}32 \\
24\end{array}$ & $\begin{array}{l}3 / 16 \\
1 / 8\end{array}$ & $\begin{array}{l}20 \\
10\end{array}$ \\
\hline
\end{tabular}

1 Anchored at each vertical.

${ }^{2}$ The sampling bottle had a cap without the isokinetic nozzle; the diameter of the threaded hole in the cap was $\frac{1}{2}$ inch.

${ }^{3}$ Anchored at one-half of the verticals--see Data Listings. 
Table 12.--Water volumes processed and masses of silt/clay and colloid fractions recovered from samples of Mississippi River and some of its tributaries for March-April 1989 cruise

[L, liter; g, gram; $\mu m$, micrometer; <, less than; integ. or pump indicates that samples were collected by depth-integration or pumping method; -- indicates that colloid was not collected and total is unknown]

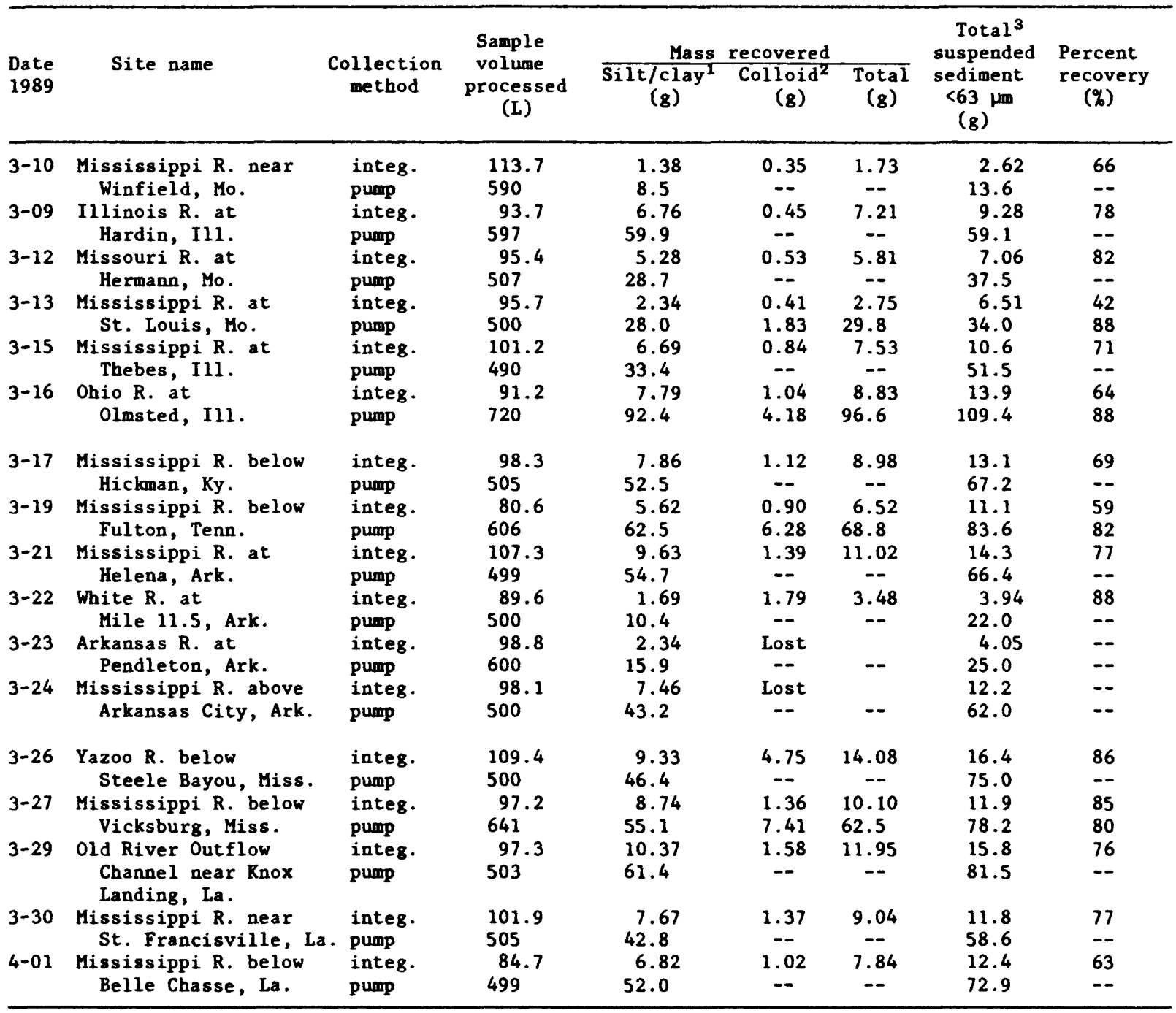

${ }^{1}$ Mass recovered from a centrifuge bowl (approximate radius is $5.2 \mathrm{~cm}$ and length is $71 \mathrm{~cm}$ ) operating at about 16,000 revolutions per minute with a flow rate of about $2 \mathrm{~L} / \mathrm{min}$.

${ }^{2}$ Mass that had passed through the centrifuge and was recovered from ultrafilter membranes (0.005- $\mu \mathrm{m}$ pore size). This includes mineral and organic colloid.

${ }^{3}$ Suspended-sediment concentration (average of $A$ and $B$ composite in table 19 for $<63-\mu m$ material) times the sample volume processed. 
Table 13.--Volumes of river water collected and processed and associated sampling parameters for June 1989 cruise

[ $V_{p}$ is the total volume collected by pumping; $V_{c}$ is the total volume collected by depth integration; $V_{d}$ is the total volume of depth-integrated sample that was dewatered by processing through the centrifuge and ultrafilter; L, liter; $\mathrm{cm} / \mathrm{s}$, centimeter per second]

\begin{tabular}{|c|c|c|c|c|c|c|c|}
\hline \multirow[b]{2}{*}{$\begin{array}{l}\text { Date } \\
1989\end{array}$} & \multirow[b]{2}{*}{ Site name } & \multicolumn{3}{|c|}{ Sample volumes } & \multicolumn{3}{|c|}{ Sampling parameters } \\
\hline & & $\begin{array}{l}\text { Samg } \\
V_{p} \\
(L)\end{array}$ & $\begin{array}{c}\frac{\text { le volu }}{v_{c}} \\
(\mathrm{~L})\end{array}$ & $\frac{v_{d}^{n e s}}{(L)}$ & $\begin{array}{l}\text { Number } \\
\text { of } \\
\text { verticals }\end{array}$ & $\begin{array}{l}\text { Nozzle } \\
\text { size } \\
\text { (inches) }\end{array}$ & $\begin{array}{l}\text { Transit } \\
\text { rate } \\
(\mathrm{cm} / \mathrm{s})\end{array}$ \\
\hline $\begin{array}{l}6-05 \\
6-04 \\
6-07 \\
6-08 \\
6-10\end{array}$ & $\begin{array}{l}\text { Mississippi R. near Winfield, Mo. } \\
\text { Illinois R. at Hardin, Ill. } \\
\text { Missouri R. at Hermann, Mo. } \\
\text { Mississippi R. at St. Louis, Mo. } \\
\text { Mississippi R. at Thebes, Ill. }\end{array}$ & $\begin{array}{l}584 \\
502 \\
519 \\
598 \\
500\end{array}$ & $\begin{array}{r}125.4 \\
94.6 \\
138.6 \\
118.6 \\
120.2\end{array}$ & $\begin{array}{r}99.7 \\
83.4 \\
125.4 \\
98.0 \\
100.6\end{array}$ & $\begin{array}{r}120 \\
1 \\
30 \\
30 \\
28\end{array}$ & $\begin{array}{l}1 / 4 \\
5 / 16 \\
5 / 16 \\
1 / 4 \\
1 / 4\end{array}$ & $\begin{array}{l}6 \\
\text { variable } \\
14 \\
14 \\
12\end{array}$ \\
\hline $\begin{array}{l}6-11 \\
6-12 \\
6-14 \\
6-17 \\
6-18\end{array}$ & $\begin{array}{l}\text { Ohio R. at Olmsted, Ill. } \\
\text { Mississippi R. below Hickman, Ky. } \\
\text { Mississippi R. at Fulton, Tenn. } \\
\text { Mississippi R. at Helena, Ark. } \\
\text { White R. at Mile 11.5, Ark. }\end{array}$ & $\begin{array}{l}604 \\
506 \\
500 \\
510 \\
580\end{array}$ & $\begin{array}{l}131.9 \\
126.0 \\
116.2 \\
111.3 \\
123.9\end{array}$ & $\begin{array}{r}110.2 \\
107.4 \\
97.7 \\
92.7 \\
105.1\end{array}$ & $\begin{array}{l}30 \\
30 \\
28 \\
30 \\
23\end{array}$ & $\begin{array}{l}1 / 4 \\
3 / 16 \\
3 / 16 \\
3 / 16 \\
5 / 16\end{array}$ & $\begin{array}{r}10 \\
10 \\
15 \\
18 \\
4-9\end{array}$ \\
\hline $\begin{array}{l}6-19 \\
6-20 \\
6-22 \\
6-23 \\
6-25\end{array}$ & $\begin{array}{l}\text { Arkansas R. at Pendleton, Ark. } \\
\text { Mississippi R. above Arkansas City, Ark. } \\
\text { Yazoo R. below Steele Bayou, Miss. } \\
\text { Mississippi R. below Vicksburg, Miss. } \\
\text { Old River Outflow Channel near } \\
\text { Knox Landing, La. }\end{array}$ & $\begin{array}{l}499 \\
502 \\
611 \\
629 \\
501\end{array}$ & $\begin{array}{r}77.6 \\
103.3 \\
122.3 \\
126.6 \\
106.3\end{array}$ & $\begin{array}{r}74.3 \\
87.8 \\
111.9 \\
106.0 \\
89.7\end{array}$ & $\begin{array}{l}30 \\
28 \\
26 \\
36 \\
30\end{array}$ & $\begin{array}{l}3 / 16 \\
1 / 8 \\
1 / 4 \\
1 / 8 \\
1 / 4\end{array}$ & $\begin{array}{c}13 \\
12 \\
2-14 \\
10 \\
13\end{array}$ \\
\hline $\begin{array}{l}6-26 \\
6-28\end{array}$ & $\begin{array}{l}\text { Mississippi R. near St. Francisville, La. } \\
\text { Mississippi R. below Belle Chasse. La. }\end{array}$ & $\begin{array}{l}505 \\
508\end{array}$ & $\begin{array}{r}124.6 \\
59.3\end{array}$ & $\begin{array}{r}104.5 \\
52.9\end{array}$ & $\begin{array}{l}30 \\
14\end{array}$ & $\begin{array}{l}3 / 16 \\
1 / 8\end{array}$ & $\begin{array}{l}16 \\
10\end{array}$ \\
\hline
\end{tabular}

${ }^{1}$ Anchored at each vertical.

${ }^{2}$ Equal-discharge increments collected while anchored at each vertical. 
Table 14.--Water volumes processed and masses of silt/clay and colloid fractions recovered from samples of the Mississippi River and its principal tributaries for June 1989 cruise

[L, liter; $8, g \mathrm{ram} ; \mu \mathrm{m}$, micrometer; <, less than; integ. or pump indicates that samples were collected by depth integration or pumping method; -- indicates that colloid was not collected and total is unknown]

\begin{tabular}{|c|c|c|c|c|c|c|c|c|}
\hline $\begin{array}{l}\text { Date } \\
1989\end{array}$ & Site name & $\begin{array}{l}\text { Collection } \\
\text { method }\end{array}$ & $\begin{array}{l}\text { Sample } \\
\text { volume } \\
\text { processed } \\
\text { (I) }\end{array}$ & $\begin{array}{c}\text { Mass } \\
\text { Silt/clay } \\
(g)\end{array}$ & $\begin{array}{c}\text { recovered } \\
\text { Colloid } \\
(8)\end{array}$ & $\begin{array}{c}\text { Total } \\
(\mathrm{g})\end{array}$ & $\begin{array}{l}\text { Total }{ }^{3} \\
\text { suspended } \\
\text { sediment } \\
<63 \mu \mathrm{m} \\
(\mathrm{g})\end{array}$ & $\begin{array}{l}\text { Percent } \\
\text { recovery } \\
(\%)\end{array}$ \\
\hline \multirow[t]{2}{*}{$6-05$} & Mississippi $R$. near & integ. & 99.7 & 4.62 & 0.59 & 5.21 & 6.98 & 75 \\
\hline & Winfield, Mo. & pump & 584 & 32.9 & - & $\cdots$ & 40.9 & -- \\
\hline \multirow[t]{2}{*}{$6-04$} & Illinois $R$. at & integ. & 83.4 & 49.87 & 0.66 & 50.53 & 59.0 & 86 \\
\hline & Hardin, Ill. & pump & 502 & 303.2 & -- & - & 355.0 & -- \\
\hline \multirow[t]{2}{*}{$6-07$} & Missouri $R$. at & integ. & 125.4 & 54.46 & 2.20 & 56.66 & 59.2 & 96 \\
\hline & Hermann, Mo. & pump & 519 & 219.2 & - & - & 245.0 & -- \\
\hline \multirow[t]{2}{*}{$6-08$} & Mississippi R. at & integ. & 98.0 & 7.89 & 0.76 & 8.65 & 12.0 & 72 \\
\hline & St. Louis, Mo. & pump & 598 & 63.9 & 3.36 & 67.3 & 73.0 & 92 \\
\hline \multirow[t]{2}{*}{$6-10$} & Mississippi R. at & integ. & 100.6 & 9.02 & 0.74 & 9.76 & 11.8 & 83 \\
\hline & Thebes, I11. & pump & 500 & 47.0 & -- & $\cdots$ & 58.5 & -- \\
\hline \multirow{2}{*}{$6-11$} & Ohio R, at & integ. & 110.2 & 8.97 & 0.64 & 9.61 & 12.7 & 76 \\
\hline & Olmsted, Ill. & pump & 604 & 56.1 & 3.23 & 59.3 & 69.5 & 85 \\
\hline \multirow[t]{2}{*}{$6-12$} & Mississippi R. below & integ. & 107.4 & 9.87 & 0.73 & 10.60 & 14.0 & 76 \\
\hline & Hickman, Ky. & pump & 506 & 56.3 & - & - & 65.8 & -- \\
\hline \multirow[t]{2}{*}{$6-14$} & Mississippi $R$. below & integ. & 97.7 & 14.33 & 1.04 & 15.37 & 17.8 & 86 \\
\hline & Fulton, Tenn. & pump & 500 & 75.5 & -- & -- & 91.0 & -- \\
\hline \multirow[t]{2}{*}{$6-17$} & Mississippi R. at & integ. & 92.7 & 14.58 & 1.12 & 15.70 & 19.8 & 79 \\
\hline & Helena, Ark. & pump & 510 & 90.6 & -- & - & 109.1 & -- \\
\hline \multirow{2}{*}{$6-18$} & White R. at & integ. & 105.1 & 5.85 & 1.42 & 7.27 & 9.67 & 75 \\
\hline & Mile 11.5, Ark. & pump & 580 & 42.7 & -- & -- & 53.4 & -- \\
\hline \multirow[t]{2}{*}{$6-19$} & Arkansas $R$. at & integ. & 74.3 & 2.90 & 0.69 & 3.59 & 5.05 & 71 \\
\hline & Pendleton, Ark. & pump & 499 & 23.2 & $\cdots$ & $\cdots$ & 33.9 & - \\
\hline \multirow[t]{2}{*}{$6-20$} & Mississippi R. above & integ. & 87.8 & 10.51 & 1.00 & 11.51 & 14.9 & 77 \\
\hline & Arkansas City, Ark. & pump & 502 & 67.8 & - & $\cdots$ & 85.3 & -- \\
\hline \multirow[t]{2}{*}{$6-22$} & Yazoo R. below & integ. & 111.9 & 21.10 & 5.67 & 26.77 & 30.4 & 88 \\
\hline & Steele Bayou, Miss. & pump & 611 & 137.5 & -- & $\cdots$ & 166.0 & -- \\
\hline \multirow[t]{2}{*}{$6-23$} & Mississippi $R$. below & integ. & 106.0 & 10.04 & 1.45 & 11.49 & 16.2 & 71 \\
\hline & Vicksburg, Miss. & pump & 629 & 68.6 & 5.25 & 73.9 & 96.2 & 77 \\
\hline \multirow[t]{2}{*}{$6-24$} & old River Outflow & integ. & 89.7 & 8.03 & 1.22 & 9.25 & 14.4 & 64 \\
\hline & $\begin{array}{l}\text { Channel near Knox } \\
\text { Landing, La. }\end{array}$ & pump & 501 & 54.1 & -- & -- & 80.2 & -- \\
\hline \multirow[t]{2}{*}{$6-26$} & Mississippi $R$. near & integ. & 104.5 & 10.73 & 1.19 & 11.92 & 16.1 & 74 \\
\hline & St. Francisville, La. & pump & 505 & 63.7 & -- & -- & 77.8 & -- \\
\hline \multirow[t]{2}{*}{$6-28$} & Mississippi R. below & integ. & 52.9 & 6.37 & 0.51 & 6.88 & 8.99 & 77 \\
\hline & Belle Chasse, La. & pump & 508 & 66.9 & -- & -- & 86.4 & -- \\
\hline
\end{tabular}

${ }^{1}$ Mass recovered from a centrifuge bowl (approximate radius is $5.2 \mathrm{~cm}$ and length is $71 \mathrm{~cm}$ ) operating at about 16,000 revolutions per minute with a flow rate of about $2 \mathrm{~L} / \mathrm{min}$.

${ }^{2}$ Mass which had passed through the centrifuge and was recovered from ultrafilter membranes (0.005- $\mu m$ pore size). This includes mineral and organic colloid.

${ }^{3}$ Suspended-sediment concentration (average of $A$ and $B$ composite in table 20 for $<63-\mu \mathrm{m}$ material) times the sample volume processed. 
Table 15.--Volumes of river water collected and processed and associated sampling parameters for February-March 1990 cruise

[ $V_{p}$ is the total volume collected by pumping; $V_{c}$ is the total volume collected by depth integration; $V_{d}$ is the total volume of depth-integrated sample that was dewatered by processing through the centrifuge and ultrafilter; L, liter; $\mathrm{cm} / \mathrm{s}$, centimeter per second]

\begin{tabular}{|c|c|c|c|c|c|c|c|}
\hline \multirow[b]{2}{*}{$\begin{array}{l}\text { Date } \\
1990\end{array}$} & \multirow[b]{2}{*}{ Site name } & \multicolumn{3}{|c|}{ Sample volumes } & \multicolumn{3}{|c|}{ Sampling parameters } \\
\hline & & $\begin{array}{l}v_{p} \\
(L)\end{array}$ & $\begin{array}{l}v_{c} \\
(L)\end{array}$ & $\begin{array}{l}v_{d} \\
(L)\end{array}$ & $\begin{array}{l}\text { Number } \\
\text { of } \\
\text { verticals }\end{array}$ & $\begin{array}{l}\text { Nozzle } \\
\text { size } \\
\text { (inches) }\end{array}$ & $\begin{array}{l}\text { Transit } \\
\text { rate } \\
(\mathrm{cm} / \mathrm{s})\end{array}$ \\
\hline $\begin{array}{l}2-25 \\
3-01 \\
2-28 \\
2-23 \\
2-24\end{array}$ & $\begin{array}{l}\text { Mississippi } R \text {. near Cache, Ill. } \\
\text { Ohio R. at Uniontown, Ky. } \\
\text { Wabash } R \text {, near New Haven, Ill. } \\
\text { Cumberland } R \text {. near Smithland, Ky. } \\
\text { Tennessee } R \text {. near Calvert City, Ky. }\end{array}$ & $\begin{array}{l}598 \\
503 \\
497 \\
499 \\
500\end{array}$ & $\begin{array}{r}21.5 \\
114.3 \\
119.0 \\
74.3 \\
117.9\end{array}$ & $\begin{array}{r}0 \\
95.9 \\
104.6 \\
67.5 \\
110.0\end{array}$ & $\begin{array}{r}5 \\
29 \\
14 \\
10 \\
14\end{array}$ & $\begin{array}{l}1 / 4 \\
1 / 4 \\
1 / 4 \\
3 / 16 \\
3 / 16\end{array}$ & $\begin{array}{r}5 \\
9 \\
7 \\
9 \\
15\end{array}$ \\
\hline $\begin{array}{l}3-03 \\
3-04 \\
3-05 \\
3-07 \\
3-08\end{array}$ & $\begin{array}{l}\text { Ohio R. at Olmsted, Ill. } \\
\text { Mississippi R. below Hickman, Ky. } \\
\text { Mississippi R. below Fulton, Tenn. } \\
\text { Mississippi R. at Helena, Ark. } \\
\text { Mississippi R. above Arkansas City, Ark. }\end{array}$ & $\begin{array}{l}510 \\
520 \\
501 \\
512 \\
509\end{array}$ & $\begin{array}{l}119.8 \\
132.1 \\
122.3 \\
126.8 \\
120.5\end{array}$ & $\begin{array}{l}104.2 \\
116.0 \\
106.2 \\
110.7 \\
104.4\end{array}$ & $\begin{array}{l}15 \\
15 \\
15 \\
15 \\
16\end{array}$ & $\begin{array}{l}3 / 16 \\
3 / 16 \\
3 / 16 \\
3 / 16 \\
1 / 8\end{array}$ & $\begin{array}{l}12 \\
14 \\
16 \\
22 \\
17\end{array}$ \\
\hline $\begin{array}{l}3-10 \\
3-12 \\
3-14\end{array}$ & $\begin{array}{l}\text { Mississippi R. below Vicksburg, Miss. } \\
\text { Mississippi R. near St. Francisville, La. } \\
\text { Mississippi R. below Belle Chasse, La. }\end{array}$ & $\begin{array}{l}524 \\
499 \\
505\end{array}$ & $\begin{array}{l}123.1 \\
138.9 \\
113.5\end{array}$ & $\begin{array}{r}106.9 \\
122.5 \\
98.9\end{array}$ & $\begin{array}{l}18 \\
15 \\
13\end{array}$ & $\begin{array}{l}1 / 8 \\
1 / 8 \\
1 / 8\end{array}$ & $\begin{array}{l}15 \\
10 \\
13\end{array}$ \\
\hline
\end{tabular}


Table 16.--Water volumes processed and masses of silt/clay and colloid fractions recovered from samples of the Mississippi River and some of its tributaries for February-March 1990 cruise

[L, liter; $8,8 \mathrm{ram} ; \mu \mathrm{m}$, micrometer; <, less than; integ. or pump indicates that samples were collected by depth-integration or pumping method; -- indicates that colloid was not collected and total is unknown]

\begin{tabular}{|c|c|c|c|c|c|c|c|c|}
\hline $\begin{array}{l}\text { Date } \\
1990\end{array}$ & Site name & $\begin{array}{l}\text { Collection } \\
\text { method }\end{array}$ & $\begin{array}{l}\text { Sample } \\
\text { volume } \\
\text { processed } \\
\text { (I) }\end{array}$ & 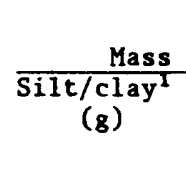 & $\begin{array}{c}\text { recovered } \\
\text { Colloid } \\
(\mathrm{g})\end{array}$ & $\frac{}{\text { Total }}$ s & $\begin{array}{l}\text { Total }^{3} \\
\text { suspended } \\
\text { sediment } \\
<63 \mu \mathrm{m} \\
(\mathrm{g})\end{array}$ & $\begin{array}{l}\text { Percent } \\
\text { recovery } \\
(\%)\end{array}$ \\
\hline $2-25$ & Mississippi $R$. near & integ. & $\begin{array}{r}0 \\
508\end{array}$ & \multicolumn{2}{|c|}{ not processed for } & suspended & d sediment & \\
\hline $3-01$ & $\begin{array}{l}\text { Ohio R. at } \\
\text { Uniontown, Ky. }\end{array}$ & $\begin{array}{l}\text { puip } \\
\text { integ. } \\
\text { pump }\end{array}$ & $\begin{array}{c}390 \\
95.9 \\
503\end{array}$ & $\begin{array}{l}14.70 \\
81.2\end{array}$ & 1.40 & 16.10 & $\begin{array}{r}19.6 \\
102.6\end{array}$ & $\begin{array}{l}82 \\
--\end{array}$ \\
\hline $2-28$ & $\begin{array}{l}\text { Wabash } R \text {. near } \\
\text { New Haven, III. }\end{array}$ & $\begin{array}{l}\text { integ. } \\
\text { pump }\end{array}$ & $\begin{array}{l}104.6 \\
497\end{array}$ & $\begin{array}{l}11.38 \\
62.1\end{array}$ & $\begin{array}{r}2.7 \\
--\end{array}$ & 14.1 & $\begin{array}{l}15.5 \\
73.6\end{array}$ & $\begin{array}{l}91 \\
--\end{array}$ \\
\hline $2-23$ & $\begin{array}{l}\text { Cumberland } R \text {, near } \\
\text { Smithland, Ky. }\end{array}$ & $\begin{array}{l}\text { integ. } \\
\text { pump }\end{array}$ & $\begin{array}{l}67.5 \\
499\end{array}$ & $\begin{array}{l}0.88 \\
12.9\end{array}$ & 0.33 & 1.17 & $\begin{array}{l}2.16 \\
16.0\end{array}$ & $\begin{array}{l}54 \\
--\end{array}$ \\
\hline $2-24$ & $\begin{array}{r}\text { Tennessee } R \text {. near } \\
\text { Calvert City, Ky. }\end{array}$ & $\begin{array}{l}\text { integ. } \\
\text { pump }\end{array}$ & $\begin{array}{l}110.0 \\
500\end{array}$ & $\begin{array}{l}3.61 \\
18.5\end{array}$ & $\begin{array}{r}1.2 \\
--\end{array}$ & $\begin{array}{l}4.8 \\
--\end{array}$ & $\begin{array}{l}5.28 \\
24.0\end{array}$ & $\begin{array}{l}91 \\
--\end{array}$ \\
\hline $3-03$ & $\begin{array}{l}\text { Ohio R. at } \\
\text { Olmsted, IIl. }\end{array}$ & $\begin{array}{l}\text { integ. } \\
\text { pump }\end{array}$ & $\begin{array}{l}104.2 \\
510\end{array}$ & $\begin{array}{l}10.00 \\
53.8\end{array}$ & 1.50 & 11.50 & $\begin{array}{l}15.0 \\
73.4\end{array}$ & $\begin{array}{l}77 \\
--\end{array}$ \\
\hline $3-04$ & $\begin{array}{l}\text { Mississippi R. below } \\
\text { Hickman, } \mathrm{Ky} \text {. }\end{array}$ & $\begin{array}{l}\text { integ. } \\
\text { pump }\end{array}$ & $\begin{array}{l}116.0 \\
520\end{array}$ & $\begin{array}{l}12.09 \\
59.8\end{array}$ & $\begin{array}{r}1.8 \\
--\end{array}$ & $\begin{array}{c}13.9 \\
--\end{array}$ & $\begin{array}{l}18.3 \\
82.2\end{array}$ & $\begin{array}{l}76 \\
--\end{array}$ \\
\hline $3-05$ & $\begin{array}{l}\text { Mississippi R. below } \\
\text { Fulton, Tenn. }\end{array}$ & $\begin{array}{l}\text { integ. } \\
\text { pump }\end{array}$ & $\begin{array}{l}106.2 \\
501\end{array}$ & $\begin{array}{l}9.68 \\
61.9\end{array}$ & $\begin{array}{c}1.52 \\
-.-\end{array}$ & 11.20 & $\begin{array}{l}15.0 \\
70.6\end{array}$ & $\begin{array}{l}75 \\
--\end{array}$ \\
\hline $3-07$ & $\begin{array}{l}\text { Mississippi R. at } \\
\text { Helena, Ark. }\end{array}$ & $\begin{array}{l}\text { integ. } \\
\text { pump }\end{array}$ & $\begin{array}{l}110.7 \\
512\end{array}$ & $\begin{array}{l}10.67 \\
58.1\end{array}$ & $\begin{array}{r}1.3 \\
--\end{array}$ & 12.0 & $\begin{array}{l}16.2 \\
74.8\end{array}$ & $\begin{array}{l}74 \\
--\end{array}$ \\
\hline $3-08$ & $\begin{array}{l}\text { Mississippi R. above } \\
\text { Arkansas City, Ark. }\end{array}$ & $\begin{array}{l}\text { integ. } \\
\text { pump }\end{array}$ & $\begin{array}{l}104.4 \\
509\end{array}$ & $\begin{array}{l}9.03 \\
56.2\end{array}$ & $\begin{array}{c}1.51 \\
--\end{array}$ & $\begin{array}{l}10.54 \\
--\end{array}$ & $\begin{array}{l}13.2 \\
64.1\end{array}$ & $\begin{array}{l}80 \\
--\end{array}$ \\
\hline $3-10$ & $\begin{array}{c}\text { Mississippi R. below } \\
\text { Vicksburg, Miss. }\end{array}$ & $\begin{array}{l}\text { integ. } \\
\text { pump }\end{array}$ & $\begin{array}{l}106.9 \\
524\end{array}$ & $\begin{array}{l}9.60 \\
55.6\end{array}$ & $\begin{array}{c}1.67 \\
--\end{array}$ & 11.27 & $\begin{array}{l}13.5 \\
66.0\end{array}$ & $\begin{array}{l}83 \\
--\end{array}$ \\
\hline $3-12$ & $\begin{array}{l}\text { Mississippi R. near } \\
\text { St. Francisville, La. }\end{array}$ & $\begin{array}{l}\text { integ. } \\
\text { pump }\end{array}$ & $\begin{array}{l}122.5 \\
499\end{array}$ & $\begin{array}{l}8.18 \\
36.5\end{array}$ & $\begin{array}{c}4.40 \\
-.\end{array}$ & $\begin{array}{l}9.58 \\
--\end{array}$ & $\begin{array}{l}12.0 \\
48.9\end{array}$ & $\begin{array}{l}79 \\
--\end{array}$ \\
\hline $3-14$ & $\begin{array}{l}\text { Mississippi R. below } \\
\text { Belle Chasse, La. }\end{array}$ & $\begin{array}{l}\text { integ. } \\
\text { pump }\end{array}$ & $\begin{array}{l}98.9 \\
505\end{array}$ & 10.15 & $\begin{array}{c}4.33 \\
--\end{array}$ & $\begin{array}{c}11.48 \\
--\end{array}$ & $\begin{array}{l}13.8 \\
70.7\end{array}$ & $\begin{array}{r}83 \\
--\end{array}$ \\
\hline
\end{tabular}

${ }^{1}$ Mass recovered from a centrifuge bowl (approximate radius is $5.2 \mathrm{~cm}$ and length is $71 \mathrm{~cm}$ ) operating at about 16,000 revolutions per minute with a flow rate of about $2 \mathrm{~L} / \mathrm{min}$.

${ }^{2}$ Mass which had passed through the centrifuge and was recovered from ultrafilter membranes $(0.005-\mu \mathrm{m}$ pore size). This includes mineral and organic colloids.

${ }^{3}$ Suspended-sediment concentration (average of $A$ and $B$ composite in table 21 for $<63-\mu \mathrm{m}$ material) times the sample volume processed.

The mass includes only mineral colloids. 
Table 17.--Volumes of river, water collected and processed and associated sampling parameters for second leg of the May-June 1990 cruise

[ $V_{p}$ is the total volume collected by pumping; $V_{c}$ is the total volume collected by depth integration; $V_{d}$ is the total volume of depth-integrated sample that was dewatered by processing through the centrifuge and ultrafilter; L, liter; $\mathrm{cm} / \mathrm{s}$, centimeter per second]

\begin{tabular}{|c|c|c|c|c|c|c|c|}
\hline \multirow[b]{2}{*}{$\begin{array}{l}\text { Date } \\
1990\end{array}$} & \multirow[b]{2}{*}{ Site name } & \multicolumn{3}{|c|}{ Sample volumes } & \multicolumn{3}{|c|}{ Sampling parameters } \\
\hline & & $\mathrm{v}_{\mathrm{p}}$ & $V_{c}$ & $\begin{array}{c}V_{d} \\
(L)\end{array}$ & $\begin{array}{l}\text { Number } \\
\text { of } \\
\text { verticals }\end{array}$ & $\begin{array}{c}\text { Nozzle } \\
\text { size } \\
\text { (inches) }\end{array}$ & $\begin{array}{l}\text { Transit } \\
\text { rate } \\
(\mathrm{cm} / \mathrm{s})\end{array}$ \\
\hline $\begin{array}{l}6-07 \\
6-11 \\
6-10 \\
6-13 \\
6-14\end{array}$ & $\begin{array}{l}\text { Illinois R. at Valley City, Ill. } \\
\text { Mississippi R. below Grafton, I1l. } \\
\text { Missouri R. at St. Charles, Mo. } \\
\text { Mississippi R. at Thebes, Ill. } \\
\text { Ohio R. at Olmsted, Ill. }\end{array}$ & $\begin{array}{r}500 \\
516 \\
0 \\
307 \\
393\end{array}$ & $\begin{array}{r}78.3 \\
64.9 \\
8.5 \\
71.5 \\
87.2\end{array}$ & $\begin{array}{r}61.1 \\
54.5 \\
0 \\
62.4 \\
70.3\end{array}$ & $\begin{array}{r}15 \\
20 \\
1 \\
20 \\
20\end{array}$ & $\begin{array}{l}1 / 4,5 / 16 \\
1 / 4 \\
3 / 16 \\
3 / 16 \\
1 / 4\end{array}$ & $\begin{array}{c}4-19 \\
8 \\
12,15 \\
20 \\
11\end{array}$ \\
\hline $\begin{array}{l}6-18 \\
6-20 \\
6-22 \\
6-23 \\
6-25 \\
6-27\end{array}$ & $\begin{array}{l}\text { Mississippi R. below Memphis, Tenn. } \\
\text { Mississippi R. below Arkansas City, Ark. } \\
\text { Yazoo R. below Steele Bayou, Miss. } \\
\text { Mississippi R. below Vicksburg, Miss. } \\
\text { Mississippi R. near St. Francisville, La. } \\
\text { Mississippi R. below Belle Chasse, La. }\end{array}$ & $\begin{array}{r}349 \\
299 \\
0 \\
349 \\
351 \\
350\end{array}$ & $\begin{array}{l}58.7 \\
59.3 \\
47.6 \\
57.3 \\
61.1 \\
51.5\end{array}$ & $\begin{array}{l}42.0 \\
48.5 \\
30.8 \\
40.5 \\
50.3 \\
40.6\end{array}$ & $\begin{array}{l}20 \\
20 \\
15 \\
20 \\
20 \\
20\end{array}$ & $\begin{array}{c}1 / 8 \\
1 / 8 \\
1 / 4-5 / 16 \\
1 / 8 \\
1 / 8 \\
1 / 8\end{array}$ & $\begin{array}{c}13 \\
16 \\
7-12 \\
15 \\
15 \\
20\end{array}$ \\
\hline
\end{tabular}

${ }^{1}$ Equal-discharge increments collected while anchored at each vertical.

${ }^{2}$ Single $7.2-\mathrm{L}$ sample was collected at vertical 17 to represent the Mississippi River upstream from the Illinois River. 
Table 18.--Water volumes processed and masses of silt/clay and colloid fractions recovered from samples of the Mississippi River and some of its tributaries for second leg of the

May-June 1990 cruise

[L, liter; $8,8 \mathrm{ram} ; \mu \mathrm{m}$, micrometer; <, less than; integ. or pump indicates that samples were collected by depth-integration or pumping method; -- indicates that colloid was not collected and total is unknown]

\begin{tabular}{|c|c|c|c|c|c|c|c|c|}
\hline $\begin{array}{l}\text { Date } \\
1990\end{array}$ & Site name & $\begin{array}{l}\text { Collection } \\
\text { method }\end{array}$ & $\begin{array}{c}\text { Sample } \\
\text { volume } \\
\text { processed } \\
\text { (L) }\end{array}$ & $\begin{array}{c}\text { Mass } \\
\text { Silt/clay } \\
\text { (8) }\end{array}$ & $\begin{array}{c}\text { recovered } \\
\text { Colloid } \\
(8)\end{array}$ & $\begin{array}{c}\text { Total } \\
(\mathrm{g})\end{array}$ & $\begin{array}{l}\text { Total } \\
\text { suspended } \\
\text { sediment } \\
<63 \mu \mathrm{m} \\
(8)\end{array}$ & $\begin{array}{l}\text { Percent } \\
\text { recovery } \\
(\%)\end{array}$ \\
\hline $\begin{array}{l}6-07 \\
6-11 \\
6-13 \\
6-14 \\
6-18\end{array}$ & $\begin{array}{l}\text { Illinois R. at } \\
\text { Valley City, Ill } \\
\text { Mississippi R. below } \\
\text { Grafton, Ill. } \\
\text { Mississippi R. at } \\
\text { Thebes, Ill. } \\
\text { Ohio R. at } \\
\text { Olmsted, Ill. } \\
\text { Mississippi R. below } \\
\text { Memphis, Tenn. }\end{array}$ & $\begin{array}{l}\text { integ. } \\
\text { pump } \\
\text { integ. } \\
\text { pump } \\
\text { integ. } \\
\text { pump } \\
\text { integ. } \\
\text { pump } \\
\text { integ. } \\
\text { pump }\end{array}$ & $\begin{array}{l}61.1 \\
500 \\
54.5 \\
516 \\
62.4 \\
307 \\
70.3 \\
393 \\
42.0 \\
349\end{array}$ & $\begin{array}{c}4.81 \\
44.6 \\
21.2 \\
219.7 \\
58.93 \\
309.7 \\
9.43 \\
64.7 \\
16.20 \\
---\end{array}$ & $\begin{array}{l}0.26 \\
-. \\
1.20 \\
-- \\
3.05 \\
-- \\
0.84 \\
-. \\
0.91 \\
--\end{array}$ & $\begin{array}{c}5.07 \\
-. \\
22.4 \\
-- \\
61.98 \\
-- \\
10.27 \\
-- \\
17.11 \\
--\end{array}$ & $\begin{array}{l}5.99 \\
49.0 \\
25.3 \\
239 \\
69.6 \\
342 \\
12.4 \\
69.6 \\
18.1 \\
151\end{array}$ & $\begin{array}{l}85 \\
-- \\
89 \\
-- \\
89 \\
-\overline{83} \\
-- \\
95 \\
--\end{array}$ \\
\hline $\begin{array}{l}6-20 \\
6-22 \\
6-23 \\
6-25 \\
6-27\end{array}$ & $\begin{array}{l}\text { Mississippi R. below } \\
\text { Arkansas City, Ark. } \\
\text { Yazoo R. below } \\
\text { Steele Bayou, Miss. } \\
\text { Mississippi R. below } \\
\text { Vicksburg, Miss. } \\
\text { Mississippi R. near } \\
\text { St. Francisville, La. } \\
\text { Mississippi R. below } \\
\text { Belle Chasse, La. }\end{array}$ & $\begin{array}{l}\text { integ. } \\
\text { pump } \\
\text { integ. } \\
\text { pump } \\
\text { integ. } \\
\text { pump } \\
\text { integ. } \\
\text { pump } \\
\text { integ. } \\
\text { pump }\end{array}$ & $\begin{array}{c}48.5 \\
299 \\
30.8 \\
0 \\
40.5 \\
349 \\
50.3 \\
351 \\
40.6 \\
350\end{array}$ & $\begin{array}{c}13.08 \\
-. \\
1.80 \\
-. \\
8.10 \\
89.0 \\
6.88 \\
56.7 \\
2.77 \\
54.7\end{array}$ & $\begin{array}{c}0.92 \\
-- \\
0.91 \\
-- \\
0.63 \\
-- \\
0.60 \\
-- \\
0.50 \\
--\end{array}$ & $\begin{array}{l}14.00 \\
-- \\
2.71 \\
-- \\
8.73 \\
-- \\
7.48 \\
-\therefore \\
3.27 \\
--\end{array}$ & $\begin{array}{c}16.2 \\
99.9 \\
3.57 \\
-- \\
11.1 \\
95.3 \\
9.26 \\
64.6 \\
7.43 \\
64.1\end{array}$ & $\begin{array}{l}86 \\
-- \\
75 \\
-- \\
79 \\
-- \\
81 \\
-- \\
44 \\
--\end{array}$ \\
\hline
\end{tabular}

${ }^{1}$ Mass recovered from a centrifuge bowl (approximate radius is $5.2 \mathrm{~cm}$ and length is $71 \mathrm{~cm}$ ) operating at about 16,000 revolutions per minute with a flow rate of about $2 \mathrm{~L} / \mathrm{min}$.

2 Mass which had passed through the centrifuge and was recovered from ultrafilter membranes $(0.005-\mu m$ pore size). No organic colloids are included in these masses.

${ }^{3}$ Suspended-sediment concentration (average of $A$ and $B$ composite in table 22 for $<63-\mu \mathrm{m}$ material) times the sample volume processed. 
The suspended sediment collected by using the depth-integration method was split into a sand fraction consisting of particle diameters greater than $63 \mu \mathrm{m}$ and a fine fraction consisting of silt and clay-type particles with diameters finer than $63 \mu \mathrm{m}$. The concentrations of the sand and the silt/clay fractions were measured separately and then added together to determine the total concentration.

The particle-size analysis of the sand and the silt/clay fraction used different methods. However, the results were combined to give particle-size distribution for the entire depth-integrated composite suspended-sediment sample collected at each sampling site.

\section{Concentration}

There were two replicate suspended-sand samples (composites A and B) for each sampling site. If there was not enough sand in either the $A$ or $B$ composite samples, the two samples were combined to form a single suspendedsand sample. The sands were transferred to glass jars and transported to a USGS laboratory in Denver, Colo., where they were dried at $80^{\circ} \mathrm{C}$, weighed to within $0.1 \mathrm{mg}$, and stored in polypropylene bottles for particle-size analysis. The mass ranged from $1 \mathrm{mg}$ to about $5,000 \mathrm{mg}$. The collected volume of water was about $50 \mathrm{~L}$ (measured to $0.1 \mathrm{~L}$ ) so that the sand concentrations in tables 19-22 have analytical errors ranging from about 2 percent for concentrations of $0.1 \mathrm{mg} / \mathrm{L}$ to less than 0.1 percent for concentrations of $100 \mathrm{mg} / \mathrm{L}$. The mean percent difference between the two replicate sand concentrations was 9 percent of the mean sand concentration for the March-April 1989 cruise, 10 percent for the June 1989 cruise, 6 percent for the February-March 1990 cruise, and 18 percent for the June 1990 cruise.

Two or three subsamples (about $150-250 \mathrm{~mL}$ ) were taken from composite A and composite $B$ for determining the suspended-sediment concentration of material finer than $63 \mu \mathrm{m}$. Each of the four to six subsamples was filtered through paired, preweighed Millipore HA filters (0.45- $\mathrm{mm}$ pore size) and dried on board the research vessel. Later, in a laboratory ashore, the filter with sediment was dried to $110^{\circ} \mathrm{C}$ and reweighed to within $0.1 \mathrm{mg}$, and the preweight of the filter was subtracted to obtain the mass of silt and clay, which typically was 20 to $40 \mathrm{mg}$. The volume of each subsample was measured to within $\pm 1 \mathrm{~mL}$, so that the concentrations in tables 19-22 have an analytical error of \pm 1 percent, or typically $1 \mathrm{mg} / \mathrm{L}$.

The mean range in silt and clay concentration for all sampling sites (calculated from two to four filters per sampling site) was $3 \pm 2$ percent of the mean silt and clay concentration for the March-April 1989 cruise, $2 \pm 2$ percent for the June 1989 cruise, $2 \pm 1$ percent for the February-March 1990 cruise, and $2 \pm 1$ percent for the June 1990 cruise. The mean percent difference between the silt and clay concentrations of the two replicates (composite $A$ and $B$ ) was about $1 \pm 1$ percent of the mean silt and clay concentration for all four cruises.

The suspended-sediment concentrations were multiplied by the water discharges (listed in tables 7-10), and by a unit-conversion constant $(0.0864)$ to obtain the sediment discharges in metric tons per day listed in the last columns of tables 19-22. 
Table 19.--Suspended-sediment concentrations in deptb-integrated, composite samples collected from the Mississippi River and some of its tributaries and the corresponding sediment discharges for March-April 1989 cruise

(Total sediment concentration of composites $A$ and $B$ were averaged in order to calculate a single value for sediment discharge $\mathrm{m}^{3} / \mathrm{s}$, cubic meter per second; mg/L, milligram per liter; $\mu m$, micrometer; <, less than; >, greater than; data for 5-vertical composite were not used to calculate the total concentration or the sediment discharge; C, collected concurrently with larger composite; nd indicates not determined. Analyses by R.H. Meade]

\begin{tabular}{|c|c|c|c|c|c|c|c|c|c|c|}
\hline \multirow{2}{*}{$\begin{array}{l}\text { Date } \\
1989\end{array}$} & \multirow[b]{2}{*}{ Site name } & \multirow[b]{2}{*}{ Composite } & \multirow{2}{*}{$\begin{array}{c}\text { Number } \\
\text { of } \\
\text { verticals }\end{array}$} & \multirow{2}{*}{$\begin{array}{c}\text { Water } \\
\text { discharge } \\
\left(\mathrm{m}^{3} / \mathrm{s}\right)\end{array}$} & \multicolumn{3}{|c|}{$\begin{array}{c}\text { Sediment concentration } \\
(\mathrm{mg} / \mathrm{L})\end{array}$} & \multicolumn{3}{|c|}{$\begin{array}{r}\text { Sediment discharge } \\
\text { (metric tons/day) }\end{array}$} \\
\hline & & & & & $\begin{array}{c}\text { Silt and } \\
\text { clay } \\
<63 \mu \mathrm{m}\end{array}$ & $\begin{array}{l}\text { Sand } \\
>63 \mu \mathrm{m}\end{array}$ & Total & $\begin{array}{c}\text { Silt and } \\
\text { clay } \\
<63 \mu \mathrm{m}\end{array}$ & $\begin{array}{l}\text { Sand } \\
>63 \mu \mathrm{m}\end{array}$ & Total \\
\hline $3-10$ & $\begin{array}{l}\text { Mississippi R. near } \\
\text { Winfield, Mo. }\end{array}$ & $\begin{array}{c}A+B \\
C\end{array}$ & $\begin{array}{r}20 \\
5\end{array}$ & 850 & $\begin{array}{l}23 \\
24\end{array}$ & $\begin{array}{l}0 \\
0\end{array}$ & 23 & 1,700 & 0 & 1,700 \\
\hline $3-09$ & $\begin{array}{l}\text { Illinois } R \text {. at } \\
\text { Hardin, I } 11.1\end{array}$ & $\begin{array}{l}\text { A } \\
\text { B }\end{array}$ & $\begin{array}{l}1 \\
1\end{array}$ & 410 & $\begin{array}{r}97 \\
101\end{array}$ & 0.3 & $\begin{array}{r}97 \\
101\end{array}$ & 3,500 & 10 & 3,510 \\
\hline $3-12$ & $\begin{array}{l}\text { Missouri R. at } \\
\text { Hermann, Mo. }\end{array}$ & $\begin{array}{l}\mathrm{A} \\
\mathrm{B} \\
\mathrm{C}\end{array}$ & $\begin{array}{r}15 \\
15 \\
5\end{array}$ & 1,480 & $\begin{array}{l}74 \\
73 \\
\text { nd }\end{array}$ & $\begin{array}{r}100 \\
87 \\
93\end{array}$ & $\begin{array}{l}174 \\
160\end{array}$ & 9,400 & 12,000 & 21,400 \\
\hline $3-13$ & $\begin{array}{l}\text { Mississippi R. at } \\
\text { St. Louis, Mo. }\end{array}$ & $\begin{array}{l}A \\
B \\
C\end{array}$ & $\begin{array}{r}15 \\
15 \\
5\end{array}$ & 3,940 & $\begin{array}{l}69 \\
68 \\
69\end{array}$ & $\begin{array}{l}78 \\
69 \\
77\end{array}$ & $\begin{array}{l}147 \\
137\end{array}$ & 23,000 & 25,000 & 48,000 \\
\hline $3-15$ & $\begin{array}{l}\text { Mississippi R. at } \\
\text { Thebes, I11. }\end{array}$ & $\begin{array}{l}\mathrm{A} \\
\mathrm{B} \\
\mathrm{C}\end{array}$ & $\begin{array}{r}14 \\
13 \\
5\end{array}$ & 4,890 & $\begin{array}{l}105 \\
105 \\
107\end{array}$ & $\begin{array}{l}50 \\
49\end{array}$ & 155 & 44,400 & 21,000 & 65,000 \\
\hline $3-16$ & $\begin{array}{l}\text { Obio R. at } \\
\text { Olmsted, IlI. }\end{array}$ & $\begin{array}{l}\mathrm{A} \\
\mathrm{B} \\
\mathrm{C}\end{array}$ & $\begin{array}{r}15 \\
15 \\
5\end{array}$ & 20,400 & $\begin{array}{l}152 \\
153 \\
150\end{array}$ & $\begin{array}{l}16 \\
16 \\
18\end{array}$ & $\begin{array}{l}168 \\
169\end{array}$ & 269,000 & 28,000 & 297,000 \\
\hline $3-17$ & $\begin{array}{l}\text { Mississippi R. below } \\
\text { Hickman, Ky. }\end{array}$ & $\begin{array}{l}\mathbf{A} \\
\mathbf{B} \\
\mathrm{C}\end{array}$ & $\begin{array}{r}15 \\
15 \\
5\end{array}$ & 24,700 & $\begin{array}{l}133 \\
133 \\
136\end{array}$ & $\begin{array}{l}75 \\
76 \\
86\end{array}$ & $\begin{array}{l}208 \\
209\end{array}$ & 284,000 & 161,000 & 445,000 \\
\hline $3-19$ & $\begin{array}{l}\text { Mississippi R. below } \\
\text { Fulton, Tenn. }\end{array}$ & $\begin{array}{l}\text { A } \\
\text { B } \\
\text { C }\end{array}$ & $\begin{array}{r}15 \\
15 \\
5\end{array}$ & 24,800 & $\begin{array}{l}137 \\
138 \\
136\end{array}$ & $\begin{array}{l}80 \\
90 \\
94\end{array}$ & $\begin{array}{l}217 \\
228\end{array}$ & 295,000 & 182,000 & 477,000 \\
\hline $3-21$ & $\begin{array}{l}\text { Mississippi R. at } \\
\text { Helena, Ark. }\end{array}$ & $\begin{array}{l}\text { A } \\
\text { B } \\
\text { C }\end{array}$ & $\begin{array}{r}15 \\
15 \\
5\end{array}$ & 25,900 & $\begin{array}{l}133 \\
133 \\
134\end{array}$ & $\begin{array}{l}68 \\
57 \\
68\end{array}$ & $\begin{array}{l}201 \\
190\end{array}$ & 298,000 & 140,000 & 438,000 \\
\hline $3-22$ & $\begin{array}{l}\text { White R. at } \\
\text { Mile 11.5, Ark. }\end{array}$ & $\begin{array}{r}{ }^{2} \mathrm{~A} \\
{ }^{2} \mathrm{~B} \\
\mathrm{C}\end{array}$ & $\begin{array}{l}5 \\
5 \\
5\end{array}$ & 1,500 & $\begin{array}{l}43 \\
44 \\
43\end{array}$ & $\begin{array}{l}0.4 \\
0.4 \\
0.4\end{array}$ & $\begin{array}{l}43 \\
44\end{array}$ & 5,600 & 50 & 5,600 \\
\hline $3-23$ & $\begin{array}{l}\text { Arkansas R. at } \\
\text { Pendleton, Ark. }\end{array}$ & $\begin{array}{r}{ }^{2} \mathrm{~A} \\
2_{\mathrm{B}} \\
\mathrm{C}\end{array}$ & $\begin{array}{r}10 \\
10 \\
5\end{array}$ & 1,900 & $\begin{array}{l}41 \\
41 \\
42\end{array}$ & $\begin{array}{l}1.5 \\
1.7 \\
1.6\end{array}$ & $\begin{array}{l}43 \\
43\end{array}$ & 6,730 & 260 & 6,800 \\
\hline $3-24$ & $\begin{array}{l}\text { Mississippi R. above } \\
\text { Arkansas City, Ark. }\end{array}$ & $\begin{array}{l}A \\
B \\
C\end{array}$ & $\begin{array}{r}15 \\
15 \\
5\end{array}$ & 26,800 & $\begin{array}{l}126 \\
123 \\
124\end{array}$ & $\begin{array}{l}69 \\
76 \\
89\end{array}$ & $\begin{array}{l}195 \\
199\end{array}$ & 288,000 & 168,000 & 456,000 \\
\hline $3-26$ & $\begin{array}{l}\text { Yazoo R. below } \\
\text { Steele Bayou, Miss. } 1\end{array}$ & $\begin{array}{r}{ }^{2} \mathrm{~A} \\
{ }^{2} \mathrm{~B} \\
\mathrm{C}\end{array}$ & $\begin{array}{r}5 \\
\times \quad 5 \\
5\end{array}$ & 1,500 & $\begin{array}{l}150 \\
149 \\
153\end{array}$ & $\begin{array}{l}0.7 \\
0.6\end{array}$ & $\begin{array}{l}151 \\
150\end{array}$ & 19,400 & 90 & 19,500 \\
\hline $3-27$ & $\begin{array}{l}\text { Mississippi R. below } \\
\text { Vicksburg, Miss. }\end{array}$ & $\begin{array}{l}\text { A } \\
\text { B } \\
\text { C }\end{array}$ & $\begin{array}{r}18 \\
18 \\
5\end{array}$ & 26,600 & $\begin{array}{l}122 \\
122 \\
123\end{array}$ & $\begin{array}{l}88 \\
73 \\
76\end{array}$ & $\begin{array}{l}210 \\
195\end{array}$ & 280,000 & 185,000 & 465,000 \\
\hline $3-29$ & $\begin{array}{l}\text { Old River Outflow Channel } \\
\text { near Knox Landing, La. }\end{array}$ & $\begin{array}{l}\text { A } \\
\text { B } \\
\text { C }\end{array}$ & $\begin{array}{r}15 \\
15 \\
5\end{array}$ & 6,160 & $\begin{array}{l}163 \\
160 \\
151\end{array}$ & $\begin{array}{l}18 \\
19 \\
18\end{array}$ & $\begin{array}{l}181 \\
179\end{array}$ & 86,000 & 9,800 & 95,800 \\
\hline $3-30$ & $\begin{array}{l}\text { Mississippi R. near } \\
\text { St. Francisville, La. }\end{array}$ & $\begin{array}{l}A \\
B \\
C\end{array}$ & $\begin{array}{r}16 \\
16 \\
5\end{array}$ & 23,100 & $\begin{array}{l}117 \\
116 \\
118\end{array}$ & $\begin{array}{l}77 \\
74 \\
64\end{array}$ & $\begin{array}{l}194 \\
190\end{array}$ & 233,000 & 151,000 & 384,000 \\
\hline 4-01 & $\begin{array}{l}\text { Mississippi R. below } \\
\text { Belle Chasse, La. }\end{array}$ & $\begin{array}{l}A \\
B \\
C\end{array}$ & $\begin{array}{r}12 \\
12 \\
4\end{array}$ & 22,500 & $\begin{array}{l}147 \\
145 \\
147\end{array}$ & $\begin{array}{l}72 \\
72 \\
84\end{array}$ & $\begin{array}{l}219 \\
217\end{array}$ & 284,000 & 140,000 & 424,000 \\
\hline
\end{tabular}

\footnotetext{
TSand in composites $A$ and $B$ were combined to give one value listed opposite composite A.
}

${ }^{2} A$ and $B$ composites were collected at the same verticals. 
Table 20.--Suspended-sediment concentrations in depth-integrated, composite samples collected from the Mississippi River and some of its tributaries and the corresponding sediment discharges for June 1989 cruise

ITotal sediment concentration of composites A and B were averaged in order to calculate a single value of sediment discharge; <, less than; >, greater than; $\mathrm{m}^{3} / \mathrm{s}$, cubic meter per second; $\mu m$, micrometer; data for 5 -vertical composite were not used to calculate the total concentration or the sediment discharge; $C$, collected concurrently with larger composite; NC, not collected concurrently with larger composite; ad indicates not determined. Analyses by R.H. Meade]

\begin{tabular}{|c|c|c|c|c|c|c|c|c|c|c|}
\hline \multirow{2}{*}{$\begin{array}{l}\text { Date } \\
1989\end{array}$} & \multirow[b]{2}{*}{ Site name } & \multirow[b]{2}{*}{ Composite } & \multirow{2}{*}{$\begin{array}{c}\text { Number } \\
\text { of } \\
\text { verticals }\end{array}$} & \multirow{2}{*}{$\begin{array}{c}\text { Water } \\
\text { discharge } \\
\left(\mathrm{m}^{3} / \mathrm{s}\right)\end{array}$} & \multicolumn{3}{|c|}{$\begin{array}{c}\text { Sediment concentration } \\
(\mathrm{mg} / \mathrm{L})\end{array}$} & \multicolumn{3}{|c|}{$\begin{array}{r}\text { Sediment discharge } \\
\text { (metric tons/day) }\end{array}$} \\
\hline & & & & & $\begin{array}{c}\text { Silt and } \\
\text { clay } \\
<63 \text { Nm }\end{array}$ & $\begin{array}{l}\text { Sand } \\
>63 \mu \mathrm{m}\end{array}$ & Total & $\begin{array}{c}\text { Silt and } \\
\text { c1 ay } \\
<63 \mu \mathrm{m}\end{array}$ & $\begin{array}{l}\text { Sand } \\
>63 \mu \mathrm{mm}\end{array}$ & Total \\
\hline $6-05$ & $\begin{array}{l}\text { Mississippi R. near } \\
\text { Winfield, Mo. }{ }^{1}\end{array}$ & $\begin{array}{r}{ }^{3} \mathrm{~A} \\
{ }^{3} \mathrm{~B} \\
\mathrm{C}\end{array}$ & $\begin{array}{r}20 \\
20 \\
5\end{array}$ & 2,320 & $\begin{array}{l}71 \\
68 \\
67\end{array}$ & $\begin{array}{l}0.1 \\
\text { ad }\end{array}$ & $\begin{array}{l}71 \\
68\end{array}$ & 14,000 & 20 & 14,000 \\
\hline $6-04$ & Illinois R. at Hardin, Ill. & & 1 & 780 & 708 & 0.5 & 709 & 48,000 & 30 & 48,000 \\
\hline $6-07$ & $\begin{array}{l}\text { Missouri R. at } \\
\text { Hermann, Mo. }\end{array}$ & $\begin{array}{l}\text { A } \\
\text { B } \\
\text { C }\end{array}$ & $\begin{array}{r}15 \\
15 \\
5\end{array}$ & 1,760 & $\begin{array}{l}471 \\
472 \\
467\end{array}$ & $\begin{array}{l}64 \\
76 \\
56\end{array}$ & $\begin{array}{l}535 \\
548\end{array}$ & 71,700 & 11,000 & 83,000 \\
\hline $6-08$ & $\begin{array}{l}\text { Mississippi R. at } \\
\text { St. Louis, Mo. }\end{array}$ & $\begin{array}{l}\text { A } \\
\text { B } \\
\text { C }\end{array}$ & $\begin{array}{r}15 \\
15 \\
5\end{array}$ & 4,760 & $\begin{array}{l}122 \\
122 \\
120\end{array}$ & $\begin{array}{l}34 \\
37 \\
37\end{array}$ & $\begin{array}{l}156 \\
159\end{array}$ & 50,200 & 15,000 & 65,000 \\
\hline $6-10$ & $\begin{array}{l}\text { Mississippi R. at } \\
\text { Thebes, Ill. }\end{array}$ & $\begin{array}{l}\text { A } \\
\text { B } \\
\text { C }\end{array}$ & $\begin{array}{r}14 \\
14 \\
5\end{array}$ & 5,230 & $\begin{array}{l}117 \\
117 \\
117\end{array}$ & $\begin{array}{l}50 \\
49 \\
53\end{array}$ & $\begin{array}{l}167 \\
166\end{array}$ & 52,900 & 22,000 & 75,000 \\
\hline $6-11$ & $\begin{array}{l}\text { Ohio R. at } \\
\text { Olmsted, I11. }\end{array}$ & $\begin{array}{l}\text { A } \\
\text { B } \\
\text { C }\end{array}$ & $\begin{array}{r}15 \\
15 \\
5\end{array}$ & 8,760 & $\begin{array}{l}115 \\
115 \\
112\end{array}$ & $\begin{array}{l}1.4 \\
1.5 \\
1.5\end{array}$ & $\begin{array}{l}116 \\
116\end{array}$ & 87,000 & 1,100 & 88,100 \\
\hline $6-12$ & $\begin{array}{l}\text { Mississippi R. below } \\
\text { Hickman, Ky. }\end{array}$ & $\begin{array}{r}A \\
B \\
N C\end{array}$ & $\begin{array}{r}15 \\
15 \\
5\end{array}$ & 14,100 & $\begin{array}{l}129 \\
130 \\
124\end{array}$ & $\begin{array}{l}13 \\
15 \\
13\end{array}$ & $\begin{array}{l}142 \\
145\end{array}$ & 158,000 & 17,000 & 175,000 \\
\hline $6-14$ & $\begin{array}{l}\text { Mississippi R. at } \\
\text { Fulton, Tena. }\end{array}$ & $\begin{array}{r}A \\
B \\
N C\end{array}$ & $\begin{array}{r}14 \\
14 \\
5\end{array}$ & 15,300 & $\begin{array}{l}182 \\
181 \\
186\end{array}$ & $\begin{array}{l}26 \\
27 \\
26\end{array}$ & $\begin{array}{l}208 \\
208\end{array}$ & 240,000 & 35,000 & 275,000 \\
\hline $6-17$ & $\begin{array}{l}\text { Mississippi R. at } \\
\text { Helena, Ark. }\end{array}$ & $\begin{array}{r}A \\
B \\
N C\end{array}$ & $\begin{array}{r}15 \\
15 \\
5\end{array}$ & 16,900 & $\begin{array}{l}216 \\
213 \\
212\end{array}$ & $\begin{array}{l}45 \\
44 \\
40\end{array}$ & $\begin{array}{l}261 \\
257\end{array}$ & 313,000 & 65,000 & 378,000 \\
\hline $6-18$ & $\begin{array}{l}\text { White R. at } \\
\text { Mile } 11.5 \text {, Ark. } 1\end{array}$ & $\begin{array}{l}{ }^{3} \mathrm{~A} \\
{ }^{3} \mathrm{~B}\end{array}$ & $\begin{array}{l}3 \\
3\end{array}$ & 770 & $\begin{array}{l}91 \\
92\end{array}$ & 0.1 & $\begin{array}{l}91 \\
92\end{array}$ & 6,100 & 7 & 6,100 \\
\hline $6-19$ & $\begin{array}{l}\text { Arkansas R. at } \\
\text { Pendleton, Ark. }{ }^{2}\end{array}$ & $\begin{array}{r}\mathrm{A} \\
\mathrm{B} \\
\mathrm{C} \\
\mathrm{NC}\end{array}$ & $\begin{array}{r}15 \\
15 \\
5 \\
5\end{array}$ & 3,600 & $\begin{array}{l}68 \\
69 \\
65\end{array}$ & $\begin{array}{l}81 \\
89 \\
81 \\
93\end{array}$ & $\begin{array}{l}149 \\
157\end{array}$ & 21,000 & 26,000 & 47,000 \\
\hline $6-20$ & $\begin{array}{l}\text { Mississippi R. above } \\
\text { Arkansas City, Ark. }\end{array}$ & $\begin{array}{r}A \\
B \\
N C\end{array}$ & $\begin{array}{r}14 \\
14 \\
5\end{array}$ & 23,300 & $\begin{array}{l}170 \\
169 \\
172\end{array}$ & $\begin{array}{l}72 \\
62 \\
67\end{array}$ & $\begin{array}{l}242 \\
231\end{array}$ & 341,000 & 135,000 & 476,000 \\
\hline $6-22$ & $\begin{array}{l}\text { Yazoo R. below } \\
\text { Steele Bayou, Miss. }\end{array}$ & $\begin{array}{r}{ }^{3} \mathrm{~A} \\
{ }^{3} \mathrm{~B} \\
\mathrm{C}\end{array}$ & $\begin{array}{l}6 \\
6 \\
6\end{array}$ & 1,070 & $\begin{array}{l}271 \\
272 \\
270\end{array}$ & $\begin{array}{l}1.2 \\
1.1 \\
1.1\end{array}$ & $\begin{array}{l}272 \\
273\end{array}$ & 25,100 & 110 & 25,200 \\
\hline $6-23$ & $\begin{array}{l}\text { Mississippi R. below } \\
\text { Vicksburg, Miss. }\end{array}$ & $\begin{array}{r}\text { A } \\
B \\
N C\end{array}$ & $\begin{array}{r}18 \\
18 \\
6\end{array}$ & 24,800 & $\begin{array}{l}154 \\
152 \\
153\end{array}$ & $\begin{array}{l}58 \\
56 \\
52\end{array}$ & $\begin{array}{l}212 \\
208\end{array}$ & 328,000 & 122,000 & 450,000 \\
\hline $6-25$ & $\begin{array}{l}\text { Old R. Outflow Channel near } \\
\text { Knox Landing, La. }\end{array}$ & $\begin{array}{r}\mathrm{A} \\
\mathrm{B} \\
\mathrm{NC}\end{array}$ & $\begin{array}{r}15 \\
15 \\
5\end{array}$ & 4,890 & $\begin{array}{l}162 \\
158 \\
160\end{array}$ & $\begin{array}{l}62 \\
57 \\
57\end{array}$ & $\begin{array}{l}224 \\
215\end{array}$ & 67,600 & 25,000 & 92,600 \\
\hline $6-26$ & $\begin{array}{l}\text { Mississippi R. near } \\
\text { St. Francisville, La. }\end{array}$ & $\begin{array}{r}\text { A } \\
\mathrm{B} \\
\mathrm{NC}\end{array}$ & $\begin{array}{r}15 \\
15 \\
5\end{array}$ & 19,000 & $\begin{array}{l}155 \\
153 \\
152\end{array}$ & $\begin{array}{l}54 \\
47 \\
43\end{array}$ & $\begin{array}{l}209 \\
200\end{array}$ & 253,000 & 83,000 & 336,000 \\
\hline $6-28$ & $\begin{array}{l}\text { Mississippi R. below } \\
\text { Belle Chasse, La. }\end{array}$ & $\begin{array}{r}A \\
B \\
N C\end{array}$ & $\begin{array}{l}7 \\
7 \\
5\end{array}$ & 20,100 & $\begin{array}{l}169 \\
172 \\
168\end{array}$ & $\begin{array}{l}36 \\
44 \\
27\end{array}$ & $\begin{array}{l}205 \\
216\end{array}$ & 296,000 & 69,000 & 365,000 \\
\hline
\end{tabular}

${ }^{1}$ Sand in composites $A$ and B were combined to give one value listed opposite composite A.

${ }^{2}$ Silt and clay in composites $A$ and $B$ were combined to give one value listed opposite composite A.

${ }^{3} A$ and $B$ composites were collected at the same verticals. 
Table 21.--Suspended-sediment concentrations in depth-integrated, composite samples collected from the Mississippi

River and some of its tributaries and the corresponding sediment discharges for February-March 1990 cruise

[Total sediment concentration of composites A and B were averaged in order to calculate a single value of sediment discharge; <, less than; >, greater than; $\mathrm{m}^{3} / \mathrm{s}$, cubic meter per second; mg/L, milligram per liter; Hm, micrometer; data for 5-vertical composite were not used to calculate the total concentration or the sediment discharge; $C$, collected concurrently with large composite; NC, not collected concurrently with larger composite; nd indicates not determined. Analyses by R.H. Meadel

\begin{tabular}{|c|c|c|c|c|c|c|c|c|c|c|}
\hline \multirow{2}{*}{$\begin{array}{l}\text { Date } \\
1990\end{array}$} & \multirow[b]{2}{*}{ Site name } & \multirow[b]{2}{*}{ Composite } & \multirow{2}{*}{$\begin{array}{c}\text { Number } \\
\text { of } \\
\text { verticals }\end{array}$} & \multirow{2}{*}{$\begin{array}{c}\text { Water } \\
\text { discharge } \\
\left(m^{3} / s\right)\end{array}$} & \multicolumn{3}{|c|}{$\begin{array}{c}\text { Sediment concentration } \\
\left(\mathrm{mg}_{\mathrm{g}} \mathrm{L}\right)\end{array}$} & \multicolumn{3}{|c|}{$\begin{array}{l}\text { Sediment discharge } \\
\text { (metric tons/day) }\end{array}$} \\
\hline & & & & & $\begin{array}{c}\text { Silt and } \\
\text { clay } \\
<63 \mu \text { m }\end{array}$ & $\begin{array}{l}\text { Sand } \\
>63 \mathrm{Nm}\end{array}$ & Total & $\begin{array}{c}\text { Silt and } \\
\text { clay } \\
<63 \text { Ha }\end{array}$ & $\begin{array}{l}\text { Sand } \\
>63 \mathrm{\mu m}\end{array}$ & Total \\
\hline $2-25$ & $\begin{array}{l}\text { Mississippi R. near } \\
\text { Cache, Ill. }\end{array}$ & One & 5 & 4,240 & 108 & 1.2 & 109 & 39,600 & 440 & 40,000 \\
\hline $3-01$ & $\begin{array}{l}\text { Ohio R. at } \\
\text { Uniontown, Ky. }{ }^{1}\end{array}$ & $\begin{array}{r}A \\
B \\
N C\end{array}$ & $\begin{array}{r}15 \\
14 \\
5\end{array}$ & 6,620 & $\begin{array}{l}205 \\
204 \\
208\end{array}$ & $\begin{array}{l}4.8 \\
4.9 \\
4.2\end{array}$ & $\begin{array}{l}210 \\
209\end{array}$ & 117,000 & 2,800 & 120,000 \\
\hline $2-28$ & $\begin{array}{l}\text { Wabash } R \text {. near } \\
\text { New Haven, I } 11.1\end{array}$ & $\begin{array}{l}{ }^{3} \mathrm{~A} \\
{ }^{3} \mathrm{~B} \\
\mathrm{NC}\end{array}$ & $\begin{array}{r}14 \\
14 \\
5\end{array}$ & $1_{2,340}$ & $\begin{array}{r}148 \\
147 \\
\text { nd }\end{array}$ & $\begin{array}{l}25 \\
25 \\
30\end{array}$ & $\begin{array}{l}173 \\
172\end{array}$ & 30,000 & 5,100 & 35,100 \\
\hline $2-23$ & $\begin{array}{l}\text { Cumberland R. near } \\
\text { Smithland, Ky. } 1,2\end{array}$ & $\begin{array}{l}{ }^{3} \mathrm{~A} \\
{ }^{3} \mathrm{~B} \\
\mathrm{NC}\end{array}$ & $\begin{array}{r}10 \\
10 \\
5\end{array}$ & $1_{2,170}$ & $\begin{array}{l}32 \\
32 \\
32\end{array}$ & $\begin{array}{l}0.05 \\
0.0\end{array}$ & $\begin{array}{l}32 \\
32\end{array}$ & 6,000 & 9 & 6,000 \\
\hline $2-24$ & $\begin{array}{l}\text { Tennessee } R \text {. near } \\
\text { Calvert City, Ky. } 1,2\end{array}$ & $\begin{array}{l}{ }^{3} \mathrm{~A} \\
{ }^{3} \mathrm{~B} \\
\mathrm{NC}\end{array}$ & $\begin{array}{r}14 \\
14 \\
5\end{array}$ & ${ }^{1} 6,570$ & $\begin{array}{l}49 \\
48 \\
37\end{array}$ & $\begin{array}{l}0.03 \\
0.0\end{array}$ & $\begin{array}{l}49 \\
48\end{array}$ & 28,000 & 20 & 28,000 \\
\hline $3-03$ & $\begin{array}{l}\text { Ohio R. at } \\
\text { Olmsted, III. }\end{array}$ & $\begin{array}{l}{ }^{3} \mathrm{~A} \\
{ }^{3} \mathrm{~B} \\
\mathrm{NC}\end{array}$ & $\begin{array}{r}15 \\
15 \\
5\end{array}$ & 16,100 & $\begin{array}{l}143 \\
145 \\
141\end{array}$ & $\begin{array}{l}5.3 \\
5.6 \\
4.4\end{array}$ & $\begin{array}{l}148 \\
151\end{array}$ & 200,000 & 7,600 & 208,000 \\
\hline $3-04$ & $\begin{array}{l}\text { Mississippi R. below } \\
\text { Hickman, Ky. }\end{array}$ & $\begin{array}{l}{ }^{3} \mathbf{A} \\
{ }^{3} \mathbf{B}\end{array}$ & $\begin{array}{l}15 \\
15\end{array}$ & 21,000 & $\begin{array}{l}158 \\
158\end{array}$ & $\begin{array}{l}48 \\
48\end{array}$ & $\begin{array}{l}206 \\
206\end{array}$ & 287,000 & 87,000 & 374,000 \\
\hline $3-05$ & $\begin{array}{l}\text { Mississippi R. below } \\
\text { Fulton, Tenn. }\end{array}$ & $\begin{array}{l}{ }^{3} \mathrm{~A} \\
{ }^{3} \mathrm{~B} \\
\mathrm{NC}\end{array}$ & $\begin{array}{r}15 \\
15 \\
5\end{array}$ & 22,800 & $\begin{array}{l}141 \\
141 \\
138\end{array}$ & $\begin{array}{l}90 \\
95 \\
83\end{array}$ & $\begin{array}{l}231 \\
236\end{array}$ & 278,000 & 182,000 & 460,000 \\
\hline $3-07$ & $\begin{array}{l}\text { Mississippi R. at } \\
\text { Helena, Ark. }\end{array}$ & $\begin{array}{l}{ }^{3} \mathrm{~A} \\
{ }^{3} \mathrm{~B} \\
\mathrm{NC}\end{array}$ & $\begin{array}{r}15 \\
15 \\
5\end{array}$ & 23,300 & $\begin{array}{l}147 \\
146 \\
142\end{array}$ & $\begin{array}{l}70 \\
59 \\
65\end{array}$ & $\begin{array}{l}217 \\
205\end{array}$ & 295,000 & 130,000 & 425,000 \\
\hline $3-08$ & $\begin{array}{c}\text { Mississippi R. above } \\
\text { Arkansas City, Ark. }\end{array}$ & $\begin{array}{l}{ }^{3} \mathrm{~A} \\
{ }^{3} \mathrm{~B}\end{array}$ & $\begin{array}{l}16 \\
16\end{array}$ & 33,200 & $\begin{array}{l}126 \\
126\end{array}$ & $\begin{array}{l}94 \\
84\end{array}$ & $\begin{array}{l}220 \\
210\end{array}$ & 361,000 & 255,000 & 616,000 \\
\hline $3-10$ & $\begin{array}{l}\text { Mississippi R. below } \\
\text { Vicksburg, Miss. }\end{array}$ & $\begin{array}{l}{ }^{3} \mathrm{~A} \\
{ }^{3} \mathrm{~B} \\
\mathrm{NC}\end{array}$ & $\begin{array}{r}18 \\
18 \\
6\end{array}$ & 34,100 & $\begin{array}{l}126 \\
125 \\
124\end{array}$ & $\begin{array}{l}117 \\
108 \\
111\end{array}$ & $\begin{array}{l}243 \\
233\end{array}$ & 370,000 & 331,000 & 701,000 \\
\hline $3-12$ & $\begin{array}{l}\text { Mississippi R. near } \\
\text { St. Francisville, La. } 1\end{array}$ & $\begin{array}{l}{ }^{3} \mathrm{~A} \\
{ }^{3} \mathrm{~B} \\
\mathrm{NC}\end{array}$ & $\begin{array}{r}15 \\
15 \\
5\end{array}$ & 26,300 & $\begin{array}{l}99 \\
98 \\
97\end{array}$ & $\begin{array}{l}85 \\
82 \\
62\end{array}$ & $\begin{array}{l}184 \\
180\end{array}$ & 224,000 & 190,000 & 414,000 \\
\hline $3-14$ & $\begin{array}{l}\text { Mississippi R. below } \\
\text { Belle Chasse, La. }\end{array}$ & $\begin{array}{l}{ }^{3} \mathrm{~A} \\
{ }^{3} \mathrm{~B} \\
\mathrm{NC}\end{array}$ & $\begin{array}{r}13 \\
13 \\
4\end{array}$ & 26,700 & $\begin{array}{l}140 \\
139 \\
135\end{array}$ & $\begin{array}{l}83 \\
79 \\
77\end{array}$ & $\begin{array}{l}223 \\
218\end{array}$ & 322,000 & 187,000 & 509,000 \\
\hline
\end{tabular}

1Water discharge was measured from left edge of flow to right edge of flow. No overbank measurement was made.

${ }^{2}$ Sand in composites $A$ and $B$ was combined to give one value listed opposite composite $A$.

${ }^{3} A$ and $B$ composites were collected at the same verticals. 
Table 22.--Suspended-sediment concentrations in depth-integrated, composite samples collected from the Mississippi River and some of its tributaries and the corresponding sediment discharges for second leg of the May-June 1990 cruise

[Total sediment concentration of composites $A$ and $B$ were averaged on order to calculate a single value of sediment discharge; $<$, less than; >, greater than; $\mathrm{m}^{3} / \mathrm{s}$, cubic meter per second; mg/L, milligram per liter; $\mu m$, micrometer. Analyses by $R . H$. Meade]

\begin{tabular}{|c|c|c|c|c|c|c|c|c|c|c|}
\hline \multirow[b]{2}{*}{$\begin{array}{l}\text { Date } \\
1990\end{array}$} & \multirow[b]{2}{*}{ Site name } & \multirow[b]{2}{*}{ Composite } & \multirow{2}{*}{$\begin{array}{c}\text { Number } \\
\text { of } \\
\text { verticals }\end{array}$} & \multirow{2}{*}{$\begin{array}{c}\text { Water } \\
\text { discharge } \\
\left(\mathrm{m}^{3} / \mathrm{s}\right)\end{array}$} & \multicolumn{3}{|c|}{$\begin{array}{c}\text { Sediment concentration } \\
\left(\mathrm{mg}_{\mathrm{g}} / \mathrm{L}\right)\end{array}$} & \multicolumn{3}{|c|}{$\begin{array}{l}\text { Sediment discharge } \\
\text { (metric tons/day) }\end{array}$} \\
\hline & & & & & $\begin{array}{l}\overline{\text { Silt and }} \\
\text { clay } \\
<63 \mu \mathrm{m}\end{array}$ & $\begin{array}{l}\text { Sand } \\
>63 \mu \mathrm{m}\end{array}$ & Total & $\begin{array}{l}\text { Silt and } \\
\text { clay } \\
<63 \mu \mathrm{m}\end{array}$ & $\begin{array}{l}\text { Sand } \\
>63 \mu \mathrm{m}\end{array}$ & Total \\
\hline $6-07$ & $\begin{array}{l}\text { Illinois R. at } \\
\text { Valley City, Ill. }\end{array}$ & $\begin{array}{l}{ }^{1} \mathbf{A} \\
{ }^{1} \mathbf{B}\end{array}$ & $\begin{array}{l}5 \\
5\end{array}$ & 1,230 & $\begin{array}{l}97 \\
99\end{array}$ & $\begin{array}{l}4.6 \\
3.8\end{array}$ & $\begin{array}{l}102 \\
103\end{array}$ & 10,400 & 440 & 10,800 \\
\hline $6-11$ & $\begin{array}{l}\text { Mississippi } R \text {, below } \\
\text { Grafton, I } 11 \text {. }\end{array}$ & $\begin{array}{l}\mathbf{A} \\
\mathbf{B}\end{array}$ & $\begin{array}{l}10 \\
10\end{array}$ & 5,040 & $\begin{array}{l}459 \\
469\end{array}$ & $\begin{array}{l}2.5 \\
2.2\end{array}$ & $\begin{array}{l}462 \\
471\end{array}$ & 202,000 & 1,000 & 203,000 \\
\hline $6-10$ & $\begin{array}{l}\text { Missouri R, at } \\
\text { St. Charles, Mo. }\end{array}$ & None & 1 & not measured & 1,940 & 392 & 2,332 & \multicolumn{3}{|c|}{ no water-discharge data } \\
\hline $6-13$ & $\begin{array}{l}\text { Mississippi } R \text {, at } \\
\text { Thebes, Ill. }\end{array}$ & $\begin{array}{l}\mathbf{A} \\
\mathbf{B}\end{array}$ & $\begin{array}{l}10 \\
10\end{array}$ & 12,600 & $\begin{array}{l}1,110 \\
1,120\end{array}$ & $\begin{array}{l}101 \\
122\end{array}$ & $\begin{array}{l}1,211 \\
1,242\end{array}$ & $1,210,000$ & 121,000 & $1,330,000$ \\
\hline $6-14$ & $\begin{array}{l}\text { Ohio R. at } \\
\text { Olmsted, I1l. }\end{array}$ & $\begin{array}{l}\mathbf{A} \\
\mathbf{B}\end{array}$ & $\begin{array}{l}10 \\
10\end{array}$ & 9,550 & $\begin{array}{l}180 \\
174\end{array}$ & $\begin{array}{l}0.2 \\
0.3\end{array}$ & $\begin{array}{l}180 \\
174\end{array}$ & 146,000 & 200 & 146,000 \\
\hline $6-18$ & $\begin{array}{l}\text { Mississippi R. below } \\
\text { Memphis, Tenn. }\end{array}$ & $\begin{array}{l}\mathbf{A} \\
\mathbf{B}\end{array}$ & $\begin{array}{l}10 \\
10\end{array}$ & 20,800 & $\begin{array}{l}432 \\
432\end{array}$ & $\begin{array}{l}38 \\
27\end{array}$ & $\begin{array}{l}470 \\
459\end{array}$ & 776,000 & 58,000 & 834,000 \\
\hline $6-20$ & $\begin{array}{l}\text { Mississippi R. below } \\
\text { Arkansas City, Ark. }\end{array}$ & $\begin{array}{l}\mathbf{A} \\
\mathbf{B}\end{array}$ & $\begin{array}{l}10 \\
10\end{array}$ & 25,500 & $\begin{array}{l}336 \\
332\end{array}$ & $\begin{array}{l}67 \\
45\end{array}$ & $\begin{array}{l}403 \\
377\end{array}$ & 736,000 & 123,000 & 859,000 \\
\hline $6-22$ & $\begin{array}{l}\text { Yazoo R. st } \\
\text { Mile } 9.0 \text {, Miss. }\end{array}$ & ${ }^{1} \mathbf{A}$ & $\begin{array}{l}5 \\
5\end{array}$ & 1,250 & $\begin{array}{l}116 \\
117\end{array}$ & $\begin{array}{l}0.2 \\
0.2\end{array}$ & $\begin{array}{l}116 \\
117\end{array}$ & 12,600 & 20 & 12,600 \\
\hline $6-23$ & $\begin{array}{l}\text { Mississippi R. below } \\
\text { Vicksburg, Miss. }\end{array}$ & $\begin{array}{l}\mathbf{A} \\
\mathbf{B}\end{array}$ & $\begin{array}{l}10 \\
10\end{array}$ & 27,300 & $\begin{array}{l}273 \\
273\end{array}$ & $\begin{array}{l}31 \\
28\end{array}$ & $\begin{array}{l}304 \\
301\end{array}$ & 644,000 & 70,000 & 714,000 \\
\hline $6-25$ & $\begin{array}{l}\text { Mississippi R. near } \\
\text { St. Frsncisville, La. }\end{array}$ & $\begin{array}{l}\mathbf{A} \\
\mathbf{B}\end{array}$ & $\begin{array}{l}10 \\
10\end{array}$ & 23,200 & $\begin{array}{l}184 \\
183\end{array}$ & $\begin{array}{l}47 \\
48\end{array}$ & $\begin{array}{l}231 \\
231\end{array}$ & 368,000 & 95,000 & 463,000 \\
\hline $6-27$ & $\begin{array}{c}\text { Mississippi R. below } \\
\text { Belle Chasse, La. }\end{array}$ & $\begin{array}{l}\text { A } \\
\text { B }\end{array}$ & $\begin{array}{l}10 \\
10\end{array}$ & 23,300 & $\begin{array}{l}183 \\
183\end{array}$ & $\begin{array}{l}50 \\
47\end{array}$ & $\begin{array}{l}233 \\
230\end{array}$ & 368,000 & 98,000 & 466,000 \\
\hline
\end{tabular}

${ }^{1} A$ and $B$ composites were collected at the same verticals. 
Our usual sampling procedure, wherein we collected duplicate composite samples (A and $B$ ) at each sampling site, allowed us to estimate the precision or reproducibility of the suspended-sediment concentrations in relation to the number of verticals sampled. The percent difference in the concentration of the composite $A$ and $B$ samples (relative to the average concentration of composites $A$ and $B$ ) in tables 19-22 were grouped by the number of verticals. For the verticals having five or more comparisons, the average percent difference is listed in table 23. Concentrations of suspended silt and clay in depth-integrated composite samples are reproducible within about 1 percent, while concentrations of sand are reproducible within about 16 percent for 10-vertical composites, within 10 percent for 15 -vertical composites, and 7 percent for 18-vertical composites.

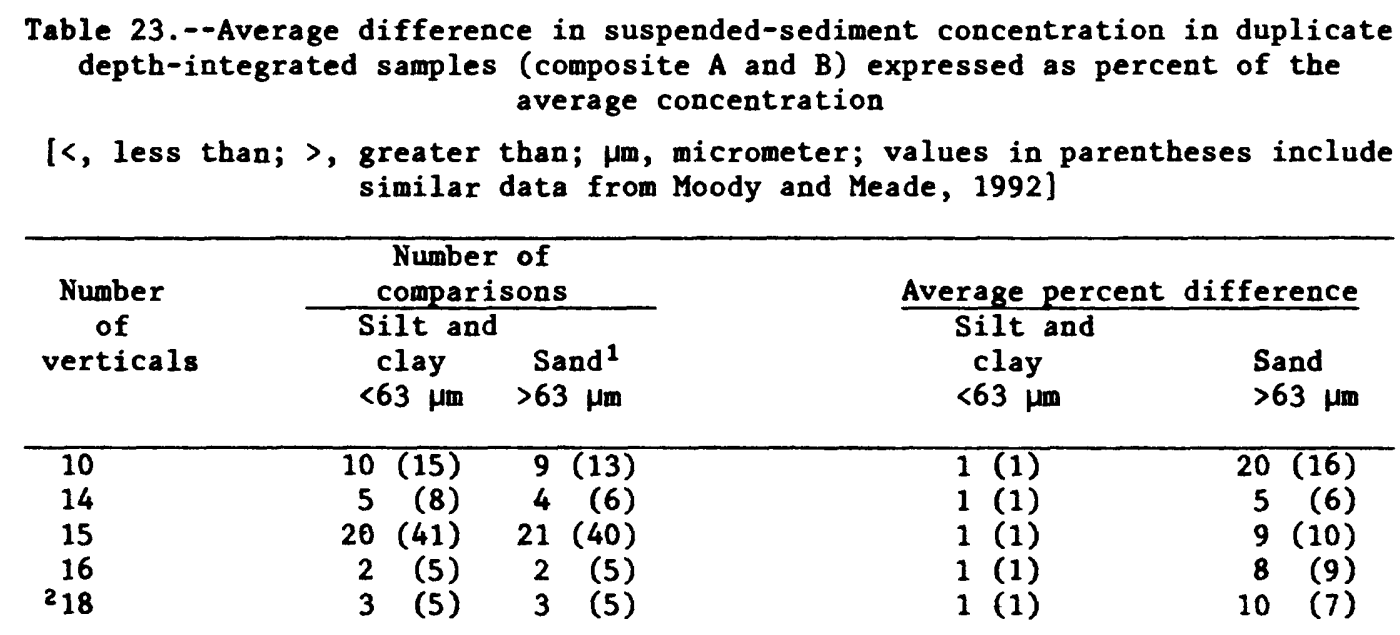

\footnotetext{
${ }^{1}$ Does not include samples with no sand, samples for which composite $A$ and $B$ were combined to obtain enough sand for analysis, or samples noted as having mostly organic material.

${ }^{2}$ All comparisons are for Mississippi River below Vicksburg, Mississippi.
}

The accuracy in measuring suspended-sediment concentration using the depth-integration method as a function of the number of verticals was estimated from data collected at only those sites listed in tables 19-20 where a 5-vertical composite was collected concurrently with two 15-vertical composites (denoted by $A$ and $B$ in the composite column of tables 19-20). The $A$ and $B$ composites were collected at different verticals; thus, by averaging the composite $A$ and $B$ concentration values, a 30-vertical, suspended-sediment concentration was determined. This 30-vertical concentration was assumed to represent the actual concentration for calculating the percent difference of the 5- and 15-vertical composite concentrations. The concentrations of silt and clay in these comparisons of concurrent samples ranged from 68 to $472 \mathrm{mg} / \mathrm{L}$ and the sand ranged from 1 to $94 \mathrm{mg} / \mathrm{L}$. For samples collected concurrently, the concentration of sand from the 15-vertical composites differed by about 5 percent and the concentration from the 5-vertical composites differed by about 9 percent from the concentrations of the 30-vertical composites. However, the concentration of suspended sediment finer than $63 \mu \mathrm{m}$ from 15-vertical composites differed by 1 percent or less and concentrations from the 5-vertical composites differed by 2 percent or less from the concentrations of the 30 -vertical composites. 
Particle-size distributions of the suspended sediment (from 1 to $1,000 \mu \mathrm{m}$ ) for the four cruises are listed in tables 24-27. The particle-size distributions of the suspended sand (greater than $63 \mu \mathrm{m}$ ) were analyzed by the visual-accumulation tube method, and the particle-size distribution of the suspended silt and clay (finer than $63 \mu \mathrm{m}$ ) were analyzed by the Sedigraph method. Both methods are based on settling properties of the particles. Both methods involve preliminary chemical treatment that disaggregates the particles as they exist in the river. Therefore, the size analyses reported here are more representative of the assemblages of individual particles available to interact with the dissolved matter in the river and less representative of the hydraulic properties of the grains as they are transported by the river. Stallard and Martin (1989) noted that settling velocities of suspended sediments that were chemically dispersed for analysis, such as those reported here, were markedly slower than settling velocities of suspended sediments that were allowed to settle in native river water immediately after sampling.

The sand samples that were saved in separate polypropylene bottles were sent to the USGS Sediment Laboratory in Iowa City, Iowa, for size analysis by the visual-accumulation-tube method (Guy, 1969). Replicate samples were run and if the results did not agree within 5 percent for all size classes, the analysis was repeated (Matthes and others, 1992). A complication arose during the particle-size analysis when the sand samples were treated with hydrogen peroxide, the standard technique for removing organic matter (Guy, 1969, p. 52). This treatment apparently disaggregated silt particles from sand-size aggregates. In most of the suspended-sand samples, the hydrogen peroxide treatment released silt grains from sand-size aggregates. Although the quantities of released silt grains were almost always (in all samples but one) 2 percent or less of the total concentration of suspended sediment, they were subtracted, where appropriate, from the sand concentrations and added to the concentration of the coarsest silt fraction $(32$ to $63 \mu \mathrm{m})$ in the data reported in tables 19-22 and tables 24-27.

The reproducibility of the sampling and size-analysis procedures was previously addressed by Moody and Meade (1992, table 18) for the sand fractions of the $A$ and $B$ composites of 12 suspended-sediment samples collected during 1987-88. When results were expressed as percentages of the sand fraction, differences between percents finer than certain sizes were as great as 7 percent. When results were expressed as percentages of the total sample, these differences were never more than 2 percent.

Only the samples collected by the depth-integration method were used in the particle-size analysis of suspended sediment finer than $63 \mu \mathrm{m}$. The essential problem in preparing a silt and clay fraction sample for particle-size analysis is one of converting the dilute suspensions collected in the field into the concentrated suspensions required for Sedigraph analysis. In our procedure, the suspended-sediment sample remained wet from the time it was collected to the time it was analyzed. The two subsamples of about 4-11 L of water and sediment (finer than $63 \mu \mathrm{m}$ ) that were taken from the $A$ and $B$ churn splitters on the research vessel were combined in a polyethylene carboy to which 10-15 $\mathrm{mL}$ of chloroform was added to retard organic growth. 
The carboys from all sampling sites were transported to the USGS laboratory in Denver, where the suspended sediment was allowed to settle undisturbed for at least 15 days. At the maximum vertical settling distance of $36 \mathrm{~cm}$ in the carboys, this time was sufficient for all particles coarser than about $0.5 \mu \mathrm{m}$ to settle (assuming Stokesian settling of quartz spherical particles). After 15 days or longer, the supernatant water was siphoned from the carboys, and the settled sediment was transferred to 1-L glass jars (maximum settling distance, $15 \mathrm{~cm}$ ) where it was allowed to settle for another 10 days or more. The supernatant was siphoned off once more, and the settled sediment was transferred to $250-\mathrm{mL}$ polyethylene bottles.

All analyses of the size distribution of particles finer than $63 \mu \mathrm{m}$ were made in the USGS sediment laboratory in Iowa City, Iowa, by the Sedigraph method as described by Lara and Matthes (1986). At least 1 in 10 Sedigraph samples were reanalyzed, and if the results did not agree within 5 percent for all size classes, the analysis was repeated (Matthes and others, 1992). After the particle-size distribution is determined, the sample is dried and weighed. The dry weight is compared with the weight of sediment that is calculated from the concentration of suspended sediment finer than $63 \mu \mathrm{m}$ determined by the filtering-and-weighing procedure (silt and clay column in tables 19-22) and the known volume (8-22 L) of river water from which the analyzed sample is allowed to settle. The differences between the calculated dry weights and the measured dry weights are assumed to represent the material finer than $0.5 \mu \mathrm{m}$ that remained in suspension and was siphoned from the carboys along with the supernatant water. These differences have been added to the mass of material finer than $1 \mu \mathrm{m}$ for purposes of calculating the percentages listed in tables 24-27. The amount of siphoned material finer than $0.5 \mu \mathrm{m}$ averaged 15 percent for the March-April 1989 cruise, 7 percent for the June 1989 cruise, 10 percent for the February-March 1990 cruise, and 6 percent for the May-June 1990 cruise. The largest amounts of siphoned material finer than $0.5 \mu \mathrm{m}$ (21-57 percent) were measured in samples collected from the White, Arkansas, and Yazoo Rivers for the March-April 1989 cruise; from the Yazoo River for the June 1989 cruise and the second leg of the May-June 1990 cruise; and from the Cumberland and Tennessee Rivers for the February-March 1990 cruise. The amount of siphoned material finer than $0.5 \mu \mathrm{m}$ ranged between 1 and 12 percent for the remaining sampling sites.

To evaluate the differences incurred by using different collecting methods and processing procedures, two additional suspended-sediment subsamples were obtained at selected sampling sites for the March-April 1989 and the June 1989 cruises: (1) samples collected by the pumping method and processed by continuous-flow centrifugation, and (2) samples collected by depth integration and processed by churning and continuous-flow centrifugation. For these selected river sampling sites and cruises, a size distribution of the suspended sediment was obtained for each of these samples. The concentration of sediment for individual size classes is listed in table 28 , in addition to the concentration of sediment for individual size classes from the depth-integrated, churned and settled sample, which is also listed in tables 24 and 25 as cumulative percent by weight. 
Table 24.--Particle-size distribution of suspended-sediment samples collected from the Mississippi River and some of its tributaries for March-April 1989 croise

[Finer than $63 \mu \mathrm{m}$ determined by Sedigraph; coarser than $63 \mu \mathrm{m}$ determined by visual-accumulation tube; <, less than; $\mathrm{mg} / \mathrm{L}$, milligram per liter; $\mu \mathrm{m}$, micrometer. Analyses by C.J. Anderson and J.A. Moody]

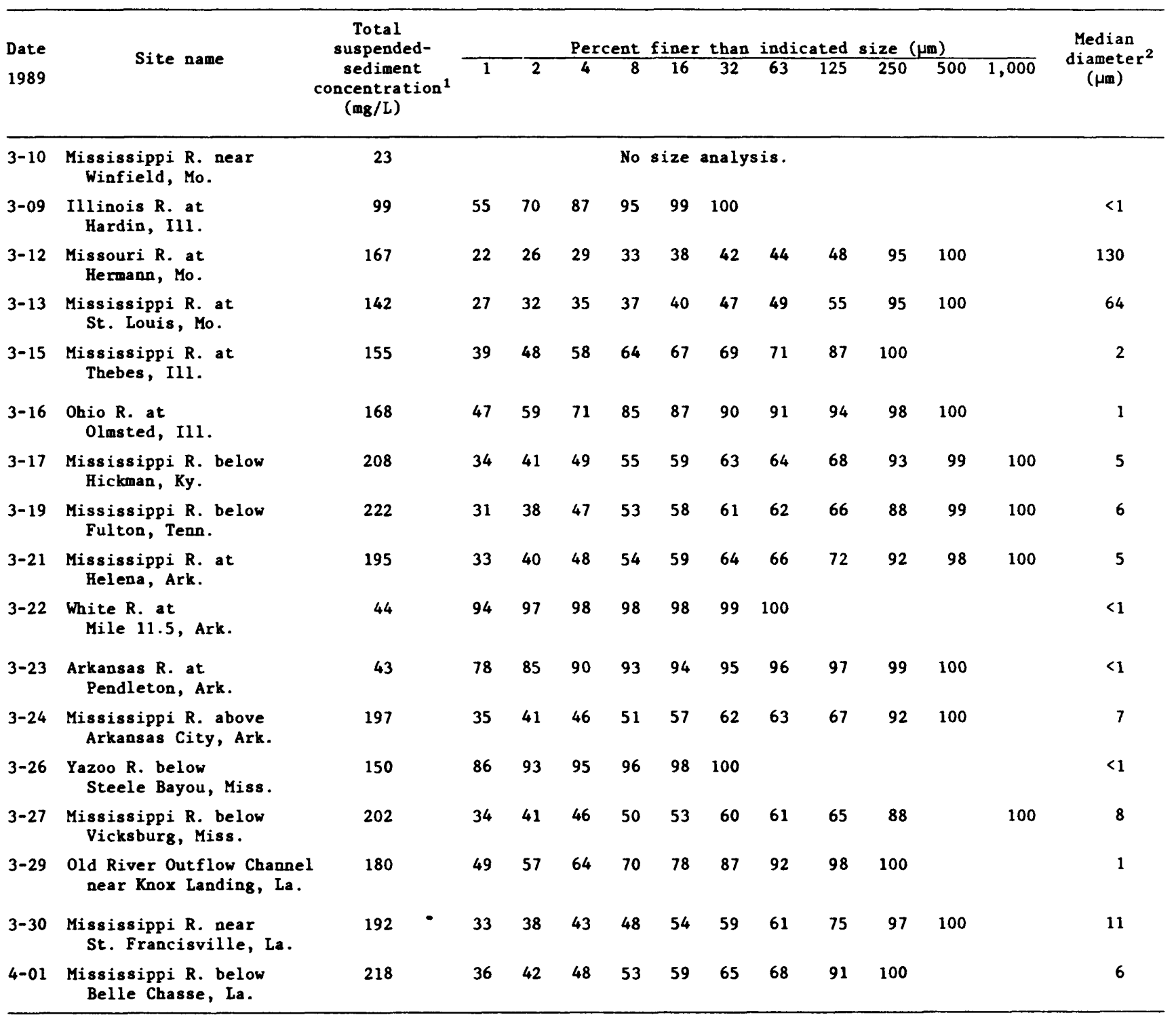

${ }^{1}$ Total suspended-sediment concentration is the average of the composite $A$ and $B$ values in table 19.

${ }^{2}$ Median diameter was computed by linear interpolation. 
Table 25.--Particle-size distribution of suspended-sediment samples collected from the Mississippi River and some of its tributaries for June 1989 cruise

[Finer than $63 \mu \mathrm{m}$ determined by Sedigraph; coarser than $63 \mu \mathrm{m}$ determined by visual-accumulation tube; <, less than; $\mathrm{mg} / \mathrm{L}$, milligram per liter; $\mu \mathrm{m}$, micrometer. Analyses by C.J. Anderson and J.A. Moody]

\begin{tabular}{|c|c|c|c|c|c|c|c|c|c|c|c|c|c|c|}
\hline $\begin{array}{l}\text { Date } \\
1989\end{array}$ & Site name & $\begin{array}{c}\text { Total } \\
\text { suspended- } \\
\text { sediment } \\
\text { concentration } 1 \\
(\mathrm{mg} / \mathrm{L})\end{array}$ & 1 & 2 & $\frac{\text { Per }}{4}$ & $\frac{\text { ent }}{8}$ & $\frac{\text { finer }}{16}$ & $\frac{\text { than }}{32}$ & $\frac{\text { indi }}{63}$ & $\frac{\text { ated }}{125}$ & $\frac{\text { size }}{250}$ & (m) & 1,000 & $\begin{array}{l}\text { Median } \\
\text { diameter }{ }^{2} \\
(\mu \mathrm{m})\end{array}$ \\
\hline $6-05$ & $\begin{array}{l}\text { Mississippi R. near } \\
\text { Winfield, Mo. }\end{array}$ & 70 & 61 & 71 & 81 & 88 & 95 & 98 & 100 & & & & & $<1$ \\
\hline $6-04$ & $\begin{array}{l}\text { Illinois R. at } \\
\text { Hardin, Ill. }\end{array}$ & 709 & 42 & 53 & 64 & 77 & 91 & 99 & 100 & & & & & 2 \\
\hline $6-07$ & $\begin{array}{l}\text { Missouri R. at } \\
\text { Hermann, Mo. }\end{array}$ & 542 & 44 & 55 & 68 & 78 & 83 & 86 & 88 & 89 & 99 & 100 & & 2 \\
\hline $6-08$ & $\begin{array}{l}\text { Mississippi R. at } \\
\text { St. Louis, Mo. }\end{array}$ & 158 & 34 & 42 & 52 & 61 & 69 & 76 & 77 & 79 & 95 & 100 & & 4 \\
\hline $6-10$ & $\begin{array}{l}\text { Mississippi R. at } \\
\text { Thebes, Ill. }\end{array}$ & 166 & 29 & 37 & 45 & 52 & 59 & 68 & 70 & 74 & 94 & 100 & & 7 \\
\hline $6-11$ & $\begin{array}{l}\text { Ohio R. at } \\
\text { Olmsted, III. }\end{array}$ & 116 & 53 & 63 & 73 & 82 & 91 & 97 & 99 & 100 & & & & $<1$ \\
\hline $6-12$ & $\begin{array}{l}\text { Mississippi R. below } \\
\text { Hickman, Ky. }\end{array}$ & 144 & 43 & 53 & 64 & 74 & 82 & 87 & 89 & 91 & 98 & 100 & & 2 \\
\hline $6-14$ & $\begin{array}{l}\text { Mississippi R. at } \\
\text { Fulton, Tenn. }\end{array}$ & 208 & 45 & 54 & 63 & 71 & 79 & 86 & 88 & 90 & 98 & 100 & & 2 \\
\hline $6-17$ & $\begin{array}{l}\text { Mississippi R. at } \\
\text { Helena, Ark. }\end{array}$ & 259 & 33 & 45 & 57 & 67 & 75 & 80 & 83 & 85 & 96 & 100 & & 3 \\
\hline $6-18$ & $\begin{array}{l}\text { White R. at } \\
\text { Mile 11.5, Ark. }\end{array}$ & 92 & 53 & 64 & 75 & 85 & 93 & 98 & 100 & & & & & $<1$ \\
\hline $6-19$ & $\begin{array}{l}\text { Arkansas } R \text {. at } \\
\text { Pendleton, Ark. }\end{array}$ & 153 & 29 & 32 & 35 & 37 & 39 & 43 & 45 & 54 & 93 & 100 & & 97 \\
\hline $6-20$ & $\begin{array}{l}\text { Mississippi } R \text {. above } \\
\text { Arkansas City, Ark. }\end{array}$ & 236 & 29 & 38 & 48 & 55 & 62 & 69 & 72 & 78 & 89 & 100 & & 5 \\
\hline $6-22$ & $\begin{array}{l}\text { Yazoo R. below } \\
\text { Steele Bayou, Miss. }\end{array}$ & 272 & 78 & 88 & 92 & 94 & 96 & 99 & 100 & & & & & $<1$ \\
\hline $6-23$ & $\begin{array}{l}\text { Mississippi R. below } \\
\text { Vicksburg, Miss. }\end{array}$ & 210 & 39 & 46 & 53 & 60 & 66 & 71 & 73 & 79 & 92 & 99 & 100 & 3. \\
\hline $6-25$ & $\begin{array}{l}\text { Old River Outflow Channel } \\
\text { near Knox Landing, La. }\end{array}$ & 220 & 39 & 46 & 52 & 57 & 63 & 70 & 75 & 100 & & & & 3 \\
\hline $6-26$ & $\begin{array}{l}\text { Mississippi R. near } \\
\text { St. Francisville, La. }\end{array}$ & 204 & 41 & 47 & 54 & 59 & 67 & 74 & 77 & 86 & 98 & 100 & & 3 \\
\hline $6-28$ & $\begin{array}{l}\text { Mississippi R. below } \\
\text { Belle Chasse, La. }\end{array}$ & 210 & 42 & 50 & 57 & 63 & 70 & 78 & 82 & 94 & 99 & 100 & & 2 \\
\hline
\end{tabular}

${ }^{1}$ Total suspended-sediment concentration is the average of the composite $A$ and $B$ values in table 20 .

${ }^{2}$ Median diameter was computed by linear interpolation. 
Table 26.--Particle-size distribution of suspended-sediment samples collected from the Mississippi River and some of its tributaries for February-March 1990 cruise

[Finer than $63 \mu \mathrm{m}$ determined by Sedigraph; coarser than $63 \mu \mathrm{m}$ determined by visual-accumulation tube; <, less than; mg/L, milligram per liter; $\mu \mathrm{m}$, micrometer. Analyses by C.J. Anderson and J.A. Moody]

\begin{tabular}{|c|c|c|c|c|c|c|c|c|c|c|c|c|c|c|}
\hline \multirow{3}{*}{$\begin{array}{l}\text { Date } \\
1989 \\
2-25\end{array}$} & \multirow{2}{*}{ Site name } & \multirow{2}{*}{$\begin{array}{c}\text { Total } \\
\text { suspended- } \\
\text { sediment } \\
\text { concentration } 1 \\
(m g / L)\end{array}$} & \multicolumn{4}{|c|}{ Percent } & \multirow{2}{*}{$\frac{\text { finer }}{16}$} & \multirow{2}{*}{$\frac{\text { than }}{32}$} & \multirow{2}{*}{\multicolumn{2}{|c|}{$\frac{\text { indicated }}{63 \quad 125}$}} & \multicolumn{3}{|c|}{ size $(\mu m)$} & \multirow{2}{*}{$\begin{array}{l}\text { Median } \\
\text { diameter } 2 \\
(\mu \mathrm{m})\end{array}$} \\
\hline & & & 1 & 2 & 4 & 8 & & & & & 250 & 500 & 1,000 & \\
\hline & $\begin{array}{l}\text { Mississippi R. near } \\
\text { Cache, I11. }\end{array}$ & 109 & 70 & 80 & 90 & 94 & 96 & 98 & 100 & & & & & $<1$ \\
\hline $3-01$ & $\begin{array}{l}\text { Ohio R. at } \\
\text { Uniontown, Ky. }\end{array}$ & 210 & 44 & 55 & 67 & 80 & 90 & 96 & 99 & 100 & & & & 2 \\
\hline $2-28$ & $\begin{array}{l}\text { Wabash } R \text {. near } \\
\text { New Haven, I11. }\end{array}$ & 172 & 56 & 66 & 76 & 79 & 82 & 83 & 86 & 93 & 98 & 100 & & $<1$ \\
\hline $2-23$ & $\begin{array}{c}\text { Cumberland } R \text {. near } \\
\text { Smithland, Ky. }\end{array}$ & 32 & 68 & 78 & 86 & 91 & 95 & 98 & 100 & & & & & $<1$ \\
\hline $2-24$ & $\begin{array}{r}\text { Tennessee } R \text {. near } \\
\text { Calvert City, Ky. }\end{array}$ & 49 & 71 & 80 & 89 & 94 & 96 & 98 & 100 & & & & & $<1$ \\
\hline $3-03$ & $\begin{array}{l}\text { Ohio R. at } \\
\text { Olmsted, I1l. }\end{array}$ & 150 & 55 & 63 & 73 & 80 & 88 & 95 & 98 & 99 & 100 & & & $<1$ \\
\hline $3-04$ & $\begin{array}{l}\text { Mississippi R. below } \\
\text { Hickman, Ky. }\end{array}$ & 206 & 42 & 50 & 59 & 65 & 71 & 75 & 78 & 80 & 95 & 100 & & 2 \\
\hline $3-05$ & $\begin{array}{l}\text { Mississippi R. below } \\
\text { Fulton, Tenn. }\end{array}$ & 234 & 30 & 37 & 44 & 49 & 55 & 59 & 61 & 64 & 89 & 98 & 100 & 9 \\
\hline $3-07$ & $\begin{array}{l}\text { Mississippi R. at } \\
\text { Helena, Ark. }\end{array}$ & 211 & 41 & 47 & 53 & 59 & 64 & 68 & 70 & 75 & 92 & 98 & 100 & 3 \\
\hline $3-08$ & $\begin{array}{c}\text { Mississippi R. above } \\
\text { Arkansas City, Ark. }\end{array}$ & 215 & 30 & 35 & 41 & 46 & 51 & 56 & 58 & 63 & 90 & 98 & 100 & 14 \\
\hline $3-10$ & $\begin{array}{l}\text { Mississippi R. below } \\
\text { Vicksburg, Miss. }\end{array}$ & 238 & 30 & 35 & 41 & 45 & 49 & 52 & 54 & 59 & 93 & 100 & & 21 \\
\hline $3-12$ & $\begin{array}{l}\text { Mississippi R. near } \\
\text { St. Francisville, La. }\end{array}$ & 182 & 38 & 43 & 48 & 51 & 53 & 54 & 55 & 69 & 96 & 100 & & 7 \\
\hline $3-14$ & $\begin{array}{c}\text { Mississippi R. below } \\
\text { Belle Chasse, La. }\end{array}$ & 220 & 35 & 40 & 46 & 50 & 55 & 61 & 67 & 90 & 99 & 100 & & 8 \\
\hline
\end{tabular}

${ }^{1}$ Total suspended-sediment concentration is the average of the composite A and B values in table 21 .

Median diameter was computed by linear interpolation. 
Table 27.--Particle-size distribution of suspended-sediment samples collected from the Mississippi River and some of its tributaries for second leg of the May-June 1990 cruise

[Finer than $63 \mu \mathrm{m}$ determined by Sedigraph; coarser than $63 \mu \mathrm{m}$ determined by visual-accumulation tube; <, less than; mg/L, milligram per liter; $\mu \mathrm{m}$, micrometer. Analyses by C.J. Anderson and J.A. Hoody]

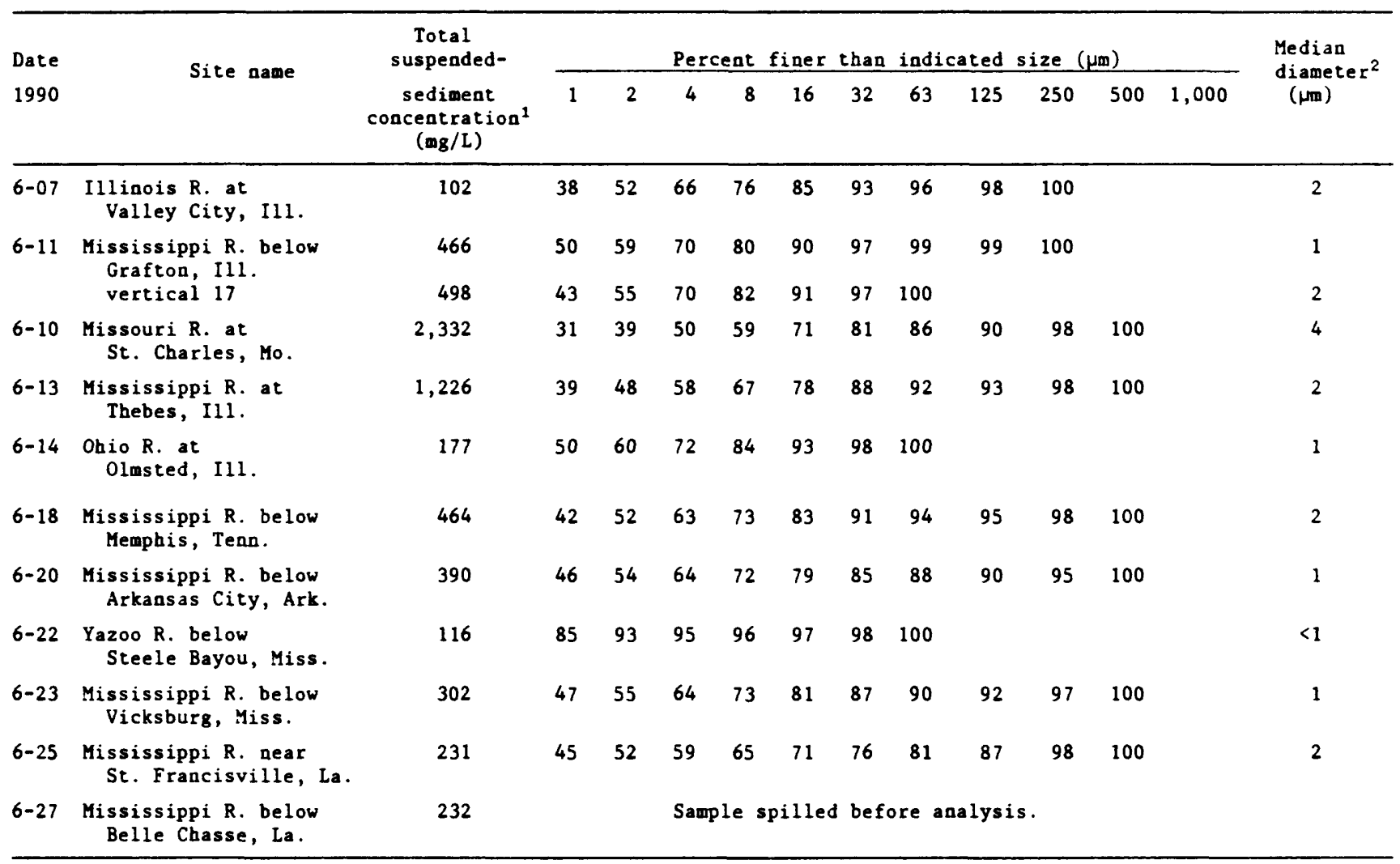

1 Total suspended-sediment concentration is the average of the composite $A$ and $B$ values in table 22 .

${ }^{2}$ Median diameter was computed by linear interpolation. 
Table 28.--Suspended-sedieent concentration, by particle-size cless, obtained by different collectins methods and concentrated by differeat processing procedures

[Abbreviations for collecting and processing methods are: CS, depth integrated, churned, and settled; CC, depth integrated, churned, and centrifuged; PC, puped and centrifuged; <l eiphoned off, estinate of aupended anterial that did not settle in at least 15 days and was siphoned off, does not epply to CC or PC saples; ag/L, ailligras per liter]

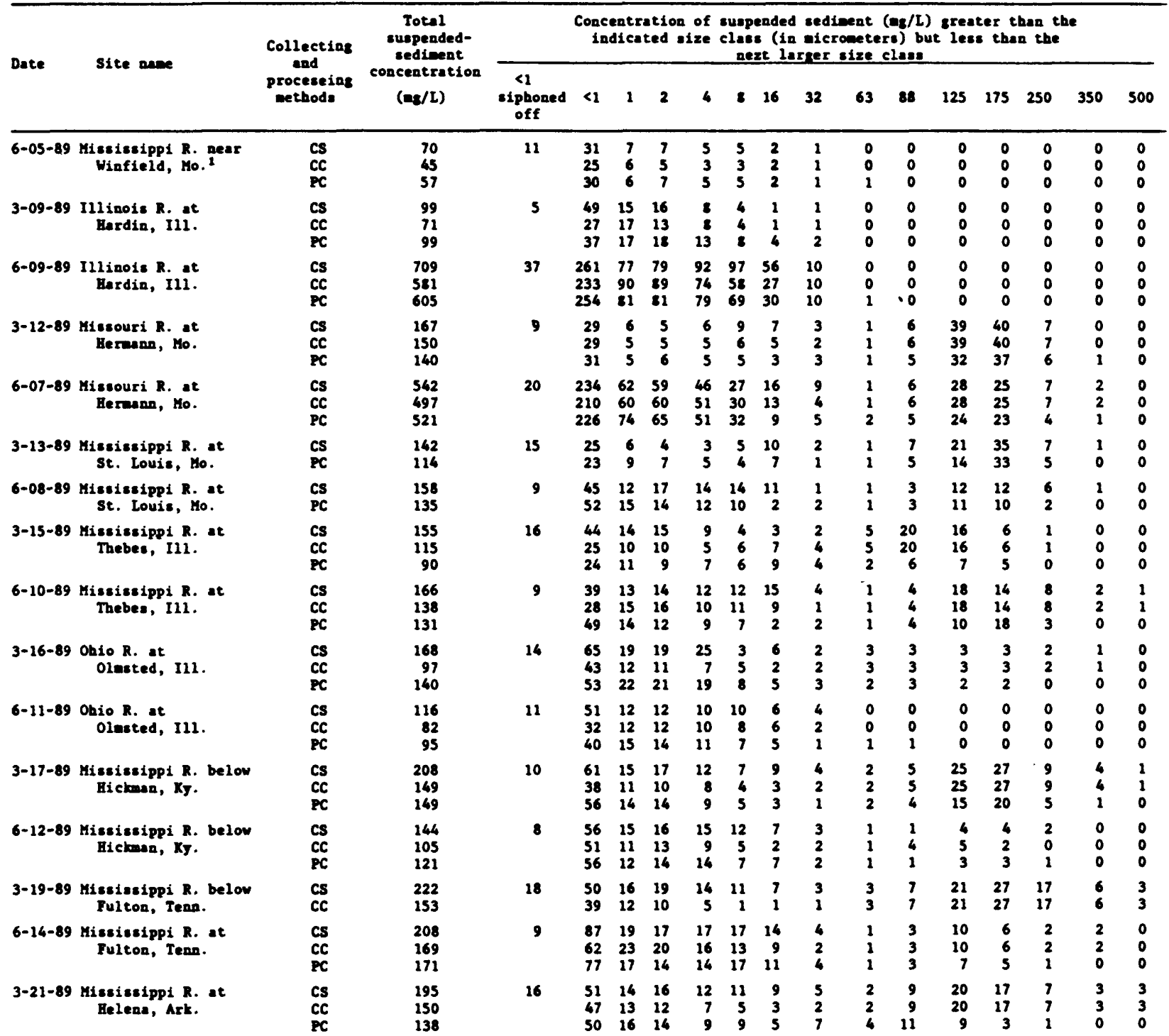


Table 28.--Suspeaded-sedimeat coaceatration, by particle-size class, obtained by differeat collectios nethods and conceatrated by different processios procedures--Coatiaued

\begin{tabular}{|c|c|c|c|c|c|c|c|c|c|c|c|c|c|c|c|c|c|c|}
\hline \multirow{2}{*}{ Date } & \multirow{2}{*}{ Site nane } & \multirow{2}{*}{$\begin{array}{l}\text { Collectins } \\
\text { and } \\
\text { processing } \\
\text { nethods }\end{array}$} & \multirow{2}{*}{$\begin{array}{l}\text { Total } \\
\text { suspeaded- } \\
\text { sedineat } \\
\text { coacestratios } \\
(\text { ag/L) }\end{array}$} & \multicolumn{5}{|c|}{$\begin{array}{r}\text { Conceatration of } \\
\text { indicated size }\end{array}$} & \multicolumn{9}{|c|}{$\begin{array}{l}\text { suspeaded sediment }(\mathrm{mg} / \mathrm{L}) \text { sreater thas the } \\
\text { class (in aicrometers) but less thas the } \\
\text { next larger size class }\end{array}$} & \multirow[b]{2}{*}{500} \\
\hline & & & & $\begin{array}{l}<1 \\
\text { siphooed } \\
\text { off }\end{array}$ & $<1$ & 1 & 2 & 4 & 8 & 16 & 32 & 63 & 88 & 125 & 175 & 250 & 350 & \\
\hline $6-18-89$ & $\begin{array}{l}\text { White R. at } \\
\text { Mile 11.5, Ark. }\end{array}$ & CS & $\begin{array}{l}92 \\
74\end{array}$ & 5 & $\begin{array}{l}44 \\
43\end{array}$ & $\begin{array}{r}10 \\
8\end{array}$ & $\begin{array}{r}10 \\
8\end{array}$ & $\begin{array}{l}9 \\
5\end{array}$ & 5 & $\begin{array}{l}5 \\
2\end{array}$ & $\begin{array}{l}1 \\
3\end{array}$ & 0 & $\begin{array}{r}\text { Too } \\
0\end{array}$ & $\underset{0}{\text { little }}$ & $\begin{array}{r}\text { saod } \\
0\end{array}$ & for 0 & $\underset{0}{a 0 a l y s i s}$ & 0 \\
\hline $3-23-89$ & $\begin{array}{l}\text { Arkansas R. at } \\
\text { Peodletos, Ark. }\end{array}$ & $\begin{array}{l}\text { CS } \\
\text { PC }\end{array}$ & $\begin{array}{l}43 \\
29\end{array}$ & 14 & $\begin{array}{l}19 \\
19\end{array}$ & $\begin{array}{l}3 \\
3\end{array}$ & $\begin{array}{l}2 \\
2\end{array}$ & $\begin{array}{l}1 \\
1\end{array}$ & $\begin{array}{l}1 \\
1\end{array}$ & $\begin{array}{l}1 \\
1\end{array}$ & $\begin{array}{l}1 \\
1\end{array}$ & $\begin{array}{l}0 \\
0\end{array}$ & $\begin{array}{l}0 \\
0\end{array}$ & $\begin{array}{l}0 \\
0\end{array}$ & 1 & $\begin{array}{l}0 \\
0\end{array}$ & $\begin{array}{l}0 \\
0\end{array}$ & $\begin{array}{l}0 \\
0\end{array}$ \\
\hline $6-19-89$ & $\begin{array}{l}\text { Arkansas R. at } \\
\text { Peadleton, Ark. }\end{array}$ & $\begin{array}{l}\text { CS } \\
\text { PC }\end{array}$ & $\begin{array}{l}153 \\
117\end{array}$ & 16 & $\begin{array}{l}33 \\
29\end{array}$ & $\begin{array}{l}5 \\
6\end{array}$ & $\begin{array}{l}4 \\
3\end{array}$ & $\begin{array}{l}3 \\
5\end{array}$ & $\begin{array}{l}3 \\
2\end{array}$ & $\begin{array}{l}6 \\
2\end{array}$ & $\begin{array}{l}3 \\
3\end{array}$ & $\begin{array}{l}5 \\
8\end{array}$ & 8 & $\begin{array}{l}25 \\
21\end{array}$ & $\begin{array}{l}34 \\
27\end{array}$ & $\begin{array}{r}10 \\
4\end{array}$ & $\begin{array}{l}0 \\
0\end{array}$ & $\begin{array}{l}0 \\
0\end{array}$ \\
\hline $3-24-89$ & $\begin{array}{l}\text { Mississippi R. above } \\
\text { Arkansas City, Ark. }\end{array}$ & $\begin{array}{l}\text { CS } \\
\text { CC } \\
\text { PC }\end{array}$ & $\begin{array}{l}197 \\
144 \\
115\end{array}$ & 12 & $\begin{array}{l}57 \\
35 \\
44\end{array}$ & $\begin{array}{r}11 \\
11 \\
9\end{array}$ & $\begin{array}{l}10 \\
12 \\
10\end{array}$ & $\begin{array}{r}10 \\
7 \\
8\end{array}$ & $\begin{array}{r}13 \\
5 \\
6\end{array}$ & $\begin{array}{r}10 \\
2 \\
6\end{array}$ & $\begin{array}{l}\mathbf{5} \\
\mathbf{3} \\
\mathbf{3}\end{array}$ & $\begin{array}{l}2 \\
2 \\
2\end{array}$ & $\begin{array}{l}5 \\
5 \\
5\end{array}$ & $\begin{array}{r}18 \\
18 \\
8\end{array}$ & $\begin{array}{l}29 \\
29 \\
10\end{array}$ & $\begin{array}{r}13 \\
13 \\
4\end{array}$ & $\begin{array}{l}2 \\
2 \\
0\end{array}$ & $\begin{array}{l}0 \\
0 \\
0\end{array}$ \\
\hline $6-20-89$ & $\begin{array}{l}\text { Mississippi R, above } \\
\text { Arkanas City, Ark. }\end{array}$ & $\begin{array}{l}\text { CS } \\
\text { CC } \\
\text { PC }\end{array}$ & $\begin{array}{l}236 \\
183 \\
172\end{array}$ & 12 & $\begin{array}{l}56 \\
53 \\
55\end{array}$ & $\begin{array}{l}22 \\
17 \\
22\end{array}$ & $\begin{array}{l}24 \\
17 \\
22\end{array}$ & $\begin{array}{l}16 \\
12 \\
16\end{array}$ & $\begin{array}{r}17 \\
8 \\
10\end{array}$ & $\begin{array}{r}17 \\
7 \\
7\end{array}$ & $\begin{array}{l}8 \\
5 \\
7\end{array}$ & $\begin{array}{l}6 \\
6 \\
6\end{array}$ & $\begin{array}{l}7 \\
7 \\
7\end{array}$ & $\begin{array}{r}13 \\
13 \\
9\end{array}$ & $\begin{array}{r}14 \\
14 \\
7\end{array}$ & $\begin{array}{r}13 \\
13 \\
3\end{array}$ & $\begin{array}{r}11 \\
11 \\
1\end{array}$ & $\begin{array}{l}0 \\
0 \\
0\end{array}$ \\
\hline $3-26-89$ & $\begin{array}{l}\text { Yazoo R. below } \\
\text { Steele Bayou, Miss. }\end{array}$ & $\begin{array}{l}\text { CS } \\
C C \\
P C\end{array}$ & $\begin{array}{r}150 \\
86 \\
93\end{array}$ & 67 & $\begin{array}{l}62 \\
66 \\
70\end{array}$ & $\begin{array}{l}11 \\
12 \\
11\end{array}$ & $\begin{array}{l}3 \\
3 \\
4\end{array}$ & $\begin{array}{l}1 \\
0 \\
2\end{array}$ & $\begin{array}{l}2 \\
2 \\
3\end{array}$ & $\begin{array}{l}3 \\
2 \\
2\end{array}$ & $\begin{array}{l}1 \\
1 \\
1\end{array}$ & $\begin{array}{l}0 \\
0 \\
0\end{array}$ & $\begin{array}{l}0 \\
0 \\
0\end{array}$ & $\begin{array}{l}0 \\
0 \\
0\end{array}$ & $\begin{array}{l}0 \\
0 \\
0\end{array}$ & $\begin{array}{l}0 \\
0 \\
0\end{array}$ & $\begin{array}{l}0 \\
0 \\
0\end{array}$ & $\begin{array}{l}0 \\
0 \\
0\end{array}$ \\
\hline $6-22-89$ & $\begin{array}{l}\text { Yazoo R. below } \\
\text { Steele Bayou, Miss. }\end{array}$ & $\begin{array}{l}\text { CS } \\
\text { CC } \\
P C\end{array}$ & $\begin{array}{l}272 \\
175 \\
227\end{array}$ & 57 & $\begin{array}{l}154 \\
120 \\
169\end{array}$ & $\begin{array}{l}27 \\
27 \\
23\end{array}$ & $\begin{array}{l}10 \\
12 \\
10\end{array}$ & $\begin{array}{l}4 \\
3 \\
6\end{array}$ & $\begin{array}{l}6 \\
5 \\
7\end{array}$ & $\begin{array}{r}10 \\
4 \\
7\end{array}$ & $\begin{array}{l}3 \\
3 \\
4\end{array}$ & $\begin{array}{l}1 \\
1 \\
1\end{array}$ & $\begin{array}{l}0 \\
0 \\
0\end{array}$ & $\begin{array}{l}0 \\
0 \\
0\end{array}$ & $\begin{array}{l}0 \\
0 \\
0\end{array}$ & $\begin{array}{l}0 \\
0 \\
0\end{array}$ & $\begin{array}{l}0 \\
0 \\
0\end{array}$ & $\begin{array}{l}0 \\
0 \\
0\end{array}$ \\
\hline $3-27-89$ & $\begin{array}{l}\text { Mississippi R. below } \\
\text { Vicksburs, Miss. }\end{array}$ & $\begin{array}{l}\text { CS } \\
\text { CC } \\
\text { PC }\end{array}$ & $\begin{array}{l}202 \\
169 \\
119\end{array}$ & 16 & $\begin{array}{l}52 \\
49 \\
47\end{array}$ & $\begin{array}{r}15 \\
9 \\
10\end{array}$ & $\begin{array}{r}11 \\
9 \\
10\end{array}$ & $\begin{array}{l}8 \\
8 \\
9\end{array}$ & $\begin{array}{l}5 \\
7 \\
7\end{array}$ & $\begin{array}{r}14 \\
6 \\
2\end{array}$ & $\begin{array}{l}3 \\
3 \\
1\end{array}$ & $\begin{array}{l}2 \\
2 \\
2\end{array}$ & $\begin{array}{l}6 \\
6 \\
5\end{array}$ & $\begin{array}{r}15 \\
15 \\
9\end{array}$ & $\begin{array}{l}31 \\
31 \\
11\end{array}$ & $\begin{array}{r}16 \\
16 \\
5\end{array}$ & $\begin{array}{l}6 \\
6 \\
1\end{array}$ & $\begin{array}{l}2 \\
2 \\
0\end{array}$ \\
\hline $6-23-89$ & $\begin{array}{l}\text { Mississippi R. below } \\
\text { Vicksburs, Miss. }\end{array}$ & $\begin{array}{l}\text { CS } \\
C C \\
P C\end{array}$ & $\begin{array}{l}210 \\
150 \\
140\end{array}$ & 10 & $\begin{array}{l}72 \\
54 \\
61\end{array}$ & $\begin{array}{l}14 \\
11 \\
13\end{array}$ & $\begin{array}{r}14 \\
9 \\
12\end{array}$ & $\begin{array}{r}16 \\
7 \\
9\end{array}$ & $\begin{array}{r}13 \\
6 \\
9\end{array}$ & $\begin{array}{r}12 \\
6 \\
5\end{array}$ & $\begin{array}{l}5 \\
3 \\
3\end{array}$ & $\begin{array}{l}5 \\
5 \\
4\end{array}$ & $\begin{array}{l}5 \\
5 \\
7\end{array}$ & $\begin{array}{l}8 \\
8 \\
9\end{array}$ & $\begin{array}{r}15 \\
15 \\
6\end{array}$ & $\begin{array}{r}11 \\
11 \\
2\end{array}$ & $\begin{array}{l}7 \\
7 \\
0\end{array}$ & $\begin{array}{l}3 \\
3 \\
0\end{array}$ \\
\hline $3-29-89$ & $\begin{array}{l}\text { 01d R. Outflow Channel } \\
\text { gear Knox Laoding, La. }\end{array}$ & $\begin{array}{l}\text { CS } \\
\text { CC } \\
\text { PC }\end{array}$ & $\begin{array}{l}180 \\
125 \\
141\end{array}$ & 17 & $\begin{array}{l}71 \\
48 \\
62\end{array}$ & $\begin{array}{l}16 \\
19 \\
14\end{array}$ & $\begin{array}{l}13 \\
19 \\
13\end{array}$ & $\begin{array}{l}11 \\
12 \\
13\end{array}$ & $\begin{array}{r}14 \\
5 \\
10\end{array}$ & $\begin{array}{r}16 \\
3 \\
7\end{array}$ & $\begin{array}{r}10 \\
5 \\
9\end{array}$ & $\begin{array}{l}? \\
? \\
7\end{array}$ & $\begin{array}{l}4 \\
4 \\
4\end{array}$ & $\begin{array}{l}2 \\
2 \\
1\end{array}$ & $\begin{array}{l}1 \\
1 \\
1\end{array}$ & $\begin{array}{l}0 \\
0 \\
0\end{array}$ & $\begin{array}{l}0 \\
0 \\
0\end{array}$ & $\begin{array}{l}0 \\
0 \\
0\end{array}$ \\
\hline $6-25-89$ & $\begin{array}{l}\text { 01d R. Outflow Channel } \\
\text { gear Knox Laoding, La. }\end{array}$ & $\begin{array}{l}\text { CS } \\
\text { CC } \\
\text { PC }\end{array}$ & $\begin{array}{l}220 \\
148 \\
157\end{array}$ & 10 & $\begin{array}{l}76 \\
37 \\
50\end{array}$ & $\begin{array}{l}15 \\
13 \\
17\end{array}$ & $\begin{array}{l}14 \\
14 \\
17\end{array}$ & $\begin{array}{r}12 \\
11 \\
9\end{array}$ & $\begin{array}{r}13 \\
8 \\
7\end{array}$ & $\begin{array}{r}16 \\
5 \\
7\end{array}$ & $\begin{array}{r}10 \\
6 \\
15\end{array}$ & $\begin{array}{l}32 \\
32 \\
24\end{array}$ & $\begin{array}{r}21 \\
21 \\
9\end{array}$ & $\begin{array}{l}1 \\
1 \\
1\end{array}$ & $\begin{array}{l}0 \\
0 \\
1\end{array}$ & $\begin{array}{l}0 \\
0 \\
0\end{array}$ & $\begin{array}{l}0 \\
0 \\
0\end{array}$ & $\begin{array}{l}0 \\
0 \\
0\end{array}$ \\
\hline $3-30-89$ & $\begin{array}{l}\text { Mississippi R. near } \\
\text { St. Fraocisville, La. }\end{array}$ & $\begin{array}{l}\text { CS } \\
\text { CC } \\
\text { PC }\end{array}$ & $\begin{array}{l}192 \\
149 \\
121\end{array}$ & 16 & $\begin{array}{l}49 \\
33 \\
33\end{array}$ & $\begin{array}{l}10 \\
11 \\
15\end{array}$ & $\begin{array}{l}10 \\
12 \\
16\end{array}$ & $\begin{array}{r}10 \\
8 \\
9\end{array}$ & $\begin{array}{r}11 \\
6 \\
7\end{array}$ & $\begin{array}{l}9 \\
3 \\
3\end{array}$ & $\begin{array}{l}4 \\
3 \\
5\end{array}$ & $\begin{array}{l}7 \\
? \\
5\end{array}$ & $\begin{array}{r}20 \\
20 \\
9\end{array}$ & $\begin{array}{l}25 \\
25 \\
13\end{array}$ & $\begin{array}{r}16 \\
16 \\
5\end{array}$ & $\begin{array}{l}5 \\
5 \\
1\end{array}$ & $\begin{array}{l}0 \\
0 \\
0\end{array}$ & $\begin{array}{l}0 \\
0 \\
0\end{array}$ \\
\hline $6-26-89$ & $\begin{array}{l}\text { Mississippi R. near } \\
\text { St. Fraacisville, Le. }\end{array}$ & $\begin{array}{l}\text { CS } \\
C C \\
P C\end{array}$ & $\begin{array}{c}204 \\
150 \\
\text { unknown }\end{array}$ & 13 & $\begin{array}{l}70 \\
52 \\
72\end{array}$ & $\begin{array}{l}13 \\
14 \\
13\end{array}$ & $\begin{array}{l}13 \\
11 \\
14\end{array}$ & $\begin{array}{r}10 \\
8 \\
11\end{array}$ & $\begin{array}{r}17 \\
8 \\
12\end{array}$ & $\begin{array}{r}14 \\
7 \\
7\end{array}$ & $\begin{array}{l}7 \\
3 \\
3\end{array}$ & $\begin{array}{l}6 \\
6 \\
S\end{array}$ & $\begin{array}{c}12 \\
12 \\
\text { Sample }\end{array}$ & $\begin{array}{c}16 \\
16 \\
\text { not av }\end{array}$ & vailab & le $\begin{array}{l}4 \\
4\end{array}$ & $\begin{array}{c}0 \\
0 \\
\text { or analy }\end{array}$ & $\begin{array}{c}0 \\
0 \\
y \leq i s\end{array}$ \\
\hline $4-01-89$ & $\begin{array}{l}\text { Mississippi R. below } \\
\text { Belle Chasse, La. }\end{array}$ & $\begin{array}{l}\text { CS } \\
\text { CC } \\
\text { PC }\end{array}$ & $\begin{array}{l}218 \\
153 \\
134\end{array}$ & 20 & $\begin{array}{l}59 \\
44 \\
58\end{array}$ & $\begin{array}{l}13 \\
12 \\
12\end{array}$ & $\begin{array}{l}12 \\
12 \\
11\end{array}$ & $\begin{array}{r}11 \\
6 \\
9\end{array}$ & $\begin{array}{r}14 \\
4 \\
7\end{array}$ & $\begin{array}{r}14 \\
3 \\
6\end{array}$ & $\begin{array}{l}7 \\
4 \\
4\end{array}$ & $\begin{array}{r}17 \\
17 \\
9\end{array}$ & $\begin{array}{l}31 \\
31 \\
14\end{array}$ & $\begin{array}{r}14 \\
14 \\
3\end{array}$ & $\begin{array}{l}6 \\
6 \\
1\end{array}$ & $\begin{array}{l}0 \\
0 \\
0\end{array}$ & $\begin{array}{l}0 \\
0 \\
0\end{array}$ & $\begin{array}{l}0 \\
0 \\
0\end{array}$ \\
\hline $6-28-89$ & $\begin{array}{l}\text { Mississippi R. below } \\
\text { Belle Chasse, La. }\end{array}$ & $\begin{array}{l}\text { CS } \\
\text { CC } \\
\text { PC }\end{array}$ & $\begin{array}{l}210 \\
159 \\
148\end{array}$ & 15 & $\begin{array}{l}74 \\
57 \\
61\end{array}$ & $\begin{array}{l}17 \\
17 \\
17\end{array}$ & $\begin{array}{l}14 \\
17 \\
15\end{array}$ & $\begin{array}{r}13 \\
9 \\
13\end{array}$ & $\begin{array}{r}16 \\
9 \\
13\end{array}$ & $\begin{array}{r}17 \\
7 \\
11\end{array}$ & $\begin{array}{l}8 \\
7 \\
6\end{array}$ & $\begin{array}{l}7 \\
7 \\
5\end{array}$ & $\begin{array}{r}19 \\
19 \\
4\end{array}$ & $\begin{array}{l}8 \\
8 \\
3\end{array}$ & $\begin{array}{l}2 \\
2 \\
0\end{array}$ & $\begin{array}{l}0 \\
0 \\
0\end{array}$ & $\begin{array}{l}0 \\
0 \\
0\end{array}$ & $\begin{array}{l}0 \\
0 \\
0\end{array}$ \\
\hline $6-17-89$ & $\begin{array}{l}\text { Mississippi R. at } \\
\text { Heleas, Ark. }\end{array}$ & $\begin{array}{l}\text { CS } \\
\text { CC } \\
\text { PC }\end{array}$ & $\begin{array}{l}259 \\
199 \\
202\end{array}$ & 8 & $\begin{array}{l}79 \\
85 \\
67\end{array}$ & $\begin{array}{l}31 \\
26 \\
33\end{array}$ & $\begin{array}{l}32 \\
26 \\
31\end{array}$ & $\begin{array}{r}25 \\
9 \\
19\end{array}$ & $\begin{array}{r}20 \\
5 \\
17\end{array}$ & $\begin{array}{r}13 \\
2 \\
9\end{array}$ & $\begin{array}{l}7 \\
2 \\
2\end{array}$ & $\begin{array}{l}1 \\
1 \\
2\end{array}$ & $\begin{array}{l}3 \\
3 \\
4\end{array}$ & $\begin{array}{r}12 \\
12 \\
7\end{array}$ & $\begin{array}{r}16 \\
16 \\
8\end{array}$ & $\begin{array}{l}9 \\
9 \\
3\end{array}$ & $\begin{array}{l}3 \\
3 \\
0\end{array}$ & $\begin{array}{l}0 \\
0 \\
0\end{array}$ \\
\hline $3-22-89$ & $\begin{array}{l}\text { White R. at } \\
\text { Mile il.5, Ark. }\end{array}$ & $\begin{array}{l}\text { CS } \\
\text { PC }\end{array}$ & $\begin{array}{l}44 \\
20\end{array}$ & 26 & $\begin{array}{l}17 \\
17\end{array}$ & $\begin{array}{l}1 \\
2\end{array}$ & $\begin{array}{l}0 \\
1\end{array}$ & $\begin{array}{l}0 \\
0\end{array}$ & $\begin{array}{l}0 \\
0\end{array}$ & $\begin{array}{l}0 \\
0\end{array}$ & $\begin{array}{l}0 \\
0\end{array}$ & 0 & $\begin{array}{r}\text { Too } \\
0\end{array}$ & $\begin{array}{c}\text { little } \\
0\end{array}$ & $\begin{array}{r}\text { sand } \\
0\end{array}$ & $\begin{array}{c}\text { for } \\
0\end{array}$ & $\begin{array}{c}\text { adeasis } \\
0\end{array}$ & 0 \\
\hline
\end{tabular}

\footnotetext{
'Only composite B was used.
} 


\section{Surface-Water Properties}

At each sampling site, a bucket was used to collect a surface-water sample at each vertical, and the temperature, $\mathrm{pH}$, and specific conductance were measured (see "Tabulated Cruise Data" section). Temperatures and pH were measured with a Beckman meter (model $\phi 12$ ), and the specific conductance was measured with an Amberscience conductivity meter (model 604). The meters were calibrated with $\mathrm{pH}$ and specific conductance standards.

During the May-June 1990 cruise, a series of closely spaced sites was sampled in two reaches of the Lower Mississippi River downstream from the confluence of the Upper Mississippi and Ohio Rivers in order to study the mixing processes downstream from the confluence (see fig. 3). The first reach was straight and started with a sampling site at Wickliffe, Ky. (river mile 950.5), and ended with a sampling site near Columbus, Ky. (river mile 936.1). The second reach was curved and extended around the tight bend of the river at New Madrid, Mo. It started with a sampling site upstream from New Madrid (river mile 898.9) and ended with a sampling site near Point Pleasant, Mo. (river mile 879.5). The last sampling site at Caruthersville, Mo. (river mile 847.5), was selected far enough downstream from the confluence that the mixing of the Upper Mississippi and Ohio Rivers should be virtually complete. At each sampling site, the specific conductance of the surface water was measured and the specific conductance, temperature, $\mathrm{pH}$, and suspended-sediment concentration of a depth-integrated sample (about $5 \mathrm{~L}$ ) were measured at 9 to 15 verticals across the river (see May-June 1990 cruise sampling sites: Mississippi River at Wickliffe, near Columbus, above New Madrid, near Point Pleasant, and at Caruthersville in the "Tabulated Cruise Data" section).

By comparing the surface and depth-integrated specific conductances, the error in using the surface value of specific conductance as an approximation of the depth-integrated value of specific conductance for all sampling sites for all cruises could be estimated. In general, the specific conductance of the surface-water sample averaged $2-3 \mu \mathrm{S} / \mathrm{cm}$ greater than the specific conductance of the depth-integrated samples (see fig. 9). The depthintegrated water sample was aerated as it was poured through a $63-\mu \mathrm{m}$ mesh sieve. A single test indicated that the specific conductance of a depth-integrated sample decreased from $364 \mu \mathrm{S} / \mathrm{cm}$ (before being poured through the sieve) to $360 \mu \mathrm{S} / \mathrm{cm}$ (after being poured through the sieve).

The specific conductance of the surface water was measured at different locations across the Lower Mississippi River at additional sampling sites in the straight reach between Wickliffe, Ky., and Columbus, $\mathrm{Ky}$., and in the curved reach around the bend at New Madrid during June 1-4, 1990 (see fig. 3). The additional sites were sampled in the downriver direction on June 1 and June 2, 1990 (table 29), in the upriver direction on June 3 and June 4, 1990 (table 29), and for a second time in the downriver direction on June 14 and June 16, 1990 (table 30). Similar measurements were made at five sampling sites on the Upper Mississippi River on June 12, 1990 (see table 31). 


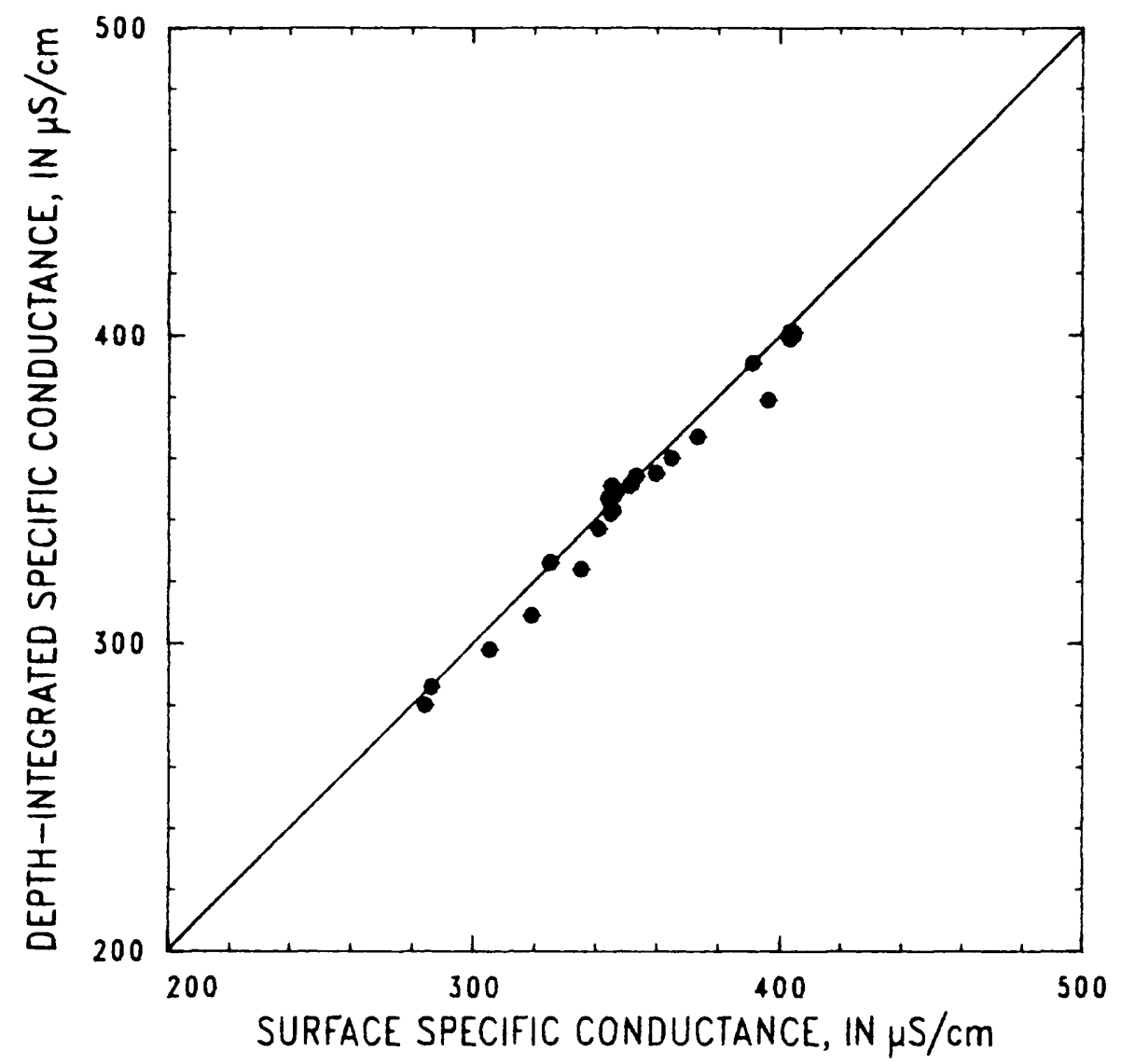

Figure 9.--Specific conductance of surface and depth-integrated water samples. Samples of surface water were collected about halfway through the depth-integration sampling process. The diagonal line indicates perfect agreement and is not a regression line. The abbreviation $\mu \mathrm{S} / \mathrm{cm}$ is microsiemens per centimeter at $25^{\circ}$ Celsius. 
Table 29.--Specific conductance of surface water at locations across the Lower Mississippi

River for the first leg of the May-June 1990 cruise, between river miles 879.5 and 946.4

[m, meter; specific conductance is in microsiemens per centimeter at 25 degrees Celsius; NS, no sample was collected; + overbank indicates that water was over the banks and could not be sampled from the research vessel]

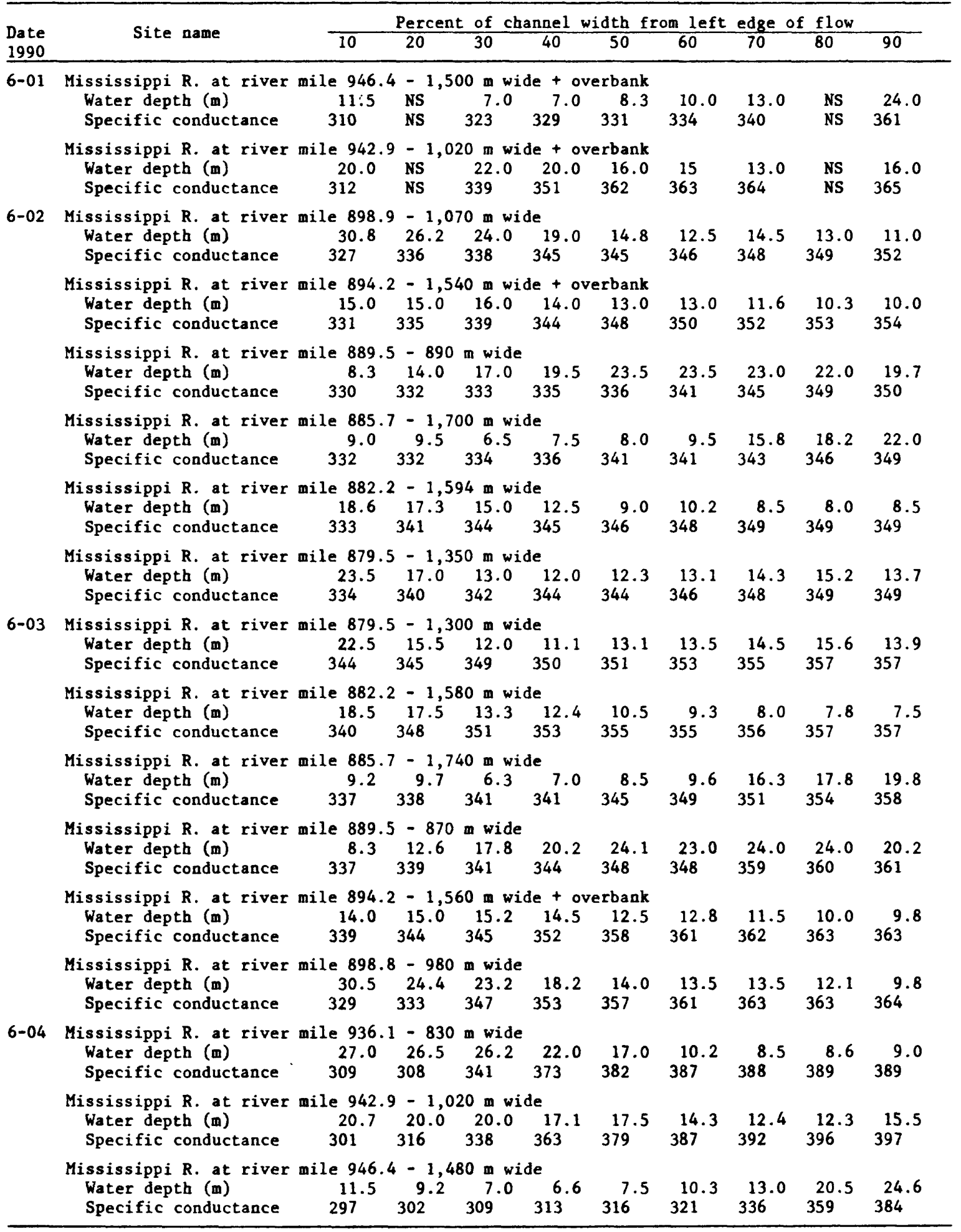


Table 30.--Specific conductance of surface water at locations across the Lower Mississippi River for the second leg of the May-June 1990 cruise, between river miles 950.5 and 736.7

[Specific conductance in microsiemens per centimeter at 25 degrees Celsius; NS, no sample collected]

\begin{tabular}{|c|c|c|c|c|c|c|c|c|}
\hline $\begin{array}{l}\text { Date } \\
1990 \\
\end{array}$ & Site name & $\frac{\text { Percent of chat }}{20}$ & $\frac{\text { nnel w }}{40}$ & $\frac{\text { idth } f_{1}}{50}$ & $\frac{\text { om left }}{60}$ & $\frac{\text { edge } 0}{70}$ & $\frac{f \text { flow }}{80}$ & 90 \\
\hline \multirow[t]{4}{*}{$6-14$} & $\begin{array}{cc}\text { Mississippi } R \text {. at river mile } 950.5 \\
\text { Water depth (m) } & 10.0 \\
\text { Specific conductance } & 280\end{array}$ & $\begin{array}{c}-740 \text { m wide } \\
27.026 .0 \\
287 \quad 310\end{array}$ & $\begin{array}{l}24.0 \\
347\end{array}$ & $\begin{array}{l}22.5 \\
370\end{array}$ & $\begin{array}{l}18.0 \\
378\end{array}$ & $\begin{array}{l}12.0 \\
379\end{array}$ & 379 & 378 \\
\hline & $\begin{array}{cc}\text { Mississippi R. at river mile } & 946.4 \\
\text { Water depth (m) } & 9.2 \\
\text { Specific conductance } & 286\end{array}$ & $\begin{array}{c}-1,500 \text { m wid } \\
6.8 \quad 5.0 \\
296 \\
297\end{array}$ & 4.5 & ${ }_{298}^{5.2}$ & $302^{7.0}$ & $\begin{array}{l}10.2 \\
313\end{array}$ & $\begin{array}{c}17.2 \\
339\end{array}$ & $\begin{array}{c}22.0 \\
353\end{array}$ \\
\hline & $\begin{array}{cc}\text { Mississippi } R \text {. at river mile } 942.9 \\
\text { Water depth (m) } & 19.3 \\
\text { Specific conductance } & 283\end{array}$ & $\begin{array}{l}-1,000 \text { m wid } \\
20.017 .5 \\
290 \quad 320\end{array}$ & 16.0 & $\begin{array}{l}15.3 \\
360\end{array}$ & $\begin{array}{l}12.8 \\
364\end{array}$ & $\begin{array}{l}11.0 \\
372\end{array}$ & $\begin{array}{l}10.8 \\
374\end{array}$ & $\begin{array}{l}13.3 \\
374\end{array}$ \\
\hline & $\begin{array}{cc}\text { Mississippi } R \text {. at river mile } 936.1 \\
\text { Water depth (m) } & 24.0 \\
\text { Specific conductance } & 280\end{array}$ & $\begin{array}{cc}- & 800 \text { m wide } \\
25.0 & 25.0 \\
310 & 324\end{array}$ & $\begin{array}{l}21.2 \\
355\end{array}$ & 361 & ${ }_{365}^{13.0}$ & $367^{7.0}$ & $367^{6.4}$ & $\begin{array}{l}6.7 \\
368\end{array}$ \\
\hline \multirow[t]{5}{*}{$6-15$} & $\begin{array}{cc}\text { Mississippi R. at river mile } 898.9 \\
\text { Water depth (m) } & 27.5 \\
\text { Specific conductance } & 310\end{array}$ & $\begin{array}{c}-980 \text { m wide } \\
23.020 .0 \\
317 \quad 326\end{array}$ & $\begin{array}{l}14.0 \\
341\end{array}$ & $\begin{array}{l}11.9 \\
347\end{array}$ & $\begin{array}{l}10.7 \\
352\end{array}$ & $\begin{array}{l}12.4 \\
355\end{array}$ & $\begin{array}{l}11.0 \\
357\end{array}$ & 358 \\
\hline & $\begin{array}{cc}\text { Mississippi } R \text {. at river mile } 894.2 \\
\text { Water depth (m) } & 12.2 \\
\text { Specific conductance } & 318\end{array}$ & $\begin{array}{c}-1,450 \text { m wide } \\
11.913 .2 \\
322328\end{array}$ & 12.4 & 338 & $344^{9.6}$ & $350^{9.2}$ & $\begin{array}{l}10.5 \\
353\end{array}$ & $\begin{array}{c}12.1 \\
357\end{array}$ \\
\hline & $\begin{array}{cc}\text { Mississippi } R \text {. at river mile } & 889.5 \\
\text { Water depth (m) } & 6.0 \\
\text { Specific conductance } & 314\end{array}$ & $\begin{array}{c}-850 \text { m wide } \\
11.8 \quad 15.5 \\
314 \quad 317\end{array}$ & $\begin{array}{l}17.1 \\
321\end{array}$ & $\begin{array}{l}19.6 \\
325\end{array}$ & $\begin{array}{l}21.0 \\
332\end{array}$ & $\begin{array}{l}21.5 \\
345\end{array}$ & $\begin{array}{l}21.0 \\
348\end{array}$ & $\begin{array}{l}18.0 \\
348\end{array}$ \\
\hline & $\begin{array}{cc}\text { Mississippi R. at river mile } & 885.7 \\
\text { Water depth (m) } & 6.9 \\
\text { Specific conductance } & 313\end{array}$ & 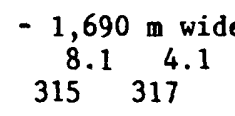 & 5.2 & 325 & $330^{7.6}$ & $\begin{array}{l}13.7 \\
335\end{array}$ & 340 & 344 \\
\hline & $\begin{array}{cc}\text { Mississippi } R \text {. at river mile } 882.2 \\
\text { Water depth (m) } & \sim 16.3 \\
\text { Specific conductance } & 315\end{array}$ & $\begin{array}{l}-1,580 \text { m wide } \\
\sim 15.312 .2 \\
331 \quad 335\end{array}$ & 9.6 & $337^{7.7}$ & 339 & $341^{6.2}$ & $341^{5.0}$ & $\begin{array}{l}5.7 \\
342\end{array}$ \\
\hline $6-15$ & $\begin{array}{cc}\text { Mississippi } R \text {. at river mile } 879.5 \\
\text { Water depth (m) } & 20.4 \\
\text { Specific conductance } & 313\end{array}$ & $\begin{array}{c}-1,300 \text { m wide } \\
12.710 .3 \\
329332\end{array}$ & 9.9 & $\begin{array}{l}12.0 \\
337\end{array}$ & 339 & $\begin{array}{l}12.3 \\
341\end{array}$ & $\begin{array}{l}12.8 \\
341\end{array}$ & $\begin{array}{l}13.1 \\
342\end{array}$ \\
\hline \multirow[t]{4}{*}{$6-16$} & $\begin{array}{cc}\text { Mississippi R. at river mile } & 847.5 \\
\text { Water depth (m) } & 7.5 \\
\text { Specific conductance } & 315\end{array}$ & $\begin{array}{cl}-780 & \text { mide } \\
\text { NS } & \text { NS } \\
\text { NS } & \text { NS }\end{array}$ & $\begin{array}{l}\text { NS } \\
\text { NS }\end{array}$ & $\begin{array}{l}15.2 \\
318\end{array}$ & $\begin{array}{l}\text { NS } \\
\text { NS }\end{array}$ & $\begin{array}{l}\text { NS } \\
\text { NS }\end{array}$ & $\begin{array}{l}\text { NS } \\
\text { NS }\end{array}$ & $\begin{array}{l}23.1 \\
324\end{array}$ \\
\hline & $\begin{array}{cc}\text { Mississippi } R \text {. at river mile } 801.0 \\
\text { Water depth (m) } & 25.8 \\
\text { Specific conductance } & 315\end{array}$ & 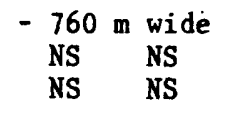 & $\begin{array}{l}\text { NS } \\
\text { NS }\end{array}$ & $\begin{array}{l}17.7 \\
320\end{array}$ & $\begin{array}{l}\text { NS } \\
\text { NS }\end{array}$ & $\begin{array}{l}\text { NS } \\
\text { NS }\end{array}$ & $\begin{array}{l}\text { NS } \\
\text { NS }\end{array}$ & $\begin{array}{l}11.0 \\
321\end{array}$ \\
\hline & $\begin{array}{cc}\text { Mississippi } R \text {, at river mile } 778.5 \\
\text { Water depth (m) } & 23.3 \\
\text { Specific conductance } & 311\end{array}$ & $\begin{array}{ll}-570 & \text { mide } \\
\text { NS } & \text { NS } \\
\text { NS } & \text { NS }\end{array}$ & $\begin{array}{l}\text { NS } \\
\text { NS }\end{array}$ & $\begin{array}{l}24.9 \\
318\end{array}$ & $\begin{array}{l}\text { NS } \\
\text { NS }\end{array}$ & $\begin{array}{l}\text { NS } \\
\text { NS }\end{array}$ & $\begin{array}{l}\text { NS } \\
\text { NS }\end{array}$ & $\begin{array}{l}6.5 \\
319\end{array}$ \\
\hline & $\begin{array}{cc}\text { Mississippi } R \text {, at river mile } & 736.7 \\
\text { Water depth (m) } & 19.8 \\
\text { Specific conductance } & 317\end{array}$ & $\begin{array}{ll}-1,110 & \mathrm{~m} \text { wide } \\
\text { NS } & \text { NS } \\
\text { NS } & \text { NS }\end{array}$ & NS & ${ }_{321}^{12.5}$ & $\begin{array}{l}\text { NS } \\
\text { NS }\end{array}$ & $\begin{array}{l}\text { NS } \\
\text { NS }\end{array}$ & $\begin{array}{l}\text { NS } \\
\text { NS }\end{array}$ & ${ }_{325}^{15.8}$ \\
\hline
\end{tabular}


Table 31.--Specific conductance of surface water at locations across the Upper Mississippi River for the second $\mathrm{leg}$ of the May-June 1990 cruise, between river miles 179.0 and 53.5

[m, meter; specific conductance in microsiemens per centimeter at 25 degrees Celsius]

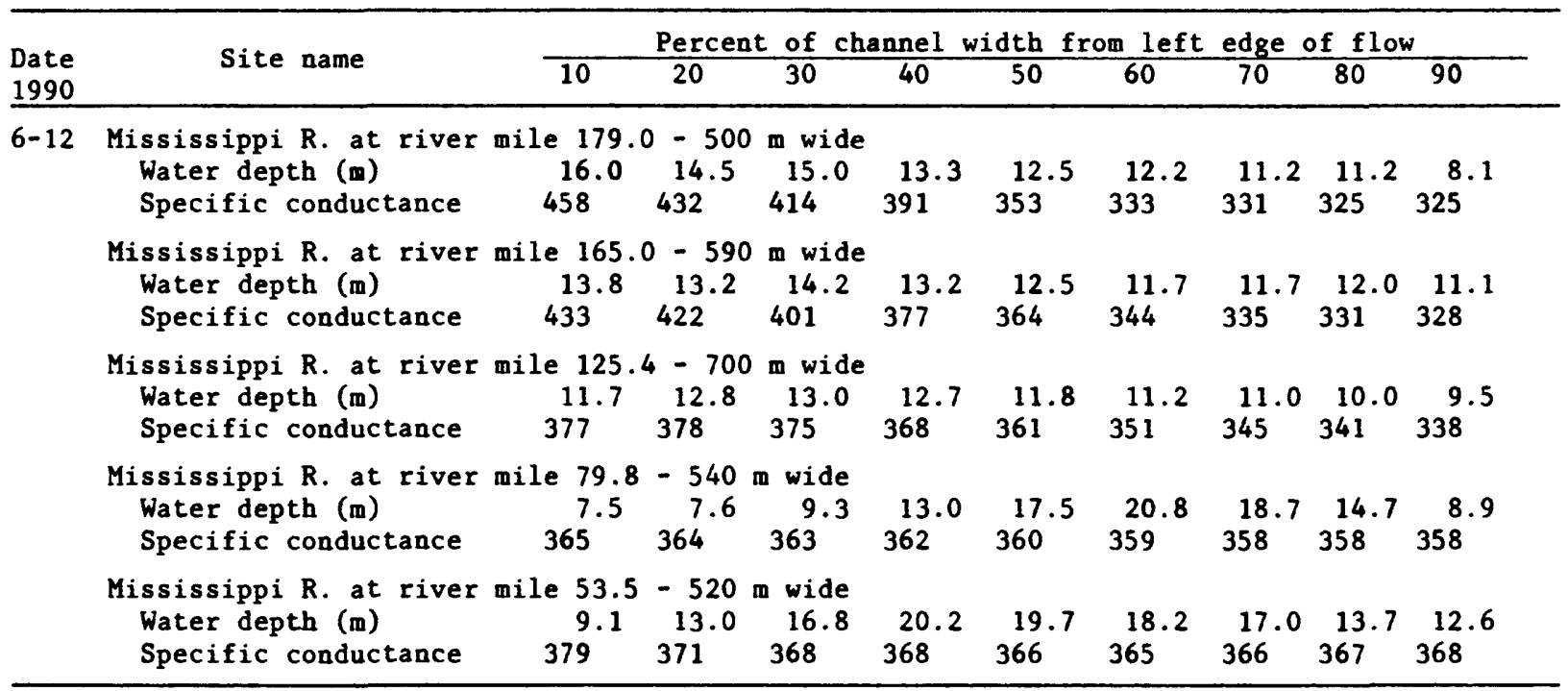




\section{REFERENCES CITED}

Bragg, Marion, 1977, Historic names and places on the Mississippi River: Vicksburg, Miss., Mississippi River Commission, 282 p.

Chow, V.T., 1959, Open-channel hydraulics: New York, McGraw-Hill, p. 680.

Guy, H.P., 1969, Laboratory theory and methods for sediment analysis: U.S. Geological Survey Techniques of Water-Resources Investigations, book 5, chap. $\mathrm{C} 1,58 \mathrm{p}$.

Guy, H.P., and Norman, V.W., 1970, Field methods for measurement of fluvial sediment: U.S. Geological Survey Techniques of Water-Resources Investigations, book 3 , chap. C2, $59 \mathrm{p}$.

Keown, M.P., Dardeau, E.A., Jr., Causey, E.M., 1981, Characterization of the suspended-sediment regime and bed-material gradation of the Mississippi

River Basin: U.S. Army Corps of Engineers, U.S. Army Engineering District, New Orleans, v. 1 and v. 2., 874 p.

Lara, 0.G., and Matthes, W.J., 1986, The Sedigraph as an alternative method to the pipet, in Proceedings of the Fourth Federal Interagency Sedimentation Conference 1986: Interagency Advisory Committee on Water Data, Subcommittee on Sedimentation, p. 1-1 to 1-12.

Leenheer, J.A., Meade, R.H., Taylor, H.E., and Pereira, W.E., 1989, Sampling, fractionation, and dewatering of suspended sediment from the Mississippi River for geochemical and trace-contaminant analysis, in Mallard, G.E., and Ragone, S.E., eds., U.S. Geological Survey Toxic Substances Hydrology Program--Proceedings of the Technical Meeting, Phoenix, Arizona, September 26-30, 1988: U.S. Geological Survey Water-Resources Investigations Report 88-4220, p. 501-511.

Matthes, W.J., Jr., Sholar, C.J., and George, J.R., 1992, Quality-assurance plan for the analysis of fluvial sediment by laboratories of the U.S. Geological Survey: U.S. Geological Survey Open-File Report 91-467, 31 p.

Meade, R.H., and Stevens, H.H., Jr., 1990, Strategies and equipment for sampling suspended sediment and associated toxic chemicals in large rivers--with emphasis on the Mississippi River: Science of the Total Envi ronment, v. 97/98, p. 125-135.

Moody, J.A., 1993, Evaluation of the Lagrangian scheme for sampling the Mississippi River during 1987-90: U.S. Geological Survey Water-Resources Investigations Report 93-4042.

Moody, J.A., and Troutman, B.M., 1992, Evaluation of the depth-integration method of measuring water discharge in large rivers: Journal of Hydrology, v. 135, p. 201-236.

Moody, J.A., and Meade, R.H., 1992, Hydrologic and sedimentologic data collected during three cruises at low water on the Mississippi River and some of its tributaries, July 1987-June 1988: U.S. Geological Survey Open-File Report 91-485, 143 p.

Nordin, C.F., Jr., Cranston, C.C., and Mejia-B.,A., 1983, New technology for measuring water and suspended-sediment discharge of large rivers, in Proceedings of the International Symposium on River Sedimentation, 2d, Nanjing: Beijing, Water Resources and Electric Power Press, p. 1145-1158.

Rees, T.F., Leenheer, J.A., and Ranville, J.F., 1991, Use of a single-bowl continuous-flow centrifuge for dewatering suspended sediment--effect on sediment physical and chemical characteristics: Hydrological Processes, v. 5 , p. 201-214. 
Richey, J.E., Meade, R.H., Salati, Eneas, Devol, A.H., Nordin, C.F., Jr., and dos Santos, Umberto, 1986, Water discharge and suspended sediment concentrations in the Amazon River, 1982-1984: Water Resources Research, v. 22 , no. 5 , p. 756-764.

Sayre, W.W., and Caro-Cordero, R., 1977, Shore-attached thermal plumes in rivers, in Shen, H.W., ed., Modeling of Rivers: New York, John Wiley, chap. 15, p. 15-1 to 15-44.

Sium, Ogbazghi, 1975, Transverse flow distribution in natural streams as influenced by cross-sectional shape: M.S. thesis, Ames, University of Iowa, $99 \mathrm{p}$.

Stallard, R.F., and Martin, D.A., 1989, Settling properties of suspended sediment from the Mississippi River [abs.], in Pederson, G.L., and Smith, M.M., compilers, U.S. Geological Survey Second National Symposium on Water Quality--Abstracts of the Technical Sessions, Orlando, Fla., November 12-17, 1989: U.S. Geological Survey Open-File Report 89-409, p. 96.

Stevens, H.H., Jr., Lutz, G.A., and Hubbell, D.W., 1980, Collapsible-bag suspended-sediment sampler: American Society of Civil Engineers Proceedings, Hydraulics Division Journal, v. 106, no. HY4, p. 611-616.

U.S. Army Corps of Engineers, [1983?], Missouri River navigation charts (Kansas City, Missouri to the mouth): Kansas City, Mo., variously paged. [1986?], Navigation charts McClellan-Kerr Arkansas River Navigation System (Catoosa, Oklahoma to mouth of White River): Little Rock, Ark., variously paged.

1987a, Ohio River navigation charts (Cairo, Illinois to Foster, Kentucky): Louisville, Ky., District, variously paged. 1987b, Charts of the Illinois waterway: Washington, D.C., U.S. Government Printing office, variously paged.

[1989a?], 1989 Flood control and navigation maps of the Mississippi River (Cairo, Illinois to the Gulf of Mexico) (57th ed.): Memphis, Tenn., variously paged.

[1989b?], Upper Mississippi River navigation charts: Rock Island, I1l., variously paged.

[1990a?], Stages and discharges of the Mississippi River and tributaries in the Vicksburg District 1989: Vicksburg, Miss., 368 p.

[1990b?], Stages and discharges of the Mississippi River and tributaries in the Memphis District 1989: Memphis, Tenn., $238 \mathrm{p}$.

[1990c?], Stages and discharges of the Mississippi River and tributaries and other watersheds in the New Orleans District for 1989: New Orleans, La . , 279 p.

[1991a?], Stages and discharges of the Mississippi River and tributaries in the Vicksburg District 1990: Vicksburg, Miss., 350 p.

[1991b?], Stages and discharges of the Mississippi River and tributaries in the Memphis District 1990: Memphis, Tenn., $236 \mathrm{p}$.

[1991c?], Stages and discharges of the Mississippi River and tributaries and other watersheds in the New Orleans District for 1990: New Orleans, La., 179 p.

U.S. Geological Survey, 1986, Water-resources data for Illinois, water years 1986-90: U.S. Geological Survey Water-Data Reports IL-86-1 to IL-90-1 (published annually).

1989-90, Water-resources data for Arkansas, water years 1989-90: U.S. Geological Survey Water-Data Reports AR-89-1 and AR-90-1 (published annually). 
1989-90, Water-resources data for Indiana, water years 1989-90: U.S. Geological Survey Water-Data Reports IN-89-1 and IN-90-1 (published annually). 1989-90, Water-resources data for Kentucky, water years 1989-90: U.S. Geological Survey Water-Data Reports KY-89-1 and KY-90-1 (published annually). 1986-90, Water-resources data for Missouri, water years 1986-90: U.S. Geological Survey Water-Data Reports M0-86-1 to MO-90-1 (published annually). 
The data in this section are organized by individual cruises. In the listings of measurements made at each vertical, the $A$ and $B$ after the vertical number identifies the sample bottle, the $X$ preceding the vertical number indicates that an extra velocity measurement was made but no sample was collected, and an $R$ indicates that the vertical was repeated because the velocity measurement was incorrect, the suspended-sediment collection bag leaked, or debris on the nozzle prevented the collection of a suitable sample. Verticals usually were occupied in numerical order. Exceptions due to weather conditions or towboat traffic are noted in the REMARKS section of each sampling site listing. Inch-pound units are used in these listings for (1) part of the name of a sampling site; (2) gage heights, which will serve as a reference for future cruises; (3) the name of a sampling weight; and (4) the designation of the nozzle size used to sample suspended sediment.

SOLID CUP refers to the standard Price AA current meter with solid, polymer bucket wheels. This current meter was used for the vertical integration measurements of discharge in this report because it does not respond to vertical velocities; however, later studies indicated that this current meter does not have a good cosine response to varying angles of attack, and its use by the U.S. Geological Survey has been discontinued.

The following abbreviations are used in the tabulated cruise data and are listed below.

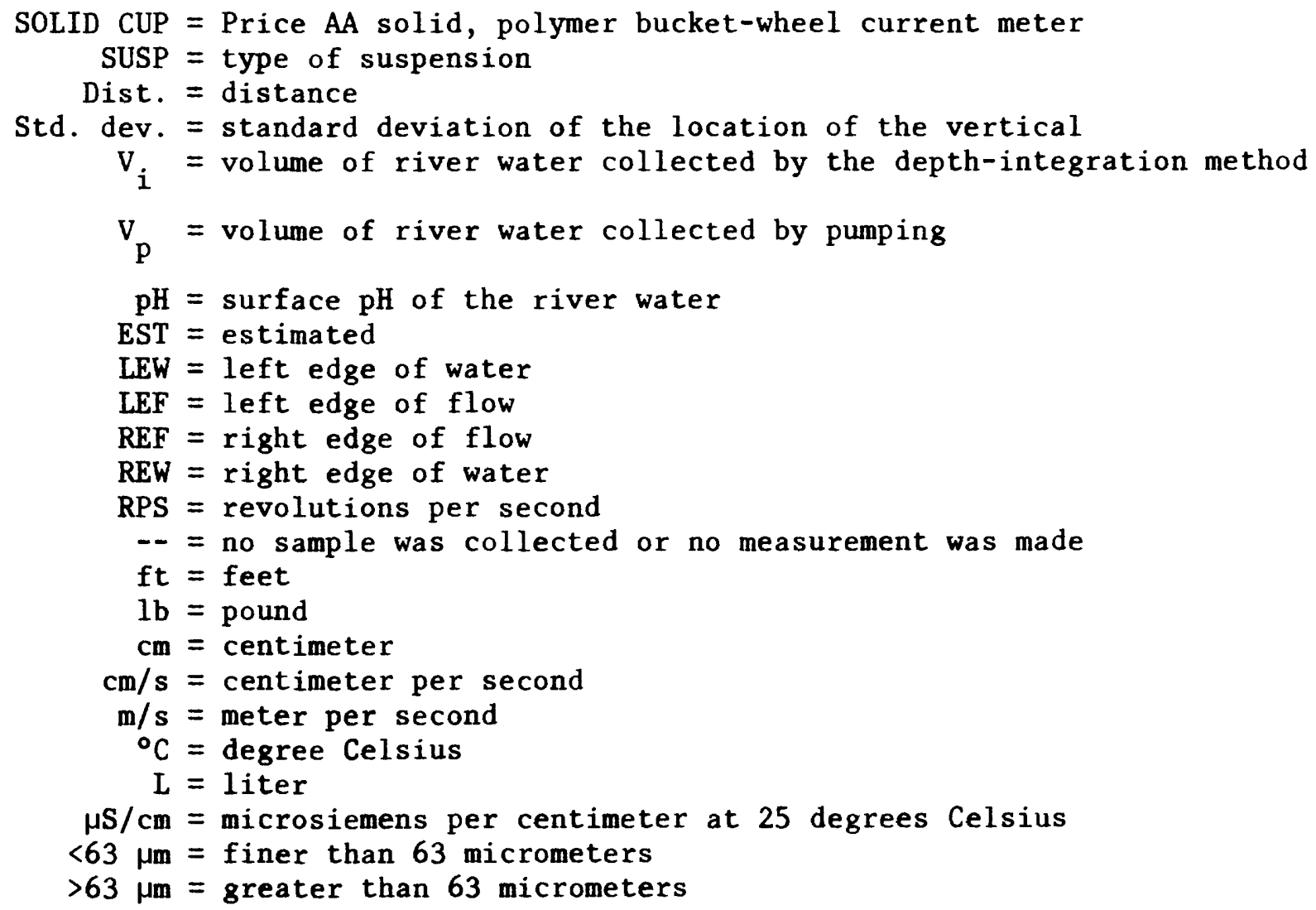


DATA LISTINGS

FOR

MARCH-APRIL 1989 CRUISE 
SITE: Mississippi River near Winfield, Missouri PARTY: Moody, Stevens, and Black

STARTING GAGE HEIGHT: -- ENDING GAGE HEIGHT

METER: SOLID CUP

SUSP: Bag sampler and 200-1b weight. Current meter $32 \mathrm{~cm}$ above nozzle.

CURRENT METER NO.: P8308282 DATE RATED: 07-08-88

CURRENT METER EQUATION: $\mathrm{V}(\mathrm{m} / \mathrm{s})=\mathrm{RPS} * 0.765+0.006$

REMARKS: Anchored at 20 verticals in the following order: $5,4,3,2,1,6-20$

Many dead or dying fish floating by the section.

Transit rate $6 \mathrm{~cm} / \mathrm{s}$ and no nozzle. Delta $x$ based on 20 -second printout.

\begin{tabular}{|c|c|c|c|c|c|c|c|c|c|}
\hline \multirow[b]{2}{*}{$\begin{array}{c}\text { Verti- } \\
\text { cal }\end{array}$} & \multirow{2}{*}{$\begin{array}{l}\text { Dist. } \\
\text { from } \\
\text { LEW } \\
(\mathrm{m})\end{array}$} & \multirow[b]{2}{*}{$\begin{array}{l}\text { Depth } \\
\text { (m) }\end{array}$} & \multirow{2}{*}{$\begin{array}{l}\text { Mean } \\
\text { veloc- } \\
\text { ity } \\
(\mathrm{m} / \mathrm{s})\end{array}$} & \multirow[b]{2}{*}{$\begin{array}{l}\text { Dis- } \\
\text { charge } \\
\left(\mathrm{m}^{3} / \mathrm{s}\right)\end{array}$} & \multicolumn{2}{|c|}{ Volume } & \multirow[b]{2}{*}{$\begin{array}{c}\text { Temper- } \\
\text { ature } \\
\left({ }^{\circ} \mathrm{C}\right)\end{array}$} & \multirow[b]{2}{*}{$\mathrm{pH}$} & \multirow{2}{*}{$\begin{array}{l}\text { Specific } \\
\text { conduct- } \\
\text { ance } \\
(\mu \mathrm{S} / \mathrm{cm})\end{array}$} \\
\hline & & & & & $\begin{array}{l}\mathrm{V}_{i} \\
(\mathrm{~L})\end{array}$ & $\begin{array}{l}V_{p} \\
(L)\end{array}$ & & & \\
\hline LEW & 0 & 0.0 & 0.00 & 0 & -- & -- & -- & -- & -- \\
\hline 01 & 23 & 5.5 & 0.12 & 15 & 0.86 & 29 & 2.7 & 9.1 & 439 \\
\hline 02 & 46 & 7.6 & 0.29 & 45 & 7.31 & 47 & 3.1 & 9.0 & 431 \\
\hline $02 \mathrm{R}$ & 54 & 7.4 & 0.40 & 53 & -- & - & - & - & -- \\
\hline 03 & 82 & 7.6 & 0.39 & 62 & 11.04 & 48 & 2.3 & 9.0 & 408 \\
\hline 04 & 106 & 7.7 & 0.42 & 60 & 11.81 & 46 & 1.7 & 8.9 & 435 \\
\hline 05 & 119 & 7.9 & 0.42 & 32 & 12.82 & 49 & 2.3 & 8.6 & 442 \\
\hline X01 & 124 & 7.8 & 0.38 & 66 & -- & -- & -- & -- & -- \\
\hline 06 & 163 & 7.2 & 0.37 & 75 & 12.00 & 46 & 3.8 & 9.0 & 448 \\
\hline 07 & 181 & 7.4 & 0.28 & 63 & 7.29 & 45 & 2.7 & 9.0 & 456 \\
\hline 08 & 224 & 6.5 & 0.32 & 58 & 8.42 & 43 & 2.8 & 9.0 & 438 \\
\hline 09 & 237 & 6.2 & 0.31 & 42 & 8.55 & 34 & 3.1 & 9.0 & 435 \\
\hline 10 & 268 & 5.9 & 0.29 & 41 & 5.77 & 34 & 3.1 & 9.1 & 432 \\
\hline $11 \mathrm{~A}$ & 285 & 5.4 & 0.29 & 17 & 5.76 & 27 & 3.1 & 9.0 & 443 \\
\hline $11 \mathrm{~B}$ & 290 & 5.4 & 0.28 & 32 & -- & -- & -- & -- & -- \\
\hline 12 & 328 & 5.0 & 0.24 & 31 & 6.48 & 23 & 2.6 & 8.9 & -- \\
\hline 13 & 342 & 4.8 & 0.27 & 27 & 3.98 & 22 & 3.2 & 8.9 & 440 \\
\hline 14 & 369 & 4.4 & 0.26 & 26 & 3.89 & 18 & 2.8 & 9.0 & 435 \\
\hline 15 & 389 & 4.9 & 0.24 & 20 & 2.20 & 18 & 2.8 & 8.9 & 448 \\
\hline $16 \mathrm{~A}$ & 403 & 3.9 & 0.26 & 12 & 4.99 & 14 & 3.3 & 9.0 & 428 \\
\hline $16 B$ & 412 & 2.7 & 0.25 & 16 & - & - & - & - & - \\
\hline 17 & 451 & 4.3 & 0.16 & 17 & 1.40 & 18 & 2.9 & 9.0 & 433 \\
\hline $18 \mathrm{C}$ & 460 & 4.0 & 0.18 & 5 & -- & -- & -- & -- & -- \\
\hline 18 & 466 & 4.1 & 0.15 & 13 & 2.26 & 13 & 3.0 & 8.9 & 433 \\
\hline 19 & 503 & 3.3 & 0.11 & 10 & 1.26 & 16 & 3.4 & 8.8 & 423 \\
\hline 20 & 521 & 2.9 & 0.08 & 6 & 1.32 & - & 3.4 & 9.0 & 430 \\
\hline REW & 552 & 0.0 & 0.00 & 0 & -- & -- & -- & -- & -- \\
\hline MEAN & & 5.3 & 0.29 & & & & & & \\
\hline TOTAL & 552 & & & 844 & 119.41 & 590 & & & \\
\hline
\end{tabular}




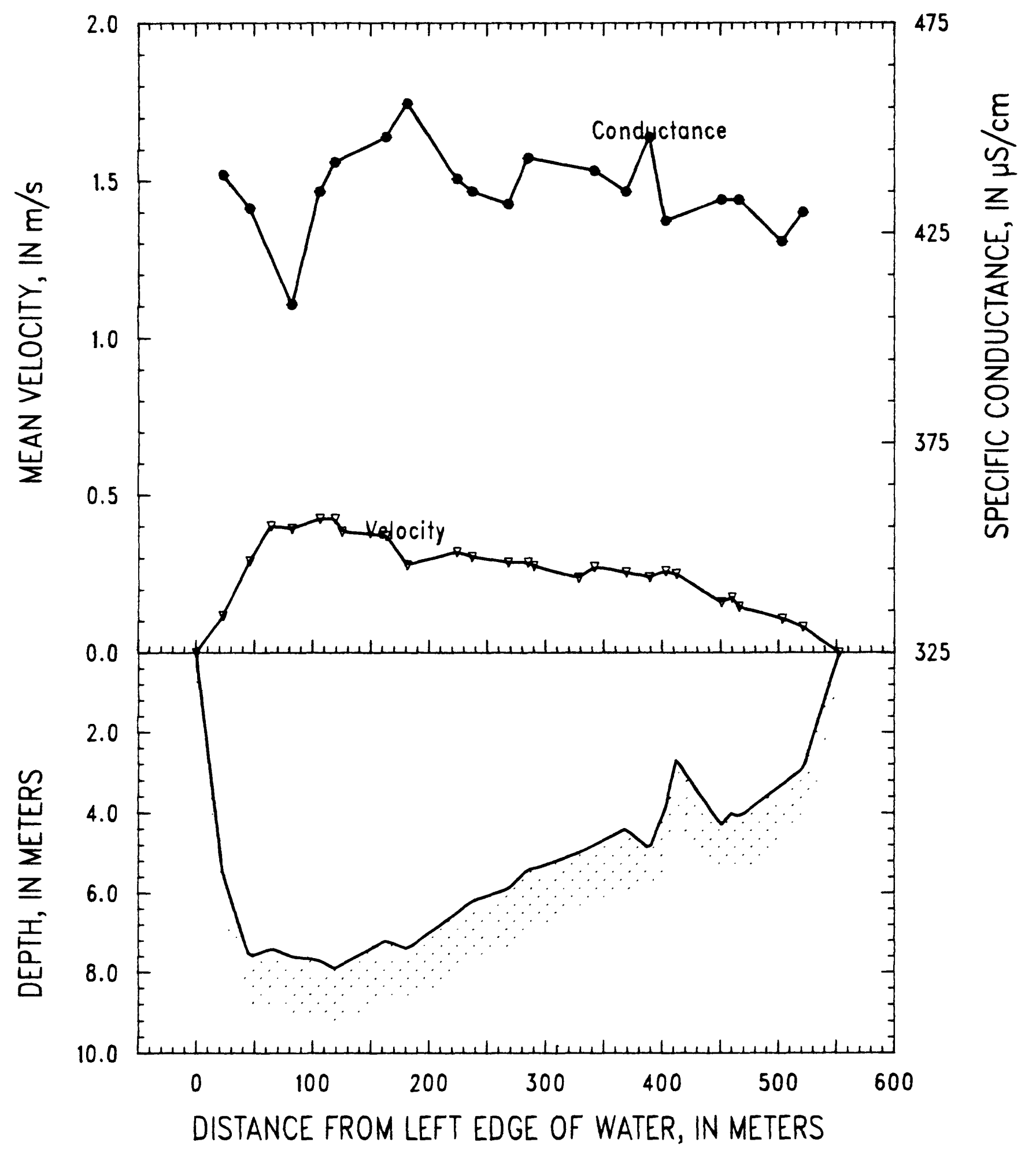

Figure 10. Mississippi River near Winfield, Missouri, on March 10,1989. 
SITE: Illinois River at Hardin, Illinois

PARTY: Moody

STARTING GAGE HEIGHT: -- ENDING GAGE HEIGHT: --

03-09-89

SUSP: $\quad 15-1 \mathrm{~b}$ weight

CURRENT METER NO.: P8308282 DATE RATED: 06-29-88 (for 15-1b weight only)

CURRENT METER EQUATION: $\mathrm{V}(\mathrm{m} / \mathrm{s})=\mathrm{RPS} * 0.751+0.016$

REMARKS: Anchored at about $120 \mathrm{~m}$ from LEW, and collected a 117.89-L depthintegrated composite and a 597-L pumped composite. No nozzle. Transit rate was $6 \mathrm{~cm} / \mathrm{s}$ down and $3 \mathrm{~cm} / \mathrm{s}$ up. Discharge was measured using a handline from small boat. Tagline stretched from R/V ACADIANA to LEW and then to REW. Water temperature was $1.6-2.2^{\circ} \mathrm{C}$.

\begin{tabular}{|c|c|c|c|c|c|c|c|c|c|}
\hline & Dist. & & Mean & & Vol & & & & Specific \\
\hline $\begin{array}{c}\text { Verti- } \\
\text { cal }\end{array}$ & $\begin{array}{l}\text { from } \\
\text { LEW } \\
\text { (m) }\end{array}$ & $\begin{array}{c}\text { Depth } \\
\text { (m) }\end{array}$ & $\begin{array}{l}\text { veloc- } \\
\text { ity } \\
(\mathrm{m} / \mathrm{s})\end{array}$ & $\begin{array}{r}\text { Dis- } \\
\text { charge } \\
\left(\mathrm{m}^{3} / \mathrm{s}\right)\end{array}$ & $\begin{array}{l}\overline{\mathrm{V}_{\mathbf{i}}} \\
(\mathrm{L})\end{array}$ & $\begin{array}{l}V_{p} \\
(L)\end{array}$ & $\begin{array}{l}\text { Temper- } \\
\text { ature } \\
\left({ }^{\circ} \mathrm{C}\right)\end{array}$ & $\mathrm{pH}$ & $\begin{array}{l}\text { conduct- } \\
\text { ance } \\
(\mu \mathrm{S} / \mathrm{cm})\end{array}$ \\
\hline
\end{tabular}

\begin{tabular}{|c|c|c|c|c|c|c|c|c|c|}
\hline LEW & 0 & 0.0 & 0.00 & 0 & -. & -- & -- & - & -. \\
\hline 01 & 10 & 0.4 & 0.10 & 0 & -. & -- & -. & -- & -. \\
\hline 02 & 20 & 1.5 & 0.22 & 3 & -- & -- & -- & -- & -- \\
\hline 03 & 30 & 2.2 & 0.29 & 6 & -- & - & -- & -- & -- \\
\hline 04 & 40 & 2.7 & 0.33 & 9 & -- & -- & -. & - & - \\
\hline 05 & 50 & 3.3 & 0.36 & 12 & -. & - & -- & - & -- \\
\hline 06 & 60 & 3.4 & 0.38 & 13 & -- & -- & -. & -. & -- \\
\hline 07 & 70 & 3.9 & 0.38 & 15 & -- & - & -- & -- & -- \\
\hline 08 & 80 & 4.0 & 0.39 & 16 & -- & - & -. & -- & - \\
\hline 09 & 90 & 4.0 & 0.37 & 15 & - & - & -. & -- & -. \\
\hline 10 & 100 & 3.7 & 0.36 & 13 & -- & -- & -- & -- & -- \\
\hline 11 & 110 & 4.0 & 0.37 & 13 & -- & - & -- & -- & -. \\
\hline 12 & 117 & 4.0 & 0.39 & 10 & -- & -- & -- & -- & -- \\
\hline 13 & 123 & 4.0 & 0.40 & 9 & -- & - & - & -- & -- \\
\hline 14 & 129 & 4.0 & 0.36 & 11 & -- & - & -- & -- & -- \\
\hline 15 & 139 & 4.8 & 0.33 & 16 & -- & - & - & -- & -. \\
\hline 16 & 149 & 5.6 & 0.43 & 24 & -- & - & -- & -- & -- \\
\hline 17 & 159 & 5.8 & 0.35 & 21 & -- & - & -- & -- & -- \\
\hline 18 & 169 & 6.0 & 0.36 & 22 & -- & - & -- & -. & -- \\
\hline 19 & 179 & 6.0 & 0.39 & 23 & -- & - & -- & -- & -- \\
\hline 20 & 189 & 5.9 & 0.41 & 24 & $\ldots$ & $\ldots$ & $\ldots$ & - & -. \\
\hline 21 & 199 & 5.9 & 0.45 & 27 & -- & $\ldots$ & -- & -- & -- \\
\hline 22 & 209 & 5.9 & 0.43 & 25 & -- & -- & -- & -- & -- \\
\hline 23 & 219 & 5.6 & 0.44 & 25 & -- & - & - & -- & -- \\
\hline 24 & 229 & 5.5 & 0.40 & 22 & -- & - & -- & -- & -- \\
\hline 25 & 239 & 5.0 & 0.35 & 18 & -- & - & -. & -- & -- \\
\hline 26 & 249 & 4.0 & 0.34 & 14 & -- & - & -- & -- & -- \\
\hline 27 & 259 & 2.8 & 0.29 & 8 & -- & -- & -- & -- & -- \\
\hline REW & 269 & 0.0 & 0.00 & 0 & -- & - & -- & -- & -- \\
\hline MEAN & & 4.1 & 0.38 & & & & & & \\
\hline TOTAL & 269 & & & 414 & & & & & \\
\hline
\end{tabular}




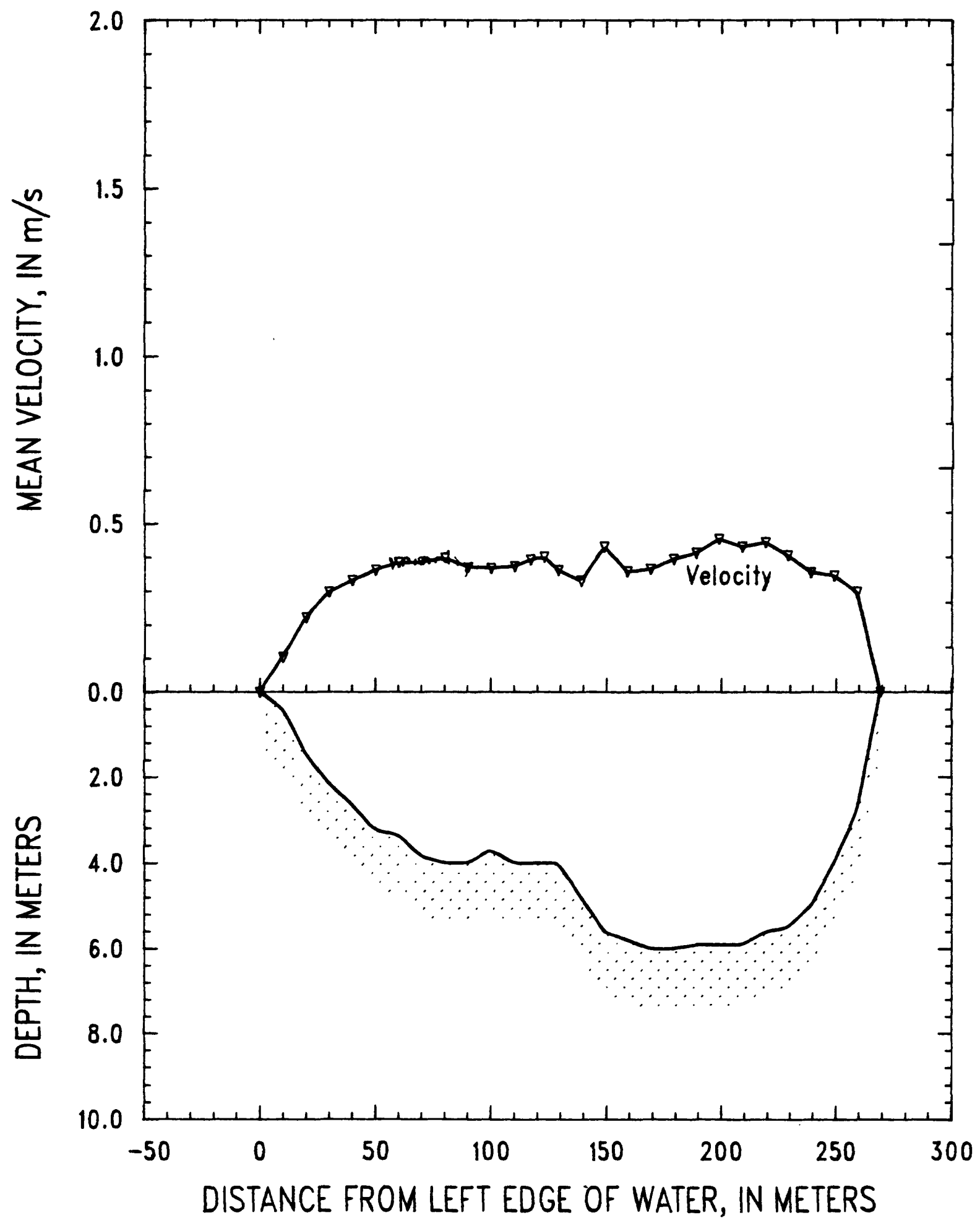

Figure 11. Illinois River at Hardin, Illinois, on March 9, 1989. 
SITE: Missouri River at Hermann, Missouri

PARTY: Moody, Stevens, and Black

$03 / 12 / 89$

STARTING GAGE HEIGHT: -- ENDING GAGE HEIGHT: --

METER: SOLID CUP

SUSP: Bag sampler and 200-lb weight. Current meter $32 \mathrm{~cm}$ above nozzle.

CURRENT METER NO.: P8308282 DATE RATED: 07-08-88

CURRENT METER EQUATION: $\mathrm{V}(\mathrm{m} / \mathrm{s})=\mathrm{RPS} * 0.765+0.006$

REMARKS: Transit rate $11 \mathrm{~cm} / \mathrm{s}$. Nozzle was $5 / 16$ inch. Slope gage was estimated at $5.5 \mathrm{ft}$. Coast Guard reported stage was $7.5 \mathrm{ft}$. Verticals occupied in order for depth-integrated sampling.

\begin{tabular}{|c|c|c|c|c|c|c|c|c|c|}
\hline \multirow[b]{2}{*}{$\begin{array}{l}\text { Verti- } \\
\text { cal }\end{array}$} & \multirow{2}{*}{$\begin{array}{l}\text { Dist. } \\
\text { from } \\
\text { LEW } \\
\text { (m) }\end{array}$} & \multirow[b]{2}{*}{$\begin{array}{c}\text { Depth } \\
\text { (m) }\end{array}$} & \multirow{2}{*}{$\begin{array}{l}\text { Mean } \\
\text { veloc- } \\
\text { ity } \\
(\mathrm{m} / \mathrm{s})\end{array}$} & \multirow[b]{2}{*}{$\begin{array}{r}\text { Dis- } \\
\text { charge } \\
\left(\mathrm{m}^{3} / \mathrm{s}\right)\end{array}$} & \multicolumn{2}{|c|}{ Volume } & \multirow[b]{2}{*}{$\begin{array}{l}\text { Temper- } \\
\text { ature } \\
\left({ }^{\circ} \mathrm{C}\right)\end{array}$} & \multirow[b]{2}{*}{$\mathrm{pH}$} & \multirow{2}{*}{$\begin{array}{l}\text { Specific } \\
\text { conduct- } \\
\text { ance } \\
(\mu \mathrm{S} / \mathrm{cm})\end{array}$} \\
\hline & & & & & $\begin{array}{l}V_{i} \\
\text { (L) }\end{array}$ & $\begin{array}{l}\overline{V_{p}} \\
(\mathrm{~L})\end{array}$ & & & \\
\hline LEW & 0 & 0.0 & 0.00 & 0 & -- & - & -- & -- & -- \\
\hline $01 \mathrm{~A}$ & 9 & 6.3 & 1.03 & 62 & 4.43 & 15 & 6.4 & 7.8 & 625 \\
\hline O2B & 19 & 6.3 & 1.18 & 67 & 5.92 & 23 & 6.8 & 8.0 & 641 \\
\hline $03 \mathrm{~A}$ & 27 & 6.2 & 1.13 & 67 & 6.23 & 28 & 6.8 & 8.0 & 636 \\
\hline 04B & 38 & 5.7 & 1.22 & 56 & 6.20 & 27 & 6.7 & 8.0 & 624 \\
\hline 05A & 43 & 6.3 & 1.29 & 73 & 5.52 & 26 & 6.7 & 8.1 & 627 \\
\hline 06B & 56 & 5.8 & 1.37 & 80 & 6.76 & 27 & 6.6 & 8.1 & 599 \\
\hline 07A & 63 & 6.2 & 1.50 & 60 & 5.89 & 24 & 6.8 & 8.1 & 581 \\
\hline 08B & 69 & 6.3 & 1.54 & 44 & 5.65 & 19 & 7.3 & 8.0 & 559 \\
\hline $\mathrm{X} 03$ & 72 & 4.7 & 1.43 & 27 & -- & -- & -- & -- & -- \\
\hline 09A & 77 & 5.2 & 1.43 & 82 & 5.09 & 19 & 7.0 & 8.0 & 583 \\
\hline $10 \mathrm{~B}$ & 94 & 4.5 & 1.44 & 71 & 4.74 & 22 & 7.0 & 8.1 & 565 \\
\hline $11 R$ & 99 & 4.3 & 1.37 & 29 & 4.31 & 16 & 7.1 & 8.1 & 549 \\
\hline $12 \mathrm{~B}$ & 104 & 4.3 & 1.51 & 58 & 4.97 & 14 & 7.1 & 8.1 & 458 \\
\hline $13 \mathrm{~A}$ & 117 & 4.8 & 1.25 & 57 & 4.95 & 16 & 7.1 & 8.1 & 446 \\
\hline $14 B$ & 123 & 5.2 & 1.30 & 37 & 4.65 & 18 & 7.1 & 8.0 & 448 \\
\hline $15 \mathrm{~A}$ & 128 & 4.6 & 1.27 & 29 & 4.10 & 15 & 7.5 & 7.9 & 431 \\
\hline X06 & 133 & 4.6 & 1.32 & 33 & - & -- & - & -- & -- \\
\hline $16 \mathrm{~B}$ & 139 & 4.4 & 1.14 & 28 & 3.93 & 14 & 7.4 & 8.0 & 434 \\
\hline $17 \mathrm{~A}$ & 144 & 4.3 & 1.16 & 50 & 3.94 & 16 & 7.4 & 8.0 & 390 \\
\hline $18 \mathrm{~B}$ & 159 & 4.4 & 1.18 & 49 & 3.90 & 15 & 7.4 & 8.0 & 375 \\
\hline $19 \mathrm{~A}$ & 163 & 4.2 & 1.22 & 36 & 3.46 & 13 & 7.5 & 8.0 & 350 \\
\hline $20 \mathrm{~B}$ & 173 & 3.6 & 1.04 & 41 & 3.41 & 13 & 7.5 & 8.0 & 354 \\
\hline $21 \mathrm{~A}$ & 185 & 3.3 & 1.16 & 36 & 3.22 & 13 & 7.6 & 8.0 & 328 \\
\hline 22B & 192 & 3.4 & 0.96 & 29 & 2.86 & 12 & 7.6 & 7.9 & 326 \\
\hline $23 \mathrm{~A}$ & 203 & 3.4 & 1.10 & 32 & 2.76 & 11 & 7.5 & 8.0 & 330 \\
\hline $24 B$ & 209 & 3.4 & 1.29 & 42 & 3.33 & 14 & 7.9 & 8.0 & 326 \\
\hline $25 \mathrm{~A}$ & 222 & 3.1 & 1.11 & 34 & 3.16 & 10 & 7.9 & 8.0 & 324 \\
\hline 26B & 229 & 3.2 & 1.08 & 19 & 2.94 & 11 & 7.6 & 8.0 & 319 \\
\hline $27 \mathrm{~A}$ & 233 & 3.0 & 1.02 & 20 & 2.01 & 9 & 7.8 & 8.0 & 324 \\
\hline $28 \mathrm{~B}$ & 242 & 3.3 & 1.01 & 27 & 2.06 & 11 & 7.9 & 8.1 & 319 \\
\hline $29 \mathrm{~A}$ & 249 & 5.5 & 0.85 & 40 & 2.93 & 20 & 7.7 & 8.1 & 315 \\
\hline $30 \mathrm{~B}$ & 259 & 6.3 & 0.95 & 63 & 3.80 & 16 & 7.9 & 8.1 & 315 \\
\hline REW & 270 & 0.0 & 0.00 & 0 & -- & -- & -- & -- & -- \\
\hline MEAN & & 4.5 & 1.21 & & & & & & \\
\hline TOTAL & 270 & & & 1,478 & 127.12 & 507 & & & \\
\hline
\end{tabular}




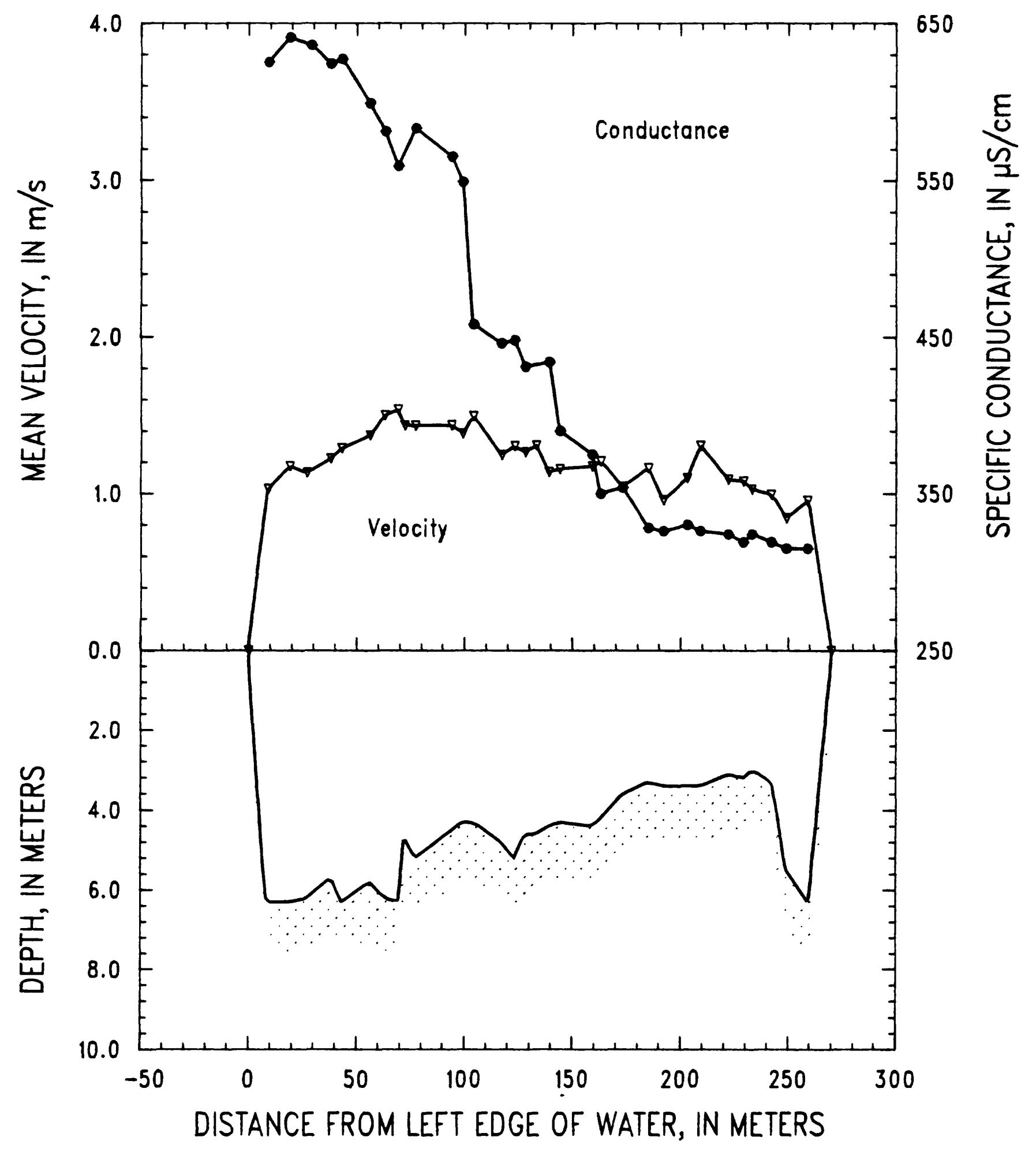

Figure 12. Missouri River at Hermann, Missouri, on March 12, 1989. 
SITE: Mississippi River at St. Louis, Missouri

03-13-89

PARTY: Moody, Stevens, and Black

STARTING GAGE HEIGHT: -- ENDING GAGE HEIGHT:

METER: SOLID CUP

SUSP: Bag sampler and 200-1b weight. Current meter $32 \mathrm{~cm}$ above nozzle.

CURRENT METER NO.: P8308282 DATE RATED: 7-08-88

CURRENT METER EQUATION: $\mathrm{V}(\mathrm{m} / \mathrm{s})=$ RPS $* 0.765+0.006$

REMARKS: Transit rate was $10 \mathrm{~cm} / \mathrm{s}$ and nozzle was $1 / 4$ inch.

Simultaneous discharge measurement made by George Gray, USGS, Missouri

District, from the Poplar Street Bridge. Verticals occupied in order for depth-integrated sampling and surface-water measurements.

\begin{tabular}{|c|c|c|c|c|c|c|c|c|c|}
\hline \multirow[b]{2}{*}{$\begin{array}{l}\text { Verti- } \\
\text { cal }\end{array}$} & \multirow{2}{*}{$\begin{array}{l}\text { Dist. } \\
\text { from } \\
\text { LEW } \\
\text { (m) }\end{array}$} & \multirow[b]{2}{*}{$\begin{array}{c}\text { Depth } \\
(\mathbf{m})\end{array}$} & \multirow{2}{*}{$\begin{array}{l}\text { Mean } \\
\text { veloc- } \\
\text { ity } \\
(\mathrm{m} / \mathrm{s})\end{array}$} & \multirow[b]{2}{*}{$\begin{array}{r}\text { Dis - } \\
\text { charge } \\
\left(\mathrm{m}^{3} / \mathrm{s}\right)\end{array}$} & \multicolumn{2}{|c|}{ Volume } & \multirow[b]{2}{*}{$\begin{array}{l}\text { Temper- } \\
\text { ature } \\
\left({ }^{\circ} \mathrm{C}\right)\end{array}$} & \multirow[b]{2}{*}{$\mathrm{pH}$} & \multirow{2}{*}{$\begin{array}{l}\text { Specific } \\
\text { conduct- } \\
\text { ance } \\
(\mu \mathrm{S} / \mathrm{cm})\end{array}$} \\
\hline & & & & & $\begin{array}{c}\overline{\mathbf{v}_{\mathbf{i}}} \\
(\mathrm{L})\end{array}$ & $\begin{array}{c}v_{p} \\
(L)\end{array}$ & & & \\
\hline LEW & 0 & 0.0 & 0.00 & 0 & -- & -- & -- & -- & -- \\
\hline $01 \mathrm{~A}$ & 25 & 10.3 & 0.79 & 150 & 4.04 & 16 & 5.4 & 8.7 & 590 \\
\hline 02B & 37 & 10.2 & 1.09 & 145 & 4.84 & 25 & 5.3 & 8.8 & 581 \\
\hline $03 \mathrm{~A}$ & 51 & 10.0 & 1.46 & 204 & 5.70 & 28 & 5.6 & 8.9 & 586 \\
\hline 04B & 65 & 10.2 & 1.43 & 212 & 6.60 & 31 & 5.9 & 8.8 & 587 \\
\hline $05 \mathrm{~A}$ & 80 & 10.6 & 1.35 & 222 & 6.34 & 32 & 5.3 & 8.8 & 585 \\
\hline 06B & 96 & 10.3 & 1.27 & 157 & 6.02 & 31 & 5.3 & 8.8 & 583 \\
\hline $\mathrm{X} 07$ & 104 & 10.2 & 1.36 & 138 & -- & -- & - & -- & -- \\
\hline $07 \mathrm{~A}$ & 116 & 9.7 & 1.23 & 125 & 4.99 & 30 & 6.0 & 8.8 & 586 \\
\hline $08 \mathrm{~B}$ & 125 & 9.3 & 1.28 & 178 & 5.46 & 26 & 6.3 & 8.9 & 579 \\
\hline $09 \mathrm{~A}$ & 146 & 8.9 & 1.27 & 170 & 5.09 & 25 & 5.5 & 8.8 & 574 \\
\hline $10 \mathrm{~B}$ & 155 & 8.8 & 1.23 & 173 & 4.98 & 22 & 5.6 & 8.8 & 568 \\
\hline $11 \mathrm{~A}$ & 178 & 7.8 & 1.21 & 175 & 4.67 & 20 & 5.7 & 8.8 & 561 \\
\hline $12 B$ & 192 & 7.2 & 1.15 & 124 & 4.02 & 17 & 5.8 & 8.7 & 562 \\
\hline $13 A$ & 208 & 7.1 & 1.10 & 117 & 3.77 & 17 & 6.0 & 8.7 & 551 \\
\hline $14 \mathrm{~B}$ & 222 & 6.6 & 1.09 & 100 & 3.74 & 15 & 6.5 & 8.7 & 550 \\
\hline $15 \mathrm{~A}$ & 236 & 6.2 & 1.24 & 123 & 3.91 & 16 & 6.2 & 8.8 & 549 \\
\hline $16 \mathrm{~B}$ & 254 & 6.5 & 1.18 & 161 & 3.71 & 14 & 6.2 & 8.7 & 538 \\
\hline $17 \mathrm{~A}$ & 278 & 6.2 & 1.22 & 132 & 3.55 & 13 & 6.3 & 8.6 & 532 \\
\hline $18 \mathrm{~B}$ & 289 & 5.8 & 1.16 & 81 & 3.15 & 12 & 6.6 & 8.6 & 533 \\
\hline $19 \mathrm{~A}$ & 302 & 5.7 & 1.17 & 84 & 3.38 & 12 & 6.5 & 8.6 & 532 \\
\hline $20 \mathrm{~B}$ & 314 & 5.3 & 1.17 & 90 & 2.77 & 11 & 7.0 & 8.5 & 527 \\
\hline $21 \mathrm{~A}$ & 331 & 5.2 & 1.04 & 103 & 2.06 & 9 & 6.6 & 8.6 & 526 \\
\hline 22B & 352 & 5.3 & 1.08 & 92 & 2.54 & 9 & 6.7 & 8.6 & 523 \\
\hline $23 A$ & 363 & 5.2 & 1.08 & 92 & 2.31 & 9 & 7.0 & 8.5 & 518 \\
\hline $24 B$ & 385 & 5.3 & 1.14 & 99 & 2.89 & 10 & 6.9 & 8.5 & 515 \\
\hline $25 \mathrm{~A}$ & 396 & 5.7 & 1.11 & 82 & 2.89 & 8 & 6.5 & 8.5 & 513 \\
\hline $26 B$ & 411 & 5.5 & 1.06 & 99 & 2.44 & 10 & 6.9 & 8.5 & 518 \\
\hline $27 A$ & 430 & 5.3 & 0.99 & 95 & 2.44 & 11 & 7.5 & 8.4 & -- \\
\hline $28 B$ & 447 & 5.1 & 0.96 & 71 & 2.45 & 9 & 7.5 & 8.4 & 520 \\
\hline $29 A$ & 459 & 5.2 & 1.06 & 72 & 2.03 & 7 & 7.3 & 8.4 & 530 \\
\hline $30 \mathrm{~B}$ & 473 & 5.9 & 0.77 & 71 & 1.72 & 5 & 7.6 & 8.4 & 527 \\
\hline REW & 490 & 0.0 & 0.00 & - & -- & - & - & -- & -- \\
\hline MEAN & & 6.9 & 1.17 & & & & & & \\
\hline TOTAL & 490 & & & 3,937 & 114.50 & 500 & & & \\
\hline
\end{tabular}




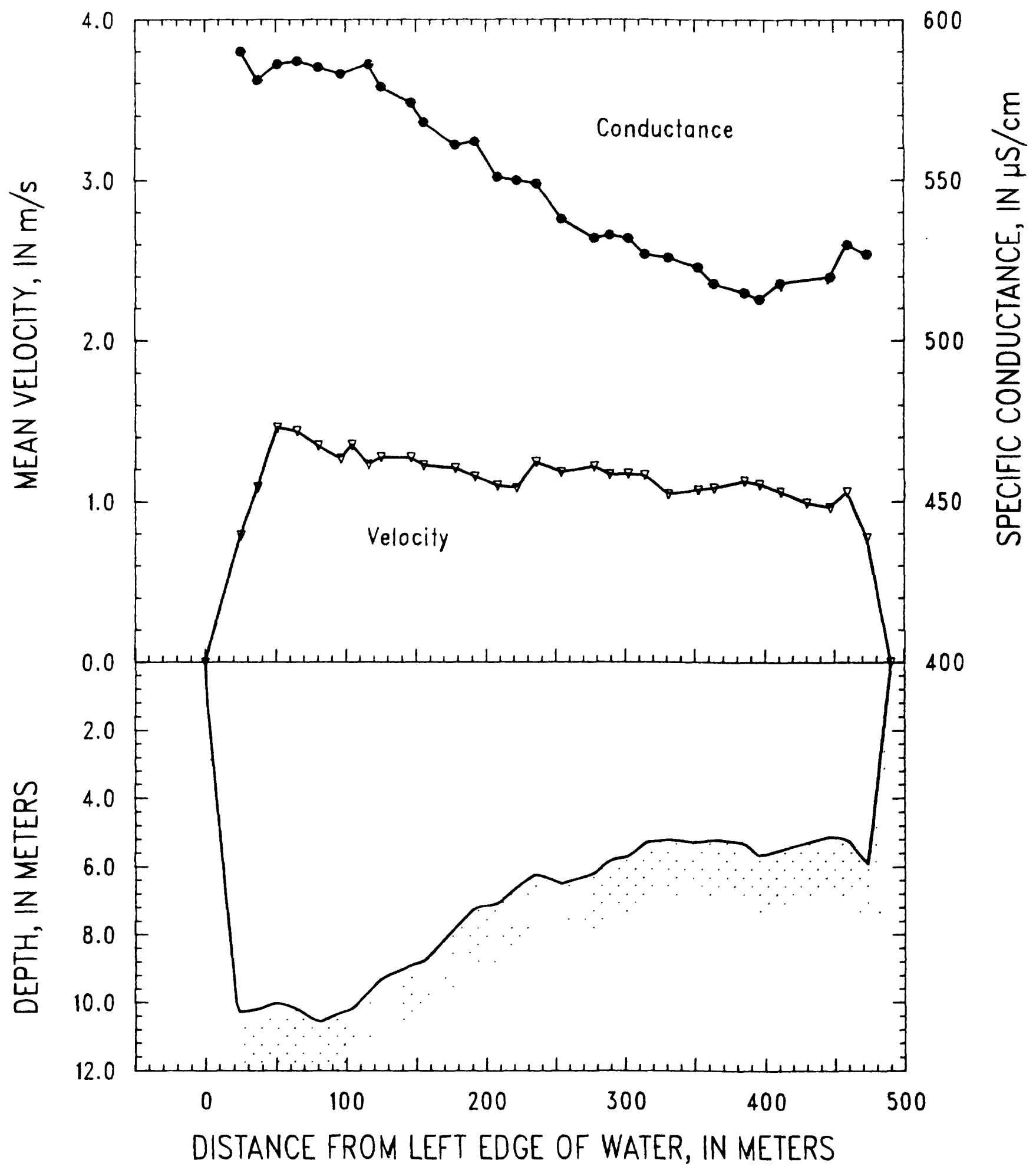

Figure 13. Mississippi River at St. Louis, Missouri, on March 13, 1989. 
SITE: Mississippi River at Thebes, Illinois 03-15-89

PARTY: Moody, Stevens, and Simoneaux

STARTING GAGE HEIGHT: -- ENDING GAGE HEIGHT: --

METER: SOLID CUP

SUSP: Bag sampler and 200-1b weight. Current meter $32 \mathrm{~cm}$ above nozzle.

CURRENT METER NO.: P8308282 DATE RATED: 07-08-88

CURRENT METER EQUATION: $\mathrm{V}(\mathrm{m} / \mathrm{s})=$ RPS $* 0.765+0.006$

REMARKS: Transit rate was $8 \mathrm{~cm} / \mathrm{s}$ and nozzle was $1 / 4$ inch. Verticals were occupied in order $01-27$; flow at verticals $28-30$ was estimated to be proportional to depth to the $2 / 3$ power. Simultaneous discharge measurement was made by George Gray, USGS, Missouri District, from Thebes railroad bridge. Backwater from the ohio River.

\begin{tabular}{|c|c|c|c|c|c|c|c|c|c|}
\hline \multirow[b]{2}{*}{$\begin{array}{c}\text { Verti- } \\
\text { cal }\end{array}$} & \multirow{2}{*}{$\begin{array}{l}\text { Dist. } \\
\text { from } \\
\text { LEW } \\
\text { (m) }\end{array}$} & \multirow[b]{2}{*}{$\begin{array}{l}\text { Depth } \\
\text { (m) }\end{array}$} & \multirow{2}{*}{$\begin{array}{l}\text { Mean } \\
\text { veloc- } \\
\text { ity } \\
(\mathrm{m} / \mathrm{s})\end{array}$} & \multirow[b]{2}{*}{$\begin{array}{r}\text { Dis- } \\
\text { charge } \\
\left(\mathrm{m}^{3} / \mathrm{s}\right)\end{array}$} & \multicolumn{2}{|c|}{ Volume } & \multirow[b]{2}{*}{$\begin{array}{l}\text { Temper- } \\
\text { ature } \\
\left({ }^{\circ} \mathrm{C}\right)\end{array}$} & \multirow[b]{2}{*}{$\mathrm{pH}$} & \multirow{2}{*}{$\begin{array}{l}\text { Specific } \\
\text { conduct- } \\
\text { ance } \\
(\mu \mathrm{S} / \mathrm{cm})\end{array}$} \\
\hline & & & & & $\begin{array}{l}\overline{\mathrm{V}_{\mathrm{i}}} \\
(\mathrm{L})\end{array}$ & $\begin{array}{l}\mathrm{V}_{\mathrm{p}} \\
(\mathrm{L})\end{array}$ & & & \\
\hline LEW & 0 & 0.0 & $0 . C 0$ & 0 & - & -- & -- & -- & -- \\
\hline $01 \mathrm{~A}$ & 32 & 4.5 & 0.38 & 42 & 0.58 & 3 & 7.5 & 8.2 & 477 \\
\hline O2B & 49 & 7.1 & 0.51 & 58 & 1.57 & 6 & 7.6 & 8.3 & 505 \\
\hline $03 \mathrm{~A}$ & 64 & 6.8 & 0.63 & 56 & 1.99 & 11 & 7.2 & 8.4 & 508 \\
\hline $\mathrm{X} 01$ & 75 & 9.8 & 0.84 & 82 & - & -- & -- & -- & -- \\
\hline $04 B$ & 84 & 8.8 & 0.98 & 86 & 4.42 & 25 & 7.1 & 8.4 & 510 \\
\hline $05 \mathrm{~A}$ & 95 & 9.0 & 1.09 & 142 & 5.43 & 21 & 7.0 & 8.5 & 514 \\
\hline 06B & 113 & 9.2 & 1.25 & 213 & 6.43 & 23 & 7.0 & 8.4 & 510 \\
\hline $07 \mathrm{~A}$ & 132 & 9.1 & 1.29 & 252 & 6.70 & 23 & 7.2 & 8.4 & 508 \\
\hline 08B & 156 & 9.4 & 1.35 & 229 & 6.78 & 23 & 7.2 & 8.4 & 505 \\
\hline $\mathrm{X} 02$ & 168 & 9.4 & 1.41 & 152 & - & -- & -- & -- & -- \\
\hline 09A & 179 & 9.4 & 1.38 & 136 & 6.74 & 23 & 7.1 & 8.5 & 511 \\
\hline $10 \mathrm{~B}$ & 189 & 9.5 & 1.39 & 258 & 6.81 & 23 & 7.7 & 8.5 & 511 \\
\hline $11 \mathrm{~A}$ & 218 & 9.4 & 1.39 & 321 & 6.97 & 23 & 7.6 & 8.6 & 517 \\
\hline $12 B$ & 238 & 9.5 & 1.33 & 152 & 6.83 & 23 & 7.9 & 8.4 & 511 \\
\hline $13 \mathrm{~A}$ & 242 & 9.5 & 1.30 & 179 & 6.58 & 23 & 7.6 & 8.5 & 519 \\
\hline $14 \mathrm{~B}$ & 267 & 9.3 & 1.28 & 274 & 6.80 & 23 & 7.5 & 8.5 & 518 \\
\hline $15 \mathrm{~A}$ & 288 & 9.3 & 1.23 & 222 & 5.92 & 23 & 7.5 & 8.5 & 522 \\
\hline 16B & 306 & 9.6 & 1.27 & 201 & 6.51 & 23 & 7.7 & 8.6 & 512 \\
\hline $17 \mathrm{~A}$ & 321 & 9.6 & 1.31 & 264 & 5.88 & 25 & 7.4 & 8.7 & 525 \\
\hline $18 \mathrm{~B}$ & 348 & 9.8 & 1.20 & 258 & 6.37 & 25 & 7.8 & 8.6 & 519 \\
\hline $19 A$ & 365 & 8.9 & 1.11 & 222 & 5.49 & 19 & 7.8 & 8.5 & 532 \\
\hline $20 \mathrm{~B}$ & 393 & 7.5 & 1.05 & 174 & 4.23 & 19 & 7.2 & 8.5 & 519 \\
\hline $21 \mathrm{~A}$ & 409 & 8.0 & 1.05 & 160 & 4.98 & 17 & 7.5 & 8.5 & 519 \\
\hline 22B & 431 & 7.1 & 1.02 & 152 & 4.02 & 11 & 7.4 & 8.6 & 523 \\
\hline $23 \mathrm{~A}$ & 451 & 6.7 & 1.02 & 103 & 3.68 & 13 & 7.7 & 8.6 & 511 \\
\hline $24 B$ & 461 & 6.7 & 0.98 & 108 & 3.60 & 13 & 7.8 & 8.5 & 511 \\
\hline $25 \mathrm{~A}$ & 484 & 6.4 & 0.95 & 106 & 2.64 & 11 & 7.6 & 8.5 & 519 \\
\hline $26 B$ & 496 & 6.0 & 1.00 & 60 & 2.65 & 11 & 7.5 & 8.6 & 511 \\
\hline $26 R$ & 504 & 6.0 & 0.83 & 48 & - & - & - & - & - \\
\hline $27 \mathrm{~A}$ & 515 & 5.3 & 0.93 & 59 & 2.41 & 7 & 7.9 & 8.5 & 515 \\
\hline $\mathrm{X} 05$ & 528 & 3.5 & 0.80 & 40 & -- & -- & -- & -- & -- \\
\hline 28 & 544 & 2.6 & 0.58 & 26 & -- & -- & -- & - & -- \\
\hline 29 & 563 & 3.0 & 0.51 & 30 & -- & -- & -- & -- & -- \\
\hline 30 & 583 & 3.0 & 0.32 & 19 & -- & -- & -- & -- & -- \\
\hline REW & 602 & 0.0 & 0.00 & 0 & -- & -- & -- & -- & -- \\
\hline MEAN & & 7.3 & 1.11 & & & & & & \\
\hline TOTAL & 602 & & & 4,884 & 133.01 & 490 & & & \\
\hline
\end{tabular}




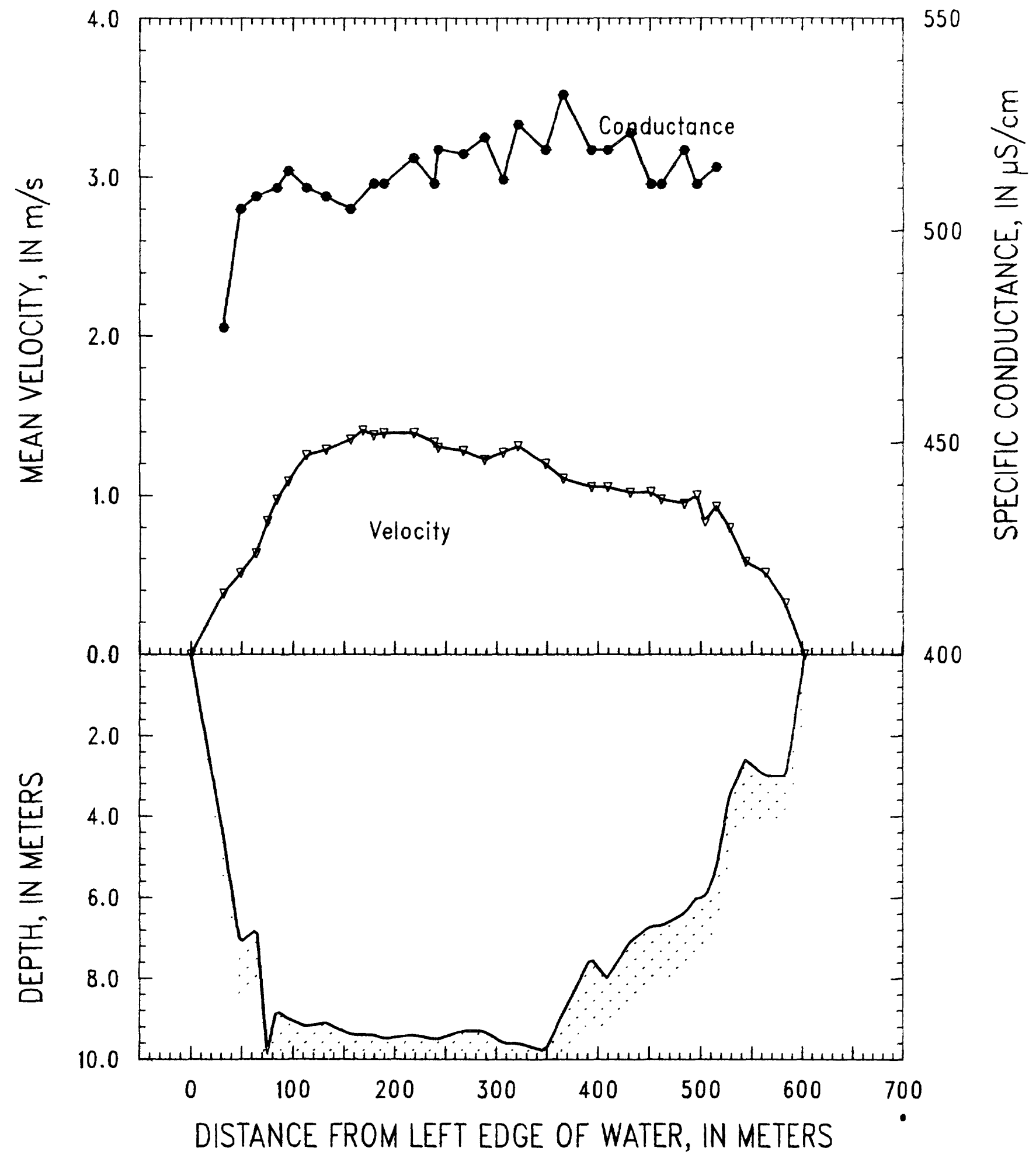

Figure 14. Mississippi River at Thebes, Illinois, on March 15, 1989. 
SITE: Ohio River at Olmsted, Illinois

03-16-89

PARTY: Moody, Stevens, and Simoneaux

METER: SOLID CUP

STARTING GAGE HEIGHT: $44.9 \mathrm{ft}$ at Lock and Dam 53

SUSP: Bag sampler and 300-1b weight. Current meter $32 \mathrm{~cm}$ above nozzle.

CURRENT METER NO.: P8308282 DATE RATED: 07-08-88

CURRENT METER EQUATION: $V(\mathrm{~m} / \mathrm{s})=\mathrm{RPS} \star 0.765+0.006$

REMARKS: Transit rate was $16 \mathrm{~cm} / \mathrm{s}$ and $3 / 16$-inch nozzle. Verticals were occupied from 31 to 1 . Vertical 31 was the right edge of flow.

\begin{tabular}{|c|c|c|c|c|c|c|c|c|c|}
\hline \multirow[b]{2}{*}{$\begin{array}{c}\text { Verti- } \\
\text { cal }\end{array}$} & \multirow{2}{*}{$\begin{array}{l}\text { Dist. } \\
\text { from } \\
\text { LEW } \\
\text { (m) }\end{array}$} & \multirow[b]{2}{*}{$\begin{array}{c}\text { Depth } \\
\text { (m) }\end{array}$} & \multirow{2}{*}{$\begin{array}{l}\text { Mean } \\
\text { veloc- } \\
\text { ity } \\
(\mathrm{m} / \mathrm{s})\end{array}$} & \multirow[b]{2}{*}{$\begin{array}{r}\text { Dis - } \\
\text { charge } \\
\left(\mathrm{m}^{3} / \mathrm{s}\right)\end{array}$} & \multicolumn{2}{|c|}{ Volume } & \multirow[b]{2}{*}{$\begin{array}{l}\text { Temper- } \\
\text { ature } \\
\left({ }^{\circ} \mathrm{C}\right)\end{array}$} & \multirow[b]{2}{*}{$\mathrm{pH}$} & \multirow{2}{*}{$\begin{array}{l}\text { Specific } \\
\text { conduct- } \\
\text { ance } \\
(\mu \mathrm{S} / \mathrm{cm})\end{array}$} \\
\hline & & & & & $\begin{array}{l}\overline{V_{i}} \\
(L)\end{array}$ & $\begin{array}{l}V_{p} \\
(L)\end{array}$ & & & \\
\hline LEW & 0 & 0.0 & 0.00 & 0 & -- & -- & -- & -- & -- \\
\hline $01 \mathrm{~A}$ & 39 & 9.0 & 0.72 & 233 & 1.01 & 5 & 10.5 & 7.5 & 163 \\
\hline 02B & 72 & 10.0 & 1.06 & 328 & 1.71 & 10 & 10.6 & 7.4 & 164 \\
\hline 03A & 101 & 10.8 & 1.29 & 417 & 2.61 & 13 & 10.6 & 7.4 & 164 \\
\hline 04B & 132 & 11.3 & 1.24 & 456 & 2.35 & 17 & 10.5 & 7.5 & 169 \\
\hline 05A & 166 & 11.5 & 1.33 & 589 & 2.72 & 16 & 10.5 & 7.4 & 173 \\
\hline 06B & 209 & 12.0 & 1.25 & 632 & 2.90 & 17 & 10.7 & 7.5 & 176 \\
\hline 07A & 250 & 12.4 & 1.33 & 468 & 3.22 & 18 & 10.3 & 7.3 & 182 \\
\hline 08B & 266 & 12.8 & 1.22 & 360 & 2.85 & 19 & 10.3 & 7.4 & 189 \\
\hline X04 & 296 & 13.1 & 1.30 & 340 & -- & -- & -- & - & -- \\
\hline 09A & 306 & 13.3 & 1.26 & 377 & 3.36 & 20 & 10.3 & 7.4 & 194 \\
\hline $10 \mathrm{~B}$ & 341 & 14.1 & 1.45 & 603 & 3.67 & 23 & 10.1 & 7.5 & 199 \\
\hline $11 \mathrm{~A}$ & 365 & 14.1 & 1.41 & 469 & 3.95 & 21 & 9.9 & 7.4 & 200 \\
\hline $12 R$ & 388 & 14.8 & 1.37 & 293 & - & - & $\ldots$ & $\ldots$ & $\ldots$ \\
\hline $12 B$ & 394 & 14.9 & 1.40 & 438 & 3.82 & 21 & 9.8 & 7.5 & 206 \\
\hline $13 \mathrm{~A}$ & 430 & 14.7 & 1.36 & 569 & 3.59 & 22 & 9.9 & 7.5 & 210 \\
\hline $14 \mathrm{~B}$ & 451 & 14.5 & 1.43 & 643 & 3.61 & 24 & 9.5 & 7.4 & 223 \\
\hline $15 R$ & 492 & 14.6 & 1.60 & 573 & -- & -- & -- & -- & -- \\
\hline $15 \mathrm{~A}$ & 500 & 14.9 & 1.61 & 156 & 4.47 & 26 & 9.2 & 7.4 & 229 \\
\hline $16 R$ & 505 & 14.9 & 1.59 & 414 & -- & -- & -- & -- & -- \\
\hline $16 \mathrm{~B}$ & 535 & 15.8 & 1.57 & 621 & 4.21 & 27 & 9.3 & 7.5 & 234 \\
\hline $17 \mathrm{~A}$ & 555 & 15.8 & 1.68 & 808 & 5.18 & 26 & 8.8 & 7.3 & 241 \\
\hline $18 \mathrm{~B}$ & 596 & 15.3 & 1.44 & 747 & 4.48 & 31 & 8.8 & 7.4 & 237 \\
\hline $19 A$ & $623^{\circ}$ & 16.2 & 1.57 & 687 & 4.46 & 27 & 8.6 & 7.4 & 250 \\
\hline $20 \mathrm{~B}$ & 650 & 16.3 & 1.65 & 848 & 4.35 & 25 & 8.6 & 7.4 & 254 \\
\hline $21 \mathrm{~A}$ & 686 & 16.4 & 1.45 & 762 & 4.41 & 28 & 10.4 & 7.3 & 249 \\
\hline $22 B$ & 714 & 16.6 & 1.67 & 804 & 4.85 & 30 & 8.4 & 7.5 & 237 \\
\hline $23 \mathrm{~A}$ & 744 & 17.9 & 1.40 & 837 & 4.85 & 30 & 8.4 & 7.4 & 242 \\
\hline $24 B$ & 781 & 18.7 & 1.34 & 879 & 4.39 & 33 & 8.3 & 7.5 & 254 \\
\hline $25 \mathrm{~A}$ & 814 & 18.3 & 1.22 & 760 & 4.41 & 34 & 8.5 & 7.4 & 254 \\
\hline 26B & 849 & 18.3 & 1.49 & 925 & 4.48 & 34 & 9.0 & 7.5 & 266 \\
\hline $27 \mathrm{~A}$ & 882 & 18.3 & 1.58 & 837 & 5.21 & 34 & 8.4 & 7.3 & 265 \\
\hline $28 B$ & 907 & 17.8 & 1.52 & 878 & 4.39 & 33 & 8.4 & 7.4 & 248 \\
\hline $29 A$ & 947 & 18.4 & 1.38 & 901 & 4.47 & 35 & 8.3 & 7.5 & 235 \\
\hline $30 \mathrm{~B}$ & 978 & 13.6 & 1.25 & 597 & 2.91 & 17 & 8.4 & 7.3 & 255 \\
\hline 31 & 1,017 & 5.6 & 0.65 & 168 & - & 4 & 8.3 & 7.3 & 263 \\
\hline REW & 1,070 & 0.0 & 0.00 & 0 & -- & -- & -- & -- & -- \\
\hline MEAN & & 13.7 & 1.39 & & & & & & \\
\hline TOTAL & 1,070 & & & 20,417 & 112.89 & 720 & & & \\
\hline
\end{tabular}




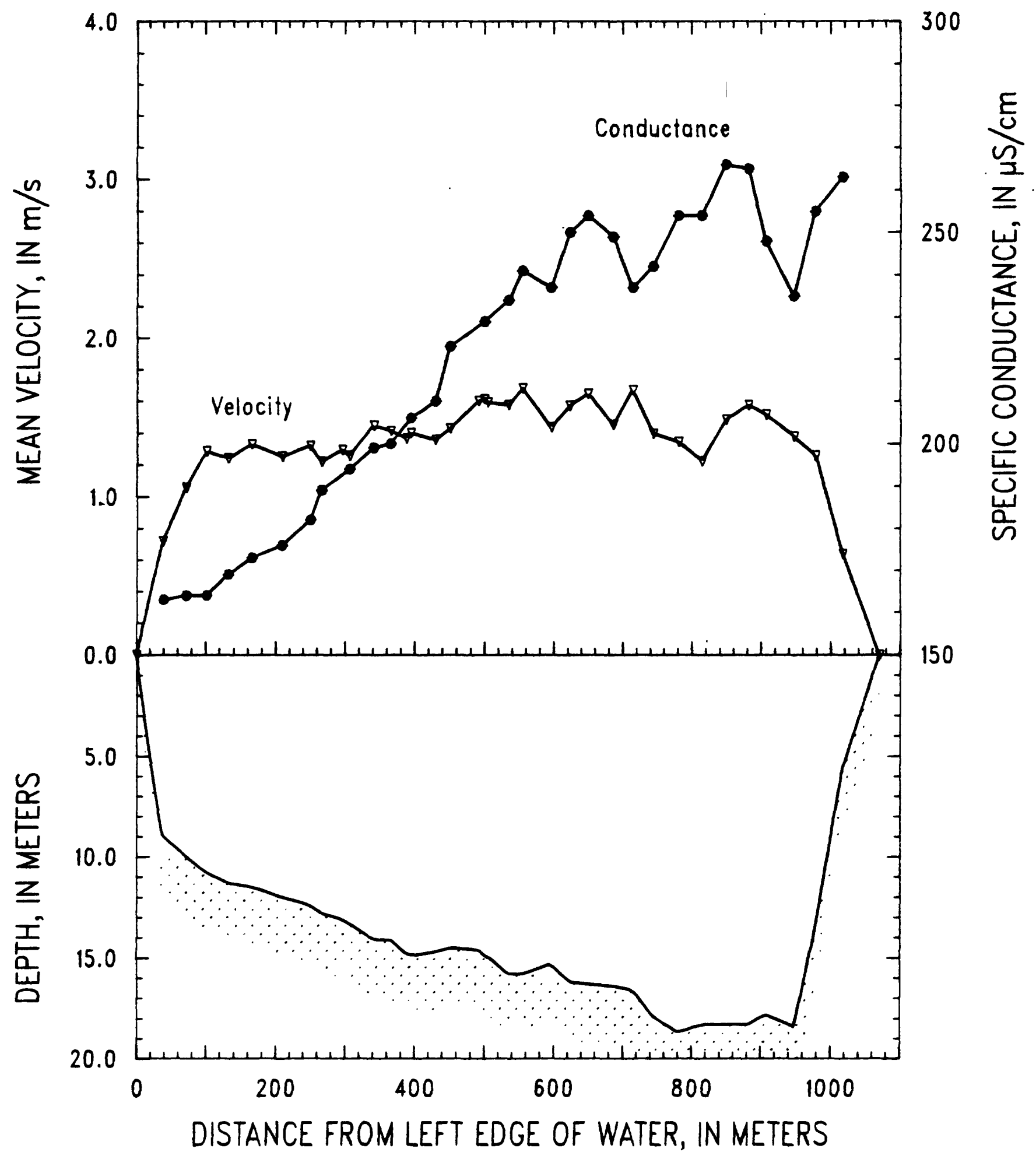

Figure 15. Ohio River at Olmsted, Illinois, on March 16, 1989. 
SITE: Mississippi River below Hickman, Kentucky

PARTY: Moody, Stevens, and Simoneaux

03-17-89

STARTING GAGE HEIGHT: $35.33 \mathrm{ft}$ at Hickman Harbor gage

SUSP: Bag sampler and 300-1b weight. Current meter $32 \mathrm{~cm}$ above nozzle.

CURRENT METER NO.: P8308282 DATE RATED: 07-08-88

CURRENT METER EQUATION: $V(\mathrm{~m} / \mathrm{s})=$ RPS $* 0.765+0.006$

REMARKS: Transit rate was $17 \mathrm{~cm} / \mathrm{s}$ and 3/16-inch nozzle. Verticals were occupied in in order 1-30. Windy. B-52 buzzed ship.

\begin{tabular}{|c|c|c|c|c|c|c|c|c|c|}
\hline \multirow[b]{2}{*}{$\begin{array}{c}\text { Verti- } \\
\text { cal }\end{array}$} & \multirow{2}{*}{$\begin{array}{l}\text { Dist. } \\
\text { from } \\
\text { LEW } \\
\text { (m) }\end{array}$} & \multirow[b]{2}{*}{$\begin{array}{l}\text { Depth } \\
\text { (m) }\end{array}$} & \multirow{2}{*}{$\begin{array}{l}\text { Mean } \\
\text { veloc- } \\
\text { ity } \\
(\mathrm{m} / \mathrm{s})\end{array}$} & \multirow[b]{2}{*}{$\begin{array}{r}\text { Dis- } \\
\text { charge } \\
\left(\mathrm{m}^{3} / \mathrm{s}\right)\end{array}$} & \multicolumn{2}{|c|}{ Volume } & \multirow[b]{2}{*}{$\begin{array}{l}\text { Temper- } \\
\text { ature } \\
\left({ }^{\circ} \mathrm{C}\right)\end{array}$} & \multirow[b]{2}{*}{$\mathrm{pH}$} & \multirow{2}{*}{$\begin{array}{c}\text { Specific } \\
\text { conduct- } \\
\text { ance } \\
(\mu \mathrm{S} / \mathrm{cm})\end{array}$} \\
\hline & & & & & $\begin{array}{l}\overline{V_{i}} \\
(L)\end{array}$ & $\begin{array}{c}\overline{V_{p}} \\
(\mathrm{~L})\end{array}$ & & & \\
\hline LEW & 0 & 0.0 & 0.00 & 0 & -- & -- & -- & -- & -- \\
\hline $01 \mathrm{~A}$ & 48 & 12.6 & 0.99 & 562 & 2.15 & 7 & 9.9 & 7.3 & 217 \\
\hline O2B & 90 & 13.4 & 1.71 & 791 & 3.83 & 15 & 10.7 & 7.3 & 207 \\
\hline $03 A$ & 117 & 13.1 & 1.70 & 814 & 3.91 & 18 & 9.7 & 7.3 & 212 \\
\hline 04B & 163 & 13.5 & 1.81 & 975 & 4.37 & 20 & 10.1 & 7.3 & 221 \\
\hline 05A & 197 & 13.5 & 1.87 & 769 & 4.18 & 22 & 9.9 & 7.2 & 222 \\
\hline 06B & 224 & 13.3 & 1.69 & 371 & 3.72 & 23 & 9.9 & 7.2 & 225 \\
\hline X07 & 230 & 13.2 & 1.80 & 487 & -- & -- & - & -- & -- \\
\hline $07 \mathrm{~A}$ & 265 & 13.8 & 1.58 & 763 & 4.66 & 23 & 10.0 & 7.5 & 223 \\
\hline 08B & 300 & 13.2 & 1.39 & 689 & 4.45 & 20 & 10.1 & 7.3 & 223 \\
\hline 09A & 340 & 13.6 & 1.75 & 573 & 3.73 & 18 & 9.8 & 7.4 & 234 \\
\hline $\mathrm{X} 02$ & 348 & 13.5 & 1.88 & 560 & -- & -- & -- & -- & -- \\
\hline $10 \mathrm{~B}$ & 384 & 12.4 & 1.81 & 786 & 3.61 & 16 & 9.8 & 7.4 & 245 \\
\hline $11 \mathrm{~A}$ & 418 & 12.0 & 1.60 & 671 & 3.55 & 17 & 10.0 & 7.4 & 233 \\
\hline $12 B$ & 454 & 11.6 & 1.84 & 928 & 3.80 & 14 & 10.2 & 7.5 & 237 \\
\hline $13 A$ & 505 & 11.6 & 1.69 & 725 & 3.47 & 15 & 9.6 & 7.5 & 254 \\
\hline $14 \mathrm{~B}$ & 528 & 11.2 & 1.85 & 705 & 3.52 & 16 & 9.5 & 7.6 & 265 \\
\hline $15 \mathrm{~A}$ & 573 & 11.4 & 1.74 & 604 & 3.47 & 15 & 9.5 & 7.6 & 278 \\
\hline $\mathrm{X} 03$ & 589 & 10.6 & 1.90 & 413 & -- & -- & - & -- & -- \\
\hline 16B & 614 & 11.7 & 1.66 & 487 & 4.09 & 16 & 9.7 & 7.6 & 286 \\
\hline $17 \mathrm{~A}$ & 639 & 11.9 & 1.76 & 744 & 4.03 & 16 & 10.1 & 7.6 & 291 \\
\hline $18 \mathrm{~B}$ & 685 & 12.7 & 2.00 & 1,067 & 4.39 & 16 & 9.7 & 7.5 & 293 \\
\hline 19A & 723 & 13.4 & 2.04 & 970 & 5.36 & 17 & 9.3 & 7.7 & 300 \\
\hline $20 \mathrm{~B}$ & 756 & 13.8 & 2.05 & 594 & 4.78 & 20 & 9.5 & 7.7 & 305 \\
\hline $20 \mathrm{R}$ & 765 & 13.8 & 1.98 & 382 & -- & -- & -- & - & -- \\
\hline X04 & 784 & 13.8 & 2.02 & 558 & -- & -- & -- & -- & -- \\
\hline $21 \mathrm{~A}$ & 805 & 14.9 & 1.95 & 407 & 4.89 & 19 & 10.0 & 7.8 & 329 \\
\hline $21 R$ & 812 & 13.9 & 1.89 & 368 & -- & -- & -- & -- & -- \\
\hline $22 B$ & 833 & 13.5 & 1.99 & 698 & 4.85 & 18 & 10.0 & 7.8 & 346 \\
\hline $23 A$ & 864 & 13.0 & 1.88 & 980 & 4.19 & 18 & 8.8 & 7.9 & 364 \\
\hline $24 B$ & 913 & 14.5 & 1.64 & 832 & 4.27 & 16 & 8.9 & 7.9 & 356 \\
\hline X06 & 934 & 14.9 & 1.49 & 311 & -- & -- & - & .- & -- \\
\hline $25 A$ & 941 & 15.4 & 1.55 & 429 & 3.92 & 17 & 9.4 & 7.9 & 374 \\
\hline 26B & 970 & 16.3 & 1.31 & 599 & 3.73 & 19 & 9.2 & 7.9 & 381 \\
\hline X05 & 997 & 16.1 & 1.43 & 413 & - & -- & -- & -- & - \\
\hline $27 \mathrm{~A}$ & 1,006 & 16.2 & 1.25 & 365 & 3.68 & 19 & 9.1 & 7.9 & 379 \\
\hline
\end{tabular}


SITE: Mississippi River below Hickman, Kentucky--continued

\begin{tabular}{|c|c|c|c|c|c|c|c|c|c|}
\hline \multirow[b]{2}{*}{$\begin{array}{c}\text { Verti- } \\
\text { cal }\end{array}$} & \multirow{2}{*}{$\begin{array}{l}\text { Dist. } \\
\text { from } \\
\text { LEW } \\
\text { (m) }\end{array}$} & \multirow[b]{2}{*}{$\begin{array}{l}\text { Depth } \\
\text { (m) }\end{array}$} & \multirow{2}{*}{$\begin{array}{l}\text { Mean } \\
\text { veloc- } \\
\text { ity } \\
(\mathrm{m} / \mathrm{s})\end{array}$} & \multirow[b]{2}{*}{$\begin{array}{r}\text { Dis - } \\
\text { charge } \\
\left(\mathrm{m}^{3} / \mathrm{s}\right)\end{array}$} & \multicolumn{2}{|c|}{ Volume } & \multirow[b]{2}{*}{$\begin{array}{l}\text { Temper- } \\
\text { ature } \\
\left({ }^{\circ} \mathrm{C}\right)\end{array}$} & \multirow[b]{2}{*}{$\mathrm{pH}$} & \multirow{2}{*}{$\begin{array}{c}\text { Specific } \\
\text { conduct- } \\
\text { ance } \\
(\mu \mathrm{S} / \mathrm{cm})\end{array}$} \\
\hline & & & & & $\begin{array}{l}\bar{V}_{i} \\
(L)\end{array}$ & $\begin{array}{l}v_{p} \\
(L)\end{array}$ & & & \\
\hline $28 B$ & 1,033 & 16.3 & 1.40 & 1,076 & 3.57 & 17 & 9.1 & 8.0 & 386 \\
\hline $29 A$ & 1,100 & 14.4 & 1.10 & 728 & 2.72 & 13 & 9.5 & 8.1 & 386 \\
\hline $30 \mathrm{~B}$ & 1,125 & 14.0 & 1.07 & 488 & 2.08 & 5 & 9.4 & 8.0 & 396 \\
\hline REW & 1,165 & 0.0 & 0.00 & 0 & - & - & - & - & - \\
\hline MEAN & & 12.8 & 1.65 & & & & & & \\
\hline TOTAL & 1,165 & & & 24,682 & 116.97 & 505 & & & \\
\hline
\end{tabular}




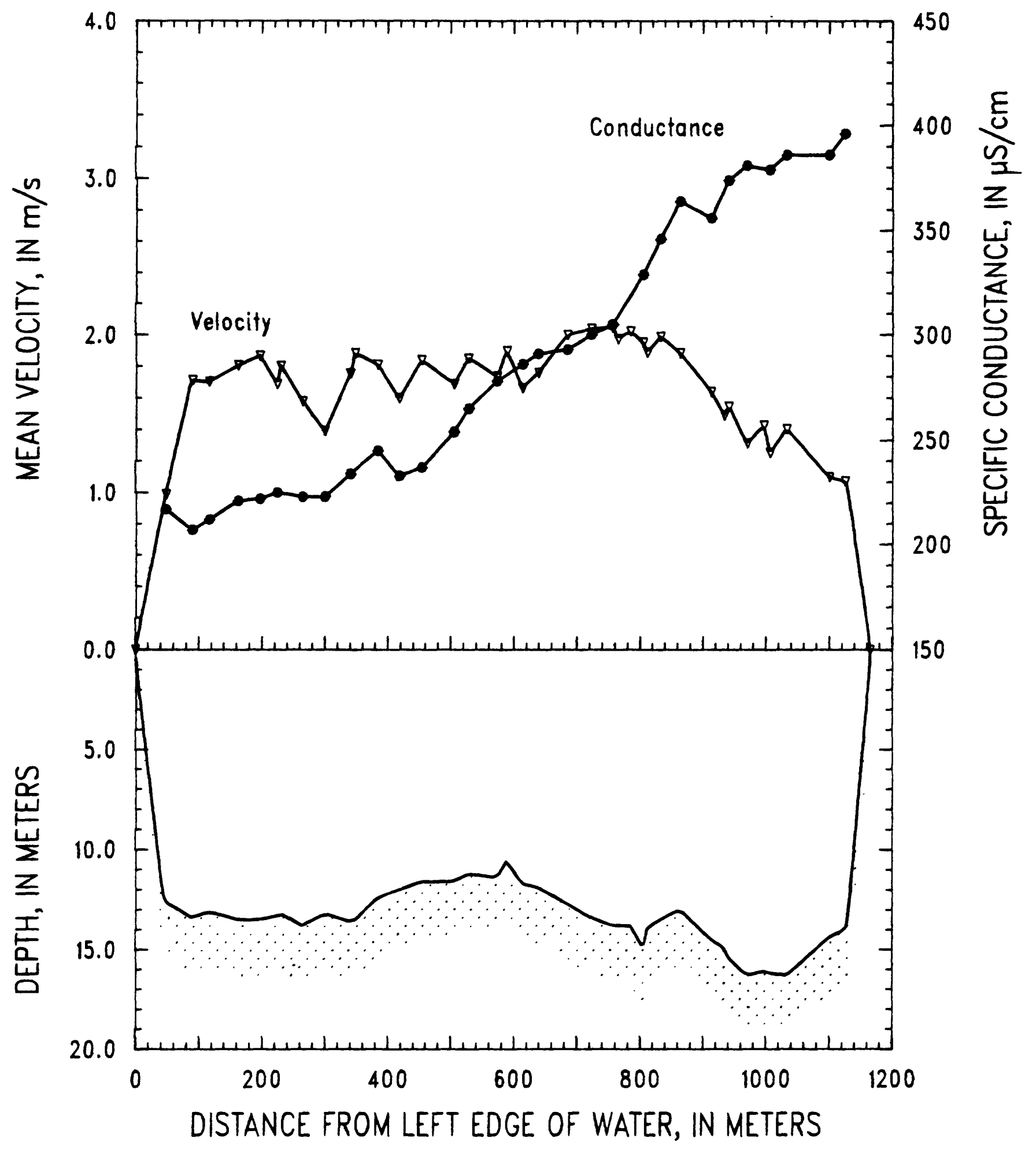

Figure 16. Mississippi River below Hickman, Kentucky, on March 17, 1989 
SITE: Mississippi River below Fulton, Tennessee

METER: $\begin{array}{r}03-19-89 \\ \text { SOLID CUP }\end{array}$

PARTY: Moody, Stevens, and Simoneaux

STARTING GAGE HEIGHT: Stage fell about $0.3 \mathrm{~m}$ during sampling.

SUSP: Bag sampler and 300-lb weight. Current meter $32 \mathrm{~cm}$ above nozzle.

CURRENT METER NO.: P8308282 DATE RATED: 07-08-88

CURRENT METER EQUATION: $V(\mathrm{~m} / \mathrm{s})=$ RPS $* 0.765+0.006$

REMARKS: Transit rate was $17 \mathrm{~cm} / \mathrm{s}$ and the nozzle was $3 / 16$ inch.

Verticals were occupied in reverse order (30-1) for the integrated sample. Site was below Sunrise Towhead but above Hatchie River.

\begin{tabular}{|c|c|c|c|c|c|c|c|c|c|}
\hline \multirow[b]{2}{*}{$\begin{array}{c}\text { Verti- } \\
\text { cal }\end{array}$} & \multirow{2}{*}{$\begin{array}{l}\text { Dist. } \\
\text { from } \\
\text { LEW } \\
\text { (m) }\end{array}$} & \multirow[b]{2}{*}{$\begin{array}{l}\text { Depth } \\
\text { (m) }\end{array}$} & \multirow{2}{*}{$\begin{array}{l}\text { Mean } \\
\text { veloc- } \\
\text { ity } \\
(\mathrm{m} / \mathrm{s})\end{array}$} & \multirow[b]{2}{*}{$\begin{array}{r}\text { Dis- } \\
\text { charge } \\
\left(\mathrm{m}^{3} / \mathrm{s}\right)\end{array}$} & \multicolumn{2}{|c|}{ Volume } & \multirow[b]{2}{*}{$\begin{array}{l}\text { Temper- } \\
\text { ature } \\
\left({ }^{\circ} \mathrm{C}\right)\end{array}$} & \multirow[b]{2}{*}{$\mathrm{pH}$} & \multirow{2}{*}{$\begin{array}{c}\text { Specific } \\
\text { conduct- } \\
\text { ance } \\
(\mu \mathrm{S} / \mathrm{cm})\end{array}$} \\
\hline & & & & & $\begin{array}{l}\overline{V_{i}} \\
(\mathrm{~L})\end{array}$ & $\begin{array}{l}v_{p} \\
(L)\end{array}$ & & & \\
\hline LEW & 0 & 0.0 & 0.00 & 0 & -- & -- & -- & -- & -- \\
\hline $01 \mathrm{~A}$ & 45 & 7.1 & 0.57 & 173 & 0.62 & 13 & 9.5 & 7.8 & 287 \\
\hline $02 \mathrm{~B}$ & 86 & 10.7 & 0.90 & 371 & 1.62 & 11 & 9.3 & 7.7 & 283 \\
\hline 03A & 122 & 11.3 & 0.95 & 495 & 1.58 & 12 & 9.1 & 7.8 & 287 \\
\hline 04B & 178 & 11.1 & 1.13 & 565 & 1.65 & 13 & 9.3 & 7.8 & 284 \\
\hline $05 \mathrm{~A}$ & 212 & 11.5 & 1.13 & 506 & 2.31 & 14 & 9.5 & 7.8 & 288 \\
\hline 06B & 256 & 12.0 & 1.21 & 639 & 2.50 & 14 & 9.4 & 7.8 & 288 \\
\hline $07 \mathrm{~A}$ & 300 & 11.9 & 1.33 & 696 & 2.63 & 16 & 9.4 & 7.8 & 288 \\
\hline $08 \mathrm{R}$ & 344 & 11.8 & 1.31 & 579 & 2.56 & 16 & 9.4 & 7.9 & 289 \\
\hline $09 A$ & 375 & 11.9 & 1.30 & 293 & 2.66 & 16 & 9.4 & 7.7 & 290 \\
\hline X09 & 382 & 12.1 & 1.31 & 357 & -- & -- & -- & -- & - \\
\hline $10 \mathrm{~B}$ & 420 & 11.7 & 1.48 & 693 & 3.04 & 18 & 9.3 & 7.7 & 289 \\
\hline $11 \mathrm{~A}$ & 462 & 11.8 & 1.45 & 781 & 2.69 & 18 & 9.4 & 7.7 & 283 \\
\hline $12 B$ & 511 & 11.7 & 1.36 & 797 & 2.80 & 18 & 9.5 & 7.7 & 288 \\
\hline $13 \mathrm{~A}$ & 562 & 11.7 & 1.51 & 644 & 3.05 & 18 & 9.7 & 7.7 & 290 \\
\hline $14 \mathrm{~B}$ & 584 & 11.6 & 1.53 & 513 & 3.08 & 16 & 9.5 & 7.7 & 290 \\
\hline $15 \mathrm{~A}$ & 620 & 11.0 & 1.44 & 397 & 2.90 & 16 & 9.5 & 7.7 & 290 \\
\hline $\mathrm{X} 08$ & 634 & 10.5 & 1.61 & 177 & -- & - & - & - & -. \\
\hline $\mathrm{X} 07$ & 641 & 11.3 & 1.55 & 262 & -. & -- & -- & -- & -- \\
\hline $16 \mathrm{~B}$ & 664 & 11.2 & 1.62 & 661 & 2.97 & 18 & 9.5 & 7.7 & 286 \\
\hline $17 \mathrm{~A}$ & 714 & 11.2 & 1.71 & 861 & 3.87 & 21 & 9.4 & 7.7 & 290 \\
\hline $18 \mathrm{~B}$ & 754 & 11.7 & 1.78 & 616 & 3.71 & 23 & 9.4 & 7.8 & 289 \\
\hline $18 \mathrm{R}$ & 773 & 11.5 & 1.82 & 428 & - & $\ldots$ & - & -- & - \\
\hline $19 \mathrm{~A}$ & 795 & 11.5 & 1.92 & 474 & 4.27 & 25 & 9.3 & 7.7 & 286 \\
\hline $\mathrm{X} 02$ & 816 & 11.4 & 1.91 & 576 & - & -- & - & - & - \\
\hline 20B & 848 & 11.7 & 1.94 & 841 & 4.46 & 23 & 9.4 & 7.7 & 288 \\
\hline $\mathrm{X} 06$ & 890 & 12.1 & 1.98 & 648 & -- & -- & - & -- & -- \\
\hline $21 \mathrm{~A}$ & 902 & 12.0 & 2.07 & 558 & 4.36 & 23 & 9.5 & 7.7 & 291 \\
\hline $22 B$ & 935 & 12.4 & 1.93 & 861 & 4.79 & 24 & 9.2 & 7.6 & 291 \\
\hline $23 \mathrm{~A}$ & 974 & 13.5 & 1.88 & 1,240 & 4.64 & 26 & 9.3 & 7.6 & 287 \\
\hline $24 B$ & 1,033 & 15.0 & 1.88 & 1,577 & 4.98 & 26 & 9.5 & 7.7 & 295 \\
\hline $25 R$ & 1,086 & 15.7 & 1.73 & 1,249 & -- & -- & -- & - & - \\
\hline $\mathrm{X} 01$ & 1,125 & 17.0 & 1.88 & 784 & 5.48 & 29 & 9.3 & 7.7 & 285 \\
\hline $26 \mathrm{~B}$ & 1,135 & 16.8 & 1.85 & 450 & 5.68 & 31 & 9.3 & 7.7 & 292 \\
\hline
\end{tabular}


SITE: Mississippi River below Fulton, Tennessee--continued

\begin{tabular}{|c|c|c|c|c|c|c|c|c|c|}
\hline \multirow[b]{2}{*}{$\begin{array}{c}\text { Verti- } \\
\text { cal }\end{array}$} & \multirow{2}{*}{$\begin{array}{l}\text { Dist. } \\
\text { from } \\
\text { LEW } \\
\text { (m) }\end{array}$} & \multirow[b]{2}{*}{$\begin{array}{l}\text { Depth } \\
\text { (m) }\end{array}$} & \multirow{2}{*}{$\begin{array}{l}\text { Mean } \\
\text { veloc- } \\
\text { ity } \\
(\mathrm{m} / \mathrm{s})\end{array}$} & \multirow{2}{*}{$\begin{array}{r}\text { Dis- } \\
\text { charge } \\
\left(\mathrm{m}^{3} / \mathrm{s}\right)\end{array}$} & \multicolumn{2}{|c|}{ Volume } & \multirow[b]{2}{*}{$\begin{array}{l}\text { Temper- } \\
\text { ature } \\
\left({ }^{\circ} \mathrm{C}\right)\end{array}$} & \multirow[b]{2}{*}{$\mathrm{pH}$} & \multirow{2}{*}{$\begin{array}{c}\text { Specific } \\
\text { conduct- } \\
\text { ance } \\
(\mu \mathrm{S} / \mathrm{cm})\end{array}$} \\
\hline & & & & & $\begin{array}{l}\overline{\mathbf{v}_{\mathbf{i}}} \\
(\mathrm{L})\end{array}$ & $\begin{array}{l}v_{p} \\
(L)\end{array}$ & & & \\
\hline $27 \mathrm{~A}$ & 1,154 & 17.2 & 1.69 & 947 & 4.91 & 34 & 9.3 & 7.7 & 295 \\
\hline $28 \mathrm{~B}$ & 1,200 & 18.0 & 1.57 & 1,318 & 5.18 & 35 & 9.3 & 7.7 & 295 \\
\hline $29 \mathrm{~A}$ & 1,247 & 18.2 & 1.51 & 1,345 & 4.51 & 33 & 9.1 & 7.7 & 295 \\
\hline $30 \mathrm{~B}$ & 1,298 & 9.7 & 1.00 & 439 & 1.84 & 6 & 9.3 & 7.7 & 301 \\
\hline REW & 1,337 & 0.0 & 0.00 & 0 & -- & -- & -- & -- & -- \\
\hline MEAN & & 12.1 & 1.53 & & & & & & \\
\hline TOTAL & 1,337 & & & 24,811 & 101.34 & 606 & & & \\
\hline
\end{tabular}




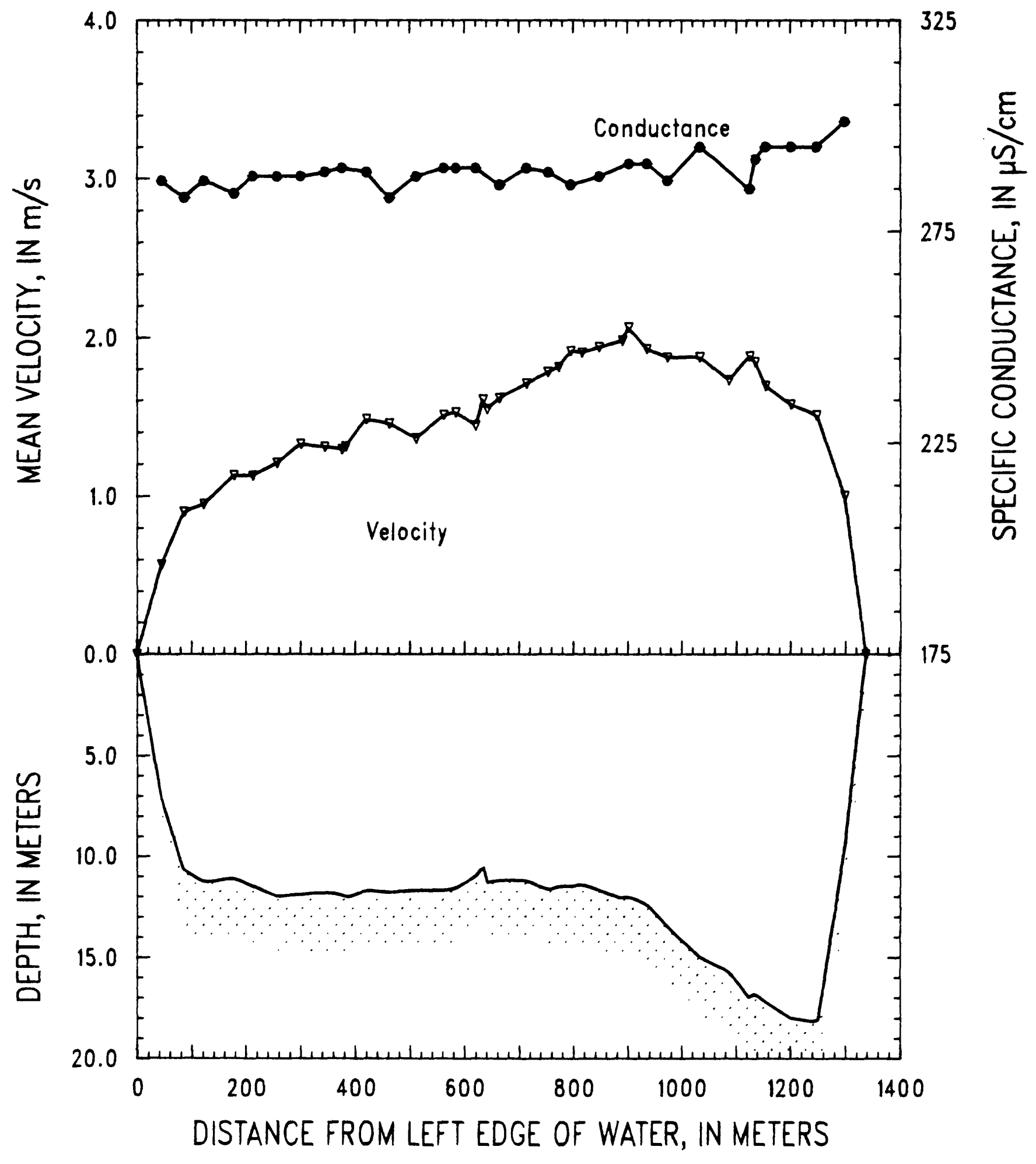

Figure 17. Mississippi River below Fulton, Tennessee, on March 19, 1989 
SITE: Mississippi River at Helena, Arkansas

PARTY: Moody, Stevens, and Black

03-21-89

STARTING GAGE HEIGHT: $33.35 \mathrm{ft}$ ENDING GAGE HEIGHT: $32.78 \mathrm{ft}$

SUSP: Bag sampler and 300-lb weight. Current meter $32 \mathrm{~cm}$ above nozzle.

CURRENT METER NO.: P8308282 DATE RATED: 07-08-88

CURRENT METER EQUATION: $V(\mathrm{~m} / \mathrm{s})=$ RPS $* 0.765+0.006$

REMARKS: Transit rate was $22 \mathrm{~cm} / \mathrm{s}$ and nozzle was $3 / 16$ inch. Verticals occupied from 30-1. Greatest velocities are not located in deepest water.

Sand transport may be visible on depth recorder, especially at vertical 13 .

\begin{tabular}{|c|c|c|c|c|c|c|c|c|c|}
\hline \multirow[b]{2}{*}{$\begin{array}{l}\text { Verti- } \\
\text { cal }\end{array}$} & \multirow{2}{*}{$\begin{array}{l}\text { Dist. } \\
\text { from } \\
\text { LEW } \\
\text { (m) }\end{array}$} & \multirow[b]{2}{*}{$\begin{array}{c}\text { Depth } \\
(\mathrm{m})\end{array}$} & \multirow{2}{*}{$\begin{array}{l}\text { Mean } \\
\text { veloc- } \\
\text { ity } \\
(\mathrm{m} / \mathrm{s})\end{array}$} & \multirow[b]{2}{*}{$\begin{array}{r}\text { Dis- } \\
\text { charge } \\
\left(m^{3} / s\right)\end{array}$} & \multicolumn{2}{|c|}{ Volume } & \multirow[b]{2}{*}{$\begin{array}{c}\text { Temper- } \\
\text { ature } \\
\left({ }^{\circ} \mathrm{C}\right)\end{array}$} & \multirow[b]{2}{*}{$\mathrm{pH}$} & \multirow{2}{*}{$\begin{array}{l}\text { Specific } \\
\text { conduct- } \\
\text { ance } \\
(\mu S / \mathrm{cm})\end{array}$} \\
\hline & & & & & $\begin{array}{l}\overline{v_{i}} \\
(L)\end{array}$ & $\begin{array}{l}\bar{v}_{p} \\
(\mathrm{~L})\end{array}$ & & & \\
\hline LEW & 0 & 0.0 & 0.00 & -- & -- & -- & -- & -- & -- \\
\hline $01 \mathrm{~A}$ & 34 & 7.2 & 0.66 & 129 & 0.78 & 3 & 9.2 & 7.5 & 295 \\
\hline $02 B$ & 54 & 11.7 & 1.12 & 314 & 1.73 & 9 & 9.1 & 7.5 & 296 \\
\hline $03 \mathrm{~A}$ & 82 & 12.9 & 1.76 & 409 & 3.48 & 9 & 9.2 & 7.5 & 292 \\
\hline X04 & 90 & 12.6 & 1.92 & 423 & -- & -- & -- & -- & -- \\
\hline 04B & 117 & 13.3 & 2.03 & 580 & 3.72 & 11 & 9.1 & 7.5 & 296 \\
\hline $05 A$ & 133 & 13.8 & 1.94 & 749 & 4.95 & 12 & 9.2 & 7.5 & 296 \\
\hline $06 \mathrm{~B}$ & 173 & 15.0 & 2.28 & 1,178 & 4.83 & 13 & 9.2 & 7.5 & 292 \\
\hline $07 \mathrm{~A}$ & 202 & 15.6 & 2.13 & 1,232 & 4.98 & 15 & 9.1 & 7.6 & 295 \\
\hline 08B & 247 & 16.8 & 2.03 & 921 & 5.38 & 15 & 9.1 & 7.5 & 296 \\
\hline 09A & 256 & 16.9 & 2.11 & 624 & 4.43 & 16 & 9.0 & 7.5 & 297 \\
\hline $10 \mathrm{~B}$ & 282 & 17.8 & 2.09 & 1,099 & 5.31 & 18 & 9.0 & 7.5 & 296 \\
\hline $11 \mathrm{~A}$ & 315 & 18.1 & 2.04 & 1,347 & 5.92 & 18 & 9.2 & 7.5 & 290 \\
\hline $12 B$ & 355 & 18.8 & 2.24 & 1,202 & 5.60 & 19 & 9.1 & 7.5 & 291 \\
\hline $13 \mathrm{~A}$ & 372 & 18.8 & 2.27 & 769 & 6.86 & 19 & 9.0 & 7.5 & 291 \\
\hline $13 R$ & 391 & 18.7 & 2.30 & 924 & - & -- & - & - & - \\
\hline $14 \mathrm{~B}$ & 415 & 18.8 & 2.14 & 805 & 6.08 & 20 & 9.1 & 7.5 & 295 \\
\hline $14 R$ & 431 & 19.5 & 2.15 & 714 & -- & -- & -- & -- & -- \\
\hline $15 \mathrm{~A}$ & 449 & 20.0 & 2.16 & 843 & 5.33 & 22 & 9.1 & 7.5 & 285 \\
\hline $16 \mathrm{~B}$ & 470 & 19.6 & 1.93 & 831 & 5.39 & 20 & 9.2 & 7.5 & 289 \\
\hline $17 \mathrm{~A}$ & 493 & 19.2 & 1.64 & 900 & 5.38 & 19 & 9.2 & 7.5 & 291 \\
\hline $18 \mathrm{~B}$ & 527 & 20.2 & 1.58 & 1,289 & 4.11 & 21 & 9.0 & 7.5 & 291 \\
\hline $19 \mathrm{~A}$ & 574 & 20.3 & 1.38 & 801 & 3.64 & 21 & 9.3 & 7.5 & 279 \\
\hline $20 \mathrm{R}$ & 584 & 20.2 & 1.45 & 411 & -- & -- & -- & - & -- \\
\hline $20 \mathrm{~B}$ & 602 & 20.3 & 1.43 & 391 & 4.29 & 22 & 9.2 & 7.5 & 291 \\
\hline $21 \mathrm{~A}$ & 611 & 20.7 & 1.42 & 791 & 4.27 & 24 & 9.3 & 7.4 & 284 \\
\hline $22 B$ & 656 & 23.6 & 1.37 & 969 & 4.50 & 27 & 9.3 & 7.5 & 277 \\
\hline $23 \mathrm{~A}$ & 671 & 24.1 & 1.47 & 477 & 4.87 & 31 & 9.2 & 7.4 & 265 \\
\hline $24 B$ & 683 & 25.1 & 1.39 & 735 & 4.33 & 30 & 9.5 & 7.4 & 274 \\
\hline $25 \mathrm{~A}$ & 713 & 24.6 & 1.36 & 1,224 & 4.42 & 26 & 9.4 & 7.5 & 260 \\
\hline $26 B$ & 756 & 20.0 & 1.44 & 1,167 & 3.63 & 12 & 9.6 & 7.3 & 262 \\
\hline $27 R$ & 794 & 12.2 & 1.40 & 589 & 2.72 & 9 & 9.5 & 7.4 & 240 \\
\hline $28 B$ & 825 & 11.5 & 1.50 & 484 & 2.30 & 8 & 9.4 & 7.4 & 253 \\
\hline $29 \mathrm{~A}$ & 850 & 10.6 & 1.33 & 361 & 1.98 & 6 & 9.5 & 7.4 & 243 \\
\hline $30 \mathrm{~B}$ & 876 & 10.1 & 0.68 & 215 & 0.88 & 4 & 9.5 & 7.5 & 251 \\
\hline REW & 913 & 0.0 & 0.00 & 0 & -- & -- & -- & -- & -- \\
\hline MEAN & & 16.5 & 1.72 & & & & & & \\
\hline TOTAL & 913 & & & 25,897 & 126.09 & 499 & & & \\
\hline
\end{tabular}




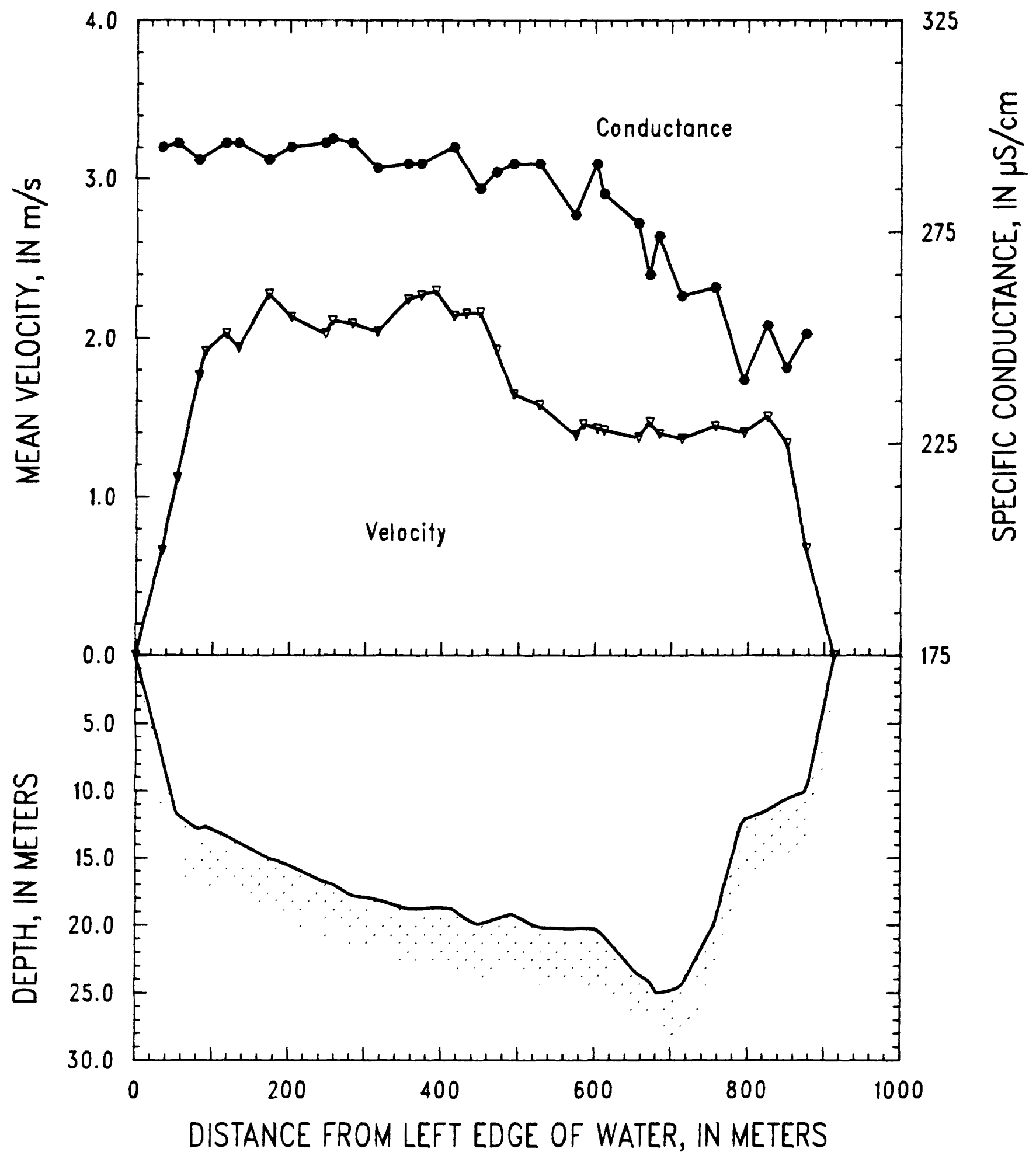

Figure 18. Mississippi River at Helena, Arkansas, on March 21, 1989. 
SITE: White River at Mile 11.5, Arkansas

03-22-89

PARTY: Moody, Stevens, and Black

METER: SOLID CUP

STARTING GAGE HEIGHT: -- ENDING GAGE HEIGHT: --

SUSP: Bag sampler and 150-1b weight. Current meter $32 \mathrm{~cm}$ above nozzle.

CURRENT METER NO.: P8308282 DATE RATED: 07-08-88

CURRENT METER EQUATION: $V(\mathrm{~m} / \mathrm{s})=$ RPS $* 0.765+0.006$

REMARKS: Transit rate was $6 \mathrm{~cm} / \mathrm{s}$ and nozzle was $1 / 4$ inch. Water is over the bank and 2-3 m deep.

\begin{tabular}{|c|c|c|c|c|c|c|c|c|c|}
\hline \multirow[b]{2}{*}{$\begin{array}{c}\text { Verti- } \\
\text { cal }\end{array}$} & \multirow{2}{*}{$\begin{array}{l}\text { Dist. } \\
\text { from } \\
\text { LEF } \\
\text { (m) }\end{array}$} & \multirow[b]{2}{*}{$\begin{array}{c}\text { Depth } \\
(\mathrm{m})\end{array}$} & \multirow{2}{*}{$\begin{array}{l}\text { Mean } \\
\text { veloc- } \\
\text { ity } \\
(\mathrm{m} / \mathrm{s})\end{array}$} & \multirow[b]{2}{*}{$\begin{array}{r}\text { Dis- } \\
\text { charge } \\
\left(\mathrm{m}^{3} / \mathrm{s}\right)\end{array}$} & \multicolumn{2}{|c|}{ Volume } & \multirow[b]{2}{*}{$\begin{array}{l}\text { Temper- } \\
\text { ature } \\
\left({ }^{\circ} \mathrm{C}\right)\end{array}$} & \multirow[b]{2}{*}{$\mathrm{pH}$} & \multirow{2}{*}{$\begin{array}{c}\text { Specific } \\
\text { conduct- } \\
\text { ance } \\
(\mu \mathrm{S} / \mathrm{cm})\end{array}$} \\
\hline & & & & & $\begin{array}{l}\overline{v_{i}} \\
(L)\end{array}$ & $\begin{array}{l}\mathrm{v}_{\mathrm{p}} \\
(\mathrm{L})\end{array}$ & & & \\
\hline LEF & 0 & 3.0 & 0.00 & 0 & $-\infty$ & -- & -- & $\cdots$ & - \\
\hline 01 & 37 & 13.3 & 0.69 & 346 & 24.88 & 117 & 11.6 & 7.1 & 150 \\
\hline 02 & 75 & 13.4 & 0.85 & 374 & 26.15 & 111 & 11.6 & 7.2 & 152 \\
\hline 03 & 103 & 12.5 & 0.83 & 320 & 24.87 & 104 & 11.5 & 7.1 & 151 \\
\hline 04 & 137 & 11.7 & 0.76 & 302 & 21.22 & 91 & 11.5 & 7.1 & 150 \\
\hline 05 & 171 & 10.5 & 0.50 & 165 & 11.47 & 77 & 11.5 & 7.0 & 152 \\
\hline REF & 200 & 2.0 & 0.00 & 0 & -- & $\cdots$ & -- & -- & -- \\
\hline MEAN & & 10.3 & 0.73 & & & & & & \\
\hline TOTAL & 200 & & & 1,507 & 108.59 & 500 & & & \\
\hline
\end{tabular}




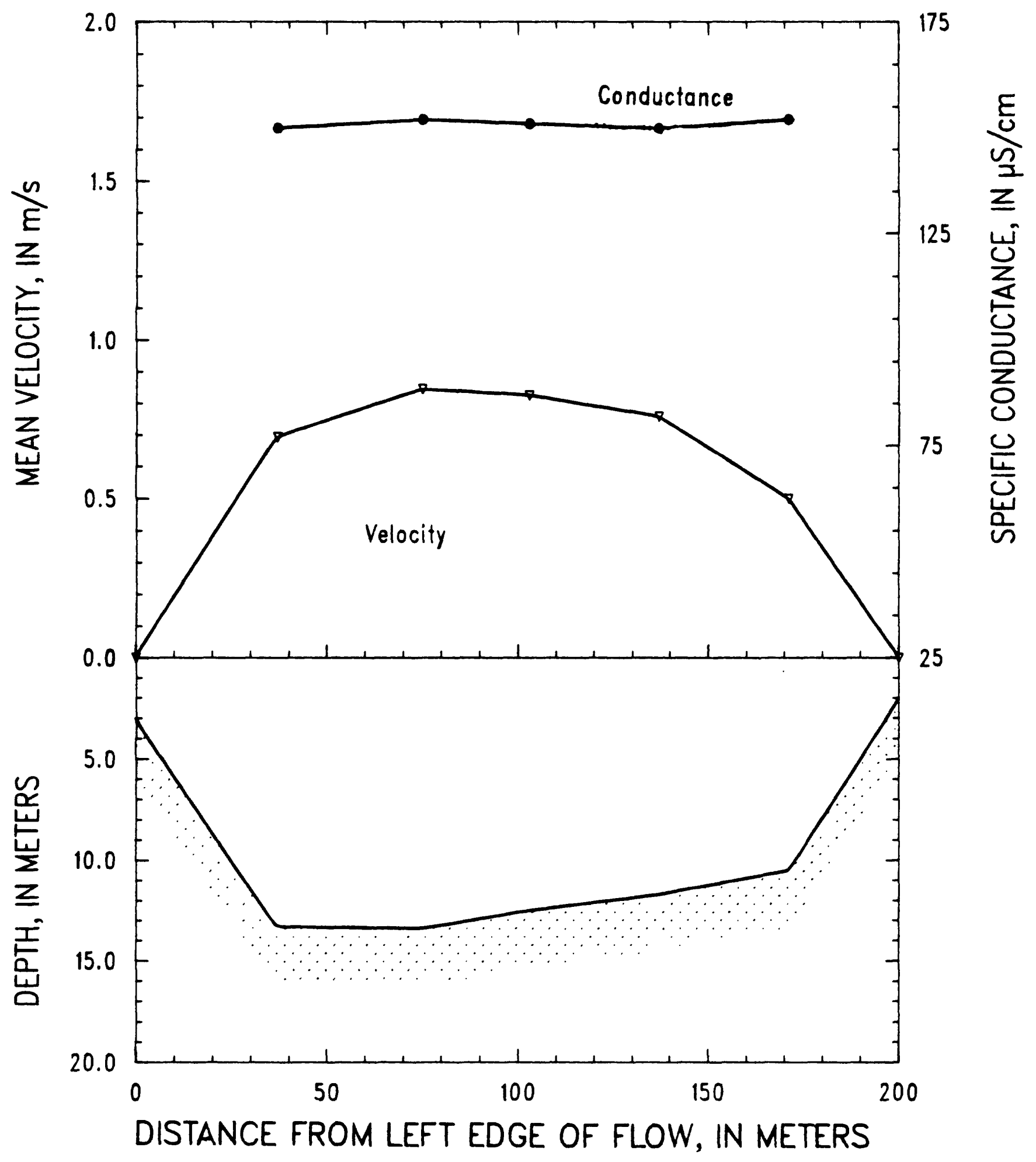

Figure 19. White River at Mile 11.5, Arkansas, on March 22, 1989. 
SITE: Arkansas River at Pendleton, Arkansas

03-23-89

PARTY: Moody, Stevens, and Black

STARTING GAGE HEIGHT: -- ENDING GAGE HEIGHT:

METER: SOLID CUP

SUSP: Bag sampler and 150-1b weight. Current meter $32 \mathrm{~cm}$ above nozzle.

CURRENT METER NO.: P8308282 DATE RATED: 07-08-88

CURRENT METER EQUATION: $V(m / s)=R P S \div 0.765+0.006$

REMARKS: Ten equal discharge verticals. Used various transit rates

$(4-12 \mathrm{~cm} / \mathrm{s})$ and various nozzles $(1 / 4,5 / 16)$. Verticals occupied in reverse order 10-1. Anchoted at verticals.

\begin{tabular}{|c|c|c|c|c|c|c|c|c|c|}
\hline \multirow[b]{2}{*}{$\begin{array}{l}\text { Verti- } \\
\text { cal }\end{array}$} & \multirow{2}{*}{$\begin{array}{l}\text { Dist. } \\
\text { from } \\
\text { LEW } \\
\text { (m) }\end{array}$} & \multirow[b]{2}{*}{$\begin{array}{c}\text { Depth } \\
\text { (m) }\end{array}$} & \multirow{2}{*}{$\begin{array}{l}\text { Mean } \\
\text { veloc- } \\
\text { ity } \\
(\mathrm{m} / \mathrm{s})\end{array}$} & \multirow[b]{2}{*}{$\begin{array}{r}\text { Dis - } \\
\text { charge } \\
\left(\mathrm{m}^{3} / \mathrm{s}\right)\end{array}$} & \multicolumn{2}{|c|}{ Volume } & \multirow[b]{2}{*}{$\begin{array}{l}\text { Temper- } \\
\text { ature } \\
\left({ }^{\circ} \mathrm{C}\right)\end{array}$} & \multirow[b]{2}{*}{$\mathrm{pH}$} & \multirow{2}{*}{$\begin{array}{l}\text { Specific } \\
\text { conduct- } \\
\text { ance } \\
(\mu \mathrm{S} / \mathrm{cm})\end{array}$} \\
\hline & & & & & $\begin{array}{c}\overline{V_{i}} \\
(\mathrm{~L})\end{array}$ & $\begin{array}{l}\mathrm{v}_{\mathrm{p}} \\
(\mathrm{L})\end{array}$ & & & \\
\hline LEW & 0 & 0.0 & 0.00 & 0 & - & -- & -- & -- & -- \\
\hline 01 & 58 & 3.7 & 0.62 & 120 & 12.55 & 60 & 11.8 & 7.9 & 275 \\
\hline X04 & 104 & 5.7 & 0.61 & 149 & -. & -- & - & - & $\ldots$ \\
\hline 02 & 144 & 5.8 & 0.66 & 184 & 10.28 & 60 & 11.9 & 7.9 & 298 \\
\hline 03 & 200 & 6.1 & 0.60 & 196 & 10.33 & 60 & 11.6 & 7.8 & 326 \\
\hline 04 & 250 & 8.2 & 0.82 & 261 & 12.43 & 60 & 11.5 & 8.0 & 341 \\
\hline 05 & 278 & 9.5 & 0.71 & 108 & 11.93 & 60 & 11.6 & 8.0 & 354 \\
\hline X02 & 282 & 10.7 & 0.58 & 89 & - & -- & -- & -- & - \\
\hline 06 & 307 & 14.5 & 0.78 & 225 & 10.73 & 60 & 11.7 & 8.0 & 348 \\
\hline 07 & 322 & 15.2 & 0.82 & 156 & 10.13 & 60 & 11.6 & 7.9 & 350 \\
\hline 08 & 332 & 15.0 & 0.85 & 141 & 11.27 & 60 & 11.5 & 7.9 & 353 \\
\hline 09 & 344 & 15.7 & 0.74 & 99 & 10.69 & 60 & 11.1 & 7.2 & 354 \\
\hline X05 & 349 & 13.5 & 0.74 & 70 & -- & -- & -- & -- & -- \\
\hline 10 & 358 & 9.5 & 0.69 & 102 & 10.38 & 60 & 11.1 & 7.3 & 351 \\
\hline REW & 380 & 0.0 & 0.00 & 0 & -- & -- & -- & -- & -- \\
\hline MEAN & & 7.1 & 0.71 & & & & & & \\
\hline TOTAL & 380 & & & 1,900 & 110.72 & 600 & & & \\
\hline
\end{tabular}




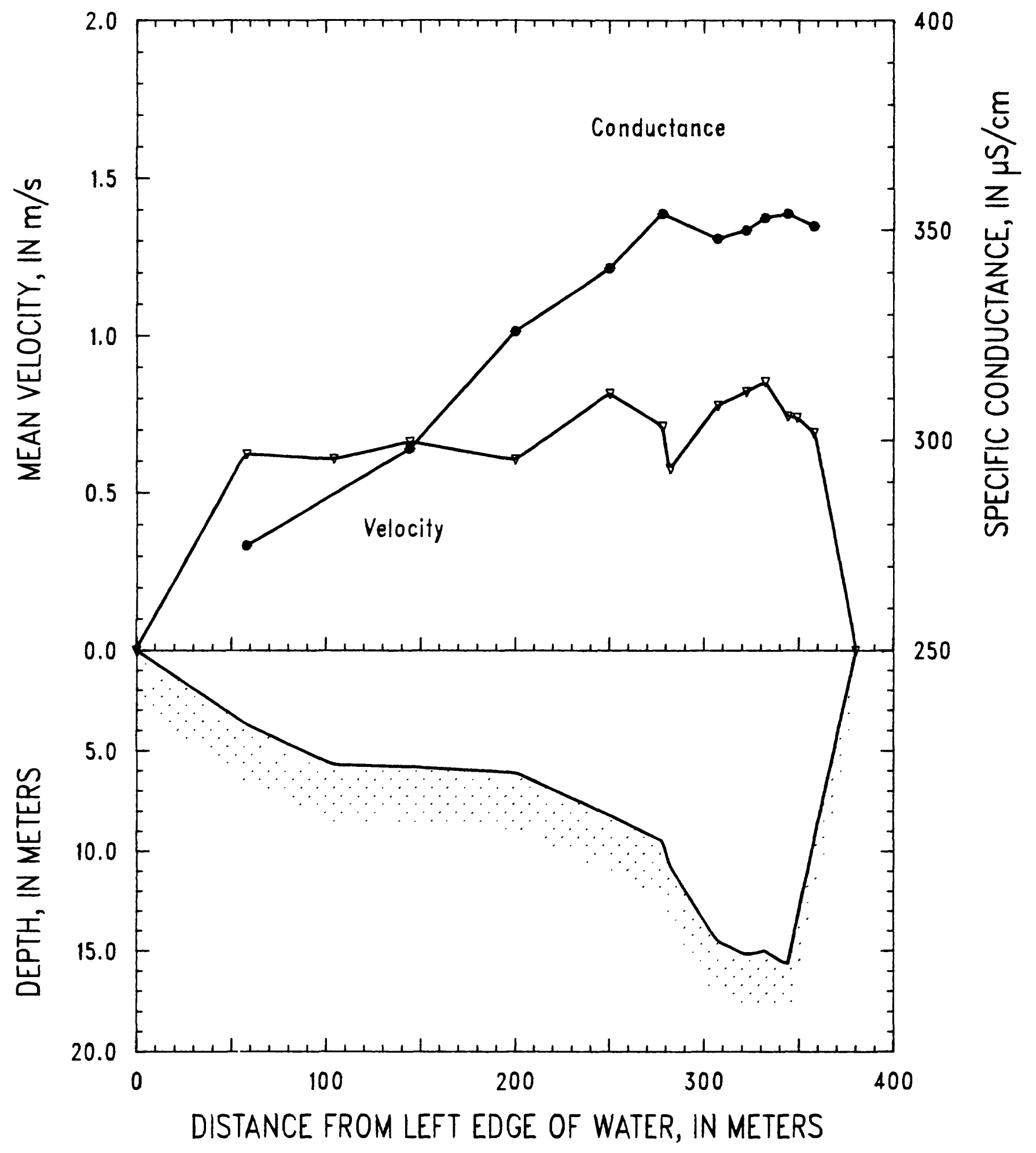

Figure 20. Arkansas River at Pendleton, Arkansas, on March 23, 1989. 
SITE: Mississippi River above Arkansas City, Arkansas

PARTY: Moody, Stevens, and Black

METER: SOLID CUP

STARTING GAGE HEIGHT: $29.23 \mathrm{ft}$ is mean gage height from 0600 to 1200 .

SUSP: Bag sampler and 300-1b weight. Current meter $32 \mathrm{~cm}$ above nozzle.

CURRENT METER NO.: P8308282 DATE RATED: 07-08-88

CURRENT METER EQUATION: $V(\mathrm{~m} / \mathrm{s})=$ RPS $* 0.765+0.006$

REMARKS: Transit rate was $22 \mathrm{~cm} / \mathrm{s}$ and nozzle was $3 / 16$ inch. Velocity was estimated to be $1 \mathrm{~cm} / \mathrm{s}$ in the trees at station 39 . Velocity reported at vertical 17 was the average of verticals 16 and 18 . Narrow band of velocity in center 300 meters. Low velocities on side may explain high mud deposits that exist there.

\begin{tabular}{|c|c|c|c|c|c|c|c|c|c|}
\hline & Dist. & & Mean & & Vol & & & & Specific \\
\hline $\begin{array}{c}\text { Verti- } \\
\text { cal }\end{array}$ & $\begin{array}{l}\text { from } \\
\text { LEW } \\
(\mathrm{m})\end{array}$ & $\begin{array}{l}\text { Depth } \\
\text { (m) }\end{array}$ & $\begin{array}{l}\text { veloc- } \\
\text { ity } \\
(\mathrm{m} / \mathrm{s})\end{array}$ & $\begin{array}{l}\text { Dis- } \\
\text { charge } \\
\left(\mathrm{m}^{3} / \mathrm{s}\right)\end{array}$ & $\begin{array}{l}\overline{V_{i}} \\
(\mathrm{~L})\end{array}$ & $\begin{array}{l}\overline{v_{p}} \\
(\mathrm{~L})\end{array}$ & $\begin{array}{l}\text { Temper- } \\
\text { ature } \\
\left({ }^{\circ} \mathrm{C}\right)\end{array}$ & $\mathrm{pH}$ & $\begin{array}{l}\text { conduct- } \\
\text { ance } \\
(\mu S / c m)\end{array}$ \\
\hline
\end{tabular}

\begin{tabular}{lrrrrrrrrr}
\hline LEW & 0 & 0.0 & 0.00 & 0 & -- & -- & -- & -- & -- \\
EST & 39 & 5.0 & 0.01 & 2 & -- & -- & -- & -- & -- \\
01A & 118 & 6.4 & 0.07 & 22 & 0.19 & 3 & 10.6 & 7.7 & 308 \\
02B & 140 & 8.6 & 0.20 & 56 & 0.25 & 6 & 11.3 & 8.0 & 300 \\
03A & 184 & 11.2 & 0.33 & 132 & 0.43 & 7 & 10.8 & 7.9 & 303 \\
04B & 211 & 13.9 & 0.49 & 158 & 0.70 & 10 & 10.9 & 7.9 & 311 \\
05A & 230 & 15.7 & 0.82 & 161 & 1.45 & 14 & 10.9 & 7.9 & 306 \\
X01 & 236 & 16.1 & 0.92 & 200 & -- & -- & -- & -- & -- \\
06B & 257 & 17.2 & 0.82 & 505 & 1.40 & 16 & 10.5 & 7.9 & 306 \\
07A & 308 & 17.5 & 1.06 & 714 & 2.08 & 16 & 10.5 & 7.9 & 304 \\
08B & 334 & 16.3 & 1.34 & 436 & 3.16 & 16 & 10.9 & 8.0 & 306 \\
09A & 348 & 16.5 & 1.32 & 522 & 3.49 & 15 & 11.0 & 8.0 & 305 \\
X02 & 382 & 16.7 & 1.52 & 522 & -- & -- & -- & -- & -- \\
10B & 389 & 16.6 & 1.53 & 431 & 3.81 & 16 & 11.3 & 8.0 & 305 \\
11A & 416 & 18.5 & 1.53 & 905 & 4.45 & 17 & 10.8 & 8.0 & 303 \\
12B & 453 & 18.2 & 2.00 & 1,255 & 5.43 & 17 & 10.5 & 8.0 & 305 \\
13A & 485 & 19.4 & 2.03 & 1,360 & 6.43 & 19 & 10.4 & 8.0 & 309 \\
14B & 522 & 21.4 & 2.41 & 1,416 & 7.03 & 19 & 10.3 & 8.0 & 304 \\
15A & 540 & 22.6 & 2.53 & 1,228 & 6.74 & 20 & 10.4 & 8.0 & 305 \\
16B & 565 & 22.7 & 2.51 & 1,764 & 5.05 & 25 & 10.7 & 7.9 & 291 \\
17A & 602 & 23.0 & 2.52 & 2,119 & 7.11 & 28 & 10.4 & 7.9 & 300 \\
18B & 638 & 23.9 & 2.34 & 1,372 & 6.98 & 28 & 10.3 & 8.0 & 301 \\
19A & 651 & 22.9 & 2.47 & 1,360 & 6.95 & 28 & 10.5 & 7.9 & 300 \\
20B & 686 & 21.2 & 2.44 & 1,214 & 6.92 & 22 & 10.6 & 7.9 & 302 \\
X04 & 698 & 20.8 & 2.26 & 939 & -- & -- & -- & -- & -- \\
21A & 726 & 20.3 & 2.35 & 906 & 6.91 & 21 & 10.4 & 8.0 & 305 \\
22B & 736 & 19.7 & 2.42 & 1,358 & 6.47 & 19 & 10.5 & 7.9 & 301 \\
23A & 783 & 18.6 & 2.04 & 1,766 & 5.70 & 18 & 10.6 & 7.9 & 308 \\
24B & 829 & 17.9 & 1.95 & 1,134 & 5.12 & 18 & 10.5 & 7.9 & 309 \\
X05 & 848 & 18.0 & 1.84 & 415 & -- & -- & -- & -- & --
\end{tabular}


SITE: Mississippi River above Arkansas City, Arkansas--continued 03-24-89

\begin{tabular}{|c|c|c|c|c|c|c|c|c|c|}
\hline \multirow[b]{2}{*}{$\begin{array}{c}\text { Verti- } \\
\text { cal }\end{array}$} & \multirow{2}{*}{$\begin{array}{l}\text { Dist. } \\
\text { from } \\
\text { LEW } \\
\text { (m) }\end{array}$} & \multirow[b]{2}{*}{$\begin{array}{l}\text { Depth } \\
\text { (m) }\end{array}$} & \multirow{2}{*}{$\begin{array}{l}\text { Mean } \\
\text { veloc- } \\
\text { ity } \\
(\mathrm{m} / \mathrm{s})\end{array}$} & \multirow[b]{2}{*}{$\begin{array}{r}\text { Dis- } \\
\text { charge } \\
\left(\mathrm{m}^{3} / \mathrm{s}\right)\end{array}$} & \multicolumn{2}{|c|}{ Volume } & \multirow[b]{2}{*}{$\begin{array}{l}\text { Temper- } \\
\text { ature } \\
\left({ }^{\circ} \mathrm{C}\right)\end{array}$} & \multirow[b]{2}{*}{$\mathrm{pH}$} & \multirow{2}{*}{$\begin{array}{l}\text { Specific } \\
\text { conduct- } \\
\text { ance } \\
(\mu \mathrm{S} / \mathrm{cm})\end{array}$} \\
\hline & & & & & $\begin{array}{l}\overline{V_{i}} \\
(\mathrm{~L})\end{array}$ & $\begin{array}{l}V_{p} \\
(\mathrm{~L})\end{array}$ & & & \\
\hline $25 A$ & 854 & 17.9 & 1.60 & 444 & 4.45 & 17 & 10.5 & 7.9 & 302 \\
\hline $26 B$ & 879 & 18.0 & 1.43 & 890 & 3.45 & 17 & 10.6 & 7.9 & 302 \\
\hline $27 \mathrm{~A}$ & 923 & 17.9 & 0.94 & 481 & 2.04 & 17 & 10.5 & 8.0 & 307 \\
\hline $28 B$ & 936 & 17.7 & 0.93 & 453 & 1.69 & 15 & 10.5 & 7.9 & 308 \\
\hline $29 A$ & 978 & 13.2 & 0.47 & 204 & 0.63 & 11 & 10.5 & 8.0 & 301 \\
\hline $30 \mathrm{~B}$ & 1,001 & 8.8 & 0.01 & 2 & 0.18 & 5 & 10.6 & 8.0 & 305 \\
\hline REW & 1,035 & 0.0 & 0.00 & 0 & -- & -- & -- & -- & -- \\
\hline MEAN & & 15.9 & 1.63 & & & & & & \\
\hline TOTAL & 1,035 & & & 26,846 & 116.69 & 500 & & & \\
\hline
\end{tabular}




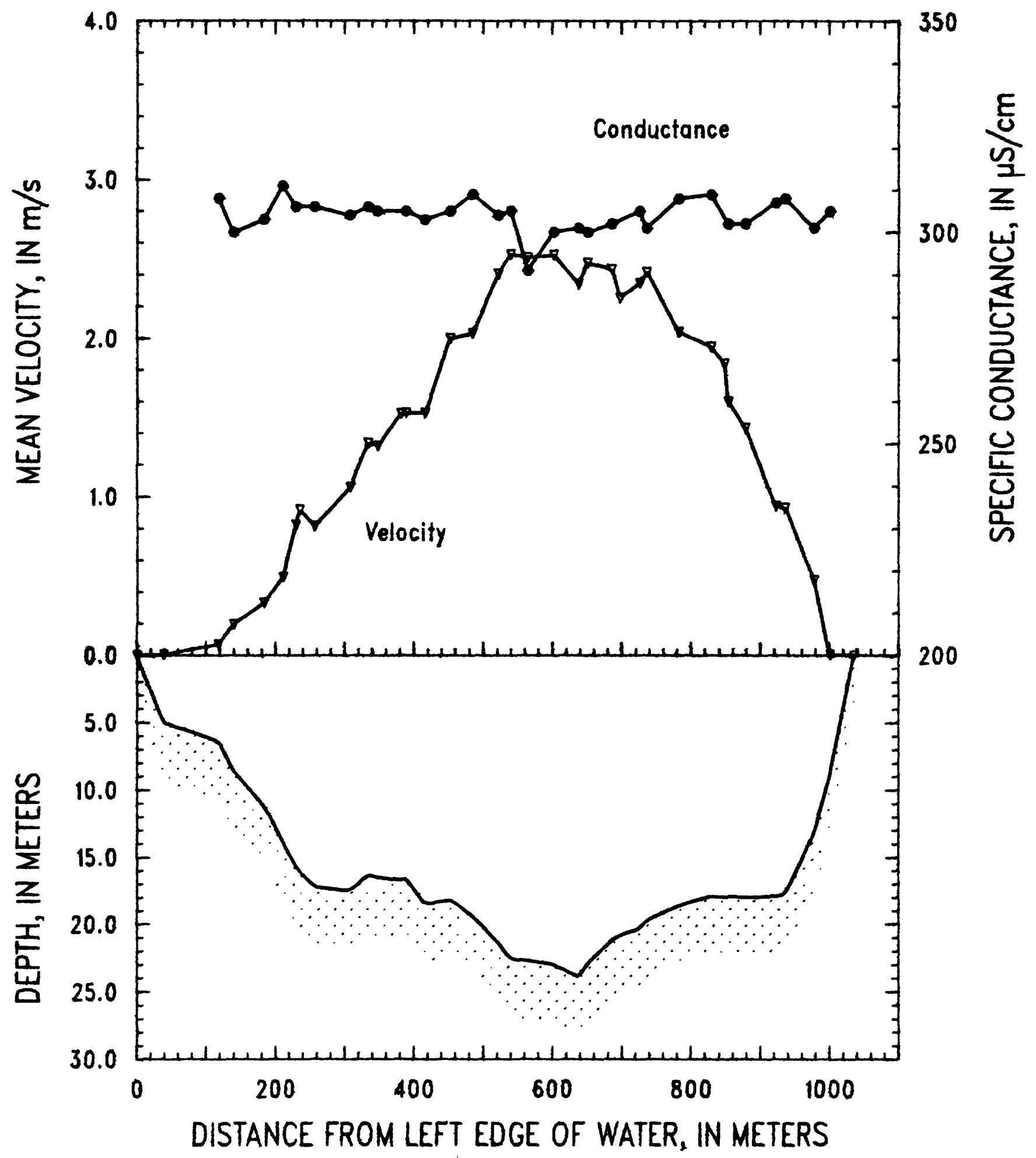

Figure 21. Mississippi River above Arkansas City, Arkansas, on March 24, 1989.

(PAGe 90 followS) 
SITE: Yazoo River below Steele Bayou, Mississippi PARTY: Moody, Stevens, and Black

STARTING GAGE HEIGHT: -- ENDING GAGE HEIGHT:

SUSP: Bag sampler and 150-1b weight. Current meter $22 \mathrm{~cm}$ above nozzle. CURRENT METER NO.: P8308282 DATE RATED: 07-08-88

CURRENT METER EQUATION: $V(\mathrm{~m} / \mathrm{s})=$ RPS $\div 0.765+0.006$

REMARKS: Sampled five equal-discharge-increment verticals with various transit rates $(7-16 \mathrm{~cm} / \mathrm{s})$ and nozzles $(1 / 4,3 / 16$, and $5 / 16$ inch). Stage is dropping at Vicksburg, creating higher discharge than measured here previously. About $50 \mathrm{~m}$ of trees on the left bank so that LEW is about $-50 \mathrm{~m}$; assumed no flow.

\begin{tabular}{|c|c|c|c|c|c|c|c|c|c|}
\hline \multirow[b]{2}{*}{$\begin{array}{c}\text { Verti- } \\
\text { cal }\end{array}$} & \multirow{2}{*}{$\begin{array}{l}\text { Dist. } \\
\text { from } \\
\text { LEW } \\
\text { (m) }\end{array}$} & \multirow[b]{2}{*}{$\begin{array}{c}\text { Depth } \\
\text { (m) }\end{array}$} & \multirow{2}{*}{$\begin{array}{l}\text { Mean } \\
\text { veloc- } \\
\text { ity } \\
(\mathrm{m} / \mathrm{s})\end{array}$} & \multirow[b]{2}{*}{$\begin{array}{r}\text { Dis- } \\
\text { charge } \\
\left(\mathrm{m}^{3} / \mathrm{s}\right)\end{array}$} & \multicolumn{2}{|c|}{ Volume } & \multirow[b]{2}{*}{$\begin{array}{l}\text { Temper- } \\
\text { ature } \\
\left({ }^{\circ} \mathrm{C}\right)\end{array}$} & & \multirow{2}{*}{$\begin{array}{l}\text { Specific } \\
\text { conduct- } \\
\text { ance } \\
(\mu \mathrm{S} / \mathrm{cm})\end{array}$} \\
\hline & & & & & $\begin{array}{l}\bar{V}_{\mathbf{i}} \\
(L)\end{array}$ & $\begin{array}{l}\mathrm{V}_{\mathrm{p}} \\
(\mathrm{L})\end{array}$ & & $\mathrm{pH}$ & \\
\hline
\end{tabular}

\begin{tabular}{lrrrrrrrrr}
\hline LEF & 0 & 5.0 & 0.00 & 0 & -- & -- & -- & -- & -- \\
1A1 & 23 & 9.1 & 0.34 & 50 & -- & -- & -- & -- & -- \\
01 & 33 & 11.8 & 0.75 & 80 & 23.80 & 100 & 15.3 & 7.7 & 75 \\
X05 & 41 & 13.0 & 0.70 & 127 & -- & -- & -- & -- & -- \\
02 & 61 & 15.8 & 0.95 & 263 & 22.51 & 100 & 15.5 & 7.4 & 83 \\
03 & 76 & 15.7 & 0.97 & 175 & 22.50 & 100 & 14.1 & 7.5 & 75 \\
X03 & 84 & 15.7 & 0.86 & 163 & -- & -- & -- & -- & -- \\
04 & 100 & 15.5 & 1.00 & 257 & 22.50 & 100 & 14.9 & 7.5 & 80 \\
X11 & 117 & 13.5 & 0.97 & 131 & -- & -- & -- & -- & -- \\
05 & 120 & 12.7 & 0.93 & 35 & 24.83 & 100 & 14.5 & 7.6 & 84 \\
$05 B$ & 123 & 11.9 & 0.83 & 34 & -- & -- & -- & -- & -- \\
X10 & 127 & 10.8 & 0.81 & 92 & -- & -- & -- & -- & -- \\
X01 & 144 & 8.0 & 0.43 & 95 & -- & -- & -- & -- & -- \\
REW & 182 & 0.0 & 0.00 & 0 & -- & -- & -- & -- & -- \\
MEAN & & 10.4 & 0.79 & & & & & & \\
TOTAL & 182 & & & 1,502 & 116.14 & 500 & & & \\
\hline
\end{tabular}




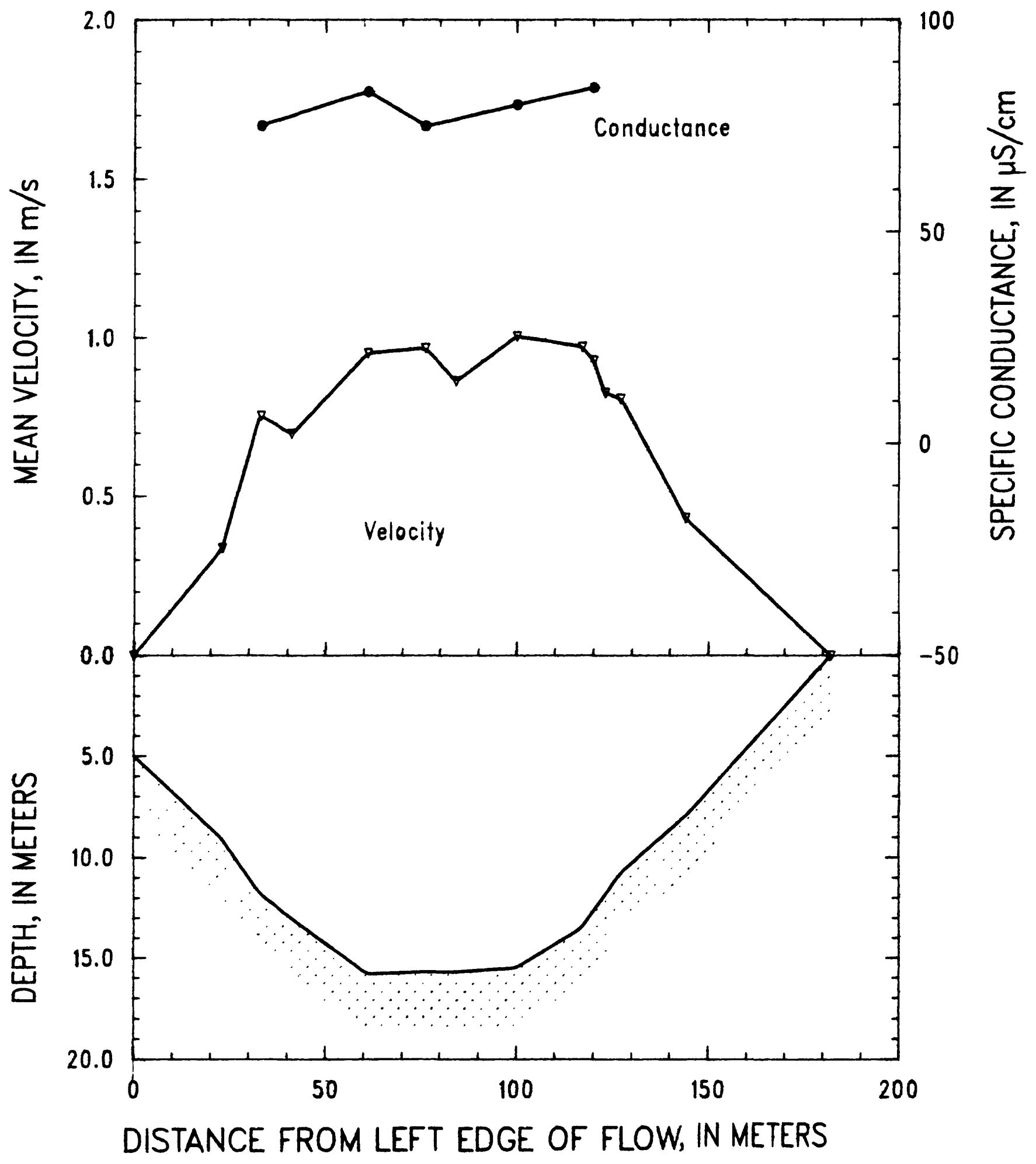

Figure 22. Yazoo River below Steele Bayou, Mississippi, on March 26, 1989. 
STATION: Mississippi River below Vicksburg, Mississippi

PARTY: Moody, Stevens, and Black

03-27-89

STARTING GAGE HEIGHT: $32.5 \mathrm{ft}$ ENDING GAGE HEIGHT:

SUSP: Bag sampler and 300-1b weight. Current meter $22 \mathrm{~cm}$ above nozzle.

CURRENT METER NO.: P8308282 DATE RATED: 07-08-88

CURRENT METER EQUATION: $V(\mathrm{~m} / \mathrm{s})=$ RPS $* 0.765+0.006$

REMARKS: Transit rate was $11 \mathrm{~cm} / \mathrm{s}$ and the nozzle was $1 / 8$ inch.

Verticals occupied in order 1-36. Highest velocities are not in the deep water but over moving sand bed.

\begin{tabular}{|c|c|c|c|c|c|c|c|c|c|}
\hline \multirow[b]{2}{*}{$\begin{array}{l}\text { Verti- } \\
\text { cal }\end{array}$} & \multirow{2}{*}{$\begin{array}{l}\text { Dist. } \\
\text { from } \\
\text { LEW } \\
\text { (m) }\end{array}$} & \multirow[b]{2}{*}{$\begin{array}{c}\text { Depth } \\
\text { (m) }\end{array}$} & \multirow{2}{*}{$\begin{array}{c}\text { Mean } \\
\text { veloc- } \\
\text { ity } \\
(\mathrm{m} / \mathrm{s})\end{array}$} & \multirow[b]{2}{*}{$\begin{array}{r}\text { Dis- } \\
\text { charge } \\
\left(\mathrm{m}^{3} / \mathrm{s}\right)\end{array}$} & \multicolumn{2}{|c|}{ Volume } & \multirow[b]{2}{*}{$\begin{array}{l}\text { Temper- } \\
\text { ature } \\
\left({ }^{\circ} \mathrm{C}\right)\end{array}$} & \multirow[b]{2}{*}{$\mathrm{pH}$} & \multirow{2}{*}{$\begin{array}{c}\text { Specific } \\
\text { conduct- } \\
\text { ance } \\
(\mu \mathrm{S} / \mathrm{cm})\end{array}$} \\
\hline & & & & & $\begin{array}{l}\mathrm{V}_{\mathbf{i}} \\
(\mathrm{L})\end{array}$ & $\begin{array}{l}\mathrm{V}_{\mathrm{p}} \\
(\mathrm{L})\end{array}$ & & & \\
\hline LEW & 0 & 0.0 & 0.00 & 0 & -- & -- & -- & -- & -- \\
\hline $01 \mathrm{~A}$ & 45 & 11.2 & 0.49 & 182 & 0.43 & 6 & 12.3 & 7.8 & 262 \\
\hline O2B & 66 & 14.2 & 0.73 & 326 & 0.94 & 11 & 13.1 & 7.8 & 260 \\
\hline $03 \mathrm{~A}$ & 125 & 20.5 & 1.02 & 733 & 3.06 & 20 & 12.5 & 7.8 & 262 \\
\hline 04B & 136 & 21.5 & 1.37 & 926 & 4.19 & 25 & 12.6 & 7.7 & 268 \\
\hline $05 \mathrm{~A}$ & 171 & 21.2 & 1.52 & 724 & 3.75 & 25 & 12.3 & 7.7 & 272 \\
\hline $\mathrm{X} 03$ & 181 & 21.3 & 1.58 & 471 & -- & -- & -- & -- & -- \\
\hline 06B & 199 & 21.1 & 1.42 & 777 & 4.39 & 26 & 12.2 & 7.7 & 279 \\
\hline 07A & 234 & 20.6 & 1.56 & 1,096 & 4.89 & 25 & 12.8 & 7.6 & 279 \\
\hline $08 \mathrm{~B}$ & 267 & 20.3 & 1.73 & 1,230 & 5.31 & 25 & 12.0 & 7.7 & 285 \\
\hline 09A & 303 & 20.1 & 1.62 & 913 & 5.30 & 24 & 11.9 & 7.8 & 289 \\
\hline $10 B$ & 323 & 20.1 & 1.76 & 1,149 & 4.59 & 26 & 12.5 & 7.7 & 295 \\
\hline $11 \mathrm{~A}$ & 368 & 19.2 & 1.72 & 992 & 5.00 & 31 & 12.0 & 7.7 & 289 \\
\hline X04 & 383 & 19.4 & 1.80 & 523 & -- & -- & -- & -- & -- \\
\hline $12 B$ & 398 & 19.5 & 1.79 & 577 & 5.01 & 27 & 12.5 & 7.7 & 305 \\
\hline $13 \mathrm{~A}$ & 416 & 19.5 & 2.03 & 1,109 & 5.51 & 39 & 12.0 & 7.7 & 312 \\
\hline $14 \mathrm{~B}$ & 454 & 17.7 & 1.96 & 1,234 & 6.01 & 23 & 11.8 & 7.7 & 309 \\
\hline $15 \mathrm{~A}$ & 487 & 18.0 & 1.98 & 979 & 5.17 & 21 & 12.2 & 7.7 & 317 \\
\hline X08 & 509 & 17.7 & 2.06 & 673 & -- & -- & -- & -- & -- \\
\hline $16 \mathrm{~B}$ & 524 & 17.5 & 2.14 & 749 & 5.66 & 25 & 11.9 & 7.8 & 317 \\
\hline $17 \mathrm{~A}$ & 549 & 17.6 & 2.06 & 833 & 4.60 & 27 & 11.7 & 7.7 & 316 \\
\hline $18 \mathrm{R}$ & 570 & 16.5 & 2.24 & 685 & 3.68 & 27 & 12.5 & 7.7 & 316 \\
\hline X09 & 586 & 16.5 & 2.07 & 870 & -. & -- & - & -- & -- \\
\hline $19 \mathrm{~A}$ & 621 & 15.5 & 2.06 & 1,136 & 5.12 & 23 & 12.1 & 7.7 & 316 \\
\hline $20 \mathrm{~B}$ & 657 & 15.5 & 1.95 & 950 & 4.99 & 22 & 12.3 & 7.7 & 316 \\
\hline $21 \mathrm{~A}$ & 684 & 14.0 & 1.75 & 822 & 3.80 & 23 & 12.3 & 7.7 & 317 \\
\hline $22 B$ & 724 & 13.1 & 1.65 & 766 & 3.20 & 18 & 12.0 & 7.7 & 317 \\
\hline $23 \mathrm{~A}$ & 755 & 12.8 & 1.43 & 694 & 2.92 & 15 & 11.8 & 7.7 & 314 \\
\hline $24 B$ & 800 & 11.7 & 1.35 & 474 & 2.27 & 13 & 11.9 & 7.7 & 319 \\
\hline $25 \mathrm{~A}$ & 815 & 11.5 & 1.39 & 231 & 1.64 & 12 & 11.7 & 7.7 & 320 \\
\hline $\mathrm{X} 10$ & 829 & 11.4 & 1.29 & 316 & -- & - & - & - & -- \\
\hline $26 B$ & 858 & 10.7 & 1.40 & 426 & 1.89 & 10 & 11.9 & 7.7 & 315 \\
\hline $27 \mathrm{~A}$ & 886 & 10.5 & 1.26 & 402 & 1.89 & 10 & 12.0 & 7.7 & 318 \\
\hline $28 \mathrm{~B}$ & 919 & 10.2 & 1.14 & 390 & 1.33 & 9 & 11.8 & 7.7 & 317 \\
\hline $29 A$ & 953 & 9.9 & 1.07 & 340 & 0.99 & 7 & 12.0 & 7.7 & 316 \\
\hline $30 \mathrm{~B}$ & 983 & 9.7 & 1.01 & 294 & 1.20 & 7 & 11.7 & 7.7 & 316 \\
\hline $31 \mathrm{~A}$ & 1,013 & 9.8 & 0.98 & 246 & 1.14 & 8 & 11.7 & 7.7 & 318 \\
\hline X01 & 1,034 & 9.9 & 0.99 & 190 & -- & -- & -- & -- & -- \\
\hline $\mathrm{X} 11$ & 1,052 & 9.8 & 0.90 & 120 & -- & -- & -- & -- & -- \\
\hline
\end{tabular}


STATION: Mississippi River below Vicksburg, Mississippi--continued 03-27-89

\begin{tabular}{|c|c|c|c|c|c|c|c|c|c|}
\hline \multirow[b]{2}{*}{$\begin{array}{c}\text { Verti- } \\
\text { cal }\end{array}$} & \multirow{2}{*}{$\begin{array}{l}\text { Dist. } \\
\text { from } \\
\text { LEW } \\
(\mathrm{m})\end{array}$} & \multirow[b]{2}{*}{$\begin{array}{c}\text { Depth } \\
(\mathrm{m})\end{array}$} & \multirow{2}{*}{$\begin{array}{l}\text { Mean } \\
\text { veloc- } \\
\text { ity } \\
(\mathrm{m} / \mathrm{s})\end{array}$} & \multirow[b]{2}{*}{$\begin{array}{r}\text { Dis- } \\
\text { charge } \\
\left(m^{3} / s\right)\end{array}$} & \multicolumn{2}{|c|}{ Volume } & \multirow[b]{2}{*}{$\begin{array}{l}\text { Temper- } \\
\text { ature } \\
\left({ }^{\circ} \mathrm{C}\right)\end{array}$} & \multirow[b]{2}{*}{$\mathrm{pH}$} & \multirow{2}{*}{$\begin{array}{c}\text { Specific } \\
\text { conduct- } \\
\text { ance } \\
(\mu \mathrm{S} / \mathrm{cm})\end{array}$} \\
\hline & & & & & $\begin{array}{l}\overline{v_{i}} \\
(L)\end{array}$ & $\begin{array}{c}\mathrm{V}_{\mathrm{p}} \\
(\mathrm{L})\end{array}$ & & & \\
\hline $32 \mathrm{~B}$ & 1,061 & 9.5 & 0.89 & 156 & 1.13 & 7 & 11.7 & 7.7 & 316 \\
\hline $33 A$ & 1,089 & 9.5 & 0.85 & 233 & 0.66 & 7 & 11.8 & 7.7 & 316 \\
\hline $34 \mathrm{~B}$ & 1,119 & 10.1 & 0.85 & 259 & 0.86 & 6 & 11.8 & 7.7 & 315 \\
\hline $35 \mathrm{~A}$ & 1,149 & 10.1 & 0.78 & 210 & 0.82 & 6 & 11.9 & 7.7 & 315 \\
\hline $36 \mathrm{~B}$ & 1,172 & 9.4 & 0.51 & 145 & 0.57 & 5 & 11.8 & 7.7 & 315 \\
\hline REW & 1,210 & 0.0 & 0.00 & 0 & -- & -- & -- & -- & - \\
\hline MEAN & & 14.5 & 1.52 & & & & & & \\
\hline TOTAL & 1,210 & & & 26,551 & 113.91 & 641 & & & \\
\hline
\end{tabular}




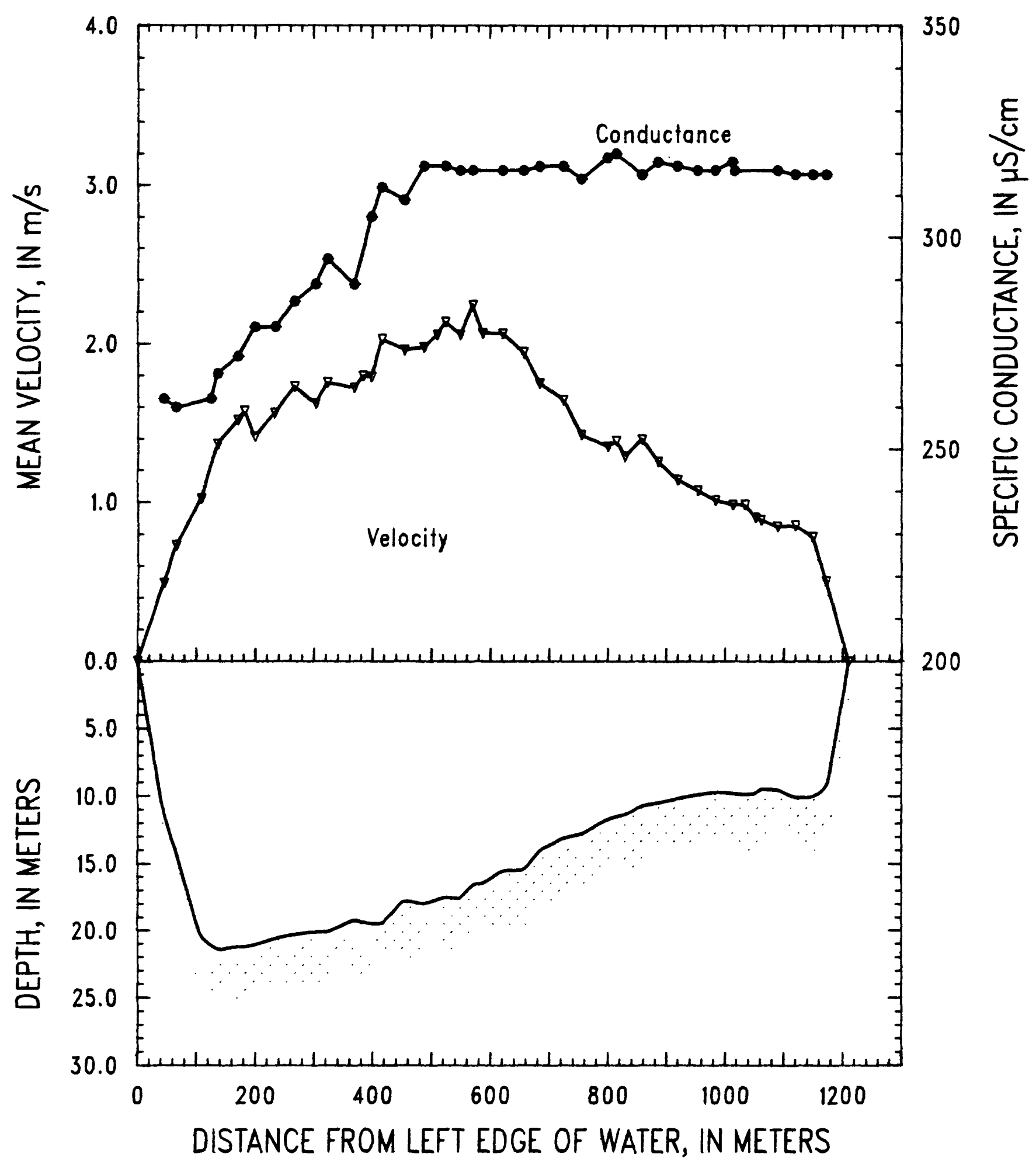

Figure 23. Mississippi River below Vicksburg, Mississippi, on March 27, 1989. 
SITE: Old River Outflow Channel near Knox Landing, Louisiana 03-29-89

PARTY: Moody, Stevens, and Black

METER: SOLID CUP

STARTING GAGE HEIGHT: -- ENDING GAGE HEIGHT: --

SUSP: Bag sampler and 200-1b weight. Current meter $20 \mathrm{~cm}$ above nozzle.

CURRENT METER NO.: P8308282 DATE RATED: 07-08-88

CURRENT METER EQUATION: $V(\mathrm{~m} / \mathrm{s})=$ RPS $* 0.765+0.006$

REMARKS: Transit rate was $15 \mathrm{~cm} / \mathrm{s}$ and nozzle was $1 / 4$ inch. Verticals were occupied in order 1-30. Heavy rain in the morning--about $5 \mathrm{~cm}$.

\begin{tabular}{|c|c|c|c|c|c|c|c|c|c|}
\hline \multirow[b]{2}{*}{$\begin{array}{c}\text { Verti- } \\
\text { cal }\end{array}$} & \multirow{2}{*}{$\begin{array}{l}\text { Dist. } \\
\text { from } \\
\text { LEW } \\
(\mathrm{m})\end{array}$} & \multirow[b]{2}{*}{$\begin{array}{l}\text { Depth } \\
(\mathrm{m})\end{array}$} & \multirow{2}{*}{$\begin{array}{l}\text { Mean } \\
\text { veloc- } \\
\text { ity } \\
(\mathrm{m} / \mathrm{s})\end{array}$} & \multirow[b]{2}{*}{$\begin{array}{r}\text { Dis- } \\
\text { charge } \\
\left(\mathrm{m}^{3} / \mathrm{s}\right)\end{array}$} & \multicolumn{2}{|c|}{ Volume } & \multirow[b]{2}{*}{$\begin{array}{l}\text { Temper- } \\
\text { ature } \\
\left({ }^{\circ} \mathrm{C}\right)\end{array}$} & \multirow[b]{2}{*}{$\mathrm{pH}$} & \multirow{2}{*}{$\begin{array}{l}\text { Specific } \\
\text { conduct- } \\
\text { ance } \\
(\mu \mathrm{S} / \mathrm{cm})\end{array}$} \\
\hline & & & & & $\begin{array}{c}\mathrm{V}_{\mathrm{i}} \\
(\mathrm{L})\end{array}$ & $\begin{array}{l}V_{p} \\
(\mathrm{~L})\end{array}$ & & & \\
\hline LEW & 0 & 0.0 & 0.00 & 0 & - & -- & -- & -- & -- \\
\hline $01 R$ & 20 & 6.9 & 0.60 & 46 & -- & -- & -- & -- & -- \\
\hline $01 \mathrm{~A}$ & 22 & 8.7 & 0.60 & 49 & 1.59 & 5 & 13.2 & 7.8 & 291 \\
\hline 02B & 39 & 13.2 & 0.87 & 184 & 3.71 & 19 & 13.2 & 7.7 & 286 \\
\hline $03 \mathrm{~A}$ & 54 & 13.1 & 0.94 & 123 & 4.19 & 19 & 13.0 & 7.7 & 293 \\
\hline 03R & 59 & 13.2 & 1.00 & 99 & -- & -- & -- & -- & -- \\
\hline $04 R$ & 69 & 13.1 & 1.06 & 145 & -- & -- & -- & -- & -- \\
\hline $04 \mathrm{~B}$ & 80 & 13.2 & 1.06 & 189 & 4.58 & 19 & 12.7 & 7.7 & 293 \\
\hline $05 \mathrm{~A}$ & 96 & 13.2 & 1.02 & 223 & 4.74 & 19 & 12.7 & 7.7 & 295 \\
\hline $06 \mathrm{~B}$ & 113 & 13.3 & 1.07 & 221 & 4.68 & 20 & 12.6 & 7.7 & 293 \\
\hline $07 \mathrm{~A}$ & 127 & 13.2 & 1.01 & 206 & 4.68 & 19 & 12.6 & 7.7 & 296 \\
\hline $08 B$ & 144 & 13.3 & 1.05 & 265 & 4.68 & 19 & 12.7 & 7.7 & 295 \\
\hline $09 \mathrm{~A}$ & 165 & 13.0 & 1.05 & 274 & 4.81 & 18 & 12.7 & 7.7 & 295 \\
\hline $10 B$ & 184 & 13.0 & 0.99 & 233 & 4.39 & 18 & 12.8 & 7.8 & 298 \\
\hline $11 \mathrm{~A}$ & 201 & 12.9 & 1.01 & 234 & 4.16 & 18 & 13.1 & 7.7 & 297 \\
\hline $12 B$ & 220 & 12.7 & 1.12 & 284 & 4.84 & 20 & 12.9 & 7.7 & 296 \\
\hline $13 \mathrm{~A}$ & 241 & 12.5 & 1.01 & 208 & 4.30 & 19 & 12.9 & 7.7 & 297 \\
\hline $14 \mathrm{~B}$ & 253 & 12.4 & 1.06 & 197 & 4.64 & 19 & 12.8 & 7.8 & 297 \\
\hline $15 \mathrm{~A}$ & 271 & 12.0 & 1.03 & 217 & 4.32 & 19 & 12.5 & 7.7 & 297 \\
\hline $16 \mathrm{~B}$ & 288 & 11.8 & 1.06 & 275 & 3.84 & 18 & 13.0 & 7.7 & 297 \\
\hline $17 \mathrm{~A}$ & 315 & 11.1 & 1.02 & 225 & 4.16 & 17 & 12.6 & 7.8 & 298 \\
\hline $18 \mathrm{~B}$ & 328 & 10.9 & 1.06 & 168 & 3.91 & 17 & 13.2 & 7.8 & 301 \\
\hline $19 \mathrm{~A}$ & 344 & 10.7 & 1.03 & 143 & 3.95 & 16 & 12.8 & 7.8 & 305 \\
\hline $20 \mathrm{~B}$ & 354 & 10.4 & 1.12 & 145 & 3.41 & 16 & 13.1 & 7.8 & 305 \\
\hline $21 \mathrm{~A}$ & 369 & 10.4 & 1.04 & 231 & 3.36 & 16 & 12.9 & 7.8 & 305 \\
\hline $22 B$ & 397 & 10.2 & 1.02 & 230 & 3.27 & 16 & 13.0 & 7.8 & 303 \\
\hline $23 \mathrm{~A}$ & 413 & 10.2 & 0.97 & 133 & 2.84 & 16 & 13.0 & 7.8 & 302 \\
\hline $24 B$ & 424 & 10.0 & 1.03 & 98 & 3.95 & 16 & 13.1 & 7.8 & 306 \\
\hline $\mathrm{X} 01$ & 432 & 10.2 & 1.10 & 90 & -- & - & - & -- & -- \\
\hline $25 \mathrm{~A}$ & 440 & 10.2 & 1.01 & 165 & 3.57 & 16 & 13.0 & 7.8 & 302 \\
\hline $26 B$ & 464 & 10.4 & 0.98 & 178 & 3.28 & 16 & 13.1 & 7.8 & 304 \\
\hline $27 \mathrm{~A}$ & 475 & 10.5 & 0.95 & 160 & 3.22 & 15 & 13.4 & 7.8 & 307 \\
\hline $28 \mathrm{~B}$ & 496 & 10.6 & 0.94 & 189 & 3.28 & 16 & 13.1 & 7.8 & 304 \\
\hline $29 \mathrm{~A}$ & 513 & 10.8 & 0.88 & 186 & 3.19 & 17 & 13.5 & 7.8 & 304 \\
\hline $30 \mathrm{~B}$ & 535 & 9.4 & 0.71 & 143 & 2.47 & 5 & 13.7 & 7.8 & 306 \\
\hline REW & 556 & 0.0 & 0.00 & 0 & -- & - & -- & -- & -- \\
\hline MEAN & & 11.1 & 1.00 & & & & & & \\
\hline TOTAL & 556 & & & 6,156 & 116.01 & 503 & & & \\
\hline
\end{tabular}




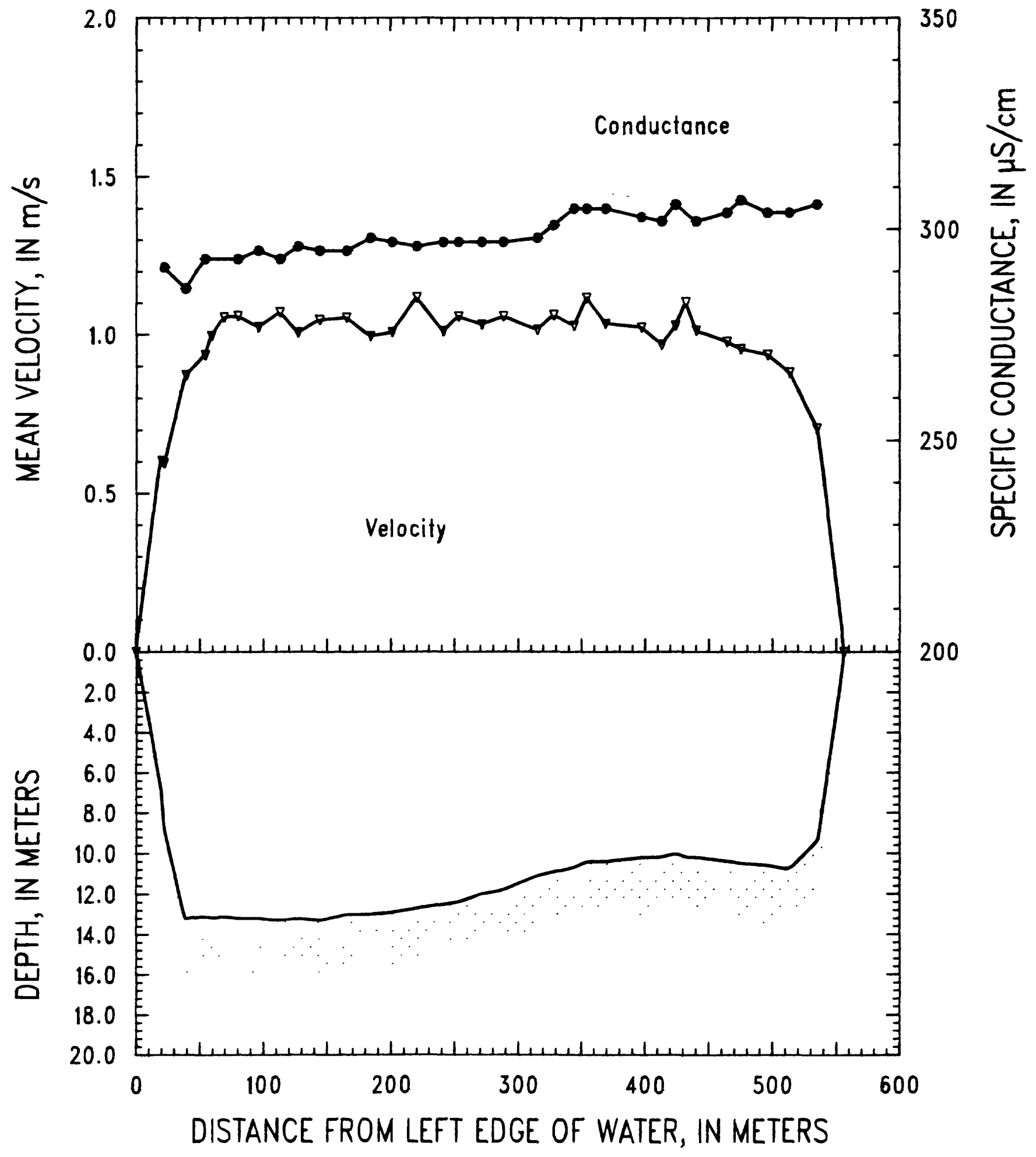

Figure 24. Old River Outflow Channel near Knox Landing, Louisiana, on March 29, 1989. 
SITE: Mississippi River near St. Francisville, Louisiana PARTY: Moody, Stevens, and Black

STARTING GAGE HEIGHT: -- ENDING GAGE HEIGHT: --

METER: SOLID CUP

SUSP: Bag sampler and $300-1 \mathrm{~b}$ weight at verticals $32-18$ and $200-1 \mathrm{~b}$ weight at verticals $17-1$.

CURRENT METER NO.: P8308282 DATE RATED: 07-08-88

CURRENT METER EQUATION: $V(\mathrm{~m} / \mathrm{s})=$ RPS $* 0.765+0.006$

REMARKS: Transit rate was $20 \mathrm{~cm} / \mathrm{s}$ and nozzle was $3 / 16$ inch. Vertical $1 \mathrm{~A}$ was at a transit rate of $10 \mathrm{~cm} / \mathrm{s}$ to represent $1 \mathrm{~A}$ and $3 \mathrm{~A}$.

\begin{tabular}{|c|c|c|c|c|c|c|c|c|c|}
\hline & Dist. & & Me & & & & & & Specific \\
\hline $\begin{array}{l}\text { Jerti- } \\
\text { cal }\end{array}$ & $\begin{array}{l}\text { from } \\
\text { LEW } \\
\text { (m) }\end{array}$ & $\begin{array}{c}\text { Depth } \\
\text { (m) }\end{array}$ & $\begin{array}{c}\text { veloc- } \\
\text { ity } \\
(\mathrm{m} / \mathrm{s})\end{array}$ & $\begin{array}{r}\text { Dis- } \\
\text { charge } \\
\left(\mathrm{m}^{3} / \mathrm{s}\right)\end{array}$ & $\begin{array}{l}\overline{V_{i}} \\
(L)\end{array}$ & $\begin{array}{l}\bar{V}_{\mathbf{p}} \\
(\mathrm{L})\end{array}$ & $\begin{array}{l}\text { Temper- } \\
\text { ature } \\
\left({ }^{\circ} \mathrm{C}\right)\end{array}$ & $\mathrm{pH}$ & $\begin{array}{l}\text { conduct- } \\
\text { ance } \\
(\mu \mathrm{S} / \mathrm{cm})\end{array}$ \\
\hline
\end{tabular}

\begin{tabular}{|c|c|c|c|c|c|c|c|c|c|}
\hline LEF & 0 & 0.0 & 0.00 & 0 & -- & -- & - & -- & -- \\
\hline $01 \mathrm{~A}$ & 33 & 8.5 & 0.34 & 99 & 0.62 & 5 & 14.9 & 7.6 & 297 \\
\hline O2B & 69 & 10.2 & 0.21 & 56 & 0.48 & 3 & 15.7 & 7.6 & 287 \\
\hline X01 & 85 & 10.4 & 0.45 & 146 & -- & -- & -- & -- & -- \\
\hline 04B & 131 & 11.1 & 0.85 & 288 & 1.04 & 3 & 13.7 & 7.6 & 300 \\
\hline $05 \mathrm{~A}$ & 146 & 11.8 & 1.02 & 217 & 1.90 & 4 & 13.5 & 7.6 & 301 \\
\hline X10 & 167 & 13.9 & 1.06 & 310 & -- & -- & -- & -- & -- \\
\hline 06B & 188 & 15.5 & 1.12 & 372 & 2.79 & 13 & 13.7 & 7.6 & 301 \\
\hline $07 \mathrm{~A}$ & 210 & 16.1 & 1.20 & 475 & 2.19 & 13 & 13.3 & 7.6 & 301 \\
\hline $\mathrm{X} 02$ & 237 & 16.0 & 1.20 & 345 & - & -- & -- & -- & -- \\
\hline 08B & 246 & 15.7 & 1.42 & 470 & 3.56 & 14 & 13.5 & 7.6 & 304 \\
\hline 09A & 279 & 14.8 & 1.65 & 758 & 3.95 & 12 & 13.7 & 7.6 & 302 \\
\hline $10 \mathrm{~B}$ & 308 & 15.8 & 1.69 & 734 & 4.19 & 17 & 13.5 & 7.6 & 303 \\
\hline $11 \mathrm{~A}$ & 334 & 16.1 & 1.72 & 749 & 3.74 & 19 & 13.9 & 7.6 & 302 \\
\hline $12 R$ & 362 & 16.8 & 1.86 & 359 & - & - & -- & -- & -- \\
\hline X09 & 357 & 18.2 & 1.42 & 103 & - & -. & -- & -- & - \\
\hline 12B & 370 & 17.6 & 1.67 & 499 & 4.95 & 21 & 13.5 & 7.6 & 302 \\
\hline X03 & 391 & 16.9 & 1.71 & 461 & -- & -- & -- & -- & -- \\
\hline $13 \mathrm{~A}$ & 402 & 18.3 & 1.77 & 438 & 5.31 & 22 & 13.5 & 7.6 & 302 \\
\hline $14 B$ & 418 & 17.6 & 1.56 & 824 & 4.09 & 21 & 13.7 & 7.6 & 300 \\
\hline $15 A$ & 462 & 16.5 & 1.75 & 1,008 & 4.94 & 19 & 14.0 & 7.6 & 302 \\
\hline $16 \mathrm{~B}$ & 488 & 16.4 & 1.77 & 696 & 3.97 & 18 & 13.5 & 7.6 & 301 \\
\hline $17 \mathrm{~A}$ & 510 & 16.3 & 1.84 & 962 & 4.93 & 20 & 13.7 & 7.6 & 304 \\
\hline $18 B$ & 552 & 16.6 & 1.79 & 995 & 4.72 & 19 & 14.2 & 7.6 & 302 \\
\hline $19 A$ & 577 & 17.8 & 1.64 & 874 & 4.75 & 18 & 14.9 & 7.6 & 302 \\
\hline $20 \mathrm{~B}$ & 612 & 16.5 & 1.63 & 871 & 4.68 & 17 & 15.3 & 7.6 & 300 \\
\hline $21 \mathrm{~A}$ & 642 & 17.8 & 1.53 & 791 & 4.42 & 21 & 15.0 & 7.6 & 304 \\
\hline $22 B$ & 670 & 18.6 & 1.56 & 987 & 4.46 & 23 & 14.1 & 7.6 & 300 \\
\hline $23 \mathrm{~A}$ & 710 & 18.7 & 1.61 & 857 & 4.86 & 23 & 14.6 & 7.7 & 311 \\
\hline $24 B$ & 727 & 19.1 & 1.63 & 623 & 4.91 & 25 & 14.0 & 7.7 & 301 \\
\hline X07 & 750 & 19.3 & 1.52 & 646 & -- & -- & -- & -- & - \\
\hline $25 A$ & 771 & 19.7 & 1.56 & 769 & 6.08 & 19 & 13.8 & 7.7 & 301 \\
\hline $26 \mathrm{~B}$ & 800 & 19.7 & 1.57 & 928 & 4.83 & 19 & 13.6 & 7.7 & 299 \\
\hline $27 A$ & 831 & 19.5 & 1.61 & 628 & 5.14 & 19 & 13.8 & 7.7 & 300 \\
\hline X05 & 840 & 19.4 & .1 .48 & 330 & -- & -- & -- & -- & -- \\
\hline $28 \mathrm{~B}$ & 854 & 19.5 & 1.64 & 830 & 4.98 & 19 & 13.5 & 7.7 & 301 \\
\hline
\end{tabular}


SITE: Mississippi River near St. Francisville, Louisiana--continued 03-30-89

\begin{tabular}{|c|c|c|c|c|c|c|c|c|c|}
\hline \multirow[b]{2}{*}{$\begin{array}{c}\text { Verti- } \\
\text { cal }\end{array}$} & \multirow{2}{*}{$\begin{array}{l}\text { Dist. } \\
\text { from } \\
\text { LEW } \\
\text { (m) }\end{array}$} & \multirow[b]{2}{*}{$\begin{array}{l}\text { Depth } \\
\text { (m) }\end{array}$} & \multirow{2}{*}{$\begin{array}{l}\text { Mean } \\
\text { veloc- } \\
\text { ity } \\
(\mathrm{m} / \mathrm{s})\end{array}$} & \multirow[b]{2}{*}{$\begin{array}{r}\text { Dis- } \\
\text { charge } \\
\left(\mathrm{m}^{3} / \mathrm{s}\right)\end{array}$} & \multicolumn{2}{|c|}{ Volume } & \multirow[b]{2}{*}{$\begin{array}{l}\text { Temper- } \\
\text { ature } \\
\left({ }^{\circ} \mathrm{C}\right)\end{array}$} & \multirow[b]{2}{*}{$\mathrm{pH}$} & \multirow{2}{*}{$\begin{array}{c}\text { Specific } \\
\text { conduct- } \\
\text { ance } \\
(\mu \mathrm{S} / \mathrm{cm})\end{array}$} \\
\hline & & & & & $\begin{array}{l}\overline{V_{i}} \\
(\mathrm{~L})\end{array}$ & $\begin{array}{l}\mathrm{V}_{\mathrm{p}} \\
(\mathrm{L})\end{array}$ & & & \\
\hline $29 \mathrm{~A}$ & 892 & 19.7 & 1.46 & 946 & 4.75 & 20 & 13.8 & 7.7 & 299 \\
\hline $30 \mathrm{~B}$ & 920 & 19.1 & 1.48 & 832 & 4.85 & 19 & 14.0 & 7.7 & 298 \\
\hline $31 \mathrm{~A}$ & 951 & 15.6 & 1.48 & 727 & 3.96 & 16 & 13.5 & 7.7 & 304 \\
\hline $32 \mathrm{~B}$ & 983 & 6.3 & 0.62 & 117 & 0.49 & 4 & 14.7 & 7.7 & 311 \\
\hline REW & 1,011 & 0.0 & 0.00 & 0 & - & -- & -- & -- & -- \\
\hline MEAN & & 15.5 & 1.47 & & & & & & \\
\hline TOTAL & 1,011 & & & 23,120 & 120.53 & 505 & & & \\
\hline
\end{tabular}




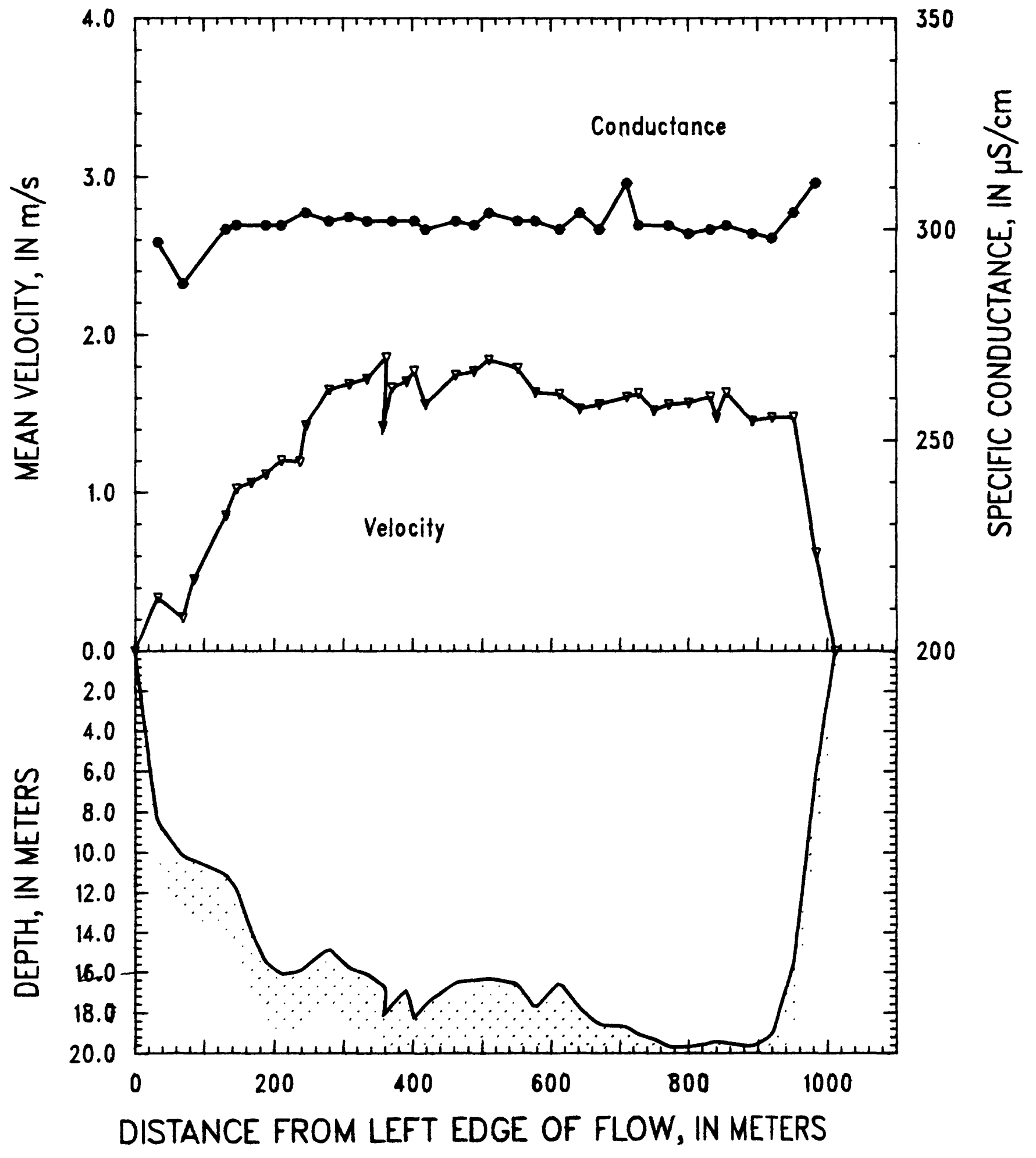

Figure 25. Mississippi River near St. Francisville, Louisiana, on March 30, 1989. 
SITE: Mississippi River below Belle Chasse, Louisiana PARTY: Moody, Stevens, and Black

STARTING GAGE HEIGHT: -- ENDING GAGE HEIGHT: --

METER: SOLID CUP

SUSP: Bag sampler and 300-1b weight. Current meter $20 \mathrm{~cm}$ above nozzle.

CURRENT METER NO.: P8308282 DATE RATED: 07-08-88

CURRENT METER EQUATION: $\mathrm{V}(\mathrm{m} / \mathrm{s})=$ RPS $* 0.765+0.006$

REMARKS: Transit rate was $10 \mathrm{~cm} / \mathrm{s}$ and the nozzle was $1 / 8$ inch.

Verticals were occupied in the following order: 1-10, 24-20, and 11-19.

\begin{tabular}{|c|c|c|c|c|c|c|c|c|c|}
\hline \multirow[b]{2}{*}{$\begin{array}{l}\text { Verti- } \\
\text { cal }\end{array}$} & \multirow{2}{*}{$\begin{array}{l}\text { Dist. } \\
\text { from } \\
\text { LEW } \\
\text { (m) }\end{array}$} & \multirow[b]{2}{*}{$\begin{array}{c}\text { Depth } \\
\text { (m) }\end{array}$} & \multirow{2}{*}{$\begin{array}{l}\text { Mean } \\
\text { veloc- } \\
\text { ity } \\
(\mathrm{m} / \mathrm{s})\end{array}$} & \multirow[b]{2}{*}{$\begin{array}{r}\text { Dis- } \\
\text { charge } \\
\left(m^{3} / \mathrm{s}\right)\end{array}$} & \multicolumn{2}{|c|}{ Volume } & \multirow[b]{2}{*}{$\begin{array}{l}\text { Temper- } \\
\text { a ture } \\
\left({ }^{\circ} \mathrm{C}\right)\end{array}$} & \multirow[b]{2}{*}{$\mathrm{pH}$} & \multirow{2}{*}{$\begin{array}{c}\text { Specific } \\
\text { conduct- } \\
\text { ance } \\
(\mu \mathrm{S} / \mathrm{cm})\end{array}$} \\
\hline & & & & & $\begin{array}{c}\overline{V_{i}} \\
(L)\end{array}$ & $\begin{array}{l}\mathrm{V}_{\mathrm{p}} \\
(\mathrm{L})\end{array}$ & & & \\
\hline LEW & 0 & 0.0 & 0.00 & 0 & -- & -- & -- & -- & - \\
\hline $01 \mathrm{~A}$ & 35 & 8.4 & 0.79 & 256 & 0.72 & 4 & 13.7 & 7.8 & 305 \\
\hline 02B & 77 & 16.4 & 1.14 & 633 & 2.39 & 11 & 13.5 & 7.8 & 304 \\
\hline 03A & 103 & 18.8 & 1.05 & 634 & 3.34 & 15 & 13.4 & 7.7 & 304 \\
\hline $04 B$ & 141 & 23.5 & 1.44 & 1,170 & 3.87 & 20 & 13.4 & 7.7 & 304 \\
\hline $05 \mathrm{~A}$ & 172 & 24.4 & 1.50 & 1,173 & 4.76 & 23 & 13.4 & 7.7 & 304 \\
\hline 06B & 205 & 24.7 & 1.68 & 1,533 & 4.25 & 23 & 13.5 & 7.7 & 304 \\
\hline 07A & 246 & 25.0 & 1.53 & 1,144 & 4.28 & 25 & 13.5 & 7.7 & 304 \\
\hline 08B & 265 & 25.8 & 1.54 & 951 & 6.25 & 25 & 13.6 & 7.7 & 305 \\
\hline $09 \mathrm{~A}$ & 294 & 25.5 & 1.56 & 1,295 & 5.27 & 25 & 13.6 & 7.7 & 305 \\
\hline $10 \mathrm{~B}$ & 330 & 25.4 & 1.55 & 1,497 & 5.80 & 25 & 13.7 & 7.7 & 305 \\
\hline $11 \mathrm{~A}$ & 370 & 25.5 & 1.49 & 1,176 & 5.95 & 25 & 13.8 & 7.7 & 307 \\
\hline $12 \mathrm{~B}$ & 392 & 26.0 & 1.48 & 1,154 & 6.23 & 26 & 13.8 & 7.7 & 307 \\
\hline $13 \mathrm{~A}$ & 430 & 27.0 & 1.47 & 1,334 & 6.54 & 28 & 13.7 & 7.6 & 307 \\
\hline $14 B$ & 459 & 26.5 & 1.42 & 1,090 & 5.26 & 27 & 13.7 & 7.7 & 307 \\
\hline $15 \mathrm{~A}$ & 488 & 25.7 & 1.39 & 1,123 & 4.80 & 26 & 13.7 & 7.7 & 307 \\
\hline $16 \mathrm{~B}$ & 522 & 25.0 & 1.25 & 1,027 & 4.99 & 24 & 13.5 & 7.7 & 307 \\
\hline $17 \mathrm{~A}$ & 554 & 24.6 & 1.14 & 704 & 4.10 & 23 & 13.8 & 7.7 & 309 \\
\hline $18 \mathrm{~B}$ & 572 & 24.5 & 1.13 & 651 & 4.10 & 23 & 13.9 & 7.7 & 309 \\
\hline $19 \mathrm{~A}$ & 601 & 24.1 & 1.09 & 896 & 3.95 & 22 & 13.9 & 7.7 & 310 \\
\hline $20 \mathrm{~B}$ & 640 & 23.6 & 1.05 & 582 & 3.06 & 21 & 13.6 & 7.7 & 307 \\
\hline X04 & 648 & 23.4 & 0.97 & 499 & -- & -- & -- & -- & -- \\
\hline $21 \mathrm{~A}$ & 684 & 23.2 & 1.00 & 583 & 2.58 & 21 & 13.7 & 7.7 & 307 \\
\hline $22 B$ & 698 & 23.3 & 0.98 & 626 & 3.02 & 22 & 13.6 & 7.7 & 307 \\
\hline $23 \mathrm{~A}$ & 739 & 17.6 & 0.93 & 607 & 2.28 & 11 & 13.7 & 7.7 & 307 \\
\hline $24 B$ & 772 & 6.7 & 0.68 & 139 & 0.50 & 4 & 14.6 & 7.7 & 308 \\
\hline REW & 800 & 0.1 & 0.00 & 0 & -- & -- & -- & -- & -- \\
\hline MEAN & & 21.5 & 1.31 & & & & & & \\
\hline TOTAL & 800 & & & 22,477 & 98.29 & 499 & & & \\
\hline
\end{tabular}




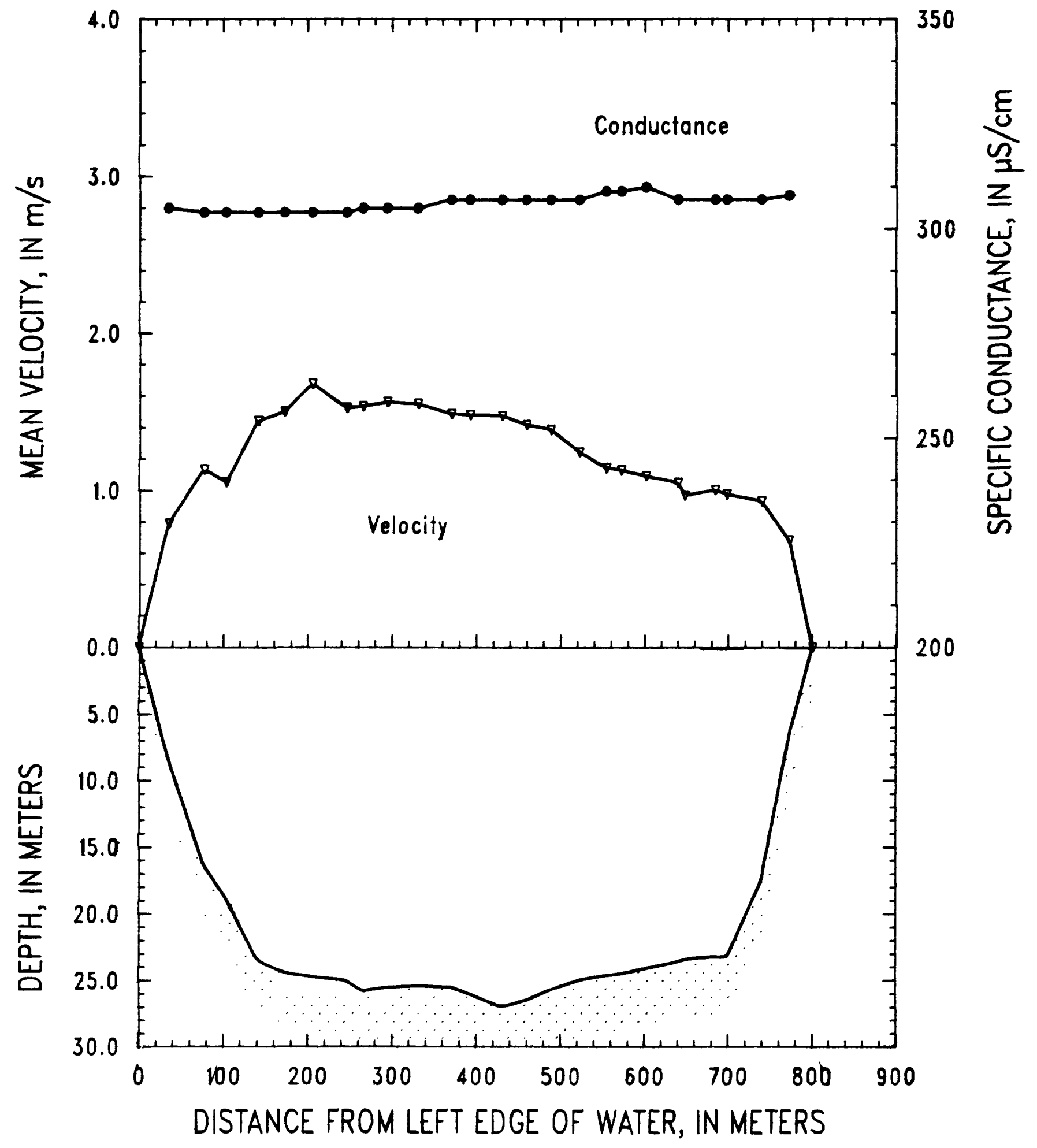

Figure 26. Mississippi River below Belle Chasse, Louisiana, on April 1, 1989. 
DATA LISTINGS

FOR

JUNE 1989 CRUISE 
SITE: Mississippi River near Winfield, Missouri

PARTY: Moody, Stevens, and Black

STARTING GAGE HEIGHT: -- ENDING GAGE HEIGHT: --

METER: SOLID CUP

SUSP: Bag sampler and $150-1 \mathrm{~b}$ weight

CURRENT METER NO.: P8308282 DATE RATED: 07-08-88

CURRENT METER EQUATION: $V(\mathrm{~m} / \mathrm{s})=\mathrm{RPS} * 0.765+0.006$

REMARKS: Transit rate was $6 \mathrm{~cm} / \mathrm{s}$ and nozzle $1 / 4$ inch. Anchored at each vertical from 1 to 20 . A and $B$ sample collected at each vertical.

\begin{tabular}{|c|c|c|c|c|c|c|c|c|c|}
\hline \multirow[b]{2}{*}{$\begin{array}{c}\text { Verti- } \\
\text { cal }\end{array}$} & \multirow{2}{*}{$\begin{array}{l}\text { Dist. } \\
\text { from } \\
\text { LEW } \\
\text { (m) }\end{array}$} & \multirow[b]{2}{*}{$\begin{array}{c}\text { Depth } \\
\text { (m) }\end{array}$} & \multirow{2}{*}{$\begin{array}{l}\text { Mean } \\
\text { veloc- } \\
\text { ity } \\
(\mathrm{m} / \mathrm{s})\end{array}$} & \multirow[b]{2}{*}{$\begin{array}{r}\text { Dis- } \\
\text { charge } \\
\left(\mathrm{m}^{3} / \mathrm{s}\right)\end{array}$} & \multicolumn{2}{|c|}{ Volume } & \multirow[b]{2}{*}{$\begin{array}{l}\text { Temper- } \\
\text { ature } \\
\left({ }^{\circ} \mathrm{C}\right)\end{array}$} & \multirow[b]{2}{*}{$\mathrm{pH}$} & \multirow{2}{*}{$\begin{array}{l}\text { Specific } \\
\text { conduct- } \\
\text { ance } \\
(\mu S / \mathrm{cm})\end{array}$} \\
\hline & & & & & $\begin{array}{l}\mathrm{V}_{\mathrm{i}} \\
(\mathrm{L})\end{array}$ & $\begin{array}{l}\mathrm{V}_{\mathrm{p}} \\
(\mathrm{L})\end{array}$ & & & \\
\hline LEW & 0 & 0.0 & 0.00 & 0 & -- & -- & -- & -- & -- \\
\hline 01 & 27 & 5.4 & 0.27 & 38 & 1.33 & 19 & 23.2 & 7.0 & 366 \\
\hline 02 & 51 & 8.1 & 0.69 & 136 & 8.55 & 47 & 23.2 & 7.0 & 365 \\
\hline 03 & 76 & 8.3 & 0.85 & 124 & 11.41 & 46 & 23.4 & 6.9 & 364 \\
\hline $\mathrm{X} 03$ & 86 & 8.4 & 0.80 & 115 & -- & -- & -- & -- & -- \\
\hline 04 & 110 & 8.8 & 0.75 & 168 & 10.38 & 49 & 23.4 & 7.0 & 381 \\
\hline 05 & 137 & 8.6 & 0.78 & 181 & 12.31 & 47 & 23.2 & 6.9 & 366 \\
\hline 06 & 164 & 8.3 & 0.74 & 163 & 9.68 & 44 & 23.8 & 7.0 & 367 \\
\hline 07 & 190 & 7.9 & 0.73 & 135 & 8.22 & 42 & 23.7 & 7.0 & 365 \\
\hline 08 & 211 & 7.7 & 0.67 & 72 & 7.53 & 38 & 23.6 & 7.2 & 365 \\
\hline $\mathrm{X} 02$ & 218 & 7.8 & 0.65 & 89 & -- & -- & -- & -- & -- \\
\hline 09 & 246 & 7.2 & 0.67 & 138 & 7.12 & 34 & 23.6 & 7.1 & 364 \\
\hline 10 & 275 & 6.9 & 0.70 & 120 & 5.35 & 33 & 23.7 & 6.8 & 364 \\
\hline 11 & 296 & 6.4 & 0.70 & 119 & 7.00 & 29 & 23.7 & 7.2 & 364 \\
\hline 12 & 328 & 6.1 & 0.67 & 114 & 5.99 & 26 & 24.1 & 7.2 & 363 \\
\hline 13 & 352 & 5.8 & 0.66 & 107 & 5.98 & 24 & 23.9 & 7.1 & 363 \\
\hline 14 & 364 & 5.3 & 0.67 & 89 & 5.23 & 20 & 23.9 & 7.1 & 363 \\
\hline 15 & 402 & 5.3 & 0.66 & 87 & 4.83 & 19 & 24.0 & 7.1 & 364 \\
\hline 16 & 434 & 4.7 & 0.62 & 77 & 4.20 & 17 & 24.0 & 7.2 & 365 \\
\hline 17 & 455 & 5.2 & 0.59 & 43 & 3.40 & 18 & 23.9 & 7.2 & 366 \\
\hline $\mathrm{X} 01$ & 462 & 4.9 & 0.51 & 27 & -- & -- & -- & -- & -- \\
\hline 18 & 477 & 4.4 & 0.58 & 57 & 3.31 & 14 & 24.0 & 7.3 & 364 \\
\hline 19 & 507 & 4.8 & 0.55 & 67 & 2.10 & 18 & 24.1 & 7.2 & 365 \\
\hline 20 & 528 & 4.1 & 0.46 & 52 & 1.48 & - & 23.9 & 7.3 & 364 \\
\hline REW & 562 & 0.0 & 0.00 & 0 & -- & -- & -- & -- & -- \\
\hline MEAN & & 6.2 & 0.67 & & & & & & \\
\hline TOTAL & 562 & & & 2,318 & 125.40 & 584 & & & \\
\hline
\end{tabular}




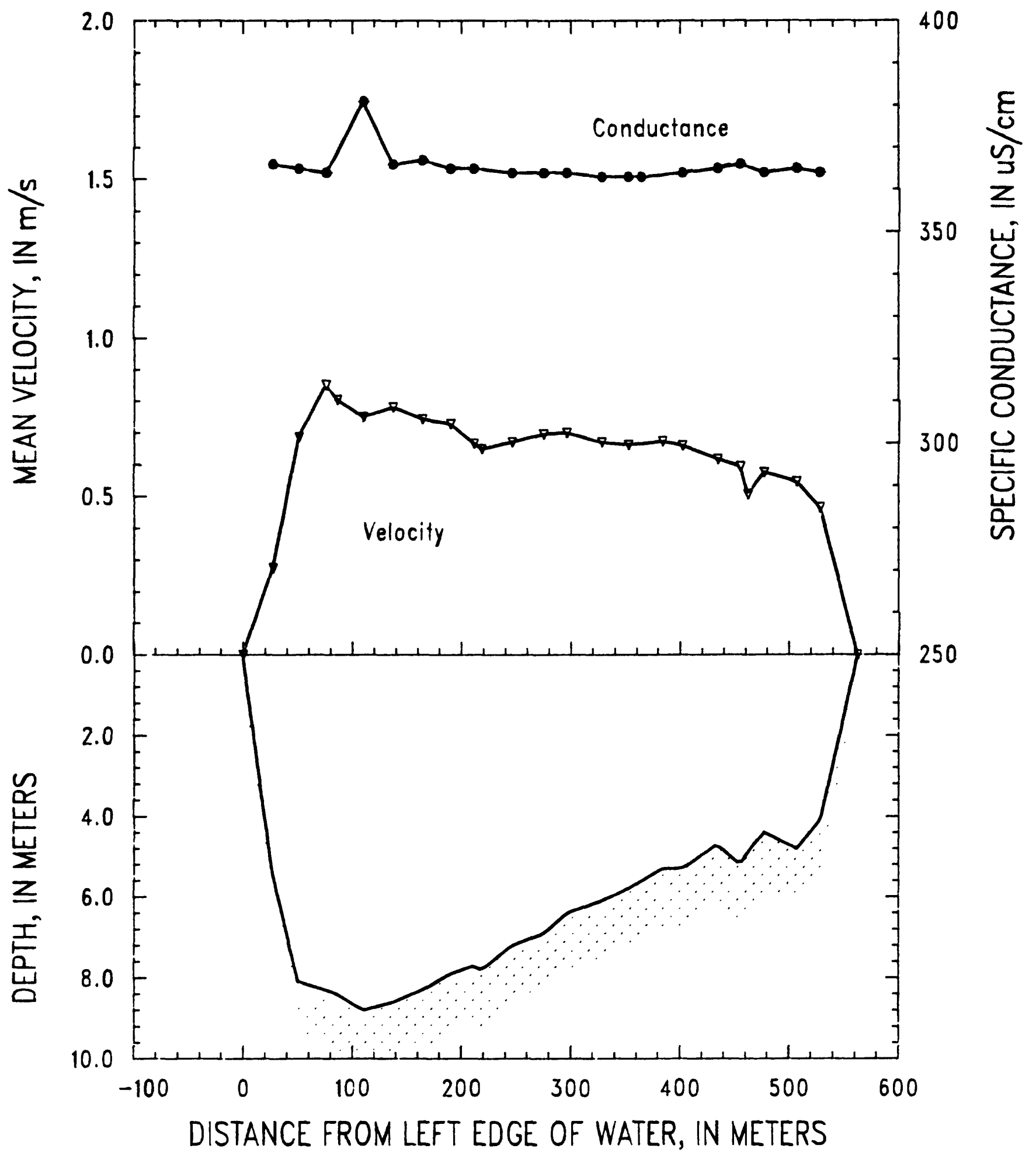

Figure 27. Mississippi River near Winfield, Missouri, on June 5, 1989. 
SITE: Illinois River at Hardin, Illinois

PARTY: Moody, Stevens, and Black

METER: SOLID CUP

STARTING GAGE HEIGHT: -- ENDING GAGE HEIGHT: --

SUSP: Bag sampler and 150-1b weight

METER NO.: P8308282 DATE RATED: 07-08-88

CURRENT METER EQUATION: $V(\mathrm{~m} / \mathrm{s})=$ RPS $* 0.765+0.006$

REMARKS: Transit rate was variable and nozzle was 5/16 inch. Suspendedsediment sample collected from vertical 1 at $158 \mathrm{~m}$ from the LEW.

\begin{tabular}{|c|c|c|c|c|c|c|c|c|c|}
\hline \multirow[b]{2}{*}{$\begin{array}{c}\text { Verti- } \\
\text { cal }\end{array}$} & \multirow{2}{*}{$\begin{array}{l}\text { Dist. } \\
\text { from } \\
\text { LEW } \\
\text { (m) }\end{array}$} & \multirow[b]{2}{*}{$\begin{array}{c}\text { Depth } \\
(\mathrm{m})\end{array}$} & \multirow{2}{*}{$\begin{array}{l}\text { Mean } \\
\text { veloc- } \\
\text { ity } \\
(\mathrm{m} / \mathrm{s})\end{array}$} & \multirow[b]{2}{*}{$\begin{array}{l}\text { Dis- } \\
\text { charge } \\
\left(\mathrm{m}^{3} / \mathrm{s}\right)\end{array}$} & \multicolumn{2}{|c|}{ Volume } & \multirow[b]{2}{*}{$\begin{array}{l}\text { Temper- } \\
\text { ature } \\
\left({ }^{\circ} \mathrm{C}\right)\end{array}$} & \multirow[b]{2}{*}{$\mathrm{pH}$} & \multirow{2}{*}{$\begin{array}{c}\text { Specific } \\
\text { conduct- } \\
\text { ance } \\
(\mu \mathrm{S} / \mathrm{cm})\end{array}$} \\
\hline & & & & & $\begin{array}{l}\overline{\mathbf{v}_{\mathbf{i}}} \\
(\mathrm{L})\end{array}$ & $\begin{array}{l}\overline{v_{p}} \\
(L)\end{array}$ & & & \\
\hline LEW & 0 & 0.0 & 0.00 & 0 & -- & - & -- & -- & -- \\
\hline 06 & 53 & 3.3 & 0.59 & 109 & -- & -- & -- & -- & -- \\
\hline 05 & 112 & 4.2 & 0.63 & 139 & - & - & -- & -- & - \\
\hline 01 & 158 & 5.6 & 0.70 & 172 & 94.56 & 502 & 24.1 & 7.51 & 729 \\
\hline 04 & 199 & 6.2 & 0.79 & 170 & -- & -- & -- & -- & -- \\
\hline 03 & 227 & 5.9 & 0.77 & 108 & -- & - & - & -- & -- \\
\hline 02 & 247 & 4.7 & 0.72 & 78 & -- & - & -- & -- & -- \\
\hline REW & 273 & 0.0 & 0.00 & 0 & - & - & - & -- & -- \\
\hline MEAN & & 4.1 & 0.70 & & & & & & \\
\hline TOTAL & 273 & & & 776 & 94.56 & 502 & & & \\
\hline
\end{tabular}


SITE: Missouri River at Hermann, Missouri

06-07-89

PARTY: Moody, Stevens, and Black

METER: SOLID CUP

STARTING GAGE HEIGHT: $7.85 \mathrm{ft}$ ENDING GAGE HEIGHT: --

SUSP: Bag sampler and $200-1 \mathrm{~b}$ weight

CURRENT METER NO.: P8308282 DATE RATED: 07-08-88

CURRENT METER EQUATION: $V(\mathrm{~m} / \mathrm{s})=$ RPS $* 0.765+0.006$

REMARKS: Transit rate was $14 \mathrm{~cm} / \mathrm{s}$ and nozzle 5/16 inch. Rising stage-$7.87 \mathrm{ft}$ at 0845 Central. Daylight Time and $9.05 \mathrm{ft}$ at approximately 1100 Central Daylight Time.

\begin{tabular}{|c|c|c|c|c|c|c|c|c|c|}
\hline \multirow[b]{2}{*}{$\begin{array}{c}\text { Verti- } \\
\text { cal }\end{array}$} & \multirow{2}{*}{$\begin{array}{l}\text { Dist. } \\
\text { from } \\
\text { LEW } \\
\text { (m) }\end{array}$} & \multirow[b]{2}{*}{$\begin{array}{l}\text { Depth } \\
\text { (m) }\end{array}$} & \multirow{2}{*}{$\begin{array}{c}\text { Mean } \\
\text { veloc- } \\
\text { ity } \\
(\mathrm{m} / \mathrm{s})\end{array}$} & \multirow[b]{2}{*}{$\begin{array}{l}\text { Dis- } \\
\text { charge } \\
\left(\mathrm{m}^{3} / \mathrm{s}\right)\end{array}$} & \multicolumn{2}{|c|}{ Volume } & \multirow[b]{2}{*}{$\begin{array}{l}\text { Temper- } \\
\text { ature } \\
\left({ }^{\circ} \mathrm{C}\right)\end{array}$} & \multirow[b]{2}{*}{$\mathrm{pH}$} & \multirow{2}{*}{$\begin{array}{c}\text { Specific } \\
\text { conduct- } \\
\text { ance } \\
(\mu \mathrm{S} / \mathrm{cm})\end{array}$} \\
\hline & & & & & $\begin{array}{l}\mathrm{V}_{\mathbf{i}} \\
(\mathrm{L})\end{array}$ & $\begin{array}{l}\mathrm{V}_{\mathrm{p}} \\
(\mathrm{L})\end{array}$ & & & \\
\hline LEW & 0 & 0.0 & 0.00 & 0 & -- & -- & -- & -- & -- \\
\hline $01 \mathrm{~A}$ & 12 & 6.9 & 1.11 & 61 & 5.92 & 19 & 23.5 & 6.3 & 720 \\
\hline $02 B$ & 16 & 6.8 & 1.22 & 67 & 5.82 & 21 & 23.3 & 7.0 & 764 \\
\hline $03 \mathrm{~A}$ & 28 & 6.8 & 1.27 & 104 & 6.11 & 20 & 23.2 & 7.5 & 697 \\
\hline $04 B$ & 40 & 6.5 & 1.48 & 96 & 6.98 & 25 & 23.3 & 7.8 & 699 \\
\hline $05 \mathrm{~A}$ & 48 & 6.2 & 1.44 & 67 & 5.99 & 23 & 23.3 & 7.8 & 683 \\
\hline $06 \mathrm{~B}$ & 55 & 6.1 & 1.33 & 57 & 5.72 & 22 & 23.2 & 7.9 & 692 \\
\hline $07 \mathrm{~A}$ & 62 & 5.7 & 1.32 & 45 & 5.77 & 21 & 23.4 & 7.9 & 678 \\
\hline 08B & 67 & 5.7 & 1.27 & 72 & 5.75 & 19 & 23.4 & 7.9 & 671 \\
\hline $09 \mathrm{~A}$ & 82 & 6.0 & 1.44 & 69 & 4.92 & 20 & 23.3 & 8.1 & 652 \\
\hline $10 B$ & 83 & 5.6 & 1.38 & 42 & 5.50 & 22 & 23.2 & 8.1 & 651 \\
\hline $11 \mathrm{~A}$ & 93 & 5.7 & 1.34 & 84 & 4.75 & 22 & 23.9 & 8.1 & 643 \\
\hline $12 B$ & 105 & 4.8 & 1.27 & 58 & 4.78 & 22 & 23.5 & 8.1 & 631 \\
\hline $13 \mathrm{~A}$ & 112 & 5.0 & 1.46 & 73 & 5.17 & 16 & 23.3 & 8.1 & 617 \\
\hline $14 \mathrm{~B}$ & 125 & 5.3 & 1.36 & 54 & 5.03 & 16 & 23.4 & 8.1 & 615 \\
\hline $15 \mathrm{~A}$ & 127 & 5.2 & 1.28 & 23 & 4.92 & 16 & 22.9 & 8.1 & 599 \\
\hline $\mathrm{X} 04$ & 132 & 5.4 & 1.35 & 40 & -- & - & - & -. & $\ldots$ \\
\hline $16 \mathrm{~B}$ & 138 & 5.2 & 1.26 & 49 & 4.88 & 17 & 23.8 & 8.1 & 597 \\
\hline $17 \mathrm{~A}$ & 147 & 5.2 & 1.28 & 70 & 4.50 & 15 & 23.1 & 8.1 & 588 \\
\hline $18 \mathrm{~B}$ & 159 & 4.9 & 1.25 & 43 & 4.33 & 17 & 23.8 & 8.1 & 581 \\
\hline $19 \mathrm{~A}$ & 161 & 5.1 & 1.12 & 48 & 3.32 & 16 & 22.9 & 7.8 & 579 \\
\hline $20 \mathrm{~B}$ & 176 & 4.8 & 1.25 & 57 & 4.16 & 13 & 22.8 & 7.5 & 570 \\
\hline $21 \mathrm{~A}$ & 180 & 4.7 & 1.30 & 55 & 3.99 & 14 & 22.7 & 7.3 & 565 \\
\hline $22 \mathrm{~B}$ & 194 & 4.3 & 1.11 & 48 & 3.79 & 13 & 22.6 & 7.5 & 557 \\
\hline $23 \mathrm{~A}$ & 200 & 4.3 & 1.06 & 32 & 3.42 & 14 & 22.9 & 7.6 & 557 \\
\hline $24 B$ & 208 & 4.3 & 1.34 & 60 & 3.86 & 17 & 22.8 & 7.6 & 555 \\
\hline $25 \mathrm{~A}$ & 221 & 4.2 & 1.07 & 34 & 3.23 & 15 & 22.6 & 7.6 & 546 \\
\hline $26 \mathrm{~B}$ & 223 & 4.2 & 1.03 & 28 & 2.96 & 13 & 22.9 & 7.6 & 545 \\
\hline $27 \mathrm{~A}$ & 234 & 4.4 & 1.09 & 48 & 3.19 & 13 & 22.9 & 7.5 & 548 \\
\hline $28 \mathrm{~B}$ & 243 & 4.8 & 1.03 & 40 & 3.08 & 12 & 23.0 & 7.5 & 548 \\
\hline $29 \mathrm{~A}$ & 250 & 6.2 & 0.99 & 52 & 3.80 & 11 & 22.9 & 7.6 & 547 \\
\hline $30 \mathrm{~B}$ & 260 & 6.8 & 1.16 & 79 & 4.55 & 15 & 22.9 & 7.6 & 548 \\
\hline REW & 270 & 0.0 & 0.00 & 0 & -- & $\ldots$ & -- & -- & -- \\
\hline MEAN & & 5.2 & 1.26 & & & & & & \\
\hline TOTAL & 270 & & & 1,755 & 138.56 & 519 & & & \\
\hline
\end{tabular}




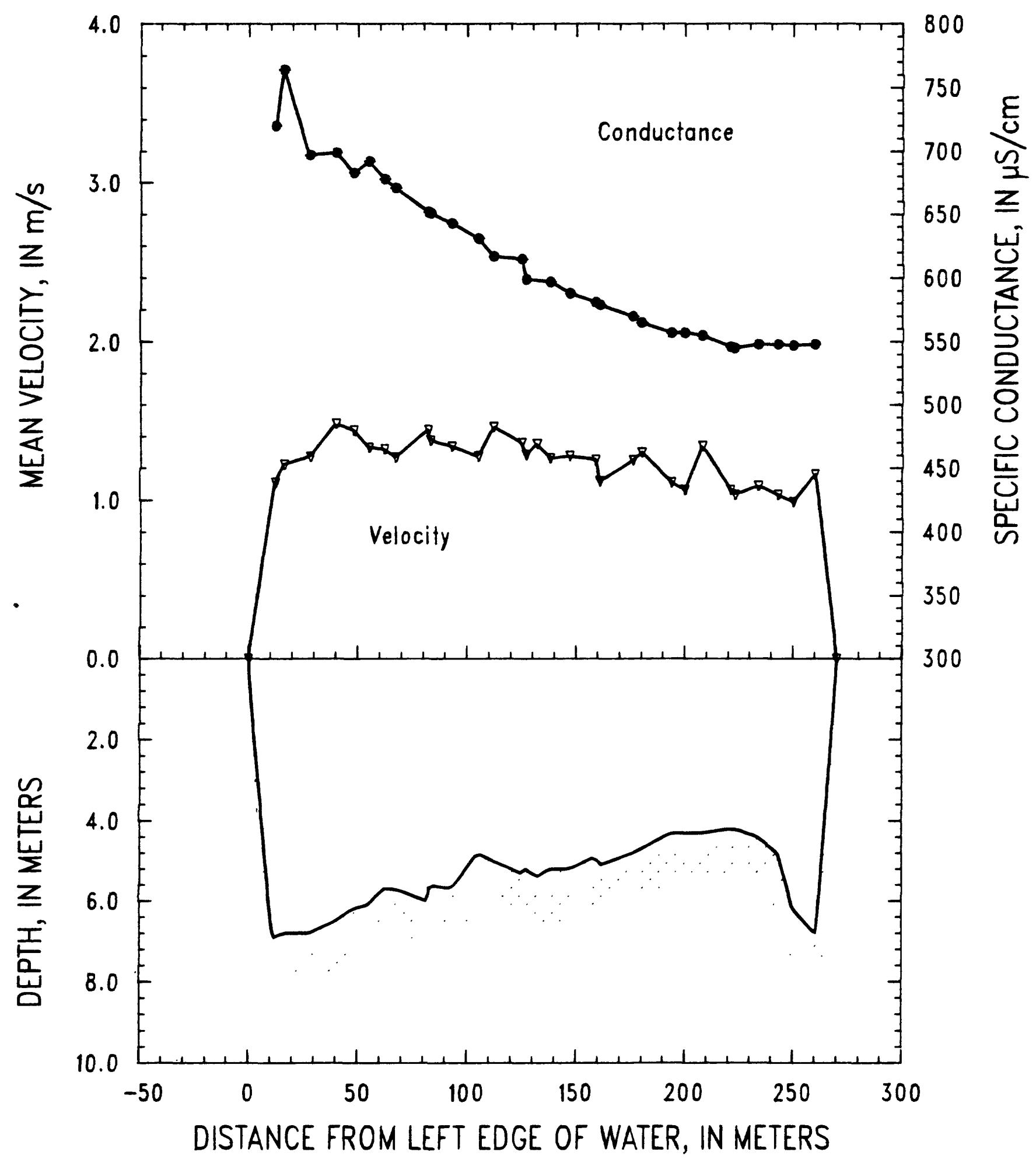

Figure 28. Missouri River at Hermann, Missouri, on June 7, 1989. 
SITE: Mississippi River at St. Louis, Missouri

06-08-89

PARTY: Moody, Stevens, and Simoneaux

METER: SOLID CUP

STARTING GAGE HEIGHT: -- ENDING GAGE HEIGHT: --

SUSP: Bag sampler and 150-1b weight

CURRENT METER NO.: P8308282 DATE RATED: 07-08-88

CURRENT METER EQUATION: $\mathrm{V}(\mathrm{m} / \mathrm{s})=$ RPS $* 0.765+0.006$

REMARKS: Transit rate was $14 \mathrm{~cm} / \mathrm{s}$ and nozzle was $1 / 4$ inch. Simultaneous discharge measurement made by George Gray, USGS Missouri District, from the Poplar Street Bridge. Verticals occupied in order for depth-integrated sampling and surface-water measurements .

\begin{tabular}{|c|c|c|c|c|c|c|c|c|c|}
\hline \multirow[b]{2}{*}{$\begin{array}{c}\text { Verti- } \\
\text { cal }\end{array}$} & \multirow{2}{*}{$\begin{array}{l}\text { Dist. } \\
\text { from } \\
\text { LEW } \\
\text { (m) }\end{array}$} & \multirow[b]{2}{*}{$\begin{array}{l}\text { Depth } \\
\text { (m) }\end{array}$} & \multirow{2}{*}{$\begin{array}{l}\text { Mean } \\
\text { veloc- } \\
\text { ity } \\
(\mathrm{m} / \mathrm{s})\end{array}$} & \multirow[b]{2}{*}{$\begin{array}{r}\text { Dis- } \\
\text { charge } \\
\left(\mathrm{m}^{3} / \mathrm{s}\right)\end{array}$} & \multicolumn{2}{|c|}{ Volume } & \multirow[b]{2}{*}{$\begin{array}{l}\text { Temper- } \\
\text { ature } \\
\left({ }^{\circ} \mathrm{C}\right)\end{array}$} & \multirow[b]{2}{*}{$\mathrm{pH}$} & \multirow{2}{*}{$\begin{array}{c}\text { Specific } \\
\text { conduct- } \\
\text { ance } \\
(\mu \mathrm{S} / \mathrm{cm})\end{array}$} \\
\hline & & & & & $\begin{array}{l}\mathrm{V}_{i} \\
(\mathrm{~L})\end{array}$ & $\begin{array}{l}\mathrm{v}_{\mathrm{p}} \\
(\mathrm{L})\end{array}$ & & & \\
\hline LEW & 0 & 0.0 & 0.00 & 0 & - & - & -- & -- & -- \\
\hline $\mathrm{X} 01$ & 15 & 1.7 & 0.25 & 6 & -- & -- & -- & -- & -- \\
\hline $\mathrm{X} 02$ & 30 & 9.1 & 0.36 & 57 & -- & -- & -- & -- & -- \\
\hline $01 \mathrm{~A}$ & 50 & 11.0 & 1.22 & 276 & 4.96 & 18 & 24.6 & 6.3 & 467 \\
\hline $02 B$ & 71 & 10.8 & 1.43 & 279 & 6.52 & 25 & 24.6 & 6.5 & 466 \\
\hline $03 \mathrm{~A}$ & 86 & 10.8 & 1.50 & 210 & 5.59 & 38 & 24.7 & 6.7 & 462 \\
\hline $04 \mathrm{~B}$ & 97 & 10.8 & 1.47 & 222 & 6.30 & 32 & 24.6 & 6.7 & 465 \\
\hline $05 \mathrm{~A}$ & 114 & 10.7 & 1.42 & 258 & 5.85 & 30 & 24.7 & 6.8 & 466 \\
\hline 06B & 131 & 10.6 & 1.33 & 218 & 5.67 & 27 & 24.7 & 6.8 & 465 \\
\hline 07A & 145 & 10.1 & 1.36 & 185 & 5.32 & 26 & 24.8 & 6.9 & 466 \\
\hline $08 \mathrm{~B}$ & 158 & 9.6 & 1.22 & 182 & 4.93 & 25 & 24.7 & 7.0 & 472 \\
\hline $09 A$ & 176 & 9.0 & 1.39 & 168 & 4.87 & 23 & 24.7 & 7.0 & 480 \\
\hline $10 \mathrm{~B}$ & 185 & 8.8 & 1.34 & 189 & 4.85 & 21 & 24.7 & 7.0 & 480 \\
\hline $11 \mathrm{~A}$ & 208 & 8.3 & 1.44 & 197 & 4.85 & 20 & 24.8 & 7.0 & 501 \\
\hline $12 B$ & 218 & 8.1 & 1.12 & 136 & 3.93 & 26 & 24.8 & 7.0 & 511 \\
\hline $13 \mathrm{~A}$ & 238 & 7.7 & 1.40 & 178 & 4.28 & 18 & 24.6 & 7.0 & 507 \\
\hline $14 B$ & 251 & 7.5 & 1.24 & 121 & 3.60 & 17 & 24.6 & 7.1 & 534 \\
\hline $15 \mathrm{~A}$ & 264 & 7.3 & 1.40 & 123 & 3.98 & 20 & 24.8 & 7.1 & 530 \\
\hline $16 \mathrm{~B}$ & 275 & 7.3 & 1.32 & 158 & 3.84 & 18 & 24.7 & 7.1 & 557 \\
\hline $17 \mathrm{~A}$ & 292 & 7.1 & 1.21 & 150 & 3.70 & 18 & 24.8 & 7.2 & 561 \\
\hline $18 \mathrm{~B}$ & 310 & 6.9 & 1.16 & 124 & 3.14 & 18 & 24.7 & 7.2 & 577 \\
\hline $19 \mathrm{~A}$ & 328 & 6.7 & 1.22 & 126 & 3.37 & 18 & 24.6 & 7.2 & 598 \\
\hline $20 \mathrm{~B}$ & 341 & 6.7 & 1.17 & 114 & 2.82 & 18 & 24.8 & 7.2 & 606 \\
\hline $21 \mathrm{~A}$ & 357 & 6.6 & 1.13 & 130 & 2.83 & 15 & 24.7 & 7.3 & 613 \\
\hline 22B & 376 & 6.3 & 1.33 & 155 & 2.62 & 15 & 24.7 & 7.3 & 629 \\
\hline $23 \mathrm{~A}$ & 394 & 6.3 & 1.19 & 101 & 3.06 & 15 & 24.8 & 7.4 & 634 \\
\hline $24 B$ & 403 & 6.2 & 1.06 & 79 & 2.33 & 15 & 24.7 & 7.4 & 640 \\
\hline $25 \mathrm{~A}$ & 418 & 6.2 & 1.20 & 104 & 2.69 & 14 & 24.7 & 7.4 & 652 \\
\hline $26 B$ & 431 & 6.7 & 1.09 & 109 & 2.50 & 14 & 24.6 & 7.4 & 655 \\
\hline $27 \mathrm{~A}$ & 448 & 6.8 & 1.11 & 117 & 3.05 & 15 & 24.9 & 7.4 & 659 \\
\hline $28 \mathrm{~B}$ & 462 & 6.7 & 1.18 & 118 & 2.90 & 15 & 24.6 & 7.4 & 667 \\
\hline $29 A$ & 478 & 6.8 & 1.02 & 101 & 2.96 & 17 & 24.6 & 7.5 & 669 \\
\hline $30 \mathrm{~B}$ & 491 & 6.4 & 0.66 & 64 & 1.29 & 7 & 24.6 & 7.5 & 672 \\
\hline REW & 508 & 0.0 & 0.00 & 0 & -- & -- & -- & -- & -- \\
\hline MEAN & & 7.7 & 1.22 & & & & & & \\
\hline TOTAL & 508 & & & 4,755 & 118.60 & 598 & & & \\
\hline
\end{tabular}




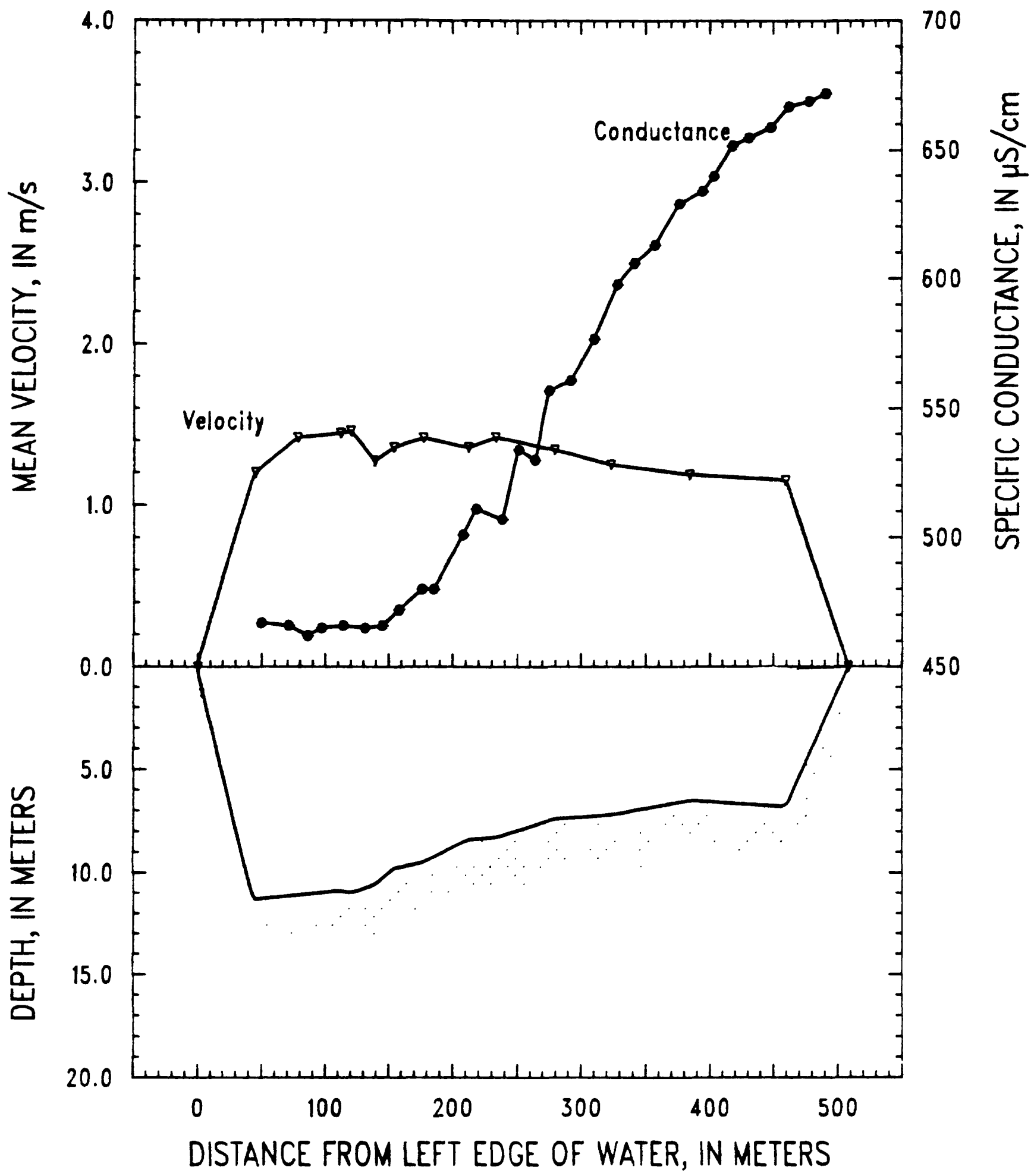

Figure 29. Mississippi River at St. Louis, Missouri, on June 8, 1989. 
SITE: Mississippi River at St. Louis, Missouri

PARTY: Moody, Stevens, and Simoneaux

06-09-89

STARTING GAGE HEIGHT: -- ENDING GAGE HEIGHT

METER: SOLID CUP

SUSP: Bag sampler and 150-1b weight

CURRENT METER NO.: P8308282 DATE KATED: 07-08-88

CURRENT METER EQUATION: $\mathrm{V}(\mathrm{m} / \mathrm{s})=$ RPS $* 0.765+0.006$

REMARKS: Nozzle was $1 / 4$ inch. Values to the right of the $S$ represent the surface-water sample and values to the right of the $D$ represent the depthintegrated sample.

Verticals $1-10$ are spaced at equal-discharge increments.

Used variable transit rates to collect full bottles at each vertical.

Larger suspended-sediment concentrations from Missouri River rise of

June 06-07 may have arrived on section since 06-08-89.

\begin{tabular}{|c|c|c|c|c|c|c|c|c|c|c|c|}
\hline \multirow{2}{*}{$\begin{array}{l}\text { Verti- } \\
\text { cal }\end{array}$} & \multirow{2}{*}{$\begin{array}{l}\text { Dist. } \\
\text { from } \\
\text { LEW } \\
\text { (m) }\end{array}$} & \multirow{2}{*}{$\begin{array}{l}\text { Depth } \\
\text { (m) }\end{array}$} & \multirow{2}{*}{$\begin{array}{l}\text { Mean } \\
\text { veloc- } \\
\text { ity } \\
(\mathrm{m} / \mathrm{s})\end{array}$} & \multirow{2}{*}{$\begin{array}{c}\text { Dis- } \\
\text { charge } \\
\left(\mathrm{m}^{3} / \mathrm{s}\right)\end{array}$} & \multirow{2}{*}{\multicolumn{2}{|c|}{$\begin{array}{l}\mathrm{v}_{\mathrm{i}} \\
(\mathrm{L})\end{array}$}} & \multicolumn{2}{|c|}{$\begin{array}{c}\text { Suspended } \\
\text { sediment } \\
(\mathrm{mg} / \mathrm{L})\end{array}$} & \multirow{2}{*}{$\begin{array}{c}\text { Temper- } \\
\text { ature } \\
\left({ }^{\circ} \mathrm{C}\right)\end{array}$} & \multirow[b]{2}{*}{$\mathrm{pH}$} & \multirow{2}{*}{$\begin{array}{c}\text { Specific } \\
\text { conduct- } \\
\text { ance } \\
(\mu \mathrm{S} / \mathrm{cm})\end{array}$} \\
\hline & & & & & & & $<63 \mu \mathrm{m}$ & $>63 \mu \mathrm{m}$ & & & \\
\hline LEW & 0 & 0.0 & 0.00 & 0 & & -- & -- & -- & -- & -- & -- \\
\hline \multirow[t]{2}{*}{01} & 45 & 11.3 & 1.20 & 529 & $S$ & -- & -- & -- & 24.0 & 6.6 & 445 \\
\hline & & & & & D & 5.63 & 101 & 8 & 23.7 & 7.3 & 456 \\
\hline \multirow[t]{2}{*}{02} & 78 & 11.1 & 1.42 & 527 & $\mathrm{~S}$ & -- & - & - & 23.6 & 6.7 & 465 \\
\hline & & & & & D & 5.95 & 104 & 23 & 23.2 & 7.7 & 451 \\
\hline \multirow[t]{2}{*}{03} & 112 & 10.9 & 1.45 & 331 & $\mathrm{~S}$ & -- & -- & -- & 23.9 & 7.6 & 456 \\
\hline & & & & & $\mathrm{D}$ & 5.74 & 111 & 33 & 23.5 & 7.9 & 451 \\
\hline \multirow[t]{2}{*}{13} & 120 & 11.0 & 1.46 & 217 & $\mathrm{~S}$ & -- & -- & -- & 23.6 & 8.1 & 455 \\
\hline & & & & & D & 5.92 & 117 & 25 & 23.4 & 8.1 & 452 \\
\hline \multirow[t]{2}{*}{04} & 139 & 10.6 & 1.27 & 229 & $\mathrm{~S}$ & -- & -- & -- & 23.5 & 7.7 & 458 \\
\hline & & & & & D & 4.85 & 123 & 40 & 23.1 & 8.0 & 456 \\
\hline \multirow[t]{2}{*}{12} & 154 & 9.8 & 1.36 & 253 & $\mathrm{~S}$ & -- & -- & -- & 23.7 & 8.1 & 470 \\
\hline & & & & & D & 5.16 & 128 & 51 & 23.3 & 8.0 & 458 \\
\hline \multirow[t]{2}{*}{05} & 177 & 9.5 & 1.42 & 391 & $\mathrm{~S}$ & -- & -- & -- & 23.6 & 7.9 & 499 \\
\hline & & & & & $\mathrm{D}$ & 5.37 & 136 & 41 & 23.6 & 8.0 & 466 \\
\hline \multirow[t]{2}{*}{11} & 212 & 8.4 & 1.36 & 319 & $\mathrm{~S}$ & -- & -- & -- & 24.0 & 8.1 & 493 \\
\hline & & & & & D & 5.91 & 170 & 67 & 23.7 & 8.1 & 485 \\
\hline \multirow[t]{2}{*}{06} & 233 & 8.3 & 1.42 & 395 & $\mathrm{~S}$ & -- & -- & -- & 22.9 & 8.0 & 504 \\
\hline & & & & & $\mathrm{D}$ & 4.87 & 190 & 77 & 23.4 & 8.1 & 503 \\
\hline \multirow[t]{2}{*}{07} & 279 & 7.4 & 1.34 & 447 & $S$ & -- & -- & -- & 23.3 & 8.0 & 525 \\
\hline & & & & & D & 4.40 & 221 & 73 & 23.6 & 8.1 & 525 \\
\hline \multirow[t]{2}{*}{08} & 323 & 7.2 & 1.25 & 473 & $\mathrm{~S}$ & -- & -- & - & 23.2 & 8.0 & 556 \\
\hline & & & & & D & 5.41 & 258 & 50 & 23.5 & 8.1 & 553 \\
\hline \multirow[t]{2}{*}{09} & 384 & 6.5 & 1.19 & 524 & $\mathrm{~S}$ & -- & -- & -- & 23.1 & 8.1 & 578 \\
\hline & & & & & D & 6.37 & 344 & 33 & 23.5 & 8.1 & 583 \\
\hline \multirow[t]{2}{*}{10} & 459 & 6.8 & 1.15 & 484 & $\mathrm{~S}$ & -- & -- & -- & 23.1 & 8.1 & 604 \\
\hline & & & & & D & 5.02 & 309 & 20 & 23.1 & 8.1 & 605 \\
\hline REW & 508 & 0.0 & 0.00 & 0 & & -- & -- & -- & -- & -- & -- \\
\hline MEAN & & 7.7 & 1.31 & & & & & & & & \\
\hline TOTAL & 508 & & & 5,119 & & & & & & & \\
\hline
\end{tabular}




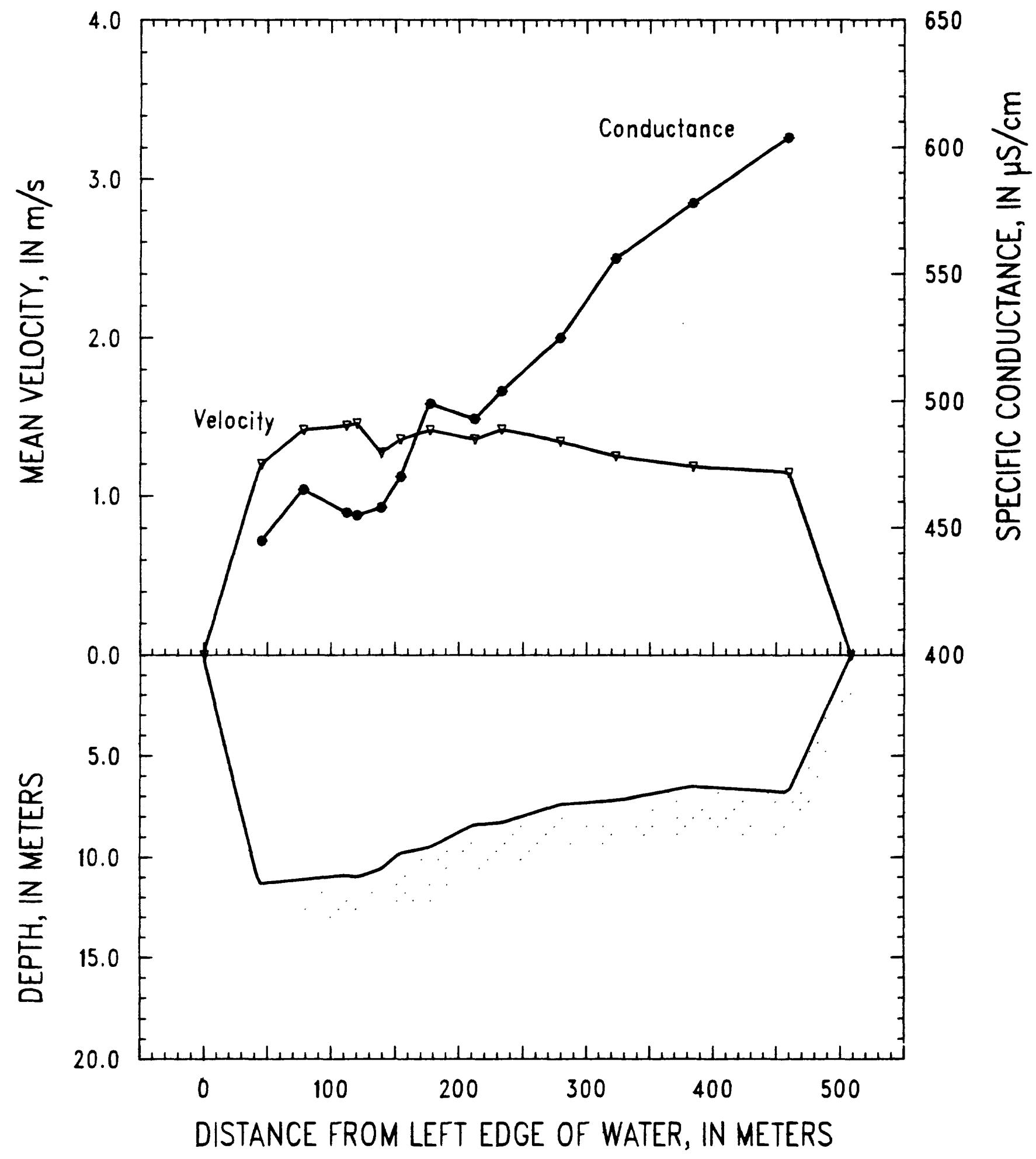

Figure 30. Mississippi River at St. Louis, Missouri, on June 9, 1989. 


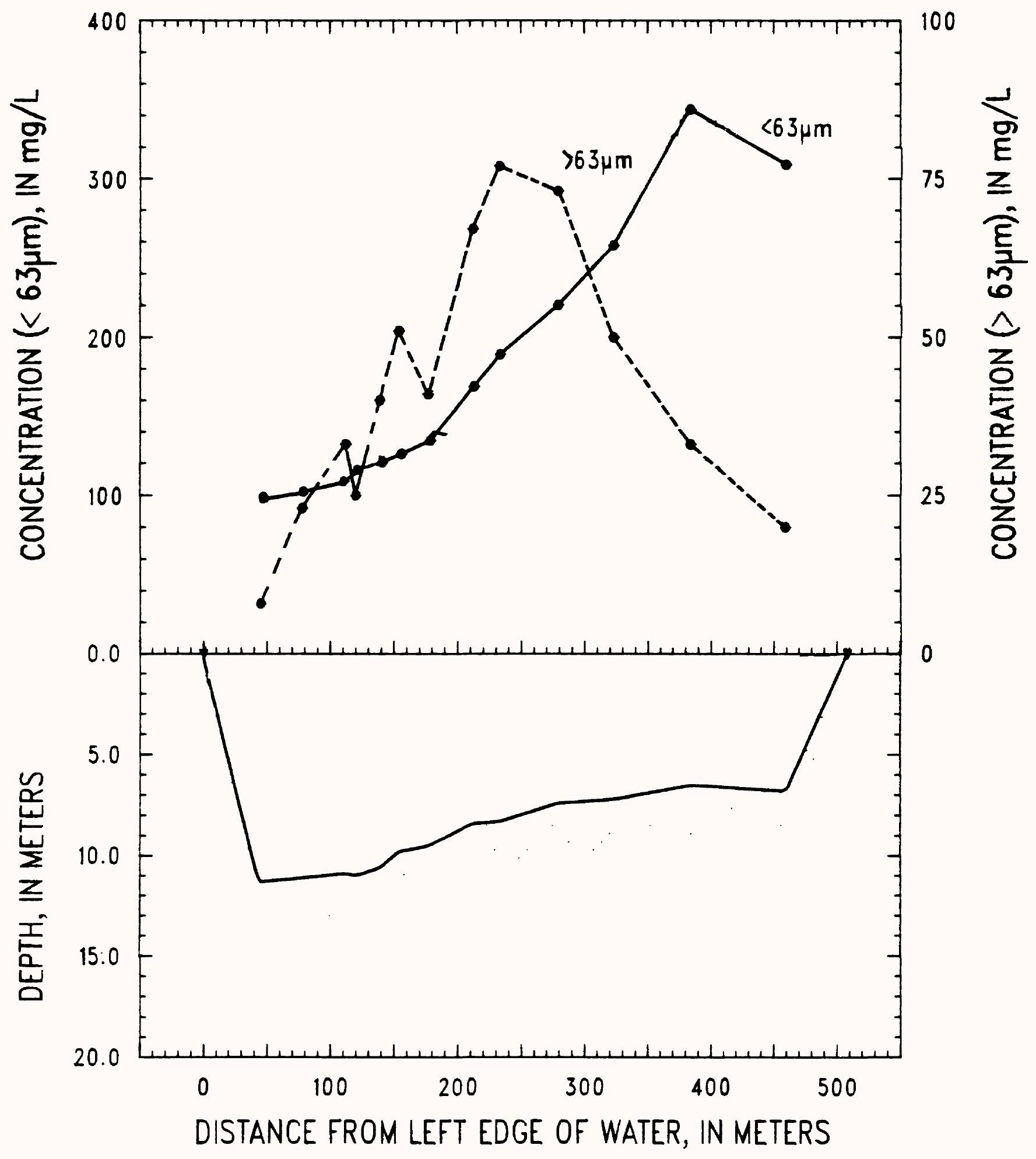

Figure 31. Mississippi River at St. Louis, Missouri, on June 9, 1989. Depth-integrated suspended-sediment concentration. 
SITE: Mississippi River at Thebes, Illinois

06-10-89

PARTY: Moody, Stevens, and Simoneaux

METER: SOLID CUP

STARTING GAGE HEIGHT: -- ENDING GAGE HEIGHT: --

SUSP: Bag sampler and 150-1b weight

CURRENT METER NO.: P8308282 DATE RATED: 07-08-88

CURRENT METER EQUATION: $V(\mathrm{~m} / \mathrm{s})=$ RPS $* 0.765+0.006$

REMARKS: Transit rate was $12 \mathrm{~cm} / \mathrm{s}$ and nozzle was $1 / 4$ inch. Verticals 28 , 29 , and 30 collected at 28 . Used distances and depths from cross-section profile for verticals 28,29 , and 30 .

\begin{tabular}{|c|c|c|c|c|c|c|c|c|c|}
\hline \multirow[b]{2}{*}{$\begin{array}{c}\text { Verti- } \\
\text { cal }\end{array}$} & \multirow{2}{*}{$\begin{array}{l}\text { Dist. } \\
\text { from } \\
\text { LEW } \\
(\mathrm{m})\end{array}$} & \multirow[b]{2}{*}{$\begin{array}{l}\text { Depth } \\
(\mathrm{m})\end{array}$} & \multirow{2}{*}{$\begin{array}{l}\text { Mean } \\
\text { veloc- } \\
\text { ity } \\
(\mathrm{m} / \mathrm{s})\end{array}$} & \multirow[b]{2}{*}{$\begin{array}{r}\text { Dis- } \\
\text { charge } \\
\left(m^{3} / s\right)\end{array}$} & \multicolumn{2}{|c|}{ Volume } & \multirow[b]{2}{*}{$\begin{array}{l}\text { Temper- } \\
\text { ature } \\
\left({ }^{\circ} \mathrm{C}\right)\end{array}$} & \multirow[b]{2}{*}{$\mathrm{pH}$} & \multirow{2}{*}{$\begin{array}{l}\text { Specific } \\
\text { conduct- } \\
\text { ance } \\
(\mu \mathrm{S} / \mathrm{cm})\end{array}$} \\
\hline & & & & & $\begin{array}{l}\mathrm{V}_{\mathrm{i}} \\
(\mathrm{L})\end{array}$ & $\begin{array}{l}\bar{v}_{p} \\
(I)\end{array}$ & & & \\
\hline$\overline{L E W}$ & 0 & 0.0 & 0.00 & -- & -- & -- & -- & -- & -- \\
\hline $01 \mathrm{~A}$ & 35 & 5.3 & 0.57 & 62 & 0.85 & 3 & 24.2 & 6.90 & 493 \\
\hline $02 B$ & 41 & 5.2 & 0.74 & 54 & 1.61 & 6 & 24.1 & 6.97 & 507 \\
\hline $03 \mathrm{~A}$ & 63 & 7.1 & 0.83 & 121 & 2.37 & 11 & 24.3 & 7.02 & 484 \\
\hline $04 \mathrm{~B}$ & 82 & 8.2 & 1.12 & 175 & 4.12 & 17 & 24.4 & 7.04 & 508 \\
\hline $05 \mathrm{~A}$ & 101 & 8.4 & 1.37 & 196 & 5.06 & 19 & 24.6 & 7.01 & 506 \\
\hline 06B & 116 & 8.6 & 1.55 & 281 & 6.02 & 23 & 24.7 & 7.04 & 512 \\
\hline $07 \mathrm{~A}$ & 143 & 8.9 & 1.47 & 256 & 6.20 & 26 & 24.7 & 7.08 & 521 \\
\hline 08B & 155 & 8.9 & 1.53 & 251 & 6.00 & 28 & 25.0 & 7.11 & 513 \\
\hline $09 \mathrm{~A}$ & 180 & 8.4 & 1.60 & 316 & 6.24 & 27 & 25.9 & 7.12 & 511 \\
\hline $10 \mathrm{~B}$ & 202 & 8.4 & 1.59 & 254 & 6.19 & 26 & 25.3 & 7.20 & 512 \\
\hline $11 \mathrm{~A}$ & 218 & 8.4 & 1.56 & 269 & 6.16 & 25 & 25.1 & 7.20 & 514 \\
\hline $12 \mathrm{~B}$ & 243 & 8.3 & 1.56 & 272 & 6.09 & 25 & 24.9 & 7.20 & 521 \\
\hline $13 \mathrm{~A}$ & 260 & 8.1 & 1.46 & 207 & 5.49 & 23 & 25.0 & 7.20 & 516 \\
\hline $14 \mathrm{~B}$ & 278 & 7.7 & 1.59 & 245 & 5.46 & 21 & 25.1 & 7.35 & 518 \\
\hline X03 & 295 & 7.3 & 1.48 & 87 & -- & -- & -- & -- & -- \\
\hline $15 \mathrm{~A}$ & 300 & 8.3 & 1.66 & 117 & 4.78 & 21 & 25.2 & 7.31 & 520 \\
\hline $16 \mathrm{~B}$ & 316 & 7.7 & 1.44 & 200 & 4.94 & 20 & 24.9 & 7.26 & 524 \\
\hline $17 \mathrm{~A}$ & 331 & 7.2 & 1.52 & 247 & 5.45 & 21 & 25.3 & 7.17 & 518 \\
\hline $18 \mathrm{~B}$ & 361 & 7.7 & 1.39 & 198 & 4.70 & 20 & 25.2 & 7.28 & 528 \\
\hline $19 \mathrm{~A}$ & 368 & 7.5 & 1.34 & 156 & 4.77 & 18 & 25.4 & 7.32 & 519 \\
\hline $20 \mathrm{~B}$ & 392 & 7.8 & 1.27 & 218 & 4.15 & 19 & 25.2 & 7.33 & 521 \\
\hline $21 \mathrm{~A}$ & 412 & 7.7 & 1.13 & 170 & 4.30 & 17 & 25.4 & 7.34 & 529 \\
\hline $22 B$ & 431 & 7.8 & 1.17 & 182 & 3.83 & 19 & 25.6 & 7.33 & 521 \\
\hline $23 \mathrm{~A}$ & 452 & 7.0 & 1.05 & 155 & 3.13 & 17 & 25.1 & 7.38 & 527 \\
\hline $24 \mathrm{~B}$ & 473 & 6.3 & 1.15 & 159 & 2.98 & 15 & 25.0 & 7.38 & 527 \\
\hline $25 \mathrm{~A}$ & 496 & 5.2 & 1.03 & 96 & 2.56 & 10 & 25.1 & 7.39 & 528 \\
\hline $26 \mathrm{~B}$ & 509 & 5.0 & 1.11 & 78 & 2.02 & 8 & 24.9 & 7.40 & 527 \\
\hline $27 \mathrm{~A}$ & 524 & 4.1 & 0.78 & 33 & 2.93 & 6 & 24.8 & 7.46 & 528 \\
\hline X05 & 530 & 3.9 & 0.91 & 46 & - & - & - & -- & - \\
\hline $28 \mathrm{~B}$ & 550 & 2.6 & 0.77 & 40 & 1.77 & 9 & 25.0 & 7.42 & 527 \\
\hline $29 \mathrm{~A}$ & 570 & 3.0 & 0.77 & 45 & - & -- & - & -- & -- \\
\hline $30 \mathrm{~B}$ & 589 & 3.0 & 0.77 & 45 & -- & -- & -- & -- & -- \\
\hline REW & 609 & 0.0 & 0.00 & 0 & -- & -- & -- & -- & -- \\
\hline MEAN & & 6.6 & 1.30 & & & & & & \\
\hline TOTAL & 609 & & & 5,231 & 120.17 & 500 & & & \\
\hline
\end{tabular}




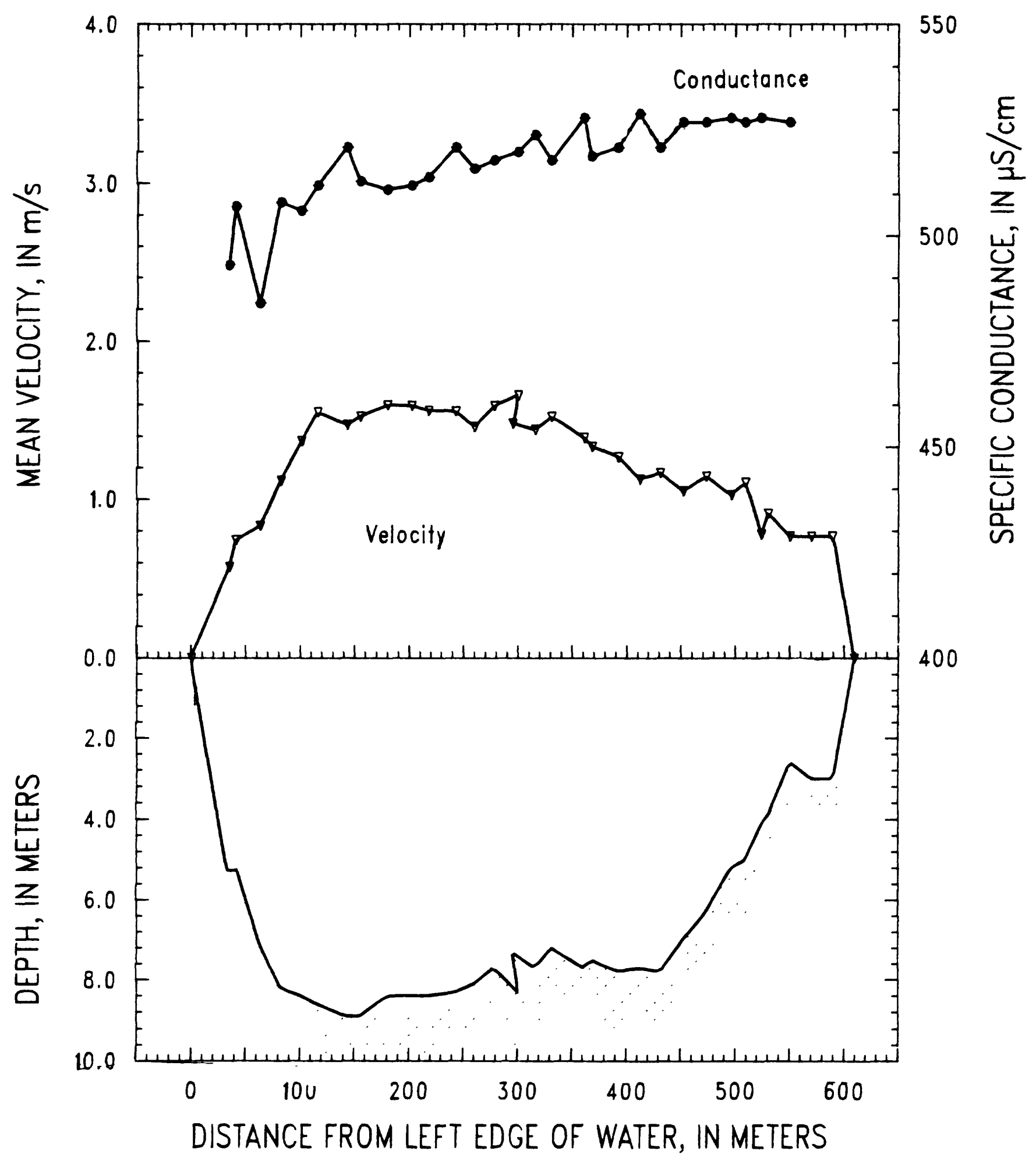

Figure 32. Mississippi River at Thebes, Illinois, on June 10, 1989. 
SITE: Ohio River at Olmsted, Illinois

06-11-89

PARTY: Moody, Stevens, and Simoneaux

METER: SOLID CUP

STARTING GAGE HEIGHT: -- ENDING GAGE HEIGHT: --

SUSP: Bag sampler and 200-1b weight

CURRENT METER NO.: P8308282 DATE RATED: 07-08-88

CURRENT METER EQUATION: $V(\mathrm{~m} / \mathrm{s})=$ RPS $* 0.765+0.006$

REMARKS: Transit rate was $10 \mathrm{~cm} / \mathrm{s}$ and nozzle was $1 / 4$ inch. $X$ verticals measured first, then verticals 30 to 1 .

\begin{tabular}{|c|c|c|c|c|c|c|c|c|c|}
\hline \multirow[b]{2}{*}{$\begin{array}{c}\text { Verti- } \\
\text { cal }\end{array}$} & \multirow{2}{*}{$\begin{array}{l}\text { Dist. } \\
\text { from } \\
\text { LEW } \\
\text { (m) }\end{array}$} & \multirow[b]{2}{*}{$\begin{array}{c}\text { Depth } \\
\text { (m) }\end{array}$} & \multirow{2}{*}{$\begin{array}{l}\text { Mean } \\
\text { veloc- } \\
\text { ity } \\
(\mathrm{m} / \mathrm{s})\end{array}$} & \multirow[b]{2}{*}{$\begin{array}{r}\text { Dis- } \\
\text { charge } \\
\left(\mathrm{m}^{3} / \mathrm{s}\right)\end{array}$} & \multicolumn{2}{|c|}{ Volume } & \multirow[b]{2}{*}{$\begin{array}{l}\text { Temper- } \\
\text { ature } \\
\left({ }^{\circ} \mathrm{C}\right)\end{array}$} & \multirow[b]{2}{*}{$\mathrm{pH}$} & \multirow{2}{*}{$\begin{array}{l}\text { Specific } \\
\text { conduct- } \\
\text { ance } \\
(\mu \mathrm{S} / \mathrm{cm})\end{array}$} \\
\hline & & & & & $\begin{array}{l}\overline{V_{i}} \\
(L)\end{array}$ & $\begin{array}{l}\mathrm{V}_{\mathrm{p}} \\
(\mathrm{L})\end{array}$ & & & \\
\hline LEW & 0 & 0.0 & 0.00 & 0 & -- & -- & - & -- & -- \\
\hline $01 \mathrm{~A}$ & 37 & 4.9 & 0.47 & 78 & 0.85 & 4 & 24.2 & 7.0 & 202 \\
\hline O2B & 68 & 5.4 & 0.54 & 96 & 1.02 & 7 & 24.2 & 7.6 & 205 \\
\hline $03 A$ & 96 & 6.7 & 0.64 & 73 & 1.88 & 9 & 24.2 & 7.6 & 208 \\
\hline X09 & 103 & 6.8 & 0.66 & 63 & -- & -- & -- & - & -- \\
\hline 04B & 137 & 6.8 & 0.73 & 157 & 2.32 & 10 & 24.1 & 7.6 & 215 \\
\hline 05A & 159 & 7.1 & 0.76 & 159 & 2.81 & 11 & 24.2 & 7.6 & 224 \\
\hline O6B & 196 & 7.4 & 0.83 & 203 & 2.83 & 12 & 24.1 & 7.6 & 231 \\
\hline 07A & 225 & 7.8 & 0.82 & 214 & 2.50 & 12 & 24.2 & 7.6 & 238 \\
\hline O8B & 263 & 7.8 & 0.83 & 257 & 3.17 & 13 & 24.2 & 7.6 & 257 \\
\hline 09A & 304 & 7.8 & 0.80 & 215 & 3.34 & 13 & 24.1 & 7.6 & 252 \\
\hline $10 \mathrm{~B}$ & 332 & 8.2 & 0.90 & 210 & 3.84 & 14 & 24.0 & 7.6 & 267 \\
\hline $11 \mathrm{~A}$ & 361 & 8.7 & 0.95 & 222 & 4.12 & 17 & 24.0 & 7.6 & 270 \\
\hline X03 & 386 & 9.2 & 0.96 & 160 & - & -- & - & -- & -- \\
\hline $12 \mathrm{~B}$ & 397 & 9.3 & 1.00 & 224 & 4.64 & 20 & 24.2 & 7.6 & 276 \\
\hline $13 \mathrm{~A}$ & 434 & 11.6 & 0.89 & 321 & 4.32 & 27 & 23.8 & 7.6 & 294 \\
\hline $14 B$ & 459 & 11.8 & 0.94 & 305 & 5.56 & 28 & 24.1 & 7.6 & 302 \\
\hline $15 \mathrm{~A}$ & 489 & 12.3 & 0.96 & 406 & 5.62 & 29 & 23.8 & 7.6 & 310 \\
\hline $16 \mathrm{~B}$ & 528 & 12.7 & 0.91 & 364 & 5.48 & 33 & 23.9 & 7.6 & 308 \\
\hline $17 \mathrm{~A}$ & 552 & 11.7 & 1.05 & 395 & 5.86 & 33 & 24.0 & 7.6 & 317 \\
\hline $18 \mathrm{~B}$ & 592 & 11.3 & 1.06 & 366 & 6.17 & 31 & 23.9 & 7.6 & 331 \\
\hline $19 \mathrm{~A}$ & 613 & 11.4 & 1.08 & 320 & 5.81 & 27 & 23.5 & 7.6 & 334 \\
\hline $20 \mathrm{~B}$ & 644 & 11.2 & 1.10 & 233 & 6.43 & 27 & 23.3 & 7.6 & 348 \\
\hline $\mathrm{X} 02$ & 651 & 10.9 & 1.14 & 218 & -- & -- & - & -- & - \\
\hline X06 & 679 & 11.1 & 1.01 & 185 & -- & -- & - & -- & -- \\
\hline $21 \mathrm{~A}$ & 684 & 11.2 & 1.04 & 181 & 5.16 & 27 & 23.1 & 7.6 & 367 \\
\hline $22 B$ & 710 & 10.7 & 1.15 & 357 & 6.32 & 25 & 23.0 & 7.6 & 369 \\
\hline $23 A$ & 742 & 10.6 & 1.06 & 372 & 6.21 & 23 & 23.1 & 7.6 & 381 \\
\hline $24 B$ & 776 & 10.7 & 1.08 & 392 & 5.74 & 23 & 23.2 & 7.6 & 378 \\
\hline $25 \mathrm{~A}$ & 810 & 11.6 & 1.11 & 387 & 6.40 & 24 & 23.7 & 7.5 & 389 \\
\hline 26B & 836 & 11.4 & 1.10 & 363 & 6.39 & 23 & 24.0 & 7.5 & 397 \\
\hline X05 & 868 & 11.7 & 1.07 & 256 & -- & -- & -- & -- & - \\
\hline $27 \mathrm{~A}$ & 877 & 12.0 & 1.03 & 279 & 5.45 & 24 & 24.1 & 7.5 & 402 \\
\hline $28 \mathrm{~B}$ & 913 & 12.7 & 0.91 & 324 & 5.91 & 27 & 24.0 & 7.4 & 399 \\
\hline $29 A$ & 933 & 13.3 & 0.75 & 303 & 4.66 & 26 & 24.1 & 7.2 & 399 \\
\hline $30 \mathrm{~B}$ & 974 & 5.6 & 0.49 & 103 & 1.08 & 5 & 24.2 & 6.9 & 397 \\
\hline REW & 1,008 & 0.0 & 0.00 & 0 & -- & -- & -- & -- & -- \\
\hline MEAN & & 9.3 & 0.94 & & & & & & \\
\hline TOTAL & 1,008 & & & 8,761 & 131.89 & 604 & & & \\
\hline
\end{tabular}




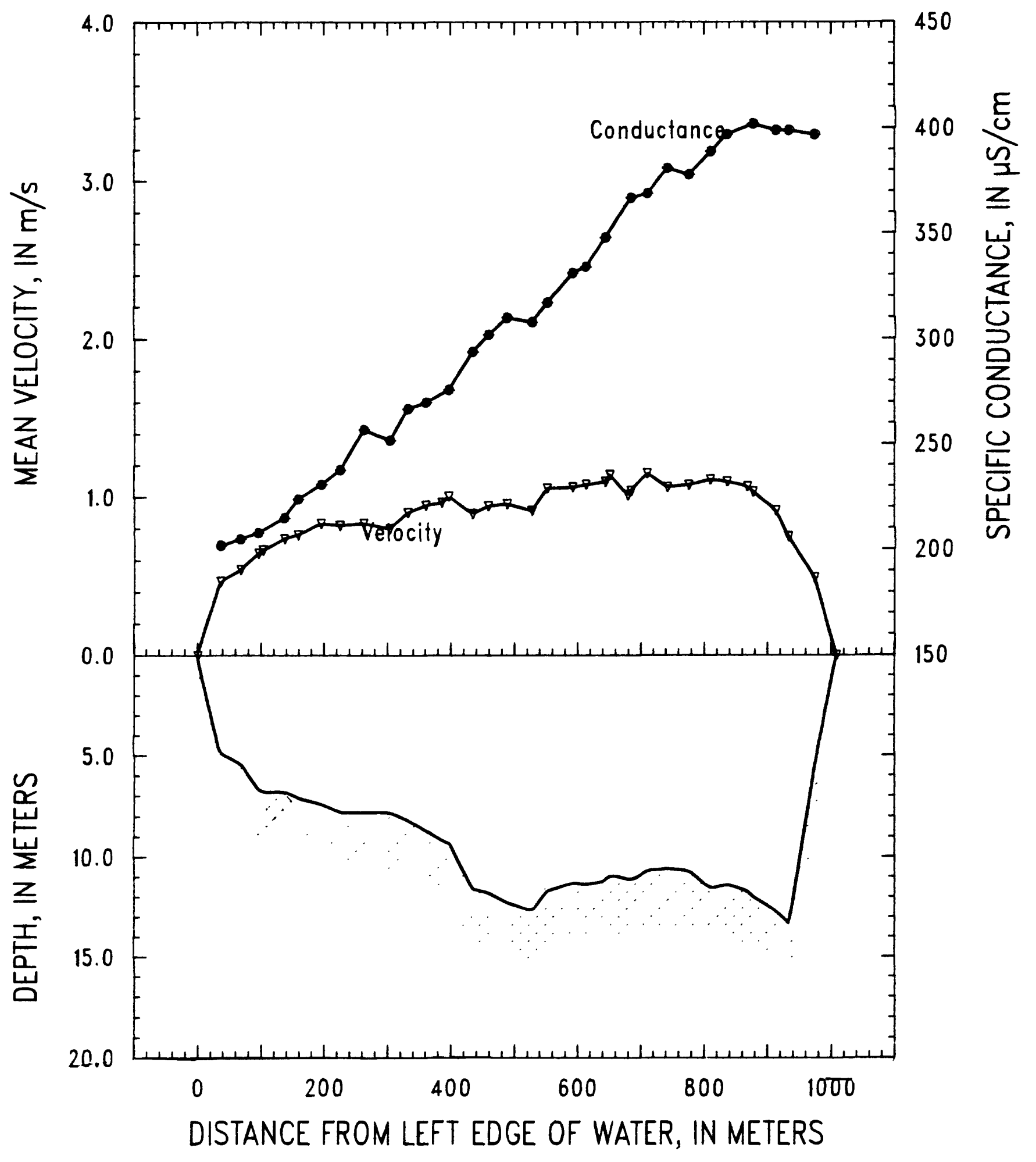

Figure 33. Ohio River at Olmsted, Illinois, on June 11, 1989. 
SITE: Mississippi River below Hickman, Kentucky 06-12-89

PARTY: Moody, Stevens, and Simoneaux METER: SOLID CUP STARTING GAGE HEIGHT: $22.84 \mathrm{ft}$ ENDING GAGE HEIGHT: $23.10 \mathrm{ft}$

SUSP: Bag sampler and 200-lb weight

CURRENT METER NO.: P8308282 DATE RATED: 07-08-88

CURRENT METER EQUATION: $V(\mathrm{~m} / \mathrm{s})=$ RPS $* 0.765+0.006$

REMARKS: Transit rate was $10 \mathrm{~cm} / \mathrm{s}$ and nozzle was $3 / 16$ inch. The 5-vertical composite sample was collected with the test velocities.

\begin{tabular}{|c|c|c|c|c|c|c|c|c|c|}
\hline \multirow[b]{2}{*}{$\begin{array}{c}\text { Verti- } \\
\text { cal }\end{array}$} & \multirow{2}{*}{$\begin{array}{l}\text { Dist. } \\
\text { from } \\
\text { LEW } \\
\text { (m) }\end{array}$} & \multirow[b]{2}{*}{$\begin{array}{l}\text { Depth } \\
(\mathrm{m})\end{array}$} & \multirow{2}{*}{$\begin{array}{l}\text { Mean } \\
\text { veloc- } \\
\text { ity } \\
(\mathrm{m} / \mathrm{s})\end{array}$} & \multirow[b]{2}{*}{$\begin{array}{r}\text { Dis- } \\
\text { charge } \\
\left(\mathrm{m}^{3} / \mathrm{s}\right)\end{array}$} & \multicolumn{2}{|c|}{ Volume } & \multirow[b]{2}{*}{$\begin{array}{c}\text { Temper- } \\
\text { ature } \\
\left({ }^{\circ} \mathrm{C}\right)\end{array}$} & \multirow[b]{2}{*}{$\mathrm{pH}$} & \multirow{2}{*}{$\begin{array}{c}\text { Specific } \\
\text { conduct- } \\
\text { ance } \\
(\mu \mathrm{S} / \mathrm{cm})\end{array}$} \\
\hline & & & & & $\begin{array}{l}\overline{v_{i}} \\
(L)\end{array}$ & $\begin{array}{l}\mathrm{V}_{\mathrm{p}} \\
(\mathrm{L})\end{array}$ & & & \\
\hline LEW & 0 & 0.0 & 0.00 & 0 & -- & -- & -- & -- & -- \\
\hline $01 \mathrm{~A}$ & 42 & 9.5 & 1.18 & 488 & 3.27 & 10 & 24.5 & 7.7 & 324 \\
\hline 02B & 87 & 9.3 & 1.44 & 535 & 4.51 & 16 & 24.9 & 7.7 & 327 \\
\hline $03 \mathrm{~A}$ & 122 & 9.5 & 1.63 & 394 & 5.49 & 18 & 25.0 & 7.7 & 332 \\
\hline X04 & 138 & 9.7 & 1.56 & 264 & -- & -- & -- & -- & -- \\
\hline 04B & 157 & 9.8 & 1.52 & 350 & 4.95 & 19 & 24.6 & 7.7 & 332 \\
\hline $05 \mathrm{~A}$ & 185 & 9.9 & 1.56 & 524 & 5.64 & 20 & 24.7 & 7.8 & 335 \\
\hline 06B & 225 & 9.5 & 1.42 & 465 & 4.69 & 19 & 24.3 & 7.8 & 335 \\
\hline $07 \mathrm{~A}$ & 254 & 9.1 & 1.50 & 486 & 4.76 & 17 & 24.2 & 7.8 & 340 \\
\hline 08B & 296 & 9.0 & 1.27 & 468 & 3.76 & 18 & 24.3 & 7.8 & 337 \\
\hline $09 \mathrm{~A}$ & 336 & 8.4 & 1.48 & 287 & 4.00 & 16 & 24.2 & 7.8 & 343 \\
\hline $\mathrm{X} 02$ & 342 & 8.7 & 1.48 & 219 & -- & -- & -- & -- & -- \\
\hline $10 \mathrm{~B}$ & 370 & 8.3 & 1.27 & 358 & 3.74 & 16 & 24.0 & 7.8 & 346 \\
\hline $11 \mathrm{~A}$ & 410 & 8.8 & 1.36 & 455 & 3.47 & 18 & 24.1 & 7.8 & 349 \\
\hline $12 B$ & 446 & 8.3 & 1.35 & 374 & 3.59 & 12 & 24.4 & 7.8 & 353 \\
\hline $13 \mathrm{~A}$ & 477 & 7.7 & 1.29 & 431 & 2.75 & 15 & 24.5 & 7.8 & 358 \\
\hline $14 \mathrm{~B}$ & 533 & 7.7 & 1.51 & 494 & 3.69 & 12 & 24.2 & 7.8 & 358 \\
\hline $15 \mathrm{~A}$ & 562 & 7.7 & 1.16 & 170 & 2.99 & 14 & 24.2 & 7.7 & 381 \\
\hline X03 & 571 & 7.8 & 1.44 & 62 & -- & -- & -- & - & -- \\
\hline 16B & 573 & 7.5 & 1.41 & 316 & 3.15 & 17 & 25.2 & 7.7 & 385 \\
\hline $17 \mathrm{~A}$ & 631 & 7.9 & 1.28 & 465 & 2.97 & 18 & 24.8 & 7.7 & 391 \\
\hline $18 \mathrm{~B}$ & 665 & 9.3 & 1.27 & 361 & 4.10 & 17 & 24.5 & 7.7 & 395 \\
\hline $19 \mathrm{~A}$ & 692 & 9.5 & 1.33 & 499 & 4.28 & 17 & 25.2 & 7.7 & 404 \\
\hline $20 \mathrm{~B}$ & 744 & 10.1 & 1.37 & 610 & 4.85 & 17 & 25.2 & 7.7 & 420 \\
\hline $21 \mathrm{~A}$ & 780 & 11.2 & 1.47 & 478 & 4.94 & 16 & 24.7 & 7.7 & 424 \\
\hline $22 \mathrm{~B}$ & 802 & 11.2 & 1.29 & 224 & 5.24 & 19 & 24.0 & 7.7 & 428 \\
\hline X08 & 811 & 11.3 & 1.36 & 361 & -- & -- & -- & -- & - \\
\hline $23 A$ & 849 & 11.7 & 1.20 & 553 & 5.19 & 24 & 27.6 & 7.7 & 439 \\
\hline $24 B$ & 890 & 12.1 & 1.23 & 491 & 4.43 & 19 & 27.6 & 7.7 & 445 \\
\hline $25 \mathrm{~A}$ & 915 & 12.2 & 1.38 & 496 & 4.81 & 19 & 27.6 & 7.7 & 448 \\
\hline $26 B$ & 949 & 12.6 & 1.26 & 579 & 5.69 & 20 & 27.7 & 7.7 & 463 \\
\hline $27 \mathrm{~A}$ & 988 & 12.7 & 1.24 & 605 & 4.70 & 19 & 27.7 & 7.7 & 467 \\
\hline X06 & 1,026 & 12.3 & 1.24 & 312 & -- & -- & -- & -- & -- \\
\hline $28 \mathrm{~B}$ & 1,029 & 12.7 & 1.11 & 190 & 5.02 & 18 & 27.6 & 7.7 & 463 \\
\hline $29 A$ & 1,053 & 11.9 & 1.04 & 377 & 3.79 & 19 & 27.6 & 7.7 & 467 \\
\hline $30 \mathrm{~B}$ & 1,090 & 8.8 & 0.97 & 350 & 2.04 & 7 & 27.8 & 7.6 & 473 \\
\hline REW & 1,135 & 0.0 & 0.00 & 0 & -- & -- & - & -- & -- \\
\hline MEAN & & 9.4 & 1.33 & & & & & & \\
\hline TOTAL & 1,135 & & & 14,091 & 126.00 & 506 & & & \\
\hline
\end{tabular}




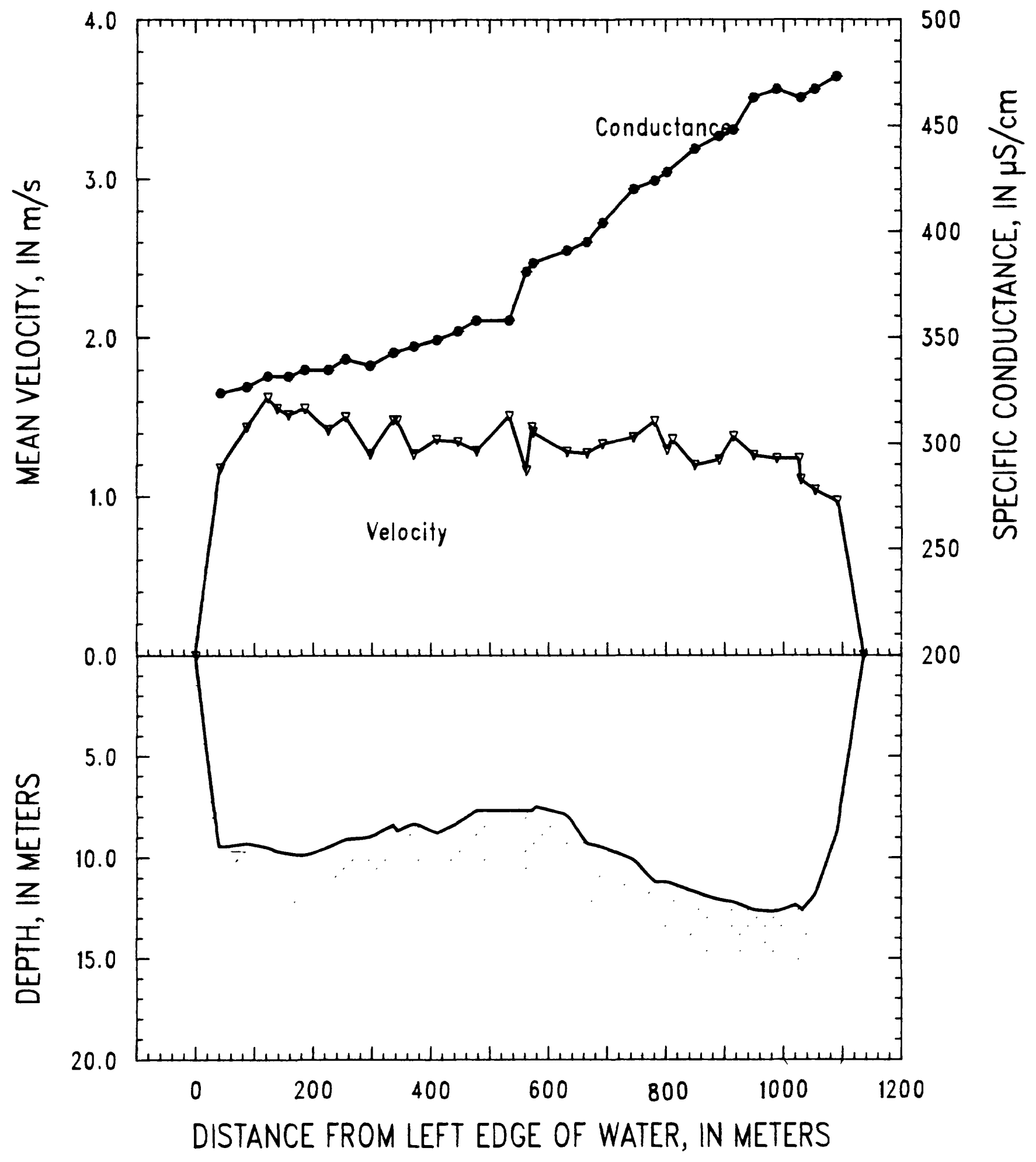

Figure 34. Mississippi River below Hickman, Kentucky, on June 12, 1989. 
SITE: Mississippi River below Hickman, Kentucky

METER: $\begin{array}{r}06-13-89 \\ \text { SOLID CUP }\end{array}$

PARTY: Moody, Stevens, and Simoneaux

STARTING GAGE HEIGHT: -- ENDING GAGE HEIGHT: --

SUSP: Bag sampler and 200-1b weight

CURRENT METER NO.: P8308282 DATE RATED: 07-08-88

CURRENT METER EQUATION: $V(\mathrm{~m} / \mathrm{s})=$ RPS $* 0.765+0.006$

REMARKS: Nozzle was $3 / 16$ inch. Varied transit rates to get full bottles at each vertical. Values to the right of the $S$ represent the surface-water sample and values to the right of the $D$ represent the depth-integrated water sample. There was a temporal change in suspended-sediment concentration of about $1 \mathrm{mg} / \mathrm{L}$ per minute.

\begin{tabular}{|c|c|c|c|c|c|c|c|c|c|c|c|}
\hline \multirow{2}{*}{$\begin{array}{l}\text { Verti- } \\
\text { cal }\end{array}$} & \multirow{2}{*}{$\begin{array}{l}\text { Dist. } \\
\text { from } \\
\text { LEW } \\
(\mathrm{m})\end{array}$} & \multirow{2}{*}{$\begin{array}{l}\text { Depth } \\
\text { (m) }\end{array}$} & \multirow{2}{*}{$\begin{array}{l}\text { Mean } \\
\text { veloc- } \\
\text { ity } \\
(\mathrm{m} / \mathrm{s})\end{array}$} & \multirow{2}{*}{$\begin{array}{l}\text { Dis- } \\
\text { charge } \\
\left(\mathrm{m}^{3} / \mathrm{s}\right)\end{array}$} & \multirow{2}{*}{\multicolumn{2}{|c|}{$\begin{array}{l}\mathrm{v}_{\mathrm{i}} \\
(\mathrm{L})\end{array}$}} & \multicolumn{2}{|c|}{$\begin{array}{l}\text { Suspended } \\
\text { sediment } \\
(\mathrm{mg} / \mathrm{L}) \\
\end{array}$} & \multirow{2}{*}{$\begin{array}{l}\text { Temper- } \\
\text { ature } \\
\left({ }^{\circ} \mathrm{C}\right)\end{array}$} & \multirow[b]{2}{*}{$\mathrm{pH}$} & \multirow{2}{*}{$\begin{array}{c}\text { Specific } \\
\text { conduct- } \\
\text { ance } \\
(\mu S / \mathrm{cm})\end{array}$} \\
\hline & & & & & & & $<63 \mu \mathrm{m}$ & $>63 \mu \mathrm{m}$ & & & \\
\hline LEW & 0 & 0.0 & 0.00 & 0 & & -- & -- & -- & -- & -- & -- \\
\hline 01 & 64 & 9.2 & 1.18 & 939 & $\begin{array}{l}S \\
D\end{array}$ & -- & -- & -- & 23.0 & $\begin{array}{l}7.0 \\
7.2\end{array}$ & 331 \\
\hline 02 & 173 & 10.2 & 1.53 & 1,750 & $\begin{array}{l}\mathrm{S} \\
\mathrm{D}\end{array}$ & 4.99 & 140 & -- & $\begin{array}{l}22.9 \\
22.9\end{array}$ & $\begin{array}{l}7.1 \\
7.2\end{array}$ & $\begin{array}{l}319 \\
335\end{array}$ \\
\hline 03 & 288 & 9.3 & 1.42 & 1,116 & $\begin{array}{l}S \\
D\end{array}$ & 6.37 & $\begin{array}{l}-- \\
140\end{array}$ & -- & $\begin{array}{l}23.2 \\
23.2\end{array}$ & $\begin{array}{l}7.2 \\
7.3\end{array}$ & $\begin{array}{l}331 \\
339\end{array}$ \\
\hline 13 & 342 & 8.8 & 1.48 & 720 & $\begin{array}{l}S \\
D\end{array}$ & 4.79 & 239 & -- & $\begin{array}{l}23.1 \\
23.2\end{array}$ & $\begin{array}{l}7.6 \\
7.6\end{array}$ & $\begin{array}{l}341 \\
352\end{array}$ \\
\hline 04 & 399 & 9.0 & 1.45 & 430 & $\begin{array}{l}S \\
D\end{array}$ & 4.62 & 154 & -- & $\begin{array}{l}23.2 \\
22.9\end{array}$ & $\begin{array}{l}7.2 \\
7.3\end{array}$ & $\begin{array}{l}352 \\
357\end{array}$ \\
\hline X01 & 408 & 9.1 & 1.36 & 272 & $\mathbf{S}$ & -- & -- & -- & -- & -- & -- \\
\hline 12 & 443 & 8.1 & 1.41 & 629 & $\begin{array}{l}S \\
D\end{array}$ & 5.75 & 242 & -- & $\begin{array}{l}23.2 \\
23.0\end{array}$ & $\begin{array}{l}7.7 \\
7.5\end{array}$ & $\begin{array}{l}347 \\
346\end{array}$ \\
\hline 05 & 518 & 7.9 & 1.41 & 700 & $\begin{array}{l}S \\
D\end{array}$ & 5.97 & 176 & -- & $\begin{array}{l}23.3 \\
23.2\end{array}$ & $\begin{array}{l}7.3 \\
7.4\end{array}$ & $\begin{array}{l}363 \\
376\end{array}$ \\
\hline 11 & 569 & 7.8 & 1.31 & 568 & $\begin{array}{l}\mathrm{S} \\
\mathrm{D}\end{array}$ & 4.30 & 231 & -- & $\begin{array}{l}23.5 \\
23.1\end{array}$ & $\begin{array}{l}7.7 \\
7.6\end{array}$ & $\begin{array}{l}358 \\
366\end{array}$ \\
\hline 06 & 629 & 8.7 & 1.28 & 667 & $\begin{array}{l}\mathrm{S} \\
\mathrm{D}\end{array}$ & 5.08 & $\begin{array}{r}-- \\
196\end{array}$ & $\overline{32}$ & $\begin{array}{l}23.3 \\
23.0\end{array}$ & $\begin{array}{l}7.4 \\
7.5\end{array}$ & $\begin{array}{l}377 \\
395\end{array}$ \\
\hline 14 & 689 & 9.6 & 1.40 & 755 & $\begin{array}{l}S \\
D\end{array}$ & 5.16 & $\begin{array}{r}-- \\
239\end{array}$ & $\begin{array}{l}-- \\
32\end{array}$ & $\begin{array}{l}23.3 \\
23.2\end{array}$ & $\begin{array}{l}7.6 \\
7.6\end{array}$ & $\begin{array}{l}399 \\
411\end{array}$ \\
\hline 07 & 741 & 10.4 & 1.37 & 806 & $\begin{array}{l}S \\
D\end{array}$ & 6.27 & $\begin{array}{r}-- \\
217\end{array}$ & $\begin{array}{l}-- \\
35\end{array}$ & $\begin{array}{l}23.4 \\
23.3\end{array}$ & $\begin{array}{l}7.4 \\
7.5\end{array}$ & $\begin{array}{l}413 \\
426\end{array}$ \\
\hline 15 & 802 & 11.4 & 1.34 & 925 & $\begin{array}{l}S \\
D\end{array}$ & 5.82 & 241 & -- & $\begin{array}{l}23.6 \\
23.4\end{array}$ & $\begin{array}{l}7.6 \\
7.5\end{array}$ & $\begin{array}{l}423 \\
437\end{array}$ \\
\hline 08 & 862 & 12.2 & 1.32 & 903 & $\begin{array}{l}S \\
D\end{array}$ & 5.44 & $\begin{array}{r}-- \\
236\end{array}$ & -- & $\begin{array}{l}23.3 \\
23.2\end{array}$ & $\begin{array}{l}7.5 \\
7.7\end{array}$ & $\begin{array}{l}439 \\
459\end{array}$ \\
\hline 16 & 914 & 12.3 & 1.24 & 792 & $\begin{array}{l}\mathrm{S} \\
\mathrm{D}\end{array}$ & 5.45 & 261 & $\begin{array}{l}-- \\
15\end{array}$ & $\begin{array}{l}23.5 \\
23.3\end{array}$ & $\begin{array}{l}7.6 \\
7.5\end{array}$ & $\begin{array}{l}443 \\
462\end{array}$ \\
\hline 09 & 966 & 12.3 & 1.15 & 1,179 & $\begin{array}{l}\mathrm{S} \\
\mathrm{D}\end{array}$ & 4.37 & 229 & -- & $\begin{array}{l}23.3 \\
23.2\end{array}$ & $\begin{array}{l}7.5 \\
7.6\end{array}$ & $\begin{array}{l}462 \\
477\end{array}$ \\
\hline 10 & 1,081 & 9.1 & 0.99 & 759 & $\begin{array}{l}S \\
D\end{array}$ & 5.03 & 208 & -- & $\begin{array}{l}23.4 \\
23.2\end{array}$ & $\begin{array}{l}7.5 \\
7.5\end{array}$ & $\begin{array}{l}474 \\
479\end{array}$ \\
\hline REW & 1,135 & 0.0 & 0.00 & 0 & & -- & -- & -- & -- & -- & -- \\
\hline $\begin{array}{l}\text { MEAN } \\
\text { TOTAL }\end{array}$ & 1,135 & 9.3 & 1.32 & 13,910 & & & & & & & \\
\hline
\end{tabular}




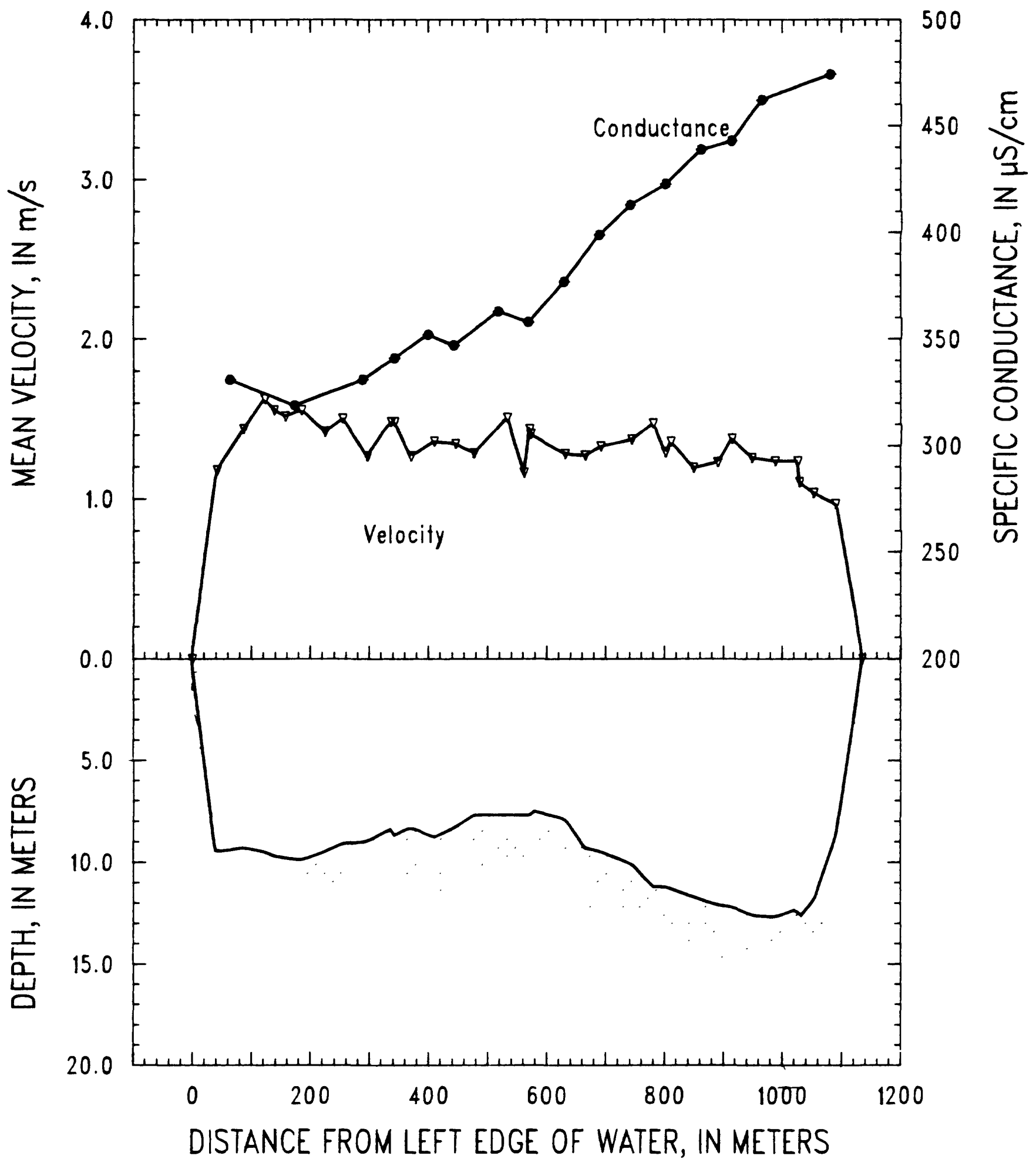

Figure 35. Mississippi River below Hickman, Kentucky, on June 13, 1989. 


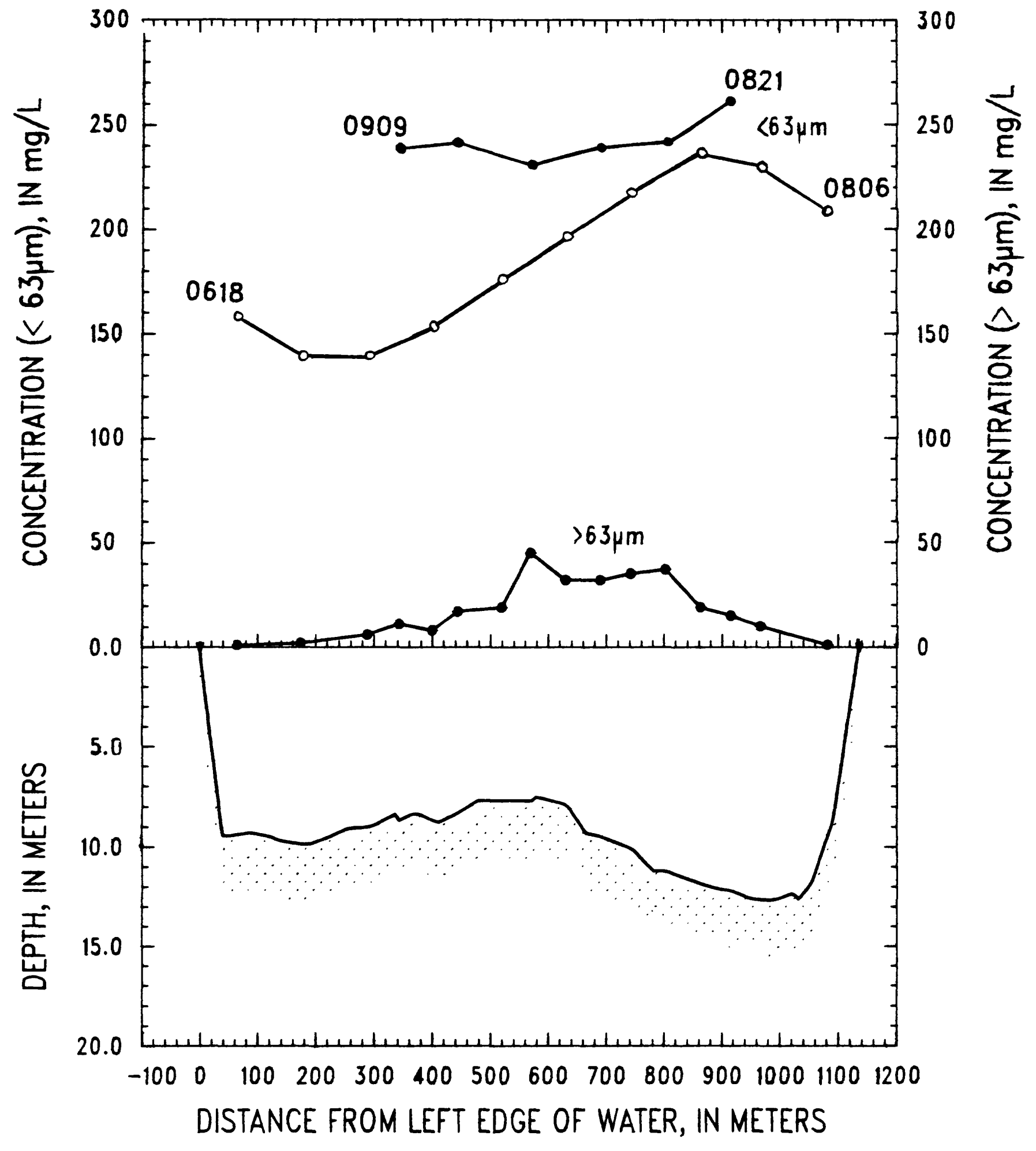

Figure 36. Mississippi River below Hickman, Kentucky, on June 13, 1989. Depth-integrated suspended-sediment concentration. Starting and ending sampling times are shown. 
SITE: Mississippi River at Fulton, Tennessee

PARTY: Moody, Stevens, and Simoneaux

06-14-89

STARTING GAGE HEIGHT: -- ENDING GAGE HEIGHT: --

SUSP: Bag sampler and 200-1b weight

CURRENT METER NO.: P8308282 DATE RATED: 07-08-88

CURRENT METER EQUATION: $\mathrm{V}(\mathrm{m} / \mathrm{s})=$ RPS $* 0.765+0.006$

REMARKS: Transit rate was $15 \mathrm{~cm} / \mathrm{s}$ and nozzle was $3 / 16$ inch. Five-vertical composite sample collected with test velocities. New solid cup in current meter.

Started depth-integrated sampling at vertical $3 \mathrm{~A}$.

Estimates for overflow channel must be added to width, area, and discharge totals below: Velocity $=0.8 \mathrm{~m} / \mathrm{s} ;$ Width $=200 \mathrm{~m} ;$ Area $=500 \mathrm{~m}^{2}$;

Discharge $=400 \mathrm{~m}^{2} / \mathrm{s}$.

\begin{tabular}{|c|c|c|c|c|c|c|c|c|c|}
\hline \multirow[b]{2}{*}{$\begin{array}{l}\text { Verti- } \\
\text { cal }\end{array}$} & \multirow{2}{*}{$\begin{array}{l}\text { Dist. } \\
\text { from } \\
\text { LEW } \\
\text { (m) }\end{array}$} & \multirow[b]{2}{*}{$\begin{array}{c}\text { Depth } \\
\text { (m) }\end{array}$} & \multirow{2}{*}{$\begin{array}{l}\text { Mean } \\
\text { veloc- } \\
\text { ity } \\
(\mathrm{m} / \mathrm{s})\end{array}$} & \multirow[b]{2}{*}{$\begin{array}{l}\text { Dis- } \\
\text { charge } \\
\left(\mathrm{m}^{3} / \mathrm{s}\right)\end{array}$} & \multicolumn{2}{|c|}{ Volume } & \multirow[b]{2}{*}{$\begin{array}{l}\text { Temper- } \\
\text { ature } \\
\left({ }^{\circ} \mathrm{C}\right)\end{array}$} & \multirow[b]{2}{*}{$\mathrm{pH}$} & \multirow{2}{*}{$\begin{array}{c}\text { Specific } \\
\text { conduct } \\
\text { ance } \\
(\mu \mathrm{S} / \mathrm{cm})\end{array}$} \\
\hline & & & & & $\begin{array}{l}\overline{\mathbf{v}_{\mathbf{i}}} \\
(\mathrm{L})\end{array}$ & $\begin{array}{l}\mathbf{v}_{\mathbf{p}} \\
(\mathrm{I})\end{array}$ & & & \\
\hline LEW & 0 & 0.0 & 0.00 & -- & -- & -- & -- & -- & -- \\
\hline 01 & -- & -- & -- & -- & -- & 3 & -- & -- & -- \\
\hline 02 & -- & -- & -- & -- & -- & 4 & & & \\
\hline $03 \mathrm{~A}$ & 87 & 8.5 & 0.72 & 410 & 1.49 & 7 & 24.0 & 6.7 & 384 \\
\hline 04B & 134 & 9.6 & 1.22 & 403 & 2.77 & 8 & 23.9 & 6.8 & 388 \\
\hline 05A & 156 & 11.2 & 1.47 & 362 & 3.76 & 9 & 24.0 & 6.9 & 374 \\
\hline 06B & 178 & 11.7 & 1.62 & 418 & 4.47 & 12 & 23.9 & 7.0 & 382 \\
\hline 07A & 200 & 12.2 & 1.77 & 724 & 4.81 & 16 & 23.6 & 7.0 & 380 \\
\hline 08B & 245 & 13.7 & 1.73 & 758 & 6.01 & 22 & 23.5 & 7.5 & 384 \\
\hline 09A & 264 & 14.3 & 1.62 & 302 & 5.43 & 27 & 23.6 & 7.3 & 370 \\
\hline $\mathrm{X02}$ & 271 & 13.4 & 1.77 & 379 & -- & - & -- & -- & -- \\
\hline $10 \mathrm{~B}$ & 296 & 14.6 & 1.70 & 582 & 5.15 & 25 & 23.7 & 7.3 & 387 \\
\hline $11 \mathrm{~A}$ & 318 & 14.3 & 1.85 & 716 & 6.28 & 24 & 24.0 & 7.3 & 385 \\
\hline X05 & 350 & 14.2 & 1.73 & 478 & -- & -- & -- & -- & -- \\
\hline $12 \mathrm{~B}$ & 357 & 14.8 & 1.72 & 395 & 5.71 & 24 & 23.8 & 7.2 & 389 \\
\hline $13 \mathrm{~A}$ & 381 & 15.5 & 1.61 & 461 & 6.48 & 26 & 23.6 & 7.1 & 386 \\
\hline $14 B$ & 394 & 16.3 & 1.67 & 720 & 6.33 & 29 & 23.5 & 7.1 & 388 \\
\hline $15 \mathrm{~A}$ & 434 & 16.8 & 1.60 & 766 & 6.26 & 25 & 23.5 & 7.1 & 387 \\
\hline $\mathrm{X} 03$ & 451 & 16.1 & 1.48 & 406 & -- & -- & -- & -- & -- \\
\hline 16B & 468 & 16.4 & 1.51 & 570 & 5.45 & 26 & 23.4 & 7.1 & 387 \\
\hline $17 \mathrm{~A}$ & 497 & 15.1 & 1.52 & 679 & 5.63 & 23 & 22.9 & 7.4 & 393 \\
\hline $18 \mathrm{~B}$ & 527 & 14.3 & 1.46 & 659 & 4.69 & 24 & 23.2 & 7.3 & 380 \\
\hline $19 A$ & 560 & 13.6 & 1.57 & 728 & 5.34 & 22 & 23.3 & 7.2 & 386 \\
\hline $20 \mathrm{~B}$ & 595 & 13.2 & 1.48 & 672 & 3.61 & 20 & 23.5 & 7.2 & 390 \\
\hline $21 \mathrm{~A}$ & 629 & 12.7 & 1.46 & 473 & 4.04 & 19 & 23.3 & 7.2 & 387 \\
\hline $22 B$ & 646 & 12.9 & 1.48 & 401 & 3.71 & 20 & 23.5 & 7.2 & 388 \\
\hline X06 & 671 & 12.9 & 1.39 & 268 & -- & -- & -- & -- & - \\
\hline $23 A$ & 676 & 13.0 & 1.33 & 172 & 3.77 & 20 & 23.0 & 7.1 & 387 \\
\hline $24 B$ & 691 & 13.4 & 1.18 & 450 & 3.75 & 22 & 23.3 & 7.2 & 384 \\
\hline $25 A$ & 733 & 13.5 & 1.21 & 563 & 3.58 & 17 & 23.3 & 7.3 & 390 \\
\hline 26B & 760 & 12.3 & 1.24 & 443 & 2.65 & 11 & 23.4 & 7.3 & 397 \\
\hline $27 A$ & 791 & 10.1 & 0.94 & 242 & 2.11 & 8 & 23.2 & 7.3 & 395 \\
\hline $28 \mathrm{~B}$ & 811 & 7.9 & 0.79 & 93 & 1.40 & 4 & 23.0 & 7.3 & 391 \\
\hline $\mathrm{X} 05$ & 821 & 6.9 & 0.67 & 76 & -- & -- & - & -- & -- \\
\hline $29 \mathrm{~A}$ & 844 & 5.4 & 0.79 & 81 & 0.88 & 2 & 23.2 & 7.3 & 388 \\
\hline $30 \mathrm{~B}$ & 859 & 4.3 & 0.66 & 83 & 0.65 & 1 & 23.3 & 7.3 & 397 \\
\hline REW & 902 & 0.0 & 0.00 & 0 & -- & -- & -- & -- & -- \\
\hline MEAN & & 11.5 & 1.43 & & & & & & \\
\hline TOTAL & 902 & & & 14,933 & 116.21 & 500 & & & \\
\hline
\end{tabular}




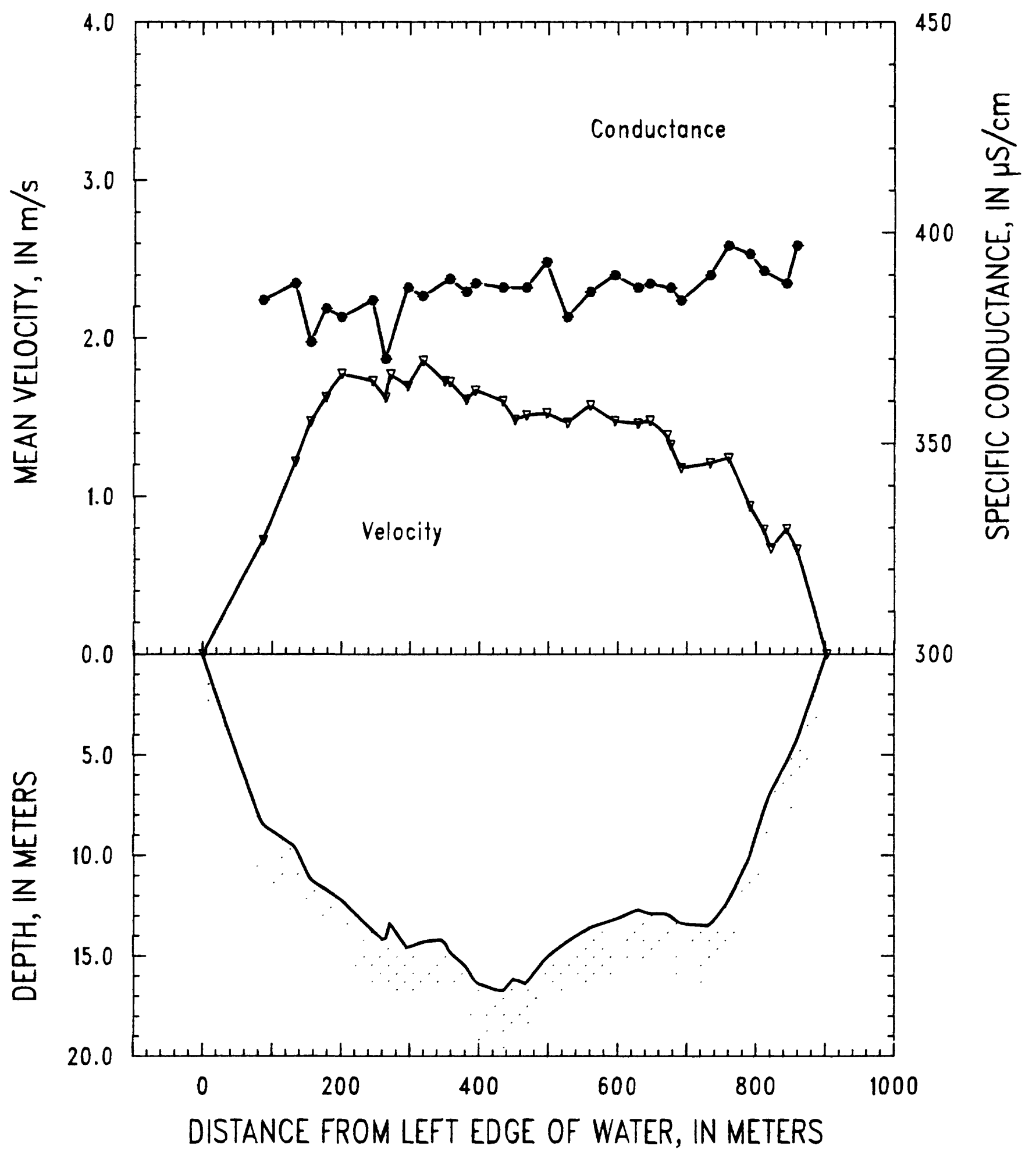

Figure 37. Mississippi River at Fulton, Tennessee, on June 14, 1989. 
SITE: Mississippi River at Helena, Arkansas

PARTY: Moody, Stevens, Rees, and Black

06-17-89

STARTING GAGE HEIGHT: $23.45 \mathrm{ft}$ ENDING GAGE HEIGHT: $23.85 \mathrm{ft}$

SUSP: Bag sampler and 200-1b weight

CURRENT METER NO.: P8308282 DATE RATED: 07-08-88

CURRENT METER EQUATION: $\mathrm{V}(\mathrm{m} / \mathrm{s})=$ RPS $* 0.765+0.006$

REMARKS: Transit rate was $18 \mathrm{~cm} / \mathrm{s}$ and nozzle was $3 / 16$ in. Verticals occupied in the order 1 to 30 .

\begin{tabular}{|c|c|c|c|c|c|c|c|c|c|}
\hline \multirow[b]{2}{*}{$\begin{array}{c}\text { Verti- } \\
\text { cal }\end{array}$} & \multirow{2}{*}{$\begin{array}{l}\text { Dist. } \\
\text { from } \\
\text { LEW } \\
(m)\end{array}$} & \multirow[b]{2}{*}{$\begin{array}{c}\text { Depth } \\
(\mathrm{m})\end{array}$} & \multirow{2}{*}{$\begin{array}{c}\text { Mean } \\
\text { veloc- } \\
\text { ity } \\
(\mathrm{m} / \mathrm{s})\end{array}$} & \multirow[b]{2}{*}{$\begin{array}{r}\text { Dis- } \\
\text { charge } \\
\left(\mathrm{m}^{3} / \mathrm{s}\right)\end{array}$} & \multicolumn{2}{|c|}{ Volume } & \multirow[b]{2}{*}{$\begin{array}{l}\text { Temper- } \\
\text { ature } \\
\left({ }^{\circ} \mathrm{C}\right)\end{array}$} & \multirow[b]{2}{*}{$\mathrm{pH}$} & \multirow{2}{*}{$\begin{array}{c}\text { Specific } \\
\text { conduct- } \\
\text { ance } \\
(\mu \mathrm{S} / \mathrm{cm})\end{array}$} \\
\hline & & & & & $\begin{array}{l}\overline{\mathrm{V}_{\mathrm{i}}} \\
(\mathrm{L})\end{array}$ & $\begin{array}{l}\mathrm{V}_{\mathbf{p}} \\
(\mathrm{L})\end{array}$ & & & \\
\hline LEW & 0 & 0.0 & 0.00 & 0 & -- & -- & -- & -- & - \\
\hline $30 \mathrm{~B}$ & 28 & 7.5 & 0.61 & 132 & 0.79 & 6 & 23.8 & 7.7 & 365 \\
\hline $29 \mathrm{~A}$ & 58 & 9.7 & 1.11 & 279 & 2.00 & 12 & 23.8 & 7.7 & 364 \\
\hline $\mathrm{X} 05$ & 80 & 10.2 & 1.39 & 212 & -- & -- & -- & -- & -- \\
\hline $28 B$ & 88 & 10.6 & 1.54 & 303 & 3.08 & 13 & 23.8 & 7.7 & 364 \\
\hline $27 \mathrm{~A}$ & 117 & 10.8 & 1.77 & 642 & 3.78 & 15 & 23.8 & 7.8 & 365 \\
\hline $26 B$ & 155 & 11.3 & 1.86 & 611 & 4.02 & 16 & 23.8 & 7.8 & 365 \\
\hline $25 \mathrm{~A}$ & 175 & 11.9 & 1.75 & 572 & 4.63 & 17 & 23.9 & 7.8 & 365 \\
\hline $24 B$ & 210 & 12.0 & 1.92 & 773 & 5.04 & 19 & 23.9 & 7.8 & 364 \\
\hline $23 \mathrm{~A}$ & 242 & 12.2 & 2.03 & 681 & 5.06 & 19 & 23.7 & 7.8 & 364 \\
\hline $22 B$ & 265 & 12.8 & 2.01 & 463 & 5.15 & 20 & 23.7 & 7.7 & 365 \\
\hline X04 & 278 & 12.8 & 1.88 & 337 & - & -- & -- & -- & -- \\
\hline $21 \mathrm{~A}$ & 293 & 13.6 & 1.91 & 635 & 5.21 & 21 & 23.8 & 7.7 & 365 \\
\hline $20 \mathrm{~B}$ & 327 & 13.7 & 2.12 & 959 & 5.31 & 24 & -- & -- & -- \\
\hline $19 \mathrm{~A}$ & 359 & 14.2 & 1.93 & 863 & 5.57 & 22 & 23.7 & 7.7 & 365 \\
\hline 18B & 390 & 13.7 & 1.80 & 739 & 4.60 & 24 & 23.8 & 7.8 & 364 \\
\hline $17 \mathrm{~A}$ & 419 & 14.1 & 1.87 & 685 & 5.62 & 22 & 23.7 & 7.8 & 365 \\
\hline $16 \mathrm{~B}$ & 442 & 14.3 & 1.73 & 446 & 4.47 & 25 & 23.8 & 7.7 & 365 \\
\hline $\mathrm{X} 03$ & 455 & 14.9 & 1.85 & 401 & -- & -- & -- & -- & -- \\
\hline $15 \mathrm{~A}$ & 471 & 15.9 & 1.63 & 636 & 4.59 & 27 & 23.6 & 7.8 & 365 \\
\hline $14 B$ & 504 & 16.9 & 1.57 & 861 & 4.79 & 25 & 23.9 & 7.7 & 365 \\
\hline $13 \mathrm{~A}$ & 536 & 17.0 & 1.30 & 619 & 4.45 & 22 & 23.8 & 7.8 & 366 \\
\hline $12 \mathrm{~B}$ & 560 & 17.0 & 1.31 & 545 & 4.07 & 20 & 23.9 & 7.8 & 365 \\
\hline $11 \mathrm{~A}$ & 585 & 17.1 & 1.22 & 563 & 3.63 & 15 & 23.8 & 7.8 & 364 \\
\hline $10 \mathrm{~B}$ & 614 & 18.6 & 1.19 & 477 & 3.50 & 18 & 24.1 & 7.9 & 363 \\
\hline $\mathrm{X} 02$ & 628 & 19.3 & 1.08 & 251 & -- & -- & - & -- & -- \\
\hline 09A & 638 & 19.8 & 0.97 & 403 & 3.57 & 20 & 23.7 & 7.9 & 362 \\
\hline 08B & 670 & 22.0 & 1.02 & 698 & 3.50 & 23 & 23.7 & 7.8 & 362 \\
\hline 07A & 700 & 21.0 & 0.95 & 487 & 3.35 & 21 & 23.8 & 7.9 & 360 \\
\hline 06B & 719 & 18.7 & 1.06 & 307 & 3.35 & 13 & 23.7 & 7.8 & 358 \\
\hline 05A & 731 & 16.8 & 0.98 & 420 & 2.99 & 9 & 23.7 & 7.9 & 358 \\
\hline 04B & 770 & 9.8 & 0.99 & 257 & 1.65 & 8 & 23.7 & 7.8 & 357 \\
\hline $\mathrm{X} 01$ & 784 & 9.1 & 0.99 & 139 & -- & -- & -- & -- & - \\
\hline $03 \mathrm{~A}$ & 801 & 7.9 & 1.02 & 173 & 1.57 & 6 & 23.7 & 7.8 & 357 \\
\hline 02B & 827 & 6.8 & 0.91 & 201 & 1.19 & 5 & 23.5 & 7.8 & 357 \\
\hline $01 \mathrm{~A}$ & 866 & 6.8 & 0.75 & 178 & 0.73 & 3 & 23.7 & 7.8 & 357 \\
\hline REW & 897 & 0.0 & 0.00 & 0 & -- & -- & -- & -- & -- \\
\hline MEAN & & 13.1 & 1.44 & & & & & & \\
\hline TOTAL & 897 & & & 16,948 & 111.32 & 510 & & & \\
\hline
\end{tabular}




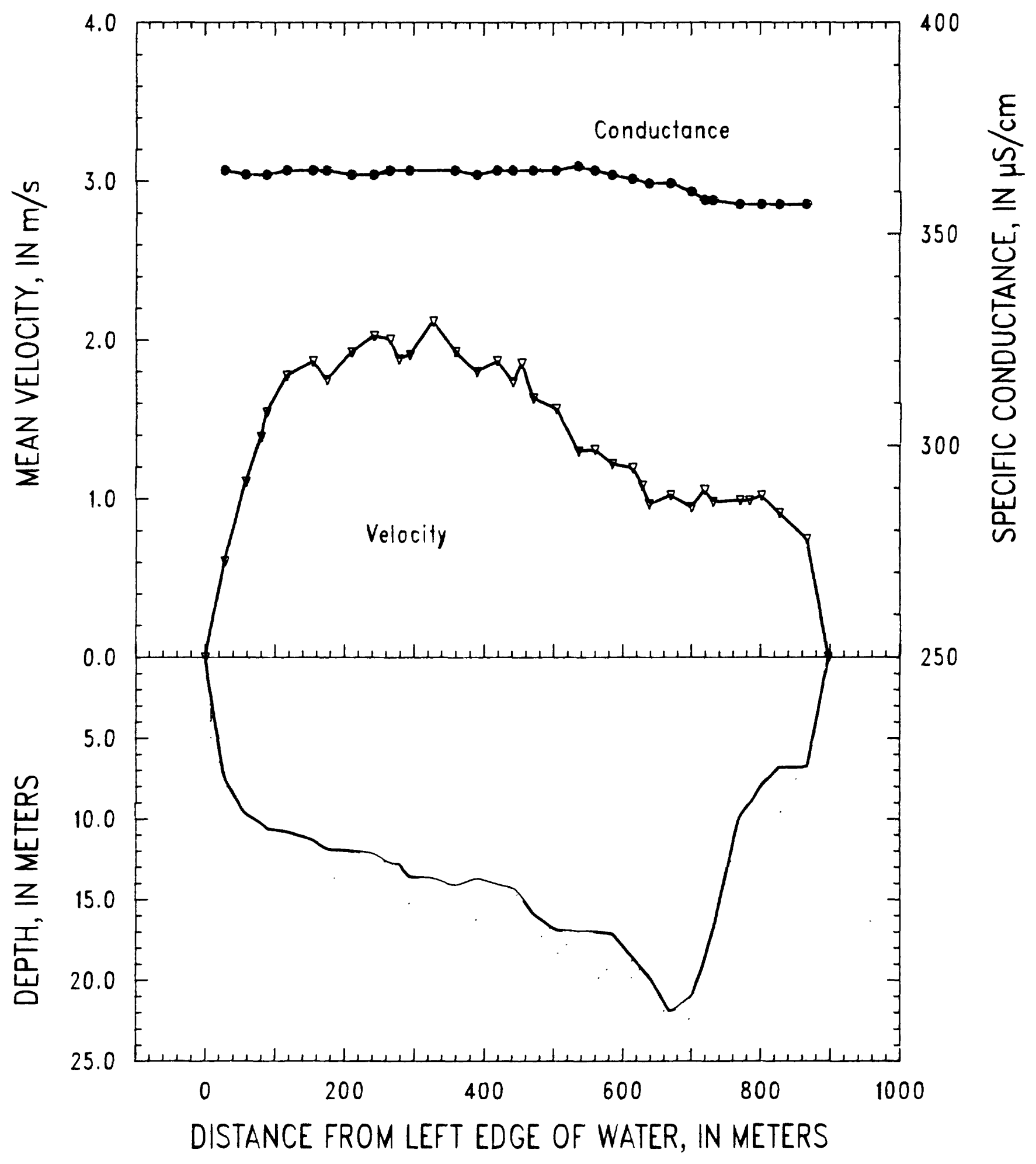

Figure 38. Mississippi River at Helena, Arkansas, on June 17, 1989. 
SITE: White River at Mile 11.5, Arkansas

06-18-89

PARTY: Moody, Stevens, Rees, and Black

METER: SOLID CUP

STARTING GAGE HEIGHT: -- ENDING GAGE HEIGHT: --

SUSP: Bag sampler and 200-1b weight

CURRENT METER NO.: P8308282 DATE RATED: 07-08-88

CURRENT METER EQUATION: $V(\mathrm{~m} / \mathrm{s})=$ RPS $* 0.765+0.006$

REMARKS: Anchored at three verticals and nozzle was 5/16 in. Varied transit rate at three centroids of equal discharge.

Discharge based on the 1 isted seven verticals $(1,2,3, \mathrm{X} 01, \mathrm{X} 02, \mathrm{X} 03$, and X04) plus 23 depths from depth profile and unit discharge proportional to depth $5 / 3$ powers was $860 \mathrm{~m}^{3} / \mathrm{s}$.

\begin{tabular}{|c|c|c|c|c|c|c|c|c|c|}
\hline \multirow[b]{2}{*}{$\begin{array}{c}\text { Verti- } \\
\text { cal }\end{array}$} & \multirow{2}{*}{$\begin{array}{l}\text { Dist. } \\
\text { from } \\
\text { LEW } \\
\text { (m) }\end{array}$} & \multirow[b]{2}{*}{$\begin{array}{l}\text { Depth } \\
\text { (m) }\end{array}$} & \multirow{2}{*}{$\begin{array}{l}\text { Mean } \\
\text { veloc- } \\
\text { ity } \\
(\mathrm{m} / \mathrm{s})\end{array}$} & \multirow[b]{2}{*}{$\begin{array}{r}\text { Dis- } \\
\text { charge } \\
\left(\mathrm{m}^{3} / \mathrm{s}\right)\end{array}$} & \multicolumn{2}{|c|}{ Volume } & \multirow[b]{2}{*}{$\begin{array}{l}\text { Temper- } \\
\text { ature } \\
\left({ }^{\circ} \mathrm{C}\right)\end{array}$} & \multirow[b]{2}{*}{$\mathrm{pH}$} & \multirow{2}{*}{$\begin{array}{c}\text { Specific } \\
\text { conduct- } \\
\text { ance } \\
(\mu \mathrm{S} / \mathrm{cm})\end{array}$} \\
\hline & & & & & $\begin{array}{l}\overline{V_{i}} \\
(L)\end{array}$ & $\begin{array}{l}\mathrm{V}_{\mathrm{p}} \\
(\mathrm{L})\end{array}$ & & & \\
\hline$\overline{L E W}$ & 0 & 0.0 & 0.00 & 0 & -- & -- & -- & -- & -- \\
\hline $03 \mathrm{~A}$ & 39 & 9.8 & 0.52 & 116 & 21.43 & 194 & 23.7 & 7.8 & 239 \\
\hline 03B & 39 & 9.8 & 0.52 & -- & 19.93 & -- & -- & -- & -- \\
\hline $\mathrm{X} 01$ & 46 & 9.8 & 0.51 & 33 & -- & -- & -- & -- & -- \\
\hline $\mathrm{X} 02$ & 52 & 9.5 & 0.52 & 122 & -- & -- & -- & -- & -- \\
\hline $\mathrm{X} 03$ & 95 & 9.1 & 0.59 & 128 & -- & - & - & -- & -- \\
\hline $02 \mathrm{~A}$ & 100 & 9.1 & 0.57 & 167 & 19.64 & 193 & 23.4 & 7.9 & 238 \\
\hline 02B & 100 & 9.1 & 0.57 & -- & 21.65 & - & -- & - & - \\
\hline $01 \mathrm{~A}$ & 159 & 8.4 & 0.51 & 141 & 20.61 & 193 & 23.1 & 7.8 & 235 \\
\hline $01 \mathrm{~B}$ & 159 & 8.4 & 0.51 & - & 20.65 & -- & - & - & -- \\
\hline X04 & 166 & 8.3 & 0.43 & 64 & -- & -- & -- & -- & -- \\
\hline REW & 195 & 0.0 & 0.00 & 0 & -- & -- & -- & -- & -- \\
\hline MEAN & & 7.5 & 0.53 & & & & & & \\
\hline TOTAL & 195 & & & 771 & 123.91 & 580 & & & \\
\hline
\end{tabular}


SITE: Arkansas River at Pendleton, Arkansas

06-19-89

PARTY: Moody, Stevens, Rees, and Black

ETER: SOLID CUP

STARTING GAGE HEIGHT: -- ENDING GAGE HEIGHT: --

SUSP: Bag sampler and 200-1b weight

CURRENT METER NO.: P8308282 DATE RATED: 07-08-88

CURRENT METER EQUATION: $\mathrm{V}(\mathrm{m} / \mathrm{s})=$ RPS $* 0.765+0.006$

REMARKS: Transit rate was $13 \mathrm{~cm} / \mathrm{s}$ and nozzle was $3 / 16$ inch. Verticals occupied from 1 to 30 .

\begin{tabular}{|c|c|c|c|c|c|c|c|c|c|}
\hline \multirow[b]{2}{*}{$\begin{array}{l}\text { Verti- } \\
\text { cal }\end{array}$} & \multirow{2}{*}{$\begin{array}{l}\text { Dist. } \\
\text { from } \\
\text { LEW } \\
\text { (m) }\end{array}$} & \multirow[b]{2}{*}{$\begin{array}{l}\text { Depth } \\
\text { (m) }\end{array}$} & \multirow{2}{*}{$\begin{array}{c}\text { Mean } \\
\text { veloc- } \\
\text { ity } \\
(\mathrm{m} / \mathrm{s})\end{array}$} & \multirow[b]{2}{*}{$\begin{array}{r}\text { Dis- } \\
\text { charge } \\
\left(\mathrm{m}^{3} / \mathrm{s}\right)\end{array}$} & \multicolumn{2}{|c|}{ Volume } & \multirow[b]{2}{*}{$\begin{array}{l}\text { Temper- } \\
\text { ature } \\
\left({ }^{\circ} \mathrm{C}\right)\end{array}$} & \multirow[b]{2}{*}{$\mathrm{pH}$} & \multirow{2}{*}{$\begin{array}{l}\text { Specific } \\
\text { conduct- } \\
\text { ance } \\
(\mu \mathrm{S} / \mathrm{cm})\end{array}$} \\
\hline & & & & & $\begin{array}{l}\overline{V_{i}} \\
(\mathrm{~L})\end{array}$ & $\begin{array}{l}V_{p} \\
(L)\end{array}$ & & & \\
\hline LEW & 0 & 0.0 & 0.00 & 0 & -- & -- & -- & -- & -- \\
\hline $30 \mathrm{~B}$ & 22 & 4.1 & 0.97 & 50 & 0.93 & 4 & 25.1 & 7.7 & 602 \\
\hline $29 \mathrm{~A}$ & 25 & 4.3 & 0.99 & 34 & 1.09 & 7 & 25.2 & 7.7 & 602 \\
\hline $28 \mathrm{~B}$ & 38 & 4.9 & 1.07 & 63 & 1.30 & 10 & 25.2 & 7.7 & 604 \\
\hline $27 \mathrm{~A}$ & 49 & 4.7 & 1.05 & 62 & 1.21 & 9 & 25.2 & 7.7 & 603 \\
\hline $26 B$ & 63 & 5.0 & 1.09 & 90 & 1.32 & 10 & 25.1 & 7.7 & 605 \\
\hline $25 A$ & 82 & 5.3 & 1.22 & 88 & 1.74 & 10 & 25.1 & 7.7 & 609 \\
\hline $24 B$ & 90 & 5.5 & 1.14 & 63 & 1.81 & 11 & 24.0 & 7.7 & 611 \\
\hline $23 \mathrm{~A}$ & 102 & 5.3 & 1.11 & 74 & 1.73 & 12 & 25.0 & 7.7 & 619 \\
\hline $22 B$ & 115 & 5.8 & 1.24 & 76 & 1.75 & 13 & 25.2 & 7.8 & 614 \\
\hline X05 & 123 & 6.4 & 1.34 & 81 & -- & -- & - & - & -- \\
\hline $21 \mathrm{~A}$ & 134 & 6.4 & 1.28 & 90 & 2.26 & 14 & 25.0 & 7.7 & 621 \\
\hline $20 \mathrm{~B}$ & 145 & 6.4 & 1.15 & 96 & 2.16 & 14 & 24.9 & 7.7 & 630 \\
\hline $19 \mathrm{~A}$ & 160 & 6.9 & 1.14 & 90 & 2.11 & 15 & 25.0 & 7.7 & 631 \\
\hline $18 \mathrm{~B}$ & 168 & 7.0 & 1.20 & 101 & 2.24 & 15 & 25.2 & 7.7 & 626 \\
\hline $17 \mathrm{~A}$ & 184 & 6.6 & 1.34 & 120 & 2.45 & 15 & 24.9 & 7.7 & 633 \\
\hline $16 \mathrm{~B}$ & 195 & 6.6 & 1.30 & 95 & 2.09 & 13 & 25.2 & 7.7 & 653 \\
\hline $15 \mathrm{~A}$ & 206 & 6.7 & 1.29 & 87 & 1.94 & 15 & 24.8 & 7.7 & 646 \\
\hline $14 \mathrm{~B}$ & 215 & 6.3 & 1.55 & 141 & 2.43 & 13 & 24.7 & 7.7 & 656 \\
\hline $13 \mathrm{~A}$ & 235 & 7.3 & 1.58 & 184 & 3.06 & 15 & 24.7 & 7.7 & 658 \\
\hline $12 \mathrm{~B}$ & 247 & 7.7 & 1.50 & 156 & 2.82 & 16 & 24.9 & 7.8 & 656 \\
\hline $11 \mathrm{~A}$ & 262 & 7.8 & 1.35 & 111 & 2.77 & 16 & 24.8 & 7.7 & 663 \\
\hline $10 \mathrm{~B}$ & 268 & 8.0 & 1.38 & 77 & 3.08 & 17 & 25.5 & 7.7 & 661 \\
\hline 09B & 276 & 8.3 & 1.35 & 73 & -- & - & - & - & -- \\
\hline $09 \mathrm{~A}$ & 281 & 8.7 & 1.30 & 102 & 2.84 & 19 & 24.7 & 7.7 & 663 \\
\hline $08 \mathrm{~B}$ & 294 & 10.5 & 1.21 & 184 & 3.37 & 22 & 24.7 & 7.7 & 660 \\
\hline $07 \mathrm{~A}$ & 310 & 13.2 & 1.27 & 227 & 4.70 & 29 & 25.1 & 7.7 & 662 \\
\hline 06B & 323 & 15.3 & 1. 31 & 210 & 5.17 & 36 & 24.6 & 7.7 & 660 \\
\hline $05 \mathrm{~A}$ & 331 & 15.4 & 1.32 & 234 & 5.31 & 39 & 24.7 & 7.7 & 656 \\
\hline $03 \mathrm{~A}$ & 344 & 14.7 & 1.33 & 147 & 5.39 & 29 & 24.6 & 7.8 & 647 \\
\hline $04 \mathrm{~B}$ & 346 & 14.3 & 1.40 & 171 & 4.81 & 44 & 24.7 & 7.7 & 650 \\
\hline O2B & 361 & 8.7 & 1.26 & 153 & 2.32 & 9 & 24.6 & 7.9 & 646 \\
\hline $01 \mathrm{~A}$ & 374 & 4.0 & 1.12 & 67 & 1.40 & 8 & 24.8 & 8.0 & 647 \\
\hline REW & 391 & 0.0 & 0.00 & 0 & -- & -- & -- & - & -- \\
\hline MEAN & & 7.2 & 1.28 & & & & & & \\
\hline TOTAL & 391 & & & 3,597 & 77.60 & 499 & & & \\
\hline
\end{tabular}




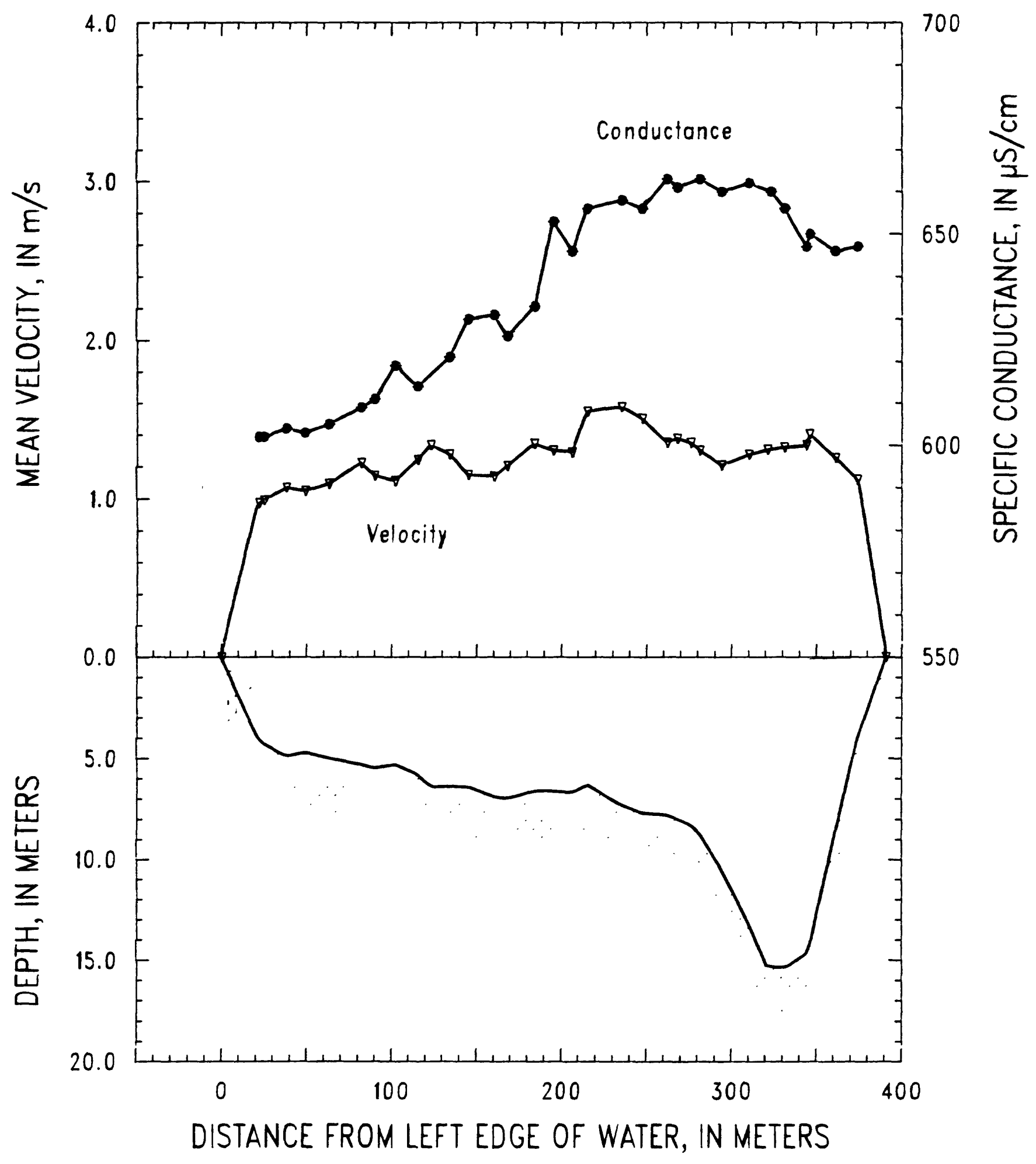

Figure 39. Arkansas River at Pendleton, Arkansas, on June 19, 1989. 
SITE: Mississippi River above Arkansas City, Arkansas 06-20-89

PARTY: Moody, Stevens, Rees, and Black

METER: SOLID CUP

STARTING GAGE HEIGHT: -- ENDING GAGE HEIGHT: --

SUSP: Bag sampler and 300-1b weight

CURRENT METER NO.: P8308282 DATE RATED: 07-08-88

CURRENT METER EQUATION: $V(\mathrm{~m} / \mathrm{s})=$ RPS $* 0.765+0.006$

REMARKS: Transit rate was $12 \mathrm{~cm} / \mathrm{s}$ and nozzle was $1 / 8$ inch. Verticals occupied from 1-29.

\begin{tabular}{|c|c|c|c|c|c|c|c|c|c|}
\hline \multirow[b]{2}{*}{$\begin{array}{l}\text { Verti- } \\
\text { cal }\end{array}$} & \multirow{2}{*}{$\begin{array}{l}\text { Dist. } \\
\text { from } \\
\text { LEW } \\
\text { (m) }\end{array}$} & \multirow[b]{2}{*}{$\begin{array}{c}\text { Depth } \\
\text { (m) }\end{array}$} & \multirow{2}{*}{$\begin{array}{l}\text { Mean } \\
\text { veloc- } \\
\text { ity } \\
(\mathrm{m} / \mathrm{s})\end{array}$} & \multirow[b]{2}{*}{$\begin{array}{r}\text { Dis- } \\
\text { charge } \\
\left(\mathrm{m}^{3} / \mathrm{s}\right)\end{array}$} & \multicolumn{2}{|c|}{ Volume } & \multirow[b]{2}{*}{$\begin{array}{l}\text { Temper- } \\
\text { ature } \\
\left({ }^{\circ} \mathrm{C}\right)\end{array}$} & \multirow[b]{2}{*}{$\mathrm{pH}$} & \multirow{2}{*}{$\begin{array}{l}\text { Specific } \\
\text { conduct- } \\
\text { ance } \\
(\mu S / \mathrm{cm})\end{array}$} \\
\hline & & & & & $\begin{array}{l}\overline{V_{i}} \\
(\mathrm{~L})\end{array}$ & $\begin{array}{l}\overline{V_{p}} \\
(\mathrm{~L})\end{array}$ & & & \\
\hline LEW & 0 & 0.0 & 0.00 & 0 & - & -- & -- & -- & -- \\
\hline $29 A$ & 62 & 5.5 & 0.31 & 83 & 0.24 & 2 & 24.9 & 7.7 & 328 \\
\hline X04 & 98 & 8.1 & 0.48 & 82 & - & - & - & $\cdots$ & -- \\
\hline $28 \mathrm{~B}$ & 104 & 8.6 & 0.59 & 84 & 0.51 & 3 & 24.8 & 7.7 & 327 \\
\hline $27 \mathrm{~A}$ & 131 & 10.7 & 0.67 & 189 & 0.84 & 5 & 24.9 & 7.6 & 328 \\
\hline $26 B$ & 157 & 13.6 & 0.88 & 363 & 1.53 & 9 & 24.7 & 7.7 & 328 \\
\hline $25 \mathrm{~A}$ & 192 & 14.8 & 0.86 & 422 & 1.48 & 12 & 24.6 & 7.7 & 331 \\
\hline $24 B$ & 223 & 15.8 & 1.03 & 553 & 2.22 & 14 & 24.7 & 7.7 & 329 \\
\hline $23 \mathrm{~A}$ & 260 & 16.7 & 1.22 & 611 & 2.65 & 16 & 24.6 & 7.7 & 332 \\
\hline $22 \mathrm{~B}$ & 283 & 17.2 & 1.35 & 686 & 2.78 & 20 & 24.5 & 7.7 & 335 \\
\hline $21 \mathrm{~A}$ & 319 & 17.6 & 1.50 & 737 & 3.66 & 18 & 24.5 & 7.7 & 333 \\
\hline $20 B$ & 339 & 18.8 & 1.61 & 785 & 4.64 & 22 & 24.6 & 7.7 & 351 \\
\hline $19 \mathrm{~A}$ & 371 & 19.5 & 1.66 & 746 & 4.63 & 25 & 24.5 & 7.7 & 352 \\
\hline $\mathrm{X} 19$ & 385 & 20.0 & 1.92 & 942 & - & - & - & -- & -- \\
\hline $18 \mathrm{~B}$ & 420 & 21.0 & 2.06 & 1,058 & 6.47 & 32 & 24.6 & 7.8 & 343 \\
\hline $17 \mathrm{~A}$ & 434 & 20.3 & 2.14 & 435 & 5.89 & 34 & 24.7 & 7.7 & 354 \\
\hline $\mathrm{X} 17$ & 440 & 19.8 & 2.20 & 435 & -- & -- & -- & - & - \\
\hline $16 \mathrm{~B}$ & 454 & 19.6 & 2.33 & 1,166 & 6.64 & 32 & 24.7 & 7.7 & 365 \\
\hline X05 & 491 & 20.3 & 2.21 & 1,012 & - & $\ldots$ & - & - & - \\
\hline $15 \mathrm{~A}$ & 499 & 21.0 & 2.19 & 919 & 6.54 & 31 & 24.6 & 7.7 & 363 \\
\hline $14 \mathrm{~B}$ & 531 & 19.8 & 1.90 & 1,014 & 5.83 & 28 & 24.5 & 7.7 & 384 \\
\hline $13 \mathrm{~A}$ & 553 & 19.4 & 1.98 & 1,443 & 5.46 & 27 & 24.6 & 7.7 & 386 \\
\hline $12 B$ & 606 & 18.2 & 2.06 & 1,553 & 5.44 & 25 & 24.7 & 7.7 & 389 \\
\hline $11 \mathrm{~A}$ & 636 & 18.1 & 1.97 & 1,014 & 5.13 & 26 & 24.8 & 7.7 & 402 \\
\hline $10 \mathrm{~B}$ & 663 & 17.8 & 1.99 & 922 & 4.86 & 25 & 24.8 & 7.7 & 404 \\
\hline $\mathrm{X} 02$ & 688 & 17.7 & 1.93 & 582 & -- & -- & -- & -- & -- \\
\hline $09 A$ & 697 & 17.8 & 2.03 & 813 & 5.35 & 22 & 24.8 & 7.7 & 403 \\
\hline $08 \mathrm{~B}$ & 733 & 17.4 & 1.77 & 1,077 & 4.89 & 20 & 24.7 & 7.7 & 408 \\
\hline $07 \mathrm{~A}$ & 767 & 17.0 & 1.71 & 859 & 4.37 & 15 & 24.7 & 7.7 & 410 \\
\hline 06B & 792 & 16.9 & 1.49 & 730 & 3.38 & 12 & 24.8 & 7.8 & 416 \\
\hline $05 \mathrm{~A}$ & 825 & 17.0 & 1.43 & 787 & 3.68 & 9 & 24.7 & 7.8 & 418 \\
\hline $04 \mathrm{~B}$ & 857 & 17.4 & 1.22 & 793 & 2.68 & 8 & 24.8 & 7.9 & 424 \\
\hline $03 \mathrm{~A}$ & 900 & 14.6 & 0.60 & 269 & 0.86 & 5 & 24.6 & 7.9 & 421 \\
\hline 02B & 918 & 11.4 & 0.49 & 174 & 0.61 & 4 & 25.2 & 7.9 & 421 \\
\hline $01 \mathrm{~A}$ & 962 & 3.7 & 0.02 & 2 & no sample & 1 & 25.1 & 7.9 & 420 \\
\hline REW & 990 & 0.0 & 0.00 & 0 & -- & -- & -- & -- & -- \\
\hline MEAN & & 15.1 & 1.56 & & & & & & \\
\hline TOTAL & 990 & & & 23,340 & 103.26 & 502 & & & \\
\hline
\end{tabular}




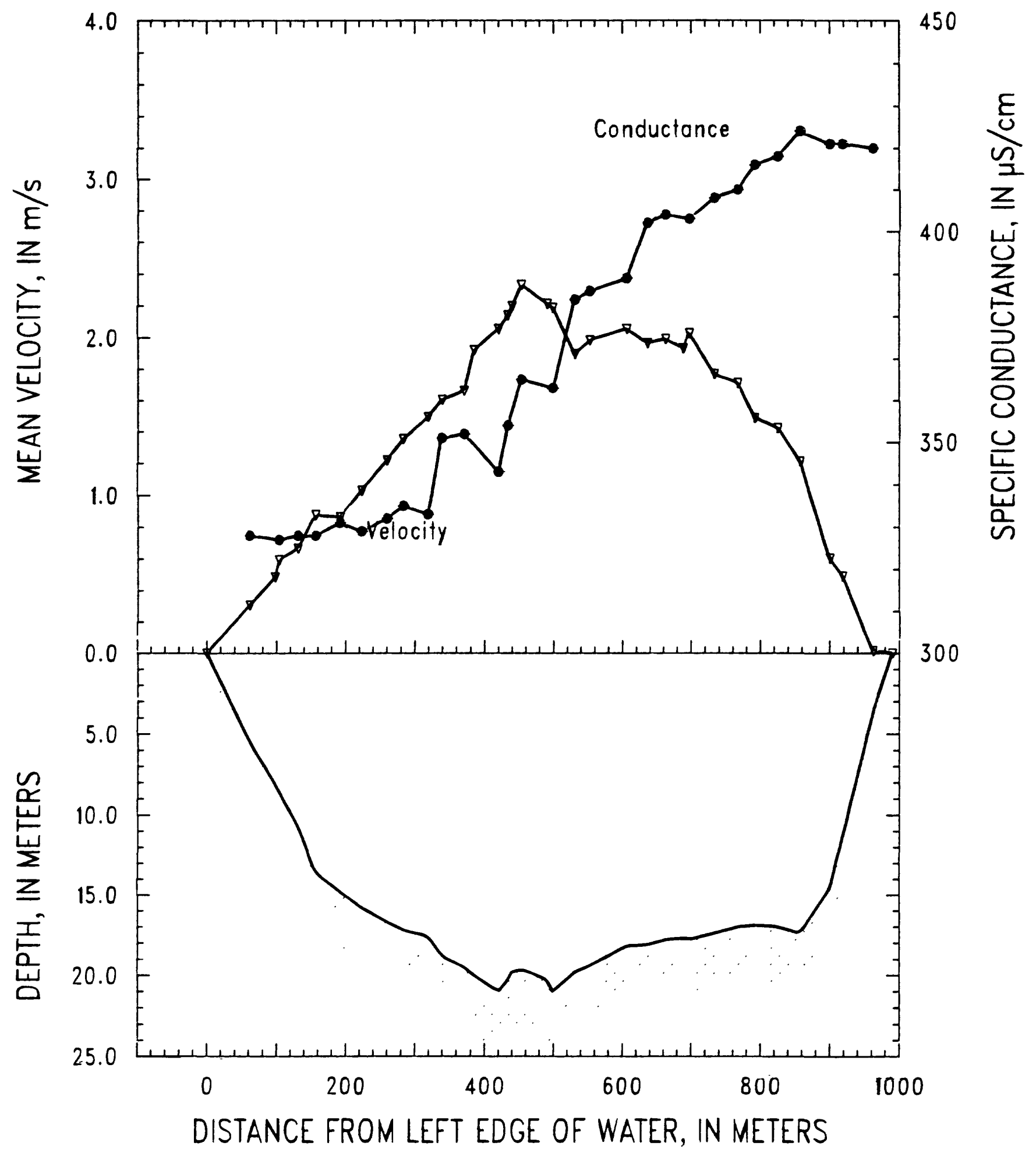

Figure 40. Mississippi River above Arkansas City, Arkansas, on June 20, 1989. 
STATION: Yazoo River below Steele Bayou, Mississippi PARTY: Moody, Stevens, Rees, and Black

STARTING GAGE HEIGHT: -- ENDING GAGE HEIGHT: --

SUSP: Bag sampler and 150-1b weight

CURRENT METER NO.: P8308282 DATE RATED: 07-08-88

CURRENT METER EQUATION: $\mathrm{V}(\mathrm{m} / \mathrm{s})=$ RPS $* 0.765+0.006$

REMARKS: Used 1/4-inch nozzle and various transit rates to get a full bottle at six equal-discharge centroids.

\begin{tabular}{|c|c|c|c|c|c|c|c|c|c|}
\hline \multirow[b]{2}{*}{$\begin{array}{l}\text { Verti- } \\
\text { cal }\end{array}$} & \multirow{2}{*}{$\begin{array}{l}\text { Dist. } \\
\text { from } \\
\text { LEW } \\
\text { (m) }\end{array}$} & \multirow[b]{2}{*}{$\begin{array}{l}\text { Depth } \\
\text { (m) }\end{array}$} & \multirow{2}{*}{$\begin{array}{l}\text { Mean } \\
\text { veloc- } \\
\text { ity } \\
(\mathrm{m} / \mathrm{s})\end{array}$} & \multirow[b]{2}{*}{$\begin{array}{r}\text { Dis- } \\
\text { charge } \\
\left(\mathrm{m}^{3} / \mathrm{s}\right)\end{array}$} & \multicolumn{2}{|c|}{ Volume } & \multirow[b]{2}{*}{$\begin{array}{l}\text { Temper- } \\
\text { ature } \\
\left({ }^{\circ} \mathrm{C}\right)\end{array}$} & \multirow[b]{2}{*}{$\mathrm{pH}$} & \multirow{2}{*}{$\begin{array}{l}\text { Specific } \\
\text { conduct- } \\
\text { ance } \\
(\mu \mathrm{S} / \mathrm{cm})\end{array}$} \\
\hline & & & & & $\begin{array}{l}\overline{V_{i}} \\
(\mathrm{~L})\end{array}$ & $\begin{array}{l}V_{p} \\
(L)\end{array}$ & & & \\
\hline$\overline{\text { LEW }}$ & 0 & 0.0 & 0.00 & 0 & -- & -- & -- & -- & -- \\
\hline X03 & 18 & 7.4 & 0.37 & 33 & -- & -- & -- & -- & -- \\
\hline 01 & 24 & 8.5 & 0.55 & 63 & 19.88 & 100 & 25.4 & 6.8 & 62 \\
\hline 02 & 45 & 13.8 & 0.78 & 211 & 21.60 & 100 & 25.5 & 6.5 & 63 \\
\hline 03 & 63 & 13.7 & 0.86 & 212 & 20.82 & 100 & 26.0 & 6.4 & 66 \\
\hline 04 & 81 & 13.8 & 0.88 & 171 & 20.05 & 111 & 26.1 & 6.4 & 65 \\
\hline 05 & 91 & 13.8 & 0.85 & 313 & 19.34 & 100 & 26.1 & 6.4 & 66 \\
\hline 06 & 134 & 6.2 & 0.30 & 42 & 20.66 & 100 & 26.2 & 6.5 & 66 \\
\hline $\mathrm{X} 04$ & 136 & 5.6 & 0.31 & 5 & -- & -- & -- & -- & -- \\
\hline X05 & 140 & 5.6 & 0.26 & 21 & -- & -- & -- & -- & -- \\
\hline REW & 165 & 0.0 & 0.00 & 0 & -- & -- & -- & -- & -- \\
\hline MEAN & & 9.2 & 0.70 & & & & & & \\
\hline TOTAL & 165 & & & 1,071 & 122.35 & 611 & & & \\
\hline
\end{tabular}


SITE: Mississippi River below Vicksburg, Mississippi

PARTY: Moody, Stevens, Rees, and Black

06-23-89

STARTING GAGE HEIGHT: -- ENDING GAGE HEIGHT: -METER: SOLID CUP

SUSP: Bag sampler and 300-1b weight

CURRENT METER NO.: P8308282 DATE RATED: 07-08-88

CURRENT METER EQUATION: $V(\mathrm{~m} / \mathrm{s})=$ RPS $* 0.765+0.006$

REMARKS: Transit rate was $10 \mathrm{~cm} / \mathrm{s}$ and nozzle was $1 / 8$ inch.

\begin{tabular}{|c|c|c|c|c|c|c|c|c|c|}
\hline \multirow[b]{2}{*}{$\begin{array}{l}\text { Verti- } \\
\text { cal }\end{array}$} & \multirow{2}{*}{$\begin{array}{l}\text { Dist. } \\
\text { from } \\
\text { LEW } \\
\text { (m) }\end{array}$} & \multirow[b]{2}{*}{$\begin{array}{l}\text { Depth } \\
\text { (m) }\end{array}$} & \multirow{2}{*}{$\begin{array}{l}\text { Mean } \\
\text { veloc- } \\
\text { ity } \\
(\mathrm{m} / \mathrm{s})\end{array}$} & \multirow[b]{2}{*}{$\begin{array}{r}\text { Dis- } \\
\text { charge } \\
\left(\mathrm{m}^{3} / \mathrm{s}\right)\end{array}$} & \multicolumn{2}{|c|}{ Volume } & \multirow[b]{2}{*}{$\begin{array}{l}\text { Temper- } \\
\text { ature } \\
\left({ }^{\circ} \mathrm{C}\right)\end{array}$} & \multirow[b]{2}{*}{$\mathrm{pH}$} & \multirow{2}{*}{$\begin{array}{c}\text { Specific } \\
\text { conduct- } \\
\text { ance } \\
(\mu \mathrm{S} / \mathrm{cm})\end{array}$} \\
\hline & & & & & $\begin{array}{l}\overline{V_{i}} \\
(L)\end{array}$ & $\begin{array}{l}\overline{V_{p}} \\
(L)\end{array}$ & & & \\
\hline LEW & 7 & 0.0 & 0.00 & 0 & -- & -- & -- & -- & -- \\
\hline $01 \mathrm{~A}$ & 38 & 9.2 & 0.60 & 193 & 0.58 & 4 & 25.6 & 6.8 & 298 \\
\hline 02B & 70 & 12.8 & 0.94 & 470 & 1.71 & 7 & 25.3 & 6.9 & 303 \\
\hline 03A & 116 & 19.7 & 1.34 & 688 & 4.43 & 11 & 25.3 & 7.1 & 303 \\
\hline $\mathrm{X} 01$ & 122 & 19.7 & 1.48 & 437 & -- & -- & -- & -- & -- \\
\hline 04B & 146 & 20.4 & 1.60 & 883 & 5.51 & 26 & 25.4 & 7.0 & 318 \\
\hline $05 \mathrm{~A}$ & 176 & 20.1 & 1.70 & 1,006 & 6.44 & 26 & 25.5 & 6.9 & 320 \\
\hline 06B & 205 & 19.6 & 1.62 & 1,114 & 5.67 & 28 & 25.7 & 7.3 & 329 \\
\hline 07A & 246 & 19.1 & 1.69 & 1,081 & 5.91 & 26 & 25.6 & 8.0 & 336 \\
\hline 08B & 272 & 19.0 & 1.76 & 938 & 6.10 & 28 & 25.2 & 8.1 & 342 \\
\hline 09A & 302 & 18.7 & 1.72 & 612 & 6.00 & 30 & 25.3 & 7.9 & 345 \\
\hline X02 & 310 & 18.6 & 1.85 & 585 & -- & -- & -- & -- & -- \\
\hline $10 B$ & 336 & 18.0 & 1.87 & 978 & 5.87 & 30 & 25.3 & 7.8 & 353 \\
\hline $11 \mathrm{~A}$ & 368 & 17.8 & 1.81 & 1,014 & 4.89 & 27 & 25.4 & 7.8 & 352 \\
\hline 12B & 399 & 17.3 & 1.83 & 1,110 & 5.30 & 27 & 25.2 & 7.8 & 354 \\
\hline $13 \mathrm{~A}$ & 438 & 16.9 & 1.79 & 1,166 & 4.93 & 27 & 25.3 & 7.7 & 364 \\
\hline $14 B$ & 476 & 17.3 & 1.84 & 1,192 & 4.90 & 29 & 25.4 & 7.8 & 360 \\
\hline $15 \mathrm{~A}$ & 513 & 15.6 & 1.90 & 905 & 4.97 & 27 & 25.3 & 7.9 & 360 \\
\hline 16B & 537 & 15.9 & 1.75 & 404 & 5.03 & 28 & 25.7 & 7.8 & 360 \\
\hline X07 & 542 & 15.6 & 1.85 & 173 & -- & -- & -- & -- & -- \\
\hline $17 \mathrm{~A}$ & 549 & 15.6 & 1.96 & 1,117 & 5.56 & 22 & 25.3 & 7.8 & 361 \\
\hline $18 \mathrm{~B}$ & 615 & 15.2 & 1.86 & 1,243 & 4.19 & 26 & 25.3 & 7.8 & 360 \\
\hline $19 \mathrm{~A}$ & 637 & 14.2 & 1.69 & 587 & 4.16 & 20 & 25.3 & 7.7 & 359 \\
\hline 20B & 664 & 13.7 & 1.56 & 576 & 3.49 & 19 & 25.3 & 7.8 & 360 \\
\hline $21 \mathrm{~A}$ & 691 & 13.5 & 1.70 & 630 & 3.60 & 17 & 25.3 & 7.8 & 360 \\
\hline 22B & 719 & 13.5 & 1.55 & 636 & 3.38 & 17 & 25.3 & 7.7 & 362 \\
\hline $23 \mathrm{~A}$ & 752 & 12.2 & 1.43 & 576 & 2.41 & 15 & 25.3 & 7.7 & 360 \\
\hline $24 B$ & 785 & 11.7 & 1.35 & 555 & 2.56 & 13 & 25.4 & 7.7 & 361 \\
\hline $25 \mathrm{~A}$ & 822 & 10.2 & 1.05 & 381 & 2.23 & 12 & 25.4 & 7.7 & 361 \\
\hline 26B & 856 & 9.7 & 1.51 & 512 & 2.12 & 12 & 25.3 & 7.7 & 360 \\
\hline $27 \mathrm{~A}$ & 892 & 9.4 & 1.26 & 343 & 2.09 & 10 & 25.6 & 7.7 & 361 \\
\hline X05 & 914 & 9.1 & 1.12 & 158 & -- & -- & -- & -- & -- \\
\hline $28 B$ & 923 & 8.8 & 1.21 & 256 & 1.58 & 10 & 25.4 & 7.7 & 360 \\
\hline $29 A$ & 962 & 8.4 & 1.13 & 346 & 1.50 & 9 & 25.5 & 7.7 & 360 \\
\hline $30 \mathrm{~B}$ & 996 & 8.3 & 1.03 & 265 & 1.42 & 8 & 25.4 & 7.7 & 360 \\
\hline $31 \mathrm{~A}$ & 1,024 & 8.4 & 0.91 & 241 & 1.15 & 8 & 25.4 & 7.7 & 362 \\
\hline $32 B$ & 1,059 & 8.6 & 1.15 & 336 & 1.51 & 8 & 25.7 & 7.8 & 360 \\
\hline $33 \mathrm{~A}$ & 1,092 & 9.0 & 1.00 & 252 & 1.35 & 8 & 25.4 & 7.8 & 362 \\
\hline $34 \mathrm{~B}$ & 1,115 & 8.8 & 1.09 & 258 & 1.48 & 6 & 25.5 & 7.9 & 361 \\
\hline $35 \mathrm{~A}$ & 1,146 & 8.9 & 1.07 & 320 & 1.40 & 5 & 25.6 & 7.8 & 361 \\
\hline $36 \mathrm{~B}$ & 1,182 & 8.0 & 0.93 & 284 & 1.14 & 3 & 25.6 & 7.8 & 362 \\
\hline REW & 1,222 & 0.0 & 0.00 & 0 & -- & -- & -- & -- & -- \\
\hline MEAN & & 13.2 & 1.53 & & & & & & \\
\hline TOTAL & 1,222 & & & 24,821 & 126.56 & 629 & & & \\
\hline
\end{tabular}




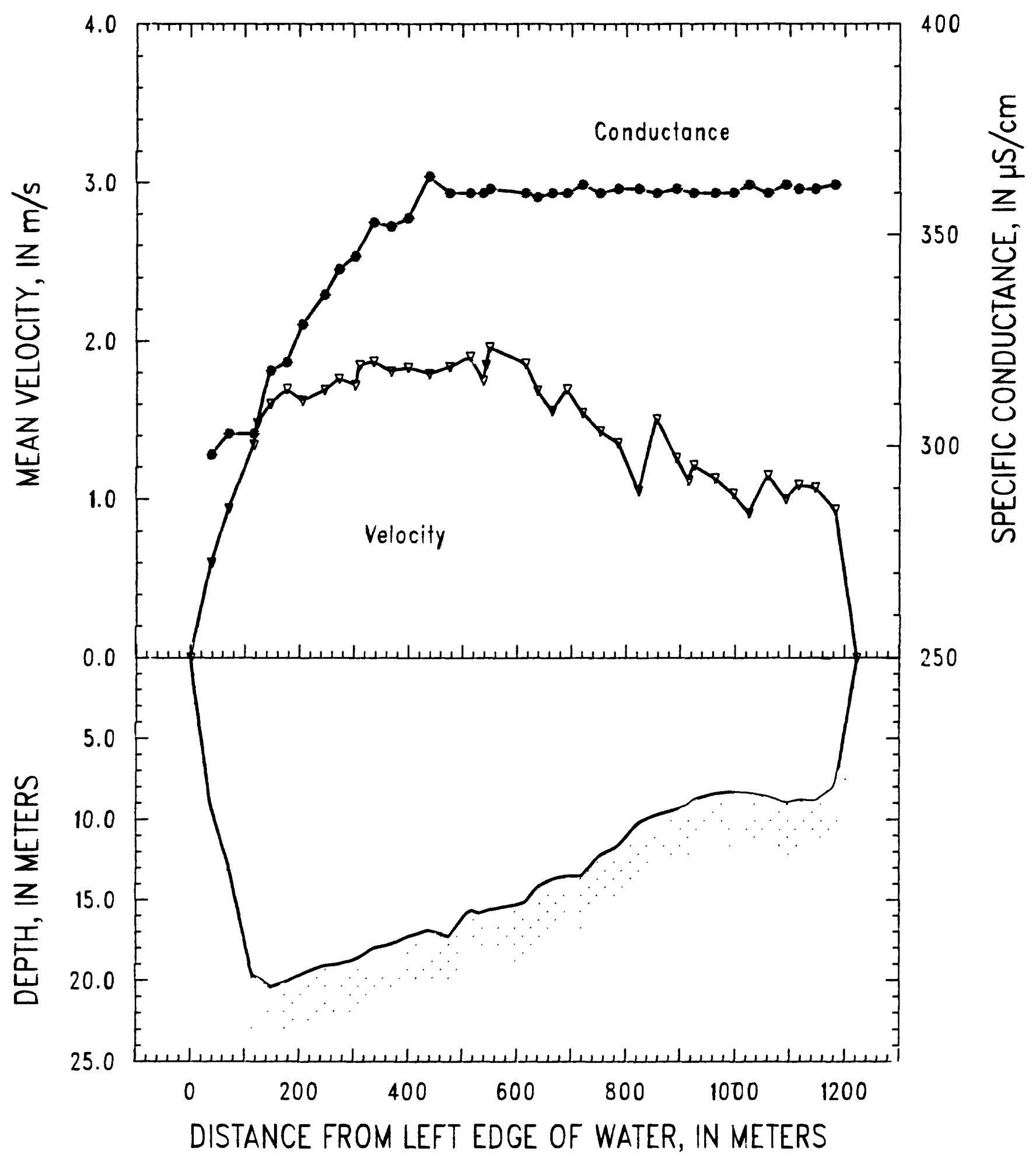

Figure 41. Mississippi River below Vicksburg, Mississippi, on June 23, 1989. 
SITE: Old River Outflow Channel near Knox Landing, Louisiana 06-25-89 PARTY: Moody, Stevens, Rees, and Simoneaux

STARTING GAGE HEIGHT: -- ENDING GAGE HEIGHT: -METER: SOLID CUP SUSP: Bag sampler and $150-1 \mathrm{~b}$ weight

CURRENT METER NO.: P8308282 DATE RATED: 07-08-88

CURRENT METER EQUATION: $V(\mathrm{~m} / \mathrm{s})=$ RPS $* 0.765+0.006$

REMARKS: Transit rate was $13 \mathrm{~cm} / \mathrm{s}$ and nozzle was $1 / 4 \mathrm{inch}$.

\begin{tabular}{|c|c|c|c|c|c|c|c|c|c|}
\hline \multirow[b]{2}{*}{$\begin{array}{c}\text { Verti- } \\
\text { cal }\end{array}$} & \multirow{2}{*}{$\begin{array}{l}\text { Dist. } \\
\text { from } \\
\text { LEW } \\
\text { (m) }\end{array}$} & \multirow[b]{2}{*}{$\begin{array}{l}\text { Depth } \\
\text { (m) }\end{array}$} & \multirow{2}{*}{$\begin{array}{l}\text { Mean } \\
\text { veloc- } \\
\text { ity } \\
(\mathrm{m} / \mathrm{s})\end{array}$} & \multirow[b]{2}{*}{$\begin{array}{c}\text { Dis- } \\
\text { charge } \\
\left(\mathrm{m}^{3} / \mathrm{s}\right)\end{array}$} & \multicolumn{2}{|c|}{ Volume } & \multirow[b]{2}{*}{$\begin{array}{l}\text { Temper- } \\
\text { ature } \\
\left({ }^{\circ} \mathrm{C}\right)\end{array}$} & \multirow[b]{2}{*}{$\mathrm{pH}$} & \multirow{2}{*}{$\begin{array}{c}\text { Specific } \\
\text { conduct- } \\
\text { ance } \\
(\mu \mathrm{S} / \mathrm{cm})\end{array}$} \\
\hline & & & & & $\begin{array}{l}\bar{V}_{i} \\
(L)\end{array}$ & $\begin{array}{l}v_{p} \\
(L)\end{array}$ & & & \\
\hline LEW & 0 & 0.0 & 0.00 & 0 & -- & - & -- & -- & -- \\
\hline $01 \mathrm{~A}$ & 26 & 6.9 & 0.62 & 92 & 1.68 & 4 & 25.8 & 7.6 & 349 \\
\hline 02B & 43 & 11.4 & 0.87 & 143 & 3.78 & 13 & 25.8 & 7.6 & 349 \\
\hline $\mathrm{X} 05$ & 55 & 12.0 & 0.93 & 94 & -- & -- & -- & -- & -- \\
\hline $03 \mathrm{~A}$ & 60 & 12.1 & 0.94 & 130 & 4.05 & 21 & 25.9 & 7.7 & 348 \\
\hline 04B & 78 & 12.2 & 0.93 & 232 & 4.60 & 23 & 25.8 & 7.7 & 348 \\
\hline $05 \mathrm{~A}$ & 101 & 12.3 & 0.92 & 176 & 3.65 & 23 & 25.8 & 7.7 & 348 \\
\hline $06 \mathrm{~A}$ & 109 & 12.3 & 0.94 & 190 & 4.92 & 23 & 25.8 & 7.7 & 348 \\
\hline $07 \mathrm{~A}$ & 134 & 12.2 & 0.99 & 247 & 4.39 & 21 & 25.8 & 7.7 & 348 \\
\hline $08 B$ & 150 & 11.9 & 0.98 & 187 & 4.76 & 21 & 25.9 & 7.7 & 348 \\
\hline $09 \mathrm{~A}$ & 166 & 11.8 & 0.94 & 166 & 3.89 & 21 & 25.9 & 7.7 & 348 \\
\hline $10 \mathrm{~B}$ & 180 & 11.8 & 0.93 & 175 & 4.79 & 21 & 25.9 & 7.7 & 348 \\
\hline $11 \mathrm{~A}$ & 198 & 11.6 & 0.91 & 191 & 3.83 & 19 & 25.8 & 7.7 & 348 \\
\hline $12 \mathrm{~B}$ & 216 & 11.4 & 0.94 & 205 & 4.53 & 21 & 25.8 & 7.7 & 347 \\
\hline $13 \mathrm{~A}$ & 236 & 11.1 & 0.92 & 133 & 3.68 & 19 & 25.7 & 7.7 & 348 \\
\hline $14 \mathrm{~B}$ & 242 & 10.8 & 0.91 & 162 & 3.92 & 18 & 25.9 & 7.7 & 348 \\
\hline $15 \mathrm{~A}$ & 269 & 10.2 & 0.91 & 162 & 3.83 & 16 & 25.9 & 7.7 & 348 \\
\hline $\mathrm{X03}$ & 277 & 10.1 & 0.99 & 70 & -- & -- & - & -- & -- \\
\hline $16 \mathrm{~B}$ & 283 & 9.8 & 0.94 & 133 & 3.74 & 16 & 25.9 & 7.7 & 349 \\
\hline $17 \mathrm{~A}$ & 306 & 8.8 & 0.88 & 136 & 3.12 & 14 & 25.9 & 7.7 & 348 \\
\hline $18 \mathrm{~B}$ & 318 & 9.1 & 0.98 & 156 & 3.67 & 15 & 25.9 & 7.7 & 347 \\
\hline $19 \mathrm{~A}$ & 341 & 8.7 & 0.88 & 157 & 3.10 & 14 & 25.8 & 7.7 & 348 \\
\hline $20 B$ & 359 & 8.6 & 0.95 & 147 & 3.14 & 15 & 25.9 & 7.7 & 347 \\
\hline $21 \mathrm{~A}$ & 377 & 8.8 & 0.90 & 110 & 3.04 & 14 & 26.1 & 7.7 & 347 \\
\hline $\mathrm{X} 02$ & 387 & 8.6 & 0.90 & 93 & -- & -- & -- & -- & -- \\
\hline $22 B$ & 401 & 8.7 & 0.88 & 99 & 2.78 & 15 & 25.8 & 7.7 & 348 \\
\hline $23 \mathrm{~A}$ & 413 & 8.8 & 0.90 & 138 & 3.01 & 16 & 26.0 & 7.7 & 347 \\
\hline $24 B$ & 436 & 8.7 & 0.92 & 164 & 3.40 & 16 & 26.1 & 7.7 & 349 \\
\hline $25 \mathrm{~A}$ & 454 & 8.9 & 0.93 & 132 & 2.98 & 16 & 26.1 & 7.7 & 348 \\
\hline $26 \mathrm{~B}$ & 468 & 9.1 & 0.95 & 142 & 3.67 & 16 & 26.1 & 7.7 & 350 \\
\hline $27 \mathrm{~A}$ & 487 & 9.1 & 0.89 & 147 & 2.58 & 15 & 26.2 & 7.7 & 348 \\
\hline $28 \mathrm{~B}$ & 504 & 9.0 & 0.93 & 155 & 3.46 & 15 & 25.9 & 7.7 & 348 \\
\hline $29 \mathrm{~A}$ & 524 & 8.9 & 0.82 & 135 & 2.55 & 13 & 25.9 & 7.7 & 349 \\
\hline $30 \mathrm{~B}$ & 541 & 8.2 & 0.56 & 87 & 1.73 & 6 & 26.0 & 7.7 & 350 \\
\hline REW & 562 & 0.0 & 0.00 & 0 & -- & -- & -- & -- & -- \\
\hline MEAN & & 9.6 & 0.90 & & & & & & \\
\hline TOTAL & 562 & & & 4,886 & 106.27 & 501 & & & \\
\hline
\end{tabular}




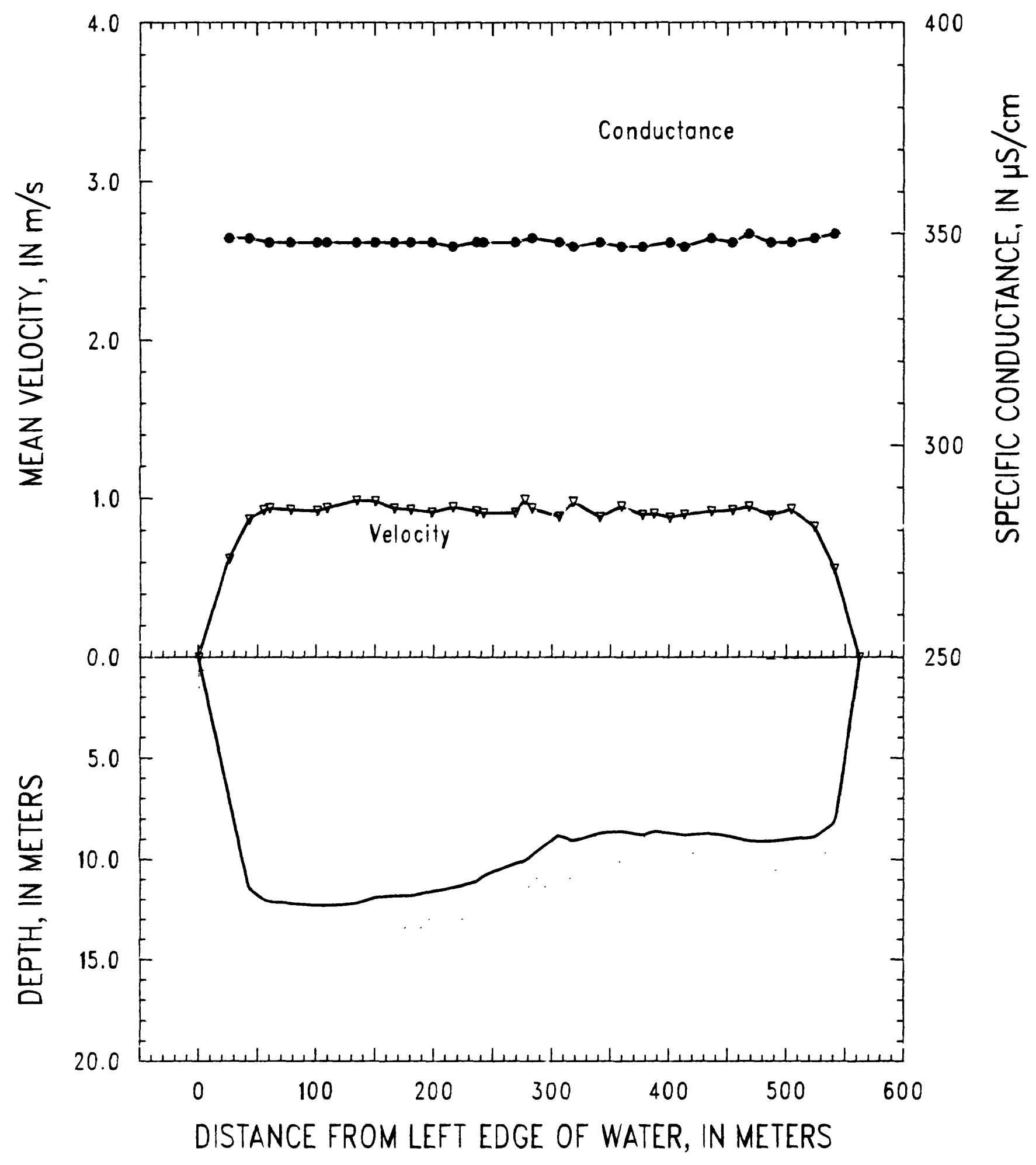

Figure 42. Old River Outflow Channel near Knox Landing, Louisiana, on June 25, 1989. 
SITE: Mississippi River near St. Francisville, Louisiana

PARTY: Moody, Stevens, and Simoneaux

06-26-89

STARTING GAGE HEIGHT: -- ENDING GAGE HEIGHT: --

SUSP: Bag sampler and 300-1b weight

CURRENT METER NO.: P8308282 DATE RATED: 07-08-88

CURRENT METER EQUATION: $V(\mathrm{~m} / \mathrm{s})=$ RPS $* 0.765+0.006$

REMARKS: Transit rate was $16 \mathrm{~cm} / \mathrm{s}$ and nozzle was $3 / 16$ inch. LEW was in the trees. Estimated depth at LEF (left edge of flow).

\begin{tabular}{|c|c|c|c|c|c|c|c|c|c|}
\hline \multirow[b]{2}{*}{$\begin{array}{c}\text { Verti- } \\
\text { cal }\end{array}$} & \multirow{2}{*}{$\begin{array}{l}\text { Dist. } \\
\text { from } \\
\text { LEW } \\
(m)\end{array}$} & \multirow[b]{2}{*}{$\begin{array}{l}\text { Depth } \\
(\mathrm{m})\end{array}$} & \multirow{2}{*}{$\begin{array}{l}\text { Mean } \\
\text { veloc- } \\
\text { ity } \\
(\mathrm{m} / \mathrm{s})\end{array}$} & \multirow[b]{2}{*}{$\begin{array}{r}\text { Dis- } \\
\text { charge } \\
\left(\mathrm{m}^{3} / \mathrm{s}\right)\end{array}$} & \multicolumn{2}{|c|}{ Volume } & \multirow[b]{2}{*}{$\begin{array}{l}\text { Temper- } \\
\text { ature } \\
\left({ }^{\circ} \mathrm{C}\right)\end{array}$} & \multirow[b]{2}{*}{$\mathrm{pH}$} & \multirow{2}{*}{$\begin{array}{l}\text { Specific } \\
\text { conduct- } \\
\text { ance } \\
(\mu \mathrm{S} / \mathrm{cm})\end{array}$} \\
\hline & & & & & $\begin{array}{l}\overline{v_{i}} \\
(L)\end{array}$ & $\begin{array}{l}\bar{v}_{p} \\
(L)\end{array}$ & & & \\
\hline$\overline{\text { LEF }}$ & 0 & 0.0 & 0.00 & 0 & -- & -- & -- & -- & -- \\
\hline $30 \mathrm{~B}$ & 31 & 8.1 & 0.57 & 149 & 0.84 & 2 & 26.0 & 7.7 & 337 \\
\hline $29 A$ & 64 & 9.9 & 0.86 & 281 & 1.75 & 6 & 26.0 & 7.7 & 337 \\
\hline $28 B$ & 97 & 10.8 & 0.92 & 369 & 1.73 & 10 & 25.8 & 7.7 & 337 \\
\hline $27 \mathrm{~A}$ & 138 & 12.3 & 1.08 & 453 & 2.67 & 11 & 25.9 & 7.7 & 337 \\
\hline $26 B$ & 165 & 13.2 & 0.97 & 412 & 2.38 & 14 & 26.0 & 7.7 & 337 \\
\hline $25 A$ & 202 & 13.7 & 1.22 & 500 & 3.43 & 14 & 25.8 & 7.7 & 337 \\
\hline $24 B$ & 225 & 13.3 & 1.16 & 534 & 3.26 & 14 & 25.8 & 7.7 & 337 \\
\hline $23 \mathrm{~A}$ & 271 & 14.6 & 1.33 & 711 & 4.01 & 16 & 25.8 & 7.6 & 337 \\
\hline X05 & 298 & 14.6 & 1.35 & 306 & -- & -- & -- & -- & -- \\
\hline $22 B$ & 302 & 12.3 & 1.52 & 205 & 4.28 & 17 & 25.8 & 7.6 & 337 \\
\hline $21 \mathrm{~A}$ & 320 & 14.1 & 1.33 & 592 & 3.43 & 15 & 25.7 & 7.6 & 341 \\
\hline $20 \mathrm{~B}$ & 365 & 14.2 & 1.49 & 700 & 4.71 & 15 & 25.9 & 7.8 & 338 \\
\hline $19 \mathrm{~A}$ & 386 & 14.6 & 1.25 & 537 & 4.27 & 14 & 25.7 & 7.8 & 337 \\
\hline $18 \mathrm{~B}$ & 424 & 14.1 & 1.45 & 642 & 3.56 & 19 & 26.0 & 7.7 & 338 \\
\hline $17 \mathrm{~A}$ & 449 & 14.0 & 1.48 & 661 & 4.36 & 16 & 26.1 & 7.6 & 338 \\
\hline $16 \mathrm{~B}$ & 488 & 13.2 & 1.34 & 522 & 4.22 & 17 & 26.2 & 7.6 & 338 \\
\hline X04 & 508 & 13.7 & 1.38 & 322 & -- & - & -- & - & -- \\
\hline $15 \mathrm{~A}$ & 522 & 13.7 & 1.34 & 412 & 4.21 & 17 & 26.1 & 7.6 & 339 \\
\hline $14 \mathrm{~B}$ & 553 & 14.2 & 1.48 & 524 & 4.39 & 17 & 26.0 & 7.6 & 339 \\
\hline $13 \mathrm{~A}$ & 572 & 14.6 & 1.35 & 413 & 4.53 & 18 & 25.9 & 7.6 & 340 \\
\hline $\mathrm{X} 13$ & 595 & 15.0 & 1.32 & 475 & - & - & -- & - & - \\
\hline $12 \mathrm{~B}$ & 620 & 15.7 & 1.24 & 534 & 4.34 & 19 & 26.0 & 7.6 & 340 \\
\hline $11 \mathrm{~A}$ & 650 & 16.2 & 1.36 & 792 & 5.12 & 19 & 25.9 & 7.6 & 340 \\
\hline $10 \mathrm{~B}$ & 692 & 17.7 & 1.36 & 709 & 5.28 & 22 & 26.0 & 7.6 & 340 \\
\hline X03 & 709 & 18.4 & 1.39 & 306 & -- & -- & - & -- & -- \\
\hline $09 \mathrm{~A}$ & 716 & 18.5 & 1.30 & 336 & 6.33 & 24 & 26.0 & 7.6 & 340 \\
\hline 08B & 737 & 18.6 & 1.43 & 839 & 5.70 & 22 & 25.9 & 7.6 & 340 \\
\hline $07 \mathrm{~A}$ & 779 & 18.1 & 1.40 & 902 & 5.57 & 26 & 25.9 & 7.6 & 340 \\
\hline $\mathrm{X} 02$ & 808 & 18.5 & 1.48 & 548 & - & -- & - & - & -- \\
\hline 06B & 819 & 20.1 & 1.43 & 676 & 6.37 & 27 & 25.8 & 7.6 & 340 \\
\hline $05 \mathrm{~A}$ & 855 & 19.2 & 1.45 & 889 & 6.03 & 26 & 25.9 & 7.6 & 339 \\
\hline $04 \mathrm{~B}$ & 883 & 18.0 & 1.42 & 766 & 5.39 & 23 & 25.9 & 7.6 & 340 \\
\hline 03A & 915 & 17.3 & 1.45 & 626 & 5.46 & 21 & 25.9 & 7.6 & 340 \\
\hline $\mathrm{X} 01$ & 933 & 17.7 & 1.42 & 426 & -- & - & - & -- & -- \\
\hline 02B & 949 & 17.4 & 1.45 & 669 & 5.39 & 18 & 25.9 & 7.7 & 340 \\
\hline $01 \mathrm{~A}$ & 986 & 9.3 & 0.93 & 306 & 1.60 & 6 & 26.1 & 7.8 & 341 \\
\hline REW & 1,020 & 0.0 & 0.00 & 0 & -- & -- & -- & -- & -- \\
\hline MEAN & & 14.3 & 1.31 & & & & & & \\
\hline Total & 1,020 & & & 19,044 & 128.61 & 505 & & & \\
\hline
\end{tabular}




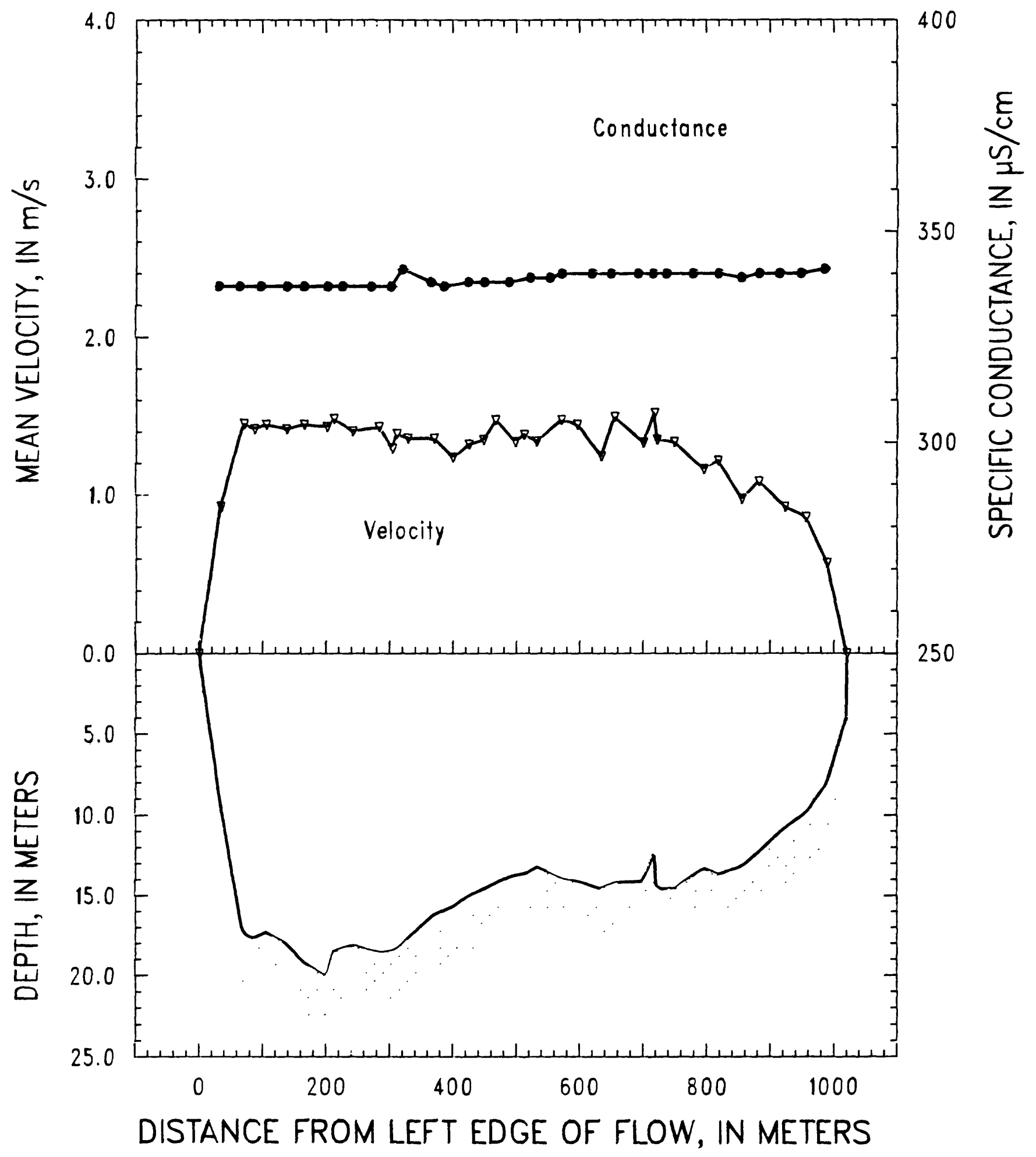

Figure 43. Mississippi River near St. Francisville, Louisiana, on June 26, 1989. 
SITE: Mississippi River below Belle Chasse, Louisiana

PARTY: Moody, Stevens, and Black

06-28-89

STARTING GAGE HEIGHT: -- ENDING GAGE HEIGHT: --

METER: SOLID CUP

SUSP: Bag sampler and 200-1b weight

CURRENT METER NO.: P8308282 DATE RATED: 07-08-88

CURRENT METER EQUATION: $\mathrm{V}(\mathrm{m} / \mathrm{s})=\operatorname{RPS} * 0.765+0.006$

REMARKS: Transit rate was $10 \mathrm{~cm} / \mathrm{s}$ and nozzle was $1 / 8$ inch.

\begin{tabular}{|c|c|c|c|c|c|c|c|c|c|}
\hline \multirow[b]{2}{*}{$\begin{array}{l}\text { Verti- } \\
\text { cal }\end{array}$} & \multirow{2}{*}{$\begin{array}{l}\text { Dist. } \\
\text { from } \\
\text { LEW } \\
\text { (m) }\end{array}$} & \multirow[b]{2}{*}{$\begin{array}{l}\text { Depth } \\
\text { (m) }\end{array}$} & \multirow{2}{*}{$\begin{array}{c}\text { Mean } \\
\text { veloc- } \\
\text { ity } \\
(\mathrm{m} / \mathrm{s})\end{array}$} & \multirow[b]{2}{*}{$\begin{array}{r}\text { Dis- } \\
\text { charge } \\
\left(\mathrm{m}^{3} / \mathrm{s}\right)\end{array}$} & \multicolumn{2}{|c|}{ Volume } & \multirow[b]{2}{*}{$\begin{array}{l}\text { Temper- } \\
\text { ature } \\
\left({ }^{\circ} \mathrm{C}\right)\end{array}$} & \multirow[b]{2}{*}{$\mathrm{pH}$} & \multirow{2}{*}{$\begin{array}{l}\text { Specific } \\
\text { conduct- } \\
\text { ance } \\
(\mu S / \mathrm{cm})\end{array}$} \\
\hline & & & & & $\begin{array}{l}\overline{V_{i}} \\
(\mathrm{~L})\end{array}$ & $\begin{array}{c}\mathrm{V}_{\mathrm{p}} \\
(\mathrm{L})\end{array}$ & & & \\
\hline LEW & 0 & 0.0 & 0.00 & 0 & -- & -- & -- & -- & -- \\
\hline $01 \mathrm{~A}$ & 63 & 11.3 & 0.83 & 388 & 1.39 & 10 & 26.2 & 7.9 & 362 \\
\hline X01 & 83 & 14.9 & 0.89 & 384 & -- & -- & -- & -- & -- \\
\hline $02 B$ & 121 & 18.5 & 1.12 & 770 & 3.31 & 27 & 26.2 & 7.9 & 364 \\
\hline $03 \mathrm{~A}$ & 157 & 19.2 & 1.21 & 1,042 & 4.17 & 33 & 26.2 & 7.9 & 362 \\
\hline $04 \mathrm{~B}$ & 211 & 20.9 & 1.24 & 1,157 & 4.23 & 37 & 26.2 & 7.9 & 364 \\
\hline X02 & 246 & 22.1 & 1.24 & 812 & -- & -- & -- & -- & -- \\
\hline $05 \mathrm{~A}$ & 270 & 23.0 & 1.37 & 1,075 & 4.90 & 43 & 26.3 & 7.9 & 361 \\
\hline 06B & 314 & 24.6 & 1.35 & 1,857 & 5.11 & 47 & 26.2 & 7.9 & 363 \\
\hline $07 \mathrm{~A}$ & 382 & 25.7 & 1.43 & 1,873 & 5.99 & 48 & 26.2 & 7.9 & 362 \\
\hline X03 & 416 & 26.3 & 1.34 & 1,237 & -- & -- & -- & -- & -- \\
\hline 08B & 452 & 26.7 & 1.33 & 1,435 & 5.63 & 40 & 26.1 & 7.9 & 363 \\
\hline 09A & 497 & 28.3 & 1.20 & 1,691 & 5.84 & 48 & 26.1 & 7.8 & 363 \\
\hline X04 & 552 & 28.5 & 1.15 & 1,095 & -- & -- & -- & -- & -- \\
\hline $10 \mathrm{~B}$ & 564 & 29.8 & 1.12 & 681 & 6.02 & 49 & 26.1 & 7.8 & 363 \\
\hline $11 \mathrm{~A}$ & 593 & 29.7 & 0.98 & 1,467 & 4.42 & 45 & 26.0 & 7.8 & 364 \\
\hline $12 \mathrm{~B}$ & 665 & 29.7 & 0.83 & 1,270 & 3.66 & 39 & 26.1 & 7.8 & 365 \\
\hline $13 \mathrm{~A}$ & 696 & 28.9 & 0.85 & 786 & 3.24 & 35 & 26.2 & 7.9 & 366 \\
\hline X05 & 729 & 24.5 & 0.79 & 501 & -- & -- & -- & -- & -- \\
\hline $14 B$ & 748 & 16.0 & 0.80 & 535 & 1.55 & 7 & 26.8 & 7.8 & 368 \\
\hline REW & 813 & 0.0 & 0.00 & 0 & -- & -- & -- & -- & -- \\
\hline MEAN & & 21.8 & 1.13 & & & & & & \\
\hline TOTAL & 813 & & & 20,056 & 59.26 & 508 & & & \\
\hline
\end{tabular}




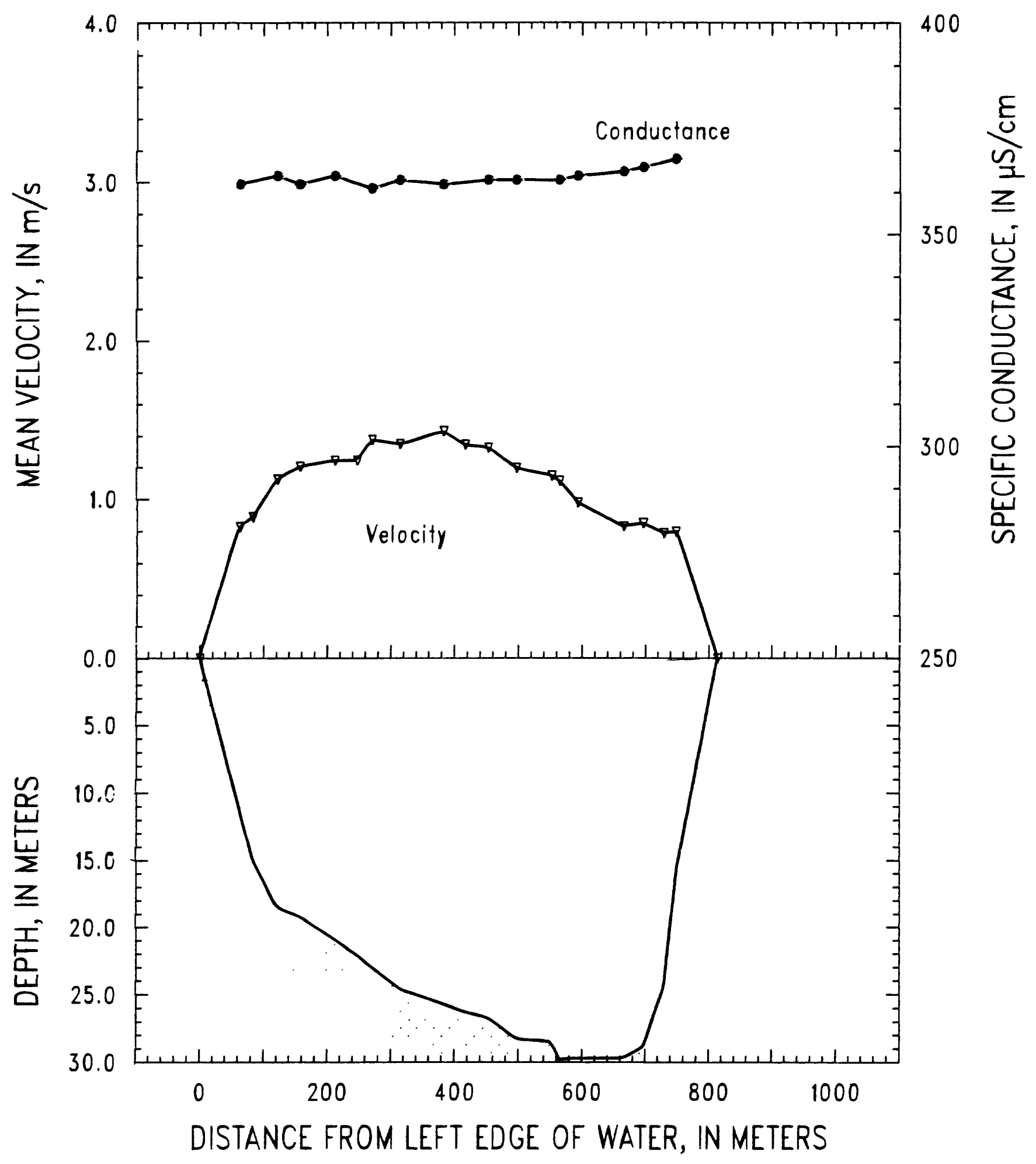

Figure 44. Mississippi River below Belle Chasse, Louisiana, on June 28, 1989. 
DATA LISTINGS

FOR

FEBRUARY-MARCH 1990 CRUISE 
SITE: Mississippi River near Cache, Illinois

$02-25-90$

PARTY: Moody, Meade, Tagg, and Simoneaux

METER: SOLID CUP

STARTING GAGE HEIGHT: -- ENDING GAGE HEIGHT: --

SUSP: Bag sampler and 150-1b weight

CURRENT METER NO.: W-297222 DATE RATED: 04-90

CURRENT METER EQUATION: $V(\mathrm{~m} / \mathrm{s})=$ RPS $* 0.744+0.011$

REMARKS: Transit rate was $5.0 \mathrm{~cm} / \mathrm{s}$, and 1/4-inch nozzle was used, no screen. Section is in last bend upstream from the confluence of the Upper

Mississippi and Ohio Rivers. Water seems to be standing in fields beyond small levees on both sides of river.

\begin{tabular}{|c|c|c|c|c|c|c|c|c|c|}
\hline \multirow[b]{2}{*}{$\begin{array}{c}\text { Verti- } \\
\text { cal }\end{array}$} & \multirow{2}{*}{$\begin{array}{l}\text { Dist. } \\
\text { from } \\
\text { LEW } \\
(\mathrm{m})\end{array}$} & \multirow[b]{2}{*}{$\begin{array}{c}\text { Depth } \\
(\mathrm{m})\end{array}$} & \multirow{2}{*}{$\begin{array}{l}\text { Mean } \\
\text { veloc- } \\
\text { ity } \\
(\mathrm{m} / \mathrm{s})\end{array}$} & \multirow[b]{2}{*}{$\begin{array}{r}\text { Dis- } \\
\text { charge } \\
\left(\mathrm{m}^{3} / \mathrm{s}\right)\end{array}$} & \multicolumn{2}{|c|}{ Volume } & \multirow[b]{2}{*}{$\begin{array}{l}\text { Temper- } \\
\text { ature } \\
\left({ }^{\circ} \mathrm{C}\right)\end{array}$} & \multirow[b]{2}{*}{$\mathrm{pH}$} & \multirow{2}{*}{$\begin{array}{c}\text { Specific } \\
\text { conduct- } \\
\text { ance } \\
(\mu \mathrm{S} / \mathrm{cm})\end{array}$} \\
\hline & & & & & $\begin{array}{l}\mathrm{V}_{\mathbf{i}} \\
(\mathrm{L})\end{array}$ & $\begin{array}{l}\overline{v_{p}} \\
(\mathrm{~L})\end{array}$ & & & \\
\hline$\overline{\text { LEW }}$ & 0 & 13.0 & 0.00 & 0 & -- & -- & -- & -- & -- \\
\hline $\mathrm{X} 01$ & 117 & 15.1 & 0.62 & 1,060 & 6.23 & 156 & 5.3 & 8.1 & -- \\
\hline X04 & 227 & 14.2 & 0.62 & 1,022 & 5.11 & 142 & 4.7 & 8.2 & -- \\
\hline X05 & 350 & 11.5 & 0.60 & 823 & 4.73 & 106 & 4.7 & 8.2 & 504 \\
\hline X06 & 467 & 10.0 & 0.59 & 716 & 3.59 & 86 & 5.0 & 8.0 & 504 \\
\hline $\mathrm{X} 07$ & 594 & 11.4 & 0.47 & 617 & 1.80 & 108 & 5.2 & 8.1 & 502 \\
\hline REW & 696 & 8.0 & 0.00 & 0 & -- & -- & -- & -- & -- \\
\hline MEAN & & 10.4 & 0.58 & & & & & & \\
\hline TOTAL & 696 & & & 4,238 & 21.46 & 598 & & & \\
\hline
\end{tabular}




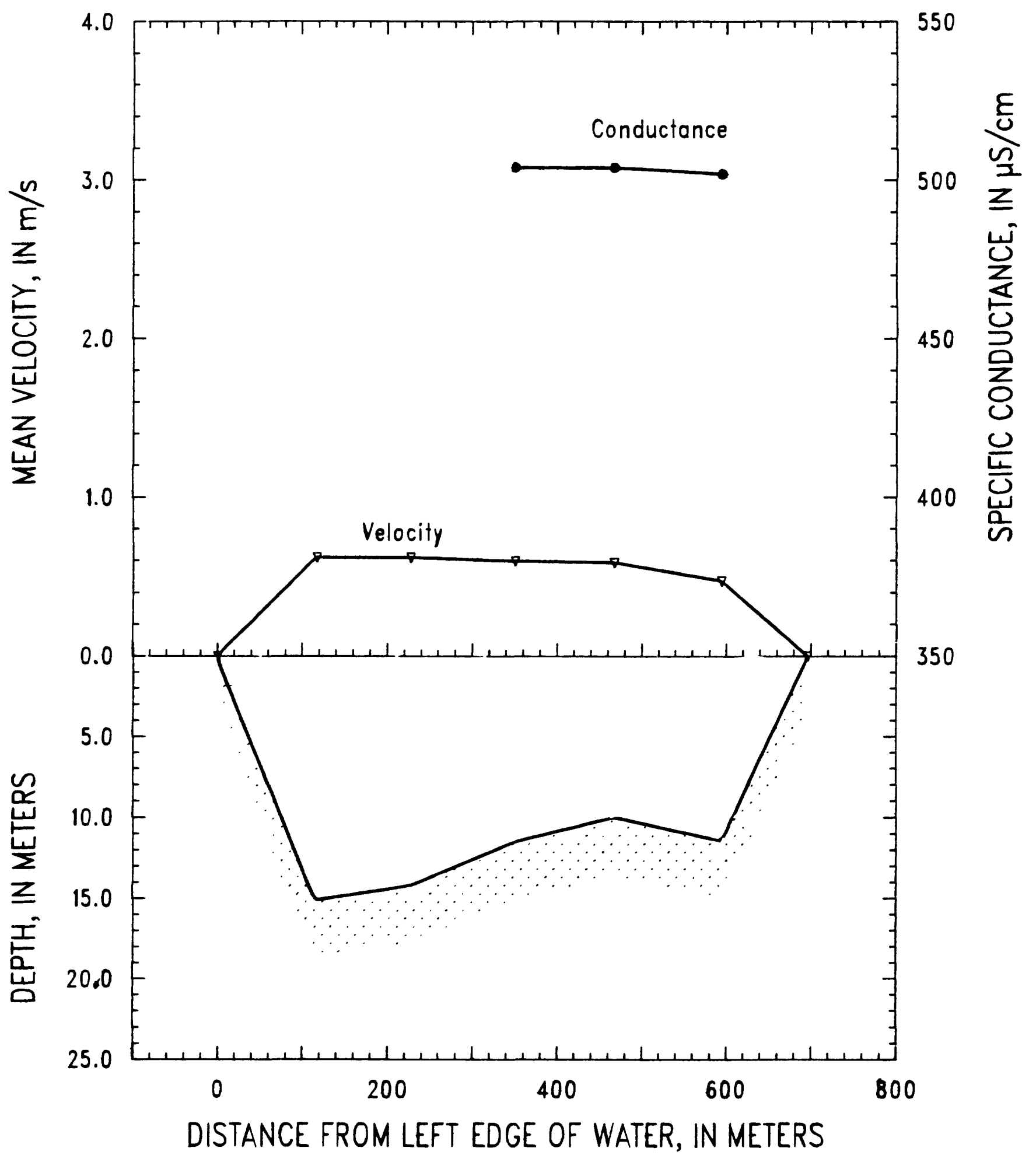

Figure 45. Mississippi River near Cache, lllinois, on February 25, 1990 
SITE: Ohio River at Uniontown, Kentucky

PARTY: Moody, Tagg, and Simoneaux

03-01-90

STARTING GAGE HEIGHT: -- ENDING GAGE HEIGHT: --

METER: SOLID CUP

SUSP: Bag sampler and 200-1b weight

CURRENT METER NO.: W-297222 DATE RATED: 04-90

CURRENT METER EQUATION: $V(\mathrm{~m} / \mathrm{s})=$ RPS $* 0.744+0.011$

REMARKS: Transit rate was $9 \mathrm{~cm} / \mathrm{s}$, and $1 / 4$-inch nozzle was used. Leaves very troublesome in middle of section. Verticals occupied from 30 to 2 .

National Weather Service forecasted discharge for Uniontown was $6,660 \mathrm{~m}^{3} / \mathrm{s}$.

\begin{tabular}{|c|c|c|c|c|c|c|c|c|c|}
\hline & Dist. & & Mean & & Vol & & & & Specific \\
\hline $\begin{array}{c}\text { Verti- } \\
\text { cal }\end{array}$ & $\begin{array}{l}\text { from } \\
\text { LEW } \\
\text { (m) }\end{array}$ & $\begin{array}{l}\text { Depth } \\
\text { (m) }\end{array}$ & $\begin{array}{l}\text { veloc- } \\
\text { ity } \\
(\mathrm{m} / \mathrm{s})\end{array}$ & $\begin{array}{r}\text { Dis- } \\
\text { charge } \\
\left(\mathrm{m}^{3} / \mathrm{s}\right)\end{array}$ & $\begin{array}{l}\overline{v_{i}} \\
(L)\end{array}$ & $\begin{array}{l}\overline{v_{p}} \\
(\mathrm{~L})\end{array}$ & $\begin{array}{l}\text { Temper- } \\
\text { ature } \\
\left({ }^{\circ} \mathrm{C}\right)\end{array}$ & $\mathrm{pH}$ & $\begin{array}{l}\text { conduct- } \\
\text { ance } \\
(\mu \mathrm{S} / \mathrm{cm})\end{array}$ \\
\hline
\end{tabular}

\begin{tabular}{|c|c|c|c|c|c|c|c|c|c|}
\hline LEW & 0 & 0.0 & 0.00 & $\overline{0}$ & -- & -- & $=$ & -- & - \\
\hline $02 \mathrm{~A}$ & 59 & 4.2 & 0.40 & 67 & 0.81 & 3 & -- & 7.3 & 273 \\
\hline 03B & 79 & 4.9 & 0.41 & 36 & 0.69 & 3 & -- & 7.4 & 275 \\
\hline $04 \mathrm{~A}$ & 95 & 5.7 & 0.42 & 43 & 0.80 & 5 & -- & 7.4 & 275 \\
\hline 05B & 115 & 6.8 & 0.45 & 82 & 1.19 & 8 & -- & 7.5 & 277 \\
\hline $06 \mathrm{~A}$ & 148 & 8.6 & 0.61 & 171 & 1.41 & 13 & 5.1 & 7.4 & 278 \\
\hline 07B & 180 & 8.9 & 0.77 & 164 & 3.43 & 13 & 5.2 & 7.5 & 278 \\
\hline $08 \mathrm{~A}$ & 196 & 9.0 & 0.71 & 128 & 2.76 & 14 & 5.3 & 7.4 & 279 \\
\hline 09B & 220 & 9.3 & 0.83 & 143 & 3.60 & 16 & 5.4 & 7.4 & 279 \\
\hline X04 & 233 & 9.7 & 0.80 & 94 & -- & -- & -- & -- & - \\
\hline $10 \mathrm{~A}$ & 244 & 9.7 & 0.90 & 175 & 3.64 & 17 & 5.5 & 7.4 & 279 \\
\hline $11 \mathrm{~B}$ & 273 & 9.8 & 0.87 & 255 & 3.70 & 17 & 5.7 & 7.4 & 280 \\
\hline $12 \mathrm{~A}$ & 304 & 10.5 & 0.95 & 255 & 2.69 & 17 & 5.8 & 7.2 & 282 \\
\hline $13 B$ & 324 & 10.8 & 0.99 & 145 & 3.72 & 19 & 6.0 & 7.3 & 280 \\
\hline $\mathrm{X} 12$ & 331 & 10.9 & 0.92 & 121 & - & -- & -- & -- & -- \\
\hline $14 \mathrm{~A}$ & 348 & 11.2 & 0.95 & 208 & 3.51 & 19 & 6.1 & 7.3 & 282 \\
\hline $15 B$ & 370 & 11.7 & 1.06 & 223 & 5.16 & 21 & 6.1 & 7.3 & 284 \\
\hline X03 & 384 & 11.8 & 0.96 & 148 & -- & -- & -- & -- & -- \\
\hline $16 \mathrm{~A}$ & 396 & 11.9 & 0.97 & 231 & 3.34 & 22 & 6.1 & 7.3 & 284 \\
\hline $17 \mathrm{~B}$ & 424 & 12.2 & 0.99 & 291 & 5.51 & 22 & 6.1 & 7.2 & 286 \\
\hline $18 \mathrm{~A}$ & 444 & 12.5 & 1.05 & 281 & 5.28 & 22 & 6.1 & 7.2 & 287 \\
\hline $19 \mathrm{~B}$ & 467 & 12.8 & 1.02 & 352 & 5.29 & 24 & 6.1 & 7.2 & 287 \\
\hline $20 \mathrm{~A}$ & 498 & 12.7 & 0.96 & 263 & 5.48 & 23 & 6.3 & 7.3 & 289 \\
\hline $21 B$ & 510 & 12.8 & 1.01 & 285 & 5.93 & 23 & 6.1 & 7.2 & 288 \\
\hline $22 \mathrm{~A}$ & 542 & 12.5 & 0.98 & 354 & 4.74 & 23 & 6.1 & 7.2 & 290 \\
\hline $23 B$ & 568 & 12.3 & 0.93 & 268 & 5.27 & 22 & 6.2 & 7.2 & 290 \\
\hline $24 \mathrm{~A}$ & 589 & 12.0 & 0.96 & 258 & 5.19 & 22 & 6.2 & 7.3 & 291 \\
\hline $25 B$ & 613 & 12.0 & 0.98 & 278 & 5.86 & 22 & 6.1 & 7.0 & 296 \\
\hline $26 \mathrm{~A}$ & 636 & 12.4 & 0.94 & 290 & 5.18 & 23 & 5.9 & 7.2 & 294 \\
\hline $27 B$ & 663 & 12.6 & 0.94 & 289 & 6.20 & 22 & 5.9 & 7.2 & 295 \\
\hline $28 \mathrm{~A}$ & 685 & 12.4 & 0.89 & 259 & 5.00 & 21 & 6.0 & 7.2 & 298 \\
\hline $29 B$ & 710 & 12.3 & 0.86 & 243 & 5.24 & 17 & 6.3 & 7.2 & 296 \\
\hline $30 \mathrm{~A}$ & 731 & 10.9 & 0.80 & 221 & 3.69 & 10 & 6.1 & 7.3 & 295 \\
\hline REW & 761 & 0.0 & 0.00 & 0 & -- & - & -- & - & -- \\
\hline MEAN & & 9.8 & 0.88 & & & & & & \\
\hline TOTAL & 761 & & & 6,621 & 114.31 & 503 & & & \\
\hline
\end{tabular}




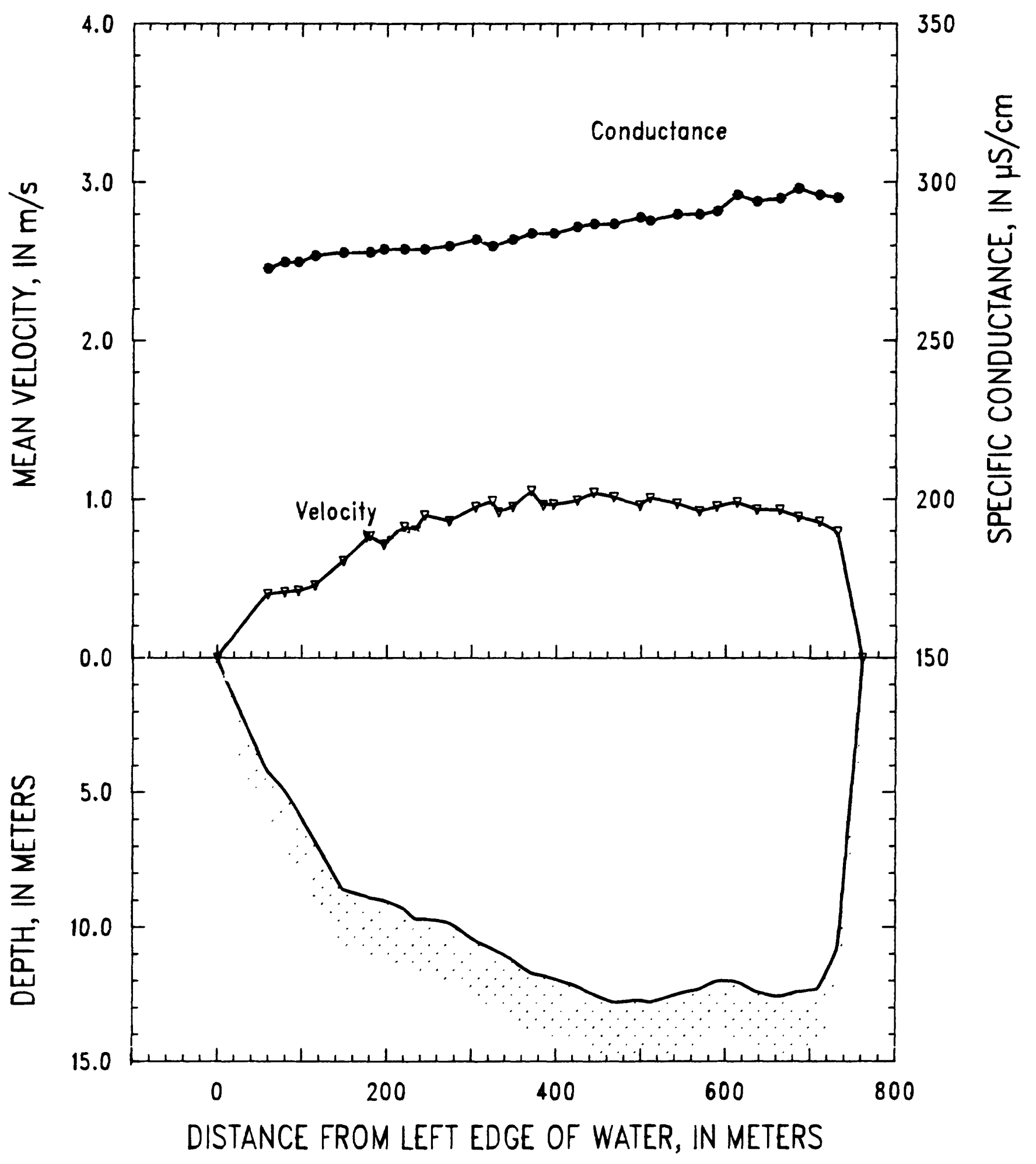

Figure 46. Ohio River at Uniontown, Kentucky, on March 1, 1990. 
SITE: Wabash River near New Haven, Illinois

PARTY: Moody, Tagg, and Simoneaux

METER: SOLID CUP

STARTING GAGE HEIGHT: -- ENDING GAGE HEIGHT: --

SUSP: Bag sampler and 150-1b weight

CURRENT METER NO.: W-297222 DATE RATED: 04-90

CURRENT METER EQUATION: $V(\mathrm{~m} / \mathrm{s})=$ RPS $* 0.744+0.011$

REMARKS: Transit rate was $7 \mathrm{~cm} / \mathrm{s}, 1 / 4-$ inch nozzle was used. REW and LEW were probably $>200 \mathrm{~m}$ from REF and LEF. There was flow across the flood plain (perhaps $0.5 \mathrm{~m}$ deep) which was not measured. National Weather Service forecast model for the Wabash River at New Harmony was $2,660 \mathrm{~m}^{3} / \mathrm{s}$.

\begin{tabular}{|c|c|c|c|c|c|c|c|c|c|}
\hline & st & & Mea & & Vol & & & & \\
\hline $\begin{array}{l}\text { Verti- } \\
\text { cal }\end{array}$ & $\begin{array}{l}\text { from } \\
\text { LEF } \\
(\mathrm{m})\end{array}$ & $\begin{array}{l}\text { Depth } \\
\text { (m) }\end{array}$ & $\begin{array}{c}\text { veloc- } \\
\text { ity } \\
(\mathrm{m} / \mathrm{s})\end{array}$ & $\begin{array}{r}\text { Dis- } \\
\text { charge } \\
\left(\mathrm{m}^{3} / \mathrm{s}\right)\end{array}$ & $\begin{array}{l}\overline{V_{i}} \\
(\mathrm{~L})\end{array}$ & $\begin{array}{l}V_{p} \\
(L)\end{array}$ & $\begin{array}{l}\text { Temper- } \\
\text { ature } \\
\left({ }^{\circ} \mathrm{C}\right)\end{array}$ & $\mathrm{pH}$ & $\begin{array}{l}\text { conduct- } \\
\text { ance } \\
(\mu \mathrm{S} / \mathrm{cm})\end{array}$ \\
\hline
\end{tabular}

\begin{tabular}{lrrrrrrrrr}
\hline LEF & 0 & 0.5 & 0.00 & 0 & -- & -- & -- & -- & -- \\
01A, B & 33 & 7.8 & 1.16 & 262 & 8.38 & 27 & 2.9 & 7.8 & 405 \\
02A,B & 58 & 7.7 & 1.08 & 133 & 9.45 & 44 & 3.1 & 7.9 & 403 \\
X01 & 65 & 7.8 & 1.08 & 51 & -- & -- & -- & -- & -- \\
X02 & 70 & 7.8 & 1.05 & 49 & -- & -- & -- & -- & -- \\
03A, B & 77 & 7.8 & 1.07 & 125 & 9.43 & 41 & 3.9 & 7.5 & 404 \\
04B & 100 & 7.9 & 1.03 & 118 & 5.57 & -- & -- & -- & -- \\
04A & 106 & 8.0 & 1.02 & 106 & 5.57 & 43 & 3.3 & 7.8 & 402 \\
05A & 126 & 8.3 & 1.01 & 105 & 4.99 & 42 & 3.5 & 7.7 & 403 \\
05B & 131 & 8.2 & 1.07 & 127 & 6.04 & -- & -- & -- & -- \\
06B & 155 & 7.9 & 1.10 & 122 & 6.00 & -- & -- & -- & -- \\
06A & 159 & 7.7 & 1.06 & 77 & 4.63 & 42 & 3.4 & 7.7 & 403 \\
07A & 174 & 7.2 & 1.09 & 74 & 4.82 & 45 & 3.5 & 7.7 & 402 \\
07B & 178 & 7.2 & 1.08 & 93 & 5.06 & -- & -- & -- & -- \\
08A,B & 198 & 6.5 & 1.07 & 150 & 8.88 & 43 & 3.0 & 7.7 & 404 \\
09A,B & 221 & 5.6 & 1.15 & 119 & 7.77 & 33 & 3.1 & 7.8 & 401 \\
X05 & 235 & 5.5 & 1.14 & 72 & -- & -- & -- & -- & -- \\
10A,B & 244 & 5.3 & 1.16 & 98 & 7.47 & 33 & 3.2 & 7.7 & 401 \\
11A,B & 267 & 5.0 & 1.05 & 120 & 6.67 & 31 & 3.1 & 7.9 & 402 \\
12A,B & 290 & 4.8 & 1.09 & 95 & 6.37 & 30 & 3.4 & 7.8 & 403 \\
13A & 303 & 4.7 & 1.13 & 72 & 3.37 & 26 & 3.5 & 7.7 & 398 \\
13B & 317 & 4.6 & 1.16 & 96 & 3.70 & -- & -- & -- & -- \\
14A,B & 339 & 4.6 & 0.87 & 76 & 4.82 & 17 & 3.6 & 7.7 & 399 \\
REF & 355 & 0.5 & 0.00 & 0 & -- & -- & -- & -- & -- \\
& & & & & & & & & \\
MEAN & & 6.1 & 1.08 & & & & & & \\
TOTAL & 355 & & & 2,340 & 118.99 & 497 & & & \\
& & & & & & & & & \\
\end{tabular}




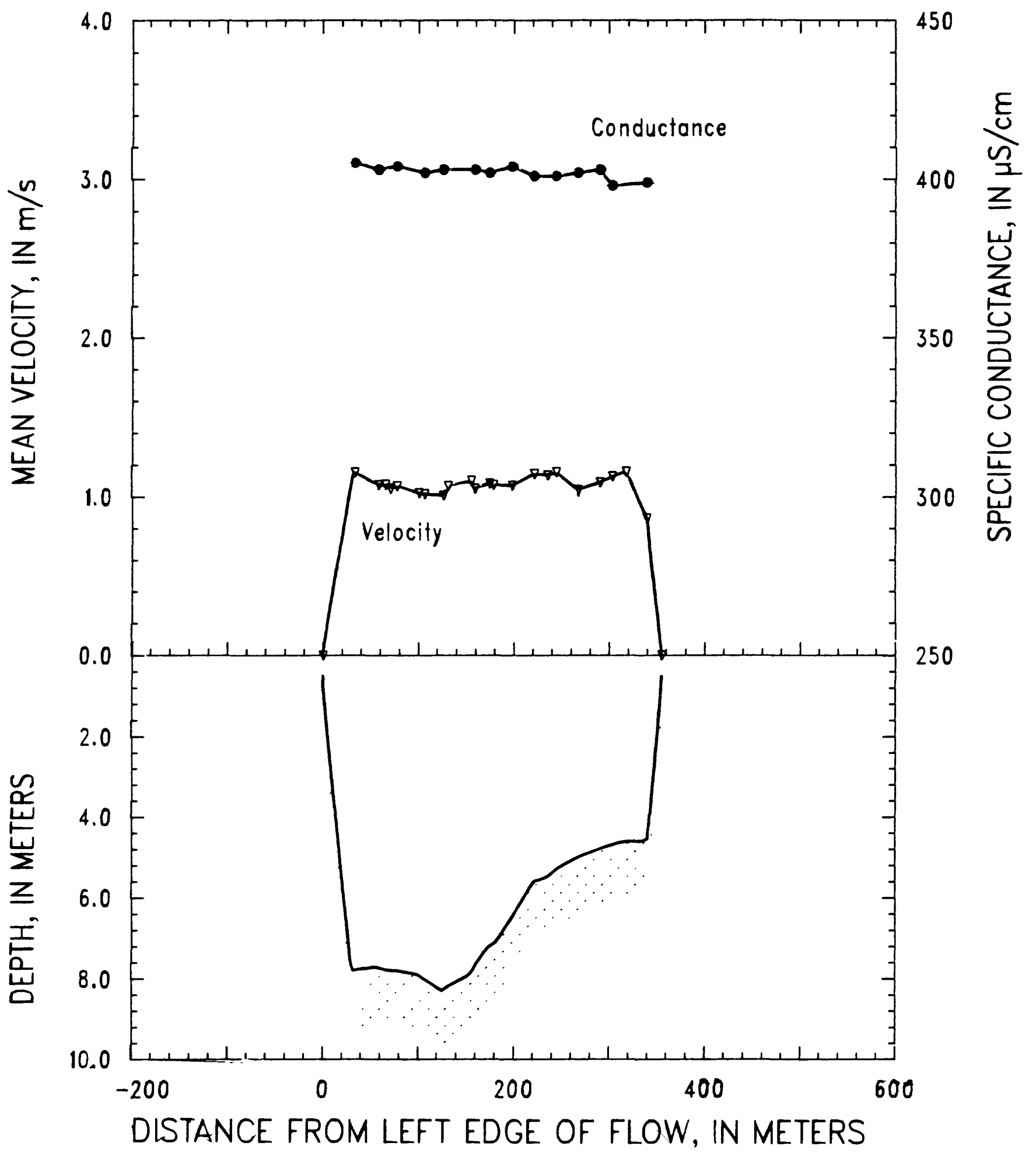

Figure 47. Wabash River near New Haven, Illinois, on February 28, 1990. 
SITE: Cumberland River near Smithland, Kentucky

$02-23-90$

PARTY: Moody, Tagg, and Simoneaux

METER: SOLID CUP

STARTING GAGE HEIGHT: -- ENDING GAGE HEIGHT: --

SUSP: Bag sampler and 200-1b weight

CURRENT METER NO.: W-297222 DATE RATED: 04-90

CURRENT METER EQUATION: $V(\mathrm{~m} / \mathrm{s})=$ RPS $* 0.744+0.011$

REMARKS: Transit rate was $9 \mathrm{~cm} / \mathrm{s}$, and $3 / 16$-inch nozzle was used. LEW $=-40 \mathrm{~m}$, $\mathrm{REW}=216 \mathrm{~m}$, and unmeasured overbank $=(40+41)=81 \mathrm{~m}$. Barkley Dam's discharge was reported as $2,270 \mathrm{~m}^{3} / \mathrm{s}$.

\begin{tabular}{|c|c|c|c|c|c|c|c|c|c|}
\hline & Dist. & & Mean & & Vol & & & & Specific \\
\hline $\begin{array}{l}\text { Verti- } \\
\text { cal }\end{array}$ & $\begin{array}{l}\text { f rom } \\
\text { LEF } \\
\text { (m) }\end{array}$ & $\begin{array}{c}\text { Depth } \\
(\mathrm{m})\end{array}$ & $\begin{array}{l}\text { veloc- } \\
\text { ity } \\
(\mathrm{m} / \mathrm{s})\end{array}$ & $\begin{array}{r}\text { Dis- } \\
\text { charge } \\
\left(\mathrm{m}^{3} / \mathrm{s}\right)\end{array}$ & $\begin{array}{l}\overline{V_{i}} \\
(\mathrm{~L})\end{array}$ & $\begin{array}{l}\overline{V_{p}} \\
(L)\end{array}$ & $\begin{array}{l}\text { Temper- } \\
\text { ature } \\
\left({ }^{\circ} \mathrm{C}\right)\end{array}$ & $\mathrm{pH}$ & $\begin{array}{l}\text { conduct- } \\
\text { ance } \\
(\mu \mathrm{S} / \mathrm{cm})\end{array}$ \\
\hline
\end{tabular}

\begin{tabular}{|c|c|c|c|c|c|c|c|c|c|}
\hline LEF & 0 & 9.0 & 0.00 & 0 & -- & -- & -- & $\cdots$ & -- \\
\hline $01 \mathrm{~B}$ & 4 & 9.7 & 0.49 & 19 & 1.12 & -- & -- & -- & -- \\
\hline $01 \mathrm{~A}$ & 8 & 10.8 & 0.56 & 46 & 1.37 & 27 & 10.3 & 7.6 & 216 \\
\hline $2 A, B$ & 19 & 13.7 & 0.55 & 49 & 3.65 & 50 & 10.4 & 7.7 & 221 \\
\hline X04 & 21 & 14.0 & 0.52 & 51 & -- & -- & -- & - & -- \\
\hline 03B & 33 & 15.3 & 0.65 & 124 & 3.49 & -- & -- & -- & -- \\
\hline $03 \mathrm{~A}$ & 46 & 15.3 & 0.74 & 107 & 3.47 & 50 & 10.7 & 7.7 & 216 \\
\hline $04 \mathrm{~A}$ & 52 & 15.3 & 0.82 & 63 & 4.36 & 48 & 10.3 & 7.5 & 215 \\
\hline 04B & 56 & 15.4 & 0.82 & 126 & 3.56 & -- & 10.3 & 7.5 & 215 \\
\hline 05B & 72 & 15.7 & 0.90 & 234 & 4.70 & -- & -- & - & - \\
\hline $05 \mathrm{~A}$ & 78 & 15.8 & -- & -- & 4.04 & 55 & 10.2 & 7.6 & 214 \\
\hline $06 \mathrm{~A}$ & 89 & 16.4 & 0.98 & 184 & 5.46 & 60 & 10.3 & 7.7 & 216 \\
\hline 06B & 95 & 16.7 & 0.97 & 113 & 5.17 & -- & -- & -- & -- \\
\hline X02 & 103 & 17.2 & 0.96 & 124 & - & -- & -- & -- & -- \\
\hline $07 \mathrm{~A}$ & 110 & 17.5 & 0.93 & 89 & 5.17 & 66 & 10.4 & 7.5 & 216 \\
\hline 07B & 114 & 17.8 & 0.94 & 142 & 5.87 & -- & -- & -- & -- \\
\hline 08B & 127 & 17.5 & 0.95 & 133 & 5.62 & -- & -- & -- & -- \\
\hline $08 \mathrm{~A}$ & 130 & 17.2 & 0.97 & 134 & 5.68 & 59 & 10.4 & 7.5 & 214 \\
\hline $\mathrm{X} 03$ & 143 & 16.6 & 0.92 & 161 & -- & -- & -- & - & -- \\
\hline $09 \mathrm{~A}$ & 151 & 16.3 & 0.87 & 71 & 3.73 & 49 & 10.2 & 7.6 & 214 \\
\hline 09B & 153 & 15.9 & 0.73 & 116 & 3.97 & - & -- & -- & - \\
\hline X08 & 171 & 13.7 & 0.51 & 73 & - & -- & -- & -- & -- \\
\hline $10 \mathrm{~A}, \mathrm{~B}$ & 174 & 13.0 & 0.50 & 13 & 3.82 & 35 & 10.4 & 7.5 & 213 \\
\hline REF & 175 & 10.0 & 0.00 & 0 & -- & - & -- & -- & - \\
\hline MEAN & & 15.3 & 0.81 & & & & & & \\
\hline TOTAL & 175 & & & 2,172 & 74.25 & 499 & & & \\
\hline
\end{tabular}




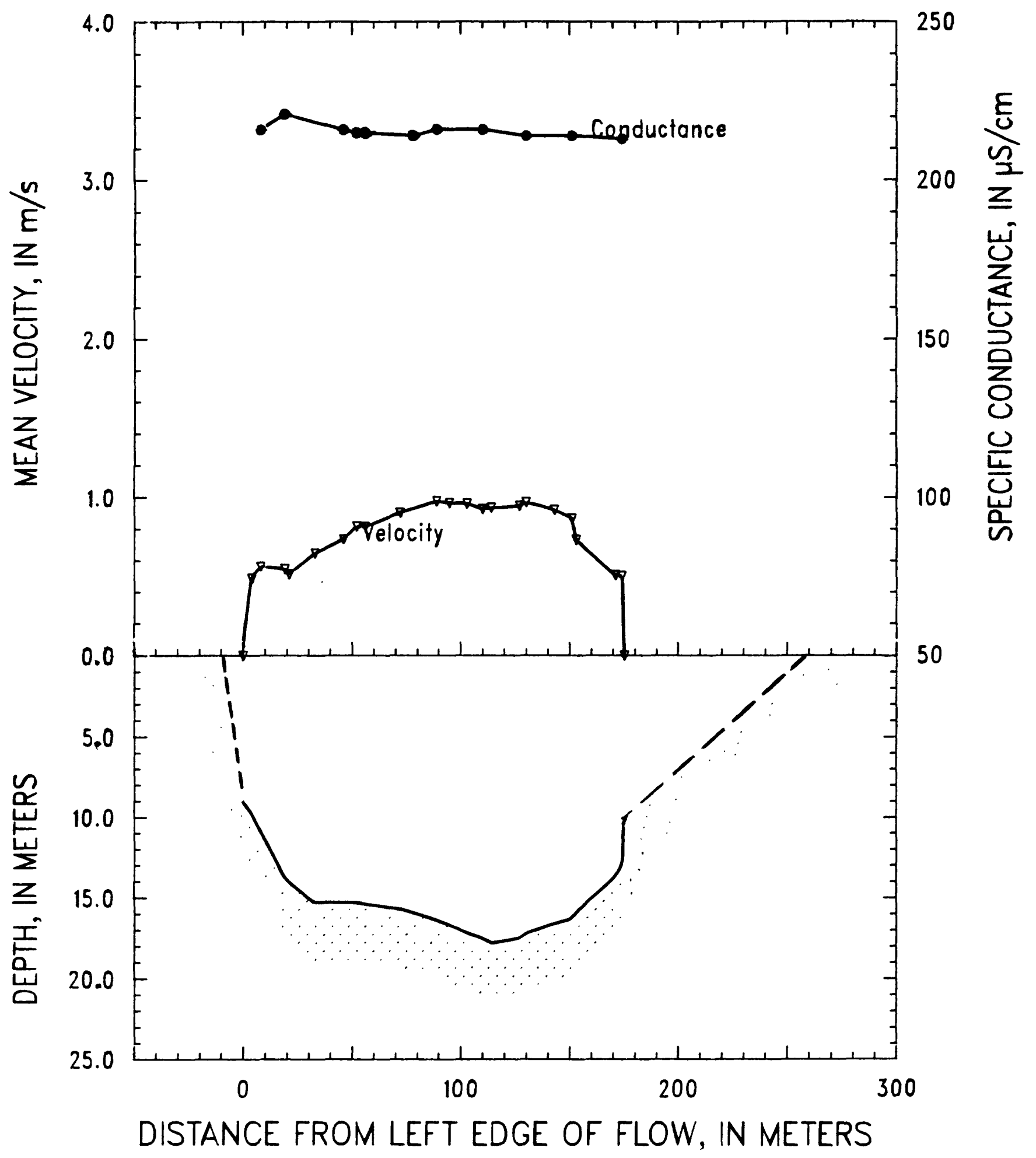

Figure 48. Cumberland River near Smithland, Kentucky, on February 23, 1990. 
SITE: Tennessee River near Calvert City, Kentucky

PARTY: Moody, Tagg, and Simoneaux

STARTING GAGE HEIGHT: --

ENDING GAGE HEIGHT:

METER: SOLID CUP

SUSP: Bag sampler and 200-1b weight

CURRENT METER NO.: W-297222 DATE RATED: 04-90

CURRENT METER EQUATION: $V(\mathrm{~m} / \mathrm{s})=$ RPS $* 0.744+0.011$

REMARKS: Transit rate was $15 \mathrm{~cm} / \mathrm{s}$, and $3 / 16$-inch nozzle was used. Cold, cold and windy day, gusts 30-40 mph from northwest. E-reel damaged.

$\mathrm{REW}=382 \mathrm{~m}$; LEW was at least $-312 \mathrm{~m}$; overbank discharge was not measured.

Discharge at Kentucky Dam was reported as $6,990 \mathrm{~m}^{3} / \mathrm{s}$.

\begin{tabular}{|c|c|c|c|c|c|c|c|c|c|}
\hline \multirow[b]{2}{*}{$\begin{array}{l}\text { Verti- } \\
\text { cal }\end{array}$} & \multirow{2}{*}{$\begin{array}{l}\text { Dist. } \\
\text { from } \\
\text { LEF } \\
\text { (m) }\end{array}$} & \multirow[b]{2}{*}{$\begin{array}{l}\text { Depth } \\
\text { (m) }\end{array}$} & \multirow{2}{*}{$\begin{array}{l}\text { Mean } \\
\text { veloc- } \\
\text { ity } \\
(\mathrm{m} / \mathrm{s})\end{array}$} & \multirow[b]{2}{*}{$\begin{array}{r}\text { Dis- } \\
\text { charge } \\
\left(\mathrm{m}^{3} / \mathrm{s}\right)\end{array}$} & \multicolumn{2}{|c|}{ Volume } & \multirow[b]{2}{*}{$\begin{array}{c}\text { Temper- } \\
\text { a ture } \\
\left({ }^{\circ} \mathrm{C}\right)\end{array}$} & \multirow[b]{2}{*}{$\mathrm{pH}$} & \multirow{2}{*}{$\begin{array}{c}\text { Specific } \\
\text { conduct- } \\
\text { ance } \\
(\mu \mathrm{S} / \mathrm{cm})\end{array}$} \\
\hline & & & & & $\begin{array}{l}\overline{\mathrm{V}_{\mathrm{i}}} \\
(\mathrm{L})\end{array}$ & $\begin{array}{l}\bar{V}_{p} \\
(I)\end{array}$ & & & \\
\hline LEF & 0 & 10.0 & 0.00 & 0 & -- & -- & -- & -- & -- \\
\hline $01 B$ & 8 & 10.7 & 0.76 & 65 & 2.16 & -- & -- & -- & -- \\
\hline $01 \mathrm{~A}$ & 14 & 13.2 & 0.93 & 153 & 2.65 & 17 & 10.4 & 7.4 & 137 \\
\hline $02 A, B$ & 33 & 15.4 & 1.19 & 448 & 8.05 & 35 & 10.6 & 7.2 & 136 \\
\hline $03 \mathrm{~A}, \mathrm{~B}$ & 33 & 16.6 & 1.29 & 449 & 8.93 & 42 & 10.4 & 7.3 & 137 \\
\hline $04 A^{\prime}$ & 75 & 17.1 & 1.34 & 286 & 5.37 & 44 & 10.5 & 7.2 & 135 \\
\hline 04B & 90 & 17.8 & 1.40 & 460 & 5.03 & -- & -- & -- & -- \\
\hline $05 \mathrm{~A}, \mathrm{~B}$ & 112 & 19.4 & 1.29 & 512 & 11.63 & 48 & 10.6 & 7.4 & 136 \\
\hline $06 \mathrm{~A}$ & 131 & 20.6 & 1.26 & 352 & 5.48 & 50 & 10.6 & 7.4 & 138 \\
\hline 06B & 139 & 20.5 & 1.33 & 341 & 5.67 & -- & -- & -- & -- \\
\hline 07B & 156 & 21.4 & 1.25 & 320 & 5.57 & -- & -- & -- & -- \\
\hline 07A & 163 & 21.6 & 1.22 & 381 & 6.61 & 53 & 10.6 & 7.3 & 137 \\
\hline $08 \mathrm{~A}, \mathrm{~B}$ & 185 & 21.8 & 1.30 & 565 & 12.08 & 54 & 10.8 & 7.3 & 137 \\
\hline $09 A, B$ & 203 & 21.3 & 1.28 & 423 & 11.97 & 51 & 10.8 & 7.4 & 138 \\
\hline $10 \mathrm{~A}$ & 216 & 20.5 & 1.28 & 368 & 5.64 & 40 & 10.7 & 7.3 & 138 \\
\hline $10 \mathrm{~B}$ & 231 & 19.2 & 1.22 & 375 & 5.06 & -- & -- & -- & -- \\
\hline $11 \mathrm{~A}$ & 240 & 18.5 & 1.15 & 223 & 3.87 & 18 & 10.6 & 7.3 & 140 \\
\hline $11 B$ & 252 & 18.2 & 1.11 & 223 & 4.31 & -- & -- & - & - \\
\hline $12 \mathrm{~B}$ & 270 & 13.8 & 0.93 & 154 & 2.47 & -- & -- & -- & -- \\
\hline $12 \mathrm{~A}$ & 276 & 13.8 & 0.86 & 207 & 2.43 & 34 & 10.7 & 7.3 & 138 \\
\hline $13 \mathrm{~A}, \mathrm{~B}$ & 305 & 9.9 & 0.76 & 189 & 2.50 & 9 & 10.7 & 7.3 & 138 \\
\hline $14 \mathrm{~A}, \mathrm{~B}$ & 326 & 6.5 & 0.69 & 78 & 1.45 & 5 & 10.7 & 7.3 & 138 \\
\hline REF & 340 & 5.0 & 0.00 & 0 & -- & -- & -- & -- & -- \\
\hline MEAN & & 16.3 & 1.18 & & & & & & \\
\hline TOTAL & 340 & & & 6,572 & 117.94 & 500 & & & \\
\hline
\end{tabular}




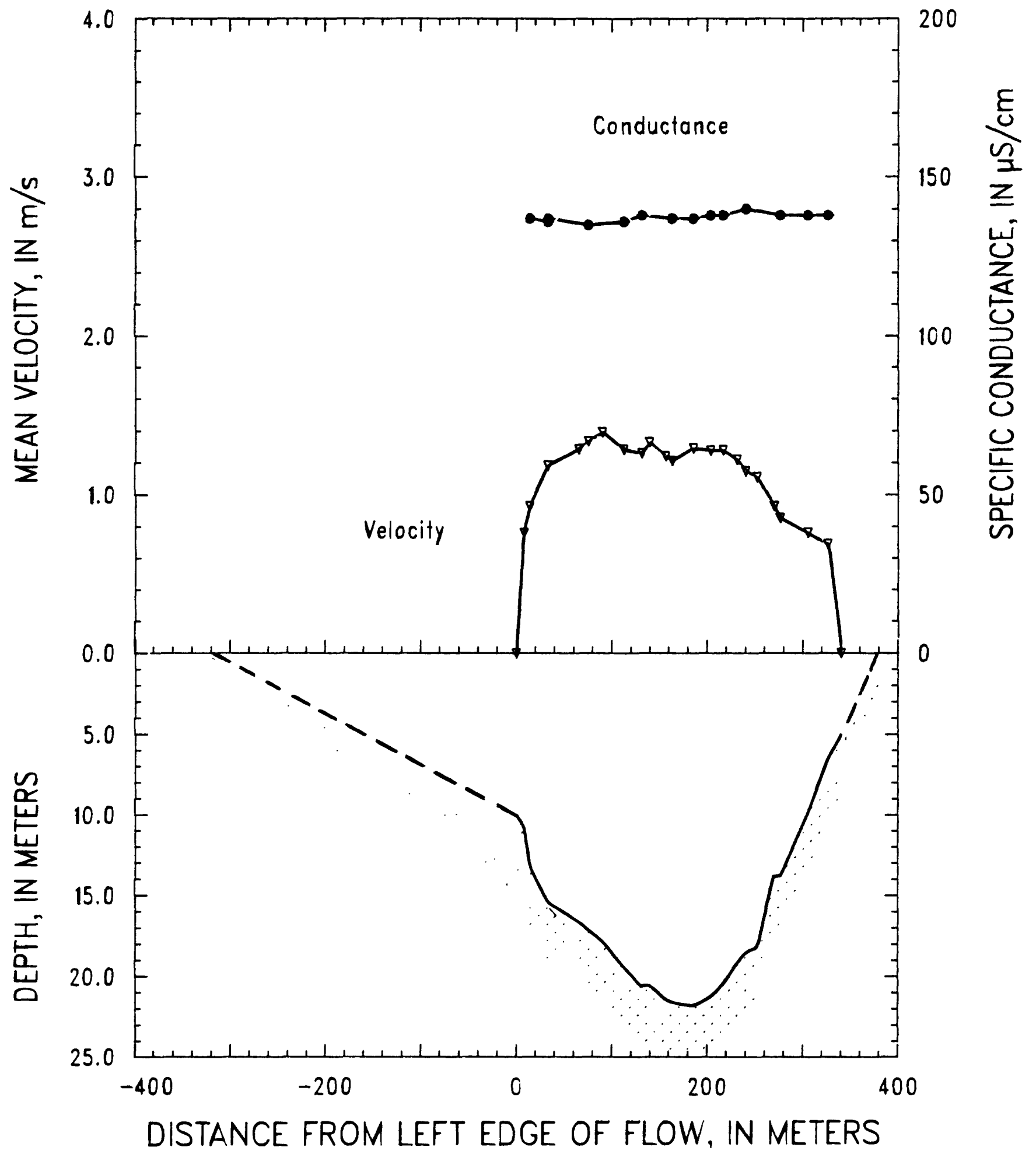

Figure 49. Tennessee River near Calvert City, Kentucky, on February 24, 1990. 
SITE: Ohio River at Olmsted, Illinois

PARTY: Moody, Tagg, and Simoneaux

STARTING GAGE HEIGHT: $41.4 \mathrm{ft}$ ENDING GAGE HEIGHT: $40.2 \mathrm{ft}$

METER: SOLID CUP

SUSP: Bag sampler and 200-1b weight

CURRENT METER NO.: W-297222 DATE RATED: 04-90

CURRENT METER EQUATION: $\mathrm{V}(\mathrm{m} / \mathrm{s})=$ RPS $* 0.744+0.011$

REMARKS: Transit rate was $12 \mathrm{~cm} / \mathrm{s}$, and 3/16-inch nozzle was used. Verticals occupied from 15 to 1 . National Weather Service forecast for Joppa was $15,750 \mathrm{~m}^{3} / \mathrm{s}$. Five drift cards marking Uniontown water passed by between 1235-1431.

\begin{tabular}{|c|c|c|c|c|c|c|c|c|c|}
\hline \multirow[b]{2}{*}{$\begin{array}{l}\text { Verti- } \\
\text { cal }\end{array}$} & \multirow{2}{*}{$\begin{array}{l}\text { Dist. } \\
\text { from } \\
\text { LEW } \\
\text { (m) }\end{array}$} & \multirow[b]{2}{*}{$\begin{array}{l}\text { Depth } \\
\text { (m) }\end{array}$} & \multirow{2}{*}{$\begin{array}{l}\text { Mean } \\
\text { veloc- } \\
\text { ity } \\
(\mathrm{m} / \mathrm{s})\end{array}$} & \multirow[b]{2}{*}{$\begin{array}{r}\text { Dis- } \\
\text { charge } \\
\left(\mathrm{m}^{3} / \mathrm{s}\right)\end{array}$} & \multicolumn{2}{|c|}{ Volume } & \multirow[b]{2}{*}{$\begin{array}{l}\text { Temper- } \\
\text { ature } \\
\left({ }^{\circ} \mathrm{C}\right)\end{array}$} & \multirow[b]{2}{*}{$\mathrm{pH}$} & \multirow{2}{*}{$\begin{array}{c}\text { Specific } \\
\text { conduct- } \\
\text { ance } \\
(\mu S / \mathrm{cm})\end{array}$} \\
\hline & & & & & $\begin{array}{l}\overline{\mathrm{V}_{\mathbf{i}}} \\
(\mathrm{L})\end{array}$ & $\begin{array}{l}\mathrm{v}_{\mathrm{p}} \\
(\mathrm{L})\end{array}$ & & & \\
\hline LEW & 0 & 0.0 & 0.00 & 0 & -- & - & -- & -- & -- \\
\hline 01B & 71 & 8.6 & 0.75 & 246 & 1.11 & -- & -- & -- & -- \\
\hline $01 \mathrm{~A}$ & 76 & 8.7 & 0.83 & 133 & 1.49 & 20 & 10.9 & 7.6 & 149 \\
\hline X05 & 108 & 9.8 & 0.93 & 279 & - & - & - & - & - \\
\hline $02 A, B$ & 137 & 9.9 & 1.03 & 495 & 4.35 & 20 & 9.3 & 7.6 & 153 \\
\hline $\mathrm{X} 07$ & 205 & 10.2 & 1.06 & 400 & - & - & -- & - & -- \\
\hline $03 A, B$ & 211 & 10.4 & 1.02 & 366 & 5.67 & 22 & 9.7 & 7.6 & 164 \\
\hline $04 \mathrm{~A}, \mathrm{~B}$ & 274 & 11.5 & 1.10 & 830 & 5.37 & 27 & 9.7 & 7.6 & 173 \\
\hline $05 \mathrm{~A}$ & 342 & 12.9 & 1.12 & 530 & 3.67 & 37 & 10.2 & 7.6 & 190 \\
\hline $05 B$ & 347 & 12.7 & 1.09 & 421 & 2.79 & - & - & - & - \\
\hline $06 \mathrm{~A}$ & 403 & 13.5 & 1.05 & 475 & 3.87 & 35 & 7.5 & 7.7 & 203 \\
\hline 06B & 414 & 13.7 & 1.21 & 623 & 3.40 & -- & -- & -- & - \\
\hline $07 \mathrm{~B}$ & 478 & 15.2 & 1.15 & 657 & 4.83 & - & -- & -- & -- \\
\hline $07 \mathrm{~A}$ & 489 & 15.5 & 1.27 & 570 & 5.25 & 43 & 9.0 & 7.7 & 235 \\
\hline D $8 A, B$ & 536 & 15.5 & 1.27 & 1,123 & 9.51 & 40 & 7.4 & 7.7 & 248 \\
\hline 09B & 603 & 15.0 & 1.22 & 724 & 4.95 & -- & -- & -- & -- \\
\hline 09A & 615 & 14.9 & 1.20 & 660 & 5.31 & 40 & 7.6 & 7.7 & 270 \\
\hline $10 \mathrm{~A}, \mathrm{~B}$ & 677 & 14.2 & 1.31 & 1,180 & 9.51 & 37 & 7.0 & 7.7 & 291 \\
\hline $11 \mathrm{~A}, \mathrm{~B}$ & 742 & 15.3 & 1.35 & 805 & 10.49 & 47 & 8.0 & 7.6 & 319 \\
\hline $\mathrm{X} 02$ & 755 & 15.6 & 1.35 & 642 & -- & -- & -- & -- & - \\
\hline $12 B$ & 803 & 14.4 & 1.35 & 552 & 5.20 & -- & -- & -- & -- \\
\hline $12 \mathrm{~A}$ & 812 & 14.4 & 1.38 & 417 & 5.29 & 39 & 7.4 & 7.6 & 328 \\
\hline$\times 09$ & 845 & 15.1 & 1.32 & 597 & - & - & -- & - & - \\
\hline $13 A, B$ & 872 & 14.6 & 1.35 & 954 & 10.18 & 41 & 6.7 & 7.6 & 329 \\
\hline $14 \mathrm{~B}^{\circ}$ & 942 & 16.0 & 1.46 & 934 & 5.88 & -- & -- & -- & - \\
\hline $14 \mathrm{~A}$ & 952 & 16.4 & 1.25 & 298 & 5.31 & 45 & 6.0 & 7.7 & 333 \\
\hline X01 & 971 & 17.0 & 1.34 & 663 & -- & - & -- & -- & - \\
\hline $15 A, B$ & 1,010 & 11.8 & 0.92 & 267 & 6.42 & 17 & 6.4 & 7.7 & 330 \\
\hline X06 & 1,020 & 9.2 & 0.79 & 255 & -- & -- & -- & -- & -- \\
\hline REW & 1,080 & 0.0 & 0.00 & 0 & -- & - & -- & -- & -- \\
\hline MEAN & & 12.5 & 1.20 & & & & & & \\
\hline TOTAL & 1,080 & & & 16,096 & 119.80 & 510 & & & \\
\hline
\end{tabular}




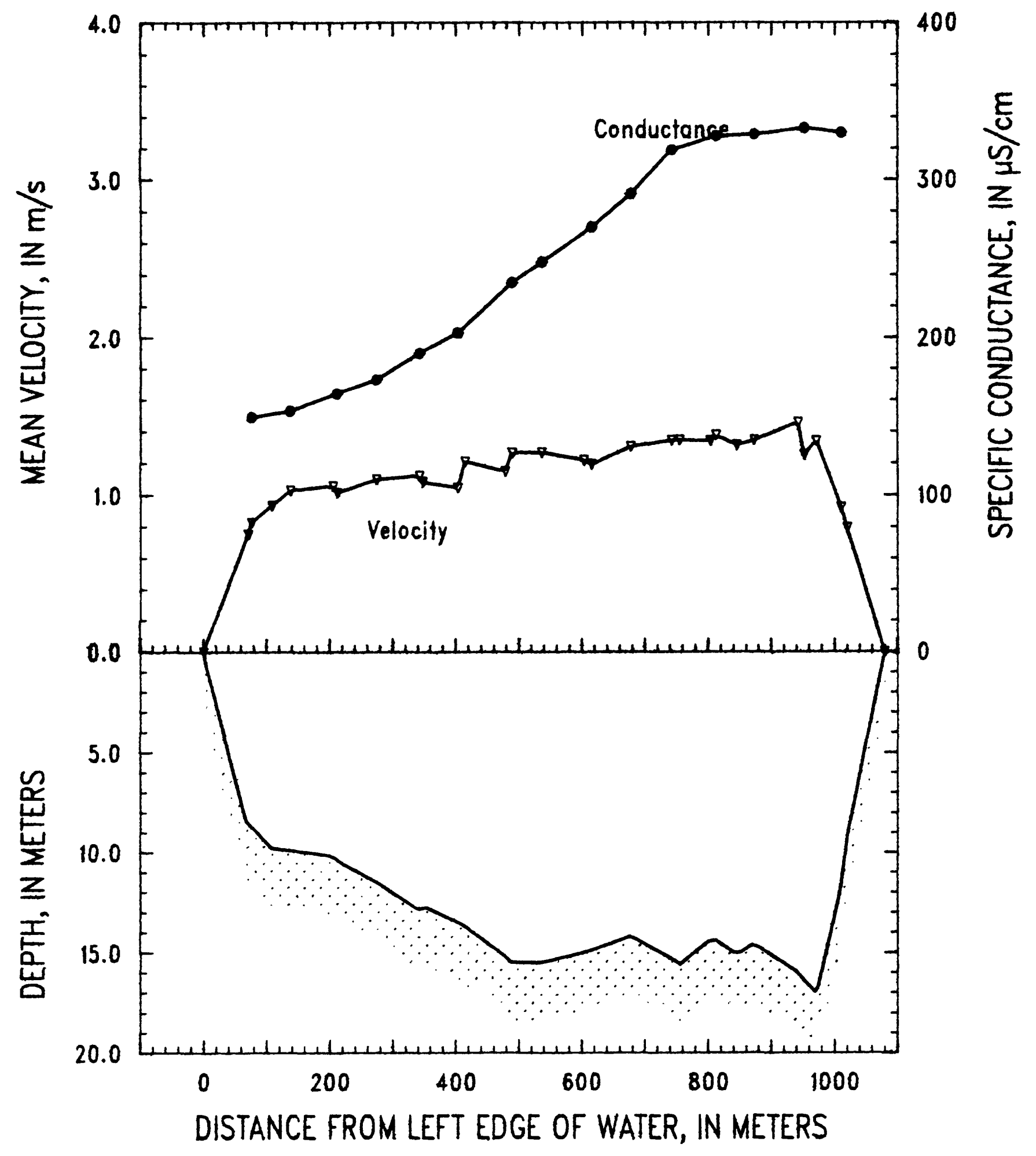

Figure 50. Ohio River at Olmsted, Illinois, on March 3, 1990. 
SITE: Mississippi River below Hickman, Kentucky

PARTY: Moody, Tagg, and Simoneaux

03-04-90

STARTING GAGE HEIGHT: $32.22 \mathrm{ft}$ On wire-line gage at Hickman.

METER: SOLID CUP

SUSP: Bag sampler and 200-lb weight

CURRENT METER NO.: W-297222 DATE RATED: 04-90

CURRENT METER EQUATION: $V(\mathrm{~m} / \mathrm{s})=$ RPS $* 0.744+0.011$

REMARKS: Transit rate was $14 \mathrm{~cm} / \mathrm{s}$, and 3/16-inch nozzle was used. Reported stage for 03-03-90 (marine radio station WJG) was $30.8 \mathrm{ft}$.

\begin{tabular}{|c|c|c|c|c|c|c|c|c|c|}
\hline \multirow[b]{2}{*}{$\begin{array}{c}\text { Verti- } \\
\text { cal }\end{array}$} & \multirow{2}{*}{$\begin{array}{l}\text { Dist. } \\
\text { from } \\
\text { LEW } \\
\text { (m) }\end{array}$} & \multirow[b]{2}{*}{$\begin{array}{l}\text { Depth } \\
\text { (m) }\end{array}$} & \multirow{2}{*}{$\begin{array}{l}\text { Mean } \\
\text { veloc- } \\
\text { ity } \\
(\mathrm{m} / \mathrm{s})\end{array}$} & \multirow[b]{2}{*}{$\begin{array}{r}\text { Dis- } \\
\text { charge } \\
\left(\mathrm{m}^{3} / \mathrm{s}\right)\end{array}$} & \multicolumn{2}{|c|}{ Volume } & \multirow[b]{2}{*}{$\begin{array}{l}\text { Temper- } \\
\text { ature } \\
\left({ }^{\circ} \mathrm{C}\right)\end{array}$} & \multirow[b]{2}{*}{$\mathrm{pH}$} & \multirow{2}{*}{$\begin{array}{l}\text { Specific } \\
\text { conduct- } \\
\text { ance } \\
(\mu \mathrm{S} / \mathrm{cm})\end{array}$} \\
\hline & & & & & $\begin{array}{l}\overline{V_{i}} \\
(L)\end{array}$ & $\begin{array}{l}\mathrm{v}_{\mathrm{p}} \\
(\mathrm{L})\end{array}$ & & & \\
\hline LEW & 0 & 0.0 & 0.00 & 0 & -- & -- & -- & -- & -- \\
\hline $01 \mathrm{~A}, \mathrm{~B}$ & 78 & 12.2 & 1.46 & 1,352 & 8.65 & 28 & 7.4 & 7.6 & 217 \\
\hline $02 \mathrm{~A}$ & 152 & 12.6 & 1.63 & 833 & 4.49 & 31 & 7.3 & 7.8 & 222 \\
\hline 02B & 159 & 12.1 & 1.75 & 488 & 5.09 & -- & -- & -- & -- \\
\hline X05 & 298 & 11.6 & 1.49 & 527 & -- & -- & -- & -- & -- \\
\hline $03 \mathrm{~A}$ & 220 & 11.9 & 1.62 & 414 & 4.13 & 31 & 7.4 & 7.8 & 228 \\
\hline 03B & 241 & 11.4 & 1.52 & 459 & 3.68 & -- & -- & -- & -- \\
\hline $04 \mathrm{~A}$ & 273 & 10.9 & 1.62 & 433 & 3.79 & 50 & 7.3 & 7.8 & 232 \\
\hline $04 B$ & 290 & 11.2 & 1.40 & 626 & 3.77 & -- & -- & -- & -- \\
\hline X04 & 353 & 11.5 & 1.69 & 680 & -- & -- & -- & -- & -- \\
\hline 05A, B & 360 & 11.3 & 1.63 & 738 & 8.55 & 31 & 7.1 & 7.8 & 240 \\
\hline $06 \mathrm{~A}$ & 433 & 11.0 & 1.54 & 670 & 4.31 & 31 & 6.7 & 7.9 & 251 \\
\hline $06 \mathrm{~B}$ & 439 & 11.1 & 1.56 & 562 & 4.23 & -- & -- & -- & - \\
\hline $07 \mathrm{~A}$ & 498 & 10.9 & 1.53 & 542 & 4.25 & 30 & 6.3 & 7.9 & 274 \\
\hline $07 \mathrm{~B}$ & 504 & 10.9 & 1.49 & 682 & 3.49 & -- & -- & -- & -- \\
\hline 08A, B & 582 & 11.1 & 1.53 & 1,338 & 7.88 & 28 & 5.9 & 7.9 & 302 \\
\hline $09 A, B$ & 662 & 12.3 & 1.63 & 1,508 & 8.50 & 33 & 5.8 & 7.9 & 326 \\
\hline $10 \mathrm{~A}, \mathrm{~B}$ & 732 & 13.4 & 1.71 & 1,386 & 10.50 & 43 & 5.6 & 8.0 & 347 \\
\hline $\mathrm{X} 02$ & 783 & 15.3 & 1.73 & 928 & $\ldots$ & - & - & - & - \\
\hline $11 \mathrm{~A}, \mathrm{~B}$ & 802 & 14.9 & 1.63 & 1,031 & 11.42 & 39 & 5.6 & 8.0 & 368 \\
\hline $12 \mathrm{~A}$ & 868 & 14.6 & 1.61 & 845 & 5.19 & 42 & 5.6 & 8.0 & 390 \\
\hline $12 \mathrm{~B}$ & 814 & 14.8 & 1.53 & 829 & 5.10 & -- & -- & -- & - \\
\hline $13 \mathrm{~A}, \mathrm{~B}$ & 941 & 16.1 & 1.39 & 1,376 & 9.68 & 40 & 5.2 & 8.0 & 404 \\
\hline $\mathrm{X01}$ & 997 & 16.8 & 1.33 & 749 & -- & -- & -- & -- & -- \\
\hline $14 B$ & 1,008 & 16.8 & 1.24 & 261 & 4.45 & -- & -- & -- & -- \\
\hline $14 \mathrm{~A}$ & 1,022 & 16.3 & 1.19 & 552 & 4.30 & 39 & 5.5 & 8.0 & 415 \\
\hline $15 \mathrm{~A}$ & 1,065 & 14.8 & 1.23 & 521 & 3.25 & 24 & 5.5 & 8.0 & 419 \\
\hline $15 \mathrm{~B}$ & 1,079 & 13.2 & 1.13 & 657 & 3.36 & -- & -- & -- & -- \\
\hline REW & 1,153 & 0.0 & 0.00 & 0 & -- & -- & $=-$ & -- & -- \\
\hline MEAN & & 12.0 & 1.51 & & & & & & \\
\hline TOTAL & 1,153 & & & 20,987 & 132.06 & 520 & & & \\
\hline
\end{tabular}




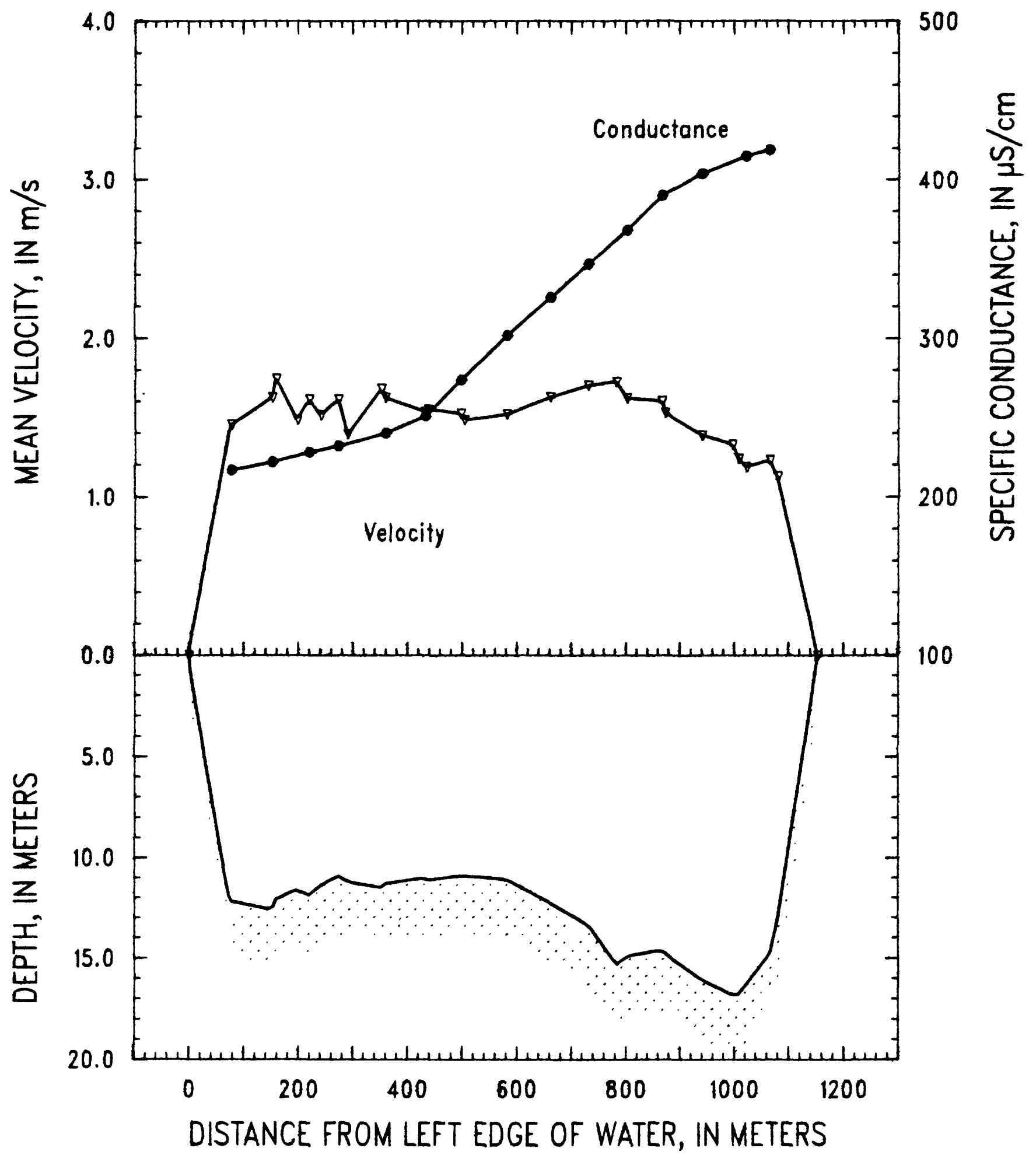

Figure 51. Mississippi River below Hickman, Kentucky, on March 4, 1990. 
SITE: Mississippi River below Fulton, Tennessee

PARTY: Moody, Garbarino, and Simoneaux

03-05-90

STARTING GAGE HEIGHT: -- ENDING GAGE HEIGHT: --

METER: SOLID CUP

SUSP: Bag sampler and 200-1b weight

CURRENT METER NO.: W-297222 DATE RATED: 04-90

CURRENT METER EQUATION: $V(\mathrm{~m} / \mathrm{s})=$ RPS $* 0.744+0.011$

REMARKS: Transit rate was $15.5 \mathrm{~cm} / \mathrm{s}$, and 3/16-inch nozzle was used.

Verticals occupied from 15 to 1 .

\begin{tabular}{|c|c|c|c|c|c|c|c|c|c|}
\hline \multirow[b]{2}{*}{$\begin{array}{c}\text { Verti- } \\
\text { cal }\end{array}$} & \multirow{2}{*}{$\begin{array}{l}\text { Dist. } \\
\text { from } \\
\text { LEW } \\
\text { (m) }\end{array}$} & \multirow[b]{2}{*}{$\underset{(m)}{\text { Depth }}$} & \multirow{2}{*}{$\begin{array}{l}\text { Mean } \\
\text { veloc- } \\
\text { ity } \\
(\mathrm{m} / \mathrm{s})\end{array}$} & \multirow[b]{2}{*}{$\begin{array}{r}\text { Dis- } \\
\text { charge } \\
\left(\mathrm{m}^{3} / \mathrm{s}\right)\end{array}$} & \multicolumn{2}{|c|}{ Volume } & \multirow[b]{2}{*}{$\begin{array}{l}\text { Temper- } \\
\text { ature } \\
\left({ }^{\circ} \mathrm{C}\right)\end{array}$} & \multirow[b]{2}{*}{$\mathrm{pH}$} & \multirow{2}{*}{$\begin{array}{l}\text { Specific } \\
\text { conduct- } \\
\text { ance } \\
(\mu S / \mathrm{cm})\end{array}$} \\
\hline & & & & & $\begin{array}{l}\overline{v_{i}} \\
(\mathrm{~L})\end{array}$ & $\begin{array}{l}\mathrm{v}_{\mathrm{p}} \\
(\mathrm{L})\end{array}$ & & & \\
\hline$\overline{\text { LEW }}$ & 0 & 0.0 & 0.00 & 0 & -- & -- & -- & -- & -- \\
\hline 01B & 81 & 11.5 & 0.89 & 474 & 2.07 & -- & - & -- & -- \\
\hline $01 \mathrm{~A}$ & 93 & 12.5 & 0.96 & 228 & 2.16 & 19 & 7.5 & 7.7 & 280 \\
\hline X06 & 119 & 12.5 & 1.05 & 413 & -- & -- & -- & -- & -- \\
\hline 02B & 156 & 12.5 & 1.06 & 310 & 2.91 & -- & -- & -- & -- \\
\hline $02 \mathrm{~A}$ & 166 & 12.5 & 1.11 & 533 & 2.67 & 25 & 7.8 & 7.7 & 280 \\
\hline X08 & 233 & 12.7 & 1.19 & 560 & -- & -- & -- & -- & -- \\
\hline $03 A, B$ & 240 & 12.6 & 1.19 & 584 & 6.33 & 26 & 7.7 & 7.8 & 283 \\
\hline $04 A$ & 311 & 12.0 & 1.33 & 622 & 3.30 & 25 & 7.9 & 7.8 & 285 \\
\hline 04B & 318 & 12.0 & 1.37 & 511 & 3.24 & -- & -- & -- & -- \\
\hline X05 & 373 & 12.0 & 1.21 & 514 & -- & -- & -- & -- & -- \\
\hline 05B & 389 & 11.7 & 1.22 & 208 & 2.91 & -- & -- & -- & -- \\
\hline 05A & 402 & 11.8 & 1.33 & 618 & 2.94 & 26 & 7.9 & 7.8 & 290 \\
\hline $06 \mathrm{~A}, \mathrm{~B}$ & 468 & 11.4 & 1.46 & 1,174 & 7.40 & 28 & 7.9 & 7.8 & 290 \\
\hline 07A, B & 543 & 12.1 & 1.54 & 1,469 & 7.94 & 33 & 7.7 & 7.9 & 290 \\
\hline 08A, B & 626 & 12.5 & 1.64 & 789 & 9.34 & 40 & 7.5 & 7.8 & 291 \\
\hline $\mathrm{X} 04$ & 620 & 12.3 & 1.78 & 820 & - & - & - & - & - \\
\hline 09A & 701 & 12.7 & 1.77 & 980 & 4.56 & 38 & 7.2 & 7.8 & 291 \\
\hline 09B & 707 & 12.7 & 1.72 & 883 & 4.72 & -- & -- & -- & -- \\
\hline $10 \mathrm{~B}$ & 782 & 12.2 & 1.93 & 954 & 5.37 & -- & -- & -- & -- \\
\hline $10 \mathrm{~A}$ & 788 & 12.5 & 1.97 & 777 & 5.28 & 39 & 7.5 & 7.8 & 292 \\
\hline $11 B$ & 845 & 12.3 & 1.92 & 781 & 5.16 & - & - & - & - \\
\hline $11 \mathrm{~A}$ & 854 & 12.4 & 1.82 & 394 & 5.28 & 39 & 7.3 & 7.8 & 293 \\
\hline X03 & 880 & 12.5 & 1.74 & 969 & -- & -- & -- & -- & -- \\
\hline $12 \mathrm{~A}$ & 943 & 13.1 & 1.82 & 837 & 5.29 & 41 & 7.6 & 7.8 & 295 \\
\hline $12 \mathrm{~B}$ & 950 & 13.1 & 1.80 & 861 & 4.85 & -- & -- & -- & -- \\
\hline $13 B$ & 1,016 & 14.3 & 1.50 & 836 & 4.63 & -- & -- & -- & -- \\
\hline $13 \mathrm{~A}$ & 1,028 & 14.3 & 1.64 & 482 & 4.56 & 47 & 8.5 & 7.8 & 296 \\
\hline $\mathrm{X02}$ & 1,057 & 15.3 & 1.72 & 1,025 & -- & -- & -- & -- & -- \\
\hline $14 \mathrm{~A}, \mathrm{~B}$ & 1,106 & 16.8 & 1.67 & 1,066 & 11.99 & 48 & 9.1 & 7.9 & 295 \\
\hline $\mathrm{X} 01$ & 1,133 & 18.0 & 1.47 & 963 & -- & -- & -- & - & -- \\
\hline $15 \mathrm{~A}$ & 1,179 & 16.7 & 1.35 & 552 & 4.00 & 27 & 7.7 & 8.0 & 298 \\
\hline $15 \mathrm{~B}$ & 1,182 & 14.5 & 1.06 & 622 & 3.41 & -- & - & - & $\ldots$ \\
\hline REW & 1,260 & 0.0 & 0.00 & 0 & -- & -- & -- & -- & -- \\
\hline MEAN & & 12.2 & 1.49 & & & & & & \\
\hline TOTAL & 1,260 & & & 22,809 & 122.31 & 501 & & & \\
\hline
\end{tabular}




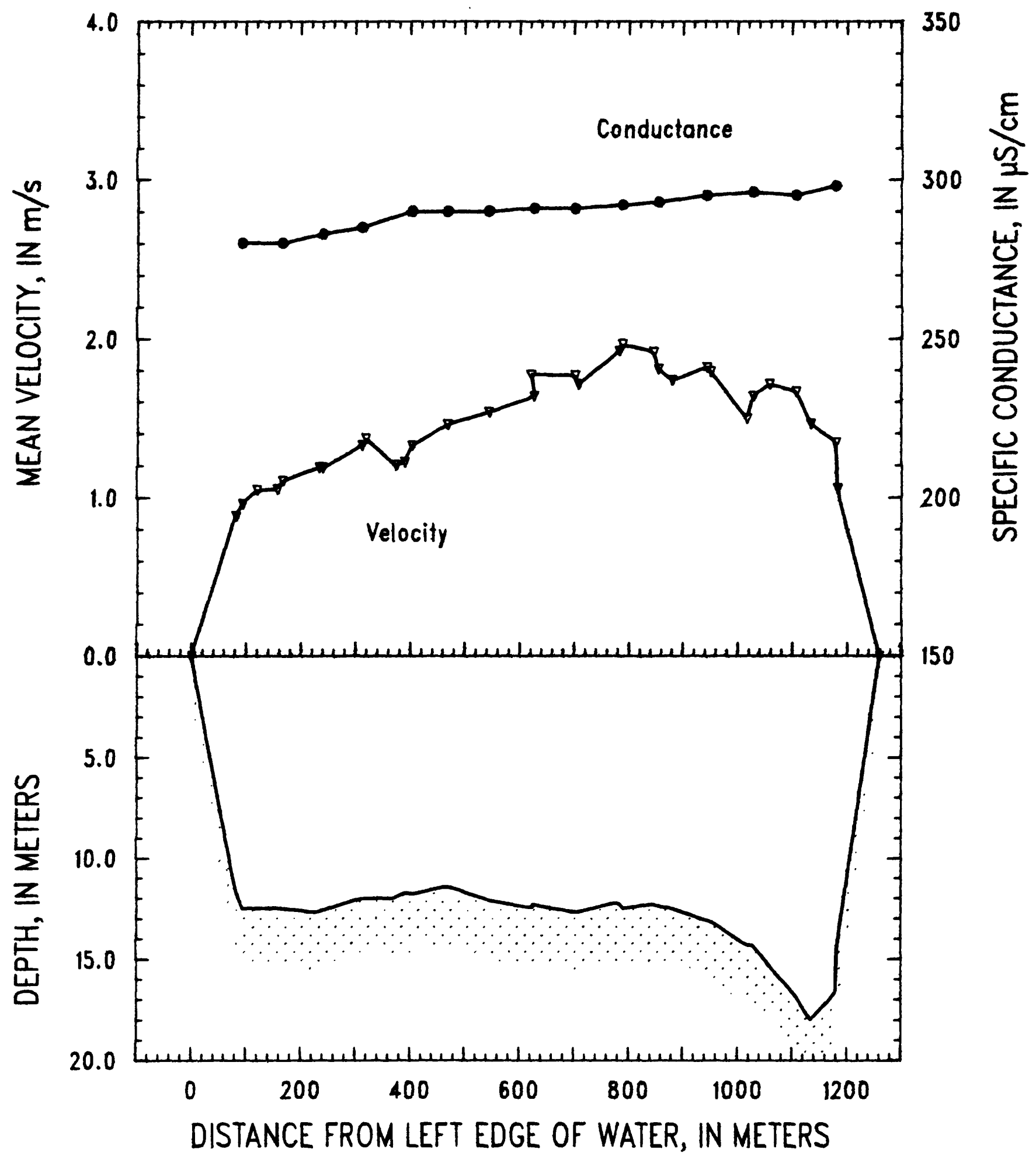

Figure 52. Mississippi River below Fulton, Tennessee, on March 5, 1990. 
SITE: Mississippi River at Helena, Arkansas

PARTY: Moody, Garbarino, and Rabalais

METER: SOLID CUP

STARTING GAGE HEIGHT: $32.75 \mathrm{ft}$ ENDING GAGE HEIGHT: $31.55 \mathrm{ft}$

SUSP: Bag sampler and $300-1 \mathrm{~b}$ weight

CURRENT METER NO.: W-297222 DATE RATED: 04-90

CURRENT METER EQUATION: $V(\mathrm{~m} / \mathrm{s})=$ RPS $* 0.744+0.011$

REMARKS: Transit rate was $22 \mathrm{~cm} / \mathrm{s}$, and 3/16-inch nozzle was used.

\begin{tabular}{|c|c|c|c|c|c|c|c|c|c|}
\hline \multirow[b]{2}{*}{$\begin{array}{l}\text { Verti- } \\
\text { cal }\end{array}$} & \multirow{2}{*}{$\begin{array}{l}\text { Dist. } \\
\text { from } \\
\text { LEW } \\
\text { (m) }\end{array}$} & \multirow[b]{2}{*}{$\begin{array}{l}\text { Depth } \\
\text { (m) }\end{array}$} & \multirow{2}{*}{$\begin{array}{l}\text { Mean } \\
\text { veloc- } \\
\text { ity } \\
(\mathrm{m} / \mathrm{s})\end{array}$} & \multirow[b]{2}{*}{$\begin{array}{r}\text { Dis- } \\
\text { charge } \\
\left(\mathrm{m}^{3} / \mathrm{s}\right)\end{array}$} & \multicolumn{2}{|c|}{ Volume } & \multirow[b]{2}{*}{$\begin{array}{l}\text { Temper- } \\
\text { ature } \\
\left({ }^{\circ} \mathrm{C}\right)\end{array}$} & \multirow[b]{2}{*}{$\mathrm{pH}$} & \multirow{2}{*}{$\begin{array}{c}\text { Specific } \\
\text { conduct- } \\
\text { ance } \\
(\mu S / \mathrm{cm})\end{array}$} \\
\hline & & & & & $\begin{array}{l}\overline{\mathrm{V}_{i}} \\
(\mathrm{~L})\end{array}$ & $\begin{array}{l}\overline{V_{p}} \\
(\mathrm{~L})\end{array}$ & & & \\
\hline LEW & 0 & 0.0 & 0.00 & 0 & -- & -- & -- & -- & -- \\
\hline $01 \mathrm{~A}$ & 43 & 10.9 & 0.82 & 223 & 1.42 & 23 & 7.7 & 7.8 & 292 \\
\hline O1B & 50 & 12.1 & 1.18 & 236 & 1.95 & -- & -- & - & -- \\
\hline O2B & 76 & 13.2 & 1.48 & 283 & 3.01 & -- & -- & -- & -- \\
\hline X06 & 79 & 13.2 & 1.60 & 328 & -- & -- & -- & -- & -- \\
\hline O2A & 107 & 13.6 & 1.56 & 701 & 2.79 & 26 & 7.7 & 7.9 & 295 \\
\hline 03B & 145 & 14.5 & 1.88 & 969 & 4.85 & -- & -- & -- & -- \\
\hline 03A & 178 & 14.8 & 1.93 & 1,001 & 5.01 & 32 & 7.7 & 7.9 & 295 \\
\hline $04 \mathrm{~A}, \mathrm{~B}$ & 215 & 16.3 & 1.99 & 890 & 10.00 & 35 & 7.5 & 7.9 & 301 \\
\hline $\mathrm{X} 05$ & 233 & 17.2 & 1.92 & 645 & -- & -- & -- & -- & -- \\
\hline 05A & 254 & 17.1 & 2.12 & 599 & 5.55 & 40 & 7.4 & 7.9 & 296 \\
\hline 05B & 268 & 18.1 & 2.11 & 1,432 & 5.20 & -- & -- & -- & -- \\
\hline 06B & 329 & 18.3 & 2.06 & 1,509 & 5.25 & -- & -- & -- & -- \\
\hline $06 \mathrm{~A}$ & 346 & 19.1 & 2.22 & 891 & 6.31 & 41 & 7.4 & 7.9 & 295 \\
\hline $07 \mathrm{~A}$ & 371 & 18.9 & 1.98 & 712 & 5.17 & 37 & 7.1 & 7.9 & 294 \\
\hline 07B & 384 & 20.1 & 1.91 & 364 & 4.05 & -- & -- & -- & -- \\
\hline X07 & 390 & 20.8 & 1.89 & 295 & -- & -- & -- & -- & -- \\
\hline X08 & 399 & 19.9 & 1.94 & 308 & -- & -- & -- & -- & -- \\
\hline $08 \mathrm{~B}$ & 406 & 21.6 & 1.94 & 419 & 5.15 & -- & -- & -- & -- \\
\hline $08 \mathrm{~A}$ & 419 & 21.6 & 1.79 & 752 & 5.82 & 46 & 7.1 & 7.9 & 294 \\
\hline$\times 09$ & 445 & 23.5 & 1.88 & 949 & -- & -- & - & - & - \\
\hline 09B & 462 & 22.1 & 1.78 & 630 & 5.51 & -- & -- & -- & -- \\
\hline $09 \mathrm{~A}$ & 477 & 21.7 & 1.62 & 1,126 & 5.22 & 43 & 7.0 & 7.9 & 294 \\
\hline $10 \mathrm{~B}$ & 526 & 21.8 & 1.37 & 928 & 4.65 & -- & - & - & - \\
\hline $10 \mathrm{~A}$ & 539 & 21.8 & 1.29 & 547 & 4.52 & 38 & 7.2 & 7.9 & 295 \\
\hline $11 \mathrm{~A}, \mathrm{~B}$ & 565 & 21.6 & 1.30 & 952 & 7.83 & 35 & 7.1 & 7.9 & 293 \\
\hline $\mathrm{X} 03$ & 607 & 23.8 & 1.24 & 857 & - & - & - & - & -- \\
\hline $12 \mathrm{~A}$ & 623 & 24.8 & 1.14 & 426 & 4.05 & 41 & 7.3 & 7.9 & 291 \\
\hline $12 \mathrm{~B}$ & 637 & 25.0 & 1.18 & 488 & 4.67 & - & - & - & - \\
\hline $\mathrm{X02}$ & 656 & 25.7 & 1.23 & 647 & -- & -- & -- & -- & -- \\
\hline $13 \mathrm{~A}$ & 678 & 23.6 & 1.34 & 569 & 4.48 & 33 & 7.2 & 7.9 & 277 \\
\hline
\end{tabular}




\begin{tabular}{|c|c|c|c|c|c|c|c|c|c|}
\hline \multirow[b]{2}{*}{$\begin{array}{c}\text { Verti- } \\
\text { cal }\end{array}$} & \multirow{2}{*}{$\begin{array}{l}\text { Dist. } \\
\text { from } \\
\text { LEW } \\
\text { (m) }\end{array}$} & \multirow[b]{2}{*}{$\begin{array}{l}\text { Depth } \\
\text { (m) }\end{array}$} & \multirow{2}{*}{$\begin{array}{c}\text { Mean } \\
\text { veloc- } \\
\text { ity } \\
(\mathrm{m} / \mathrm{s})\end{array}$} & \multirow[b]{2}{*}{$\begin{array}{r}\text { Dis- } \\
\text { charge } \\
\left(\mathrm{m}^{3} / \mathrm{s}\right)\end{array}$} & \multicolumn{2}{|c|}{ Volume } & \multirow[b]{2}{*}{$\begin{array}{c}\text { Temper- } \\
\text { ature } \\
\left({ }^{\circ} \mathrm{C}\right)\end{array}$} & \multirow[b]{2}{*}{$\mathrm{pH}$} & \multirow{2}{*}{$\begin{array}{l}\text { Specific } \\
\text { conduct- } \\
\text { ance } \\
(\mu \mathrm{S} / \mathrm{cm})\end{array}$} \\
\hline & & & & & $\begin{array}{l}\overline{v_{i}} \\
(L)\end{array}$ & $\begin{array}{l}\mathrm{v}_{\mathrm{p}} \\
(\mathrm{L})\end{array}$ & & & \\
\hline $13 B$ & 692 & 22.5 & 1.28 & 691 & 4.80 & -- & - & - & -- \\
\hline $14 \mathrm{~A}$ & 726 & 20.5 & 1.24 & 750 & 3.56 & 31 & 7.3 & 7.9 & 273 \\
\hline $14 \mathrm{~B}$ & 751 & 14.1 & 1.23 & 294 & 2.75 & - & -- & - & -- \\
\hline $\mathrm{X} 01$ & 760 & 13.3 & 1.20 & 390 & -- & -- & -- & -- & -- \\
\hline $15 \mathrm{~A}$ & 800 & 10.4 & 0.94 & 240 & 1.53 & 11 & 7.4 & 7.9 & 269 \\
\hline $15 B$ & 809 & 9.6 & 1.14 & 302 & 1.68 & - & - & - & - \\
\hline REW & 855 & 0.0 & 0.00 & 0 & -- & -- & -- & - & - \\
\hline MEAN & & 17.3 & 1.58 & & & & & & \\
\hline TOTAL & 855 & & & 23,343 & 126.78 & 512 & & & \\
\hline
\end{tabular}




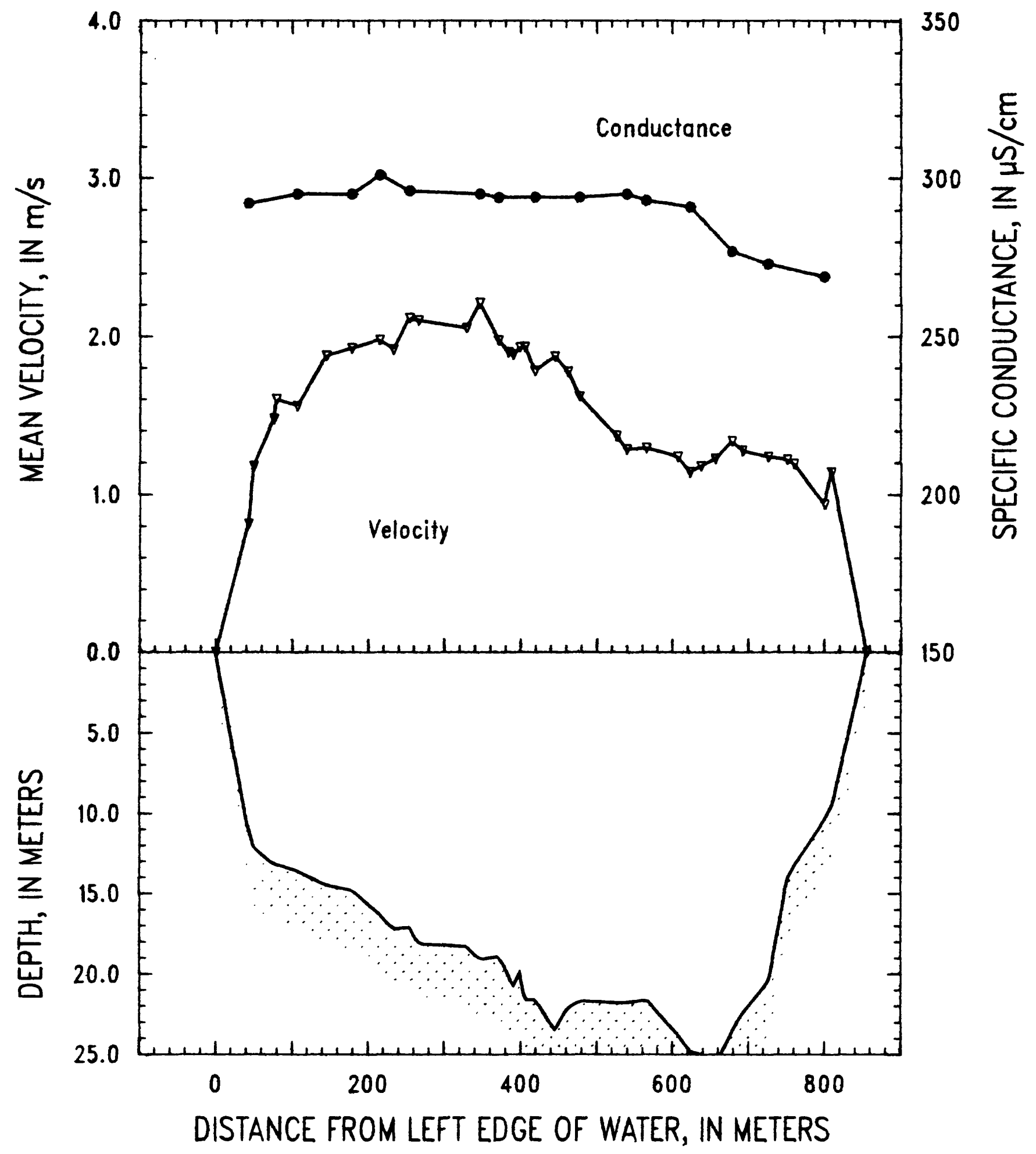

Figure 53. Mississippi River at Helena, Arkansas, on March 7, 1990. 
SITE: Mississippi River above Arkansas City, Arkansas 03-08-90

PARTY: Moody, Garbarino, and Rabalais

METER: SOLID CUP

STARTING GAGE HEIGHT: $34.8 \mathrm{ft}$ ENDING GAGE HEIGHT: $34.5 \mathrm{ft}$

SUSP: Bag sampler and 300-1b weight

CURRENT METER NO.: W-297222 DATE RATED: 04-90

CURRENT METER EQUATION: $V(\mathrm{~m} / \mathrm{s})=$ RPS $\div 0.744+0.011$

REMARKS: Transit rate was $17 \mathrm{~cm} / \mathrm{s}$, and $1 / 8$-inch nozzle was used. Water was in the trees so that LEF was estimated to be at $90 \mathrm{~m}$ and the REF to be at $1,050 \mathrm{~m}$.

\begin{tabular}{|c|c|c|c|c|c|c|c|c|c|}
\hline \multirow[b]{2}{*}{$\begin{array}{c}\text { Verti- } \\
\text { cal }\end{array}$} & \multirow{2}{*}{$\begin{array}{l}\text { Dist. } \\
\text { from } \\
\text { LEW } \\
\text { (m) }\end{array}$} & \multirow[b]{2}{*}{$\begin{array}{l}\text { Depth } \\
\text { (m) }\end{array}$} & \multirow{2}{*}{$\begin{array}{l}\text { Mean } \\
\text { veloc- } \\
\text { ity } \\
(\mathrm{m} / \mathrm{s})\end{array}$} & \multirow[b]{2}{*}{$\begin{array}{r}\text { Dis- } \\
\text { charge } \\
\left(\mathrm{m}^{3} / \mathrm{s}\right)\end{array}$} & \multicolumn{2}{|c|}{ Volume } & \multirow[b]{2}{*}{$\begin{array}{l}\text { Temper- } \\
\text { ature } \\
\left({ }^{\circ} \mathrm{C}\right)\end{array}$} & \multirow[b]{2}{*}{$\mathrm{pH}$} & \multirow{2}{*}{$\begin{array}{c}\text { Specific } \\
\text { conduct- } \\
\text { ance } \\
(\mu \mathrm{S} / \mathrm{cm})\end{array}$} \\
\hline & & & & & $\begin{array}{l}\mathrm{V}_{\mathbf{i}} \\
(\mathrm{L})\end{array}$ & $\begin{array}{l}V_{p} \\
(L)\end{array}$ & & & \\
\hline IEW & 0 & 0.0 & 0.00 & 0 & -- & -- & -- & -- & -- \\
\hline $02 \mathrm{~A}$ & 150 & 8.8 & 0.21 & 144 & 0.35 & 2 & 8.5 & 7.9 & 275 \\
\hline O2B & 153 & 9.8 & 0.30 & 56 & 0.19 & -- & -- & -- & -- \\
\hline 03A & 188 & 16.0 & 0.44 & 198 & 0.54 & 13 & 8.0 & 7.9 & 280 \\
\hline 03B & 209 & 16.8 & 0.52 & 175 & 1.26 & -- & -- & -- & -- \\
\hline $\mathrm{X01}$ & 228 & 17.2 & 0.90 & 371 & -- & -- & -- & -- & -- \\
\hline $04 \mathrm{~A}$ & 257 & 17.4 & 0.84 & 301 & 1.16 & 15 & 8.0 & 7.9 & 278 \\
\hline 04B & 269 & 17.9 & 0.76 & 198 & 1.14 & - & - & - & - \\
\hline 05 & 286 & 17.5 & 0.85 & 520 & 3.41 & 18 & 8.2 & 7.9 & 280 \\
\hline 06B & 339 & 19.3 & 1.45 & 1,263 & 2.49 & -- & - & -- & -- \\
\hline $06 \mathrm{~A}$ & 376 & 19.8 & 1.53 & 1,329 & 3.67 & 18 & 8.1 & 7.9 & 281 \\
\hline 07A & 427 & 22.1 & 2.00 & 1,417 & 5.16 & 32 & 8.0 & 7.9 & 281 \\
\hline 07B & 440 & 22.1 & 1.84 & 1,079 & 4.54 & -- & - & - & - \\
\hline 08 & 480 & 24.1 & 2.12 & 1,484 & 9.31 & 45 & 7.9 & 7.9 & 280 \\
\hline 09A & 498 & 25.0 & 2.49 & 840 & 6.51 & 53 & 8.0 & 7.9 & 278 \\
\hline 09B & 507 & 25.2 & 2.11 & 1,434 & 4.75 & - & - & - & $\ldots$ \\
\hline X03 & 552 & 25.5 & 2.66 & 2,514 & -- & -- & -- & -- & -- \\
\hline $10 \mathrm{~B}$ & 581 & 25.9 & 2.78 & 1,406 & 6.06 & -- & -- & -- & -- \\
\hline $10 \mathrm{~A}$ & 591 & 25.6 & 2.73 & 1,574 & 6.53 & 54 & 8.1 & 7.9 & 281 \\
\hline $11 \mathrm{~B}$ & 626 & 25.0 & 2.85 & 2,601 & 6.79 & - & - & -- & - \\
\hline $11 \mathrm{~A}$ & 664 & 22.1 & 2.86 & 2,088 & 6.80 & 51 & 8.4 & 7.9 & 289 \\
\hline $12 \mathrm{~A}$ & 692 & 22.1 & 2.41 & 1,120 & 6.11 & 56 & 8.6 & 7.8 & 287 \\
\hline $12 B$ & 706 & 21.6 & 2.65 & 572 & 6.62 & - & -- & -- & -- \\
\hline X04 & 712 & 20.5 & 2.76 & 509 & -- & -- & -- & -- & -- \\
\hline $13 B$ & 724 & 20.4 & 2.63 & 965 & 5.89 & -- & -- & -- & -- \\
\hline $13 \mathrm{~A}$ & 748 & 20.1 & 2.54 & 1,251 & 5.68 & 37 & 8.4 & 7.8 & 290 \\
\hline $14 \mathrm{~A}$ & 773 & 19.9 & 2.48 & 912 & 5.74 & 30 & 8.2 & 7.8 & 291 \\
\hline $14 B$ & 785 & 19.9 & 2.28 & 2,383 & 4.72 & -- & - & - & - \\
\hline $15 \mathrm{~A}$ & 878 & 18.7 & 1.74 & 1,794 & 3.65 & 27 & 8.1 & 7.9 & 292 \\
\hline $15 B$ & 895 & 19.0 & 1.65 & 565 & 3.09 & - & -- & -- & -- \\
\hline 16 & 914 & 19.4 & 1.49 & 955 & 3.26 & 26 & 8.2 & 7.8 & 291 \\
\hline $17 \mathrm{~B}$ & 961 & 18.6 & 0.88 & 419 & 1.26 & - & - & - & -- \\
\hline $17 \mathrm{~A}$ & 965 & 17.5 & 0.73 & 763 & 1.02 & 32 & -- & -- & -- \\
\hline REW & 1,080 & 0.0 & 0.00 & 0 & -- & -- & -- & -- & -- \\
\hline MEAN & & 17.0 & 1.81 & & & & & & \\
\hline TOTAL & 1,080 & & & 33,200 & 120.50 & 509 & & & \\
\hline
\end{tabular}




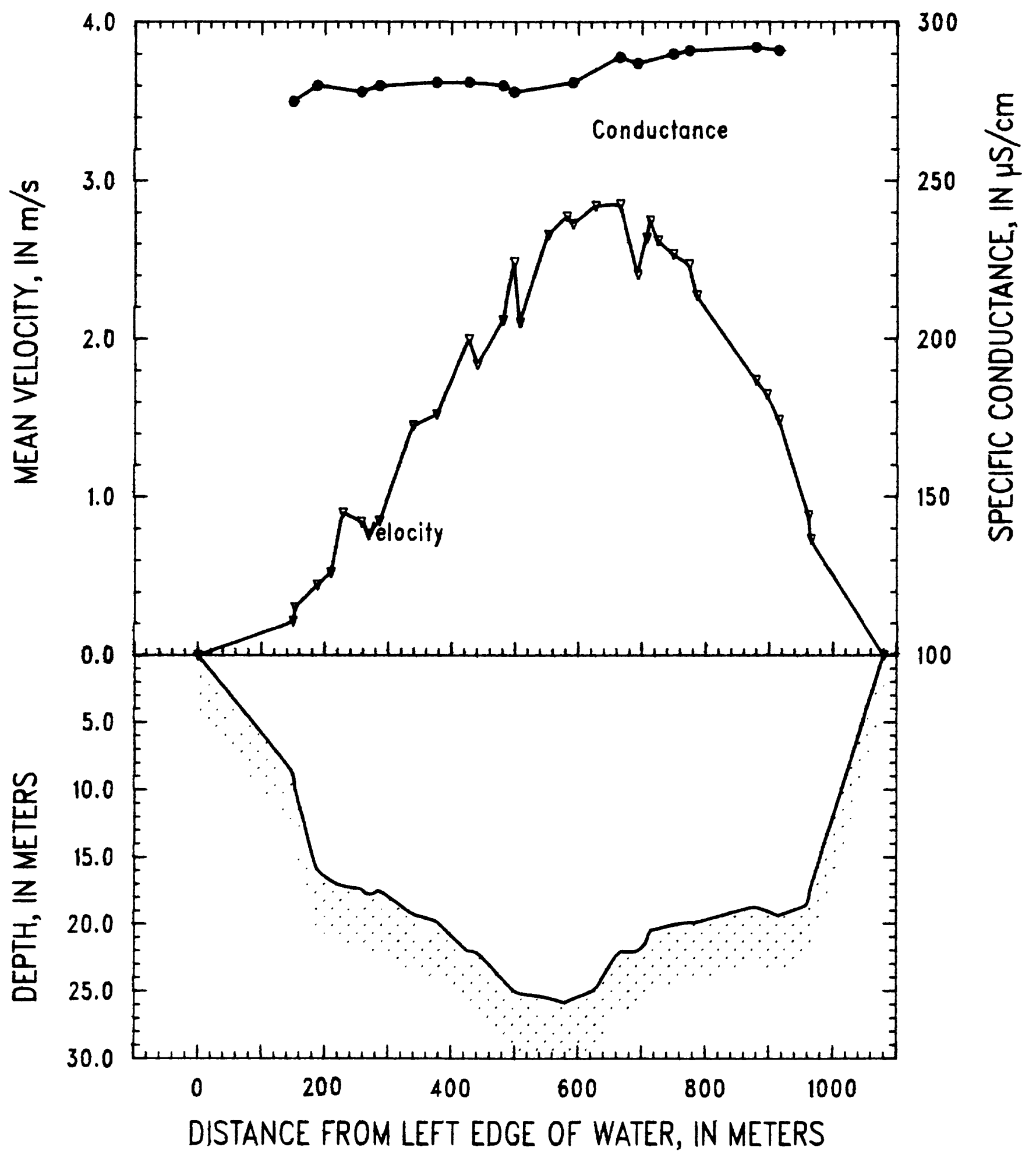

Figure 54. Mississippi River above Arkansas City, Arkansas, on March 8, 1990. 
SITE: Mississippi River below Vicksburg, Mississippi

PARTY: Moody, Garbarino, and Rabalais

03-10-90

STARTING GAGE HEIGHT: $38.95 \mathrm{ft}$ ENDING GAGE HEIGHT: --

METER: SOLID CUP

SUSP: Bag sampler and $300-1 \mathrm{~b}$ weight

CURRENT METER NO.: W-297222 DATE RATED: 04-90

CURRENT METER EQUATION: $V(\mathrm{~m} / \mathrm{s})=\operatorname{RPS} * 0.744+0.011$

REMARKS: LEF is approximately $25 \mathrm{~m}$ and REF is about $1,252 \mathrm{~m}$. REW is unknown but chosen so that the neglected flow near the bank is beyond $1,252 \mathrm{~m}$.

Yazoo River water opposite the Port of Vicksburg was $13.5^{\circ} \mathrm{C}$, with $\mathrm{pH}=7.48$ and specific conductance of $122 \mu \mathrm{S} / \mathrm{cm}$.

\begin{tabular}{|c|c|c|c|c|c|c|c|c|c|}
\hline \multirow[b]{2}{*}{$\begin{array}{l}\text { Verti- } \\
\text { cal }\end{array}$} & \multirow{2}{*}{$\begin{array}{l}\text { Dist. } \\
\text { from } \\
\text { LEW } \\
\text { (m) }\end{array}$} & \multirow[b]{2}{*}{$\begin{array}{c}\text { Depth } \\
(\mathrm{m})\end{array}$} & \multirow{2}{*}{$\begin{array}{l}\text { Mean } \\
\text { veloc- } \\
\text { ity } \\
(\mathrm{m} / \mathrm{s})\end{array}$} & \multirow[b]{2}{*}{$\begin{array}{l}\text { Dis- } \\
\text { charge } \\
\left(\mathrm{m}^{3} / \mathrm{s}\right)\end{array}$} & \multicolumn{2}{|c|}{ Volume } & \multirow[b]{2}{*}{$\begin{array}{c}\text { Temper- } \\
\text { ature } \\
\left({ }^{\circ} \mathrm{C}\right)\end{array}$} & \multirow[b]{2}{*}{$\mathrm{pH}$} & \multirow{2}{*}{$\begin{array}{l}\text { Specific } \\
\text { conduct- } \\
\text { ance } \\
(\mu S / \mathrm{cm})\end{array}$} \\
\hline & & & & & $\begin{array}{l}\overline{\mathbf{V}_{\mathbf{i}}} \\
(\mathrm{L})\end{array}$ & $\begin{array}{c}\bar{v}_{p} \\
(\mathrm{~L})\end{array}$ & & & \\
\hline LEW & 0 & 0.0 & 0.00 & 0 & -- & $=$ & - & -- & - \\
\hline $01 \mathrm{~A}$ & 53 & 11.8 & 0.21 & 75 & 0.43 & 12 & 11.1 & 7.5 & 240 \\
\hline $01 B$ & 60 & 13.1 & 0.55 & 315 & 0.56 & - & - & - & - \\
\hline $02 A, B$ & 141 & 22.8 & 1.33 & 1,755 & 6.72 & 33 & 11.3 & 7.5 & 244 \\
\hline $\mathrm{X} 06$ & 176 & 22.6 & 1.45 & 953 & -- & -- & - & - & - \\
\hline $03 \mathrm{~A}, \mathrm{~B}$ & 199 & 22.0 & 1.63 & 1,469 & 7.99 & 33 & 11.2 & 7.5 & 241 \\
\hline $04 \mathrm{~B}$ & 258 & 21.7 & 1.78 & 1,311 & 5.08 & - & - & - & - \\
\hline $04 \mathrm{~A}$ & 267 & 21.9 & 1.77 & 1,317 & 5.24 & 36 & 11.2 & 7.5 & 253 \\
\hline 05B & 326 & 22.1 & 1.81 & 1,457 & 5.24 & -- & -- & -- & $\ldots$ \\
\hline $05 \mathrm{~A}$ & 340 & 22.1 & 2.00 & 1,126 & 4.63 & 40 & 10.9 & 7.5 & 263 \\
\hline X05 & 377 & 22.0 & 1.79 & 1,303 & -- & -- & -- & -- & -- \\
\hline 06B & 406 & 21.7 & 2.08 & 881 & 6.07 & -- & - & -- & -- \\
\hline $06 \mathrm{~A}$ & 416 & 21.4 & 2.14 & 1,213 & 5.70 & 48 & 10.8 & 7.6 & 262 \\
\hline 07A, B & 459 & 22.1 & 2.21 & 1,492 & 12.02 & 42 & 10.7 & 7.5 & 276 \\
\hline$\times 07$ & 477 & 22.1 & 2.08 & 1,772 & -- & -- & -- & -- & -- \\
\hline $08 A, B$ & 536 & 20.1 & 2.25 & 2,349 & 10.97 & 42 & 11.3 & 7.6 & 286 \\
\hline $09 A$ & 581 & 17.7 & 2.52 & 1,206 & 5.23 & 36 & 10.7 & 7.7 & 287 \\
\hline 09B & 590 & 17.1 & 2.56 & 1,491 & 5.24 & - & - & - & - \\
\hline 10B & 649 & 17.5 & 2.41 & 1,600 & 4.04 & -- & -- & -- & - \\
\hline $10 \mathrm{~A}$ & 666 & 16.5 & 2.41 & 1,015 & 4.47 & 34 & 11.2 & 7.6 & 292 \\
\hline $\mathrm{X} 03$ & 700 & 16.2 & 2.27 & 973 & -- & -- & - & - & - \\
\hline $11 A, B$ & 719 & 16.5 & 2.18 & 1,724 & 7.98 & 34 & 10.7 & 7.7 & 294 \\
\hline $12 \mathrm{~A}$ & 796 & 14.1 & 2.02 & 1,224 & 3.64 & 30 & 10.4 & 7.7 & 292 \\
\hline $12 B$ & 805 & 14.1 & 2.05 & 780 & 3.81 & -- & -- & -- & -- \\
\hline $13 B$ & 850 & 14.1 & 1.87 & 750 & 3.09 & -- & -- & -- & -- \\
\hline $13 A$ & 862 & 13.4 & 1.71 & 344 & 2.47 & 28 & 11.0 & 7.7 & 292 \\
\hline $\mathrm{X} 02$ & 880 & 11.6 & 1.67 & 532 & - & - & - & -- & - \\
\hline $14 \mathrm{~A}$ & 917 & 12.0 & 1.54 & 405 & 1.85 & 23 & 10.8 & 7.7 & 293 \\
\hline $14 B$ & 924 & 11.8 & 1.55 & 569 & 2.04 & - & - & - & - \\
\hline $15 \mathrm{~A}$ & 979 & 9.9 & 1.08 & 358 & 1.28 & 18 & 10.8 & 7.7 & 293 \\
\hline $15 \mathrm{~B}$ & 991 & 10.1 & 1.21 & 390 & 1.31 & - & . - & - & - \\
\hline $16 \mathrm{~A}, \mathrm{~B}$ & 1,043 & 9.8 & 0.98 & 420 & 2.15 & 16 & 10.7 & 7.7 & 293 \\
\hline $\mathrm{XO1}$ & 1,078 & 9.6 & 0.91 & 313 & -- & - & - & - & - \\
\hline $17 \mathrm{~A}, \mathrm{~B}$ & 1,115 & 9.7 & 0.94 & 463 & 2.03 & 9 & 10.5 & 7.7 & 292 \\
\hline $18 \mathrm{~A}, \mathrm{~B}$ & 1,179 & 10.9 & 0.74 & 776 & 1.78 & 10 & 10.3 & 7.7 & 285 \\
\hline REW & 1,308 & 0.0 & 0.00 & 0 & -- & -- & -- & -- & -- \\
\hline MEAN & & 15.2 & 1.72 & & & & & & \\
\hline TOTAL & 1,308 & & & 34,121 & 123.06 & 524 & & & \\
\hline
\end{tabular}




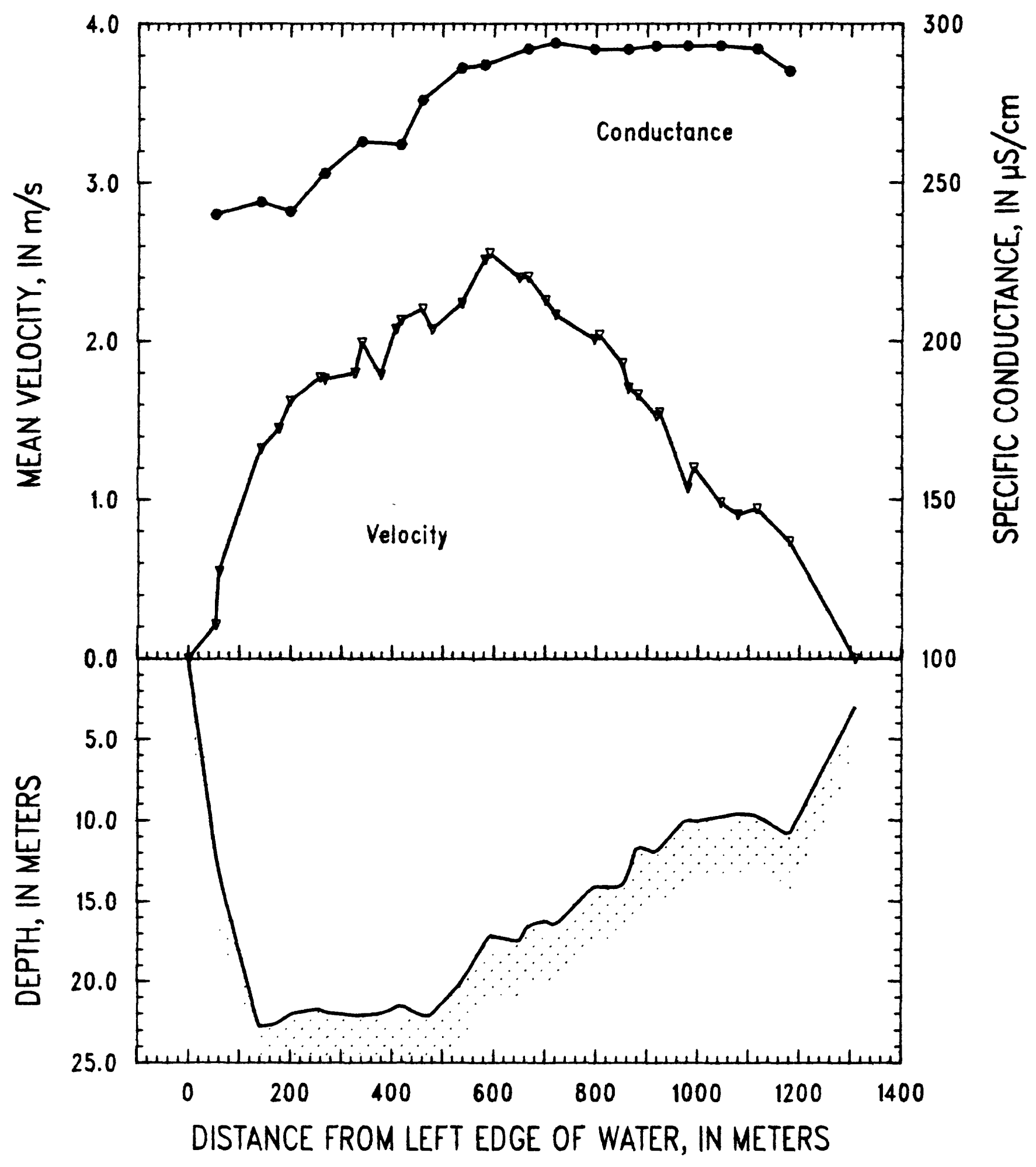

Figure 55. Mississippi River below Vicksburg, Mississippi, on March 10, 1990. 
SITE: Mississippi River near St. Francisville, Louisiana

PARTY: Moody, Garbarino, and Simoneaux

03-12-90

STARTING GAGE HEIGHT: -- ENDING GAGE HEIGHT: --

METER: SOLID CUP

SUSP: Bag sampler and 300-1b weight

CURRENT METER NO.: W-297222 DATE RATED: 04-90

CURRENT METER EQUATION: $V(\mathrm{~m} / \mathrm{s})=$ RPS $* 0.744+0.011$

REMARKS: Transit rate was $10 \mathrm{~cm} / \mathrm{s}$, and $1 / 8$-inch nozzle was used.

LEF is at $0 \mathrm{~m}$, depth of $3.0 \mathrm{~m}$ is an estimate, and LEW is unknown.

Flow coming into river from left side was not measured.

\begin{tabular}{|c|c|c|c|c|c|c|c|c|c|}
\hline \multirow[b]{2}{*}{$\begin{array}{l}\text { Verti- } \\
\text { cal }\end{array}$} & \multirow{2}{*}{$\begin{array}{l}\text { Dist. } \\
\text { from } \\
\text { LEF } \\
\text { (m) }\end{array}$} & \multirow[b]{2}{*}{$\begin{array}{l}\text { Depth } \\
\text { (m) }\end{array}$} & \multirow{2}{*}{$\begin{array}{l}\text { Mean } \\
\text { veloc- } \\
\text { ity } \\
(\mathrm{m} / \mathrm{s})\end{array}$} & \multirow[b]{2}{*}{$\begin{array}{r}\text { Dis - } \\
\text { charge } \\
\left(\mathrm{m}^{3} / \mathrm{s}\right)\end{array}$} & \multicolumn{2}{|c|}{ Volume } & \multirow[b]{2}{*}{$\begin{array}{l}\text { Temper- } \\
\text { ature } \\
\left({ }^{\circ} \mathrm{C}\right)\end{array}$} & \multirow[b]{2}{*}{$\mathrm{pH}$} & \multirow{2}{*}{$\begin{array}{l}\text { Specific } \\
\text { conduct- } \\
\text { ance } \\
(\mu \mathrm{S} / \mathrm{cm})\end{array}$} \\
\hline & & & & & $\begin{array}{l}\overline{v_{i}} \\
(\mathrm{~L})\end{array}$ & $\begin{array}{l}\mathrm{V}_{\mathrm{p}} \\
(\mathrm{L})\end{array}$ & & & \\
\hline$\overline{L E F}$ & 0 & 3.0 & 0.00 & 0 & -- & -- & -- & -- & -- \\
\hline O1B & 48 & 10.4 & 0.33 & 97 & 0.25 & -- & -- & -- & -- \\
\hline $01 \mathrm{~A}$ & 57 & 11.3 & 0.10 & 31 & 0.26 & 7 & 13.0 & 7.6 & 279 \\
\hline O2B & 103 & 13.5 & 0.55 & 224 & 0.85 & -- & -- & -- & -- \\
\hline $02 \mathrm{~A}$ & 117 & 13.6 & 0.49 & 296 & 0.62 & 12 & 12.0 & 7.7 & 280 \\
\hline 03 & 191 & 16.0 & 1.06 & 1,186 & 4.63 & 18 & 13.2 & 7.6 & 279 \\
\hline 04B & 257 & 15.7 & 1.09 & 734 & 3.26 & -- & -- & -- & -- \\
\hline $04 \mathrm{~A}$ & 277 & 15.9 & 1.06 & 557 & 2.84 & 24 & 11.9 & 7.7 & 279 \\
\hline 05 & 323 & 16.3 & 1.76 & 1,631 & 9.26 & 30 & 11.8 & 7.7 & 280 \\
\hline 06 & 391 & 16.9 & 2.06 & 2,137 & 12.14 & 36 & 12.2 & 7.6 & 280 \\
\hline $07 \mathrm{~A}$ & 446 & 19.2 & 2.08 & 1,276 & 6.80 & 56 & 12.4 & 7.6 & 280 \\
\hline $07 \mathrm{~B}$ & 455 & 19.2 & 2.09 & 1,286 & 6.36 & -- & -- & -- & -- \\
\hline 08 & 510 & 18.8 & 2.26 & 1,339 & 11.67 & 52 & 12.7 & 7.7 & 280 \\
\hline $\mathrm{X} 03$ & 518 & 18.2 & 2.15 & 1,195 & -- & -- & -- & -- & -- \\
\hline 09 & 571 & 17.9 & 1.95 & 1,933 & 12.44 & 38 & 12.6 & 7.7 & 279 \\
\hline $10 \mathrm{~B}$ & 629 & 18.1 & 1.87 & 1,187 & 6.50 & -- & -- & -- & -- \\
\hline $10 \mathrm{~A}$ & 641 & 19.7 & 2.00 & 1,302 & 6.35 & 41 & 13.4 & 7.6 & 279 \\
\hline $11 \mathrm{~B}$ & 695 & 19.8 & 1.62 & 1,028 & 5.66 & - & - & - & - \\
\hline $11 \mathrm{~A}$ & 705 & 19.8 & 1.61 & 1,038 & 5.65 & 36 & 12.6 & 7.6 & 277 \\
\hline $12 \mathrm{~A}$ & 760 & 20.8 & 1.64 & 1,059 & 5.48 & 40 & 12.2 & 7.6 & 279 \\
\hline $12 \mathrm{~B}$ & 767 & 20.8 & 1.66 & 983 & 6.89 & -- & -- & -- & -- \\
\hline $13 B$ & 817 & 21.1 & 1.58 & 1,117 & 6.39 & -- & -- & -- & - \\
\hline $13 \mathrm{~A}$ & 834 & 21.1 & 1.56 & 1,120 & 5.77 & 39 & 12.2 & 7.6 & 278 \\
\hline $14 \mathrm{~A}$ & 885 & 21.3 & 1.44 & 874 & 4.75 & 39 & 12.3 & 7.6 & 279 \\
\hline $14 \mathrm{~B}$ & 891 & 21.1 & 1.57 & 598 & 5.67 & -- & -- & -- & $\ldots$ \\
\hline $\mathrm{X} 01$ & 921 & 20.2 & 1.49 & 813 & - & -- & -- & -- & -- \\
\hline $15 \mathrm{~A}$ & 945 & 19.5 & 1.44 & 462 & 4.55 & 31 & 12.9 & 7.6 & 272 \\
\hline $15 B$ & 954 & 18.4 & 1.32 & 824 & 4.36 & -- & -- & -- & -- \\
\hline REW & 1,013 & 0.0 & 0.00 & 0 & -- & -- & -- & -- & -- \\
\hline MEAN & & 16.8 & 1.55 & & & & & & \\
\hline TOTAL & 1,013 & & & 26,327 & 138.90 & 499 & & & \\
\hline
\end{tabular}




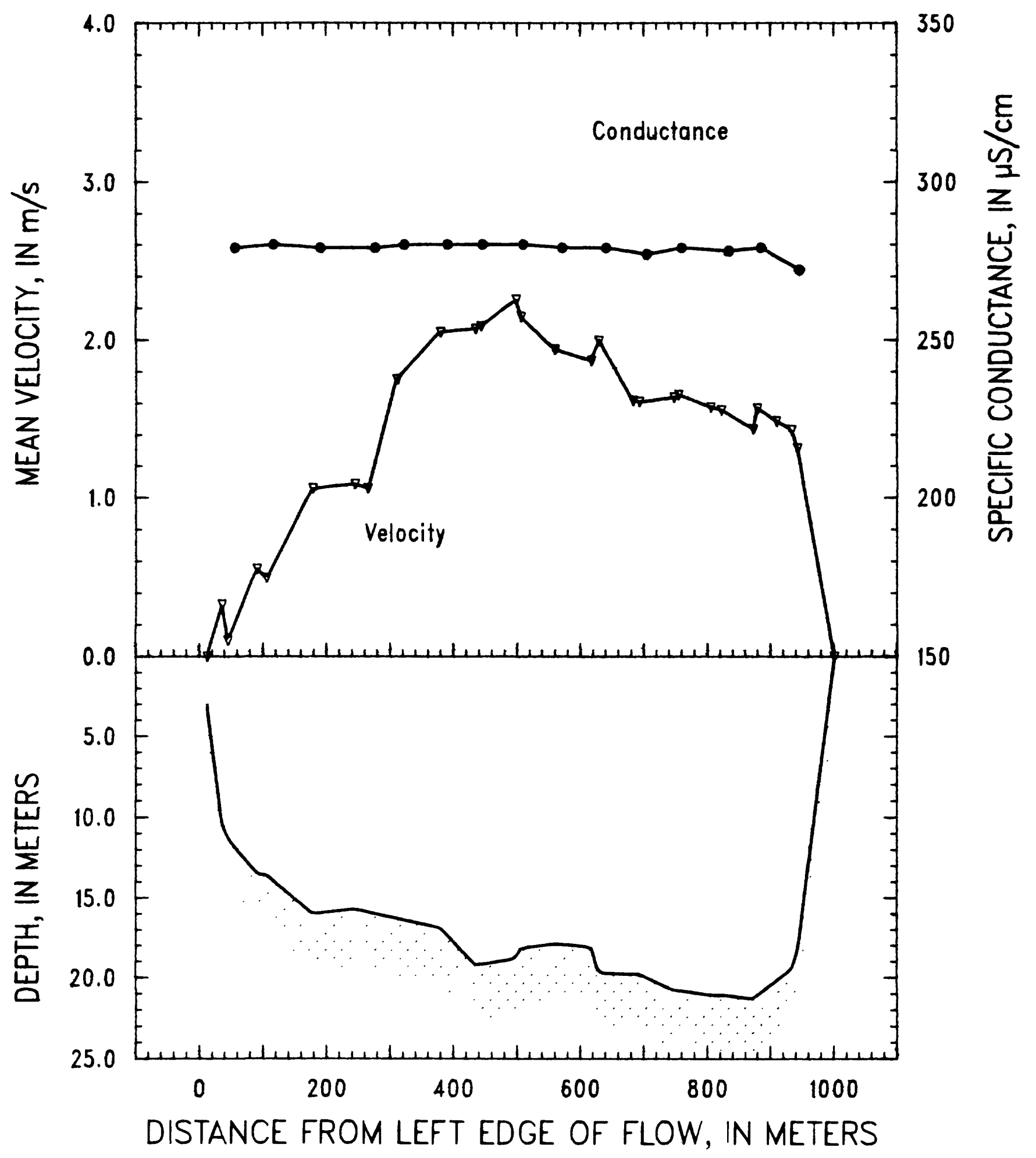

Figure 56. Mississippi River near St. Francisville, Louisiana, on March 12, 1990. 
SITE: Mississippi River below Belle Chasse, Louisiana 03-14-90

PARTY: Moody, Garbarino, and Simoneaux METER: SOLID CUP

STARTING GAGE HEIGHT: $13.8 \mathrm{ft}$ ENDING GAGE HEIGHT: --

SUSP: Bag sampler and $300-1 \mathrm{~b}$ weight

CURRENT METER NO.: W-297222 DATE RATED: 04-90

CURRENT METER EQUATION: $V(\mathrm{~m} / \mathrm{s})=$ RPS $* 0.744+0.011$

REMARKS: Transit rate was $13 \mathrm{~cm} / \mathrm{s}$, and 1/8-inch nozzle was used. LEF is at $27 \mathrm{~m}$, and REF is at $824 \mathrm{~m}$. Verticals occupied in the following order: $1-4,6-9,13,12,5,11$, then 10 .

\begin{tabular}{|c|c|c|c|c|c|c|c|c|c|}
\hline \multirow[b]{2}{*}{$\begin{array}{c}\text { Verti- } \\
\text { cal }\end{array}$} & \multirow{2}{*}{$\begin{array}{l}\text { Dist. } \\
\text { from } \\
\text { LEW } \\
\text { (m) }\end{array}$} & \multirow[b]{2}{*}{$\begin{array}{l}\text { Depth } \\
(\mathrm{m})\end{array}$} & \multirow{2}{*}{$\begin{array}{l}\text { Mean } \\
\text { veloc- } \\
\text { ity } \\
(\mathrm{m} / \mathrm{s})\end{array}$} & \multirow[b]{2}{*}{$\begin{array}{r}\text { Dis- } \\
\text { charge } \\
\left(\mathrm{m}^{3} / \mathrm{s}\right)\end{array}$} & \multicolumn{2}{|c|}{ Volume } & \multirow[b]{2}{*}{$\begin{array}{l}\text { Temper- } \\
\text { ature } \\
\left({ }^{\circ} \mathrm{C}\right)\end{array}$} & \multirow[b]{2}{*}{$\mathrm{pH}$} & \multirow{2}{*}{$\begin{array}{l}\text { Specific } \\
\text { conduct- } \\
\text { ance } \\
(\mu \mathrm{S} / \mathrm{cm})\end{array}$} \\
\hline & & & & & $\begin{array}{l}\mathrm{V}_{\mathrm{i}} \\
(\mathrm{L})\end{array}$ & $\begin{array}{l}V_{p} \\
(\mathrm{~L})\end{array}$ & & & \\
\hline LEW & 0 & 37.5 & 0.00 & 0 & -- & - & -- & -- & -- \\
\hline $01 \mathrm{~A}$ & 75 & 12.5 & 0.87 & 465 & 1.13 & 18 & 14.0 & 7.8 & 288 \\
\hline 01B & 86 & 14.9 & 0.97 & 433 & 1.74 & -- & -- & -- & -- \\
\hline $02 \mathrm{~A}$ & 135 & 21.8 & 1.38 & 845 & 3.21 & 35 & 14.4 & 7.7 & 286 \\
\hline 02B & 142 & 21.9 & 1.33 & 583 & 3.98 & -- & -- & -- & -- \\
\hline $\mathrm{X} 02$ & 175 & 23.9 & 1.56 & 935 & - & -- & -- & -- & -- \\
\hline $03 A$ & 192 & 24.1 & 1.66 & 559 & 4.01 & 46 & 13.2 & 7.7 & 284 \\
\hline 03B & 203 & 25.5 & 1.73 & 1,452 & 5.25 & -- & -- & -- & -- \\
\hline 04B & 258 & 25.6 & 1.83 & 1,450 & 5.37 & -- & -- & -- & -- \\
\hline $04 \mathrm{~A}$ & 265 & 25.3 & 1.78 & 1,327 & 5.77 & 46 & 13.3 & 7.7 & 288 \\
\hline $05 A$ & 317 & 27.8 & 1.71 & 1,404 & 5.90 & 51 & 13.7 & 7.7 & 289 \\
\hline 05B & 324 & 27.5 & 1.74 & 574 & 6.09 & -- & -- & -- & -- \\
\hline X04 & 341 & 27.6 & 1.80 & 1,265 & - & -- & -- & -- & -- \\
\hline $06 \mathrm{~A}, \mathrm{~B}$ & 375 & 27.6 & 1.65 & 1,961 & 11.49 & 51 & 12.7 & 7.8 & 290 \\
\hline $07 A, B$ & 427 & 28.1 & 1.62 & 2,525 & 12.51 & 55 & 12.8 & 7.7 & 290 \\
\hline $08 \mathrm{~A}, \mathrm{~B}$ & 486 & 27.1 & 1.59 & 1,468 & 10.43 & 49 & 12.9 & 7.7 & 293 \\
\hline X05 & 495 & 27.0 & 1.64 & 1,129 & -- & -- & -- & -- & -- \\
\hline 09A, B & 537 & 26.4 & 1.50 & 1,925 & 10.47 & 45 & 12.5 & 7.8 & 290 \\
\hline $10 \mathrm{~A}, \mathrm{~B}$ & 592 & 25.7 & 1.35 & 1,800 & 8.77 & 35 & 12.4 & 7.7 & 289 \\
\hline $11 \mathrm{~A}, \mathrm{~B}$ & 641 & 24.4 & 1.14 & 1,421 & 6.78 & 31 & 12.7 & 7.8 & 290 \\
\hline $12 \mathrm{~A}$ & 694 & 23.4 & 1.10 & 852 & 3.32 & 29 & 13.0 & 7.7 & 286 \\
\hline $12 B$ & 707 & 23.3 & 1.01 & 657 & 2.96 & -- & -- & -- & -- \\
\hline $13 \mathrm{~A}$ & 750 & 23.5 & 0.94 & 718 & 2.51 & 14 & 13.4 & 7.7 & 289 \\
\hline $13 B$ & 772 & 16.2 & 0.93 & 986 & 1.78 & -- & -- & -- & -- \\
\hline REW & 881 & 0.0 & 0.00 & 0 & -- & -- & -- & -- & -- \\
\hline MEAN & & 21.2 & 1.43 & & & & & & \\
\hline TOTAL & 881 & & & 26,732 & 113.47 & 505 & & & \\
\hline
\end{tabular}




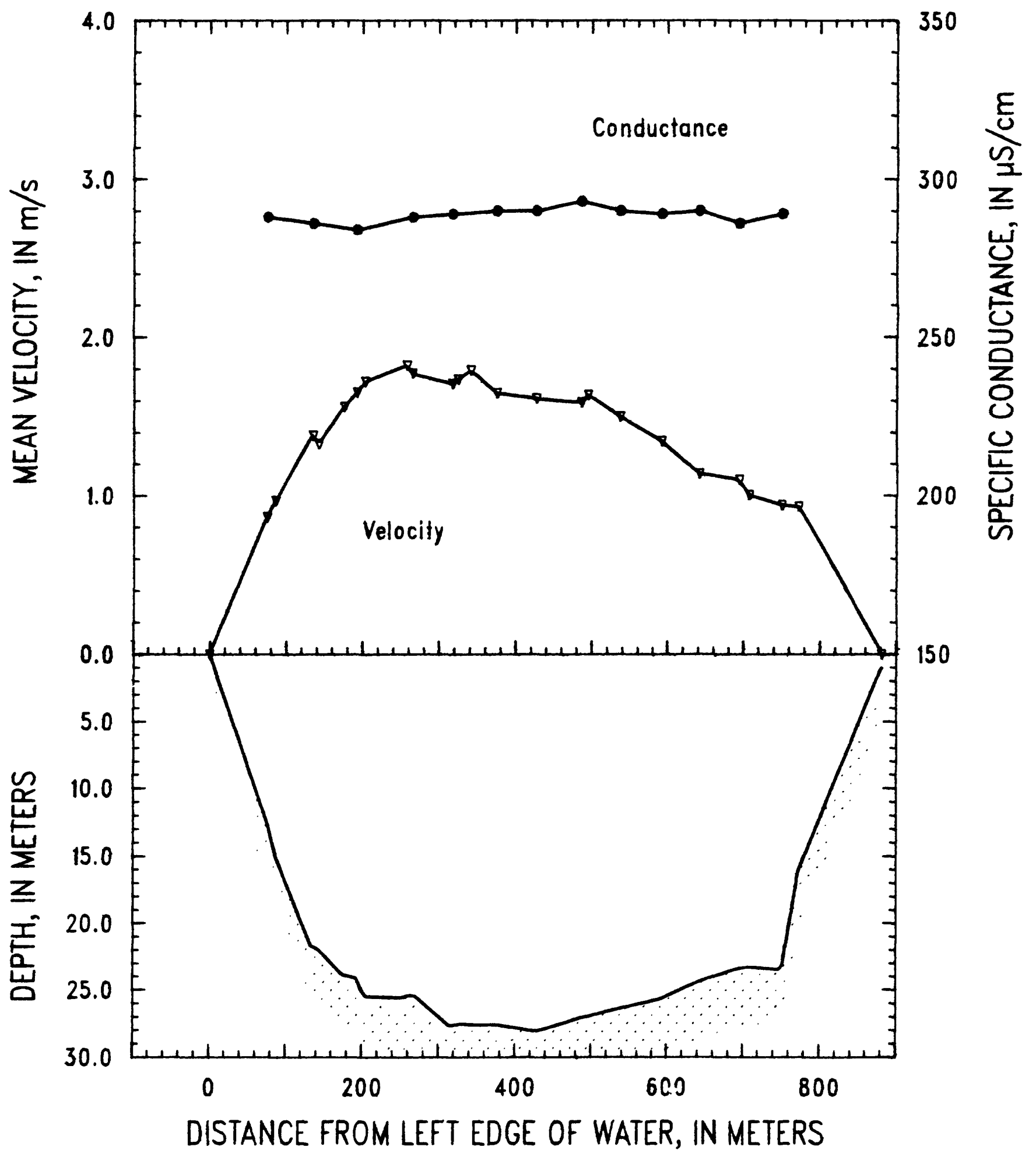

Figure 57. Mississippi River below Belle Chasse, Louisiana, on March 14, 1990. 
DATA LISTINGS

FOR

MAY-JUNE 1990 CRUISE 
SITE: Mississippi River near Cairo, Illinois

PARTY: Moody, Delaune, and Simoneaux

05-31-90

STARTING GAGE HEIGHT: --

ENDING GAGE HEIGHT: --

METER: SOLID CUP

SUSP: Bag sampler and $300-1 \mathrm{~b}$ weight

CURRENT METER NO.: W-297222 DATE RATED: 04-90

CURRENT METER EQUATION: $V(\mathrm{~m} / \mathrm{s})=$ RPS $* 0.744+0.011$

REMARKS: Flow in 100-m section west of small levee road (on right side) was not measured nor was the possible flow in a section of about $100 \mathrm{~m}$ beyond a line of trees on the left edge. Vertical 3.5 was done last, between verticals 3 and 4 . Individual verticals were collected using various nozzles and transit rates.

\begin{tabular}{|c|c|c|c|c|c|c|c|c|c|c|}
\hline \multirow[t]{2}{*}{$\begin{array}{c}\text { Verti- } \\
\text { cal }\end{array}$} & \multirow[t]{2}{*}{$\begin{array}{l}\text { Mean } \\
\text { dist. } \\
\text { from } \\
\text { LEW } \\
\text { (m) }\end{array}$} & \multirow[t]{2}{*}{$\begin{array}{l}\text { Stan- } \\
\text { dard } \\
\text { devia- } \\
\text { tion } \\
\text { (m) }\end{array}$} & \multirow[t]{2}{*}{$\begin{array}{l}\text { Mean } \\
\text { depth } \\
\text { (m) }\end{array}$} & \multirow[t]{2}{*}{$\begin{array}{l}\text { Mean } \\
\text { veloc- } \\
\text { ity } \\
(\mathrm{m} / \mathrm{s})\end{array}$} & \multirow[t]{2}{*}{$\begin{array}{c}\text { Dis- } \\
\text { charge } \\
\left(\mathrm{m}^{3} / \mathrm{s}\right)\end{array}$} & \multicolumn{2}{|c|}{$\begin{array}{c}\text { Sus- } \\
\text { pended } \\
\text { sedi- } \\
\text { ment } \\
(\mathrm{mg} / \mathrm{L})\end{array}$} & \multirow[t]{2}{*}{$\begin{array}{c}\text { Temper- } \\
\text { ature } \\
\left({ }^{\circ} \mathrm{C}\right)\end{array}$} & \multirow[t]{2}{*}{$\mathrm{pH}$} & \multirow[t]{2}{*}{$\begin{array}{c}\text { Specific } \\
\text { conduct- } \\
\text { ance } \\
(\mu S / \mathrm{cm})\end{array}$} \\
\hline & & & & & & $<63 \mu \mathrm{m}$ & $>63 \mu \mathrm{m}$ & & & \\
\hline LEW & 0 & 0 & 00.0 & 0.00 & 0 & -- & -- & -- & -- & -- \\
\hline $1 \mathrm{~B}$ & 161 & 10 & 13.5 & 0.49 & 933 & 582 & 20 & 19.1 & 7.6 & 367 \\
\hline $2 A$ & 280 & 1 & 14.3 & 1.38 & 2,540 & 655 & 46 & 19.1 & 7.7 & 365 \\
\hline $3 B$ & 418 & 1 & 15.6 & 2.03 & 2,916 & 694 & 64 & 18.9 & 7.9 & 364 \\
\hline 3.5 & 464 & 1 & 16.3 & 2.10 & 1,555 & 704 & 91 & 19.2 & 7.7 & 365 \\
\hline $4 \mathrm{~A}$ & 509 & 2 & 16.4 & 2.43 & 2,991 & 680 & 90 & 19.2 & 7.7 & 361 \\
\hline $5 B$ & 614 & 1 & 14.5 & 1.95 & 2,649 & 613 & 69 & 19.2 & 7.7 & 362 \\
\hline REW & 696 & 0 & 0.0 & 0.00 & 0 & - & - & - & - & - \\
\hline MEAN & & & 12.2 & 1.60 & & & & & & \\
\hline TOTAL & 696 & & & & 13,584 & & & & & \\
\hline
\end{tabular}




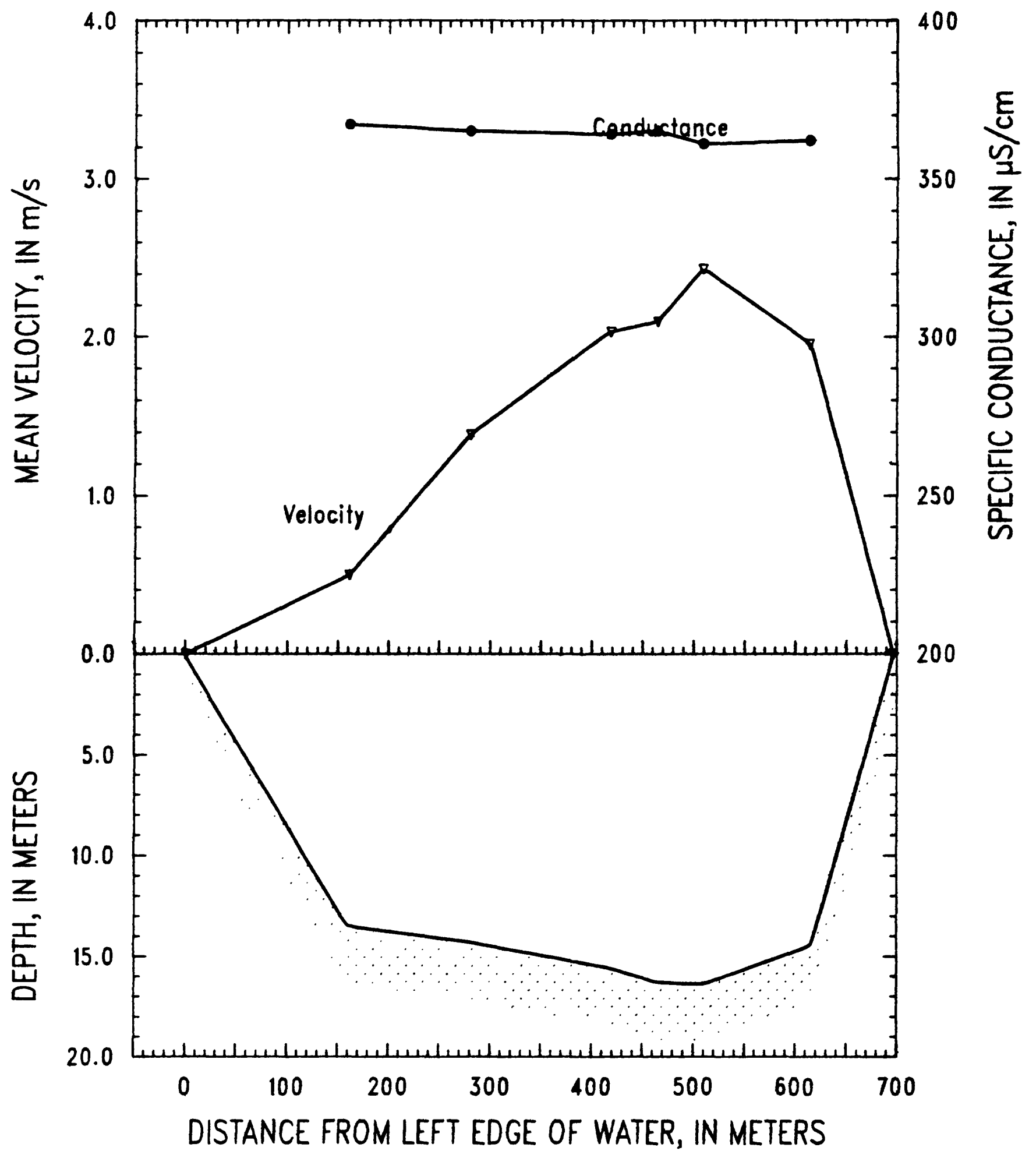

Figure 58. Mississippi River near Cairo, lllinois, on May 31, 1990. 


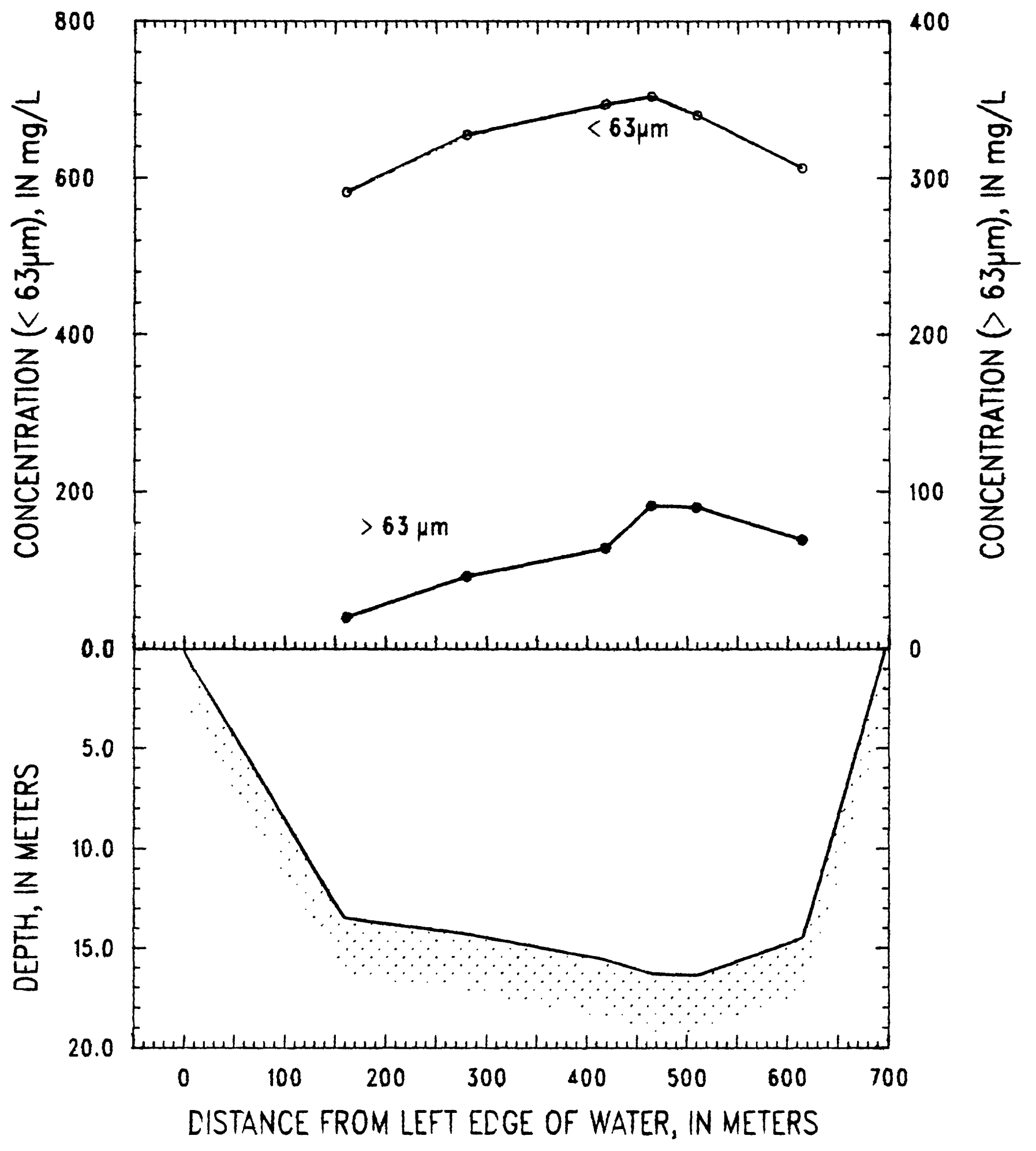

Figure 59. Mississippi River near Cairo, Illinois, on May 31, 1990-suspended-sediment concentration. 
SITE: Ohio River at Olmsted, Illinois

PARTY: Moody, Delaune, Simoneaux, and LeBoeuf

05-31-90

STARTING GAGE HEIGHT: --

ENDING GAGE HEIGHT:

METER: SOLID CUP

SUSP: Bag sampler and 300-1b weight

CURRENT METER NO.: W-297222 DATE RATED: 04-90

CURRENT METER EQUATION: $V(\mathrm{~m} / \mathrm{s})=$ RPS $* 0.744+0.011$

REMARKS: Individual verticals were collected using the $1 / 4-$ inch nozzle and various transit rates. Section is 16.5 miles upriver from the confluence of the Ohio and Mississippi Rivers.

\begin{tabular}{|c|c|c|c|c|c|c|c|c|c|c|}
\hline \multirow[t]{2}{*}{$\begin{array}{c}\text { Verti- } \\
\text { cal }\end{array}$} & \multirow[t]{2}{*}{$\begin{array}{l}\text { Mean } \\
\text { dist. } \\
\text { from } \\
\text { LEW } \\
\text { (m) }\end{array}$} & \multirow[t]{2}{*}{$\begin{array}{l}\text { Stan- } \\
\text { dard } \\
\text { devia- } \\
\text { tion } \\
(\mathrm{m})\end{array}$} & \multirow[t]{2}{*}{$\begin{array}{l}\text { Mean } \\
\text { depth } \\
(m)\end{array}$} & \multirow[t]{2}{*}{$\begin{array}{l}\text { Mean } \\
\text { veloc- } \\
\text { ity } \\
(\mathrm{m} / \mathrm{s})\end{array}$} & \multirow[t]{2}{*}{$\begin{array}{c}\text { Dis- } \\
\text { charge } \\
\left(\mathrm{m}^{3} / \mathrm{s}\right)\end{array}$} & \multicolumn{2}{|c|}{$\begin{array}{l}\text { Sus- } \\
\text { pended } \\
\text { sedi- } \\
\text { ment } \\
(\mathrm{mg} / \mathrm{L})\end{array}$} & \multirow[t]{2}{*}{$\begin{array}{l}\text { Temper- } \\
\text { ature } \\
\left({ }^{\circ} \mathrm{C}\right)\end{array}$} & \multirow[t]{2}{*}{$\mathrm{pH}$} & \multirow[t]{2}{*}{$\begin{array}{l}\text { Specific } \\
\text { conduct } \\
\text { ance } \\
(\mu \mathrm{S} / \mathrm{cm})\end{array}$} \\
\hline & & & & & & $<63 \mu \mathrm{m}$ & $>63 \mu \mathrm{m}$ & & & \\
\hline LEW & 0 & -- & 0.0 & -- & 0 & -- & -- & -- & -- & - \\
\hline $01 \mathrm{~A}$ & 74 & 3 & 11.0 & 0.65 & 501 & 87 & 4 & 19.8 & 7.6 & 249 \\
\hline O2B & 141 & 4 & 12.5 & 0.86 & 671 & 107 & 6 & 19.7 & 7.7 & 263 \\
\hline 03A & 199 & 4 & 12.9 & 0.91 & 628 & 106 & 5 & 19.8 & 7.7 & 256 \\
\hline 04B & 248 & 7 & 13.9 & 0.94 & 965 & 110 & 3 & 19.7 & 7.6 & 274 \\
\hline 05A & 347 & 8 & 15.3 & 1.02 & 1,314 & 120 & 3 & 19.6 & 7.7 & 293 \\
\hline 06B & 416 & 5 & 16.0 & 0.98 & 1,127 & 125 & 4 & 19.6 & 7.7 & 307 \\
\hline $07 \mathrm{~A}$ & 491 & 7 & 17.1 & 1.05 & 1,230 & 128 & 4 & 19.5 & 7.7 & 319 \\
\hline 08B & 553 & 4 & 15.9 & 1.11 & 1,067 & 126 & 2 & 19.3 & 7.7 & 327 \\
\hline 09A & 612 & 5 & 17.7 & 1.04 & 1,215 & 136 & 3 & 19.1 & 7.7 & 335 \\
\hline $10 \mathrm{~B}$ & 685 & 2 & 16.4 & 1.14 & 1,364 & 140 & 3 & 19.3 & 7.8 & 340 \\
\hline $11 \mathrm{~A}$ & 758 & 2 & 16.6 & 1.19 & 1,344 & 132 & 2 & 19.2 & 7.6 & 341 \\
\hline $12 \mathrm{~B}$ & 821 & 2 & 16.7 & 1.32 & 1,332 & 127 & 3 & 19.3 & 7.7 & 329 \\
\hline $13 B$ & 879 & 2 & 16.8 & 1.21 & 1,222 & 125 & 1 & 19.4 & 7.7 & 337 \\
\hline $14 \mathrm{~A}$ & 941 & 2 & 17.8 & 0.93 & 1,048 & 124 & 3 & 19.4 & 7.7 & 330 \\
\hline $15 B$ & 1,005 & 2 & 11.8 & 0.62 & 577 & 99 & 2 & 19.7 & 7.6 & 324 \\
\hline REW & 1,090 & & 0.0 & 0.00 & 0 & - & - & - & - & - \\
\hline MEAN & & & 14.0 & 1.01 & & & & & & \\
\hline TOTAL & 1,098 & & & & 15,605 & & & & & \\
\hline
\end{tabular}




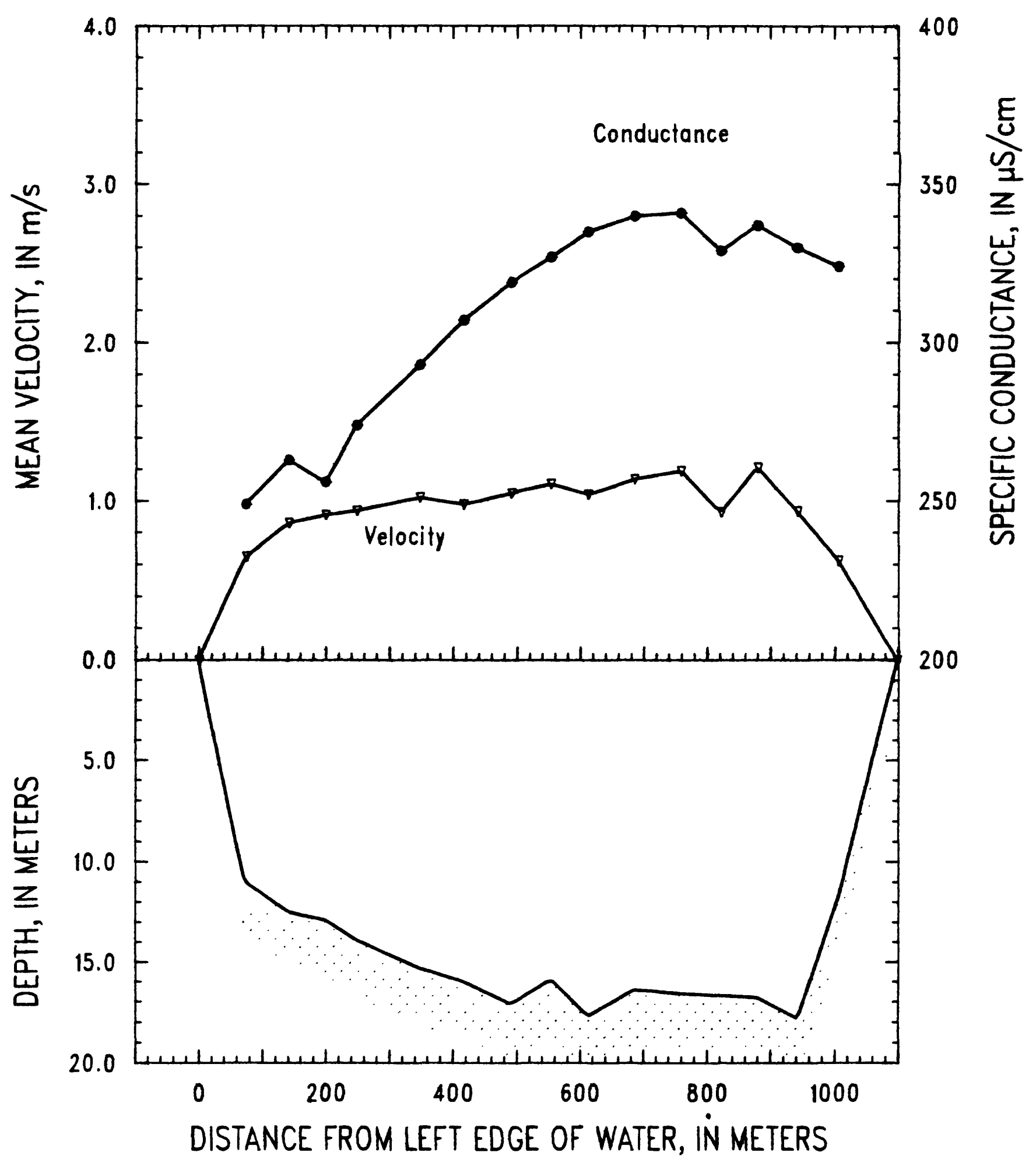

Figure 60. Ohio River at Olmsted, Illinois, on May 31, 1990. 


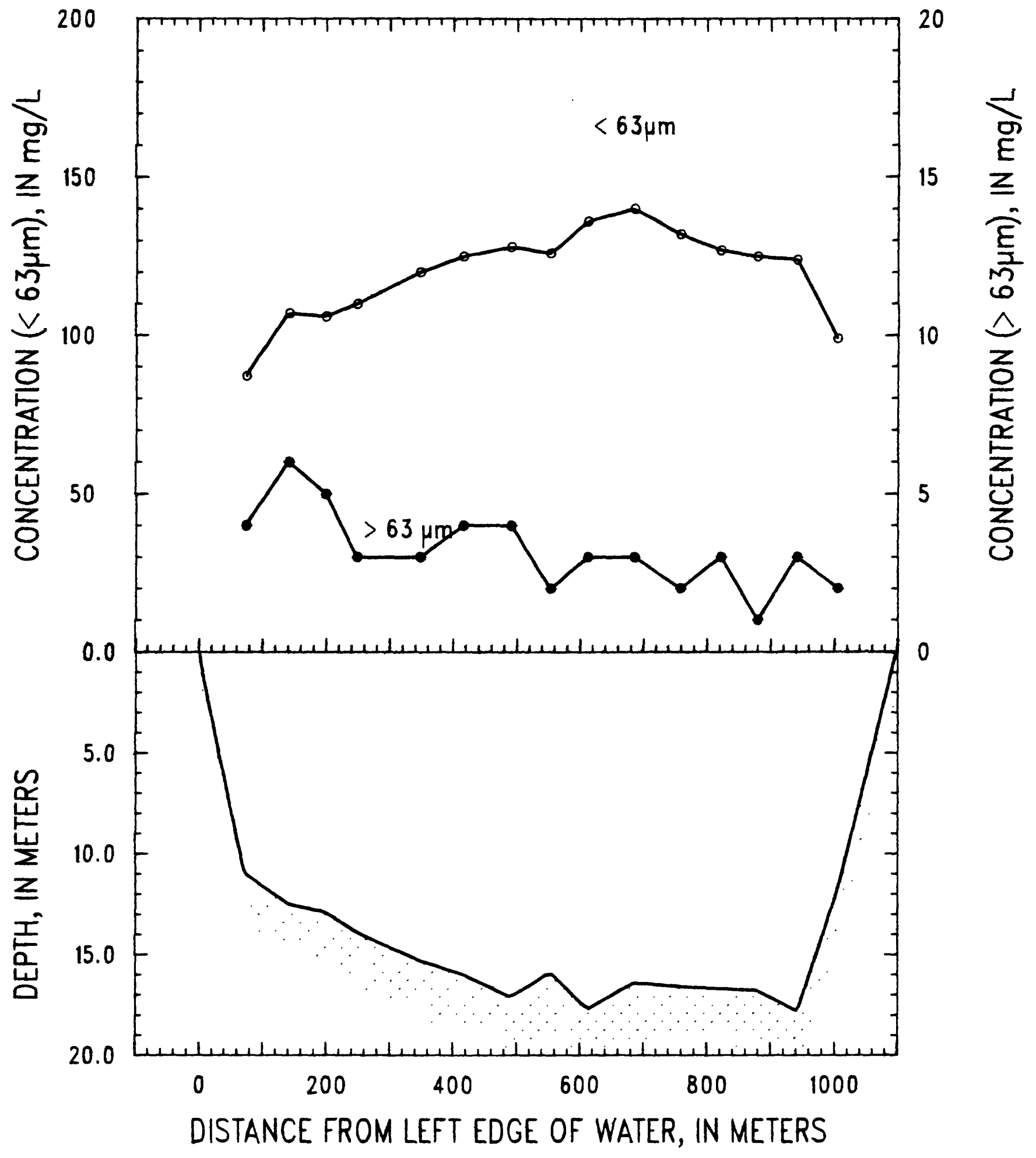

Figure 61. Ohio River at Olmsted, Illinois, on May 31, 1990-suspended-sediment concentration. 
SITE: Mississippi River at Wickliffe, Kentucky

PARTY: Moody, Delaune, and Simoneaux

$05-30-90$

STARTING GAGE HEIGHT: $47 \mathrm{ft}$ ENDING GAGE HEIGHT: --

METER: SOLID CUP

SUSP: Bag sampler and $300-1 \mathrm{~b}$ weight

CURRENT METER NO.: W-297222 DATE RATED: 04-90

CURRENT METER EQUATION: $\mathrm{V}(\mathrm{m} / \mathrm{s})=$ RPS $* 0.744+0.011$

REMARKS: Individual verticals were collected using $1 / 8$ - and $3 / 16$-inch nozzles and various transit rates. First measurement in a short time series of 3 .

\begin{tabular}{|c|c|c|c|c|c|c|c|c|c|c|}
\hline \multirow[t]{2}{*}{$\begin{array}{c}\text { Verti- } \\
\text { cal }\end{array}$} & \multirow[t]{2}{*}{$\begin{array}{l}\text { Mean } \\
\text { dist. } \\
\text { from } \\
\text { LEW } \\
\text { (m) }\end{array}$} & \multirow[t]{2}{*}{$\begin{array}{l}\text { Stan- } \\
\text { dard } \\
\text { devia- } \\
\text { tion } \\
\text { (m) }\end{array}$} & \multirow[t]{2}{*}{$\begin{array}{l}\text { Mean } \\
\text { depth } \\
\text { (m) }\end{array}$} & \multirow[t]{2}{*}{$\begin{array}{l}\text { Mean } \\
\text { veloc- } \\
\text { ity } \\
(\mathrm{m} / \mathrm{s})\end{array}$} & \multirow[t]{2}{*}{$\begin{array}{c}\text { Dis- } \\
\text { charge } \\
\left(\mathrm{m}^{3} / \mathrm{s}\right)\end{array}$} & \multicolumn{2}{|c|}{$\begin{array}{l}\text { Sus- } \\
\text { pended } \\
\text { sedi- } \\
\text { ment } \\
(\mathrm{mg} / \mathrm{L})\end{array}$} & \multirow[t]{2}{*}{$\begin{array}{l}\text { Temper- } \\
\text { ature } \\
\left({ }^{\circ} \mathrm{C}\right)\end{array}$} & \multirow[t]{2}{*}{$\mathrm{pH}$} & \multirow[t]{2}{*}{$\begin{array}{l}\text { Specific } \\
\text { conduct- } \\
\text { ance } \\
(\mu \mathrm{S} / \mathrm{cm})\end{array}$} \\
\hline & & & & & & $<63 \mu \mathrm{m}$ & $>63 \mu \mathrm{m}$ & & & \\
\hline LEW & 0 & -- & 0.0 & 0.00 & 0 & -- & -- & - & -- & -- \\
\hline $01 \mathrm{~B}$ & 58 & 5 & 10.8 & 2.20 & 1,343 & 99 & 6 & 20.0 & 7.8 & 281 \\
\hline $02 \mathrm{~A}$ & 113 & 8 & 18.2 & 2.60 & 2,812 & 105 & 5 & 20.0 & 7.8 & 288 \\
\hline 03B & 177 & 17 & 29.0 & 2.60 & 3,801 & 124 & 17 & 18.7 & 7.7 & 304 \\
\hline $04 \mathrm{~A}$ & 214 & 5 & 30.0 & 2.40 & 3,236 & 138 & 12 & 18.7 & 7.6 & 298 \\
\hline 05B & 267 & 8 & 26.9 & 2.41 & 3,148 & 270 & 35 & 18.8 & 7.7 & 330 \\
\hline $06 \mathrm{~A}$ & 311 & 9 & 26.2 & 2.39 & 2,688 & 344 & 48 & 19.5 & 7.7 & 345 \\
\hline $07 \mathrm{~B}$ & 353 & 8 & 26.2 & 2.22 & 2,876 & 470 & 54 & 19.2 & 7.8 & 334 \\
\hline 08A & 410 & 10 & 22.4 & 2.06 & 2,428 & 535 & 99 & 18.5 & 7.8 & 372 \\
\hline $09 \mathrm{~A}$ & 458 & 3 & 19.5 & 2.06 & 1,828 & 632 & 136 & 18.3 & 7.7 & 373 \\
\hline $10 \mathrm{~B}$ & 501 & 4 & 16.8 & 2.01 & 1,668 & 619 & 153 & 18.2 & 7.8 & 379 \\
\hline $11 \mathrm{~A}$ & 557 & 5 & 13.4 & 1.68 & 1,135 & 621 & 146 & 17.9 & 7.7 & 378 \\
\hline $12 \mathrm{~B}$ & 602 & 2 & 11.5 & 1.62 & 855 & 614 & 91 & 18.8 & 7.7 & 383 \\
\hline $13 \mathrm{~A}$ & 649 & 2 & 10.4 & 1.66 & 873 & 609 & 45 & 19.3 & 7.7 & 384 \\
\hline $14 \mathrm{~B}$ & 703 & 2 & 10.2 & 1.41 & 706 & 598 & 45 & 19.6 & 7.8 & 385 \\
\hline $15 \mathrm{~A}$ & 747 & 1 & 9.4 & 1.50 & 875 & 590 & 32 & 19.1 & 7.8 & 382 \\
\hline REW & 827 & -- & 0.0 & 0.00 & 0 & -- & -- & - & -- & -- \\
\hline MEAN & & & 16.9 & 2.17 & & & & & & \\
\hline TOTAL & 827 & & & & 30,272 & & & & & \\
\hline
\end{tabular}




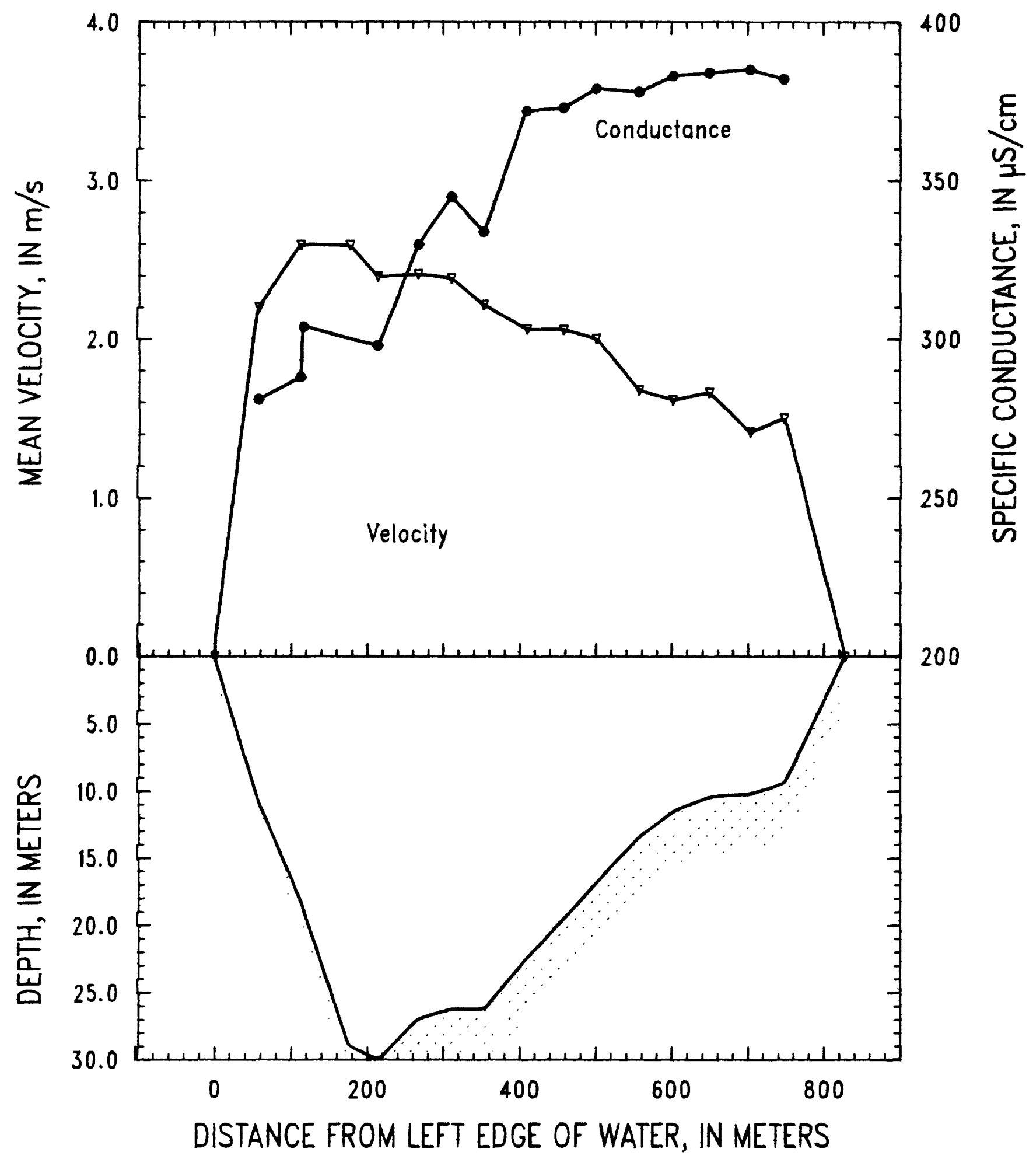

Figure 62. Mississippi River at Wickliffe, Kentucky, on May 30, 1990. 


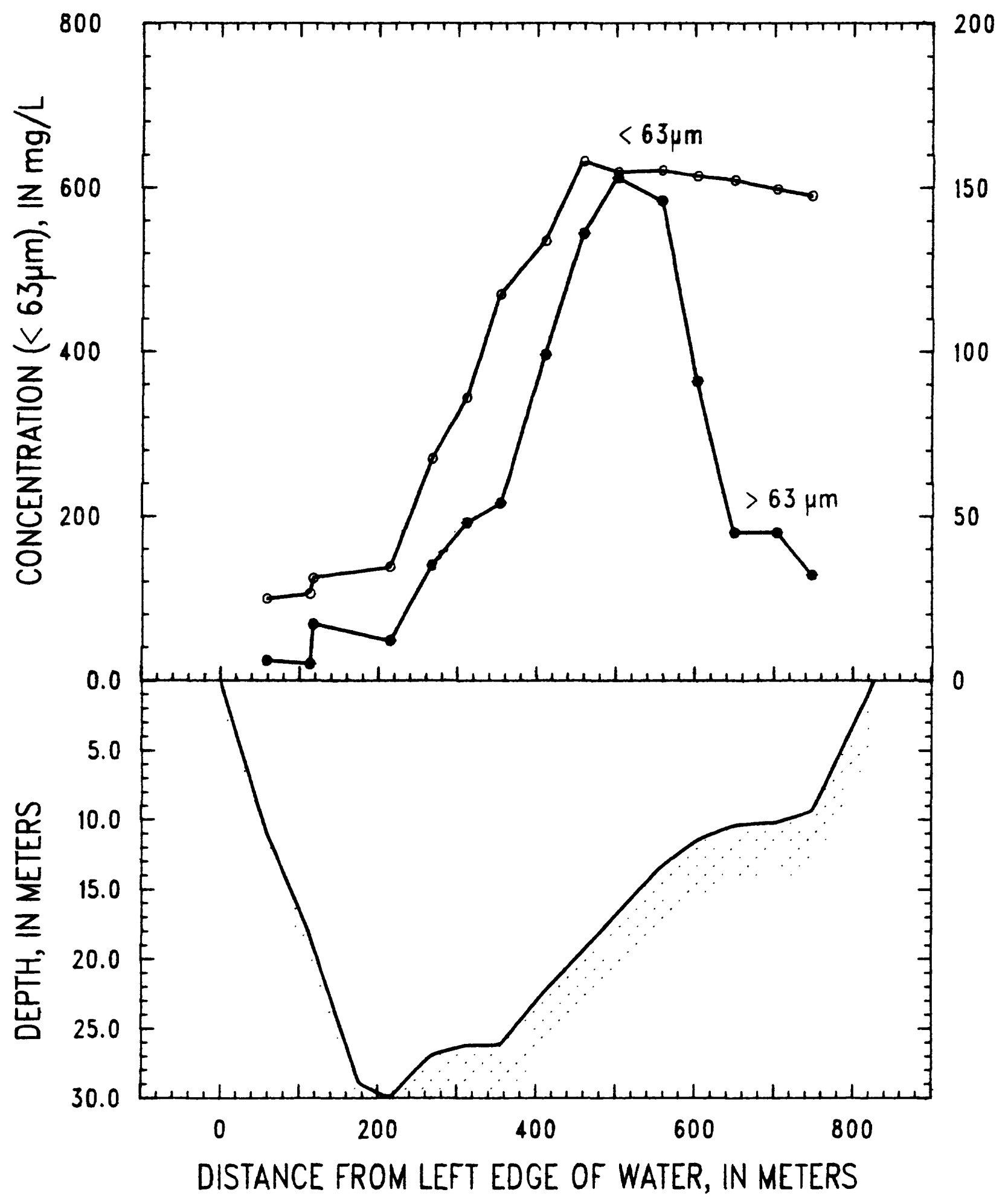

200 
SITE: Mississippi River at Wickliffe, Kentucky

PARTY: Moody, Delaune, and Simoneaux

STARTING GAGE HEIGHT: -- ENDING GAGE HEIGHT: --

METER: SOLID CUP

SUSP: Bag sampler and $300-1 b$ weight

CURRENT METER NO.: W-297222 DATE RATED: 04-90

CURRENT METER EQUATION: $V(\mathrm{~m} / \mathrm{s})=$ RPS $* 0.744+0.011$

REMARKS: Individual verticals were collected using 1/8- and 3/16-inch nozzles and various transit rates. Second sampling in the short time series of 3 . Numerous patches of wood and trash. An oily film collected on the hydrowire. Values of temperature, $\mathrm{pH}$, and specific conductance are measurements of the depth-integrated sample and measurements of the surface sample are in parentheses.

\begin{tabular}{|c|c|c|c|c|c|c|c|c|c|c|}
\hline \multirow[t]{2}{*}{$\begin{array}{l}\text { Verti- } \\
\text { cal }\end{array}$} & \multirow[t]{2}{*}{$\begin{array}{l}\text { Mean } \\
\text { dist. } \\
\text { from } \\
\text { LEW } \\
(m)\end{array}$} & \multirow[t]{2}{*}{$\begin{array}{l}\text { Stan- } \\
\text { dard } \\
\text { devia- } \\
\text { tion } \\
(\mathrm{m})\end{array}$} & \multirow[t]{2}{*}{$\begin{array}{l}\text { Mean } \\
\text { depth } \\
\text { (m) }\end{array}$} & \multirow[t]{2}{*}{$\begin{array}{l}\text { Mean } \\
\text { veloc- } \\
\text { ity } \\
(\mathrm{m} / \mathrm{s})\end{array}$} & \multirow[t]{2}{*}{$\begin{array}{l}\text { Dis- } \\
\text { charge } \\
\left(m^{3} / s\right)\end{array}$} & \multicolumn{2}{|c|}{$\begin{array}{c}\text { Sus - } \\
\text { pended } \\
\text { sedi- } \\
\text { ment } \\
(\mathrm{mg} / \mathrm{L})\end{array}$} & \multirow[t]{2}{*}{$\begin{array}{l}\text { Temper- } \\
\text { ature } \\
\left({ }^{\circ} \mathrm{C}\right)\end{array}$} & \multirow[t]{2}{*}{$\mathrm{pH}$} & \multirow[t]{2}{*}{$\begin{array}{l}\text { Specific } \\
\text { conduct- } \\
\text { ance } \\
(\mu \mathrm{S} / \mathrm{cm})\end{array}$} \\
\hline & & & & & & $<63 \mu \mathrm{m}$ & $>63 \mu \mathrm{m}$ & & & \\
\hline LEW & 0 & -- & 0.0 & 0.00 & 0 & -- & - & - & -- & -- \\
\hline $01 \mathrm{~A}$ & 65 & 4 & 11.3 & 2.15 & 1,251 & 112 & 3 & 19.5 & 7.3 & 296 \\
\hline 02B & 103 & 2 & 15.0 & 2.34 & 1,650 & 114 & 3 & 19.2 & 7.4 & 301 \\
\hline 03A & 159 & 7 & 29.1 & 2.53 & 3,527 & 130 & 3 & 19.3 & 7.5 & 307 \\
\hline 04B & 199 & 4 & 30.3 & 2.38 & 3,785 & 157 & 7 & 19.0 & 7.5 & 322 \\
\hline 05A & 264 & 7 & 26.8 & 2.34 & 3,361 & 307 & 30 & 18.7 & 7.5 & 341 \\
\hline 06B & 306 & 3 & 27.1 & 2.30 & 2,646 & 396 & 36 & 18.7 & 7.5 & 349 \\
\hline 07B & 349 & 4 & 24.4 & 2.27 & 2,937 & 419 & 63 & 18.3 & 7.6 & 352 \\
\hline 08A & 412 & 9 & 21.3 & 2.28 & 2,675 & 497 & 73 & 19.1 & 7.5 & $355(360)$ \\
\hline 09B & 459 & 8 & 19.9 & 2.01 & 1,777 & 615 & 97 & 19.0 & 7.5 & 360 \\
\hline $10 \mathrm{~A}$ & 501 & 4 & 16.8 & 1.97 & 1,537 & 610 & 149 & 18.7 & 7.6 & 361 \\
\hline $11 B$ & 552 & 3 & 14.1 & 1.77 & 1,273 & 590 & 123 & 18.9 & 7.4 & 357 \\
\hline $12 \mathrm{~A}$ & 603 & 2 & 11.7 & 1.60 & 872 & 583 & 103 & 18.5 & 7.5 & 355 \\
\hline $13 B$ & 645 & 3 & 10.3 & 1.53 & 741 & 565 & 39 & 18.5 & 7.5 & 361 \\
\hline $14 \mathrm{~A}$ & 697 & 2 & 9.6 & 1.54 & 776 & 551 & 39 & 18.7 & 7.6 & 360 \\
\hline $15 \mathrm{~A}$ & 750 & 2 & 9.6 & 1.44 & 898 & 555 & 45 & 18.8 & 7.5 & 360 \\
\hline REW & 827 & -- & 0.0 & 0.00 & 0 & -- & -- & -- & -- & -- \\
\hline MEAN & & & 16.8 & 2.14 & & & & & & \\
\hline TOTAL & 827 & & & & 29,706 & & & & & \\
\hline
\end{tabular}




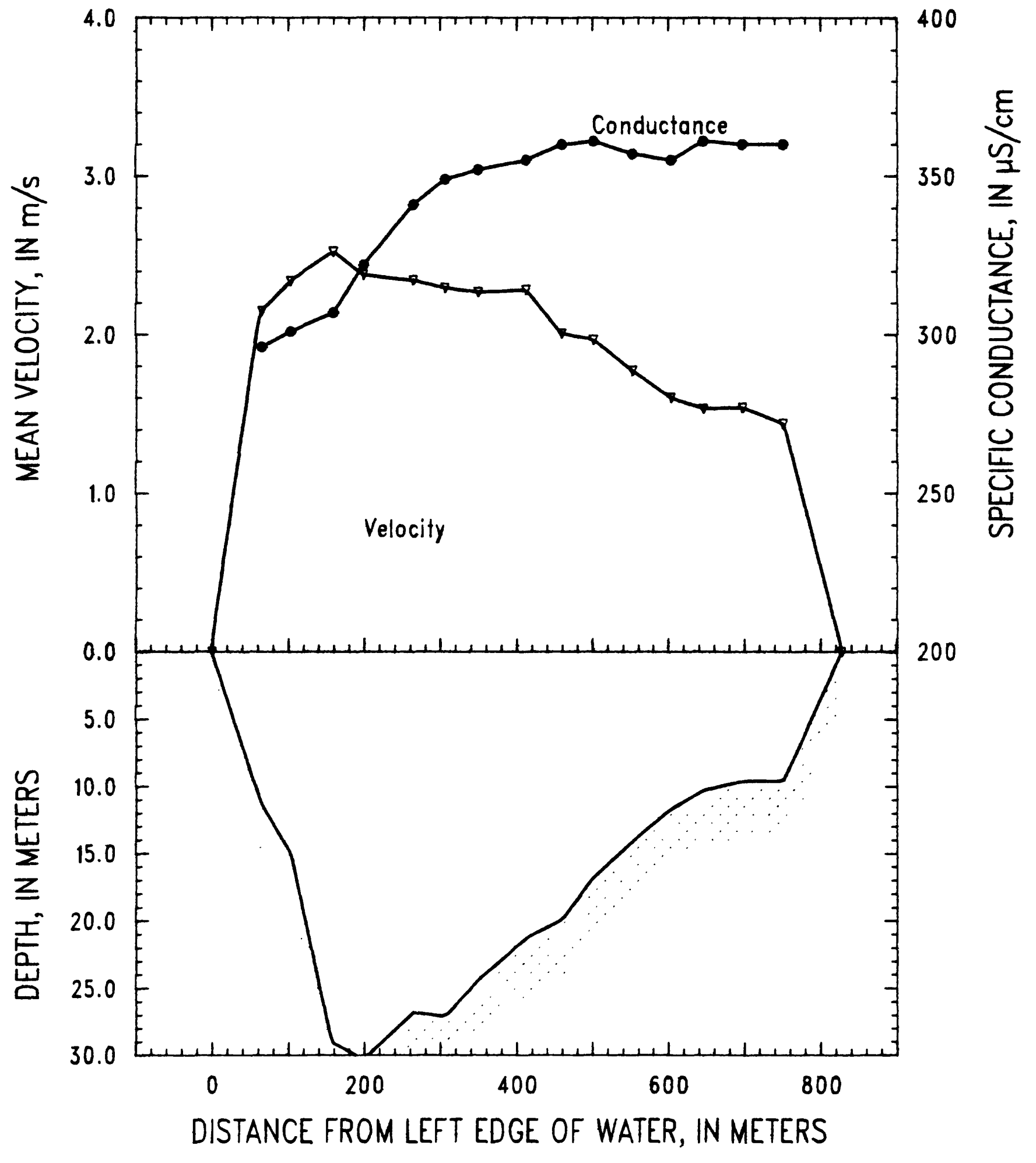

Figure 64. Mississippi River at Wickliffe, Kentucky, on June 1, 1990. 


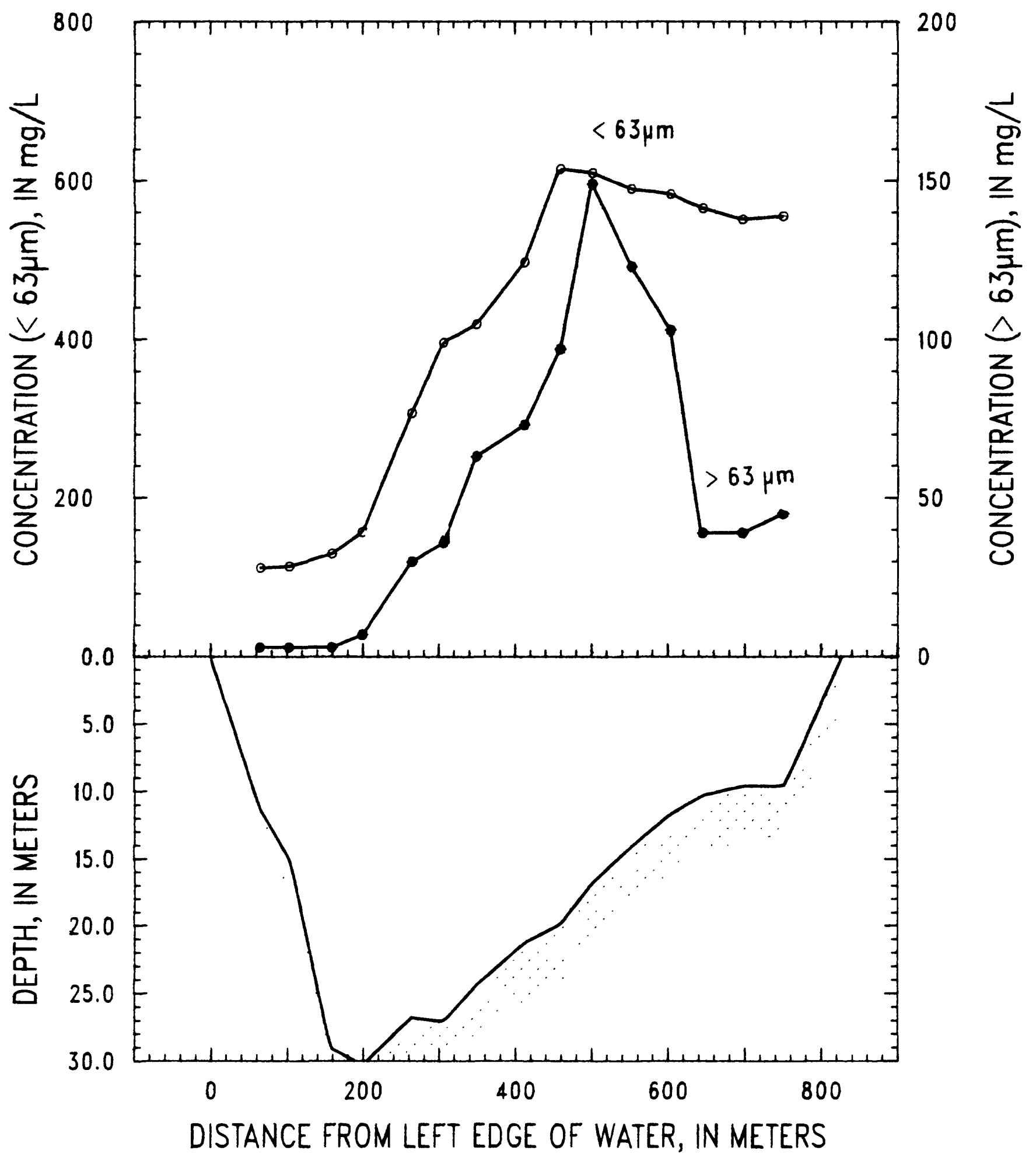

Figure 65. Mississippi River at Wickliffe, Kentucky, on June 1, 1990-suspended-sediment concentration. 
SITE: Mississippi River at Wickliffe, Kentucky

06-04-90

PARTY: Moody, Delaune, and Simoneaux

METER: SOLID CUP

STARTING GAGE HEIGHT: -- ENDING GAGE HEIGHT: $46.8 \mathrm{ft}$

SUSP: Bag sampler and 300-lb weight

CURRENT METER NO.: W-297222 DATE RATED: 04-90

CURRENT METER EQUATION: $V(\mathrm{~m} / \mathrm{s})=$ RPS $* 0.744+0.011$

REMARKS: Individual verticals were collected using $1 / 8-$ and $3 / 16$-inch nozzles and various transit rates. Third sampling in a short time series of 3 . No trash today, and no oily film collected on the hydrowire. Values of temperature, $\mathrm{pH}$, and specific conductance are measurements of the depthintegrated samples and measurements of the surface sample are in parentheses.

\begin{tabular}{|c|c|c|c|c|c|c|c|c|c|c|}
\hline \multirow[t]{2}{*}{$\begin{array}{l}\text { Verti- } \\
\text { cal }\end{array}$} & \multirow[t]{2}{*}{$\begin{array}{l}\text { Mean } \\
\text { dist. } \\
\text { from } \\
\text { LEW } \\
\text { (m) }\end{array}$} & \multirow[t]{2}{*}{$\begin{array}{l}\text { Stan- } \\
\text { dard } \\
\text { devia- } \\
\text { tion } \\
\text { (m) }\end{array}$} & \multirow[t]{2}{*}{$\begin{array}{l}\text { Mean } \\
\text { depth } \\
\text { (m) }\end{array}$} & \multirow[t]{2}{*}{$\begin{array}{l}\text { Mean } \\
\text { veloc- } \\
\text { ity } \\
(\mathrm{m} / \mathrm{s})\end{array}$} & \multirow[t]{2}{*}{$\begin{array}{l}\text { Dis- } \\
\text { charge } \\
\left(\mathrm{m}^{3} / \mathrm{s}\right)\end{array}$} & \multicolumn{2}{|c|}{$\begin{array}{c}\text { Sus- } \\
\text { pended } \\
\text { sedi- } \\
\text { ment } \\
(\mathrm{mg} / \mathrm{L})\end{array}$} & \multirow[t]{2}{*}{$\begin{array}{l}\text { Temper- } \\
\text { ature } \\
\left({ }^{\circ} \mathrm{C}\right)\end{array}$} & \multirow[t]{2}{*}{$\mathrm{pH}$} & \multirow[t]{2}{*}{$\begin{array}{l}\text { Specific } \\
\text { conduct- } \\
\text { ance } \\
(\mu S / \mathrm{cm})\end{array}$} \\
\hline & & & & & & $<63 \mu \mathrm{m}$ & $>63 \mu \mathrm{m}$ & & & \\
\hline LEW & 0 & -- & 0.0 & 0.00 & 0 & -- & -- & -- & $\cdots$ & $-\infty$ \\
\hline $01 \mathrm{~A}$ & 63 & 3 & 10.5 & 2.05 & 1,111 & 131 & 5 & 21.1 & 7.7 & $280(284)$ \\
\hline 02B & 103 & 3 & 13.5 & 2.35 & 1,537 & 141 & 6 & 20.8 & 7.9 & $286(286)$ \\
\hline 03A & 160 & 6 & 29.1 & 2.50 & 3,706 & 150 & 7 & 20.9 & 7.8 & $298(305)$ \\
\hline 04B & 205 & 5 & 29.4 & 2.37 & 3,379 & 160 & 11 & 20.8 & 7.9 & 309 (319) \\
\hline 05B & 257 & 3 & 28.6 & 2.20 & 3,119 & 168 & 17 & 20.7 & 7.9 & 324 (335) \\
\hline $06 \mathrm{~A}$ & 304 & 8 & 27.8 & 2.12 & 2,801 & 232 & 17 & 20.7 & 7.8 & 351 (345) \\
\hline 07B & 352 & 3 & 25.3 & 1.99 & 2,514 & 294 & 35 & 20.6 & 7.9 & 367 (373) \\
\hline 08A & 404 & 7 & 24.0 & 2.08 & 2,366 & 367 & 65 & 20.4 & 7.9 & $379(396)$ \\
\hline 09B & 447 & 5 & 22.0 & 2.00 & 2,095 & 430 & 57 & 20.4 & 8.0 & 391 (391) \\
\hline $10 \mathrm{~A}$ & 499 & 6 & 17.9 & 1.94 & 1,960 & 478 & 88 & 20.4 & 7.9 & $399(403)$ \\
\hline $11 \mathrm{~A}$ & 560 & 5 & 13.7 & 1.69 & 1,145 & 490 & 122 & 20.4 & 8.0 & $399(403)$ \\
\hline $12 B$ & 598 & 3 & 11.9 & 1.53 & 883 & 470 & 88 & 20.3 & 7.9 & $399(403)$ \\
\hline $13 \mathrm{~A}$ & 657 & 2 & 10.0 & 1.43 & 679 & 466 & 42 & 20.0 & 7.9 & 401 (403) \\
\hline $14 B$ & 693 & 1 & 10.0 & 1.36 & 603 & 475 & 61 & 19.8 & 7.9 & $401(404)$ \\
\hline $15 \mathrm{~A}$ & 746 & 5 & 9.6 & 1.46 & 938 & 460 & 24 & 19.8 & 8.0 & $400(403)$ \\
\hline REW & 827 & -- & 0.0 & 0.00 & 0 & -- & -- & -- & -- & -- \\
\hline MEAN & & & 17.1 & 2.04 & & & & & & \\
\hline TOTAL & 827 & & & & 28,836 & & & & & \\
\hline
\end{tabular}




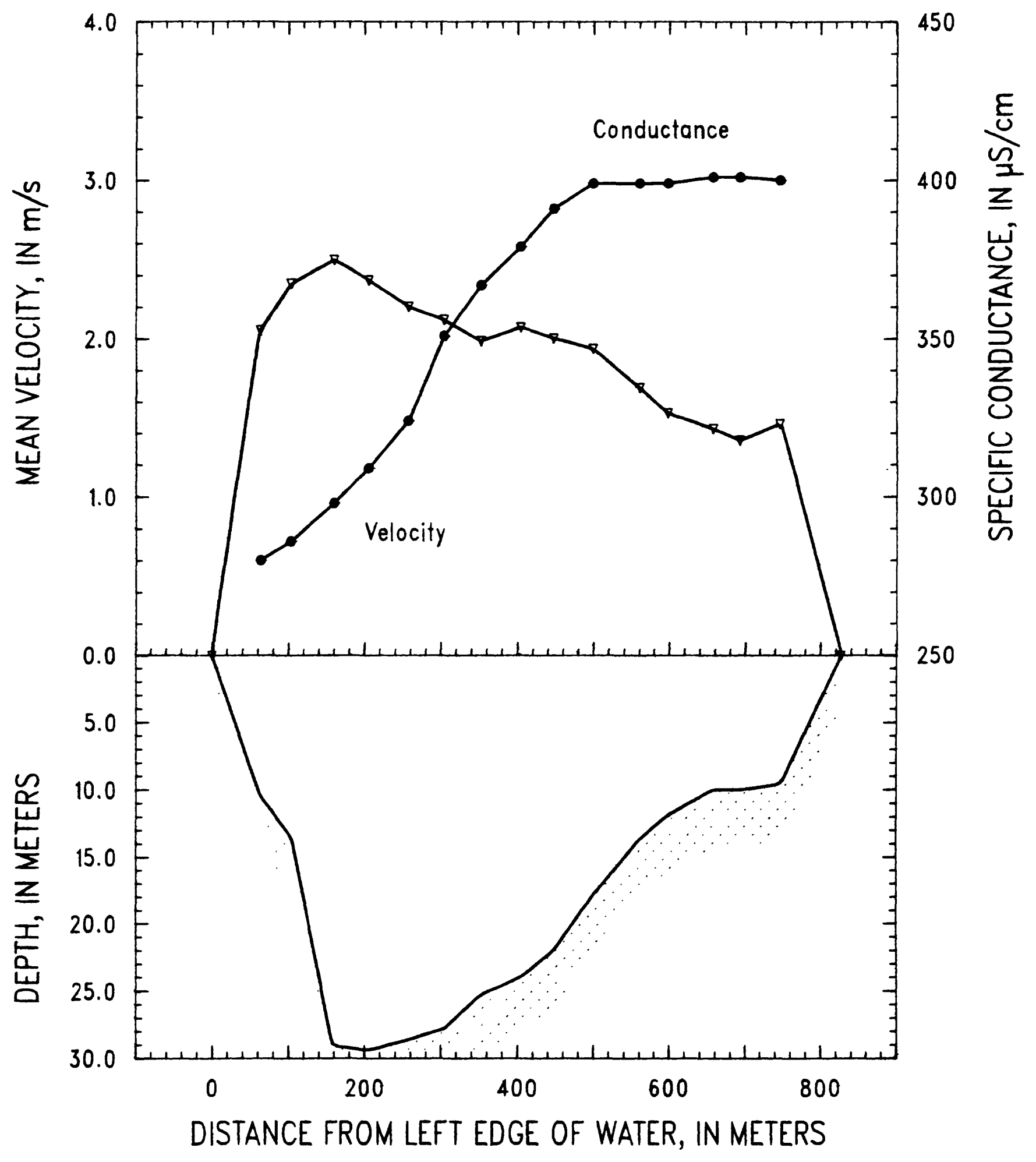

Figure 66. Mississippi River at Wickliffe, Kentucky, on June 4, 1990. 


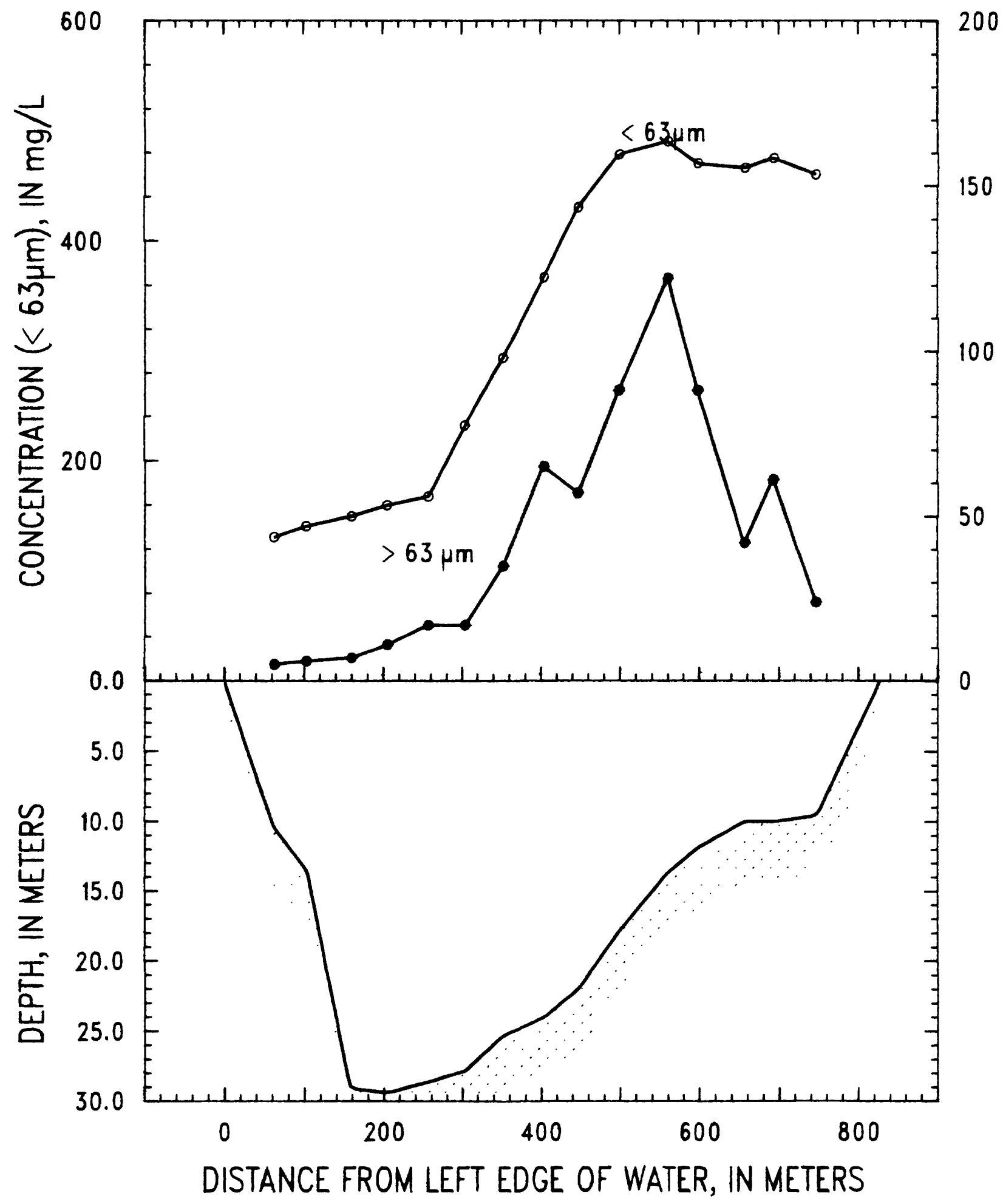

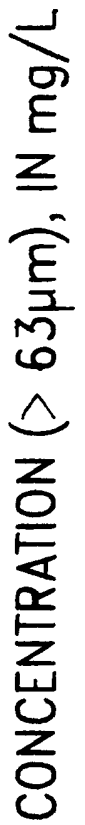

Figure 67. Mississippi River at Wickliffe, Kentucky, on June 4, 1990-suspended-sediment concentration. 
SITE: Mississippi River near Columbus, Kentucky

PARTY: Moody, Delaune, and Simoneaux

06-01-90

STARTING GAGE HEIGHT: $44.4 \mathrm{ft}$ ENDING GAGE HEIGHT: $44.4 \mathrm{ft}$

METER: SOLID CUP

SUSP: Bag sampler and 300-1b weight

CURRENT METER NO.: W-297222 DATE RATED: 04-90

CURRENT METER EQUATION: $\mathrm{V}(\mathrm{m} / \mathrm{s})=$ RPS $* 0.744+0.011$

REMARKS: Individual verticals were collected using the $1 / 8-$ and 3/16-inch nozzles and variable transit rates. Values of temperature, $\mathrm{pH}$, and specific conductance are measurements of the depth-integrated sample and measurements of the surface sample are in parenthese.

\begin{tabular}{|c|c|c|c|c|c|c|c|c|c|c|}
\hline \multirow[t]{2}{*}{$\begin{array}{c}\text { Verti- } \\
\text { cal }\end{array}$} & \multirow[t]{2}{*}{$\begin{array}{l}\text { Mean } \\
\text { dist. } \\
\text { from } \\
\text { LEW } \\
(m)\end{array}$} & \multirow[t]{2}{*}{$\begin{array}{l}\text { Stan- } \\
\text { dard } \\
\text { devia- } \\
\text { tion } \\
\quad(m)\end{array}$} & \multirow[t]{2}{*}{$\begin{array}{l}\text { Mean } \\
\text { depth } \\
\text { (m) }\end{array}$} & \multirow[t]{2}{*}{$\begin{array}{l}\text { Mean } \\
\text { veloc- } \\
\text { ity } \\
(\mathrm{m} / \mathrm{s})\end{array}$} & \multirow[t]{2}{*}{$\begin{array}{c}\text { Dis- } \\
\text { charge } \\
\left(\mathrm{m}^{3} / \mathrm{s}\right)\end{array}$} & \multicolumn{2}{|c|}{$\begin{array}{l}\text { Sus- } \\
\text { pended } \\
\text { sedi- } \\
\text { ment } \\
(\mathrm{mg} / \mathrm{L})\end{array}$} & \multirow[t]{2}{*}{$\begin{array}{l}\text { Temper- } \\
\text { ature } \\
\left({ }^{\circ} \mathrm{C}\right)\end{array}$} & \multirow[t]{2}{*}{$\mathrm{pH}$} & \multirow[t]{2}{*}{$\begin{array}{c}\text { Specific } \\
\text { conduct- } \\
\text { ance } \\
(\mu \mathrm{S} / \mathrm{cm})\end{array}$} \\
\hline & & & & & & $<63 \mu \mathrm{m}$ & $>63 \mu \mathrm{m}$ & & & \\
\hline LEW & 0 & 0 & 0.0 & 0.00 & 0 & -- & -- & -- & -- & -- \\
\hline $01 \mathrm{~A}$ & 69 & 6 & 17.8 & 2.05 & 2,150 & 108 & 10 & 20.0 & 7.7 & 304 \\
\hline 02B & 118 & 4 & 26.5 & 2.24 & 3,000 & 150 & 62 & 20.3 & 7.7 & 305 \\
\hline $03 \mathrm{~A}$ & 170 & 2 & 27.8 & 2.23 & 3,440 & 173 & 74 & 20.1 & 7.7 & 316 \\
\hline $04 \mathrm{~A}$ & 229 & 4 & 26.9 & 2.35 & 3,578 & 261 & 84 & 20.7 & 7.6 & 332 \\
\hline 05B & 283 & 4 & 27.0 & 2.57 & 3,717 & 361 & 126 & 20.2 & 7.6 & 344 \\
\hline $06 \mathrm{~A}$ & 336 & 5 & 24.3 & 2.38 & 3,725 & 442 & 80 & 20.1 & 7.7 & 352 \\
\hline 07B & 412 & 8 & 20.2 & 1.94 & 2,445 & 472 & 62 & 20.0 & 7.7 & 356 \\
\hline $08 \mathrm{~A}$ & 461 & 2 & 16.6 & 1.98 & 1,558 & 477 & 36 & 20.0 & 7.7 & $360(360)$ \\
\hline $09 B$ & 507 & 3 & 13.1 & 1.74 & 1,368 & 508 & 4 & 20.0 & 7.7 & 360 \\
\hline $10 \mathrm{~A}$ & 581 & 4 & 9.1 & 1.65 & 923 & 509 & 19 & 19.9 & 7.7 & 356 \\
\hline $11 \mathrm{~B}$ & 630 & 3 & 8.1 & 1.55 & 620 & 508 & 29 & 19.9 & 7.8 & 362 \\
\hline $12 \mathrm{~A}$ & 680 & 1 & 8.4 & 1.45 & 580 & 495 & 8 & 20.1 & 7.8 & 359 \\
\hline $13 \mathrm{~B}$ & 725 & 4 & 8.7 & 1.42 & 642 & 497 & 16 & 19.8 & 7.7 & 363 \\
\hline $14 \mathrm{~A}$ & 784 & 4 & 8.9 & 1.51 & 845 & 485 & 14 & 20.1 & 7.7 & 358 \\
\hline $15 \mathrm{~B}$ & 851 & 2 & 8.8 & 1.37 & 773 & 478 & 9 & 20.0 & 7.7 & 361 \\
\hline REW & 912 & -- & 0.0 & 0.00 & 0 & -- & -- & -- & -- & -- \\
\hline MEAN & & & 15.6 & 2.06 & & & & & & \\
\hline TOTAL & 912 & & & & 29,364 & & & & & \\
\hline
\end{tabular}




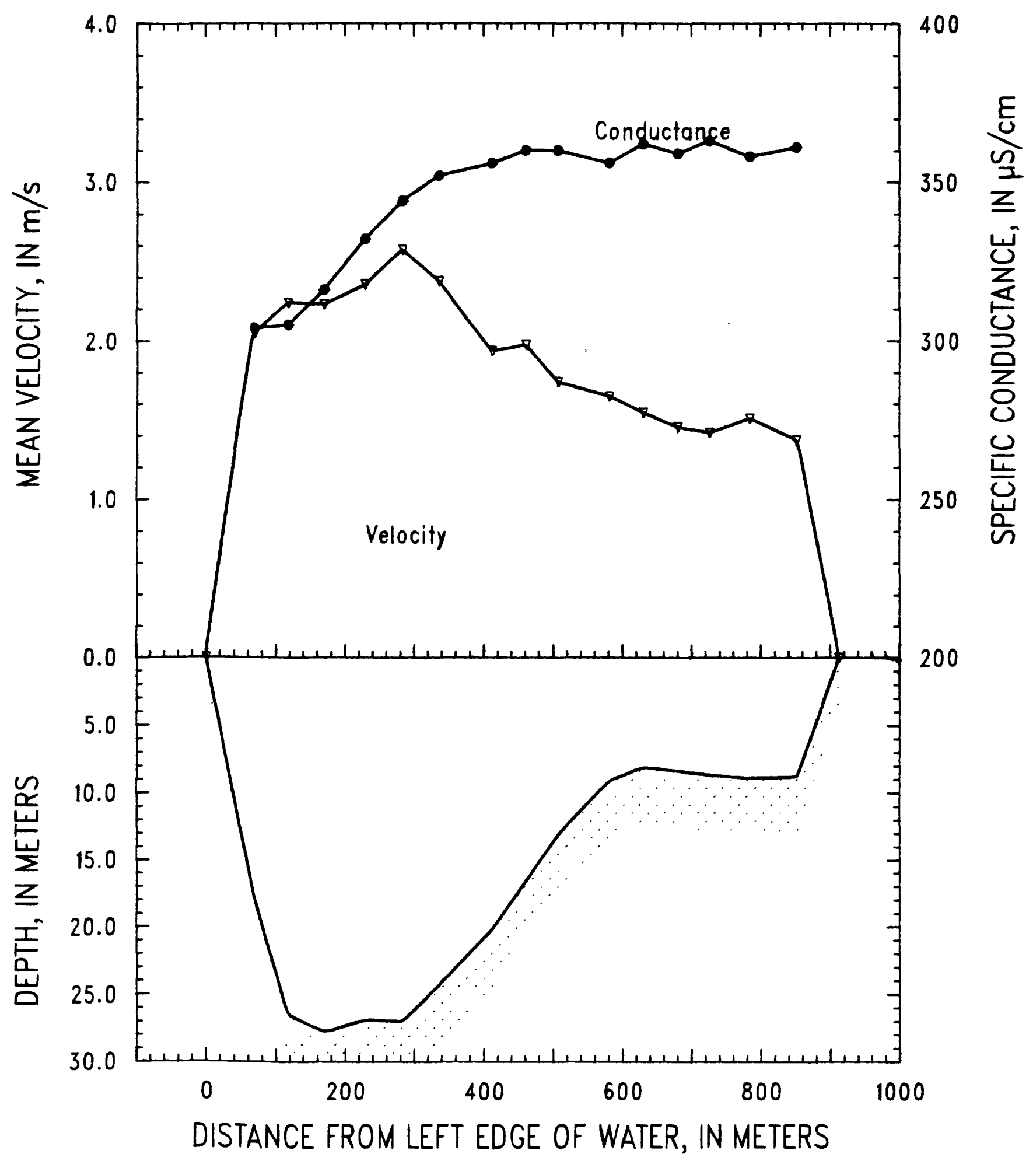

Figure 68. Mississippi River near Columbus, Kentucky, on June 1, 1990. 


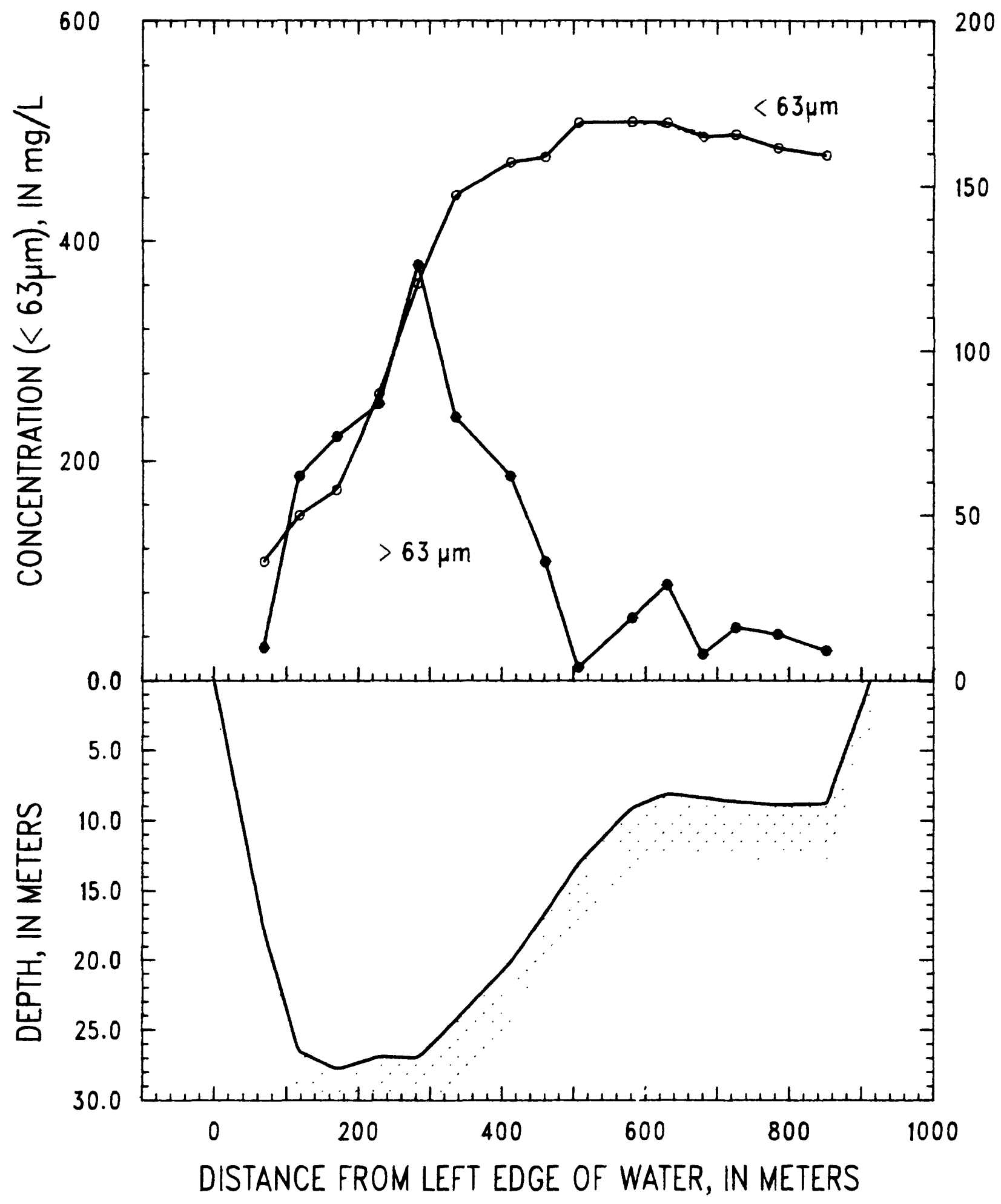

200

100

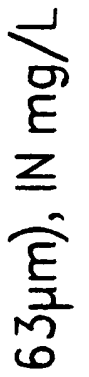

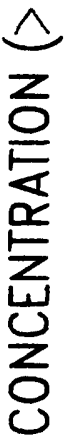

Figure 69. Mississippi River near Columbus, Kentucky, on June 1, 1990-suspended-sediment concentration. 
SITE: Mississippi River above New Madrid, Missouri

PARTY: Moody, Delaune, and Simoneaux

STARTING GAGE HEIGHT: -- ENDING GAGE HEIGHT: --

SUSP: Bag sampler and 300-1b weight

CURRENT METER NO.: W-297222 DATE RATED: 04-90

CURRENT METER EQUATION: $\mathrm{V}(\mathrm{m} / \mathrm{s})=$ RPS $* 0.744+0.011$

REMARKS: Ten-vertical section using $1 / 8-$ and $3 / 16-$ inch nozzles and variable transit rates. Strong (20-30 knots) southwest wind. Values of temperature, $\mathrm{pH}$, and specific conductance are measurements of the depth-integrated sample and measurements of the surface sample are in parentheses.

\begin{tabular}{|c|c|c|c|c|c|c|c|c|c|c|}
\hline \multirow[t]{2}{*}{$\begin{array}{c}\text { Verti- } \\
\text { cal }\end{array}$} & \multirow[t]{2}{*}{$\begin{array}{l}\text { Mean } \\
\text { dist. } \\
\text { from } \\
\text { LEW } \\
(\mathrm{m})\end{array}$} & \multirow[t]{2}{*}{$\begin{array}{l}\text { Stan- } \\
\text { dard } \\
\text { devia- } \\
\text { tion } \\
\text { (m) }\end{array}$} & \multirow[t]{2}{*}{$\begin{array}{l}\text { Mean } \\
\text { depth } \\
\text { (m) }\end{array}$} & \multirow[t]{2}{*}{$\begin{array}{l}\text { Mean } \\
\text { veloc- } \\
\text { ity } \\
(\mathrm{m} / \mathrm{s})\end{array}$} & \multirow[t]{2}{*}{$\begin{array}{l}\text { Dis- } \\
\text { charge } \\
\left(\mathrm{m}^{3} / \mathrm{s}\right)\end{array}$} & \multicolumn{2}{|c|}{$\begin{array}{c}\text { Sus- } \\
\text { pended } \\
\text { sedi- } \\
\text { ment } \\
(\mathrm{mg} / \mathrm{L})\end{array}$} & \multirow[t]{2}{*}{$\begin{array}{l}\text { Temper- } \\
\text { ature } \\
\left({ }^{\circ} \mathrm{C}\right)\end{array}$} & \multirow[t]{2}{*}{$\mathrm{pH}$} & \multirow[t]{2}{*}{$\begin{array}{c}\text { Specific } \\
\text { conduct- } \\
\text { ance } \\
(\mu \mathrm{S} / \mathrm{cm})\end{array}$} \\
\hline & & & & & & $<63 \mu \mathrm{m}$ & $>63 \mu \mathrm{m}$ & & & \\
\hline LEW & 0 & -- & 0.0 & 0.00 & 0 & -- & -- & -- & -- & $-\infty$ \\
\hline $01 \mathrm{~A}$ & 120 & 10 & 30.4 & 1.25 & 4,315 & 207 & 35 & 21.0 & 7.6 & $326(325)$ \\
\hline O2B & 228 & 12 & 25.1 & 1.38 & 3,569 & 251 & 22 & 20.7 & 7.8 & 332 \\
\hline 03A & 326 & 5 & 24.2 & 1.74 & 3,015 & 271 & 29 & 20.6 & 7.7 & 336 \\
\hline 04B & 371 & 10 & 21.6 & 1.77 & 1,915 & 281 & 62 & 20.7 & 7.9 & 337 (341) \\
\hline $05 \mathrm{~A}$ & 426 & 18 & 19.0 & 1.80 & 2,821 & 290 & 84 & 20.7 & 7.9 & 342 \\
\hline 06B & 536 & 7 & 14.5 & 1.91 & 2,964 & 320 & 64 & 20.5 & 7.8 & $347 \quad(344)$ \\
\hline $07 \mathrm{~A}$ & 640 & 7 & 14.9 & 1.98 & 3,132 & 325 & 74 & 20.6 & 7.7 & $348(346)$ \\
\hline 08B & 748 & 6 & 16.5 & 2.00 & 3,467 & 330 & 58 & 20.5 & 7.7 & 351 (351) \\
\hline 09A & 850 & 3 & 11.7 & 2.01 & 2,479 & 343 & 70 & 20.6 & 7.8 & $351(351)$ \\
\hline $10 \mathrm{~B}$ & 959 & 1 & 11.1 & 1.91 & 2,221 & 355 & 74 & 20.8 & 7.7 & $354(353)$ \\
\hline REW & 1,060 & -- & 0.0 & 0.00 & 0 & -- & -- & -- & -- & -- \\
\hline MEAN & & & 16.7 & 1.69 & & & & & & \\
\hline TOTAL & 1,060 & & & & 29,898 & & & & & \\
\hline
\end{tabular}




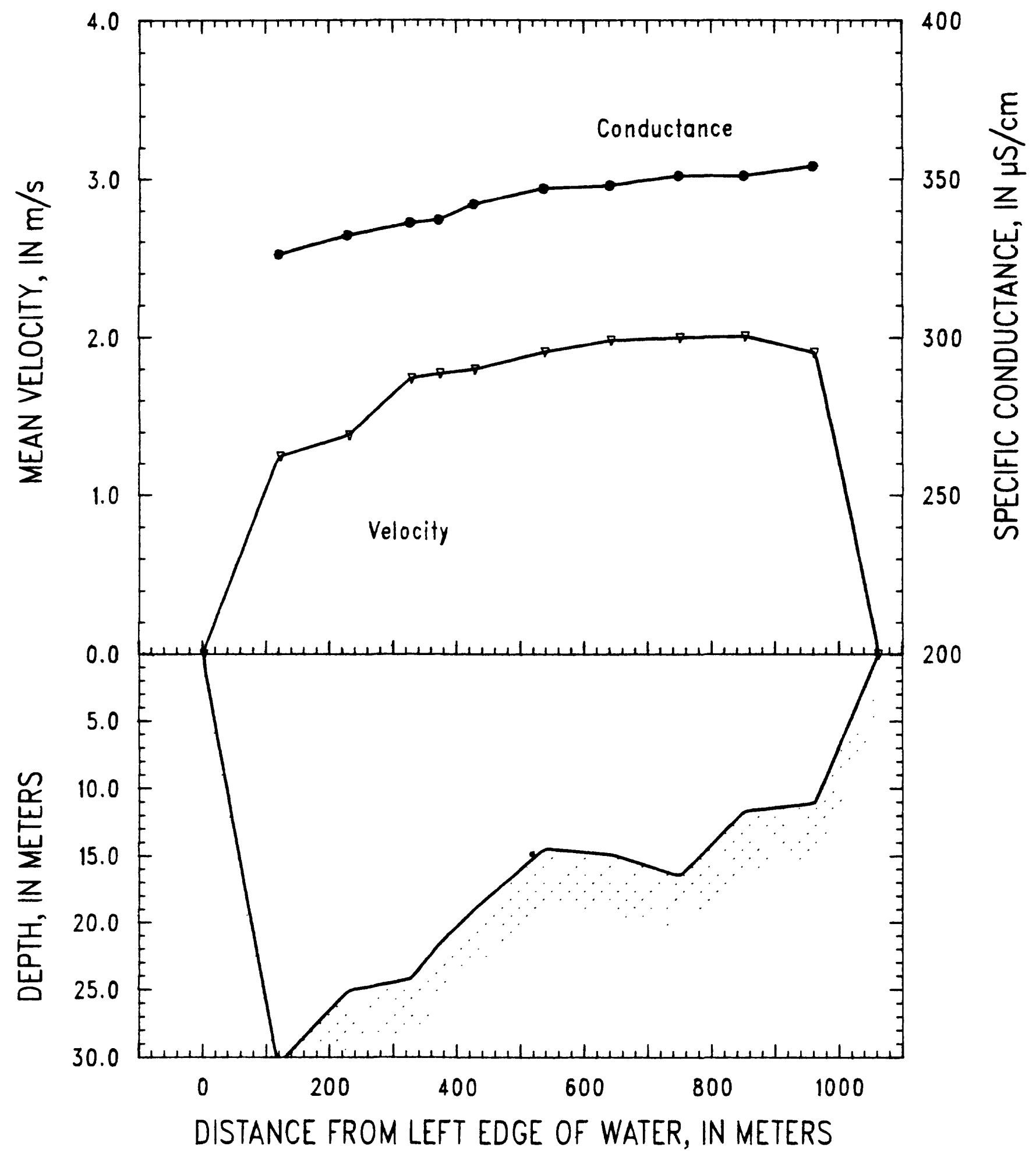

Figure 70. Mississippi River above New Madrid, Missouri, on June 2, 1990. 


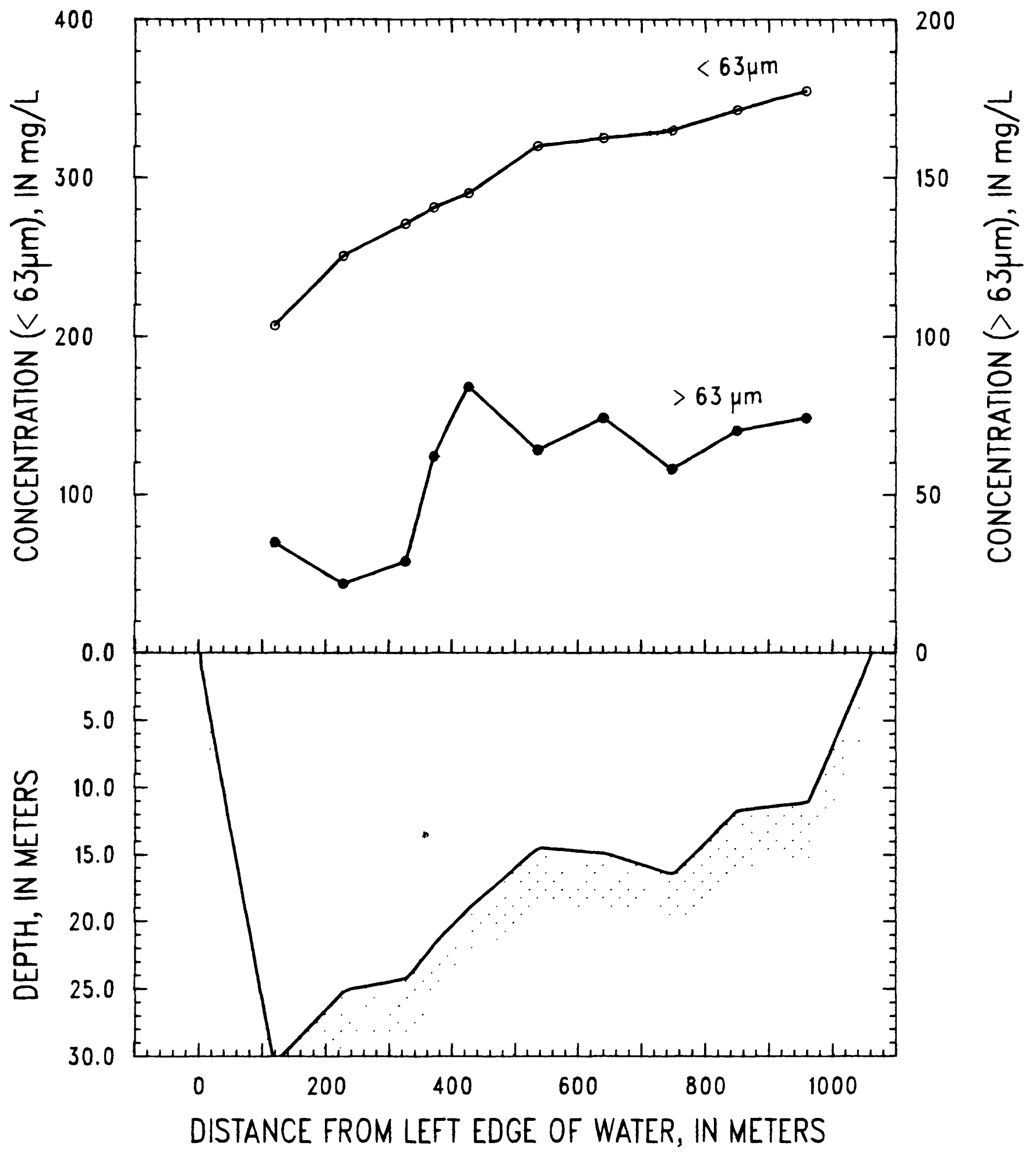

Figure 71. Mississippi River above New Madrid, Missouri, on June 2, 1990--suspended-sediment concentration. 
SITE: Mississippi River near Point Pleasant, Missouri 06-02-90

PARTY: Moody, Delaune, and Simoneaux

METER: SOLID CUP

STARTING GAGE HEIGHT: -- ENDING GAGE HEIGHT: --

SUSP: Bag sampler and $300-1 \mathrm{~b}$ weight

CURRENT METER NO.: W-297222 DATE RATED: 04-90

CURRENT METER EQUATION: $V(\mathrm{~m} / \mathrm{s})=$ RPS $* 0.744+0.011$

REMARKS: Ten verticals using $1 / 8-$ and 3/16-inch nozzles and variable transit rate. Strong southwest wind dropped off to 5-10 knots. Values of temperature, $\mathrm{pH}$, and specific conductance are measurements of the depth-integrated sample and measurements of the surface sample are in parentheses.

\begin{tabular}{|c|c|c|c|c|c|c|c|c|c|c|}
\hline \multirow[t]{2}{*}{$\begin{array}{c}\text { Verti- } \\
\text { cal }\end{array}$} & \multirow[t]{2}{*}{$\begin{array}{l}\text { Mean } \\
\text { dist. } \\
\text { from } \\
\text { LEW } \\
\text { (m) }\end{array}$} & \multirow[t]{2}{*}{$\begin{array}{l}\text { Stan- } \\
\text { dard } \\
\text { devia- } \\
\text { tion } \\
\text { (m) }\end{array}$} & \multirow[t]{2}{*}{$\begin{array}{l}\text { Mean } \\
\text { depth } \\
(\mathrm{m})\end{array}$} & \multirow[t]{2}{*}{$\begin{array}{l}\text { Mean } \\
\text { veloc- } \\
\text { ity } \\
(\mathrm{m} / \mathrm{s})\end{array}$} & \multirow[t]{2}{*}{$\begin{array}{l}\text { Dis- } \\
\text { charge } \\
\left(m^{3} / s\right)\end{array}$} & \multicolumn{2}{|c|}{$\begin{array}{c}\text { Sus- } \\
\text { pended } \\
\text { sedi- } \\
\text { ment } \\
(\mathrm{mg} / \mathrm{L}) \\
\end{array}$} & \multirow[t]{2}{*}{$\begin{array}{l}\text { Temper- } \\
\text { ature } \\
\left({ }^{\circ} \mathrm{C}\right)\end{array}$} & \multirow[t]{2}{*}{$\mathrm{pH}$} & \multirow[t]{2}{*}{$\begin{array}{c}\text { Specific } \\
\text { conduct- } \\
\text { ance } \\
(\mu \mathrm{S} / \mathrm{cm})\end{array}$} \\
\hline & & & & & & $<63 \mu \mathrm{m}$ & $>63 \mu \mathrm{m}$ & & & \\
\hline LEW & 0 & -- & 0.0 & 0.00 & 0 & -- & -- & -- & -- & -- \\
\hline $01 B$ & 149 & 4 & 22.8 & 1.93 & 4,356 & 239 & 48 & 20.9 & 7.8 & 332 \\
\hline $02 \mathrm{~A}$ & 198 & 4 & 19.7 & 1.92 & 2,496 & 257 & 131 & 20.7 & 7.8 & 334 \\
\hline $03 \mathrm{~A}$ & 281 & 4 & 15.4 & 1.79 & 2,926 & 278 & -- & 20.9 & 7.7 & 336 \\
\hline 04B & 410 & 5 & 13.0 & 1.79 & 3,207 & 296 & 133 & 20.7 & 7.7 & 341 \\
\hline 05A & 556 & 10 & 12.5 & 1.58 & 2,750 & 303 & 42 & 21.0 & 7.9 & 342 \\
\hline 06B & 689 & 6 & 12.4 & 1.57 & 2,574 & 302 & 31 & 20.7 & 7.8 & $344(345)$ \\
\hline $07 \mathrm{~A}$ & 821 & 9 & 13.7 & 1.43 & 2,527 & 309 & 25 & 20.7 & 7.7 & 346 \\
\hline 08B & 947 & 7 & 14.4 & 1.35 & 2,524 & 309 & 20 & 20.5 & 7.8 & 347 \\
\hline 09A & 1,081 & 4 & 15.1 & 1.41 & 2,815 & 318 & 15 & 20.6 & 7.7 & 349 \\
\hline $10 \mathrm{~B}$ & 1,211 & 3 & 14.2 & 1.48 & 2,823 & 317 & 16 & 20.6 & 7.7 & 347 \\
\hline REW & 1,350 & -- & 0.0 & 0.00 & 0 & -- & -- & -- & -- & - \\
\hline MEAN & & & 13.3 & 1.62 & & & & & & \\
\hline TOTAL & 1,1350 & & & & 28,998 & & & & & \\
\hline
\end{tabular}




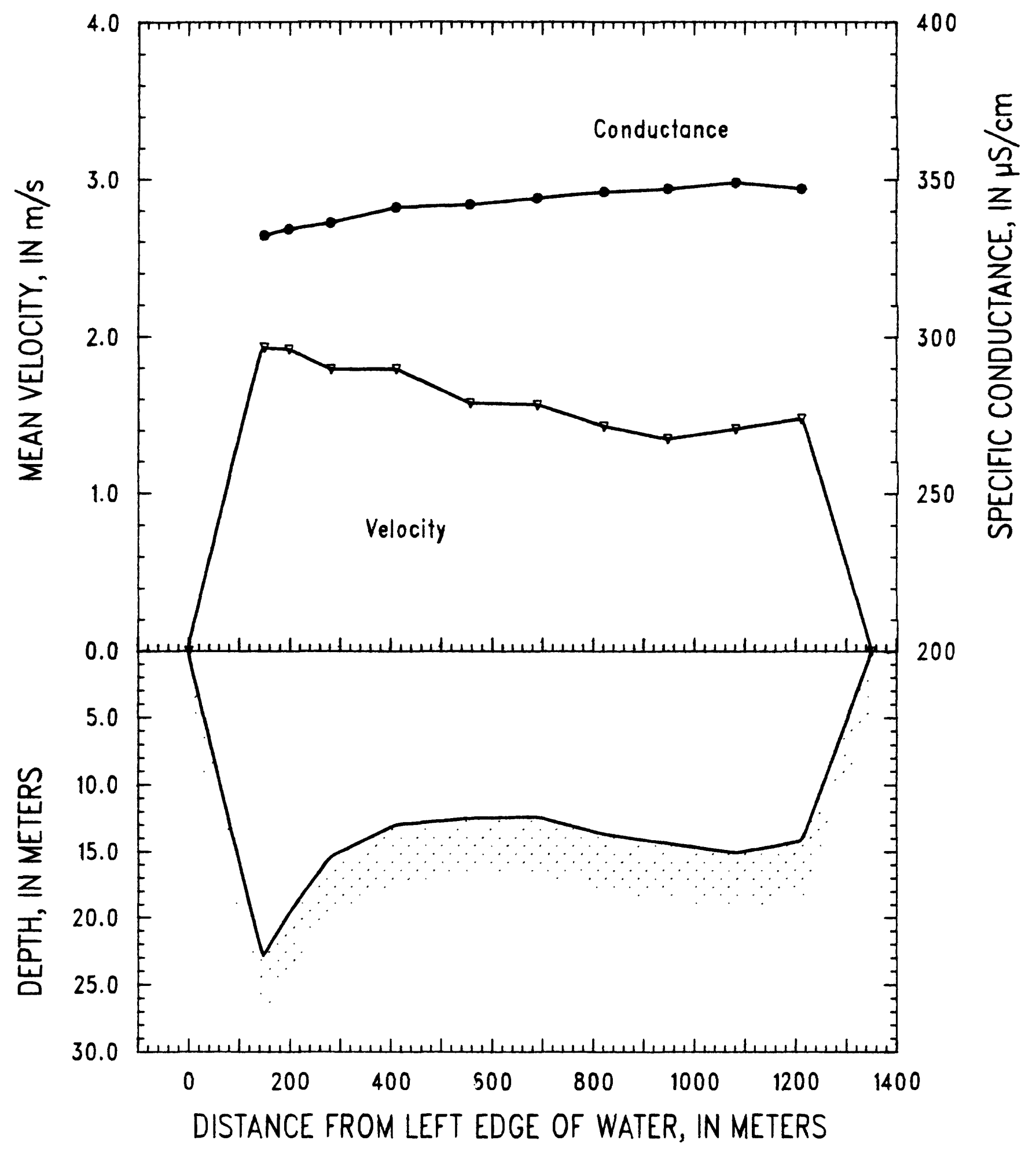

Figure 72. Mississippi River near Point Pleasant, Missouri, on June 2, 1990. 


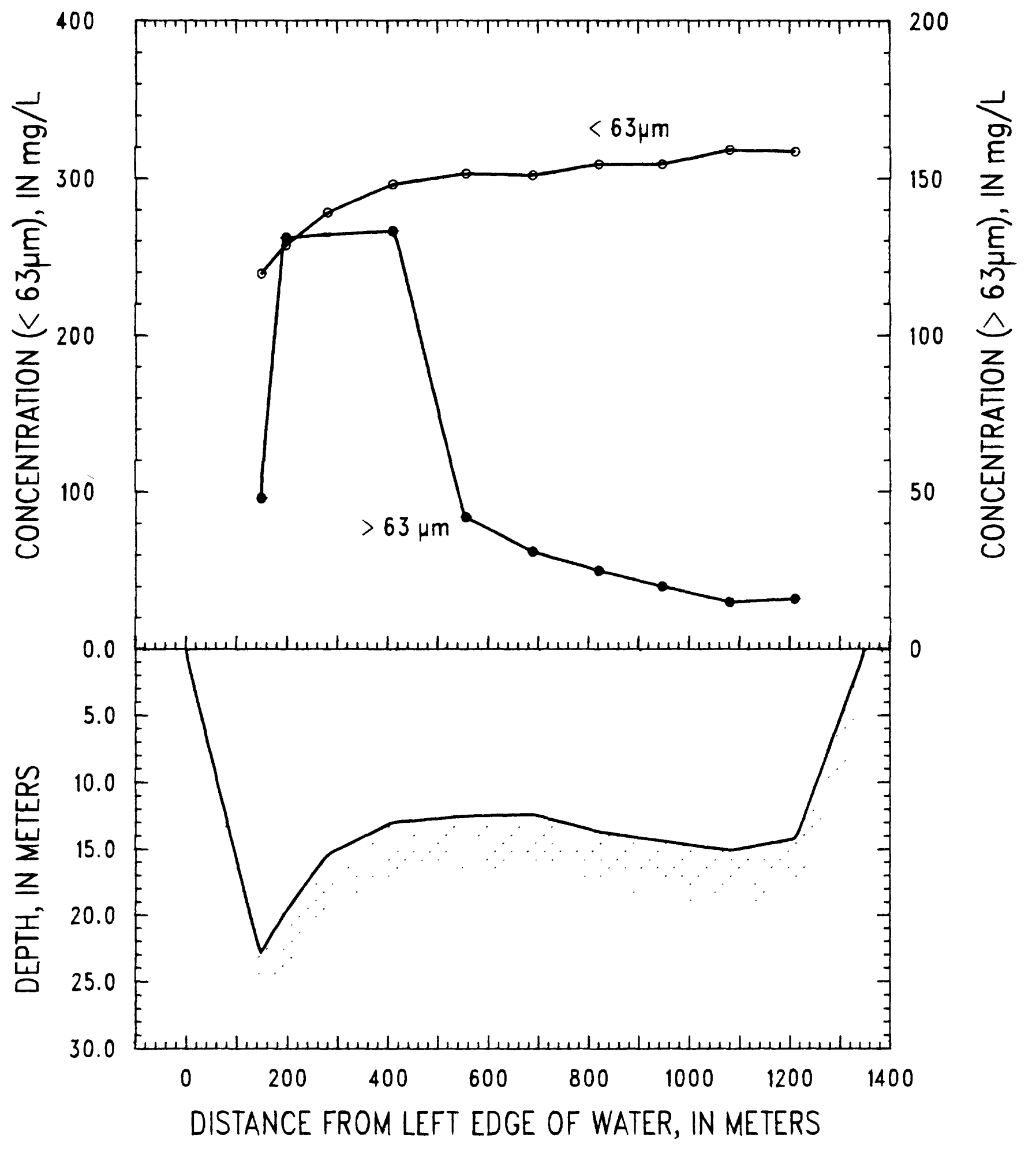

Figure 73. Mississippi River near Point Pleasant, Missouri, on June 2,1990--suspended-sediment concentration. 
SITE: Mississippi River at Caruthersville, Missouri

METER: $\begin{array}{r}06-03-90 \\ \text { SOLID CUP }\end{array}$

PARTY: Moody, Delaune, LeBoeuf, and Simoneaux

STARTING GAGE HEIGHT: --

ENDING GAGE HEIGHT: --

SUSP: Bag sampler and $300-1 \mathrm{~b}$ weight

CURRENT METER NO.: W-297222 DATE RATED: 04-90

CURRENT METER EQUATION: $\mathrm{V}(\mathrm{m} / \mathrm{s})=$ RPS $* 0.744+0.011$

REMARKS: Nine verticals collected using the 1/8- and 3/16-inch nozzles and a variable transit rate. Values of temperature, $\mathrm{pH}$, and specific conductance are measurements of the depth-integrated sample and measurements of the surface sample are in parentheses.

\begin{tabular}{|c|c|c|c|c|c|c|c|c|c|c|}
\hline \multirow[t]{2}{*}{$\begin{array}{c}\text { Verti- } \\
\text { cal }\end{array}$} & \multirow[t]{2}{*}{$\begin{array}{l}\text { Mean } \\
\text { dist. } \\
\text { from } \\
\text { LEW } \\
\text { (m) }\end{array}$} & \multirow[t]{2}{*}{$\begin{array}{l}\text { Stan- } \\
\text { dard } \\
\text { devia- } \\
\text { tion } \\
\text { (m) }\end{array}$} & \multirow[t]{2}{*}{$\begin{array}{l}\text { Mean } \\
\text { depth } \\
\quad(m)\end{array}$} & \multirow[t]{2}{*}{$\begin{array}{l}\text { Mean } \\
\text { veloc- } \\
\text { ity } \\
(\mathrm{m} / \mathrm{s})\end{array}$} & \multirow[t]{2}{*}{$\begin{array}{l}\text { Dis- } \\
\text { charge } \\
\left(\mathrm{m}^{3} / \mathrm{s}\right)\end{array}$} & \multicolumn{2}{|c|}{$\begin{array}{l}\text { Sus- } \\
\text { pended } \\
\text { sedi- } \\
\text { ment } \\
(\mathrm{mg} / \mathrm{L})\end{array}$} & \multirow[t]{2}{*}{$\begin{array}{l}\text { Temper- } \\
\text { ature } \\
\left({ }^{\circ} \mathrm{C}\right)\end{array}$} & \multirow[t]{2}{*}{$\mathrm{pH}$} & \multirow[t]{2}{*}{$\begin{array}{l}\text { Specific } \\
\text { conduct- } \\
\text { ance } \\
(\mu S / \mathrm{cm})\end{array}$} \\
\hline & & & & & & $<63 \mu \mathrm{m}$ & $>63 \mu \mathrm{m}$ & & & \\
\hline LEW & 0 & -- & 1.0 & 0.00 & 0 & -- & -- & -- & -- & - \\
\hline $01 \mathrm{~B}$ & 95 & 4 & 8.9 & 1.51 & 1,202 & 238 & 65 & 20.0 & 7.8 & 336 \\
\hline $02 \mathrm{~A}$ & 179 & 2 & 9.4 & 1.76 & 1,343 & 242 & 56 & 20.0 & 7.7 & 337 \\
\hline 03B & 257 & 1 & 11.4 & 1.86 & 1,751 & 243 & 108 & 19.8 & 7.8 & 338 \\
\hline $04 \mathrm{~A}$ & 344 & 3 & 14.2 & 2.04 & 2,537 & 246 & 100 & 19.8 & 7.8 & 339 \\
\hline 05B & 432 & 3 & 18.4 & 2.22 & 3,554 & 255 & 128 & 19.8 & 7.8 & 340 \\
\hline 06B & 518 & 2 & 22.2 & 2.46 & 4,280 & 263 & 88 & 20.1 & 7.8 & 343 \\
\hline $07 \mathrm{~A}$ & 589 & 5 & 24.3 & 2.53 & 4,219 & 268 & 39 & 20.1 & 7.8 & 342 \\
\hline 08B & 655 & 9 & 24.7 & 2.63 & 4,847 & 271 & 27 & 20.3 & 7.7 & $342(345)$ \\
\hline $09 \mathrm{~A}$ & 738 & 19 & 25.0 & 2.18 & 5,294 & 242 & 9 & 20.7 & 7.8 & $343(345)$ \\
\hline REW & 849 & -- & 0.0 & 0.00 & 0 & -- & -- & -- & -- & -- \\
\hline MEAN & & & 15.3 & 2.23 & & & & & & \\
\hline TOTAL & 849 & & & & 29,027 & & & & & \\
\hline
\end{tabular}




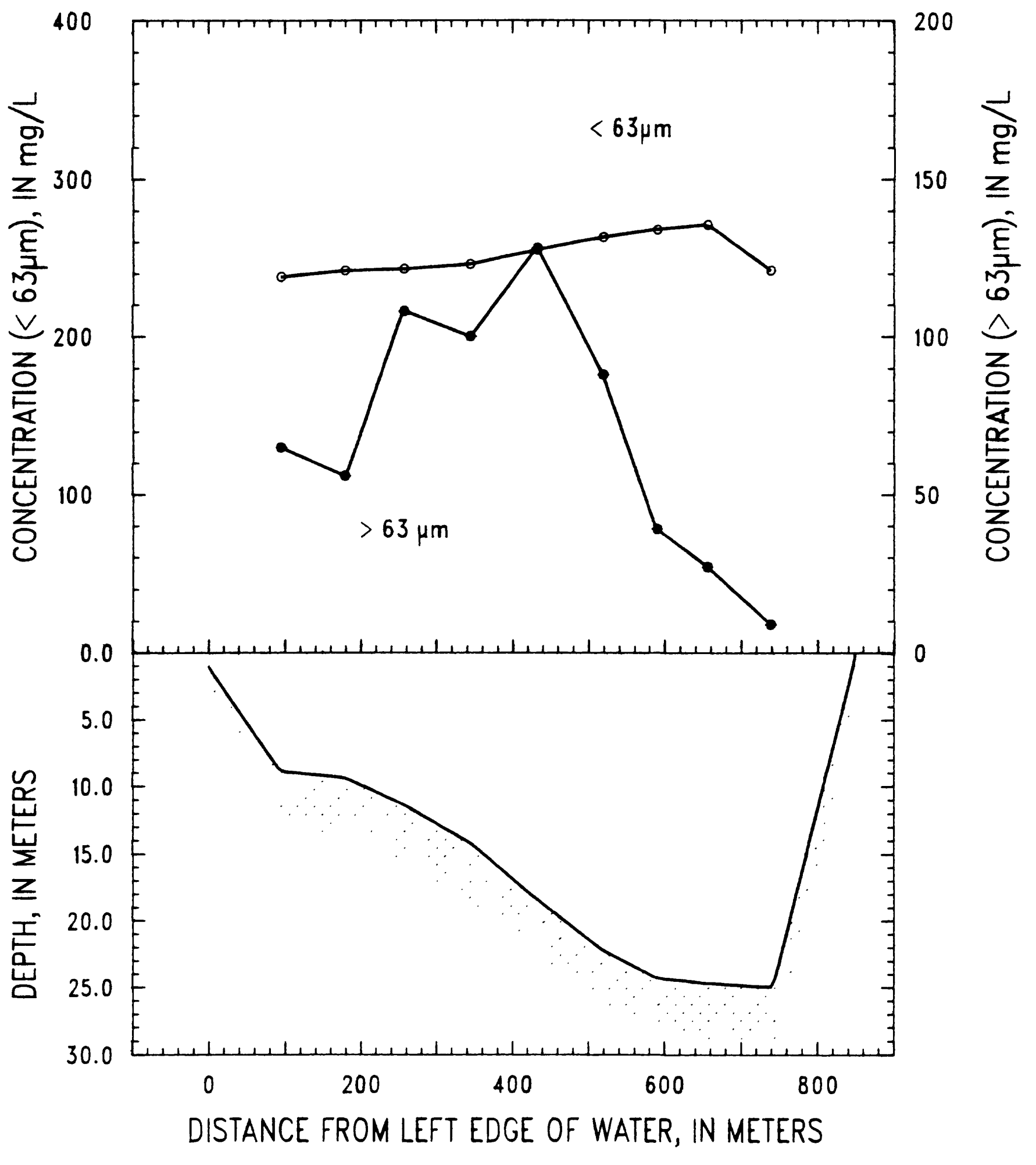

Figure 75. Mississippi River at Caruthersville, Missouri, on June 3, 1990--suspended-sediment concentration. 


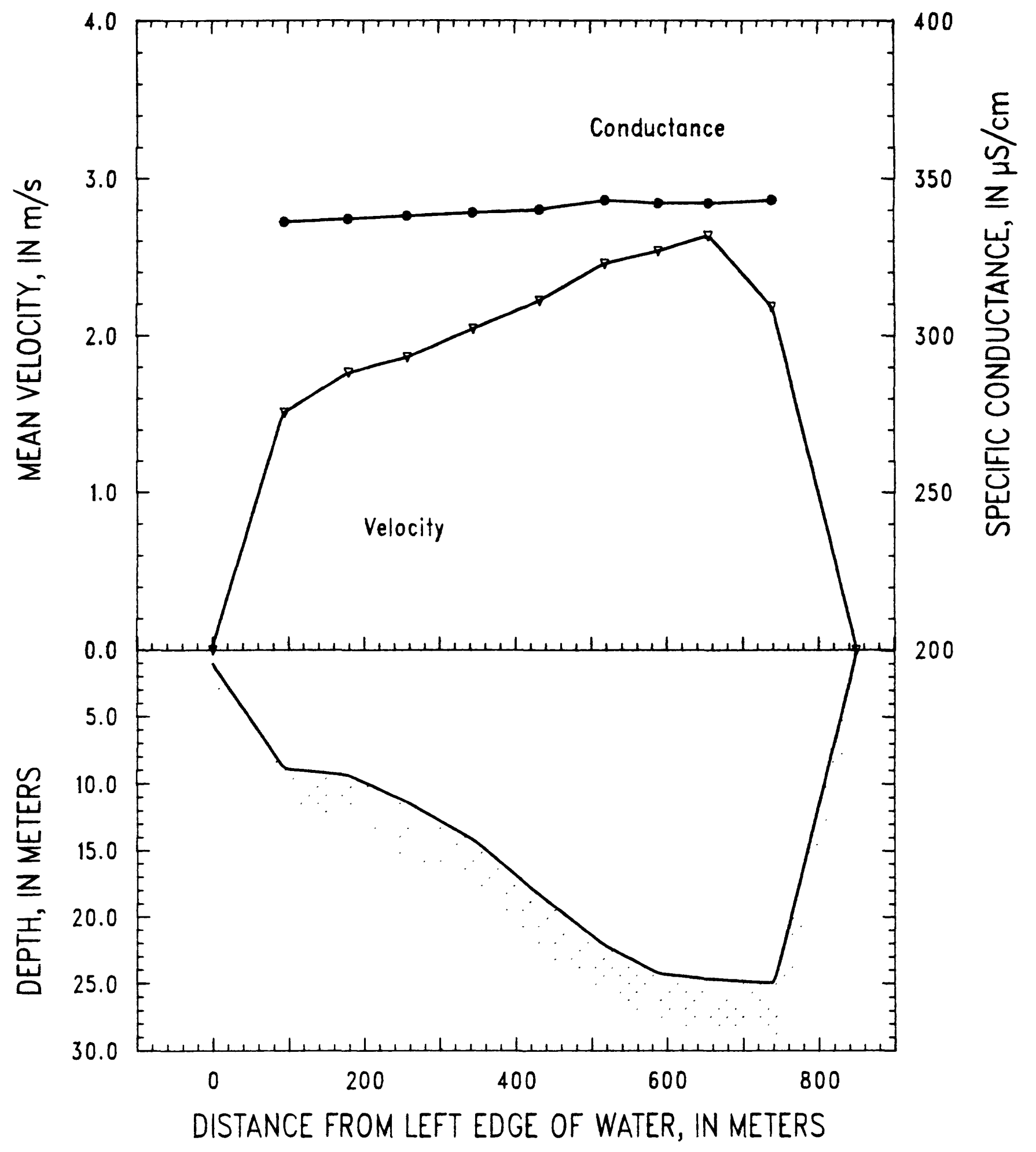

Figure 74. Mississippi River at Caruthersville, Missouri, on June 3, 1990. 
SITE: Illinois River at Valley City, Illinois

PARTY: Moody, Delaune, LeBoeuf, and Simoneaux METER: SOLID CUP

STARTING GAGE HEIGHT: $13.03 \mathrm{ft}$ ENDING GAGE HEIGHT: --

SUSP: Bag sampler and 150-1b weight

CURRENT METER NO.: W-297222 DATE RATED: 04-90

CURRENT METER EQUATION: $V(\mathrm{~m} / \mathrm{s})=$ RPS $* 0.744+0.011$

REMARKS: USGS Illinois District collected simultaneous NASQAN sample. Dense fog in the morning and heavy thunderstorm in the afternoon. Five test velocities (free boat); anchored at five verticals to collect water. Various transit rates $(4-19 \mathrm{~cm} / \mathrm{s})$ and various nozzle sizes $(1 / 4 \mathrm{inch}$ and $5 / 16$ inch) were used.

\begin{tabular}{|c|c|c|c|c|c|c|c|c|c|}
\hline \multirow[b]{2}{*}{$\begin{array}{c}\text { Verti- } \\
\text { cal }\end{array}$} & \multirow{2}{*}{$\begin{array}{l}\text { Dist. } \\
\text { from } \\
\text { LEW } \\
\text { (m) }\end{array}$} & \multirow[b]{2}{*}{$\begin{array}{l}\text { Depth } \\
\text { (m) }\end{array}$} & \multirow{2}{*}{$\begin{array}{l}\text { Mean } \\
\text { veloc- } \\
\text { ity } \\
(\mathrm{m} / \mathrm{s})\end{array}$} & \multirow[b]{2}{*}{$\begin{array}{r}\text { Dis- } \\
\text { charge } \\
\left(\mathrm{m}^{3} / \mathrm{s}\right)\end{array}$} & \multicolumn{2}{|c|}{ Volume } & \multirow[b]{2}{*}{$\begin{array}{l}\text { Temper- } \\
\text { ature } \\
\left({ }^{\circ} \mathrm{C}\right)\end{array}$} & \multirow[b]{2}{*}{$\mathrm{pH}$} & \multirow{2}{*}{$\begin{array}{c}\text { Specific } \\
\text { conduct- } \\
\text { ance } \\
(\mu \mathrm{S} / \mathrm{cm})\end{array}$} \\
\hline & & & & & $\begin{array}{l}\overline{V_{i}} \\
(L)\end{array}$ & $\begin{array}{l}V_{p} \\
(\mathrm{~L})\end{array}$ & & & \\
\hline LEW & 0 & 0.0 & 0.00 & 0 & -- & -- & - & $\ldots$ & -- \\
\hline $\mathrm{X} 05$ & 40 & 5.0 & 0.84 & 122 & -- & -- & -- & -- & -- \\
\hline $1 \mathrm{AB}$ & 58 & 7.1 & 0.93 & 123 & 16.22 & 100 & 9.4 & 7.9 & 691 \\
\hline X04 & 77 & 7.4 & 0.80 & 103 & -- & -- & -- & -- & - \\
\hline $2 \mathrm{AB}$ & 93 & 8.0 & 0.99 & 127 & 15.87 & 100 & 9.6 & 8.2 & 735 \\
\hline X03 & 109 & 8.7 & 1.09 & 105 & -- & -- & - & - & - \\
\hline $3 \mathrm{AB}$ & 115 & 8.8 & 1.04 & 196 & 15.33 & 100 & 9.5 & 8.2 & 737 \\
\hline $4 \mathrm{AB}$ & 152 & 8.8 & 1.00 & 246 & 15.42 & 100 & 9.7 & 8.2 & 736 \\
\hline 05B & 171 & 7.2 & 0.73 & 58 & 7.42 & -- & - & -- & -- \\
\hline $05 \mathrm{~A}$ & 174 & 7.0 & 0.79 & 53 & 8.04 & 100 & 9.7 & 8.2 & 735 \\
\hline $\mathrm{X} 01$ & 190 & 5.4 & 0.76 & 63 & -- & -- & -- & -- & -- \\
\hline X06 & 205 & 4.8 & 0.41 & 31 & -- & - & -- & -- & - \\
\hline REW & 221 & 1.0 & & & & & & & \\
\hline MEAN & & 6.2 & 0.90 & & & & & & \\
\hline TOTAL & 221 & & & 1,227 & 78.30 & 500 & & & \\
\hline
\end{tabular}




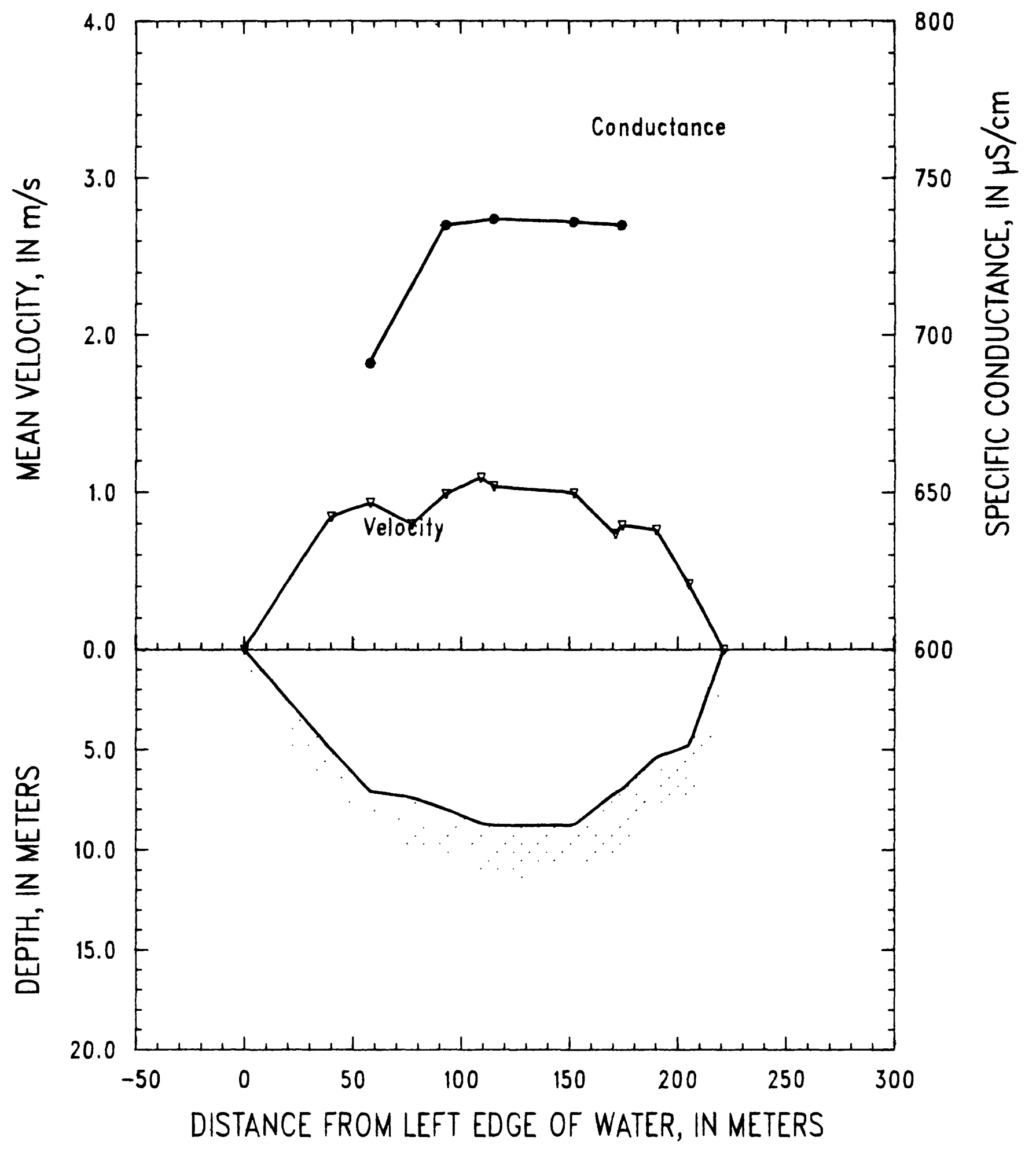

Figure 76. Illinois River at Valley City, Illinois, on June 7, 1990. 
SITE: Mississippi River below Grafton, Illinois

PARTY: Moody, Garbarino, and Simoneaux

06-11-90

STARTING GAGE HEIGHT: $16.8 \mathrm{ft}$

ENDING GAGE HEIGHT: $16.1 \mathrm{ft}$

SUSP: Bag sampler and $300-1 \mathrm{~b}$ weight

CURRENT METER NO.: W-297222 DATE RATED: 04-90

CURRENT METER EQUATION: $V(\mathrm{~m} / \mathrm{s})=$ RPS $* 0.744+0.011$

REMARKS: Bêautiful day and wonderful scenery. Transit rate was $8 \mathrm{~cm} / \mathrm{s}$ and the nozzle was $1 / 4$ inch. Open river. USGS Missouri District collected a simultaneous NASQAN sample.

\begin{tabular}{|c|c|c|c|c|c|c|c|c|c|}
\hline \multirow[b]{2}{*}{$\begin{array}{l}\text { Verti- } \\
\text { cal }\end{array}$} & \multirow{2}{*}{$\begin{array}{l}\text { Dist. } \\
\text { from } \\
\text { LEW } \\
\text { (m) }\end{array}$} & \multirow[b]{2}{*}{$\begin{array}{l}\text { Depth } \\
\text { (m) }\end{array}$} & \multirow{2}{*}{$\begin{array}{l}\text { Mean } \\
\text { veloc- } \\
\text { ity } \\
(\mathrm{m} / \mathrm{s})\end{array}$} & \multirow[b]{2}{*}{$\begin{array}{r}\text { Dis- } \\
\text { charge } \\
\left(\mathrm{m}^{3} / \mathrm{s}\right)\end{array}$} & \multicolumn{2}{|c|}{ Volume } & \multirow[b]{2}{*}{$\begin{array}{l}\text { Temper- } \\
\text { ature } \\
\left({ }^{\circ} \mathrm{C}\right)\end{array}$} & \multirow[b]{2}{*}{$\mathrm{pH}$} & \multirow{2}{*}{$\begin{array}{c}\text { Specific } \\
\text { conduct- } \\
\text { ance } \\
(\mu \mathrm{S} / \mathrm{cm})\end{array}$} \\
\hline & & & & & $\begin{array}{l}V_{i} \\
(L)\end{array}$ & $\begin{array}{l}\mathrm{V}_{\mathbf{p}} \\
(\mathrm{L})\end{array}$ & & & \\
\hline LEW & 0 & 0.0 & 0.00 & 0 & - & -- & -- & -- & -- \\
\hline $01 \mathrm{~A}$ & 53 & 8.1 & 1.05 & 376 & 4.71 & 30 & 22.0 & 8.0 & 737 \\
\hline $\mathrm{X} 01$ & 88 & 9.2 & 1.02 & 206 & - & - & - & - & -- \\
\hline 02B & 97 & 9.2 & 1.02 & 240 & 5.51 & 42 & 22.0 & 7.9 & 617 \\
\hline $03 \mathrm{~A}$ & 139 & 8.7 & 0.96 & 383 & 5.01 & 45 & 21.9 & 7.8 & 566 \\
\hline $04 \mathrm{~B}$ & 189 & 7.7 & 0.96 & 323 & 4.62 & 39 & 22.0 & 7.8 & 497 \\
\hline $\mathrm{X} 02$ & 226 & 7.3 & 1.02 & 171 & -- & -- & - & - & - \\
\hline 05A & 235 & 7.3 & 0.95 & 180 & 4.36 & 37 & 22.0 & 7.7 & 466 \\
\hline 06B & 278 & 7.4 & 0.92 & 304 & 3.77 & 33 & 21.9 & 7.8 & 416 \\
\hline $07 \mathrm{~A}$ & 324 & 6.5 & 0.81 & 227 & 3.04 & 29 & 21.8 & 7.8 & 402 \\
\hline $\mathrm{X} 10$ & 364 & 5.4 & 0.86 & 109 & -- & -- & -- & -- & $-\infty$ \\
\hline 08B & 371 & 6.1 & 0.86 & 111 & 2.57 & 29 & 21.8 & 7.7 & 401 \\
\hline $\mathrm{X} 11$ & 406 & 6.4 & 0.85 & 101 & -- & -- & -- & -- & -- \\
\hline $09 \mathrm{~A}$ & 408 & 5.1 & 0.90 & 126 & 2.46 & 25 & 21.9 & 7.7 & 396 \\
\hline $10 \mathrm{~B}$ & 461 & 5.3 & 0.78 & 179 & 2.43 & 24 & 22.0 & 7.8 & 391 \\
\hline $11 \mathrm{~A}$ & 494 & 5.0 & 0.90 & 175 & 2.28 & 22 & 22.0 & 7.7 & 389 \\
\hline $12 B$ & 539 & 5.7 & 0.72 & 127 & 2.46 & 18 & 22.1 & 7.7 & 388 \\
\hline X04 & 556 & 5.8 & 0.78 & 138 & -- & -- & -- & - & -- \\
\hline $13 \mathrm{~A}$ & 600 & 5.0 & 0.77 & 155 & 2.62 & 15 & 22.1 & 7.7 & 385 \\
\hline $14 B$ & 636 & 6.9 & 0.81 & 190 & 3.56 & 19 & 22.1 & 7.7 & 384 \\
\hline $15 \mathrm{~A}$ & 668 & 7.8 & 0.81 & 187 & 4.03 & 23 & 22.2 & 7.7 & 382 \\
\hline X05 & 695 & 9.2 & 0.52 & 131 & - & - & -- & - & - \\
\hline $16 \mathrm{~B}$ & 723 & 8.2 & 0.49 & 155 & 2.08 & 31 & 22.2 & 7.7 & 376 \\
\hline $17 \mathrm{~A}$ & 772 & 7.8 & 0.80 & 272 & 2.94 & 21 & 22.2 & 7.6 & 359 \\
\hline $18 \mathrm{~B}$ & 810 & 7.0 & 0.73 & 123 & 3.26 & 19 & 22.4 & 7.7 & 355 \\
\hline X06 & 820 & 7.1 & 0.69 & 101 & - & -- & -- & -- & -- \\
\hline $19 \mathrm{~A}$ & 851 & 5.4 & 0.71 & 144 & 2.18 & 11 & 22.4 & 7.7 & 355 \\
\hline $20 \mathrm{~B}$ & 895 & 4.2 & 0.57 & 107 & 1.03 & 4 & 22.6 & 7.7 & 353 \\
\hline REW & 941 & 0.0 & 0.00 & 0 & -- & -- & -- & -- & -- \\
\hline MEAN & & 6.4 & 0.83 & & 64.92 & 516 & & & \\
\hline TOTAL & 941 & & & 5,041 & & & & & \\
\hline
\end{tabular}




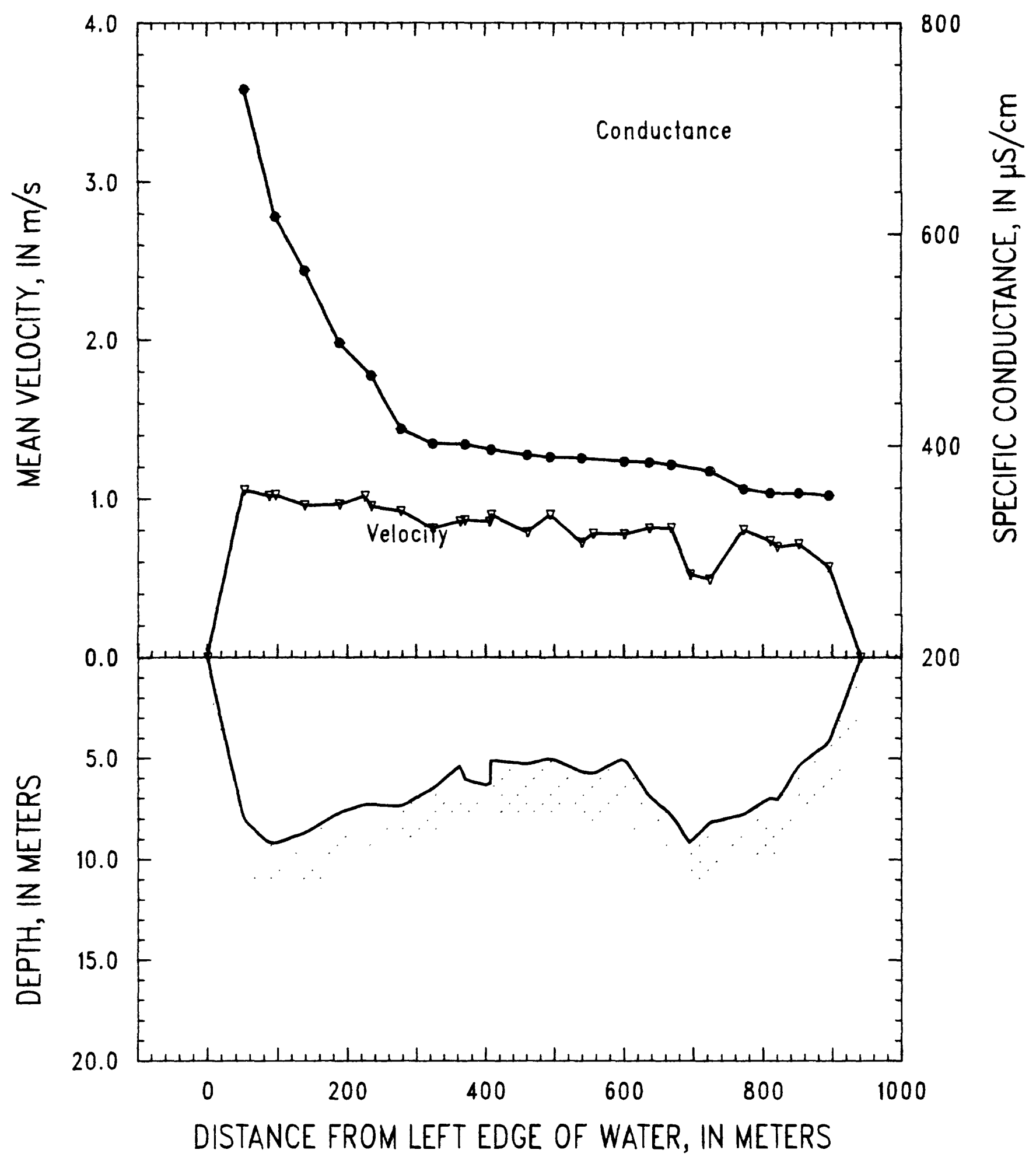

Figure 77. Mississippi River below Grafton, Illinois, on June 11, 1990. 
SITE: Mississippi River at Thebes, Illinois

06-13-90

PARTY: Moody, Garbarino, and Simoneaux

METER: SOLID CUP

STARTING GAGE HEIGHT: -- ENDING GAGE HEIGHT: $32.98 \mathrm{ft}$

SUSP: Bag sampler and 300-1b weight

CURRENT METER NO.: W-297222 DATE RATED: 04-90

CURRENT METER EQUATION: $V(\mathrm{~m} / \mathrm{s})=$ RPS $* 0.744+0.011$

REMARKS: Transit rate was $20 \mathrm{~cm} / \mathrm{s}$ and the nozzle was $3 / 16$ inch.

Measured to the right edge of flow; the edge of water was far into the

willows, but there was no significant flow. Depth was estimated at REF.

USGS Missouri District collected a simultaneous NASQAN sample from Thebes railroad bridge.

\begin{tabular}{|c|c|c|c|c|c|c|c|c|c|}
\hline \multirow[b]{2}{*}{$\begin{array}{c}\text { Verti- } \\
\text { cal }\end{array}$} & \multirow{2}{*}{$\begin{array}{l}\text { Dist. } \\
\text { from } \\
\text { LEW } \\
\text { (m) }\end{array}$} & \multirow[b]{2}{*}{$\begin{array}{c}\text { Depth } \\
(\mathrm{m})\end{array}$} & \multirow{2}{*}{$\begin{array}{l}\text { Mean } \\
\text { veloc- } \\
\text { ity } \\
(\mathrm{m} / \mathrm{s})\end{array}$} & \multirow[b]{2}{*}{$\begin{array}{r}\text { Dis- } \\
\text { charge } \\
\left(\mathrm{m}^{3} / \mathrm{s}\right)\end{array}$} & \multicolumn{2}{|c|}{ Volume } & \multirow[b]{2}{*}{$\begin{array}{l}\text { Temper- } \\
\text { ature } \\
\left({ }^{\circ} \mathrm{C}\right)\end{array}$} & \multirow[b]{2}{*}{$\mathrm{pH}$} & \multirow{2}{*}{$\begin{array}{l}\text { Specific } \\
\text { conduct- } \\
\text { ance } \\
(\mu \mathrm{S} / \mathrm{cm})\end{array}$} \\
\hline & & & & & $\begin{array}{l}\mathrm{V}_{i} \\
(\mathrm{~L})\end{array}$ & $\begin{array}{c}\overline{V_{p}} \\
(\mathrm{~L})\end{array}$ & & & \\
\hline LEW & 0 & 0.0 & 0.00 & 0 & -- & -- & -- & -- & -- \\
\hline $01 \mathrm{~A}$ & 30 & 5.5 & 0.16 & 27 & 0.31 & 7 & 22.0 & 7.5 & 363 \\
\hline O2B & 61 & 11.9 & 1.21 & 419 & 2.35 & 12 & 21.7 & 7.5 & 358 \\
\hline $03 \mathrm{~A}$ & 88 & 12.1 & 1.62 & 559 & 3.20 & 15 & 21.8 & 7.5 & 361 \\
\hline $04 \mathrm{~B}$ & 118 & 13.2 & 1.85 & 611 & 4.42 & 16 & 21.1 & 7.5 & 360 \\
\hline $05 \mathrm{~A}$ & 138 & 14.1 & 1.99 & 814 & 4.49 & 21 & 22.2 & 7.5 & 360 \\
\hline 06B & 176 & 13.7 & 2.11 & 911 & 4.68 & 19 & 22.1 & 7.4 & 359 \\
\hline $07 \mathrm{~A}$ & 201 & 14.0 & 2.12 & 905 & 4.48 & 20 & 22.1 & 7.5 & 359 \\
\hline $08 \mathrm{~B}$ & 237 & 13.8 & 2.19 & 966 & 5.52 & 21 & 21.3 & 7.8 & 359 \\
\hline $09 \mathrm{~A}$ & 265 & 13.9 & 2.21 & 954 & 5.52 & 20 & 21.8 & 7.9 & 357 \\
\hline $10 \mathrm{~B}$ & 299 & 14.3 & 2.18 & 702 & 5.10 & 24 & 22.1 & 7.9 & 356 \\
\hline $\mathrm{X} 03$ & 310 & 13.5 & 2.36 & 335 & -- & -- & -- & -- & -- \\
\hline X08 & 320 & 14.0 & 2.03 & 242 & -- & -- & -- & -- & -- \\
\hline $11 \mathrm{~A}$ & 327 & 13.5 & 2.05 & 541 & 5.17 & 22 & 20.9 & 8.0 & 357 \\
\hline $12 \mathrm{~B}$ & 359 & 13.8 & 2.30 & 938 & 4.97 & 23 & 20.5 & 7.8 & 356 \\
\hline $13 \mathrm{~A}$ & 386 & 12.6 & 2.25 & 794 & 4.81 & 21 & 21.1 & 7.7 & 355 \\
\hline $14 \mathrm{~B}$ & 415 & 12.3 & 1.99 & 637 & 4.23 & 17 & 21.7 & 7.7 & 356 \\
\hline $15 \mathrm{~A}$ & 438 & 12.1 & 1.77 & 622 & 3.15 & 12 & 20.9 & 7.7 & 356 \\
\hline $16 \mathrm{~B}$ & 473 & 12.3 & 1.41 & 562 & 3.16 & 12 & 21.9 & 7.7 & 356 \\
\hline $17 \mathrm{~A}$ & 503 & 10.7 & 1.21 & 369 & 2.18 & 9 & 21.3 & 7.7 & 356 \\
\hline $18 \mathrm{~B}$ & 530 & 10.2 & 0.98 & 270 & 1.66 & 7 & 22.1 & 7.6 & 357 \\
\hline $19 \mathrm{~A}$ & 557 & 9.7 & 0.96 & 302 & 1.61 & 6 & 21.4 & 7.6 & 357 \\
\hline $20 \mathrm{~B}$ & 595 & 6.8 & 0.57 & 129 & 0.46 & 3 & 22.7 & 7.6 & 356 \\
\hline $\mathrm{REF}$ & 623 & 2.0 & 0.00 & 0 & -- & -- & -- & -- & -- \\
\hline MEAN & & 11.4 & 1.77 & & & & & & \\
\hline TOTAL & 623 & & & 12,609 & 71.47 & 307 & & & \\
\hline
\end{tabular}




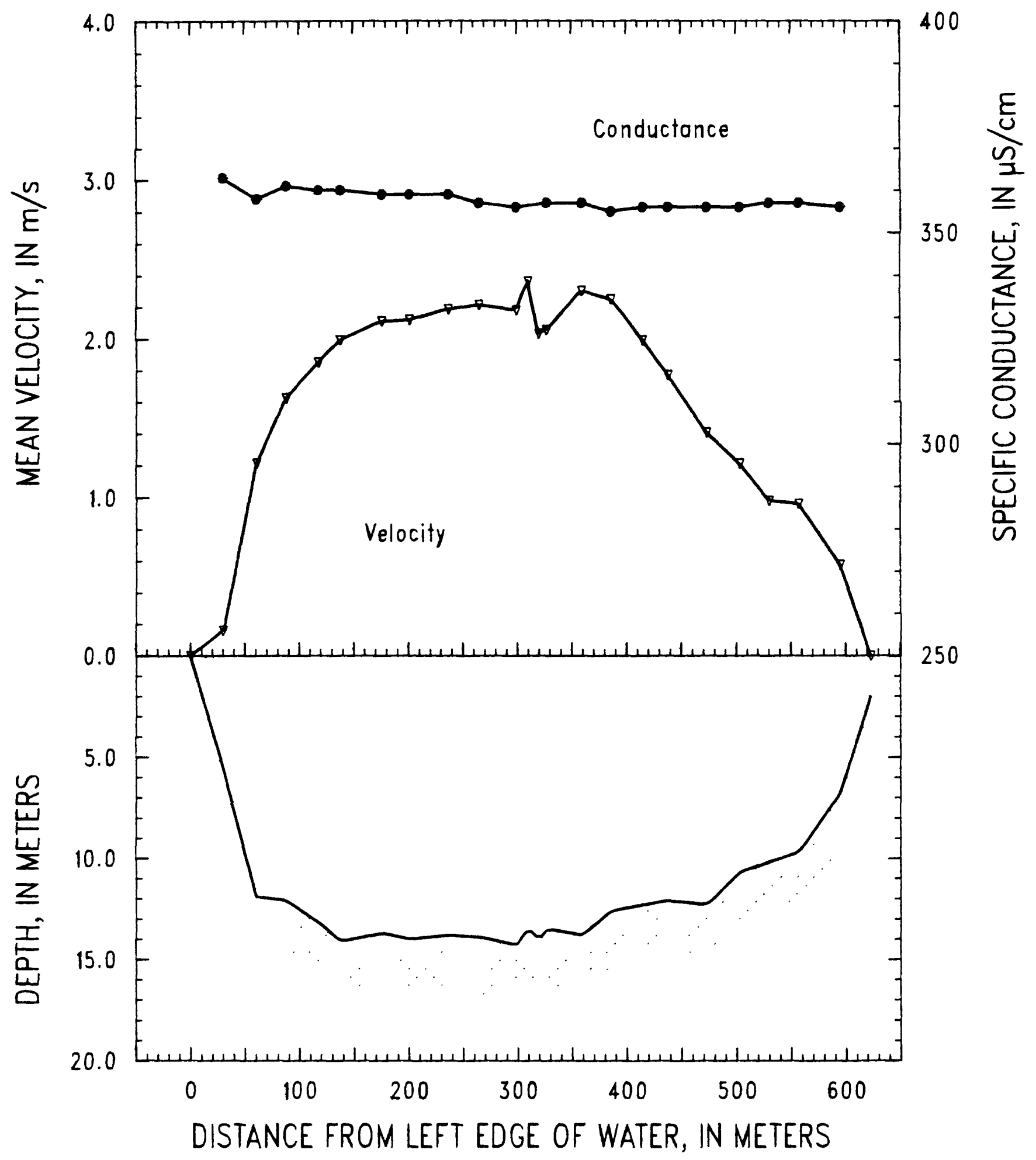

Figure 78. Mississippi River at Thebes, Illinois, on June 13, 1990. 
SITE: Ohio River at Olmsted, Illinois

PARTY: Moody, Garbarino, and Simoneaux

METER: SOLID CUP

STARTING GAGE HEIGHT: $38.0 \mathrm{ft}$

ENDING GAGE HEIGHT: $37.8 \mathrm{ft}$

SUSP: Bag sampler and 300-1b weight

CURRENT METER NO.: W-297222 DATE RATED: 04-90

CURRENT METER EQUATION: $V(\mathrm{~m} / \mathrm{s})=$ RPS $* 0.744+0.011$

REMARKS: Transit rate was $11 \mathrm{~cm} / \mathrm{s}$; nozzle was $1 / 4$ inch; order of verticals: 15, 20-1. USGS Kentucky District collected a NASQAN sample about 2 miles upriver.

\begin{tabular}{|c|c|c|c|c|c|c|c|c|c|}
\hline \multirow[b]{2}{*}{$\begin{array}{l}\text { Verti- } \\
\text { cal }\end{array}$} & \multirow{2}{*}{$\begin{array}{l}\text { Dist. } \\
\text { from } \\
\text { LEW } \\
(\mathrm{m})\end{array}$} & \multirow[b]{2}{*}{$\begin{array}{c}\text { Depth } \\
(\mathrm{m})\end{array}$} & \multirow{2}{*}{$\begin{array}{l}\text { Mean } \\
\text { veloc- } \\
\text { ity } \\
(\mathrm{m} / \mathrm{s})\end{array}$} & \multirow[b]{2}{*}{$\begin{array}{r}\text { Dis- } \\
\text { charge } \\
\left(m^{3} / s\right)\end{array}$} & \multicolumn{2}{|c|}{ Volume } & \multirow[b]{2}{*}{$\begin{array}{l}\text { Temper- } \\
\text { ature } \\
\left({ }^{\circ} \mathrm{C}\right)\end{array}$} & \multirow[b]{2}{*}{$\mathrm{pH}$} & \multirow{2}{*}{$\begin{array}{l}\text { Specific } \\
\text { conduct- } \\
\text { ance } \\
(\mu \mathrm{S} / \mathrm{cm})\end{array}$} \\
\hline & & & & & $\begin{array}{l}\mathrm{V}_{\mathbf{i}} \\
(\mathrm{L})\end{array}$ & $\begin{array}{l}\overline{V_{p}} \\
(L)\end{array}$ & & & \\
\hline LEW & 0 & 0.0 & 0.00 & 0 & -- & $-\infty$ & -- & -- & - \\
\hline $01 \mathrm{~A}$ & 53 & 7.4 & 0.53 & 202 & 1.64 & 8 & 22.0 & 7.6 & 267 \\
\hline 02B & 103 & 9.1 & 0.69 & 305 & 2.97 & 13 & 22.2 & 7.7 & 270 \\
\hline X05 & 150 & 9.7 & 0.66 & 199 & -- & $\cdots$ & - & -- & - \\
\hline 03A & 165 & 9.6 & 0.71 & 181 & 2.89 & 14 & 21.7 & 7.7 & 271 \\
\hline 04B & 203 & 10.0 & 0.81 & 391 & 3.32 & 16 & 21.6 & 7.7 & 271 \\
\hline 05A & 262 & 10.9 & 0.74 & 369 & 2.62 & 16 & 21.8 & 7.7 & 270 \\
\hline 06B & 295 & 11.8 & 0.74 & 391 & 3.70 & 19 & 22.2 & 7.7 & 270 \\
\hline X04 & 352 & 13.3 & 0.71 & 302 & $-\infty$ & -- & $\cdots$ & - & -- \\
\hline 07A & 359 & 13.2 & 0.71 & 262 & 4.39 & 20 & 22.3 & 7.7 & 271 \\
\hline 08B & 408 & 13.9 & 0.75 & 488 & 4.20 & 23 & 22.2 & 7.7 & 270 \\
\hline $09 A$ & 453 & 13.7 & 0.84 & 529 & 4.84 & 24 & 22.0 & 7.7 & 270 \\
\hline $10 \mathrm{~B}$ & 500 & 13.4 & 0.82 & 541 & 5.23 & 23 & 22.3 & 7.7 & 269 \\
\hline $\mathrm{x} 03$ & 551 & 13.4 & 0.80 & 312 & - & -- & -- & -- & -- \\
\hline $11 \mathrm{~A}$ & 558 & 13.5 & 0.81 & 258 & 5.60 & 23 & 22.4 & 7.7 & 266 \\
\hline $12 B$ & 598 & 13.7 & 0.86 & 505 & 5.58 & 24 & 22.2 & 7.7 & 267 \\
\hline $13 \mathrm{~A}$ & 644 & 13.9 & 0.83 & 581 & 5.30 & 24 & 23.2 & 7.6 & 273 \\
\hline $14 B$ & 699 & 13.8 & 0.90 & 625 & 5.75 & 27 & 21.7 & 7.7 & 276 \\
\hline $15 A$ & 745 & 14.0 & 0.89 & 306 & 5.73 & 27 & 23.2 & 7.7 & 282 \\
\hline $\mathrm{X} 02$ & 748 & 14.1 & 0.88 & 340 & -- & -- & -- & -- & $\cdots$ \\
\hline $16 \mathrm{~B}$ & 800 & 14.1 & 0.88 & 633 & 5.82 & 27 & 22.5 & 7.7 & 285 \\
\hline $17 \mathrm{~A}$ & 850 & 14.6 & 0.83 & 591 & 5.54 & 25 & 22.2 & 7.6 & 286 \\
\hline $18 \mathrm{~B}$ & 897 & 14.7 & 0.78 & 542 & 5.48 & 21 & 21.9 & 7.6 & 290 \\
\hline $19 \mathrm{~A}$ & 944 & 15.3 & 0.62 & 447 & 4.79 & 22 & 22.9 & 7.6 & 292 \\
\hline $20 \mathrm{~B}$ & 991 & 8.2 & 0.60 & 251 & 1.79 & 5 & 23.0 & 7.6 & 291 \\
\hline REW ? & 1,046 & 0.0 & 0.00 & 0 & -- & -- & -- & - & -- \\
\hline MEAN & & 11.8 & 0.78 & & & & & & \\
\hline TOTAL & 1,046 & & & 9,551 & 87.18 & 393 & & & \\
\hline
\end{tabular}




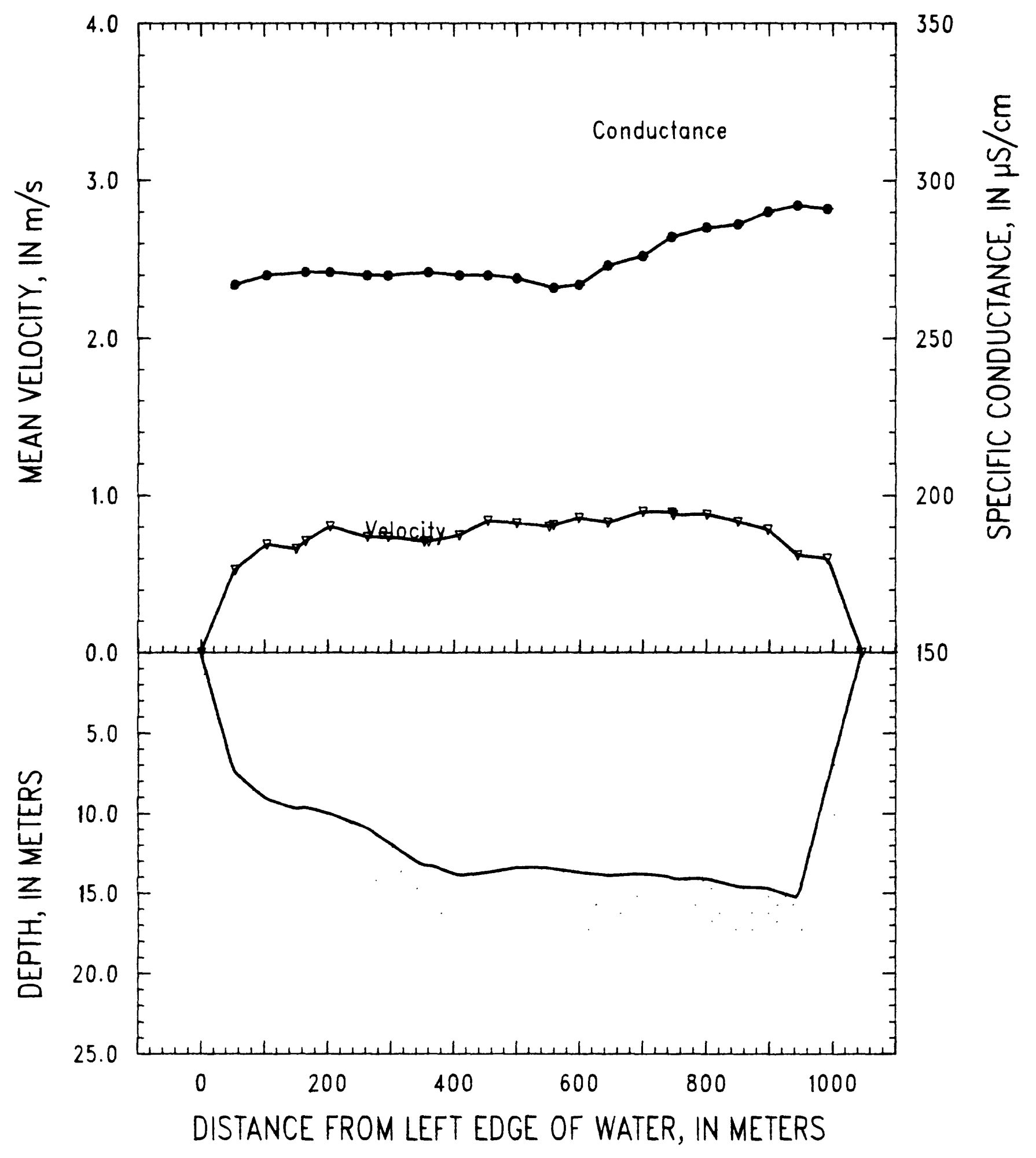

Figure 79. Ohio River at Olmsted, Illinois, on June 14, 1990. 
SITE: Mississippi River below Memphis, Tennessee

06-18-90

PARTY: Moody, Garbarino, and LeBoeuf

METER: SOLID CUP

STARTING GAGE HEIGHT: $22.3 \mathrm{ft}$

ENDING GAGE HEIGHT:

SUSP: Bag sampler and 300-1b weight

CURRENT METER NO.: W-297222 DATE RATED: 04-90

CURRENT METER EQUATION: $V(\mathrm{~m} / \mathrm{s})=$ RPS $* 0.744+0.011$

REMARKS :

Discharge was measured between the left edge of water and the right

edge of flow. Transit rate was $13 \mathrm{~cm} / \mathrm{s}$ and nozzle was $1 / 8$ inch.

Arkansas District collected a simultaneous NASQAN sample at mile 734.6.

\begin{tabular}{|c|c|c|c|c|c|c|c|c|c|}
\hline \multirow[b]{2}{*}{$\begin{array}{c}\text { Verti- } \\
\text { cal }\end{array}$} & \multirow{2}{*}{$\begin{array}{l}\text { Dist. } \\
\text { from } \\
\text { LEW } \\
\text { (m) }\end{array}$} & \multirow[b]{2}{*}{$\begin{array}{l}\text { Depth } \\
\text { (m) }\end{array}$} & \multirow{2}{*}{$\begin{array}{l}\text { Mean } \\
\text { veloc- } \\
\text { ity } \\
(\mathrm{m} / \mathrm{s})\end{array}$} & \multirow[b]{2}{*}{$\begin{array}{r}\text { Dis- } \\
\text { charge } \\
\left(\mathrm{m}^{3} / \mathrm{s}\right)\end{array}$} & \multicolumn{2}{|c|}{ Volume } & \multirow[b]{2}{*}{$\begin{array}{l}\text { Temper- } \\
\text { ature } \\
\left({ }^{\circ} \mathrm{C}\right)\end{array}$} & \multirow[b]{2}{*}{$\mathrm{pH}$} & \multirow{2}{*}{$\begin{array}{c}\text { Specific } \\
\text { conduct- } \\
\text { ance } \\
(\mu \mathrm{S} / \mathrm{cm})\end{array}$} \\
\hline & & & & & $\begin{array}{l}V_{i} \\
(L)\end{array}$ & $\begin{array}{l}\mathrm{V}_{\mathrm{p}} \\
(\mathrm{L})\end{array}$ & & & \\
\hline LEW & 0 & 0.0 & 0.00 & 0 & -- & -- & -- & -- & -- \\
\hline $01 \mathrm{~A}$ & 60 & 10.1 & 1.00 & 545 & 1.32 & 9 & 25.6 & 7.5 & 341 \\
\hline 02B & 108 & 10.3 & 1.29 & 624 & 1.49 & 12 & 25.6 & 7.5 & 342 \\
\hline $03 \mathrm{~A}$ & 154 & 10.1 & 1.63 & 675 & 2.30 & 14 & 25.6 & 7.5 & 338 \\
\hline $04 \mathrm{~B}$ & 190 & 10.3 & 1.77 & 800 & 2.61 & 15 & 25.6 & 7.5 & 337 \\
\hline $05 A$ & 242 & 11.7 & 1.91 & 1,006 & 3.42 & 19 & 25.6 & 7.5 & 338 \\
\hline O6B & 280 & 12.4 & 1.88 & 1,373 & 3.29 & 20 & 25.6 & 7.5 & 340 \\
\hline $07 \mathrm{~A}$ & 360 & 15.3 & 1.95 & 1,449 & 4.47 & 23 & 25.6 & 7.5 & 340 \\
\hline 08B & 377 & 16.0 & 1.99 & 845 & 4.46 & 24 & 26.2 & 7.5 & 341 \\
\hline X04 & 413 & 17.4 & 1.93 & 1,077 & -- & -- & -- & - & -- \\
\hline 09A & 441 & 18.2 & 1.93 & 772 & 4.29 & 27 & 25.7 & 7.5 & 344 \\
\hline $10 \mathrm{~B}$ & 457 & 19.1 & 1.83 & 1,402 & 5.11 & 29 & 26.0 & 7.5 & 344 \\
\hline $11 \mathrm{~A}$ & 521 & 20.7 & 1.84 & 1,333 & 5.06 & 29 & 25.9 & 7.4 & 345 \\
\hline $\mathrm{X} 07$ & 527 & 21.4 & 1.88 & 905 & -- & -- & - & -- & -- \\
\hline X08 & 566 & 21.0 & 1.77 & 1,041 & - & -- & -- & -- & -- \\
\hline $12 \mathrm{~B}$ & 583 & 21.2 & 1.69 & 608 & 5.29 & 30 & 25.9 & 7.4 & 347 \\
\hline X03 & 600 & 20.8 & 1.76 & 749 & -- & -- & -- & -- & -- \\
\hline $13 \mathrm{~A}$ & 624 & 20.3 & 1.57 & 1,180 & 3.84 & 25 & 25.6 & 7.4 & 346 \\
\hline $14 \mathrm{~B}$ & 674 & 18.7 & 1.40 & 1,110 & 3.90 & 21 & 25.6 & 7.4 & 344 \\
\hline $\mathrm{X} 02$ & 709 & 18.8 & 1.35 & 712 & -- & -- & -- & -- & -- \\
\hline $15 \mathrm{~A}$ & 730 & 18.8 & 1.12 & 536 & 2.46 & 17 & 26.3 & 7.4 & 346 \\
\hline $16 \mathrm{~B}$ & 760 & 18.4 & 1.09 & 683 & 2.11 & 14 & 25.7 & 7.4 & 348 \\
\hline X01 & 798 & 16.7 & 0.88 & 367 & -- & -- & -- & - & - \\
\hline $17 \mathrm{~A}$ & 810 & 16.0 & 0.81 & 370 & 1.47 & 10 & 25.7 & 7.4 & 348 \\
\hline $18 \mathrm{~B}$ & 855 & 13.0 & 0.70 & 454 & 1.22 & 6 & 25.8 & 7.4 & 348 \\
\hline $19 A$ & 910 & 9.3 & 0.31 & 153 & 0.27 & 3 & 25.5 & 7.5 & 350 \\
\hline $20 B$ & 962 & 6.7 & 0.08 & 24 & 0.36 & 2 & 26.6 & 7.5 & 349 \\
\hline REF & 1,002 & 2.0 & -- & -- & -- & -- & -- & -- & -- \\
\hline
\end{tabular}

MEAN

TOTAL 1,002
$14.1 \quad 1.47$

$20,793 \quad 58.74 \quad 349$ 


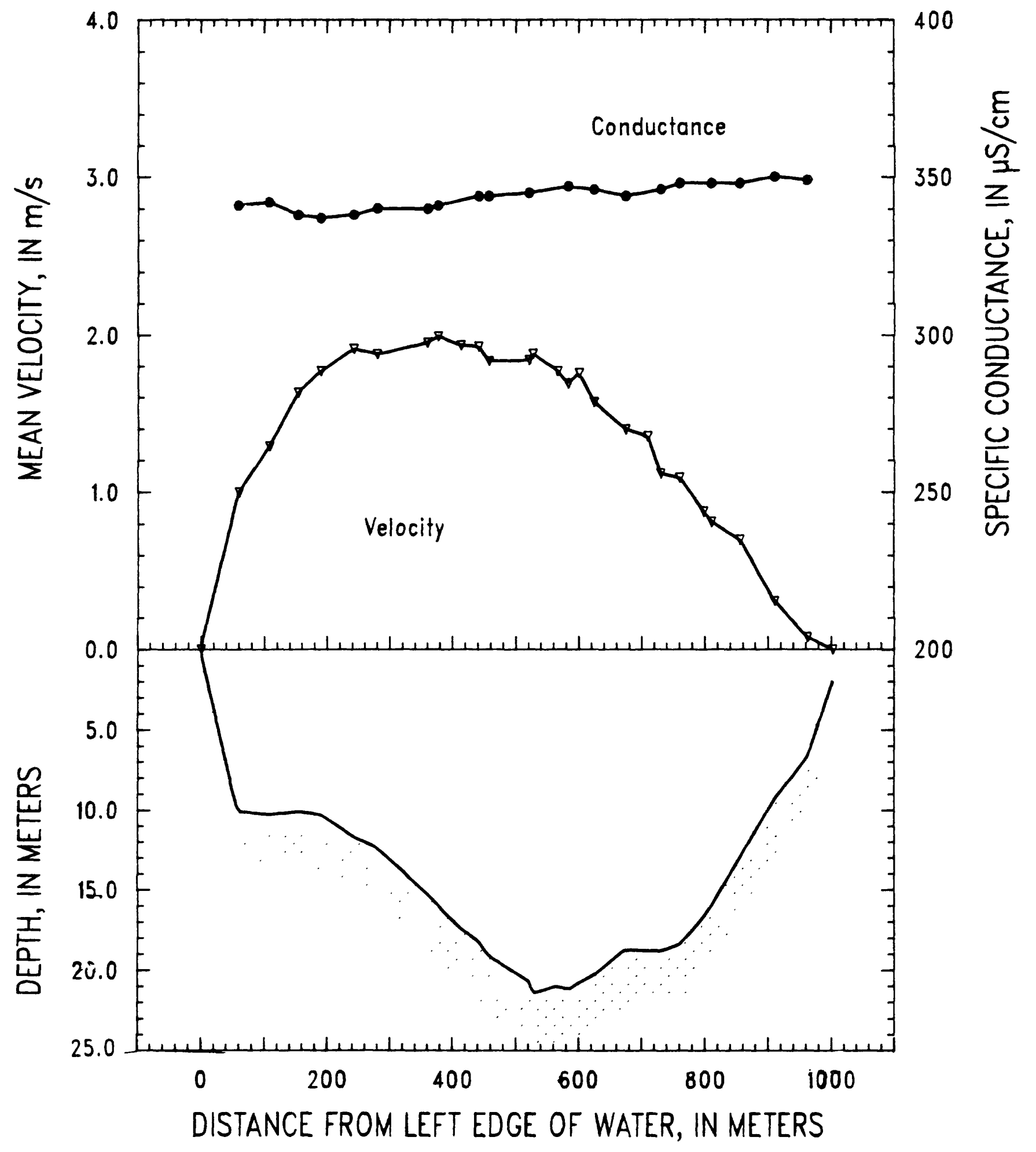

Figure 80. Mississippi River below Memphis, Tennesse, on June 18, 1990. 
SITE: Mississippi River below Arkansas City, Arkansas PARTY: Moody, Garbarino, and LeBoeuf

STARTING GAGE HEIGHT: $29.8 \mathrm{ft}$ ENDING GAGE HEIGHT:

METER: SOLID CUP

SUSP: Bag sampler and $300-1 \mathrm{~b}$ weight

CURRENT METER NO.: 90JM1 DATE RATED: 04-90

CURRENT METER EQUATION: $V(\mathrm{~m} / \mathrm{s})=$ RPS $* 0.755+0.011$

REMARKS: USGS Arkansas District collected a simultaneous NASQAN sample

$0.1 \mathrm{mile}$ below the section. Transit rate was about $16 \mathrm{~cm} / \mathrm{s}$ and the nozzle was $1 / 8$ inch.

\begin{tabular}{|c|c|c|c|c|c|c|c|c|c|}
\hline \multirow[b]{2}{*}{$\begin{array}{l}\text { Verti- } \\
\text { cal }\end{array}$} & \multirow{2}{*}{$\begin{array}{l}\text { Dist. } \\
\text { from } \\
\text { LEW } \\
\text { (m) }\end{array}$} & \multirow[b]{2}{*}{$\begin{array}{c}\text { Depth } \\
(\mathrm{m})\end{array}$} & \multirow{2}{*}{$\begin{array}{l}\text { Mean } \\
\text { veloc- } \\
\text { ity } \\
(\mathrm{m} / \mathrm{s})\end{array}$} & \multirow[b]{2}{*}{$\begin{array}{r}\text { Dis- } \\
\text { charge } \\
\left(\mathrm{m}^{3} / \mathrm{s}\right)\end{array}$} & \multicolumn{2}{|c|}{ Volume } & \multirow[b]{2}{*}{$\begin{array}{l}\text { Temper- } \\
\text { ature } \\
\left({ }^{\circ} \mathrm{C}\right)\end{array}$} & \multirow[b]{2}{*}{$\mathrm{pH}$} & \multirow{2}{*}{$\begin{array}{c}\text { Specific } \\
\text { conduct- } \\
\text { ance } \\
(\mu \mathrm{S} / \mathrm{cm})\end{array}$} \\
\hline & & & & & $\begin{array}{l}\mathrm{V}_{\mathbf{i}} \\
(\mathrm{I})\end{array}$ & $\begin{array}{l}\overline{V_{p}} \\
(\mathrm{I})\end{array}$ & & & \\
\hline LEW & 0 & 0.0 & 0.00 & 0 & -- & -- & -- & -- & -- \\
\hline $01 B$ & 56 & 4.5 & 0.45 & 97 & 0.25 & 2 & 26.1 & 7.6 & 326 \\
\hline $02 \mathrm{~A}$ & 95 & 7.4 & 0.63 & 199 & 0.52 & 4 & 26.2 & 7.7 & 325 \\
\hline $03 \mathrm{~B}$ & 141 & 8.7 & 0.82 & 275 & 0.99 & 5 & 26.0 & 7.6 & 325 \\
\hline X05 & 172 & 8.9 & 0.95 & 194 & -- & -- & -- & -- & -- \\
\hline $04 \mathrm{~A}$ & 187 & 8.7 & 0.88 & 287 & 1.02 & 5 & 25.9 & 7.6 & 326 \\
\hline $05 B$ & 247 & 9.4 & 0.99 & 511 & 1.15 & 6 & 26.0 & 7.6 & 326 \\
\hline $06 \mathrm{~A}$ & 297 & 10.5 & 1.10 & 491 & 1.42 & 8 & 26.0 & 7.6 & 326 \\
\hline $07 \mathrm{~B}$ & 332 & 11.3 & 1.19 & 704 & 1.66 & 10 & 26.3 & 7.6 & 325 \\
\hline $08 \mathrm{~A}$ & 402 & 12.6 & 1.35 & 691 & 1.91 & 12 & 26.0 & 7.6 & 325 \\
\hline X04 & 413 & 12.6 & 1.57 & 257 & -- & -- & -- & -- & -- \\
\hline 09B & 428 & 12.9 & 1.54 & 297 & 2.42 & 15 & 26.0 & 7.7 & 325 \\
\hline $\mathrm{X} 07$ & 443 & 13.4 & 1.53 & 411 & -- & -- & -- & -- & -- \\
\hline $10 \mathrm{~A}$ & 468 & 14.3 & 1.76 & 1,286 & 3.09 & 19 & 26.1 & 7.6 & 323 \\
\hline $11 \mathrm{~B}$ & 545 & 19.7 & 1.89 & 1,581 & 4.53 & 18 & 26.1 & 7.7 & 322 \\
\hline X06 & 553 & 19.2 & 2.06 & 809 & -- & -- & -- & -- & -- \\
\hline $12 \mathrm{~A}$ & 586 & 18.0 & 2.35 & 1,457 & 5.04 & 23 & 26.2 & 7.7 & 321 \\
\hline $\mathrm{X} 03$ & 622 & 22.5 & 1.86 & 1,127 & -- & - & -- & -- & -- \\
\hline $13 B$ & 640 & 22.3 & 1.74 & 1,375 & 4.49 & 23 & 26.2 & 7.6 & 320 \\
\hline $14 \mathrm{~A}$ & 693 & 21.5 & 2.02 & 2,196 & 5.28 & 24 & 26.1 & 7.6 & 321 \\
\hline $15 B$ & 741 & 21.9 & 2.03 & 1,268 & 5.27 & 27 & 26.0 & 7.6 & 320 \\
\hline $\mathrm{X} 02$ & 750 & 22.0 & 2.01 & 1,572 & - & - & - & - & - \\
\hline $16 \mathrm{~A}$ & 812 & 23.0 & 1.88 & 2,145 & 5.47 & 27 & 25.5 & 7.6 & 318 \\
\hline $17 \mathrm{~B}$ & 849 & 23.3 & 1.95 & 2,154 & 5.00 & 25 & 25.5 & 7.6 & 319 \\
\hline $18 \mathrm{~A}$ & 907 & 25.0 & 1.51 & 1,245 & 4.80 & 26 & 26.1 & 7.6 & 317 \\
\hline $\mathrm{X} 01$ & 915 & 25.0 & 1.88 & 894 & -- & -- & - & -- & -- \\
\hline $19 B$ & 945 & 23.7 & 1.33 & 1,276 & 3.60 & 18 & 26.2 & 7.7 & 313 \\
\hline $20 \mathrm{~A}$ & 996 & 14.2 & 0.84 & 659 & 1.38 & 2 & 26.1 & 7.7 & 317 \\
\hline REW & 1,055 & 0.0 & 0.00 & 0 & -- & -- & - & -- & -- \\
\hline
\end{tabular}

MEAN

$15.0 \quad 1.61$

TOTAL 1,055 


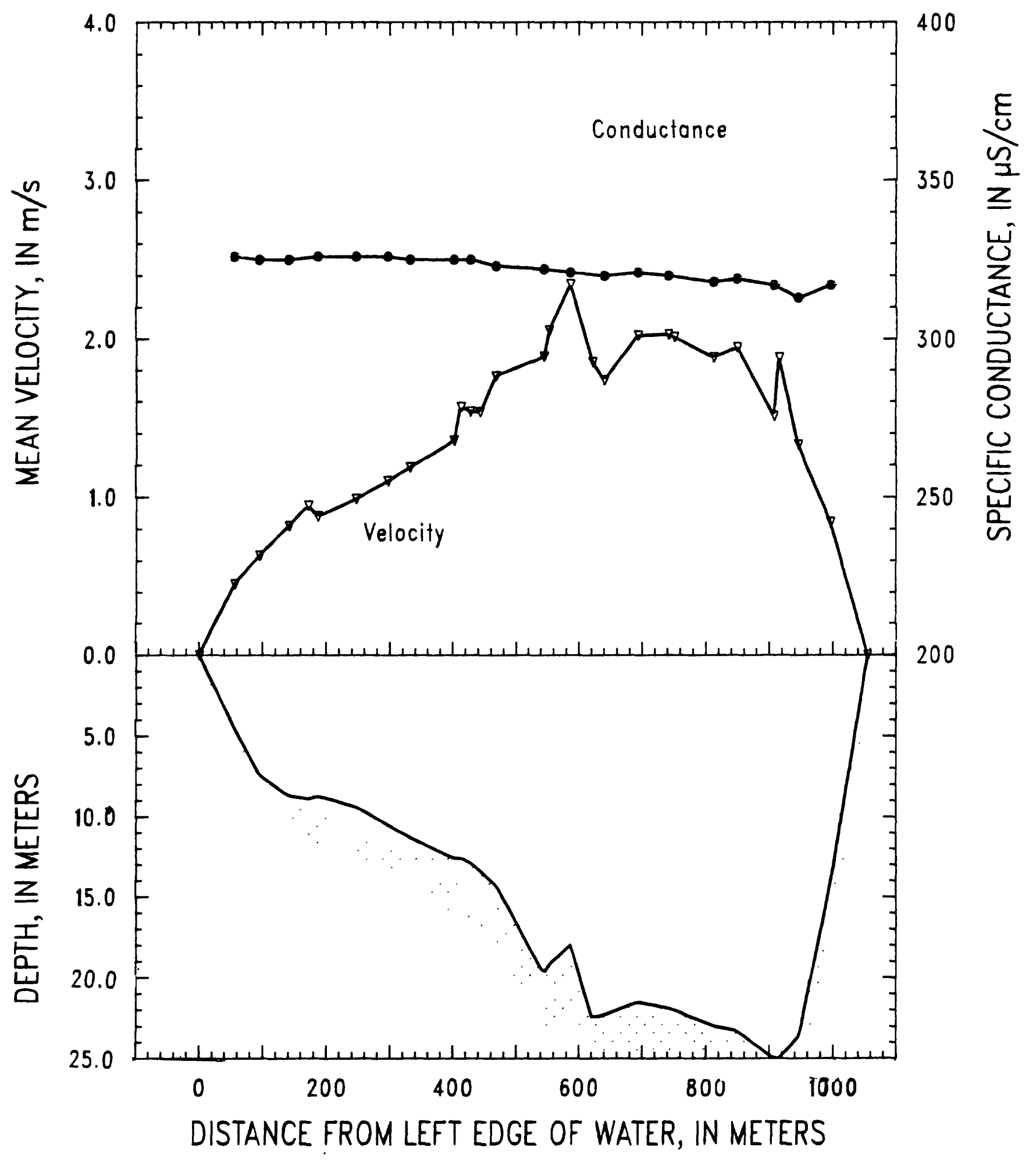

Figure 81. Mississippi River below Arkansas City, Arkansas, on June 20, 1990. 
SITE: Yazoo River below Steele Bayou, Mississippi PARTY: Moody, Garbarino, and LeBoeuf

STARTING GAGE HEIGHT: $37.7 \mathrm{ft}$ at Vicksburg bridge

METER: SOLID CUP

SUSP: Bag sampler and 300-1b weight

CURRENT METER NO.: 90JM1 DATE RATED: 04-90

CURRENT METER EQUATION: $V(\mathrm{~m} / \mathrm{s})=$ RPS $* 0.755+0.011$

REMARKS: Transit rate $(7-12 \mathrm{~cm} / \mathrm{s})$ and nozzle sizes (1/4-5/16 inch) were variable. Equal-discharge sampling at anchor stations: $31,58,79,100$, and 129. Collected $2 \mathrm{~A}-$ and $2 \mathrm{~B}$-composite samples at each vertical. The second B sample at vertical 1 (1B2) was not at $34 \mathrm{~m}$ from LEW.

Underestimated effect of bank and submerged brush on left bank. Order of verticals: 4, 3, 2, 1, 5. USGS Mississippi District collected simultaneous NASQAN sample at three verticals. Steele Bayou was flowing and entered on the right bank about mile 0.5 downstream from the station.

\begin{tabular}{|c|c|c|c|c|c|c|c|c|c|}
\hline \multirow[b]{2}{*}{$\begin{array}{c}\text { Verti- } \\
\text { cal }\end{array}$} & \multirow{2}{*}{$\begin{array}{l}\text { Dist. } \\
\text { from } \\
\text { LEF } \\
\text { (m) }\end{array}$} & \multirow[b]{2}{*}{$\begin{array}{l}\text { Depth } \\
\text { (m) }\end{array}$} & \multirow{2}{*}{$\begin{array}{l}\text { Mean } \\
\text { veloc- } \\
\text { ity } \\
(\mathrm{m} / \mathrm{s})\end{array}$} & \multirow[b]{2}{*}{$\begin{array}{r}\text { Dis- } \\
\text { charge } \\
\left(\mathrm{m}^{3} / \mathrm{s}\right)\end{array}$} & \multicolumn{2}{|c|}{ Volume } & \multirow[b]{2}{*}{$\begin{array}{c}\text { Temper- } \\
\text { ature } \\
\left({ }^{\circ} \mathrm{C}\right)\end{array}$} & \multirow[b]{2}{*}{$\mathrm{pH}$} & \multirow{2}{*}{$\begin{array}{l}\text { Specific } \\
\text { conduct- } \\
\text { ance } \\
(\mu S / \mathrm{cm})\end{array}$} \\
\hline & & & & & $\begin{array}{c}\mathrm{V}_{i} \\
(\mathrm{I})\end{array}$ & $\begin{array}{l}V_{p} \\
(I)\end{array}$ & & & \\
\hline LEF & 0 & 2.0 & -- & -- & -- & - & -- & -- & - \\
\hline $1 \mathrm{~A}, \mathrm{~B}$ & 34 & 10.3 & 0.37 & 78 & 10.08 & 0 & 27.7 & 6.9 & 118 \\
\hline $1 \mathrm{~B} 2$ & 41 & 11.2 & 0.57 & 45 & -- & -- & -- & -- & -- \\
\hline $\mathrm{X} 01$ & 48 & 14.6 & 0.74 & 119 & -- & -- & -- & -- & -- \\
\hline $2 A, B$ & 63 & 14.9 & 0.78 & 133 & 9.49 & 0 & 27.7 & 6.8 & 118 \\
\hline $3 A, B$ & 71 & 15.1 & 0.78 & 142 & 8.78 & 0 & 28.0 & 6.9 & 118 \\
\hline $\mathrm{X03}$ & 87 & 15.7 & 0.77 & 127 & -- & - & -- & -- & -- \\
\hline $4 A, B$ & 92 & 15.5 & 0.79 & 256 & 8.10 & 0 & 28.1 & 6.8 & 114 \\
\hline $05 B$ & 129 & 13.4 & 0.64 & 177 & 5.91 & - & - & - & - \\
\hline $05 \mathrm{~A}$ & 133 & 12.8 & 0.57 & 72 & 5.23 & 0 & 27.8 & 6.9 & 115 \\
\hline $\mathrm{X} 02$ & 149 & 7.6 & 0.38 & 98 & -- & -- & -- & -- & -- \\
\hline REW & 200 & 0.0 & 0.00 & 0 & -- & -- & -- & -- & -- \\
\hline MEAN & & 9.8 & 0.64 & & 47.59 & 0 & & & \\
\hline TOTAL & 200 & & & 1,247 & & & & & \\
\hline
\end{tabular}




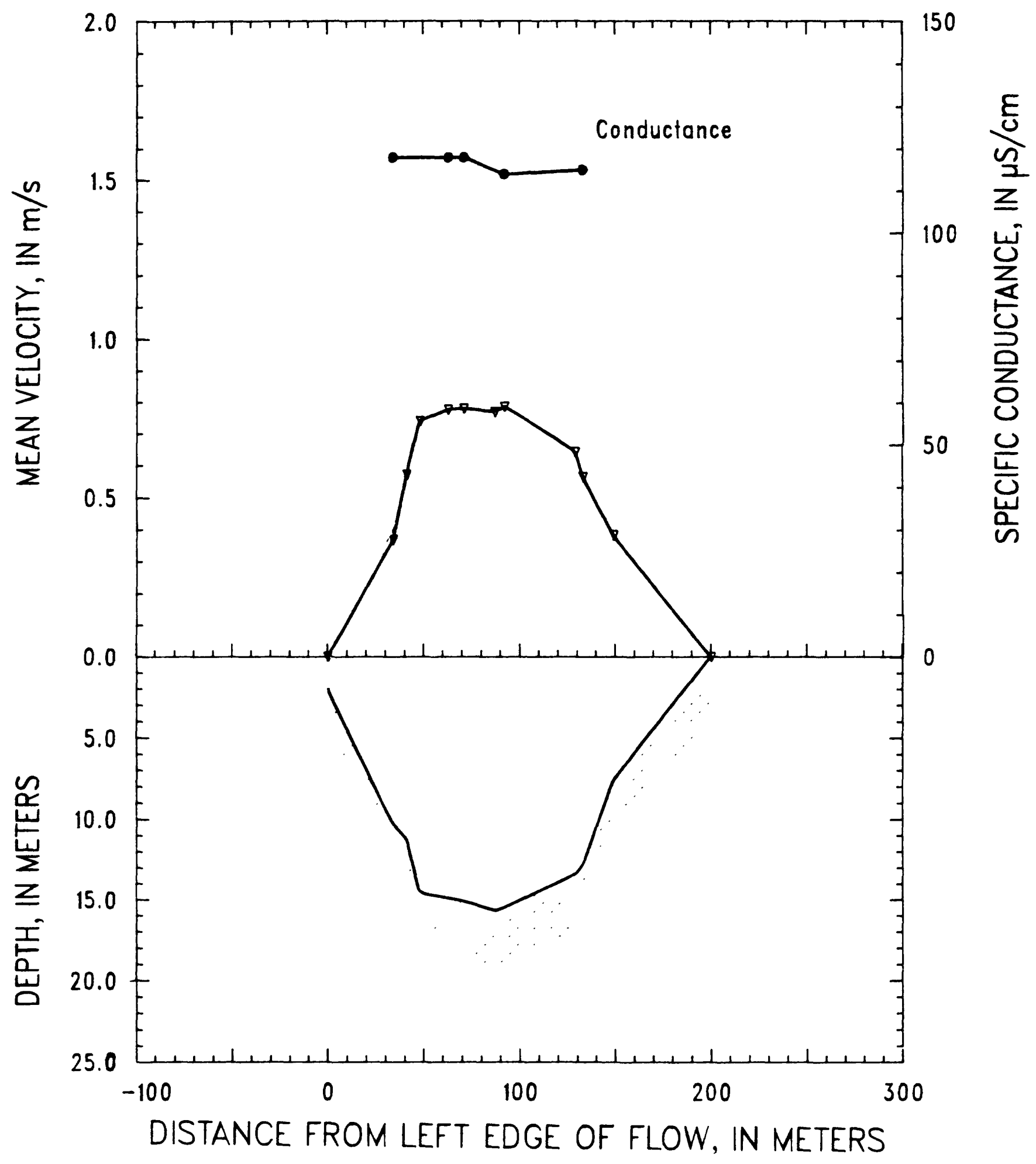

Figure 82. Yazoo River below Steele Bayou, Mississippi, on June 22, 1990. 
SITE: Mississippi River below Vicksburg, Mississippi 06-23-90

PARTY: Moody, Garbarino, and Simoneaux

STARTING GAGE HEIGHT: $36.7 \mathrm{ft}$

SUSP: Bag sampler and 300-1b weight

CURRENT METER NO.: 90JM1 DATE RATED: 04-90

CURRENT METER EQUATION: $V(\mathrm{~m} / \mathrm{s})=$ RPS $* 0.755+0.011$

REMARKS: Transit rate was $15 \mathrm{~cm} / \mathrm{s}$ and a $1 / 8$-inch nozzle was used. USGS Mississippi District collected a NASQAN sample just downriver from the bridge from ACADIANA after we sampled this site. Beautiful day (low humidity) with light breeze.

\begin{tabular}{|c|c|c|c|c|c|c|c|c|c|}
\hline \multirow[b]{2}{*}{$\begin{array}{c}\text { Verti- } \\
\text { cal }\end{array}$} & \multirow{2}{*}{$\begin{array}{l}\text { Dist. } \\
\text { from } \\
\text { LEW } \\
\text { (m) }\end{array}$} & \multirow[b]{2}{*}{$\begin{array}{l}\text { Depth } \\
\text { (m) }\end{array}$} & \multirow{2}{*}{$\begin{array}{l}\text { Mean } \\
\text { veloc- } \\
\text { ity } \\
(\mathrm{m} / \mathrm{s})\end{array}$} & \multirow[b]{2}{*}{$\begin{array}{r}\text { Dis- } \\
\text { charge } \\
\left(\mathrm{m}^{3} / \mathrm{s}\right)\end{array}$} & \multicolumn{2}{|c|}{ Volume } & \multirow[b]{2}{*}{$\begin{array}{c}\text { Temper- } \\
\text { ature } \\
\left({ }^{\circ} \mathrm{C}\right)\end{array}$} & \multirow[b]{2}{*}{$\mathrm{pH}$} & \multirow{2}{*}{$\begin{array}{c}\text { Specific } \\
\text { conduct- } \\
\text { ance } \\
(\mu \mathrm{S} / \mathrm{cm})\end{array}$} \\
\hline & & & & & $\begin{array}{l}V_{i} \\
(L)\end{array}$ & $\begin{array}{l}\bar{v}_{p} \\
(L)\end{array}$ & & & \\
\hline LEW & 0 & 0.0 & 0.00 & 0 & -- & -- & - & -- & -- \\
\hline $01 \mathrm{~A}$ & 66 & 13.6 & 0.75 & 691 & 1.23 & 7 & 26.8 & 7.5 & 288 \\
\hline 02B & 135 & 22.1 & 1.55 & 1,659 & 4.06 & 26 & 26.7 & 7.5 & 289 \\
\hline X01 & 163 & 21.6 & 1.61 & 815 & -- & -- & - & -- & -- \\
\hline $03 A$ & 182 & 21.3 & 1.73 & 1,637 & 4.39 & 27 & 26.6 & 7.6 & 295 \\
\hline $04 \mathrm{~B}$ & 252 & 20.7 & 1.64 & 1,866 & 4.59 & 26 & 26.2 & 7.6 & 303 \\
\hline $\mathrm{X} 02$ & 292 & 20.6 & 1.75 & 1,012 & - & - & - & - & -- \\
\hline $05 \mathrm{~A}$ & 308 & 20.6 & 1.74 & 608 & 4.58 & 28 & 26.5 & 7.6 & 308 \\
\hline X06 & 326 & 20.3 & 1.74 & 988 & -- & - & - & -- & -- \\
\hline $06 \mathrm{~B}$ & 364 & 20.1 & 1.82 & 1,828 & 4.46 & 27 & 26.5 & 7.7 & 318 \\
\hline $07 \mathrm{~A}$ & 426 & 19.5 & 1.69 & 1,844 & 4.10 & 25 & 26.7 & 7.6 & 315 \\
\hline $\mathrm{X} 07$ & 476 & 18.9 & 1.61 & 912 & -- & -- & -- & -- & -- \\
\hline 08B & 486 & 19.3 & 1.76 & 543 & 4.48 & 24 & 26.7 & 7.7 & 323 \\
\hline X03 & 508 & 19.6 & 1.68 & 888 & - & $\ldots$ & - & - & - \\
\hline 09A & 540 & 18.9 & 1.60 & 1,485 & 4.19 & 24 & 26.3 & 7.7 & 322 \\
\hline $10 \mathrm{~B}$ & 606 & 18.7 & 1.49 & 1,685 & 3.92 & 24 & 26.6 & 7.7 & 321 \\
\hline $11 \mathrm{~A}$ & 661 & 18.5 & 1.62 & 1,721 & 3.66 & 22 & 26.8 & 7.7 & 326 \\
\hline $12 \mathrm{~B}$ & 721 & 17.3 & 1.47 & 1,523 & 3.05 & 20 & 26.8 & 7.7 & 326 \\
\hline $13 \mathrm{~A}$ & 781 & 15.4 & 1.29 & 763 & 2.10 & 15 & 26.9 & 7.7 & 323 \\
\hline X04 & 798 & 14.7 & 1.12 & 486 & -- & -- & -- & -- & - \\
\hline $14 B$ & 840 & 12.9 & 1.21 & 809 & 1.65 & 12 & 26.9 & 7.7 & 325 \\
\hline $15 \mathrm{~A}$ & 902 & 11.1 & 1.15 & 709 & 1.21 & 9 & 27.0 & 7.7 & 322 \\
\hline $16 \mathrm{~B}$ & 951 & 10.1 & 1.16 & 635 & 1.34 & 8 & 26.8 & 7.7 & 323 \\
\hline $17 \mathrm{~A}$ & 1,010 & 8.9 & 1.09 & 447 & 1.17 & 7 & 26.8 & 7.7 & 320 \\
\hline $\mathrm{X} 05$ & 1,043 & 8.6 & 1.02 & 269 & - & - & -- & - & -- \\
\hline $18 \mathrm{~B}$ & 1,071 & 8.5 & 1.09 & 414 & 1.10 & 6 & 26.9 & 7.7 & 324 \\
\hline $19 \mathrm{~A}$ & 1,132 & 8.9 & 1.00 & 536 & 1.08 & 6 & 26.9 & 7.7 & 322 \\
\hline $20 \mathrm{~B}$ & 1,191 & 9.2 & 0.92 & 520 & 0.95 & 6 & 27.1 & 7.7 & 322 \\
\hline REW & 1,255 & 0.0 & 0.00 & 0 & -- & - & -- & -- & -- \\
\hline MEAN & & 14.9 & 1.46 & & & & & & \\
\hline TOTAL & 1,255 & & & 27,293 & 57.31 & 349 & & & \\
\hline
\end{tabular}




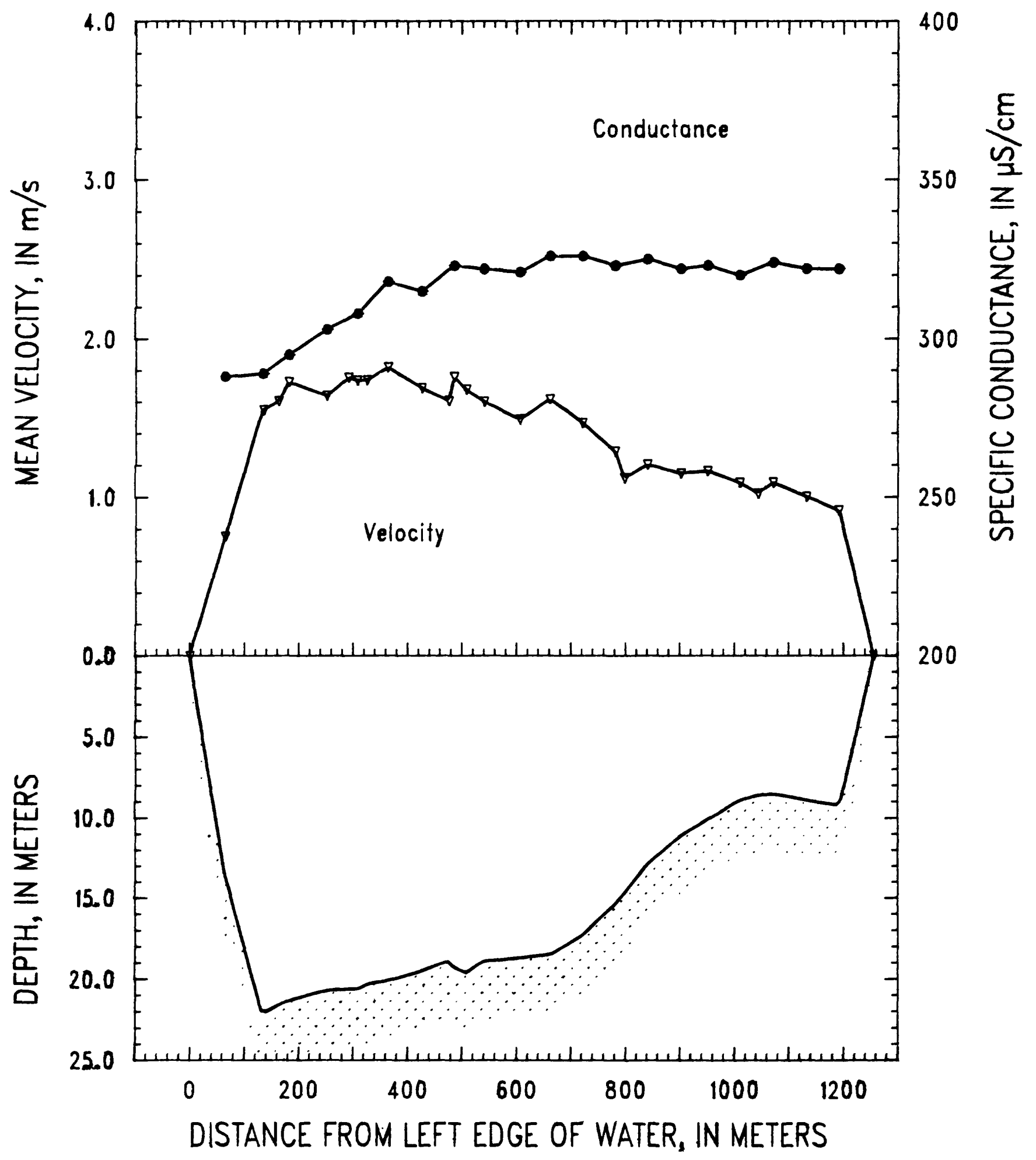

Figure 83. Mississippi River below Vicksburg, Mississippi, on June 23, 1990. 
SITE: Mississippi River near St. Francisville, Louisiana

06-25-90

PARTY: Moody, Garbarino, and Simoneaux

METER: SOLID CUP

STARTING GAGE HEIGHT: -- ENDING GAGE HEIGHT: --

SUSP: Bag sampler and $300-1 \mathrm{~b}$ weight

CURRENT METER NO.: 90JM1 DATE RATED: 04-90

CURRENT METER EQUATION: $\mathrm{V}(\mathrm{m} / \mathrm{s})=\operatorname{RPS} * 0.755+0.011$

REMARKS: Transit rate was $15 \mathrm{~cm} / \mathrm{s}$ and nozzle was $1 / 8$ inch. USGS Louisiana

District collected a simultaneous NASQAN sample. Order of verticals was $20-1$.

\begin{tabular}{|c|c|c|c|c|c|c|c|c|c|}
\hline \multirow[b]{2}{*}{$\begin{array}{c}\text { Verti- } \\
\text { cal }\end{array}$} & \multirow{2}{*}{$\begin{array}{l}\text { Dist. } \\
\text { from } \\
\text { LEW } \\
\text { (m) }\end{array}$} & \multirow[b]{2}{*}{$\begin{array}{l}\text { Depth } \\
\text { (m) }\end{array}$} & \multirow{2}{*}{$\begin{array}{l}\text { Mean } \\
\text { veloc- } \\
\text { ity } \\
(\mathrm{m} / \mathrm{s})\end{array}$} & \multirow[b]{2}{*}{$\begin{array}{r}\text { Dis- } \\
\text { charge } \\
\left(\mathrm{m}^{3} / \mathrm{s}\right)\end{array}$} & \multicolumn{2}{|c|}{ Volume } & \multirow[b]{2}{*}{$\begin{array}{l}\text { Temper- } \\
\text { ature } \\
\left({ }^{\circ} \mathrm{C}\right)\end{array}$} & \multirow[b]{2}{*}{$\mathrm{pH}$} & \multirow{2}{*}{$\begin{array}{l}\text { Specific } \\
\text { conduct- } \\
\text { ance } \\
(\mu \mathrm{S} / \mathrm{cm})\end{array}$} \\
\hline & & & & & $\begin{array}{l}\overline{V_{i}} \\
(\mathrm{~L})\end{array}$ & $\begin{array}{l}\mathrm{V}_{\mathrm{p}} \\
(\mathrm{L})\end{array}$ & & & \\
\hline LEF & 0 & 2.0 & 0.00 & 0 & -- & -- & -- & -- & -- \\
\hline $01 B$ & 60 & 11.0 & 0.09 & 54 & 0.34 & 3 & 27.1 & 7.4 & 312 \\
\hline $02 \mathrm{~A}$ & 105 & 12.1 & 0.45 & 135 & 0.51 & 4 & 27.1 & 7.6 & 313 \\
\hline X05 & 110 & 12.2 & 0.45 & 131 & -- & -- & -- & -- & -- \\
\hline O3B & 153 & 13.6 & 0.66 & 417 & 0.97 & 5 & 27.2 & 7.6 & 313 \\
\hline $04 \mathrm{~A}$ & 203 & 14.8 & 1.02 & 743 & 1.66 & 6 & 27.1 & 7.6 & 309 \\
\hline 05B & 251 & 14.9 & 1.22 & 993 & 2.28 & 10 & 27.5 & 7.6 & 311 \\
\hline $06 \mathrm{~A}$ & 312 & 15.5 & 1.53 & 865 & 2.81 & 15 & 26.4 & 7.6 & 311 \\
\hline X04 & 324 & 15.9 & 1.57 & 550 & - & -- & -- & -- & - \\
\hline $07 \mathrm{~B}$ & 356 & 17.0 & 1.58 & 900 & 3.57 & 20 & 27.4 & 7.6 & 311 \\
\hline $08 \mathrm{~A}$ & 391 & 14.0 & 1.85 & 1,221 & 3.32 & 21 & 27.2 & 7.6 & 312 \\
\hline 09B & 450 & 17.0 & 1.83 & 1,559 & 4.84 & 24 & 27.2 & 7.6 & 315 \\
\hline $10 \mathrm{~A}$ & 491 & 17.8 & 1.70 & 861 & 3.79 & 23 & 27.2 & 7.7 & 311 \\
\hline X03 & 507 & 17.7 & 1.69 & 792 & -- & -- & -- & -- & -- \\
\hline $11 \mathrm{~B}$ & 544 & 17.9 & 1.69 & 1,122 & 4.20 & 23 & 27.0 & 7.6 & 310 \\
\hline $12 \mathrm{~A}$ & 581 & 18.2 & 1.58 & 1,499 & 3.44 & 25 & 27.0 & 7.6 & 308 \\
\hline $13 B$ & 648 & 19.6 & 1.52 & 1,561 & 4.01 & 22 & 26.8 & 7.6 & 311 \\
\hline $14 \mathrm{~A}$ & 686 & 19.4 & 1.56 & 789 & 3.77 & 22 & 26.5 & 7.6 & 311 \\
\hline $\mathrm{X} 02$ & 700 & 20.2 & 1.50 & 680 & - & -- & - & - & - \\
\hline $15 B$ & 731 & 20.9 & 1.62 & 1,611 & 4.09 & 24 & 26.6 & 7.6 & 311 \\
\hline $16 \mathrm{~A}$ & 795 & 20.8 & 1.51 & 1,555 & 3.63 & 24 & 26.8 & 7.6 & 312 \\
\hline $17 \mathrm{~B}$ & 830 & 20.6 & 1.67 & 1,566 & 4.39 & 26 & 26.6 & 7.6 & 311 \\
\hline $18 \mathrm{~A}$ & 886 & 20.4 & 1.56 & 1,161 & 3.85 & 24 & 26.4 & 7.6 & 314 \\
\hline $\mathrm{X01}$ & 903 & 19.6 & 1.59 & 717 & -- & -- & -- & - & - \\
\hline $19 \mathrm{~B}$ & 932 & 19.6 & 1.58 & 1,131 & 4.13 & 22 & 26.8 & 7.6 & 313 \\
\hline $20 \mathrm{~A}$ & 976 & 12.1 & 1.04 & 586 & 1.48 & 8 & 27.1 & 7.6 & 312 \\
\hline REW & 1,025 & 0.0 & 0.00 & 0 & -- & -- & -- & -- & -- \\
\hline MEAN & & 15.9 & 1.42 & & & & & & \\
\hline TOTAL & 1,025 & & & 23,199 & 61.08 & 351 & & & \\
\hline
\end{tabular}




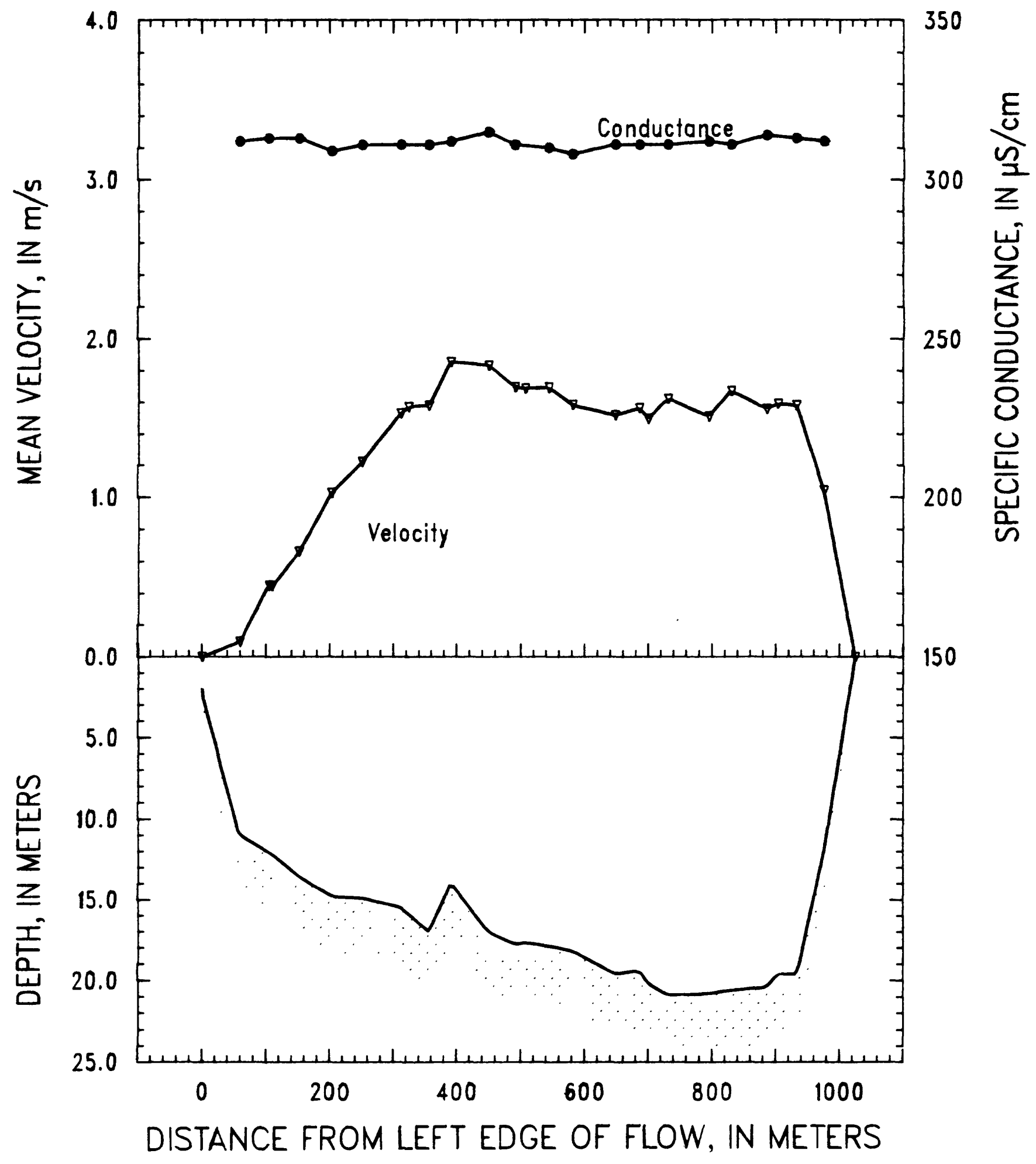

Figure 84. Mississippi River near St. Francisville, Louisiana, on June 25, 1990. 
SITE: Mississippi River below Belle Chasse, Louisiana 06-27-90

PARTY: Moody, Garbarino, and Simoneaux METER: SOLID CUP STARTING GAGE HEIGHT: $13.3 \mathrm{ft}$ SUSP: Bag sampler and 300-1b weight CURRENT METER NO.: 90JM1 DATE RATED: 04-90

CURRENT METER EQUATION: $V(\mathrm{~m} / \mathrm{s})=$ RPS $* 0.755+0.011$

REMARKS: Transit rate was $20 \mathrm{~cm} / \mathrm{s}$ and a $1 / 8$-inch nozzle was used. Section was moved about 0.4 mile upriver from usual location at river mile 73.1 because a ship anchored on section. USGS Louisiana District collected a simultaneous NASQAN sample about 1 mile upriver.

\begin{tabular}{|c|c|c|c|c|c|c|c|c|c|}
\hline \multirow[b]{2}{*}{$\begin{array}{l}\text { Verti- } \\
\text { cal }\end{array}$} & \multirow{2}{*}{$\begin{array}{l}\text { Dist. } \\
\text { from } \\
\text { LEW } \\
(\mathrm{m})\end{array}$} & \multirow[b]{2}{*}{$\begin{array}{l}\text { Depth } \\
\text { (m) }\end{array}$} & \multirow{2}{*}{$\begin{array}{l}\text { Mean } \\
\text { veloc- } \\
\text { ity } \\
(\mathrm{m} / \mathrm{s})\end{array}$} & \multirow[b]{2}{*}{$\begin{array}{c}\text { Dis- } \\
\text { charge } \\
\left(\mathrm{m}^{3} / \mathrm{s}\right)\end{array}$} & \multicolumn{2}{|c|}{ Volume } & \multirow[b]{2}{*}{$\begin{array}{l}\text { Temper- } \\
\text { ature } \\
\left({ }^{\circ} \mathrm{C}\right)\end{array}$} & \multirow[b]{2}{*}{$\mathrm{pH}$} & \multirow{2}{*}{$\begin{array}{c}\text { Specific } \\
\text { conduct- } \\
\text { ance } \\
(\mu \mathrm{S} / \mathrm{cm})\end{array}$} \\
\hline & & & & & $\begin{array}{c}\mathrm{V}_{\mathrm{i}} \\
(\mathrm{I})\end{array}$ & $\begin{array}{c}V_{p} \\
(L)\end{array}$ & & & \\
\hline LEW & 0 & 0.0 & 0.00 & 0 & -- & -- & -- & -- & -- \\
\hline $01 \mathrm{~A}$ & 47 & 10.0 & 0.78 & 361 & 0.69 & 2 & 27.3 & 7.6 & 325 \\
\hline O2B & 92 & 20.5 & 1.33 & 967 & 2.99 & 16 & 27.3 & 7.6 & 324 \\
\hline X05 & 118 & 34.0 & 1.34 & 842 & -- & -- & -- & -- & -- \\
\hline $03 \mathrm{~A}$ & 129 & 35.0 & 1.32 & 371 & 3.83 & 37 & 27.4 & 7.6 & 325 \\
\hline $\mathrm{x} 06$ & 134 & 32.4 & 1.48 & 866 & -- & -- & -- & -- & -- \\
\hline 04B & 165 & 31.2 & 1.62 & 1,770 & 4.66 & 33 & 27.5 & 7.6 & 323 \\
\hline $05 A$ & 204 & 30.6 & 1.65 & 2,070 & 3.62 & 32 & 27.7 & 7.6 & 323 \\
\hline O6B & 247 & 29.8 & 1.65 & 1,670 & 4.71 & 29 & 27.4 & 7.6 & 322 \\
\hline X04 & 272 & 28.7 & 1.67 & 935 & -- & -- & -- & -- & -- \\
\hline $07 \mathrm{~A}$ & 286 & 28.4 & 1.64 & 1,394 & 3.96 & 26 & 27.3 & 7.6 & 323 \\
\hline 08B & 332 & 25.9 & 1.64 & 1,342 & 3.98 & 25 & 27.5 & 7.6 & 322 \\
\hline $09 A$ & 349 & 25.4 & 1.59 & 769 & 3.94 & 21 & 27.4 & 7.6 & 324 \\
\hline X07 & 370 & 24.8 & 1.55 & 867 & -- & -- & -- & -- & -- \\
\hline $\mathrm{X} 03$ & 394 & 23.1 & 1.56 & 703 & -- & -- & -- & -- & -- \\
\hline $10 \mathrm{~B}$ & 409 & 22.7 & 1.46 & 764 & 3.04 & 19 & 27.7 & 7.6 & 324 \\
\hline $11 \mathrm{~A}$ & 440 & 21.3 & 1.50 & 1,180 & 2.84 & 16 & 27.5 & 7.6 & 323 \\
\hline $12 \mathrm{~B}$ & 483 & 19.6 & 1.40 & 1,238 & 2.22 & 14 & 27.5 & 7.6 & 323 \\
\hline $13 \mathrm{~A}$ & 530 & 18.3 & 1.24 & 590 & 1.92 & 14 & 26.7 & 7.6 & 323 \\
\hline $\mathrm{X} 02$ & 535 & 17.8 & 1.24 & 188 & -- & -- & -- & -- & -- \\
\hline $14 \mathrm{~B}$ & 547 & 17.6 & 1.25 & 717 & 2.07 & 12 & 27.8 & 7.6 & 324 \\
\hline $15 \mathrm{~A}$ & 600 & 15.6 & 1.16 & 789 & 1.41 & 10 & 27.7 & 7.6 & 325 \\
\hline $16 \mathrm{~B}$ & 634 & 15.3 & 1.00 & 568 & 1.20 & 10 & 27.5 & 7.6 & 323 \\
\hline $17 \mathrm{~A}$ & 674 & 14.7 & 1.08 & 452 & 1.18 & 9 & 27.5 & 7.6 & 322 \\
\hline X01 & 691 & 14.8 & 1.08 & 320 & - & -- & -- & -- & -- \\
\hline $18 \mathrm{~B}$ & 714 & 14.7 & 1.05 & 517 & 1.21 & 10 & 27.6 & 7.6 & 324 \\
\hline $19 \mathrm{~A}$ & 758 & 15.3 & 0.88 & 567 & 1.05 & 10 & 27.5 & 7.6 & 326 \\
\hline $20 \mathrm{~B}$ & 798 & 15.0 & 0.74 & 484 & 0.96 & 5 & 27.3 & 7.6 & 324 \\
\hline $\mathrm{REF}$ & 845 & 1.0 & 0.00 & 0 & -- & -- & - & -- & -- \\
\hline MEAN & & 20.0 & 1.38 & & & & & & \\
\hline TOTAL & 845 & & & 23,301 & 51.48 & 350 & & & \\
\hline
\end{tabular}




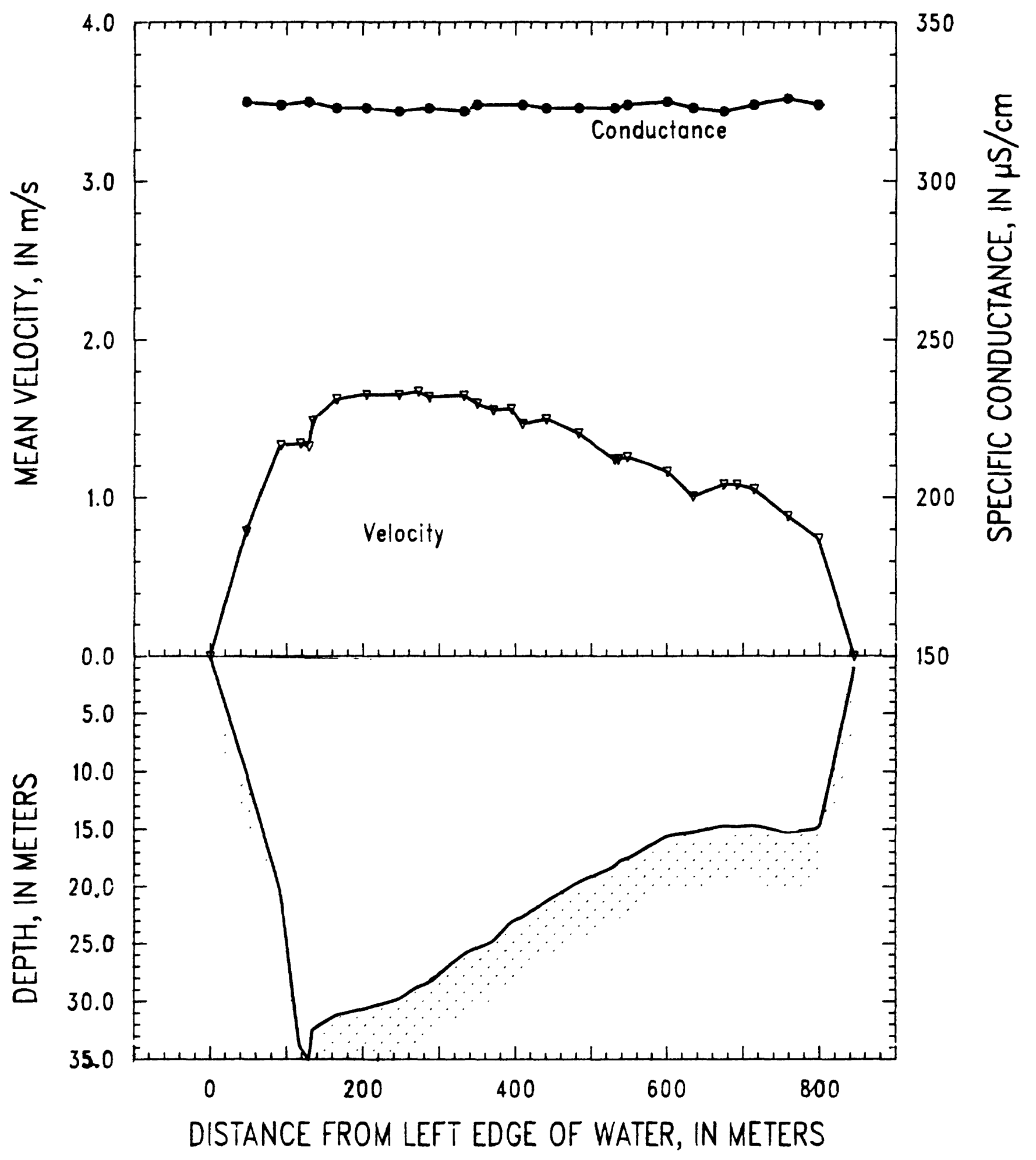

Figure 85. Mississippi River below Belle Chasse, Louisiana, on June 27, 1990. 